\title{
PATTERNS OF GENETIC CONNECTIVITY IN DEEP-SEA VULNERABLE MARINE ECOSYSTEMS AND IMPLICATIONS FOR CONSERVATION
}

BY

\section{CONG ZENG}

A thesis submitted to the Victoria University of Wellington in fulfilment of the requirements for the degree of Doctor of Philosophy in Marine Biology.

Victoria University of Wellington 



\section{Abstract}

Knowledge about and understanding of population structure and connectivity of deepsea fauna decreases with increasing depth, but such information is crucial for the management of vulnerable marine ecosystems (VMEs) in particular. As such, research using genetic markers, which does not require knowledge of ecological or environmental processes as a prerequisite for the analysis, is a practical method to investigate population connectivity of VME indicator taxa. However, population genetics studies are yet to be broadly conducted in the deep sea around New Zealand.

To provide background information and develop hypothesises for this research, 196 population genetic studies of deep-sea fauna were reviewed and analysed. Based on the collected studies, four different patterns of spatial genetic structure were observed: global homogeneous, oceanic, regional, and fine structure. These different structures were reported that they were related to depth, topography, distance between populations, temperature and other biological factors. Quantification of the relationship between these factors and the detection of barriers to gene flow (barrier detection) showed that depth, currents and topography contributed significantly to barrier detection and depth and topography were acting as a barrier to gene flow in the deep sea. Furthermore, different sampling strategies and different genetic marker types significantly influenced genetic barrier detection. Comparison amongst different habitats suggested that different conservation strategies should be developed for different habitat types (Chapter 2).

This study used different genetic markers to assess the genetic connectivity amongst VME indicator taxa Vulnerable Marine Ecosystems (VME). Seven VME indicator taxa were selected: 4 sponges (Neoaulaxinia persicum, Penares sp., Pleroma menoui and Poecillastra laminaris) and 3 corals (Goniocorella dumosa, Madrepora oculata and Solenosmilia variabilis), at different spatial scales. Due to lack of genetic information for these species, genetic markers were developed for Poecillastra laminaris (0) and S. variabilis (Chapter 4).

A geographic province (northern-southern province), region (north-central-south), and geomorphic feature hierarchical testing framework was employed to examine species-specific 
genetic variation in mitochondrial (COI, Cytb and $12 S)$ and nuclear markers (microsatellites) amongst populations of four deep-sea sponges within the New Zealand region. For Poecillastra laminaris, significant mitochondrial and nuclear DNA genetic differences were revealed amongst biogeographic provinces. In contrast, no significant structure was detected across the same area for Penares sp. Both Neoaulaxinia persicum and Pleroma menoui were only available from the northern province, in which Pleroma menoui showed no evidence of genetic structure, but $N$. persicum exhibited a geographic differentiation in $12 S$. No depthrelated isolation was observed for any of the four species at the mitochondrial markers, nor at the microsatellite loci for Poecillastra laminaris. Genetic connectivity in Poecillastra laminaris is likely to be influenced by oceanic sub-surface currents that generate routes for gene flow and may also act as barriers to dispersal. Although data are limited, these results suggest that the differences in patterns of genetic structure amongst the species can be attributed to differences in life history and reproductive strategies. The results are discussed in the context of existing marine protected areas, and the future design of spatial management measures for protecting VMEs in the New Zealand region (Chapter 5).

To better understand the vulnerability of stony corals (Goniocorella dumosa, Madrepora oculata and Solenosmilia variabilis) to disturbance within the New Zealand region, and to guide marine protected area design, genetic structure and connectivity were determined using microsatellite loci and DNA sequencing. Analyses compared population genetic differentiation between two biogeographic provinces, amongst three sub-regions (north-central-south), and amongst geomorphic features. Population genetic differentiation varied amongst species and between marker types. For G. dumosa, genetic differentiation existed amongst regions and populations on geomorphic features, but not between provinces. For M. oculata, only a north-central-south regional structure was observed. For S. variabilis, genetic differentiation was observed between provinces, amongst regions and amongst geomorphic features based on microsatellite variation. Multivariate analyses indicated that populations on the Kermadec Ridge were genetically different from Chatham Rise populations in all three coral species. Furthermore, a significant isolation-by-depth pattern was observed for both marker types in G. dumosa, and also in ITS of M. oculata. An isolation-by-distance pattern was found in microsatellites of $S$. variabilis. Migrate analysis showed that medium to high self-recruitment were detected in all geomorphic feature populations, and different species presented different genetic connectivity patterns. These different patterns of population genetic structure and connectivity at a range of spatial scales 
indicate that flexible spatial management is required for the conservation of deep-sea corals around New Zealand (Chapter 6).

Understanding the deep-sea ecological processes that shape spatial genetic patterns of species is critical for predicting evolutionary dynamics and defining significant evolutionary and/or management units. In this study, the potential role of environmental factors in shaping the genetic structure of the 7 deep-sea habit-providing study species was investigated using a seascape genetics approach. The genetic data were acquired from nuclear and mitochondrial sequences and microsatellite genotype data, and 25 environmental variables ( 5 topographic, 17 physiochemical and 3 biological variables). The results indicated that environmental factors affected genetic variation differently amongst the species. However, factors related to current and food source explained the north-central-south genetic structure in sponges and corals, and environmental variation in these parameters may be acting as a barrier to gene flow. At the geomorphic feature level, the DistLM and dbRDA analysis showed that factors related to the food source and topography were most related to genetic variation in microsatellites of sponge and corals. This study highlights the utility of seascape genetic studies to better understand the processes shaping the genetic structure of organisms (Chapter 7).

The outcomes of this study provide vital information to assist in effective management and conservation of VME indicator taxa and contribute to an understanding of evolutionary and ecological processes in the deep sea (Chapter 8). 


\section{Acknowledgments}

Four years is not a long time on the journey of life, but these four years in New Zealand will be one of the most-cherished periods in my lifetime. Since I started my New Zealand life, I have received many munificent assistances in both academic and living aspects, and here I express my appreciation to these people in this thesis.

Firstly, I am extremely indebted to my primary supervisor Professor Jonathan Gardner for bringing me here. This is an important milestone in this one-way journey, because this opportunity not only shifts my research interests into marine biology but also offers me a fabulous experience in such a beautiful and peaceful country. Besides, his great effects and contributions to my $\mathrm{PhD}$ project contained herein are indelible. I also greatly appreciate my secondary supervisors, Dr Ashley Rowden and Dr Malcolm Clark, for guiding me in the researches, providing logistical support for my research and their helpful feedback on the content of this thesis and other publications, and an opportunity to participate in research cruise TAN1402. I am also grateful to members of my committee Dr Peter Ritchie and Professor Phil Lester for the valuable advice and input in the early stages of my studies. Also, I am grateful that Associate Professor Joe Zuccarello who provided practical advice for my experiments.

This research would not have been possible without the enormous generosity of everyone who helped with sample collection. I would like to thank Ms Sadie Mills (NIWA) for supporting and caring for me in the Invertebrate Collection (NIC). Many appreciations are given to Ms Dianne Tracey for coral identifications and providing chances for academic training and an international conference. I am also thankful to Dr Michelle Kelly who is responsible for the sponge taxonomical work for my thesis. Additional thanks are for kindly assistances from other staff in NIWA.

Great thanks to Dr Karen Miller (UTAS) and Dr Sophie Arnaud Haond (Ifremer) for sharing the data and sending the specimens, and Dr Mike Williams (NIWA) for generating the environmental data as well. Thank you to Dr Everett Meredith (NOAA) and Dr Cheryl Morrison (USGS) for connecting me with other coral experts.

I would like to thank my lab mates for sharing their knowledge with me and for their friendship, especially Dr Leighton Thomas and Dr Catarina Silva for all the invaluable advice and support in the beginning of this research. I would also like to thank the staff from the 
School of Biological Sciences and the Faculty of Sciences, specially Mary Murray, Patricia Stein, Paul Marsden and Mark Stephen for all the logistical support and infinite patience.

The Ministry of Business, Innovation and Employment provided funding for this research (Vulnerable Marine Ecosystems MBIE Programme C01X1229), which gave me the privilege to conduct this research. I also received the Victoria $\mathrm{PhD}$ Submission Scholarship and VUW Faculty of Science Strategic Research Grant that allowed me to pursue my studies and to attend international conferences.

During the years in Wellington, I am very grateful to have met my officemates in KK416 and KK127, and I am very happy that I have shared a lot of good moments with them. In addition, my flatmates and friends are very important part of my Wellington life, and many thanks for you to colorize my aloneness. I would also like to thank many valuable friends overseas for their support and encourages. Additionally, a special acknowledgement is given to Qiang Chen, for your companioning during these years.

Last but not least, I would like to acknowledge my parents for respecting my decisions and fuelling my passion for completing this degree. I also take this chance to thank my previous supervisor in China, Professor Weimin Wang, for encouraging and supporting me to take this new adventure. 


\section{Table of Contents}

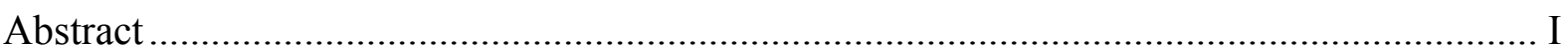

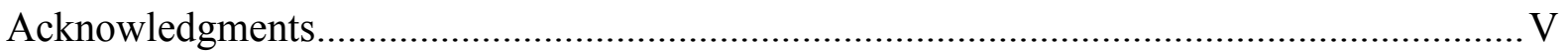

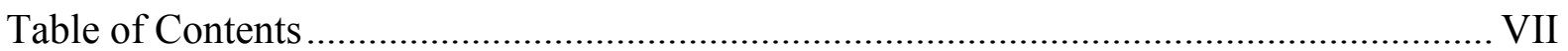

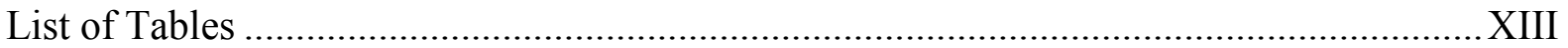

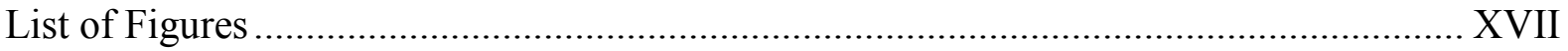

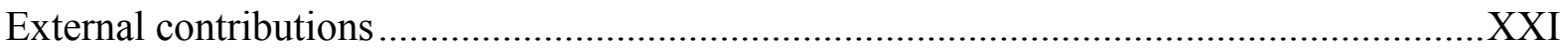

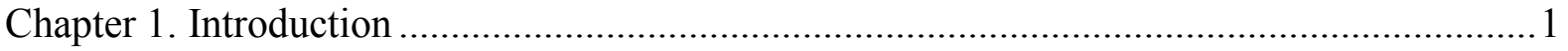

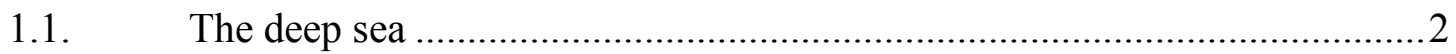

1.2. Vulnerable Marine Ecosystems (VMEs) and VME indicator taxa ..............

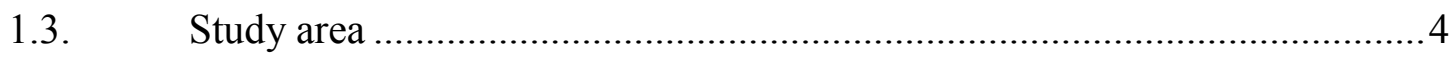

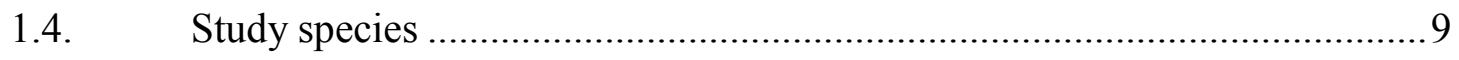

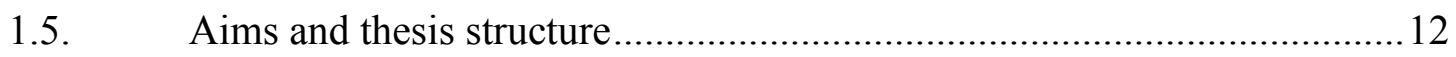

Chapter 2. Patterns of genetic connectivity in the deep sea: challenges for, and applications to, the conservation of Vulnerable Marine Ecosystems............................................................ 15

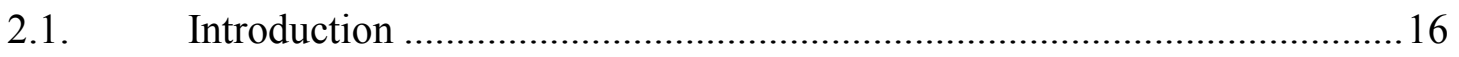

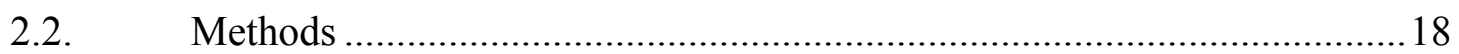

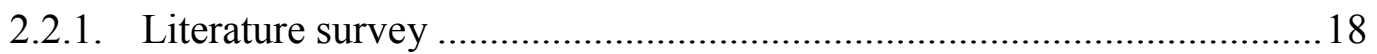

2.2.2. Database compilation and variable selection ...................................... 19

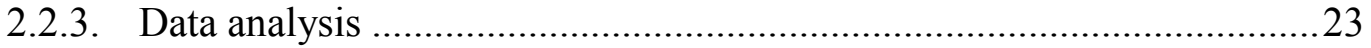

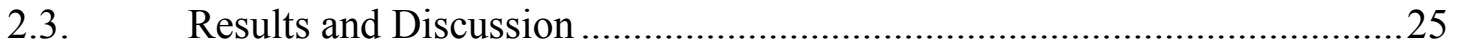

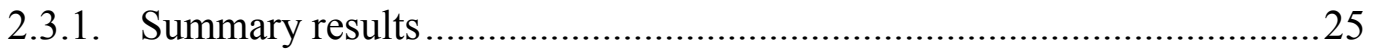

2.3.2. Patterns of genetic structure reported amongst deep-sea taxa................29

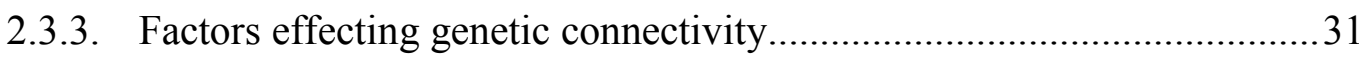

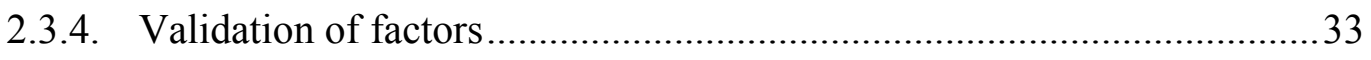

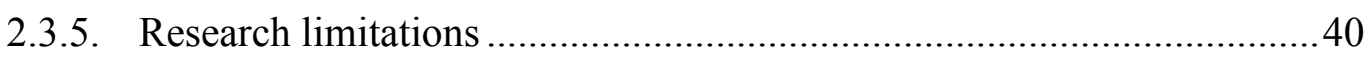


2.3.6. Barrier occurrences amongst habitat types........................................... 46

2.3.7. Barrier occurrence difference between VMEs and non-VMEs ..............48

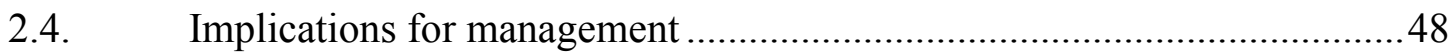

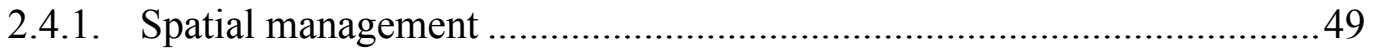

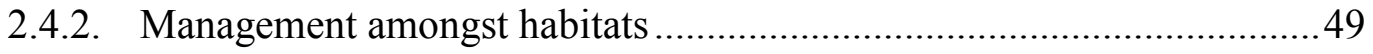

2.4.3. Management of VMEs and VME indicator taxa.................................50

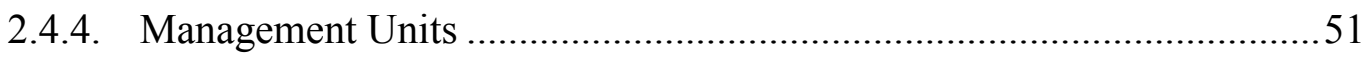

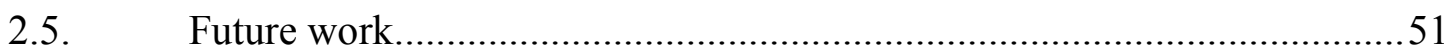

Appendix 53

Chapter 3. Data mining from Poecillastra laminaris (Demosponge) 454 sequencing data ....67

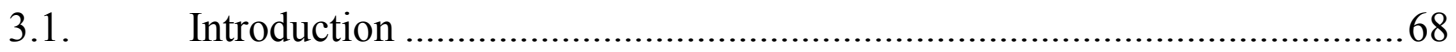

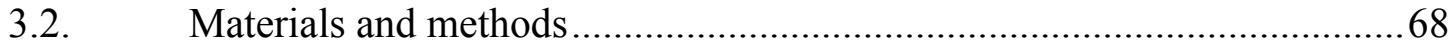

3.2.1. Samples, DNA extractions and pyrosequencing ..................................68

3.2.2. Microsatellite discovery and characterisation .....................................6 69

3.2.3. Mitochondrial genome assembly and marker design ........................... 70

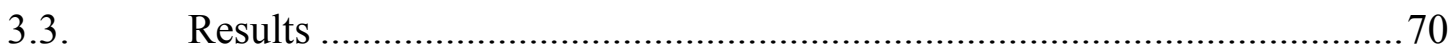

3.3.1. Next Generation Sequencing ........................................................ 70

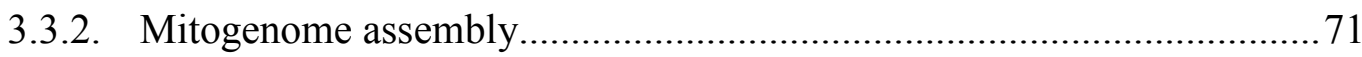

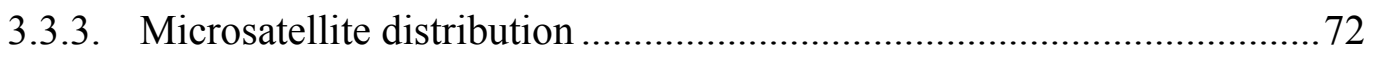

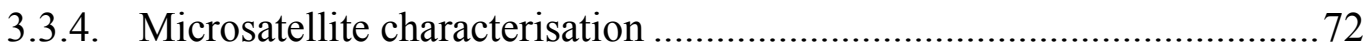

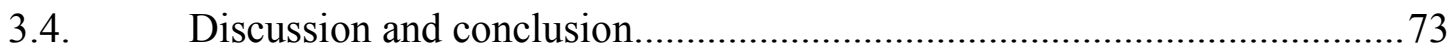

Chapter 4. Data mining from Solenosmilia variabilis (Scleractinia) Next Generation

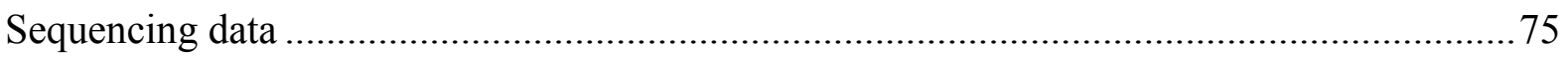

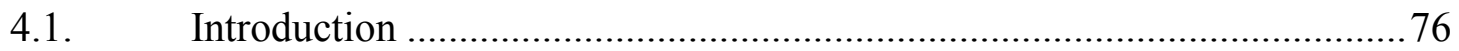

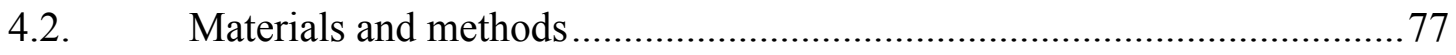

4.2.1. Samples, DNA extraction and pyrosequencing .................................... 77

4.2.2. Mitochondrial genome assembly and marker design ............................ 78 
4.2.3. Microsatellite discovery and characterisation ..................................... 79

4.2.4. Single nucleotide polymorphisms (SNPs) discovery ............................. 80

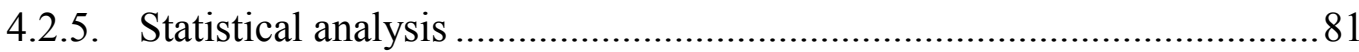

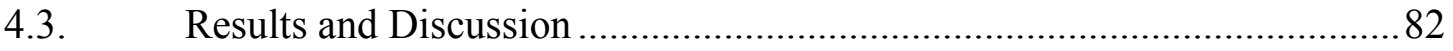

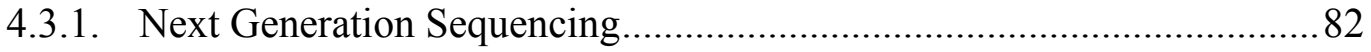

4.3.2. Mitogenome assembly and marker development.................................. 83

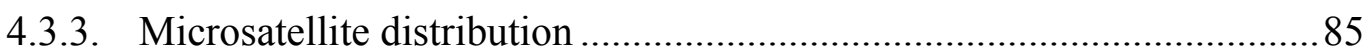

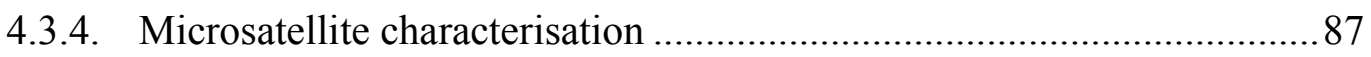

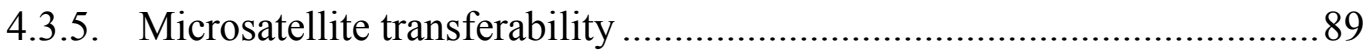

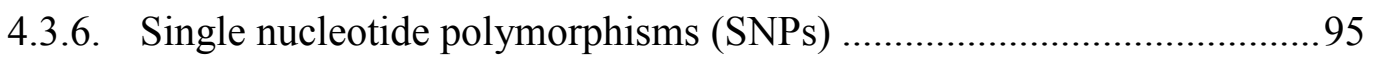

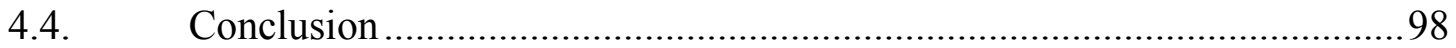

Appendix 99

Chapter 5. Patterns of genetic connectivity amongst four deep-sea demosponges in the New Zealand region: implications for the protection of vulnerable marine ecosystems ............... 105

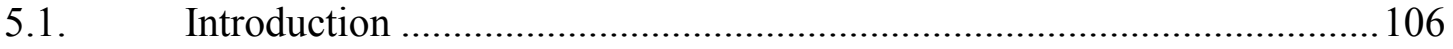

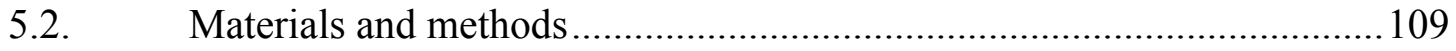

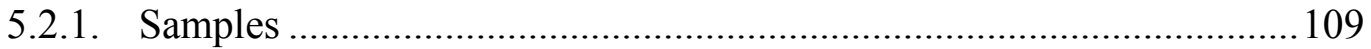

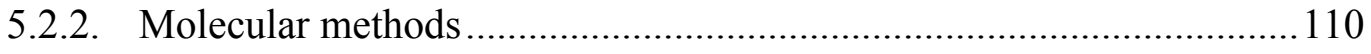

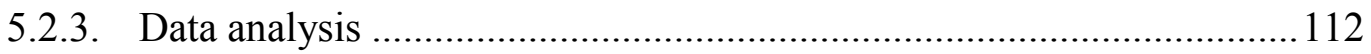

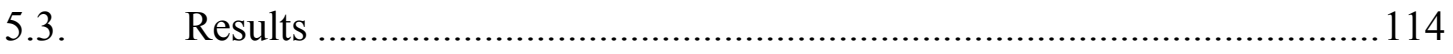

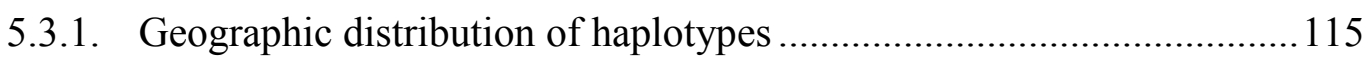

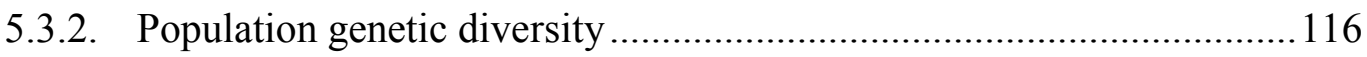

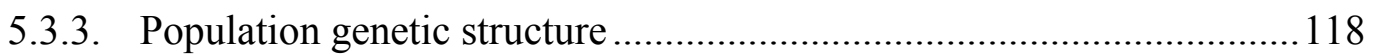

5.3.4. Isolation by distance and depth ........................................................ 120

5.3.5. Tajima's D and R2 tests of neutrality and mismatch distribution......... 121

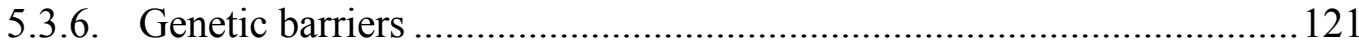

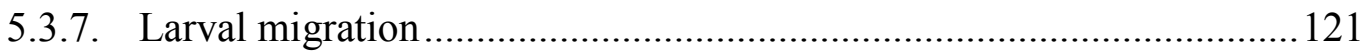


5.3.8. Microsatellite variation

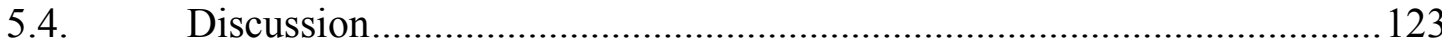

5.4.1. Sampling effect, marker selection and genetic diversity...................... 123

5.4.2. Patterns of genetic connectivity ................................................... 124

5.4.3. Life history and dispersal strategies .............................................. 125

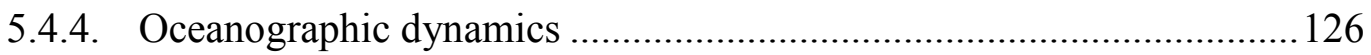

5.4.5. Depth and associated environmental gradients ................................ 127

5.4.6. Conservation and management implications..................................... 128

Appendix 132

Chapter 6. Population structure and genetic connectivity of stony corals (Scleractinia) in the New Zealand region based on analysis of microsatellite and sequence data: implications for

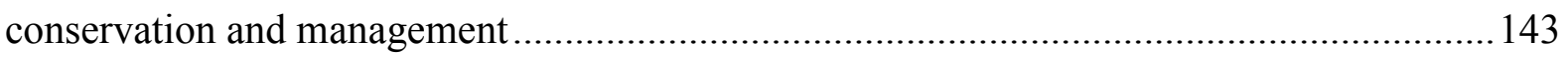

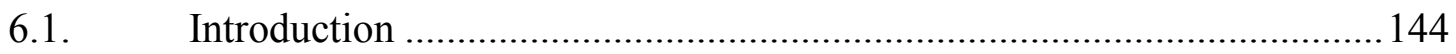

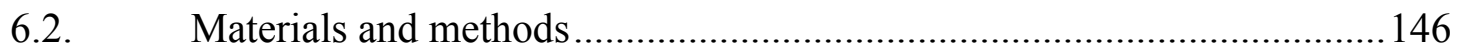

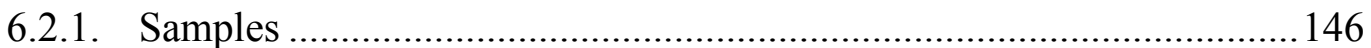

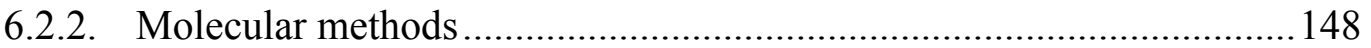

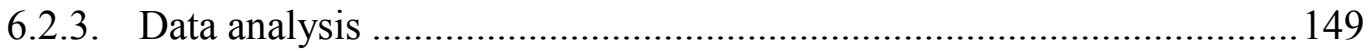

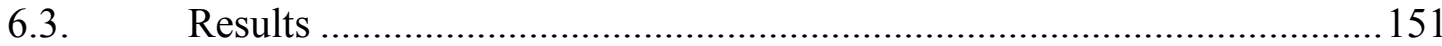

6.3.1. Population structure based on ITS and D-loop variation...................... 151

6.3.2. Population structure based on microsatellites ................................... 157

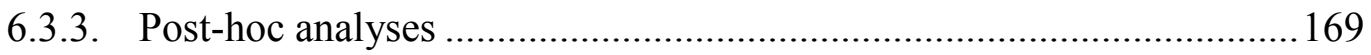

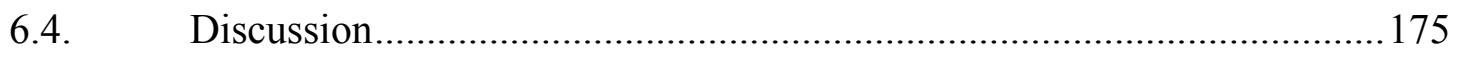

6.4.1. Influence of sample sizes and markers on structures detected............. 176

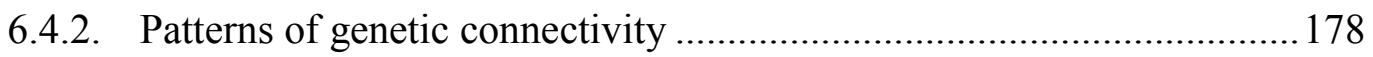

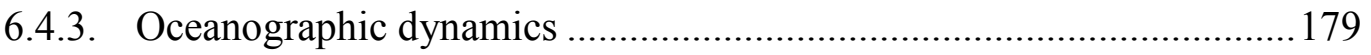

6.4.4. Depth and associated environmental gradients ................................... 180

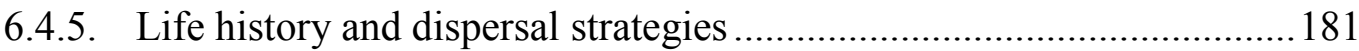

6.4.6. Conservation and management implications...................................... 182 
Appendix 185

Chapter 7. Identifying environmental factors associated with the genetic structure of Vulnerable Marine Ecosystem indicator taxa in the New Zealand region 191

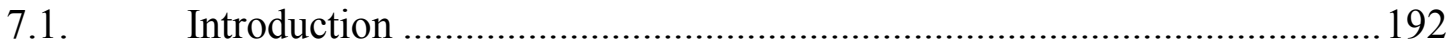

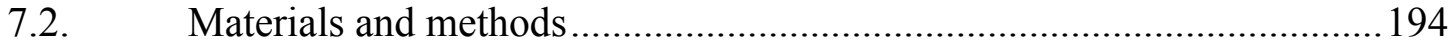

7.2.1. Sample collection and molecular experiments ................................... 194

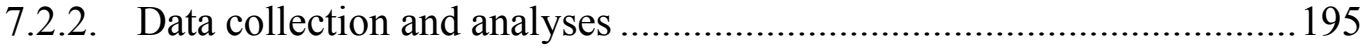

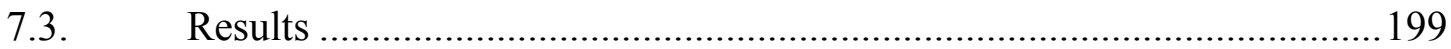

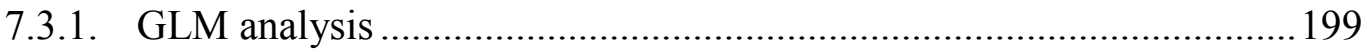

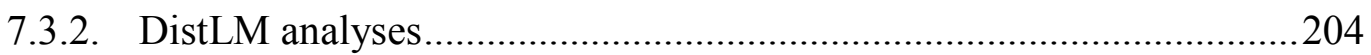

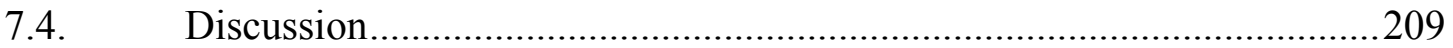

7.4.1. Choices for markers and research scales..........................................2210

7.4.2. Environmental effects and genetic variations ...................................2211

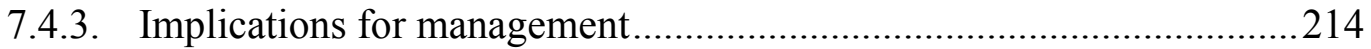

Appendix 216

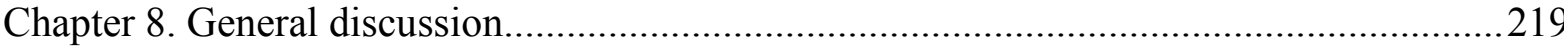

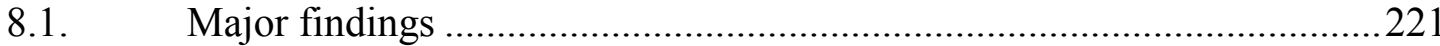

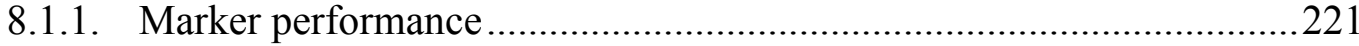

8.1.2. Common patterns of genetic structure in the New Zealand deep sea ...222

8.1.3. Connectivity amongst geomorphic feature populations .......................224

8.1.4. Factors contributing to genetic differentiation ...................................225

8.2. Management implications and future directions ..................................2229

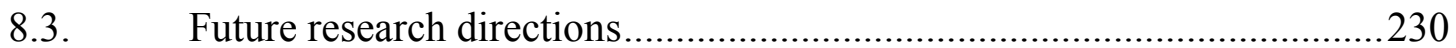

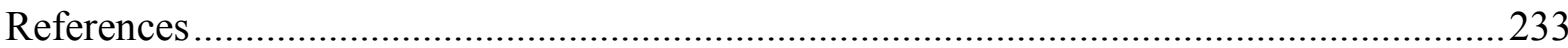




\section{List of Tables}

Table 1.1 VME indicator taxa amongst different Regional Fisheries Management Organisations

Table 2.1 Number of species for which genetic connectivity estimates have been established

Table 2.2 Logistical regression analyses of the contribution of different factors to the detection of barriers to gene flow amongst multiple deep-sea taxa........................................33

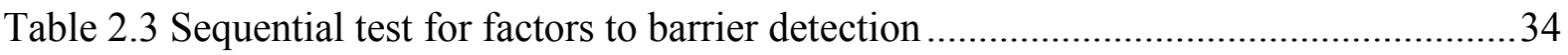

Table 2.4 General linear model analyses of sampling regime on genetic barrier detection .... 41

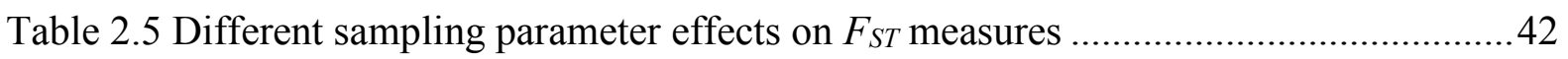

Table 2.6 Different sampling parameter effects on $\Phi_{S T}$ measures........................................ 43

Table 2.7 Average nucleotide diversity of mitochondrial and nuclear genes .........................43

Table 2.8 Detection of a barrier to gene flow as a function of depth ...................................45

Table 2.9 Comparisons of barrier detection between VMEs and non-VMEs and between

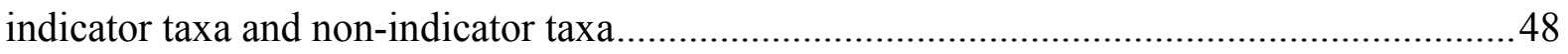

Table 3.1 Characterisation of the mitochondrial genome of Poecillastra laminaris............... 71

Table 3.2 Details of PCR primers and conditions used to amplify three DNA regions .......... 72

Table 3.3 Characterisation of 10 microsatellites for Poecillastra laminaris ........................... 73

Table 4.1 Summary information for sequencing data for Solenosmilia variabilis ................. 83

Table 4.2 Summary information for sample alignment mapping to Acropora digitifera........ 83

Table 4.3 Characterisation of the mitochondrial genome of Solenosmilia variabilis.............. 84

Table 4.4 Details of PCR primers and conditions used to amplify two DNA regions ............85

Table 4.5 Microsatellite distributions (\%) information for three species .............................. 86

Table 4.6 Primer information for Solenosmilia variabilis microsatellites................................ 87

Table 4.7 Characterisation of 27 microsatellite loci for Solenosmilia variabilis .................... 88

Table 4.8 Cross-amplification information of 27 loci in Goniocorella dumosa .......................89

Table 4.9 Cross-amplification information of 27 loci in Desmophyllum dianthus..................91

Table 4.10 Cross-amplification information of 27 loci in Madrepora oculata .......................92

Table 4.11 SNPs distributions amongst different samples .................................................96

Table 5.1 Number of partial sequences plus length of sequence (bp) for three mitochondrial genes. 110 
Table 5.2 Number of partial sequences (n) plus length of sequence (bp) for three mitochondrial genes for each of four sponge species

Table 5.3 Nucleotide diversity statistics for Poecillastra laminaris at three different spatial scales.

Table 5.4 Nucleotide diversity statistics for Penares sp. at three different spatial scales ..... 115 Table 5.5 Nucleotide diversity statistics for Neoaulaxinia persicum and Pleroma menoui at two different spatial scales. 116

Table 5.6 Hierarchical AMOVA results for Poecillastra laminaris when tested for genetic variation at mitochondrial genes

Table 5.7 Hierarchical AMOVA results for Penares sp. when tested for genetic variation at mitochondrial genes.

Table 5.8 Hierarchical AMOVA results for Neoaulaxinia persicum and Pleroma menoui when tested for genetic variation at the COI mitochondrial gene 120

Table 5.9 Mantel tests of isolation by depth and isolation by distance amongst different markers and species

Table 5.10 Theta and number of effective migrants per generation amongst pooled populations from 6 locations based on COI and Cytb sequences for Poecillastra laminaris 122 Table 6.1 ITS and D-loop diversity statistics for Goniocorella dumosa ............................... 152

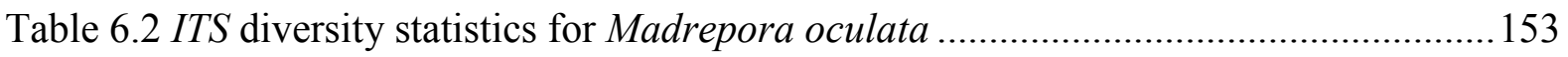
Table 6.3 ITS and D-loop diversity statistics for Solenosmilia variabilis 153 Table 6.4 Pairwise $\Phi_{S T}$ values for ITS of Madrepora oculata (above diagonal) and Solenosmilia variabilis (below diagonal) amongst geomorphic feature populations 156

Table 6.5 Hierarchical AMOVA results for Goniocorella dumosa when tested for genetic variation at the ITS and D-loop regions 156 Table 6.6 Hierarchical AMOVA results for Madrepora oculata when tested for genetic variation at the ITS and Microsatellites 157

Table 6.7 Hierarchical AMOVA results for Solenosmilia variabilis when tested for genetic variation at the ITS and D-loop 158

Table 6.8 Mantel tests of isolation by distance and isolation by depth amongst different markers in Goniocorella dumosa, Madrepora oculata and Solenosmilia variabilis..... 158 Table 6.9 Basic population genetic information of microsatellite data at three spatial scales for Goniocorella dumosa 160 Table 6.10 Basic population genetic information of microsatellite data at three spatial scales for Madrepora oculata. 162 
Table 6.11 Basic population genetic information of microsatellite data at three spatial scales for Solenosmilia variabilis.....

Table 6.12 Hierarchical AMOVA results for Goniocorella dumosa when tested for microsatellite genetic variation

Table 6.13 Hierarchical AMOVA results for Madrepora oculata when tested for microsatellite genetic variation

Table 6.14 Hierarchical AMOVA results for Solenosmilia variabilis when tested for microsatellite genetic variation

Table 6.15 Pairwise $F_{\text {ST }}$ values for all (below diagonal) and neutral (above diagonal) loci amongst Goniocorella dumosa populations.

Table 6.16 Pairwise population genetic differentiation $\left(F_{S T}\right)$ of Madrepora oculata in microsatellites 168

Table 6.17 Pairwise $F_{S T}$ values for all (below diagonal) and neutral (above diagonal) amongst Solenosmilia variabilis populations.

Table 6.18 Information about reproductive life-history characteristics for Goniocorella dumosa, Madrepora oculata and Solenosmilia variabilis .....

Table 7.1 Numbers of specimens used for each genetic marker.

Table 7.2 Detailed information of environmental variables included in this study 196

Table 7.3 General linear model analysis results of genetic variation at the individual level for Poecillastra laminaris. 202

Table 7.4 General linear model analysis results of genetic variation for Goniocorella dumosa .203

Table 7.5 General linear model analysis results of genetic variation for Madrepora oculata 204

Table 7.6 General linear model analysis results of genetic variation for Solenosmilia variabilis 205

Table 7.7 Sequential test results of the DistLM analysis for microsatellite data amongst the North-Central-South regions 205

Table 7.8 Sequential test results of the DistLM analysis for microsatellite data amongst geomorphic features for all species.

Table 8.1 Genetic differentiation patterns amongst different species in the present study ...223 Table 8.2 Summary of significant genetic differentiation amongst different geomorphic feature populations of sponges (above diagonal) and corals (below diagonal)..... .224 


\section{List of Figures}

Figure 1.1 Distribution of major geomorphic features and deep-sea lower bathyal provinces

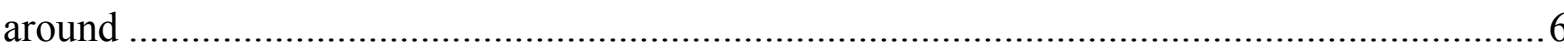

Figure 1.2 Summary of the major currents in the New Zealand region ................................ 7

Figure 1.3 Offshore protected areas within the New Zealand EEZ .....................................

Figure 1.4 Specimens of Poecillastra laminaris (A), Neoaulaxinia persicum (B), Penares sp.

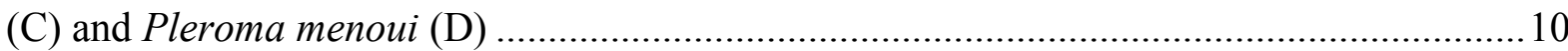

Figure 1.5 Morphology and branching of Goniocorella dumosa, Madrepora oculata and .... 11 Figure 2.1 Proposed lower bathyal (upper) and abyssal (below) provinces from Watling et al (2013)

Figure 2.2 The distribution of research covered by areas in deep-sea provinces (bar charts) and at an oceanic scale (pie chart) .26

Figure 2.3 Covered depths for each record (lines from minimum to maximum) (top) and accumulated density of examined studies (bottom) 27

Figure 2.4 Genetic barrier detection distributions across different phyla. 28

Figure 2.5 Summary of different marker types and the chronological distribution of their

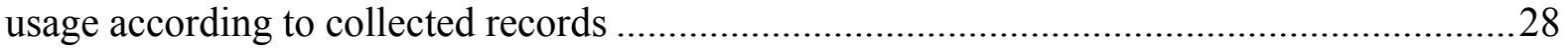

Figure 2.6 Testing structures at different spatial scales amongst collected studies ................29

Figure 2.7 Summary of contributions of individual effects amongst categorised factors for

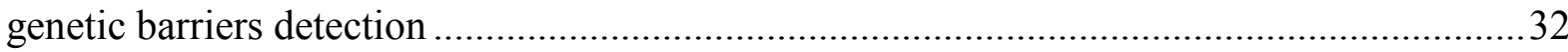

Figure 2.8 Average sample size with depth distribution of reviewed studies 44 Figure 2.9 Comparisons of the presence of barriers to gene flow as a function of habitat type

Figure 4.1 Dendrogram of sample similarity based on microsatellite variation amongst three corals . .86

Figure 4.2 Structure analyses of all loci (A) and 12 loci that amplified for all four species (B)

Figure 4.3 DAPC scatterplot (above) and complot (below) of all loci (A, 27 loci) and part (B, 12 loci) amongst four species

Figure 4.4 Distribution features of cross-amplified microsatellites of motifs (A), transferability level (B), G/C content (C) .................................................................. 95

Figure 4.5 Venn diagram of SNPs in four Solenosmilia variabilis samples ..........................97 
Figure 4.6 Dendrogram of 5 samples revealed by SNPs that were detected in all datasets ....97 Figure 4.7 Structures results of sample coral samples based on SNPs $(K=2)$.......................98

Figure 5.1 Map showing the locations of samples for the study species. 111

Figure 5.2 Haplotype map for Poecillastra laminaris COI (above line) and Cytb (below line) (A), Penares sp. COI (above line) and $12 S$ (below line) (B), Neoaulaxinia persicum COI (above line) and $12 S$ (below line) (C), Pleroma menoui COI (D)....

Figure 5.3 Haplotype map for Poecillastra laminaris COI (above line) and Cytb (below line) overlain with a summary of the major currents in the New Zealand region 128 Figure 5.4 Haplotype map for Poecillastra laminaris COI (above line) and Cytb (below line) overlain with protected areas within the New Zealand EEZ

Figure 6.1 Map showing the distribution of samples amongst biogeographic provinces, regions, andgeomorphic features used for the analysis of population structure for three species of deep-sea stony corals 147

Figure 6.2 Haplotype map for Goniocorella dumosa (left) and Madrepora oculata (right) ITS (above line) and D-loop (below line). 154 Figure 6.3 Haplotype map for Solenosmilia variabilis ITS (above line) and D-loop (below line) 155

Figure 6.4 Scatterplot (above) and complot (below) of DAPC of Goniocorella dumosa based on variation at all microsatellite loci (60 PCs and 2 DAs) 170 Figure 6.5 Scatterplot (above) and complot (below) of DAPC of Madrepora oculata based on variation at all microsatellite loci (60 PCs and 2 DAs) 170 Figure 6.6 Scatterplot (above) and complot (below) of DAPC of Solenosmilia variabilis based on variation at all microsatellite loci (154 PCs and 2 DAs) 171 Figure 6.7 Barriers to gene flow indicated by software Barriers based on the full microsatellite data set for Goniocorella dumosa (A), ITS data for Madrepora oculata (B), ITS data for Solenosmilia variabilis (C) and the full microsatellite data set for Solenosmilia variabilis (D).

Figure 6.8 Circular plots of migration (gene flow) between and within populations of Goniocorella dumosa on geomorphic features based on all microsatellite loci (left) and neutral microsatellite loci (right)

Figure 6.9 Circular plots of migration (gene flow) between and within populations of Madrepora oculata on geomorphic features based on ITS sequence data. 174 
Figure 6.10 Circular plots of migration (gene flow) between and within populations of Solenosmilia variabilis on geomorphic features based on ITS sequence data (top), all microsatellite loci (bottom left) and neutral microsatellite loci (bottom right) ..................... 175

Figure 7.1 Heatmap of correlations between environmental variables...............................200 Figure 7.2 dbRDA analyses of all (left) and neutral (right) microsatellite variation for

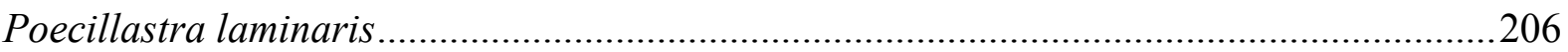

Figure 7.3 dbRDA analyses of all (above) and neutral (below) microsatellite variation for

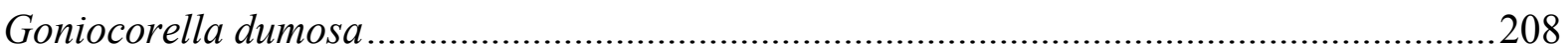

Figure 7.4 dbRDA analyses of all (left) and neutral (right) microsatellite variation for

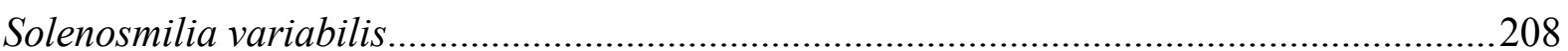

Figure 8.1 Sample collection depth distributions of all examined species ..........................226 


\section{External contributions}

This work is part of the project "Predicting the occurrence of vulnerable marine ecosystems for planning spatial management in the South Pacific region" (C01X1229) funded by the Ministry of Business, Innovation and Employment (MBIE). All specimens used throughout this thesis were obtained from the Invertebrate Collection of the National Institute of Water and Atmospheric Research (NIWA), with the assistance of Sadie Miller (NIWA). All laboratory work, analysis and writing were conducted by the author with the help of supervisors (Jonathan Gardner, Ashley Rowden and Malcolm Clark), and there were very important contributions to this thesis listed as follows:

\section{Chapter 3}

Illumina sequences data and unpublished microsatellites for Solenosmilia variabilis were provided by Karen Miller (Australian Institute of Marine Science, AIMS).

\section{Chapter 4}

Michelle Kelly (NIWA) was responsible for all sponge identifications.

\section{Chapter 5}

Dianne Tracey was in charge of all coral identifications. Unpublished microsatellites for Madrepora oculata developed and shared by Cheryl Morrison (USGS). Sophie Arnaud Haond (IFREMER, France) donated 20 M. oculata samples from Europe.

\section{Chapter 6}

Data for environmental variables were provided by NIWA from various sources and generated by Mike Williams (NIWA).

\section{Specimen Collection}

Sponges

Specimens included in this study were collected on various research cruises, details of those projects responsible for the collection of these valuable samples are provided as follow: "Seamounts: their importance to fisheries and marine ecosystems", undertaken by the NIWA and funded by the former New Zealand Foundation for Research, Science and Technology (FRST) with additional funding from the former Ministry of Fisheries (MFISH TAN0104,TAN0905), NOAA Satellite Operations Facility (TAN0413 cruise), and Census of Marine Life (CoML) field programme on seamounts, CenSeam (TAN0604 cruise); Specimens collected under the Scientific Observer Program funded by the New Zealand 
Ministry for Primary Industries (MPI); New Zealand Cold Vents: New Vents program (RV Sonne voyage SO191) conducted by IFM-GEOMAR Leibniz-Institut für Meereswissenschaften an der Universität Kiel and partners; Fisheries research trawl surveys (TAN0501, TAN0813, TAN0901) conducted by NIWA and funded by MFish (now MPI); RENEWZ I (TAN0616) voyage, were components of the project 'Exploration of Chemosynthetic Habitats of the New Zealand Region', funded by NOAA Ocean Exploration and NIWA, with co-funding from Woods Hole Oceanographic Institution (WHOI), Scripps Oceanographic Institution, and the University of Hawaii; Ocean Survey 20/20 Chatham/Challenger Biodiversity and Seabed Habitat Project, jointly funded by MFish, Land Information New Zealand (LINZ), NIWA, and Department of Conservation; New ZealandAustralian "MacRidge 2" research voyage (TAN0803), the biological component of which was part of NIWA's research project "Seamounts: their importance to fisheries and marine ecosystems" funded by FRST and CSIRO's Division of Marine and Atmospheric Research project "Biodiversity Voyages of Discovery" funded by the CSIRO Wealth from Oceans Flagship; Kermadec Arc Minerals (KARMA, TAN1007) voyage, funded by the Ministry of Business, Innovation \& Employment (MBIE), in collaboration with Auckland University, GNS Science, and WHOI; Ocean Survey 20/20 Mapping the Mineral Resources of the Kermadec Arc (voyage TAN1104), funded by LINZ, GNS, NIWA and WHOI; Biogenic Habitats on the Continental Shelf (voyages TAN1105 \& TAN1108), funded by MFish, FRST, NIWA and Oceans Survey 20/20 R/V Tangaroa days funded by LINZ; 'Impact of resource use on vulnerable deep-sea communities' project (TAN1206), funded by MBIE; Nascent Inter-Ridge Volcanic And Neotectonic Activity (NIRVANA, TAN1213) voyage, funded by MPI, in collaboration with Auckland University, GNS, and the University of New Hampshire (USA) and with funding from the program 'Impact of resource use on vulnerable deep-sea communities' project, funded by MBIE; South Pacific Vulnerable Marine Ecosystems Project funded by MBIE.

\section{Corals}

Specimens and data were provided by the NIWA Invertebrate Collection including material from the following projects: "Seamounts: their importance to fisheries and marine ecosystems", undertaken by the NIWA and funded by the former New Zealand Foundation for Research, Science and Technology (FRST) with additional funding from the former Ministry of Fisheries (MFish), NOAA Satellite Operations Facility (TAN0413) and the Census of Marine Life field programme on seamounts, CenSeam (TAN0604); RENEWZ I 
(TAN0616) voyage, were components of the project 'Exploration of Chemosynthetic Habitats of the New Zealand Region', funded by NOAA Ocean Exploration and NIWA, with co-funding from Woods Hole Oceanographic Institution (WHOI), Scripps Oceanographic Institution, and the University of Hawaii; Ocean Survey 20/20 Chatham/Challenger Biodiversity and Seabed Habitat Project, jointly funded by MFish, Land Information New Zealand (LINZ), NIWA, and Department of Conservation; New Zealand-Australian "MacRidge 2" research voyage (TAN0803), the biological component of which was part of NIWA's research project "Seamounts: their importance to fisheries and marine ecosystems" funded by FRST, and CSIRO's Division of Marine and Atmospheric Research project "Biodiversity Voyages of Discovery" funded by the CSIRO Wealth from Oceans Flagship; 'Impact of resource use on vulnerable deep-sea communities' (DSCA: TAN1004, TAN1206, TAN1503), funded by the Ministry of Business, Innovation \& Employment (MBIE); Kermadec Arc Minerals (KARMA) voyage, funded by MBIE, in collaboration with Auckland University, GNS Science, and WHOI; Ocean Survey 20/20 Mapping the Mineral Resources of the Kermadec Arc Project, funded by LINZ, Institute of Geological and Nuclear Science, NIWA, and WHOI; Biogenic Habitats on the Continental Shelf project (voyages TAN1105), funded by MPI, FRST, NIWA and Oceans Survey 20/20 shiptime (LINZ); Nascent Inter-Ridge Volcanic And Neotectonic Activity (NIRVANA, TAN1213) voyage, funded by the Ministry for Primary Industries (MPI), in collaboration with Auckland University, GNS, and the University of New Hampshire (USA) and with funding from the DSCA project, funded by MBIE; NIWA/GNS Oceans Survey 20/20 Reinga voyage (TAN1312), funded by LINZ and New Zealand Petroleum \& Minerals; South Pacific Vulnerable Marine Ecosystems Project (C01X1229) funded by MBIE; Scientific Observer Program funded by MFish/MPI; NIWA trawl surveys funded by MFish/MPI; various campaigns and cruises of the New Zealand Oceanographic Institution. 

Chapter 1. Introduction 


\subsection{The deep sea}

The deep sea, usually defined as the region beyond the continental shelf break and at depths greater than $200 \mathrm{~m}$, has a diverse topography and oceanography and encompasses a wide range of habitat types and depths. It is the single largest biome on the planet (RamirezLlodra et al., 2011). Compared with shallow-water habitats and their biotas, which are relatively well studied, information from the deep sea around the world is still scarce. Despite being largely unknown, the deep sea is nevertheless extremely important with unique ecosystems, and contains many marine resources of a biological and non-biological nature of high potential commercial value (Ramirez-Llodra et al., 2011).

In the deep-sea biome, different seafloor habitats are patchily distributed, including the continental slope, abyssal plain, trenches and troughs, seamounts, hydrothermal vents and cold seeps (Oldham et al., 2014). These habitats represent different environments, and can contain different taxa. The water column above these seafloor habitats, is the largest habitat in the ocean, and comprises all the open ocean waters and is divided into pelagic zones at different depths (Oldham et al., 2014), where most larval dispersal of deep-sea organisms is thought to occur. Some deep-sea habitats are much more studied than others (Oldham et al., 2014). The abyssal plain, the most common benthic habitat in the deep sea, covers approximately half of the Earth's surface. However, the understanding of this habitat is limited due to the spatial scale of the area, and its inaccessibility (Ebbe et al., 2010; Webb et al., 2010). Trenches and troughs are long, narrow, steep-sided depressions, and the extreme depth keeps this area largely unexplored, such that most of the fauna at these deepest depths are still unknown (Oldham et al., 2014). By comparison, the continental slope, seamounts, hydrothermal vents and cold seeps are relatively well studied (details see Sibuet \& Olu 1998; van Dover et al., 2002; Shank 2010; Vrijenhoek 2010; Levin et al., 2016), but even including all of these habitats, only a small proportion of the deep-seafloor has been well studied.

In the past decades, as human activities have extended, anthropogenic impacts have increased with direct disturbances on deep-sea communities, and also indirect disturbances through changes to the chemistry of the oceans (Davies et al., 2007; Halpen et al., 2008; Pitcher et al., 2008). In terms of direct effects, extractive resource exploitation is currently the main reason for degradation of deep-sea ecosystems (Davies et al., 2007). Deep-sea fisheries have not only caused the number and diversity of fishes to decline (Baum et al., 2003; Devine et al., 2006; Worm et al., 2005), but have also damaged structure-forming organisms (HallSpencer et al., 2002; Koslow et al., 2001; Pitcher et al., 2008; Roberts, 2002), reduced 
fisheries-associated species (by-catch) (Hareide et al., 2005) and have removed epifauna and modified the substratum (Gage et al., 2005). Re-suspension of large quantities of sediment by bottom trawling has also caused reductions in benthic abundance in deep-sea communities (Palanques et al., 2001; Palanques et al., 2006; Palanques et al., 2014). Recently, increasing demands for, and diminishing reserves of, hydrocarbons and minerals on land and in shallow water are pushing exploration into the deeper ocean (Hallock et al., 2004), with associated negative effects on deep-sea animals (Gass \& Roberts, 2006; van Dover, 2014). Moreover, the negative effects of waste disposal and pollution in the deep sea are still not well understood or widely studied. In terms of indirect effects, both physical and ecological changes caused by climate change and ocean acidification are expected to have negative implications for deep-sea habitats and organisms (Levin et al., 2001; Ruhl \& Smith, 2004; Hansen et al., 2004; Danovaro et al., 2004; Guinotte et al., 2006).

\subsection{Vulnerable Marine Ecosystems (VMEs) and VME indicator $\operatorname{taxa}$}

With the ever increasing pressure of adverse impacts and their consequences, concerns for deep-sea conservation are increasingly being raised. However, it is impossible to protect everything in the vast area of the deep sea, and conservation priorities have been set. In 2008, the United Nations Food and Agriculture Organisation (FAO) defined particular vulnerable marine ecosystems (VMEs) where organisms and their associated taxa are easily disturbed, are slow to recover or may not recover at all. Identified VMEs include seamounts, hydrothermal vents, cold seeps, deep-sea coral reefs and sponge gardens (FAO, 2009). VMEs can be associated with marine resources, for example they maybe sites of interest for particular fisheries (seamounts) and mining (vents), and are thus subject to high current or future levels of exploitation (Clark et al., 2010; Shank 2010; Boschen et al., 2013; Levin et al., 2016). As a consequence, the United Nations General Assembly Resolution 61/105 calls for states to protect VMEs. However, the first challenge is how to identify and locate VMEs because there is not much information about the distribution of VMEs in the deep sea. As such, VME indicator taxa have been identified to help locate VMEs.

The guidelines for the management of deep-sea fisheries provide examples of VME indicator taxa, (e.g. habitat-forming species of stony corals and sponges) (FAO, 2009), but regionally-specific VME indicator taxa are more relevant. Currently, different Regional 
Fisheries Management Organisations (RFMOs) have identified regional-specific indicators (Table 1.1).

Table 1.1 VME indicator taxa amongst different Regional Fisheries Management Organisations

\begin{tabular}{ccccccc}
\hline Common name & Taxonomic name & CCAMLR & NAFO & NEAFC & SPRFMO & GFCM \\
\hline Sponges & Porifera & $\mathrm{Y}$ & $\mathrm{Y}$ & $\mathrm{Y}$ & $\mathrm{Y}$ & $\mathrm{Y}$ \\
Anemones & Actiniaria & $\mathrm{Y}$ & $\mathrm{Y}$ & $\mathrm{Y}$ & $\mathrm{Y}$ & $\mathrm{Y}$ \\
Soft corals & Alcyonacea & $\mathrm{Y}$ & & $\mathrm{Y}$ & $\mathrm{Y}$ & $\mathrm{Y}$ \\
Gorgonian & Gorgonacea & $\mathrm{Y}$ & & $\mathrm{Y}$ & $\mathrm{Y}$ & $\mathrm{Y}$ \\
Sea pens & Pennatulacea & $\mathrm{Y}$ & $\mathrm{Y}$ & $\mathrm{Y}$ & $\mathrm{Y}$ & $\mathrm{Y}$ \\
Stony corals & Scleractinia & $\mathrm{Y}$ & $\mathrm{Y}$ & $\mathrm{Y}$ & $\mathrm{Y}$ & $\mathrm{Y}$ \\
Black corals & Antipatharia & $\mathrm{Y}$ & & $\mathrm{Y}$ & $\mathrm{Y}$ & $\mathrm{Y}$ \\
Stylasterid corals & Anthoathecate & $\mathrm{Y}$ & & & $\mathrm{Y}$ & $\mathrm{Y}$ \\
Bryozoans & Bryozoa & $\mathrm{Y}$ & $\mathrm{Y}$ & & & $\mathrm{Y}$ \\
Protozoans & Protozoa & $\mathrm{Y}$ & & $\mathrm{Y}$ & & \\
Foraminifera & Foraminifera & $\mathrm{Y}$ & & $\mathrm{Y}$ & & $\mathrm{Y}$ \\
Sea lilies & Stalked crinoids & $\mathrm{Y}$ & $\mathrm{Y}$ & $\mathrm{Y}$ & $\mathrm{Y}$ & $\mathrm{Y}$ \\
Armless stars & Euryalida & $\mathrm{Y}$ & & & & \\
Sea squirts & Ascidiacea & $\mathrm{Y}$ & $\mathrm{Y}$ & & & \\
\hline
\end{tabular}

CCAMLR, Commission for the Conservation of Antarctic Marine Living Resources; NAFO, Northwest Atlantic Fisheries Organization; NEAFC, North East Atlantic Fisheries Commission; SPRFMO, South Pacific Regional Fisheries Management Organisation; GFCM, General Fisheries Commission for the Mediterranean. The information of this table was summarized from FAO (http://www.fao.org/in-action/vulnerable-marineecosystems/definitions/en/).

Following their identification, with the aid of distribution records or habitat suitability models for VME indicator taxa, the question of how to manage VMEs poses a major challenge. The design of effective spatial management measures to conserve VMEs requires information that can guide, for example, the distribution, size and spacing of marine protected areas (MPAs) (Agardy, 2000; O’Leary et al., 2012). Therefore, as well as studies of the distribution of VMEs, it is also important that research is carried out that examines the evolutional and ecological processes that influence the distribution of species and populations. Such research includes the genetic structure and connectivity of populations of VME indicator taxa, and the processes that influences these patterns.

\subsection{Study area}

The deep sea around New Zealand is physiographically complex, comprising a series of parallel ridges and troughs in the north west (Lord Howe Rise, New Caledonia Basin and 
Norfolk Ridge), two island arcs of volcanic origin (Kermadec and Macquarie Ridge) in the north and south respectively, the Campbell Plateau and Chatham Rise in the southeast and east, and the Challenger Plateau and Tasman Basin in the west (Cullen, 1970) (Figure 1.1). As well as the complex topography, circulation around New Zealand is also complicated and it is mainly dominated by Subtropical Water (STW) and Subantarctic Water (SAW) (Chiswell et al., 2015) (Figure 1.2). These and other environmental differences across the region are thought to account for the deep-sea fauna of the seas around New Zealand being separated into two biogeographical provinces (Watling et al., 2013).

Currently, there are 17 seamount closures and 17 Benthic Protection Areas (BPAs) throughout the New Zealand EEZ, established in 2001 and 2007, respectively (Brodie \& Clark, 2002; Helson et al., 2010) (Figure 1.3). These areas were selected to protect the seafloor fauna, including VMEs, from bottom trawling, but the design of these BPAs did not consider the genetic connectivity of deep-sea populations.

Amongst the few deep-sea genetic studies, research illustrates a complex pattern for deep-sea connectivity at different spatial scales in the New Zealand deep sea. No population genetic structure has been found in fish, crustaceans or demosponges off New Zealand (Smith et al., 1996; Bors et al., 2012). However, significant population genetic structure exists amongst distinct populations on the Chatham Rise, Hikurangi Margin and Challenger Plateau for a polychaete (Bors et al., 2012). Furthermore, Miller et al. (2010) found that there were non-overlapping $16 \mathrm{~S}$ and ITS haplotype distributions in antipatharians, suggesting limited connections between seamounts in the north of New Zealand. They, and other authors, have also pointed out that ocean expanses of 500-1000 km may be effective barriers to gene flow for deep-sea coral species, because of topographically controlled current patterns which promote within-ridge rather than among-ridge connectivity (de Forges et al., 2000; Miller et al., 2010). In the Bay of Plenty, six populations of the antipatharian Stichopathes filiformis from six different seamounts also shared ITS haplotypes, suggesting that at scales of 50-100 $\mathrm{km}$ (Miller et al., 2010), these seamounts may be connected by gene flow through surface and near-surface current flow (Sutton \& Chereskin, 2002). Apparently, no consistent pattern for the connectivity of deep-sea fauna has yet emerged amongst different taxa and at different scales, within the New Zealand region. 


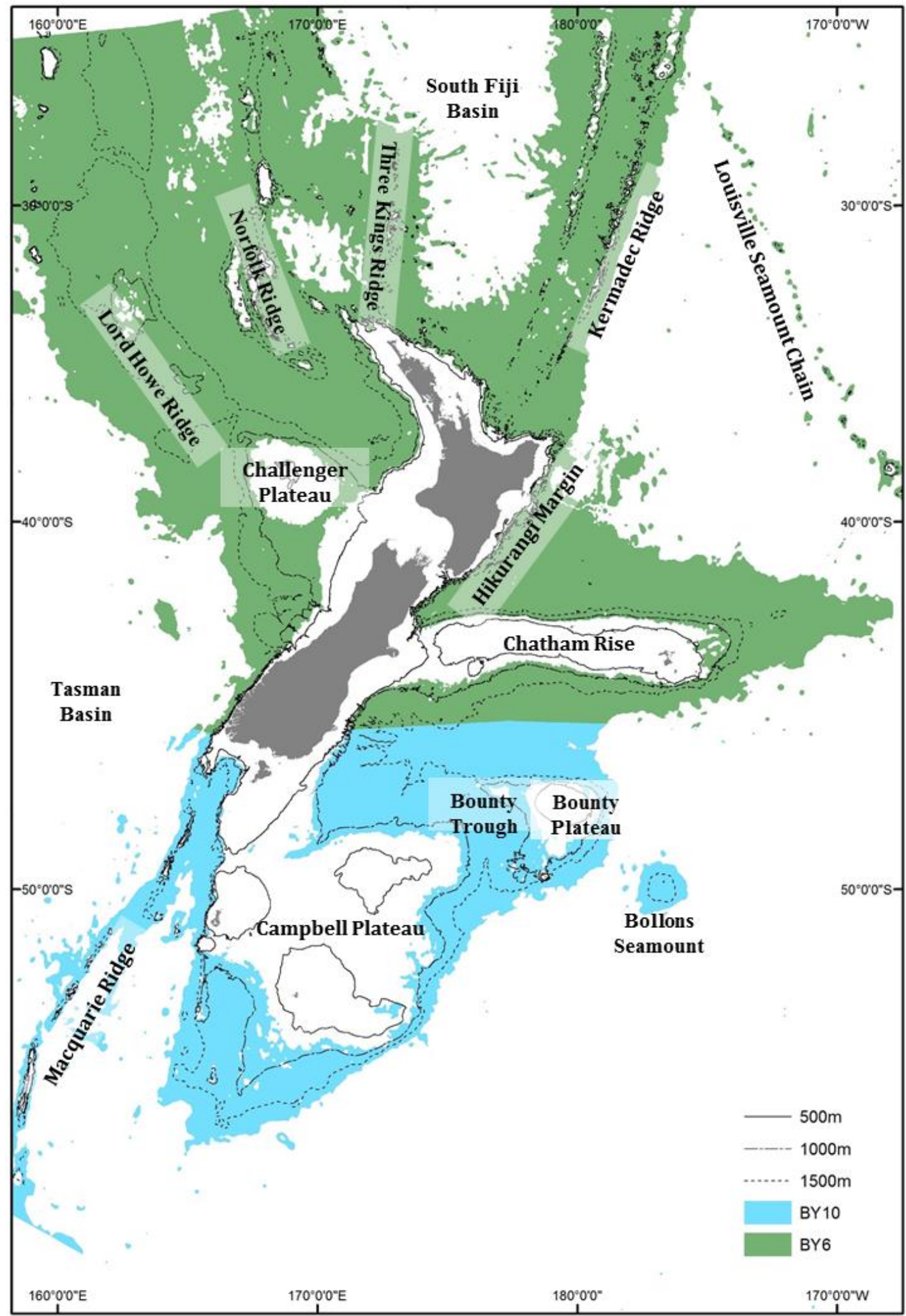

Figure 1.1 Distribution of major geomorphic features and deep-sea lower bathyal provinces around

New Zealand 


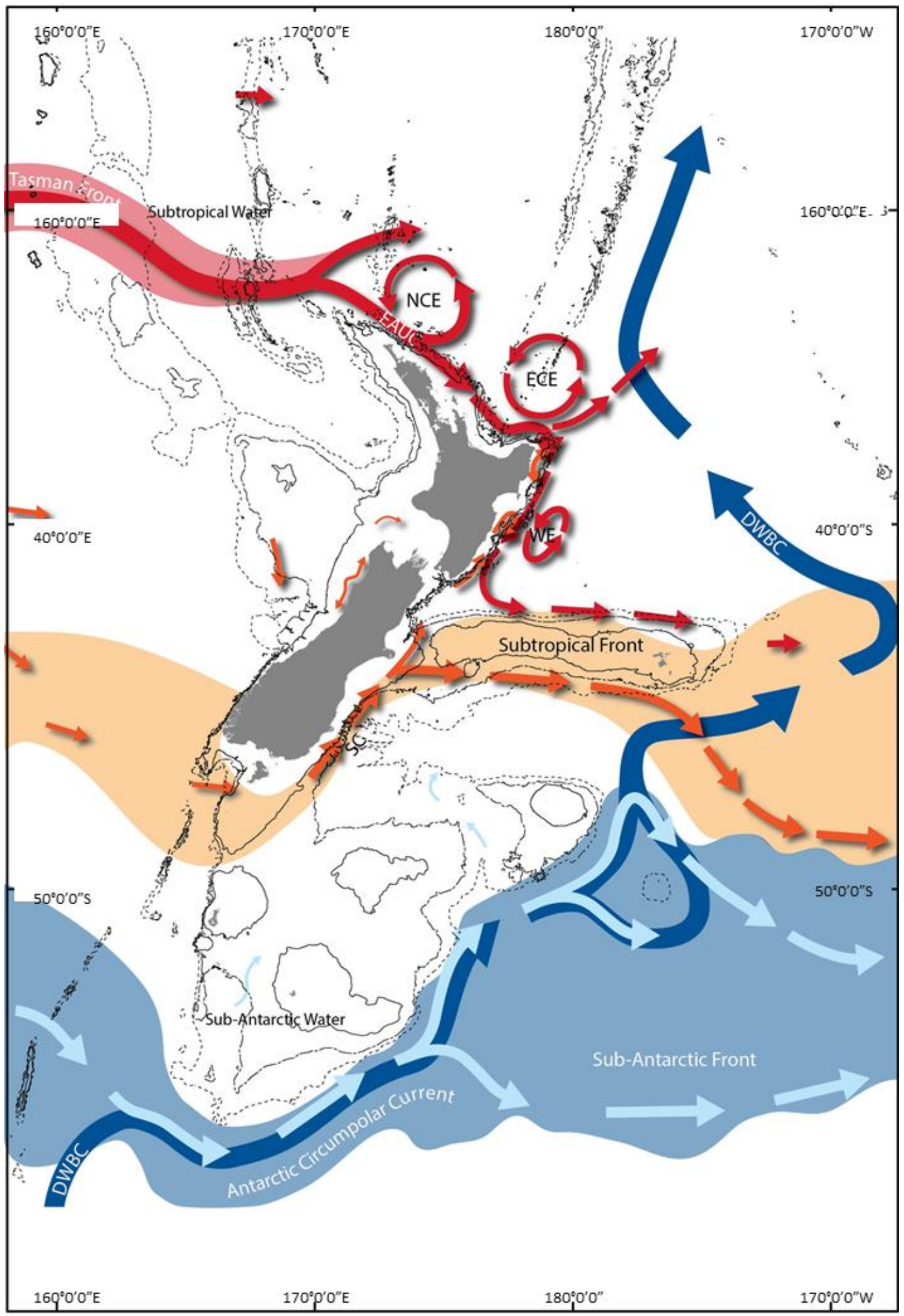

Figure 1.2 Summary of the major currents in the New Zealand region

EAUC, East Auckland Current; NCE, North Cape Eddy; ECE, East Cape Eddy; WE, Wairarapa Eddy (Leathwick et al., 2008, Bors et al., 2012). 


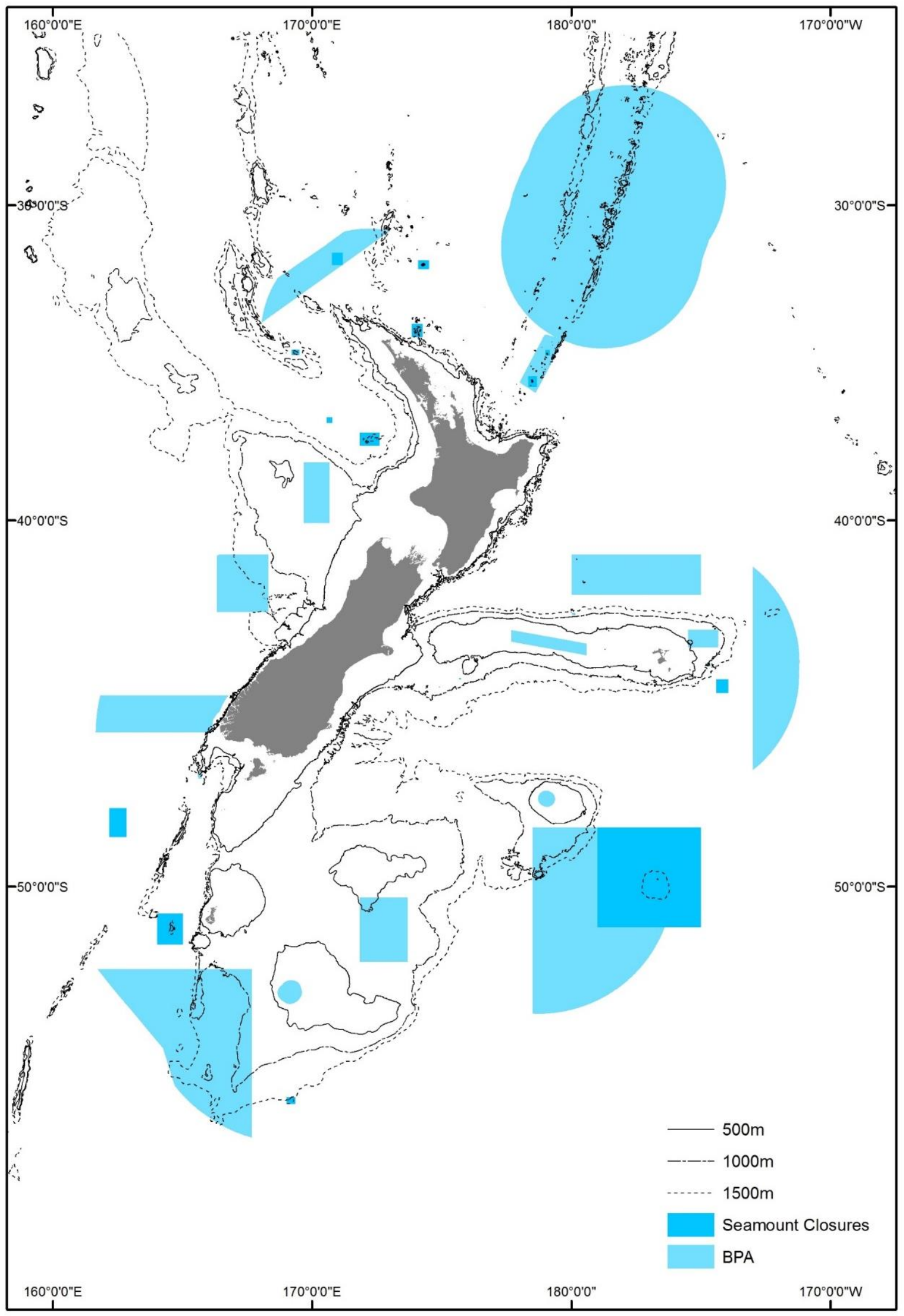

Figure 1.3 Offshore protected areas within the New Zealand EEZ 


\subsection{Study species}

Species of structure or habitat-forming sponges and corals are widely recognised as VME indicator taxa (Table 1.1), including within the New Zealand region (Parker et al., 2009). However, population genetic connectivity knowledge about deep-sea sponges is very limited. In the present study, four demosponges, Neoaulaxinia persicum, Poecillastra laminaris, Pleroma menoui and Penares sp., were selected to be tested for patterns of genetic structure (Figure 1.4).

Poecillastra laminaris, known as the fibreglass cup sponge, is very "hairy" with siliceous spicules, and has a morphological shape that is relatively variable (common as shallow cup-like or large ragged fan sponge with rippled edges) (Tracey et al., 2007). Size of Poecillastra laminaris is often about $5-10 \mathrm{~cm}$ in diameter, and it is widely distributed around New Zealand across large range of a latitudinal range $\left(\sim 30^{\circ} \mathrm{S}-\sim 60^{\circ} \mathrm{S}\right)$ and depth $(119-1615$ m) (Database of the National Institute of Water \& Atmospheric Research Invertebrate Collection (NIC), 2015).

Penares sp., known as the golden brown crater sponge, is a spherical sponge with a smooth, waxy surface indented all over with groups of oscules, and is compressible with a size of up to $15 \mathrm{~cm}$ in diameter (Tracey et al., 2007). Penares sp. is often free-living and the distribution depth around New Zealand ranges from 350-1408 m (NIC database, 2015).

Neoaulaxinia persicum is egg-shaped with a thin skirt base or encompassing branches of scleractinian corals. It is often attached to small pieces of scleractinean coral rubble and rock surfaces on the sides of seamounts. The size of $N$. persicum is about $5 \mathrm{~cm}$ in diameter (Kelly, 2007). The collected depth ranges from 581 to $1680 \mathrm{~m}$ (NIC database, 2015).

Pleroma menoui is a highly irregular multilobate encrusting plate, occasionally tubular or a thick fan composed of fused lobes. Immature sponges are thick flattened earshaped plates (Kelly, 2007). P. menoui is usually attached to hard substrata on continental shelf banks, and found with a range of other lithistid sponges, molluscs, bryozoans, brachiopods, echinoderms, hydroids, and stylasterid hydrocorals (Kelly, 2007). The maximum dimensions of this specimen are recorded as $21 \mathrm{~cm}$ from New Zealand and New Caledonia (Kelly, 2007). The depth distribution of this species is from 179 to $945 \mathrm{~m}$, and all collected species are from north of the North Island (NIC database, 2015).

Amongst stony corals in the deep sea, Goniocorella dumosa, Madrepora oculata and Solenosmilia variabilis, are the three most common species within the New Zealand 
Exclusive Economic Zone (EEZ) that can form habitat structures (Tracey et al., 2011), and were this chosen for the present study.

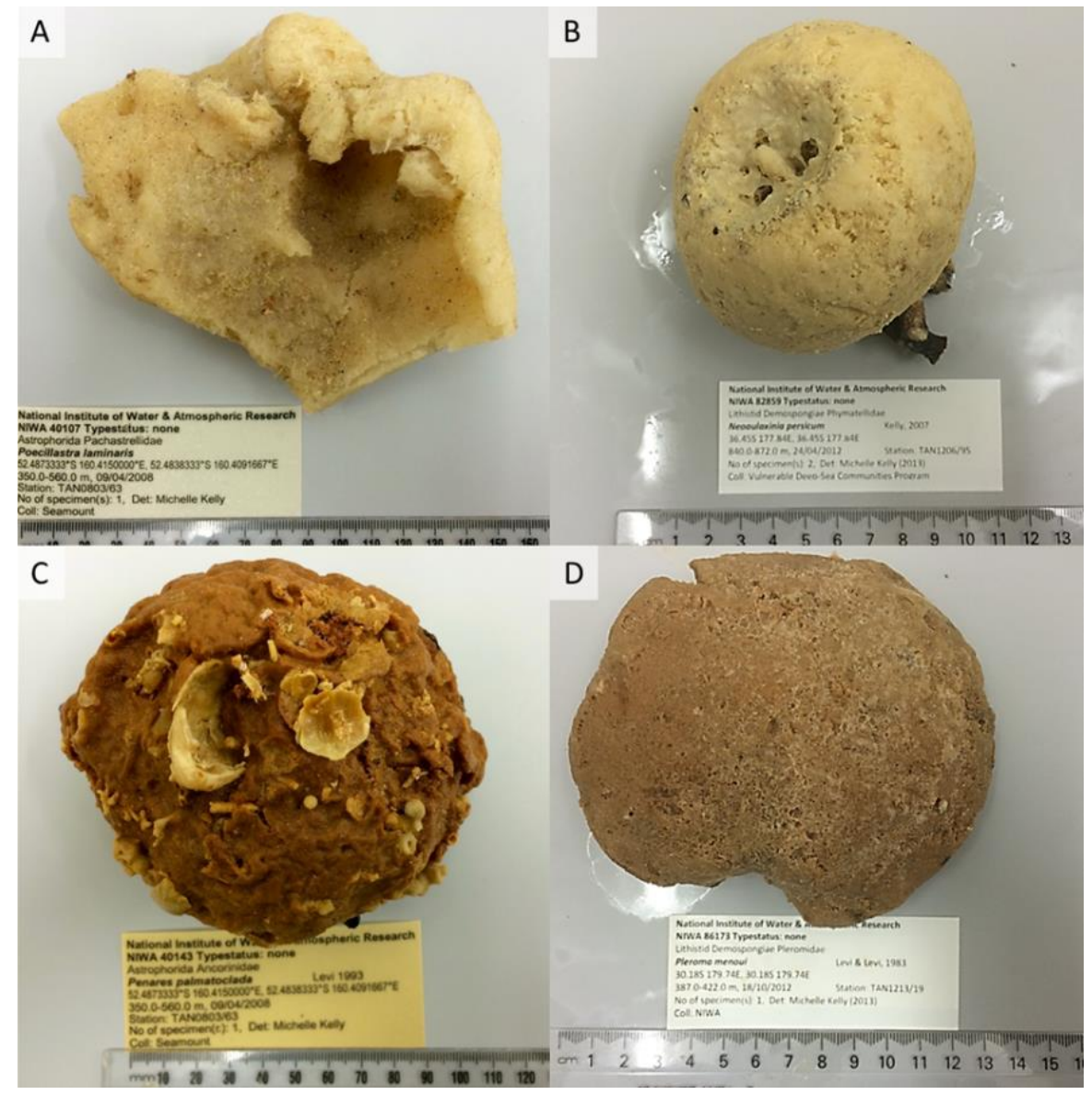

Figure 1.4 Specimens of Poecillastra laminaris (A), Neoaulaxinia persicum (B), Penares sp. (C) and Pleroma menoui (D)

Madrepora oculata forms branched colonies, and is typically a secondary framework builder, often being associated with other reef-building corals such as Lophelia pertusa (in the Northern hemisphere) and S. variabilis (in the Southern hemisphere) (Hain \& Corcoran, 2004; Hourigan et al., 2007). Morphologically, M. oculata is quite variable in its tendency to branch and in its texture (Pawson et al., 1983) (Figure 1.5), and it is usually bushy, forming small fan-shaped colonies, with thick skeletal parts that grow in a lamellar pattern (Hourigan et al., 2007). Unlike M. oculata with less extensive structures, G. dumosa and S. variabilis can form single species structures that cover larger areas. Both these species develop complex 3D branching structures, but G. dumosa tends to occur as discrete colonies in high density thickets, whilst $S$. variabilis colonies can coalesce to form larger and continuous reef-like structures (Figure 1.5). S. variabilis is widely distributed in the South Pacific and occasionally found in the Atlantic Ocean, whereas G. dumosa is mainly reported from the 
Pacific Ocean (Yabe \& Eguchi, 1936; Pawson et al., 1983; González-Romero et al., 2009). Compared to the deeper distribution of $S$. variabilis, G. dumosa is frequently found in shallow water at depths of 300 to $400 \mathrm{~m}$ (Hain \& Corcoran, 2004). S. variabilis prefers to settle on hard substrata (such as seamount summits), whereas G. dumosa predominantly forms colonies on soft bottom (O'Hara et al., 2008). The collected depth ranges in NIC of G. dumosa, M. oculata and S. variabilis were $198-1270 \mathrm{~m}, 236-1537 \mathrm{~m}$, and $322-1805 \mathrm{~m}$, respectively.

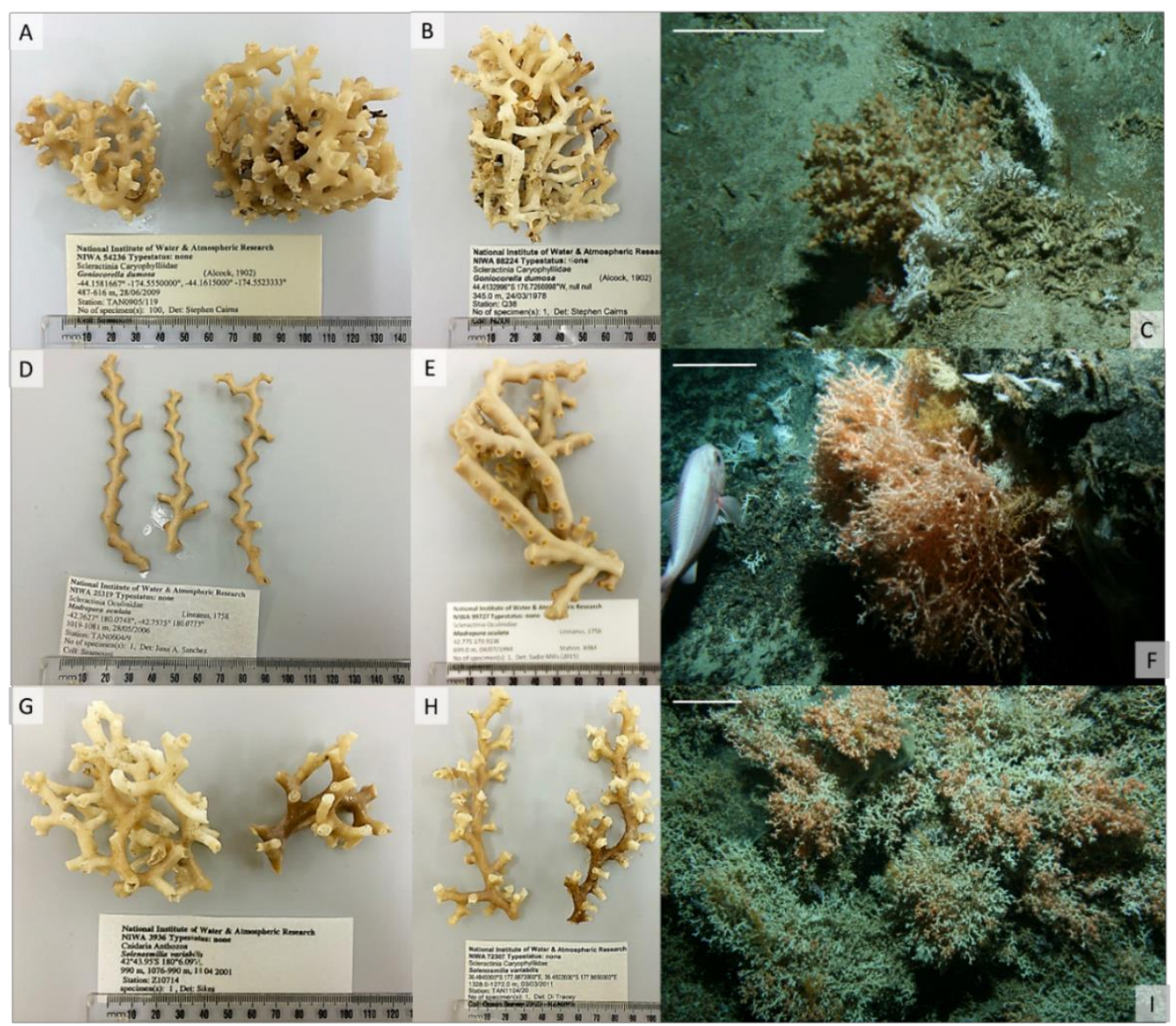

Figure 1.5 Morphology and branching of Goniocorella dumosa, Madrepora oculata and

\section{Solenosmilia variabilis}

In Figure 1.5 above, specimens labelled A (NIWA ID 54236) and G (3936) are easily misidentified when only a small proportion of skeleton is available; B (88224) is typical G. dumosa with smooth tubular bridges intersecting the branches (key feature); C (25319) and D (99727) are showed the variable branching of $M$. oculata, and the texture of D is much more fragile than that of E. G and H (72307) show the branching and polyps of S. variabilis. The colour of dry-preserved and ethanol-preserved specimens has faded out in all three corals. In situ image of G. dumosa (C), M. oculata (F), and S. variabilis (I) on the Chatham Rise (TAN1503 voyage, DTIS Camera). The white bars in C, F and I are $20 \mathrm{~cm}$. 


\subsection{Aims and thesis structure}

This $\mathrm{PhD}$ research is a part of the project "Predicting the occurrence of vulnerable marine ecosystems for planning spatial management in the South Pacific region", which is funded by the Ministry for Business, Innovation and Employment (MBIE), New Zealand. The aim of this project is to produce scientific information that that can assist New Zealand and the South Pacific Regional Fisheries Management Organisation (SPRFMO) initiatives on spatial management in the South Pacific region, and potentially the New Zealand EEZ.

The overall aim of this $\mathrm{PhD}$ thesis was to investigate the patterns of genetic structure and connectivity of VME indicator taxa. The specifics were to:

1. Define patterns of genetic population structure and genetic connectivity in demosponges and corals, and identify barriers to dispersal.

2. Identify environmental variables that might explain the observed structure/connectivity and dispersal barriers for demosponges and corals.

3. Consider how observed patterns of population genetic structure and genetic connectivity can inform deep-sea conservation for VMEs around New Zealand.

\section{Thesis Structure}

Each chapter of this thesis (except Chapter 1 and Chapter 8) has its own specific objectives and individual sections of Introduction, Methods, Results and Discussion. The purpose of Chapter 2 is to give an overview on the development of the genetic connectivity field and develop suitable hypotheses for the data testing in Chapters 5 and 6. Chapter 3 describes the mitochondrial and microsatellites markers developed for Poecillastra laminaris and corresponds to publications in the journal Mitochondrial DNA (Zeng et al., 2014a) and Marine Biodiversity (Zeng et al., 2016). Chapter 4 describes the application of mitochondrial, microsatellite and single-nucleotide polymorphism markers developed for Solenosmilia variabilis, and corresponds to a publication in Mitochondrial DNA (Zeng et al., 2014b). In Chapter 5, the genetic structures of four sponges are described, the null hypothesis of panmixia (i.e., no genetic structure) is separately tested in these species using mitochondrial sequence markers and the factors that shape the patterns observed are discussed. This chapter has been submitted for publication in the journal Biodiversity and Conservation. In Chapter 6, the genetic structures of three stony corals are described. The null hypothesis is also separately tested in these species using mitochondrial and nuclear sequences markers and microsatellites and the factors that shape the patterns observed are discussed. This chapter has 
been drafted for submission to the journal Molecular Ecology. In Chapter 7, a seascape genetics approach is used to identify associations between the genetic structures of collected sponges and corals across New Zealand and environmental variables. Finally, Chapter 8 summarises the key findings, indicates future directions and provides recommendations about the management and conservation of New Zealand's VMEs. 

Chapter 2. Patterns of genetic connectivity in the deep sea: challenges for, and applications to, the conservation of Vulnerable Marine Ecosystems 


\subsection{Introduction}

Habitats, such as trenches and troughs, seamounts, hydrothermal vents and cold seeps, which have distinctive hydrodynamic, topographic and geomorphic features, are patchily distributed in the deep sea. Therefore, genetic connectivity amongst populations could be complex for spatially fragmented populations in different habitats (Hilário et al., 2015). For the conservation of deep-sea habitats, connectivity amongst populations is an important consideration for marine protected area (MPA) designation and MPA network design (Cowen et al., 2002; Palumbi, 2003; Anadón et al., 2013). Connectivity is a critical component of marine ecology to answer the questions 'where, how far and how much do larvae dispersal?', and this provides information about identifying source and sink populations and characterising spatial units for conservation (Lowe \& Allendorf, 2010). However, it is impossible to physically monitor the movement of larval migrants in marine ecosystems, because the migrants are spread in an open and wide marine medium and are very small $(<$ $500 \mu \mathrm{m}$ in diameter) making them impossible to track (Cowen \& Sponaugle, 2009; Buston \& D'Aloia, 2013). Estimating connectivity indirectly using molecular biology is an alternative and powerful approach using genetic metrics to provide an indirect measurement of connectivity by sampling the adults without the constraint of directly monitoring larvae (Hellberg et al., 2002). Population connectivity describes the exchange of individuals of the same species between populations and, in the case of genetic connectivity (gene flow), the extent of gene exchange caused by immigrants (Boschen et al., 2016). In other words, when populations are reproductively isolated and do not exchange genes or experience limited gene exchanges, genetic differences between them will accumulate and eventually form the population genetic structure. In practice, the analysis of genetic variation components at different spatial scale provides a solution for measuring patterns of population connectivity (Hedgecock et al., 2007), often in the absence of much information about the ecosystem in question and also without large sample size from the populations in question.

Analysising the genetic connectivities in an ecosystem is not a simple task. Because patterns of genetic connectivity can operate on scales from organisms living at a special habitat, such as a vent chimney, seamount or trench, to within an ocean or even across multiple oceans, connectivity surveys should consider a nested design to investigate the multiple scales of connectivity of different species (Thaler et al., 2011; Hilário et al., 2015; Boschen et al., 2016). In addition, the dynamics of populations change over time in response to changes in the environment and the supply of recruits (Excoffier et al., 2009). Within a 
network of connected populations, source populations that contribute most recruits to subsequent generations, help maintain the genetic diversity of the regional metapopulation, and these populations are viewed as being of more importance than other (sink) populations (Boschen et al., 2016). In a management context, genetic studies are typically conducted with the ultimate objective of identifying genetic connectivity patterns to locate source populations, and with the aim of identifying factors that contribute to genetic isolation (reduced or zero genetic connectivity) between populations (Young, 2000). However, in a vast and largely unknown ecosystem such as the deep sea, perhaps the first question to ask is how to develop different spatial frames for testing genetic variation components because there is a big difference between genetic connectivity at the oceanic and at a fine (e.g., between two vent chimneys) spatial scale.

Recently, increasing physical oceanographic modelling, predictive habitat mapping, ground-truth surveys and identification of different ecosystem biota/communities (e.g., biogeographic provinces) have all contributed to an improved understanding of the scales of genetic connectivity in the deep sea. This understanding arises from new knowledge about species-specific habitat requirements, distributions and types of substrata within habitat types, as well as factors such as currents and localised topography that may act as a barrier to gene flow. Previous studies have all proposed schemes for biogeographic provinces within the deep sea (Zezina, 1997; Vinogradova, 1997; Briggs \& Bowen, 2012). Subsequently, Watling et al. (2013) defined 14 lower bathyal and 14 abyssal provinces for the deep-sea benthos combined with the results of previous studies. This new scheme mainly considers regional variations in temperature, salinity and particulate organic carbon (POC) flux. As such, distinctive environmental conditions at the proposed province boundaries may act as barriers to gene flow (in a neutral genetic sense this type of environmental discontinuity is the most commonly invoked explanation of a barrier to gene flow). On the other hand, the boundaries between provinces provide an informative framework for testing the scales of genetic connectivity and whether the provinces may be considered as management units.

Despite the recognition of the importance of connectivity and the need to identify source and sink populations, not many genetic connectivity studies have been published for deep-sea ecosystems. This lack of study does not reflect a lack of appreciation of the deep sea itself, but arises because of the many technical limitations (logistics, sampling difficulties, but also including poor state of knowledge about the taxonomy of many deep-sea taxa and the historically poor state of preservation of archived samples) associated with working in the deep sea (Wandeler et al., 2007; Miller et al., 2011). In earlier deep-sea studies, much focus 
has understandably been on the determination of taxonomic identity and evolutionary history, which are critical prerequisites for the identification of intraspecific populations for the pursuit of population genetic studies. However, a lack of biological (taxonomical identification) and distributional information for most species in deep-sea ecosystems means that genetic analyses are difficult to conduct and interpret at spatial scales that are meaningful to conservation.

To provide background information and to build hypotheses for this $\mathrm{PhD}$ project, in this chapter, a large number of deep-sea genetic studies were summarised and a large database of papers was compiled detailing population genetic connectivity of deep-sea species, from all regions of the world's oceans. The database was utilised, applying a metaanalysis approach, to determine if a general pattern of genetic structure exists and identify causative agents (factors) as barriers to gene flow amongst deep-sea taxa. Furthermore, comparisons of genetic connectivity amongst different habitats and between VME and nonVME indicator taxa were employed to help inform management decisions about the measures that may be most efficient for protecting VMEs.

\subsection{Methods}

\subsubsection{Literature survey}

Search terms "deep sea" and "genetic" were used in Google Scholar to compile a database of studies that documented studies of population genetics in the deep sea. Papers that were identified but that were not relevant research were manually excluded. Additional papers were added from the literature collections of colleagues and from the reference sections of the papers obtained through the search. All papers available for deep-sea taxa at the time of compilation (15 May 2016) were included, regardless of life-history strategy, marker type, habitat, or ecosystem. Species were considered to be deep-sea taxa if their depth range began or most samples were collected below $200 \mathrm{~m}$ depth. Microorganisms (not considered for marine conservation) and mammals (covered by a different conservation strategy) were not considered in this study.

In total, 196 population genetic or phylogeographic studies including the data from the present thesis, published from 1972 to 2016, were included in this review (publications listed in Table S2.1). When more than one species was included in a single publication (multi species cases) or when one species was examined by two or more different genetic markers 
(multi marker cases), each case was considered separately. This selection procedure resulted in a total of 503 data lines in the database. Data in this database were included in a metaanalysis to answer the following questions about the population genetic structure and connectivity of deep-sea taxa: 1) What are the patterns of genetic structure that are reported? 2) Which factors are most likely to act as barriers to gene flow in the deep sea? 3) Which factors were identified as limiting the detection of barriers to gene flow? and 4) Which deepsea habitats were genetically most isolated and therefore require the greatest attention in terms of future management and conservation?

\subsubsection{Database compilation and variable selection}

The database contained four sections - sampling, genetic markers, genetic results and factors contributing to the identification of barriers to gene flow as discussed by the paper authors (for details refer to subsections below). Information about these sections was extracted from the selected papers. If the information was not available (i.e., not given in the paper or could not be deduced or calculated from information in the paper) the parameter was recorded as a missing value. Information about DNA sequence length (e.g., the length in base pairs (bp) of a gene or DNA region) and the sample location (e.g., latitude and longitude) was checked in the National Center for Biotechnology Information (NCBI) database and on Google Earth, respectively, if the information was not stated in the paper.

Multiple factors compiled from the literature review present complex relationships, and do not work in isolation. That is, they are often interdependent such that changes in one factor may contribute to changes in another, producing cascading effects. Additionally, the interactions between environment and biology and research limitation generate obstacles to an in-depth appreciation of patterns of genetic connectivity and the identification of barriers to gene flow in the deep sea. With this in mind the data set was constructed to permit ease of identification of the most common patterns of genetic structure and also the major causes of barriers to gene flow.

\subsubsection{Sampling}

In the sampling section, minimum and maximum sampling depth, number of examined specimens (total number of specimens), number of sampled populations, and sample locations were recorded for each data line. Additionally, the code for the deep-sea 
biogeographic province(s) relevant to the site sampling locations was introduced from Watling et al. (2013) to supplement the sampling location data, and the total number of provinces covered by the study was also included to test if the presence of a province boundary acted as a barrier gene flow, that is, to test the association between genetic structure and provinces boundary (Figure 2.1). Sampled area habitat types were classified for the deep sea as described by Oldham et al. (2014). Studies that did not describe the sampling habitat type were split into two categories, either continental slope if sampled at $<3000 \mathrm{~m}$ depth, or abyssal plain. Sampled habitat types were classified as abyssal plain (including plateaus), cold seep, hydrothermal vent, seamount, slope, and water column; there were too few studies dealing with trenches for this category to be included. Two records from food fall studies were assigned to where the whale carcasses (bones) were actually found, abyssal plain and seamount. When different habitat types were sampled within anyone study, the habitat type from which most samples were collected was used to represent all samples. The reason for this was that based on the limited information provided in many publications it was not possible to allocate individual samples to specific habitat types.

\subsubsection{Genetic markers}

In this section the type of marker, number of markers, the name of the marker (e.g. COI, ITS) and the length (base pairs, bp) of DNA sequence were recorded. Markers were sorted into type as allozyme, nuclear DNA sequence, mitochondrial DNA sequence, Amplified Fragment Length Polymorphism (AFLP), Randomly Amplified Polymorphic DNA (RAPD), Restriction Fragment Length Polymorphism (RFLP), Single Nucleotide Polymorphism (SNP), Single Strand Conformation Polymorphism (SSCP), and Microsatellite (SSR).

\subsubsection{Genetic results}

In this section the detection of a barrier to gene flow (binomial, yes or no), minimum and maximum values of nucleotide diversity $(\pi)$, minimum and maximum values of genetic differentiation ( $\Phi_{S T}$ (sequence marker) or $F_{S T}$ (all other marker types)) were obtained from the studies. For the detection of a barrier to gene flow, if a publication reported a genetic barrier (the authors reported genetic structure(s) present), the barrier detection classification was "Yes", otherwise the record was "No" (barrier undetected) - note, this variable is referred to hereafter as "genetic barrier". When multiple values for genetic diversity and/or 
differentiation were available in one publication by the same species, only the minimum and maximum values were recorded.

When only one value of these parameters was available this value was used as both the minimum and the maximum. Statistically non-significant values of $F_{S T}$ (regardless of the size of the value) were set to zero because the focus here is on significant genetic differentiation. Factors that acted as genetic barriers as discussed in the publications were identified, and ranked according to their effects as either -1 or 1 , with -1 standing for the factor was not considered as a genetic barrier and 1 meaning that it was considered as a genetic barrier.

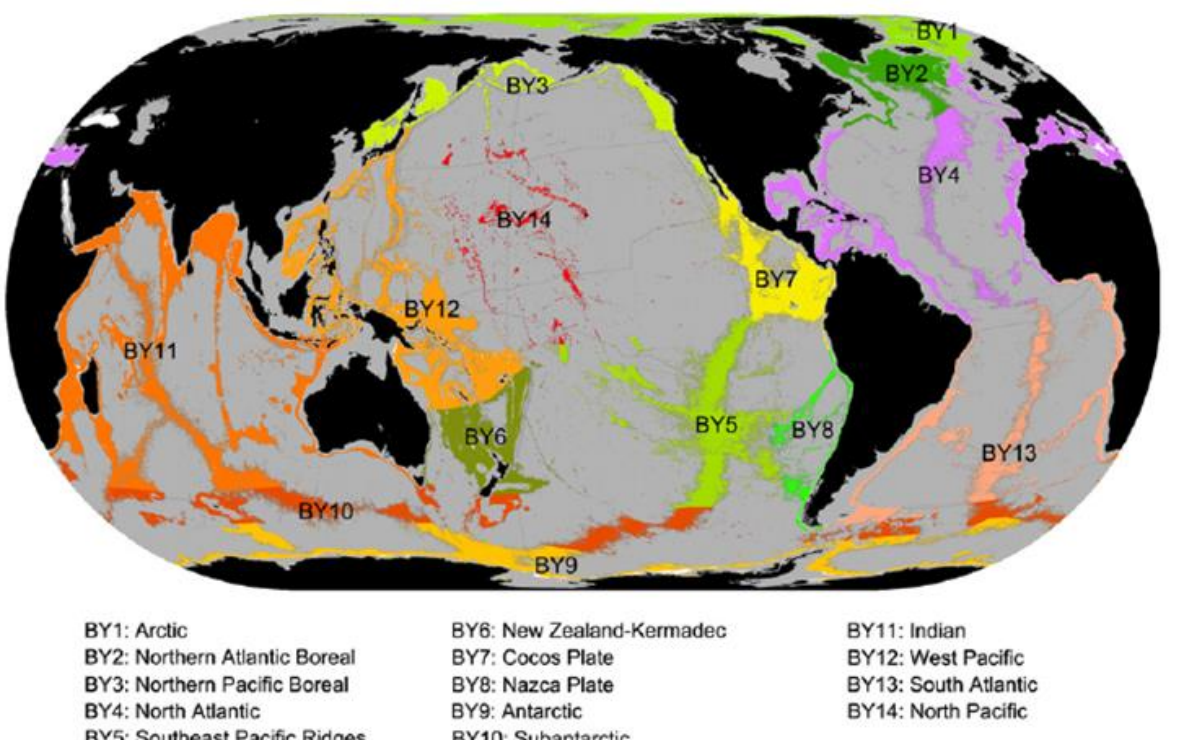

BY5: Southeast Pacific Ridges $\quad$ BY10: Subantarctic

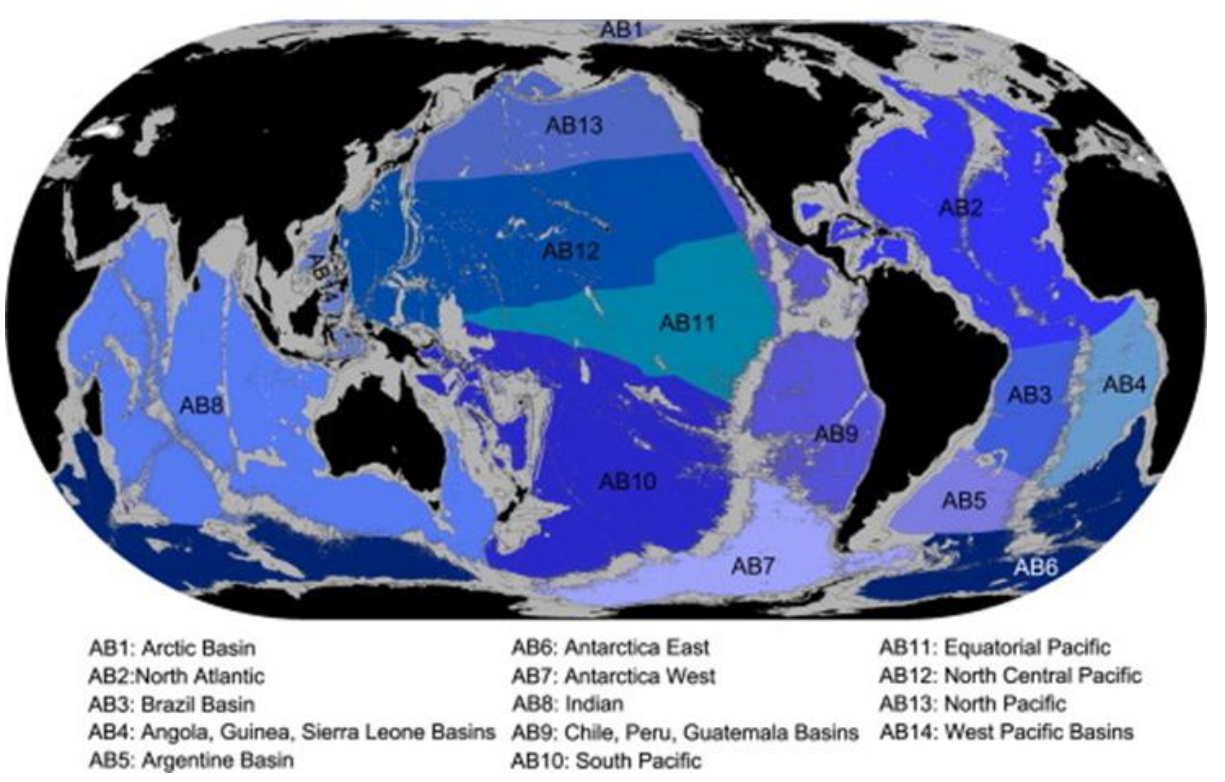

Figure 2.1 Proposed lower bathyal (upper) and abyssal (below) provinces from Watling et al (2013) 


\subsubsection{Contributing factors}

To identify factors that contributed to genetic isolation or connectivity (i.e., each factor has a binomial response, "Yes" or "No", as to whether the study authors identified it as acting as a barrier to gene flow) currents (surface and deep currents, including eddies and gyres), distance, depth, seafloor topography, and temperature (mainly caused by hydrothermal systems and latitudinal variation) were categorised as environmental factors based on a review of the collected papers. In the database, for each factor, the value was set to 1 if it was considered as acting as a barrier to gene flow to explain the genetic structure, and to -1 when the factor when promoted genetic connectivity, and to 0 when the factor did not contribute to genetic differentiation or it was not discussed.

Factors related to biological aspects, broadly defined here to include, for instance, adult mobility potential, larval dispersal potential, reproductive strategy, evolutionary and population dynamic histories, were recorded. Because there were not enough published data to cover these individual categories, they were recorded as one lumped category. Very early on in the review it became apparent that sampling constraints, including factors such as insufficient specimens and invariant genetic markers, were closely related to the failure to detect barriers to gene flow. Accordingly, research limitations as described in the individual papers were identified as a separate category.

To provide general knowledge for management, vulnerable marine ecosystems (VMEs) were also considered. Criteria of VME indicator taxa (FAO, 2009) were employed to classify the investigated organism in each published study as being indicator taxa or not based on CCAMLR VME indicator taxa Classification Guide (CCAMLR, 2009). In VME determinations, if the sampling site contained seamount, hydrothermal vent, cold seep and Canyon (NAFO, https://www.nafo.int/), it was recorded as 'Yes', otherwise 'No'. The same scoring approach was employed for the presence/absence of VME indicator taxa as recorded in each paper.

\subsubsection{Data quality control and coding}

All species' scientific names were checked in the World Register of Marine Species database (WoRMS, http://www.marinespecies.org), and the synonymised name was replaced by the corrected name where necessary. In addition, information about the phylum of the study organism(s) of each published paper was included to complement the biological information. Finally, any additional information that appeared to be relevant (e.g., about 
dispersal scale or reproductive mode) was recorded as a comment in the database to provide background knowledge for discussion, but this information was not included in the analyses, due to insufficient data.

In the database, phylum, location, deep-sea biogeographic province code, marker type, marker detail, and sampled area were all defined as categorical variables. Factors related to the detection of a barrier to gene flow (distance, depth, current, topography, temperature, biological factors, limitation), were coded as binary variables, as were VME indicator taxa (this category includes taxa such as corals and sponges that are typically associated with VMEs but in this case occur in non-VME habitats such as areas on the slope or abyss) and VME habitat. Variables such as minimum and maximum depth, nucleotide diversity, $F_{S T}$ value, $\Phi_{S T}$ value, number of specimens, number of populations and genetic markers, and sequence length were coded as continuous variables.

\subsubsection{Data analysis}

Data checking, preliminary calculations (such as depth range which was calculated as maximum minus minimum depth), basic analyses (such as means, standard deviations and summary values) and the figures (plots) were all completed in Excel 2013. The density distribution charts were completed in $\mathrm{R}$ by package 'ggplot2'. The meta-analysis was undertaken as described below.

\subsubsection{Correlation analysis}

Correlation analysis was conducted to reveal whether genetic barrier occurrence was different amongst different habitats between VME (hydrothermal vent, cold seep and seamount) and non-VME sites, and VME indicator taxa and non-VME indicator taxa (taxa list sees Chapter 1). Because the data for genetic barrier detection (binomial variable), habitat type (categorical variable), VME (binomial variable) and VME indicator taxa (binomial variable) were not numeric variables, cross tabulation (non-parametric) analysis was conducted to investigate whether barriers to gene flow occurred at different frequencies in different habitat types. All cross tabulation analyses were run in the software package IBM SPSS Statistics (v 22.0), and the $\chi^{2}$ and Cramer's V statistics were employed to test the relationships. 


\subsubsection{Logistic regression analysis}

To quantify the contribution of multiple causative factors to the presence/absence of a genetic barrier, logistic regression analysis was employed to test for genetic barrier detection (barrier detected as 1 and undetected as 0 ) as an independent variable and factors (distances, depth, currents, topography, temperature, biological factors) as predictive variables. In this analysis the variable called "research limitation" (which includes factors such as low genetic marker variability, small sample sizes, restricted range of sampling, etc) was excluded because it does not help to explain how biological and physico-chemical and geological variables contribute to barrier detection. Sequential testing was employed to predict the relative contribution of factors to explain the presence of a genetic barrier (detection). Significant factors were more likely to contribute to a barrier for genetic connectivity in the deep-sea, whilst non-significant factors may promote genetic connectivity or be independent of barrier detection. Due to their non-significance in the primary analysis, joint effects (interactions) between variables were not considered in the logistic regression, which was run in $\mathrm{R}(\mathrm{v} 3.2 .2)$.

\subsubsection{General linear model (GLM) analyses}

For all categories of genetic marker types, a general linear model was built to investigate the effects of sampling regime on genetic barrier detection. The categorical and continuous variables phylum, marker type, number of populations, number of biogeographic provinces, sample sizes, length for sequencing markers or number of loci for genotypic markers, minimum depth and depth range were defined as independent variables, and genetic barrier detection (Yes/No) was the dependent variable.

$F_{S T}$ and $\Phi_{S T}$ values (dependent variables) were analysed separately using GLM with the independent variables being sample size, number of populations, number of genetic markers as covariates and phylum and marker type as fixed factors to determine the contribution of these independent variables to explaining variation in the genetic differentiation $\left(F_{S T}\right.$ or $\left.\Phi_{S T}\right)$. This analysis is a supplementary test of genetic barrier presence based on a continuously distributed dependent value which is an estimate of genetic distance to the analysis described in the previous paragraph which is a categorical variable based on the presence or absence of a genetic barrier.

To determine if increased genetic variation is associated with a higher rate of barrier detection, Spearman (non-parametric) correlation analysis between standardised nucleotide 
diversity and barrier detection ratios amongst different phyla was employed. To obtain the standardised nucleotide diversity, phylum and marker type as fixed factors and sample size (number of specimens examined in the study) and the length of DNA sequence (bp) as covariates were utilised to build the GLM to generate the standardised data.

In the present study, it was hypothesised that the larger depth ranges covered would result in a higher probability of genetic barrier detection. Minimum, maximum and depth range (subtraction of maximum and minimum) were compared between barrier detected and undetected groups. Standardised datasets of minimum, maximum and cover depth produced by GLM analysis with phylum and marker type as fixed factors and sample size and number

of populations as covariates were compared to compensate for the variations between different studies. A t-test was used in the comparisons between detected and undetected groups, and the GLM analysis was based on the subset data that excluded the records with "invariation markers categorised in research limitations".

Joint effects (interactions) between variables were considered in all GLM analyses, and all GLM and t-test analyses were run using the software package IBM SPSS Statistics (v 22.0).

\subsection{Results and Discussion}

\subsubsection{Summary results}

Published investigations covered all oceans, but more than one third of all papers was from the Pacific Ocean (188 of 503 records, 37.38\%). The Indian Ocean was the least studied area (Figure 2.1). According to the deep-sea biogeographic province scheme of Watling et al. (2013), BY4 (North Atlantic Ocean including the Mediterranean Sea), and especially the Mid-Atlantic Ridge region, was the most researched biogeographic province (Figure 2.2) (25.05\% of all records). The region with the lowest representation was the Southeast Pacific Ridges area, BY5 (1 of 503 records, $0.20 \%$ ). Research from other, deeper water provinces (AB1-14, 32 of 503 records, 6.36\%) was much less common than research from shallow water provinces (BY1-14, 471 of 503 studies). None of the population genetics studies was conducted in the hadal provinces (HD 1-10). 


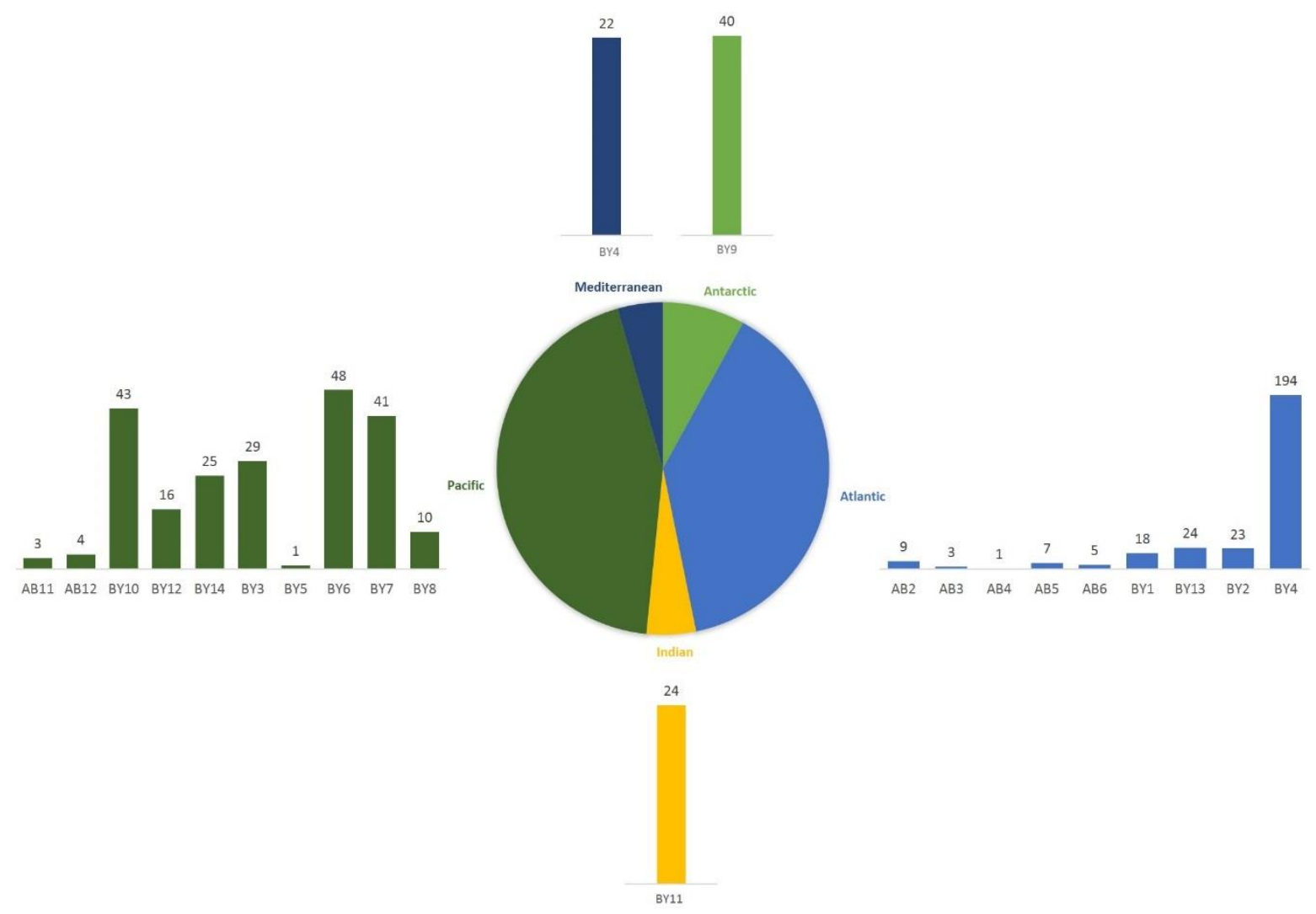

Figure 2.2 The distribution of research covered by areas in deep-sea provinces (bar charts) and at an oceanic scale (pie chart)

In terms of vertical distribution (depth), most studies covered the mesopelagic and bathypelagic zones, and published reports for taxa below $2500 \mathrm{~m}$ were sharply reduced in number (Figure 2.3). More than 200 taxa belonging to 10 phyla were covered in these studies the Annelida (56), Arthropoda (164), Chaetognatha (2), Chordata (86), Cnidaria (75), Echinodermata (28), Foraminifera (14), Mollusca (67), Nemertea (1), and Porifera (10) (Table 2.1, Figure 2.4). As both genetic marker and ship-based sampling technologies have developed profoundly during the period from 1972 to 2016, and there has been a substantial increase in the number of studies published related to population genetic connectivity in the deep sea (Figure 2.5). Early studies used allozyme electrophoresis, and as methods have developed, later studies have used DNA sequencing and fragment analysis of DNA. More recently, still studies have used microsatellites and even single nucleotide polymorphisms (SNPs), as these techniques have become increasingly available and cost-effective. 
Table 2.1 Number of species for which genetic connectivity estimates have been established

\begin{tabular}{|c|c|c|c|c|c|c|}
\hline \multirow{2}{*}{ Phylum } & \multirow{2}{*}{ Allozymes } & \multicolumn{2}{|c|}{ Sequencing } & \multirow{2}{*}{ Microsatellite } & \multirow{2}{*}{ Others } & \multirow{2}{*}{ Total } \\
\hline & & Nuclear & Mitochondrial & & & \\
\hline Nemertea & 1 & & & & & 1 \\
\hline Chaetognatha & & 1 & 1 & & & 2 \\
\hline Porifera & & 2 & 8 & & & 10 \\
\hline Foraminifera & & 8 & 6 & & & 14 \\
\hline Echinodermata & 9 & 1 & 18 & & & 28 \\
\hline Annelida & 8 & 12 & 32 & 2 & 2 & 56 \\
\hline Mollusca & 10 & 10 & 41 & 3 & 3 & 67 \\
\hline Cnidaria & 3 & 23 & 36 & 13 & & 75 \\
\hline Chordata & 5 & & 47 & 27 & 7 & 86 \\
\hline Arthropoda & 14 & 31 & 106 & 9 & 4 & 164 \\
\hline Total & 49 & 88 & 296 & 54 & 16 & 503 \\
\hline
\end{tabular}

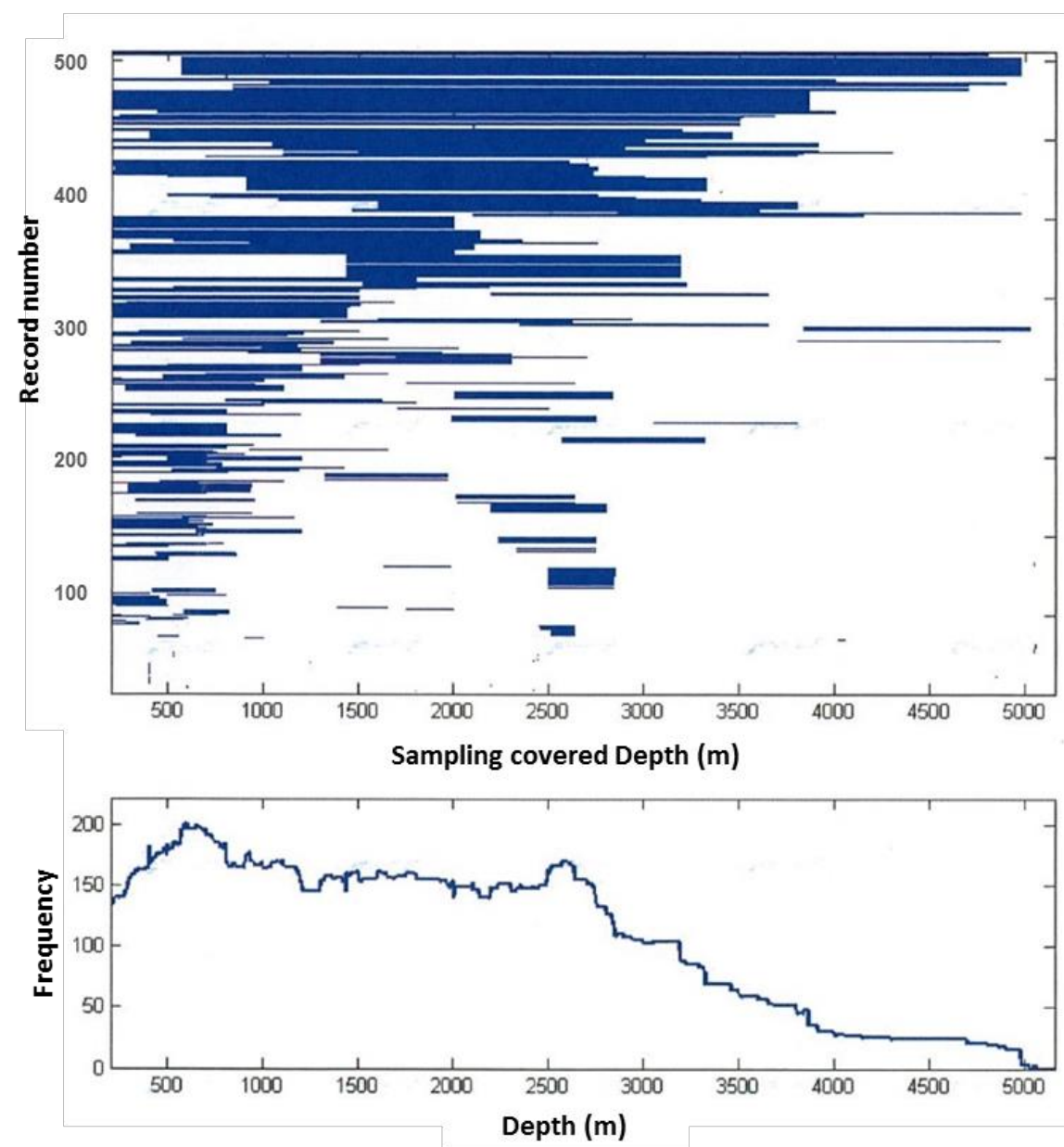

Figure 2.3 Covered depths for each record (lines from minimum to maximum) (top) and accumulated density of examined studies (bottom) 


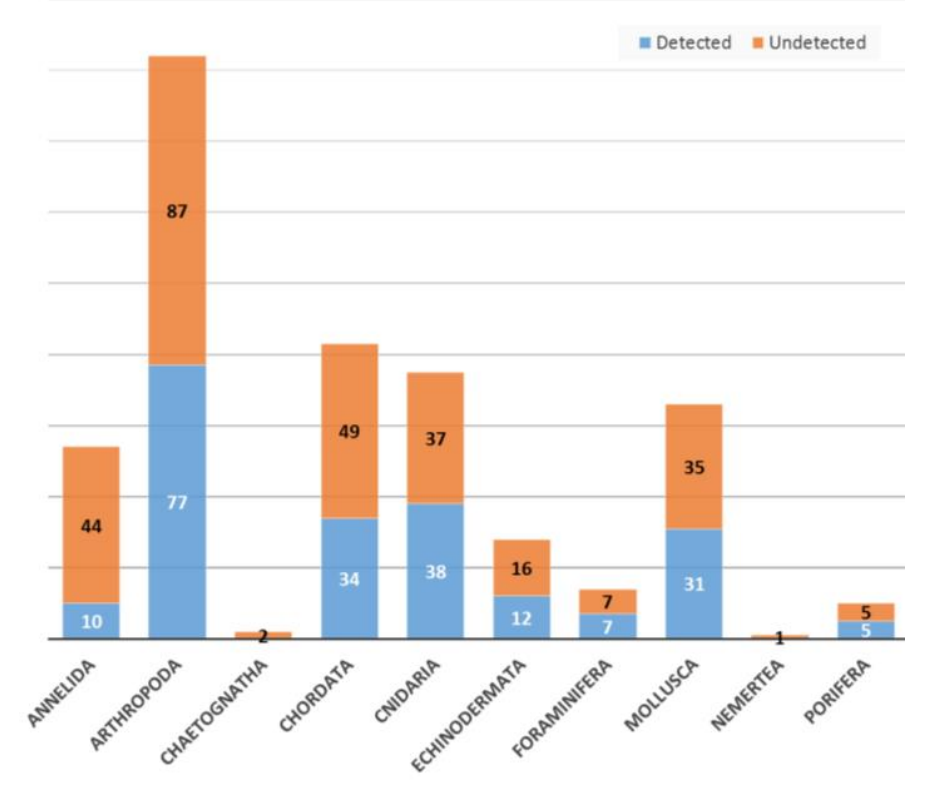

Figure 2.4 Genetic barrier detection distributions across different phyla

Blue is number of cases detected and orange is number of cases not detected

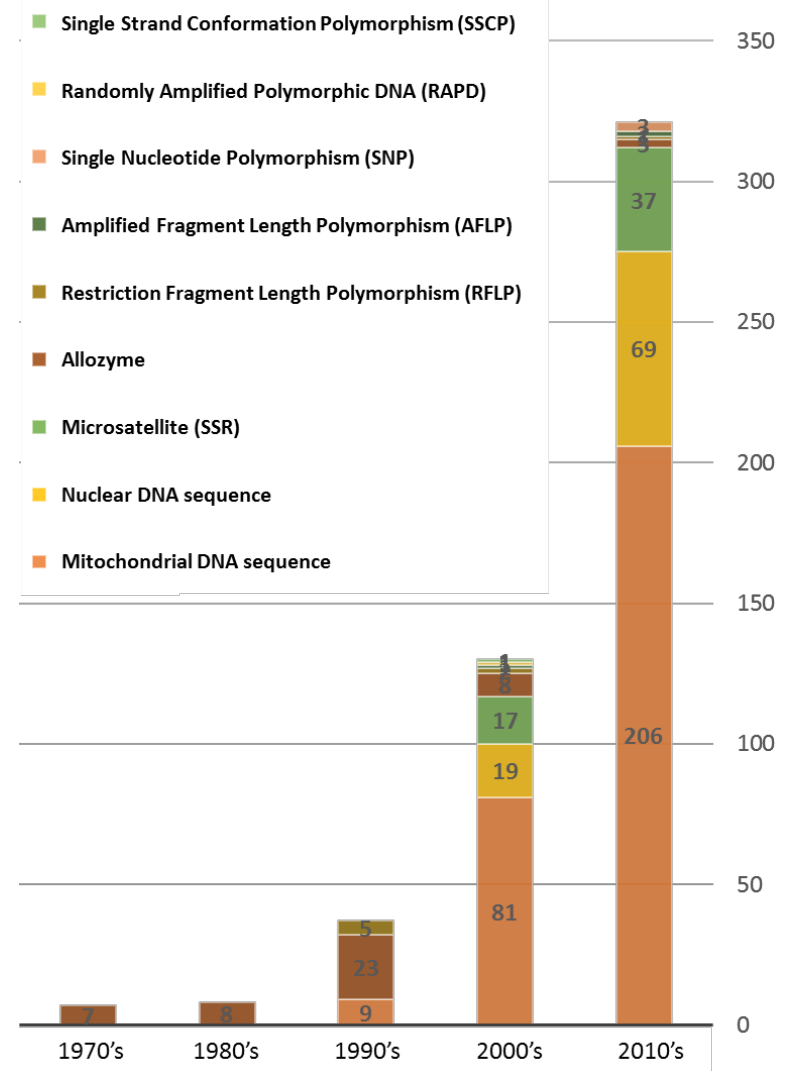

Figure 2.5 Summary of different marker types and the chronological distribution of their usage according to collected records

Numbers indicate number of records per marker type. 


\subsubsection{Patterns of genetic structure reported amongst deep-sea taxa}

It has often been pointed out that in theory global hydrodynamic currents could promote the dispersal ability of larvae (even adults) to broadcast all around the world and thereby form a global pattern of genetic connectivity (McClain \& Hardy, 2010). In addition, deep-sea species may be widely distributed because of strong adult mobility or a long larval dispersal period to overcome oceanographic and topographic barriers, and these factors may generate genetic exchange across long distance over time (Shank, 2010). Despite potential long-distance dispersal and gene flow amongst different populations, it is now recognised that the full genetic exchange of many species amongst different geographic populations is not achieved for several different reasons (this is the difference between potential versus realised dispersal (Reisser et al., 2014).

Various patterns of genetic structures amongst different species have been reported. These patterns of genetic structure at different spatial were tested based on the sampled area, that is, at different spatial scales that mainly including global, oceanic, regional (within ocean amongst provinces) and fine (within province) scale (Figure 2.6).

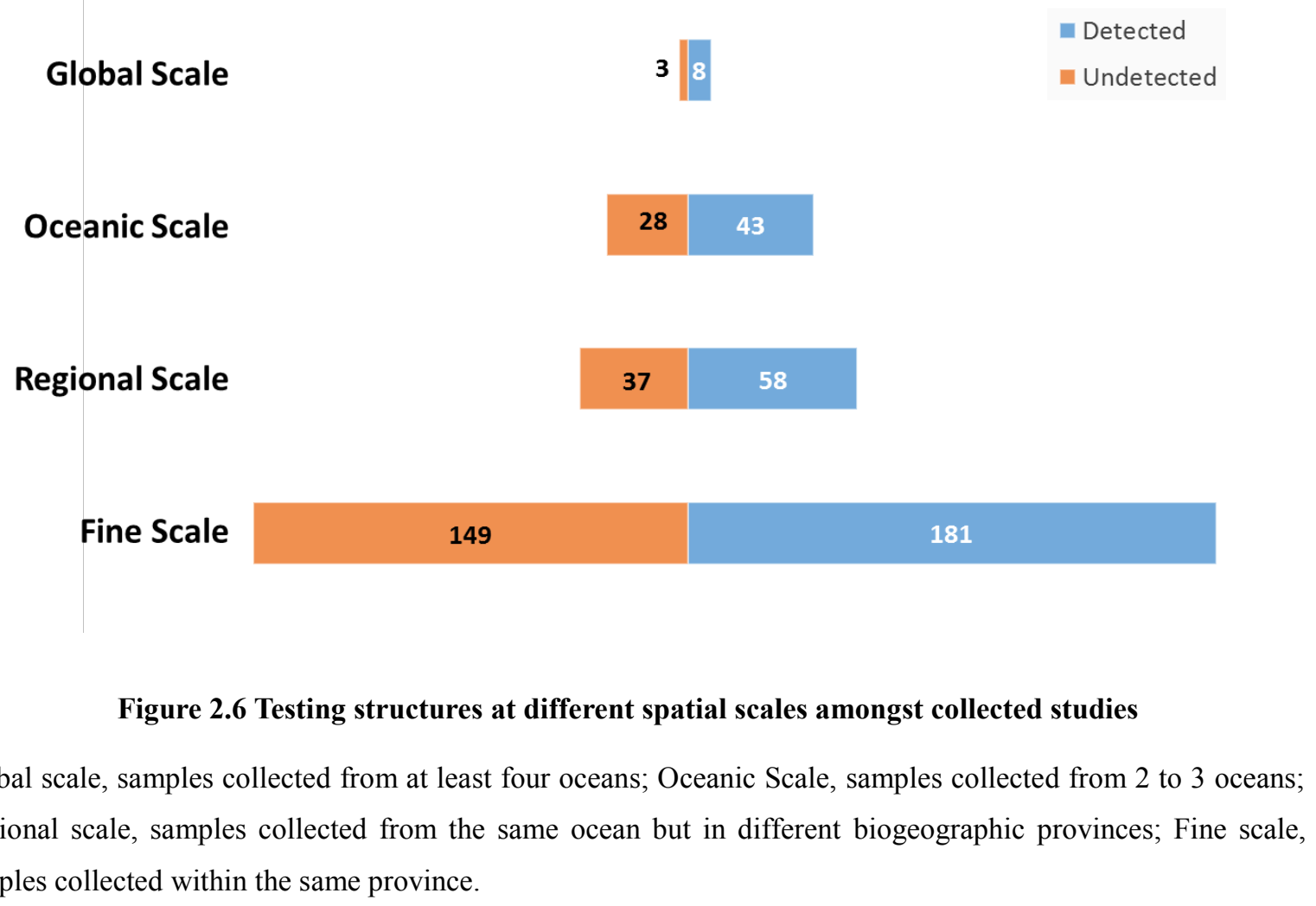




\subsubsection{Globally homogeneous - panmixia}

To test presence/absence of population genetic structure at a global scale (at least four oceans) requires a large number of samples that cover all, or at least most of, the world's oceans. Practically, this is difficult to achieve because of the challenges of sampling logistics and because of the uneven distribution of organisms at a global scale (whilst some taxa are cosmopolitan in their distribution, most are not). In the database, only 11 records were examined for genetic structure at a global scale, and all employed DNA sequencing. Three of these records (Hippasteria phrygiana revealed by ATPS $\alpha$, Epistominella exigua revealed by $12 S$, Epistominella exigua revealed by ITS) did not report evidence of genetic structuring at a global scale (Lecroq et al., 2009; Foltz et al., 2013). However, Foltz et al. (2013) did detect evidence of significant genetic structure in $H$. phrygiana for COI, suggesting that lack of genetic structure at ATPS $\alpha$ at the global scale was because the latter genetic marker was insufficiently informative. For E. exigua, no structure was also detected at an oceanic scale in other studies (Brandt et al., 2007; Pawlows et al., 2007), but all of them used 12S or ITS as the genetic markers. Overall, 9 records detected genetic differentiation at the global scale, and only 2 of 11 did not detect global structure, but more samples and different markers are required to prove globally homogeneous status, that is, panmixia.

\subsubsection{Oceanic scale}

Seventy-one records related to analyses conducted at the oceanic scale, across two or three oceans. Of these, 43 records $(60.56 \%)$ reported significant genetic differentiation across a range of phyla, including the Arthropoda, Chaetognatha, Chordata, Echinodermata, Foraminifera, Mollusca and Porifera. Amongst these studies, the population genetic structure at the oceanic scale was always reported as arising from evolutionary isolation.

Comparing between different oceans, 28 records reported found no genetic structure between oceans across various taxa and different types of markers. This pattern might reflect the fact that deep-sea animals can maintain genetic connectivity at very broad scales. However, more samples and different markers are required to exclude the possibility that the absence of genetic structure was not caused by technical issues such as invariant markers and/or limited sample sizes. 


\subsubsection{Regional scale}

At the regional scale, that is, amongst biogeographic provinces but within the same ocean, of the total 95 records, $58(61.05 \%)$ reported evidence of genetic structure between provinces. Although 37 records reported no genetic structure, of these studies 15 records reported potential issues with small sample sizes, which potentially prevented the detection of genetic structure if it existed.

\subsubsection{Fine scale structure within biogeographic provinces}

Amongst the four different spatial scales examined, studies within biogeographic provinces were the most abundant, representing nearly two of every three records $(65.61 \%)$. Significant genetic structure was reported in more than half $(54.85 \%)$ of these records. Amongst the records of no genetic structure (149 in total), 78 records of them indicated that the absence of structure might be attributable to small sample sizes.

\subsubsection{Factors effecting genetic connectivity}

Different genetic structural patterns amongst different taxa are likely to be influenced by the number and different types of habitats at different scales through a mass environmental effect (Shmida \& Wilson, 1985). It is, however, not clear how numerous environmental factors act to influence genetic connectivity in deep-sea communities.

The existence of one or more genetic barriers (Figure 2.7) was reported in $43.34 \%$ of of the 503 records that were reviewed. Of these, in $31.65 \%$ of the cases the barrier was caused by depth, $23.39 \%$ by biological factors, $20.18 \%$ by distance, $19.26 \%$ by seafloor topography, and $16.51 \%$ by currents. Amongst the genetic barrier undetected records, more than one third of the records $(39.30 \%)$ revealed that small sample sizes and/or noninformative genetic marker type may have contributed to the failure to reveal population genetic differentiation and a barrier to gene flow. About one third $(31.58 \%)$ of all of the records indicated that pelagic larval duration (PLD) contributed to genetic connectivity over different spatial scales, whilst 3.86\% reported that currents assisted larval dispersal (Figure 2.7). Furthermore, in the genetic barrier detected records, $10.49 \%$ reported that distance was not a genetic isolating factor and $7.49 \%$ indicated that depth was not a barrier to gene flow. 


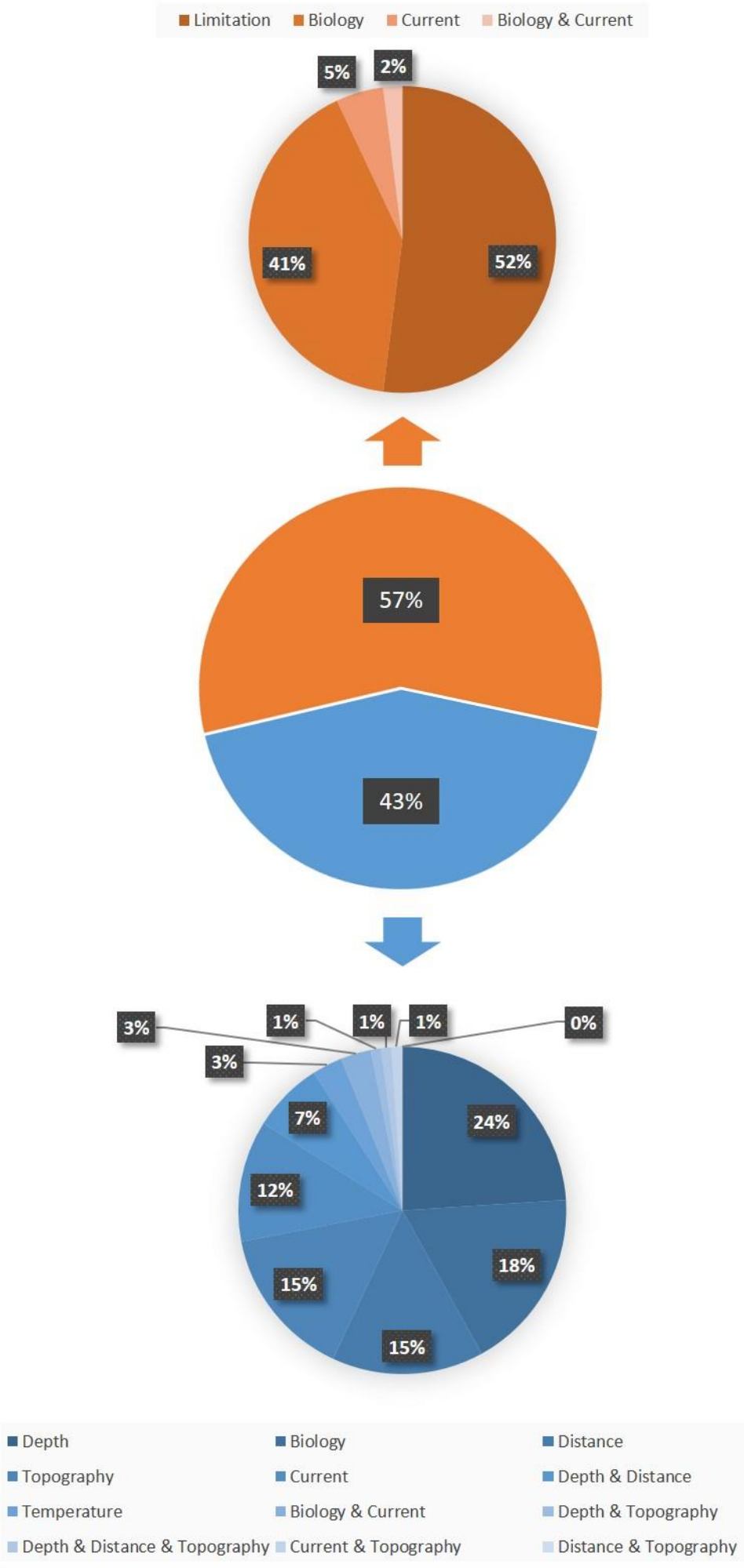

Figure 2.7 Summary of contributions of individual effects amongst categorised factors for genetic barriers detection

The number indicates the percentage of barriers detected (middle pie chart) and the colour represents the detected (blue) and undetected barrier (orange). The number indicatesthe percentage of each categorised factors contributing to genetic barrier absence (left pie chart) and presence (right pie chart). 


\subsubsection{Validation of factors}

As summarised and discussed above, the effects of each factor on genetic connectivity were complex. Whilst currents and life-history characteristics could both weaken and strengthen genetic connectivity, other factors were also reported that may cause genetic isolation or independence from shaping genetic connectivity. However, whether these factors were related to genetic barriers and how important these factors are as barriers have not been tested yet.

In logistical regression analysis, the whole model was significant $(p<0.0001)$ and the estimates for each factor except life-history characteristic were positive. This means that the factors identified in this study were significantly related with genetic barriers. The quantifying of the contribution to barrier detection indicated that depth, currents and topography were the variables that contributed to the formation of a barrier for genetic connectivity and that biological factors tended to promote genetic connectivity at different spatial. In the sequential test for the regression, depth (after adding depth, the residual deviance was greatly reduced) contributed most in the model amongst the reviewed studies, implying that depth was the most common barrier for deep-sea organismal gene flow. Although temperature and biological factors did not contribute significantly to the barrier detection, each of their residual deviances was reduced after its addition to the model (Table 2.2). Further and detailed discussions are given in the following paragraphs.

Logistic regression analysis results indicated that currents were significant $(p=0.037)$, whereas sequential testing indicated that the contribution of currents to the model was borderline non-significant ( $p=0.062$ ) (Table 2.3). Overall, these results suggest that currents do not play a major role as barriers to gene flow amongst deep-sea taxa.

Table 2.2 Logistical regression analyses of the contribution of different factors to the detection of barriers to gene flow amongst multiple deep-sea taxa

\begin{tabular}{|c|c|c|c|c|}
\hline Variables & Estimate & Std. Error & $z$ value & $\mathbf{p}$ \\
\hline Intercept & 1.443 & 0.125 & 11.523 & $<0.001 * * *$ \\
\hline Distances & 0.437 & 0.290 & 1.509 & 0.131 \\
\hline Depth & 0.937 & 0.306 & 3.060 & $0.002 * *$ \\
\hline Currents & 0.722 & 0.346 & 2.089 & $0.037 *$ \\
\hline Topography & 0.908 & 0.397 & 2.285 & $0.022 *$ \\
\hline Temperature & 0.351 & 1.098 & 0.319 & 0.749 \\
\hline Biological factors & -0.340 & 0.219 & -1.551 & 0.121 \\
\hline
\end{tabular}

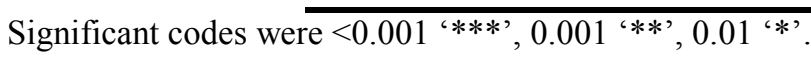


Table 2.3 Sequential test for factors to barrier detection

\begin{tabular}{|c|c|c|c|c|c|}
\hline Adding variables & Df & Deviance & Resid. Df & Resid. Dev & $\mathbf{p}$ \\
\hline Null & & & 502 & 447.310 & \\
\hline Distance & 1 & 3.300 & 501 & 444.020 & 0.069 \\
\hline Depth & 1 & 9.882 & 500 & 434.130 & $0.002 *$ \\
\hline Currents & 1 & 3.495 & 499 & 430.640 & 0.062 \\
\hline Topography & 1 & 5.820 & 498 & 424.820 & $0.016^{*}$ \\
\hline Temperature & 1 & 0.106 & 497 & 424.710 & 0.745 \\
\hline Biological factors & 1 & 2.419 & 495 & 421.590 & 0.120 \\
\hline
\end{tabular}

\subsubsection{Horizontal distance}

Of the 95 records that discussed distance effects, 28 records reported that genetic connectivity patterns did not conform to an isolation by distance pattern (i.e., distance was not a genetic barrier) (Figure 2.7). The logistical regression and sequential test showed that distance was non-significant in the model, which indicated that distance was not a main factor acting as a barrier to gene flow (Table 2.2 and Table 2.3).

Isolation by distance (IBD) is the tendency of populations that are geographically closer to be more genetically similar than populations that are further apart. Both dispersal variance and migration probabilities may contribute to local genetic differentiation (Ishida, 2009). Genetic similarity across distance for deep-sea organisms is most likely to be attributable to currents, especially deep currents (or near-bottom flow), which have pervasive effects because they can influence deep-sea larval and adult dispersal directly. This may explain why the distance was not a significant contributor for barrier detection in logistical and sequential tests. Unlike many shallow water invertebrates, large horizontal distances $(100 \mathrm{~s}$ of $\mathrm{km})$ do not act as barriers to gene flow for several deep-sea species, such as Argyropelecus hemigimnus (Chordata) (Gordeeva, 2014), Austrolaenilla pelagica (Annelida) (Neal et al., 2014), Callogorgia americana delta (Cnidaria) (Quattrini 2014), Gigantopelta chessoia (Mollusca) (Roterman et al., 2016) and Kiwa tyleri (Arthropoda) (Roterman et al., 2016). This observation may be related to the longer PLD for deep-sea species compared to shallow and eurybathic species (Hilário et al., 2015).

In the present review, 18 studies revealed an IBD pattern within a single deep-sea biogeographic province (i.e., within an environmentally similar region). The reason that fewer deep-sea than shallow water species exhibited a pattern of IBD may be explained by the double constraint of small habitat patch sizes in the deep sea (e.g., seamount, vent and seep sites) and different reproduction and dispersal strategies (Selkoe et al., 2014). 


\subsubsection{Depth and associated gradients}

In the present study, 87 records reported depth-associated genetic differentiation in various habitats, including the slope, seamounts, and hydrothermal vents. Amongst collected studies that detected genetic barriers, depth was the most common factor acting as a barrier for gene flow (solely for about $24 \%$ and jointly for about $9 \%$ of all studies) (Figure 2.7). Both the results of the logistical and sequential test agreed that depth was a significant contributing explanatory factor to the detection of a barrier to gene flow $(\mathrm{p}<0.01)$ (Table 2.2 and Table 2.3).

In the deep sea, as depth increases, so the pressure, salinity and density also increase, but the temperature, dissolved oxygen and alkalinity decrease. Eventually, at hadal depths (> $6000 \mathrm{~m}$ ) the environment is more stable, with the cold temperature, high pressure and less alkaline $\mathrm{pH}$ (Pibernat et al., 2007). This different environment from shallow seas puts deepsea organisms under strong ecological pressure, which may lead to a change in the composition of genes within a species and between shallow and deep populations. For example, whole genome comparison between a shallow-water urchin Strongylocentrotus purpuratus and a deep-sea urchin Allocentrotus fragilis has illustrated that genes have undergone selection for the deep sea (Oliver et al., 2010), supporting the hypothesis that depth-derived selection may cause divergence of morphology and genetic mutations.

Except for different environment genetic selection, the gradients associated with depth were restricted to the vertical distributions of deep-sea organisms, and this discontinuous distribution indirectly affected gene flow. For example, deep-sea stony coral survival and colonisation potential may be related to the concentrations of $\mathrm{O}_{2}$ and $\mathrm{CO}_{3}{ }^{2-}$ (Thiagarajan et al., 2013, Desmophyllum dianthus), and to temperature (Brooke et al., 2013, Lophelia pertusa). The ongoing genetic divergence and larval dispersal disconnection caused by depth and associated deep-sea environmental gradients ultimately separate the gene pools of taxa at different depths.

The depth at which genetic breaks occurred for different species varied a lot across taxa. There were two patterns of depth-associated barriers: a single barrier present and multi breaks across the vertical distribution. In both the North and South Atlantic populations of the deep-sea bivalve Deminucula atacellana above and below approximately 3,300 m were observed to be genetically distinct, whilst within these depth regimes the populations showed little divergence (Chase et al., 1998). Similar cases of depth differentiation have been detected in bathyal brittle-stars (O'Hara et al., 2014), the coral D. dianthus (Miller et al., 
2011), the amphipod Eurythenes gryllus (France \& Kocher, 1996; Havermans et al., 2013), and the seastar Zoroaster fulgens (Howell et al., 2004). For multi barriers patterns, significant differences were found amongst echinoderms at 250-m depth intervals for the Asteroschema clavigera and at 100-m depth intervals for Ophiocreas oedipus (Cho \& Shank 2010).

However, depth does not appear to be an important structuring factor in several largescale studies, including those of Paragorgia arborea (Herrera et al., 2012), Bathybembix bairdii (Siebenaller, 1978) and Frigidoalvania brychia (Quattro et al., 2001), all of which exhibited high genetic similarity amongst different depth classes. As far as it is possible to state at the moment, the relationship between depth and genetic differentiation is speciesspecific. Besides, amongst those studies that revealed that depth was not a barrier to gene flow, most had some sort sampling bias (all of them covered a sampling depth range of $<1,400 \mathrm{~m}$ ). To verify whether sampling bias caused failures to reveal depth-associated barriers, a further investigation was conducted in research limitations.

\subsubsection{Oceanic currents}

In the reviewed literature, 64 records considered that currents affected connectivity patterns, with currents having both positive and negative effects on population connectivity (Figure 2.7). The positive (promoting gene flow, 14 records) and negative (acting as a barrier to gene flow, 26 records) aspects of currents may explain why the factor currents was found to contribute significantly to barrier detection (logistical regression) but not contribute significantly to being a barrier (sequential test) to genetic connectivity in the present analysis (Table 2.2 and Table 2.3).

Oceanic currents are complex, derived from many factors, and it is this complexity that makes assessing genetic connectivity very difficult. Currents may not only bring food, nutrients and other resources to deep-sea taxa, but currents may also directly contribute to the dispersal of species through larval supply, and also at sufficiently high speeds will erode the sediments and many of the organisms on or within them (Levin et al., 2001; Watanabe et al., 2009). Furthermore, in the Horizontal Distances section, currents were considered as the factors that assisted long-distance larval dispersal. Also, ocean currents may, through their influence on larval transport and recruitment, interact with the genetic consequences of the large variance in individual reproductive success to generate patterns of fine-scale patchy genetic structure (Banks et al., 2007). 
This two-sided aspect of currents (promoting and hindering the connectivity) may be demonstrated by two examples, the Antarctic Circumpolar Current (ACC) and equatorial currents in East Pacific. The ACC is an example of how a current may act as a conveyor belt for connectivity. In the present study, 57.14\% records were reported as being genetically homogeneous in the region of the Antarctic Convergence. ACC connects the southern regions of the Atlantic, Pacific and Indian oceans, and serves as a principal pathway of exchange amongst them, promoting larval dispersal. Therefore, it is not surprising that with assistance of the ACC the taxon-specific populations were found to be genetically homogeneous within this region (biogeographic province BY9). In contrast, in the deep-sea province BY7 (the Cocos Plate), extensive genetic differentiation along the East Pacific Rise has been reported that is related to equatorial currents (Bucklin, 1988; France et al., 1992; Craddock et al., 1997; Won et al., 2003; Hurtado et al., 2004; Matabos et al., 2008; Plouviez et al., 2009, 2010, 2013; Coykendall et al., 2011). In other words, the equatorial currents impeded the bilateral connection of larval from north and south of the equator.

Arellano et al. (2014) provided direct evidence that the long-lived larvae of two coldseep molluscs (Bathymodiolus childressi and Bathynerita naticoidea) migrate hundreds of meters above the ocean floor. In this way, both deep water and shallow water currents may transport the larvae of deep-sea taxa, highlighting the interactions between biological factors such as larval vertical behaviour and physical oceanography. For example, larvae may exhibit behavioural adaptations that allow them to move up into surface waters thereby taking advantage of the faster moving surface currents (compared to deeper currents) that assist deep-sea organisms in their dispersal.

\subsubsection{Seafloor topography}

In the database, 39 records (33 solely and 6 with other factors) revealed that topography may act as a barrier to gene flow (Figure 2.7). In the logistical and sequential analyses, a significant contribution of topography was detected in both analyses (Table 2.2 and Table 2.3), which indicated that topography is an important factor acting as a genetic barrier.

Topography may shape directly the deep-sea animals' settlement and colonisation, as well as indirectly through modification of other environmental factors (such as sediments) (Gage, 1996). From the collected publications, 3 studies conducted within any one basin reported that study populations were all genetically homogeneous (but note that number of 
sampled populations and number of sampled individuals may be small): not surprisingly, populations from different basins were more likely to be genetically differentiated (but no significant IBDs detected). This result may be interpreted, at least in part, as indicating a lack of topographic features as barriers to larval dispersal within any one deep-sea basin. In contrast, topographic features, especially pronounced changes in bathymetry (e.g., ridges) are associated with barriers to genetic connectivity for deep-sea animals. For instance, the Strait of Gibraltar with its shallow depth acts as a barrier for genetic connectivity between the deeper basins either side of it, the North Atlantic Ocean and the Mediterranean Sea (e.g., Catarino et al., 2015), and the Florida Straits also act to constrict animal movements northward (e.g., Portnoy et al., 2014).

\subsubsection{Temperature}

In the present study, only 7 records made mention of the influence of temperature on genetic connectivity and the logistical and sequential tests indicated that temperature does not explain or contribute to the presence of a barrier to gene flow amongst deep-sea taxa (Figure 2.7, Table 2.2 and Table 2.3).

Temperature, which is not independent of latitude, hydrothermal system or depth, has important implications for species' distributions and genetic barrier formation (Jorde et al., 2015). In ophiuroid populations, genetic connectivity may be adapted to, or at least related to different temperatures, with phylogeographical breaks occurring between tropical, temperate and polar regions (O'Hara et al., 2014). However, a recent modelling approach focusing on ophiuroid diversity has suggested that this pattern might be driven by energy availability (Woolley et al., 2016), which in turn, may not be completely independent of sea surface temperature. These indirect contributions to genetic connections might explain why temperature was non-significant in both analyses.

\subsubsection{Biological factors}

In this study, 116 records pointed out that biological factors contribute directly to promoting genetic connectivity amongst populations at different scales. Meanwhile, 39 records reported that life-history characteristics limited the genetic connections between populations (Figure 2.7). Both the logistical and sequential tests supported the hypothesis that biological factors related to life-history characteristics were not likely to be a genetic barrier ( $>0.05)$ (Table 2.2 and Table 2.3). 
Although information about mode of reproduction and larval dispersal strategies is important to help interpret the connectivity of different populations, biological knowledge of this sort for most deep-sea species is still unknown. Although a further categorised analysis of the variables in the group "biological factor" was not possible in the present study, three aspects were identified from the collected studies to explain positive and negative effects on genetic connectivity: larval dispersal and reproduction, population history, and adult mobility and body size.

\subsection{Larval dispersal and reproduction}

Biogeographic patterns are generally considered to be linked to larval ecology, because organisms with low dispersal capabilities (and hence reduced gene flow) are expected to have restricted distributions. In the present study, a longer life-duration and a prolonged pelagic larval phase amongst most teleost fishes suggested a high potential for connectivity and gene flow between different geographic populations through dispersal (e.g., Aphanopus carbo (Longmore et al., 2014), and Beryx decadactylus (Friess \& Sedberry, 2011)). Lecithotrophic species have relatively few eggs and a short larval period compared to planktotrophic species, but planktotrophic larvae are constrained by the availability of food (Young, 2003), and it is expected that species that possess these two different larval types will ultimately have different connectivity patterns. This presumption has subsequently been demonstrated by O'Hara et al. (2014) for five deep-sea ophiuriods. Factors such as inbreeding, self-recruitment and asexual reproduction all help to maintain low genetic diversity within subpopulations and contribute to high genetic variability from site to site. For example, in Lophelia pertusa, local recruitment of larvae leads to limited gene flow amongst populations (Le Goff-Vitry et al., 2004). Also, higher levels of genetic differentiation (80\%) were detected in Annelida compared with other phyla. This finding for Annelida may be related to their trochophore larval stage, and it also could explain why distance was reported to be a genetic barrier for most annelids.

\subsection{Population history}

In the evolutionary origin of deep-sea taxa, genetic subdivision may arise across ocean expanses following radiation and dispersal events and also during major geological and mass extinction events (Herrera et al., 2012, 2015). These different evolutionary histories are important in explaining various forms of pronounced differentiation between populations in 
close geographic proximity. In addition, a relatively recent population bottleneck or speciation event followed by limited migration and the beginnings of regional divergence may result in limited differentiation or even genetic homogeneity. For example, hydrothermal shrimp, Rimicaris exoculata, was reported to have undergone a recent population expansion and connectivity along the Mid-Atlantic Ridge (Creasey et al., 1996; Petersen et al., 2010; Teixeira et al., 2012).

\subsection{Adult mobility and body size}

Mobility of adults affects genetic connectivity. In giant squid (Architeuthis dux), a cosmopolitan species with high adult mobility, there was no detectable phylogenetic structure at the mitochondrial level (Winkelmann et al., 2013). Similarly, low levels of global genetic differentiation were reported in the deep-sea teleost Hoplostethus atlanticus (Smith et al., 1996b; Varela et al., 2012, 2013). Great swimming ability of this species is expected to achieve population exchange and the formation of a metapopulation.

Small body size may make for easily transported propagules that contribute to high levels of connectivity and a global distribution. For instance, foraminifera are a common and cosmopolitan member of the microfauna in the deep sea. Epistominella vitrea was collected from a depth range of 30 to $4803 \mathrm{~m}$, but no genetic differentiation was detected at different depths or amongst geographic populations based on data from different marker types (Pawlowski et al., 2007b). Similar results were found in other foraminifera, including Epistominella exigu, Cibicides wuellerstorfi and Oridorsalis umbonatus (Brandt et al., 2007; Pawlowski et al., 2007b; Lecroq et al., 2009), suggesting that small body size contributes to easy transport and high genetic connectivity.

\subsubsection{Research limitations}

The GLM analyses of sampling regime (Table 2.4) showed that the variables depth range, number of bio geographic provinces, number of populations, phylum, marker type and sample size were significant in explaining variation in genetic barrier detection $(p<0.05)$ (Table 2.4). The GLM analyses of $F_{S T}$ (Table 2.5) and $\Phi_{S T}$ (Table 2.6) showed that not all the sampling parameters influenced the minimum value of genetic differentiation $\left(F_{S T}\right.$ for genotypic markers and $\Phi_{S T}$ for sequencing markers). However, the maximum value of $F_{S T}$ was significantly affected by phylum, marker types and their interactions, and $\Phi_{S T}$ was 
significantly affected by sample size, number of populations and phylum. As discussed below, these parameters were mainly related to two aspects of genetic markers and sampling.

Table 2.4 General linear model analyses of sampling regime on genetic barrier detection

\begin{tabular}{cccccc}
\hline Source & Type III Sum of Squares & df & Mean Square & F & Sig. \\
\hline Corrected model & 22.774 & 48 & 0.474 & 2.279 & 0.000 \\
Intercept & 1.920 & 1 & 1.920 & 9.225 & 0.003 \\
Minimum depth & 0.019 & 1 & 0.019 & 0.093 & 0.761 \\
Depth range & 0.892 & 1 & 0.892 & 4.286 & 0.039 \\
Total Samples & 0.146 & 1 & 0.146 & 0.700 & 0.404 \\
Number of populations & 0.232 & 1 & 0.232 & 1.114 & 0.292 \\
Number of provinces & 1.405 & 1 & 1.405 & 6.751 & 0.010 \\
DNA sequenced length/number of & 0.106 & 1 & 0.106 & 0.507 & 0.477 \\
loci & 6.287 & 8 & 0.786 & 3.775 & 0.000 \\
Phylum & 0.471 & 2 & 0.236 & 1.132 & 0.324 \\
Marker yype & 1.288 & 6 & 0.215 & 1.032 & 0.405 \\
Habitat & 2.858 & 7 & 0.408 & 1.961 & 0.060 \\
Phylum * Marker type & 3.831 & 12 & 0.319 & 1.533 & 0.112 \\
Phylum * Habitat & 0.546 & 4 & 0.136 & 0.655 & 0.624 \\
Marker type * Habitat & 0.581 & 3 & 0.194 & 0.930 & 0.427 \\
Phylum * Marker type * Habitat & 58.706 & 282 & 0.208 & & \\
Error & 186.000 & 331 & & & \\
Total & 81.480 & 330 & & & \\
Corrected Total & & & & &
\end{tabular}

R Squared $=0.280$ (Adjusted R Squared $=0.157$ ).

\subsection{Genetic markers}

Choice of appropriate markers is always a concern for genetic studies. In studies involving assessments of patterns of multi-species genetic variability, concerns about the comparability of genetic data have arisen where analyses of different markers in a single species have produced conflicting results (Ayala \& Valentine 1974; Craddock et al., 1995; Hensley et al., 1995; Jollivet et al., 1995; Hurtado et al., 2004; Plouviez et al., 2009, 2013) or inconsistent results (Abbiati et al., 1993; Costantini et al., 2007, 2010). These different patterns of response may arise because of differences in sample size and/or with the variability of the different marker types (i.e., the inherent properties of specific marker types).

Amongst the collected studies, no significant Spearman correlation coefficients were observed between nucleotide diversity (neither minimum nor maximum, standardised or unstandardised) and barrier detection ratios (Table 2.7). This result might indicate that genetic structures varied across the different species because the standardised nucleotide diversity values excluded sampling effects. 
Table 2.5 Different sampling parameter effects on $F_{S T}$ measures

\begin{tabular}{|c|c|c|c|c|c|c|}
\hline Source & & $\begin{array}{c}\text { Type III Sum } \\
\text { of Squares }\end{array}$ & df & $\begin{array}{c}\text { Mean } \\
\text { Square } \\
\end{array}$ & $\mathbf{F}$ & Sig. \\
\hline \multirow{2}{*}{ Corrected Model } & $F_{S T} \mathrm{Min}$ & $0.162^{\mathrm{a}}$ & 21 & 0.008 & 1.994 & 0.025 \\
\hline & $F_{S T} \operatorname{Max}$ & $2.281^{\mathrm{b}}$ & 21 & 0.109 & 4.422 & 0.000 \\
\hline \multirow{2}{*}{ Intercept } & $F_{S T}$ Min & 0.002 & 1 & 0.002 & 0.632 & 0.431 \\
\hline & $F_{S T} \operatorname{Max}$ & 0.026 & 1 & 0.026 & 1.054 & 0.310 \\
\hline \multirow{2}{*}{ Minimum depth } & $F_{S T}$ Min & 0.000 & 1 & 0.000 & 0.064 & 0.802 \\
\hline & $F_{S T} \operatorname{Max}$ & 0.002 & 1 & 0.002 & 0.082 & 0.775 \\
\hline \multirow{2}{*}{ Depth range } & $F_{S T}$ Min & 0.002 & 1 & 0.002 & 0.525 & 0.472 \\
\hline & $F_{S T} \operatorname{Max}$ & 0.001 & 1 & 0.001 & 0.031 & 0.862 \\
\hline \multirow{2}{*}{ Sample size } & $F_{S T}$ Min & 0.000 & 1 & 0.000 & 0.017 & 0.898 \\
\hline & $F_{S T}$ Max & 0.003 & 1 & 0.003 & 0.111 & 0.740 \\
\hline \multirow{2}{*}{ Number of populations } & $F_{S T}$ Min & 0.000 & 1 & 0.000 & 0.006 & 0.941 \\
\hline & $F_{S T}$ Max & 0.007 & 1 & 0.007 & 0.301 & 0.586 \\
\hline \multirow{2}{*}{ Number of provinces } & $F_{S T}$ Min & 0.001 & 1 & 0.001 & 0.168 & 0.684 \\
\hline & $F_{S T} \operatorname{Max}$ & 0.006 & 1 & 0.006 & 0.236 & 0.629 \\
\hline \multirow{2}{*}{ Number of loci } & $F_{S T}$ Min & 0.002 & 1 & 0.002 & 0.588 & 0.447 \\
\hline & $F_{S T} \operatorname{Max}$ & 0.023 & 1 & 0.023 & 0.944 & 0.336 \\
\hline \multirow{2}{*}{ Phylum } & $F_{S T}$ Min & 0.025 & 4 & 0.006 & 1.613 & 0.186 \\
\hline & $F_{S T}$ Max & 0.449 & 4 & 0.112 & 4.574 & 0.003 \\
\hline \multirow{2}{*}{ Marker type } & $F_{S T}$ Min & 0.048 & 5 & 0.010 & 2.470 & 0.045 \\
\hline & $F_{S T} \operatorname{Max}$ & 1.038 & 5 & 0.208 & 8.455 & 0.000 \\
\hline \multirow{2}{*}{ Phylum * Marker type } & $F_{S T}$ Min & 0.049 & 6 & 0.008 & 2.121 & 0.068 \\
\hline & $F_{S T}$ Max & 0.381 & 6 & 0.063 & 2.582 & 0.030 \\
\hline \multirow{2}{*}{ Error } & $F_{S T}$ Min & 0.186 & 48 & 0.004 & & \\
\hline & $F_{S T} \operatorname{Max}$ & 1.179 & 48 & 0.025 & & \\
\hline \multirow{2}{*}{ Total } & $F_{S T}$ Min & 0.406 & 70 & & & \\
\hline & $F_{S T} \operatorname{Max}$ & 5.368 & 70 & & & \\
\hline \multirow{2}{*}{ Corrected Total } & $F_{S T}$ Min & 0.349 & 69 & & & \\
\hline & $F_{S T} \operatorname{Max}$ & 3.460 & 69 & & & \\
\hline
\end{tabular}

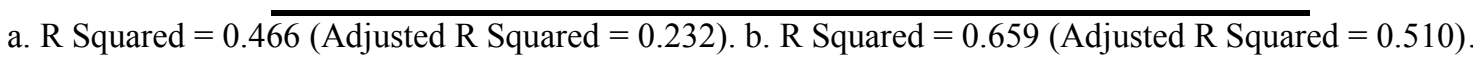


Table 2.6 Different sampling parameter effects on $\Phi_{S T}$ measures

\begin{tabular}{|c|c|c|c|c|c|c|}
\hline Source & & $\begin{array}{c}\text { Type III Sum } \\
\text { of Squares }\end{array}$ & df & $\begin{array}{c}\text { Mean } \\
\text { Square }\end{array}$ & $\mathbf{F}$ & Sig. \\
\hline \multirow{2}{*}{ Corrected Model } & $\Phi_{S T}$ Min & $.546^{\mathrm{a}}$ & 18 & 0.030 & 0.653 & 0.845 \\
\hline & $\Phi_{S T} \operatorname{Max}$ & $3.071^{\mathrm{b}}$ & 18 & 0.171 & 2.821 & 0.001 \\
\hline \multirow{2}{*}{ Intercept } & $\Phi_{S T} \operatorname{Min}$ & 0.045 & 1 & 0.045 & 0.977 & 0.326 \\
\hline & $\Phi_{S T} \operatorname{Max}$ & 0.747 & 1 & 0.747 & 12.348 & 0.001 \\
\hline \multirow{2}{*}{ Minimum depth } & $\Phi_{S T}$ Min & 0.000 & 1 & 0.000 & 0.009 & 0.926 \\
\hline & $\Phi_{S T} \operatorname{Max}$ & 0.069 & 1 & 0.069 & 1.141 & 0.289 \\
\hline \multirow{2}{*}{ Depth range } & $\Phi_{S T} \operatorname{Min}$ & 0.000 & 1 & 0.000 & 0.001 & 0.973 \\
\hline & $\Phi_{S T} \operatorname{Max}$ & 0.008 & 1 & 0.008 & 0.133 & 0.716 \\
\hline \multirow{2}{*}{ Sample size } & $\Phi_{S T} \operatorname{Min}$ & 0.096 & 1 & 0.096 & 2.067 & 0.155 \\
\hline & $\Phi_{S T} \operatorname{Max}$ & 0.720 & 1 & 0.720 & 11.901 & 0.001 \\
\hline \multirow{2}{*}{ Number of populations } & $\Phi_{S T} \operatorname{Min}$ & 0.077 & 1 & 0.077 & 1.650 & 0.203 \\
\hline & $\Phi_{S T} \operatorname{Max}$ & 0.790 & 1 & 0.790 & 13.068 & 0.001 \\
\hline \multirow{2}{*}{ Number of provinces } & $\Phi_{S T} \operatorname{Min}$ & 0.038 & 1 & 0.038 & 0.810 & 0.371 \\
\hline & $\Phi_{S T} \operatorname{Max}$ & 0.022 & 1 & 0.022 & 0.360 & 0.550 \\
\hline \multirow{2}{*}{ Sequence length } & $\Phi_{S T}$ Min & 0.001 & 1 & 0.001 & 0.011 & 0.917 \\
\hline & $\Phi_{S T} \operatorname{Max}$ & 0.001 & 1 & 0.001 & 0.013 & 0.909 \\
\hline \multirow{2}{*}{ Phylum } & $\Phi_{S T} \operatorname{Min}$ & 0.190 & 6 & 0.032 & 0.679 & 0.667 \\
\hline & $\Phi_{S T} \operatorname{Max}$ & 0.860 & 6 & 0.143 & 2.370 & 0.038 \\
\hline \multirow{2}{*}{ Marker type } & $\Phi_{S T} \operatorname{Min}$ & 0.000 & 1 & 0.000 & 0.008 & 0.929 \\
\hline & $\Phi_{S T} \operatorname{Max}$ & 0.002 & 1 & 0.002 & 0.025 & 0.874 \\
\hline \multirow{2}{*}{ Phylum * Marker type } & $\Phi_{S T}$ Min & 0.009 & 5 & 0.002 & 0.037 & 0.999 \\
\hline & $\Phi_{S T} \operatorname{Max}$ & 0.390 & 5 & 0.078 & 1.290 & 0.278 \\
\hline \multirow{2}{*}{ Error } & $\Phi_{S T}$ Min & 3.394 & 73 & 0.046 & & \\
\hline & $\Phi_{S T} \operatorname{Max}$ & 4.416 & 73 & 0.060 & & \\
\hline \multirow{2}{*}{ Total } & $\Phi_{S T}$ Min & 5.817 & 92 & & & \\
\hline & $\Phi_{S T} \operatorname{Max}$ & 20.669 & 92 & & & \\
\hline \multirow{2}{*}{ Corrected Total } & $\Phi_{S T} \operatorname{Min}$ & 3.940 & 91 & & & \\
\hline & $\Phi_{S T} \operatorname{Max}$ & 7.487 & 91 & & & \\
\hline
\end{tabular}

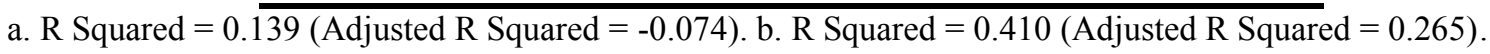

Table 2.7 Average nucleotide diversity of mitochondrial and nuclear genes

\begin{tabular}{ccccccc}
\hline \multirow{2}{*}{ Phylum } & Size & \multicolumn{2}{c}{ Reported } & \multicolumn{2}{c}{ Standardised } & Barrier detection rate \\
\cline { 3 - 6 } & & Min & Max & Min & Max & 80.95 \\
\hline Annelida & 20 & 0.0004 & 0.0584 & $0.008 \pm 0.002$ & $0.011 \pm 0.006$ & 52.90 \\
Arthropoda & 75 & 0.0000 & 0.1568 & $0.004 \pm 0.001$ & $0.020 \pm 0.004$ & 100.00 \\
Chaetognatha & 1 & 0.0220 & 0.0540 & $0.022 \pm 0.009$ & $0.054 \pm 0.023$ & 55.32 \\
Chordata & 41 & 0.0002 & 0.0728 & $0.009 \pm 0.001$ & $0.011 \pm 0.004$ & 40.68 \\
Cnidaria & 30 & 0.0000 & 0.1680 & $0.004 \pm 0.002$ & $0.020 \pm 0.004$ & 84.21 \\
Echinodermata & 17 & 0.0000 & 0.0125 & $0.004 \pm 0.005$ & $0.008 \pm 0.012$ & 50.00 \\
Foraminifera & 6 & 0.0000 & 0.0019 & $0.000 \pm 0.004$ & $0.000 \pm 0.009$ & 44.00 \\
Mollusca & 33 & 0.0000 & 0.0315 & $0.004 \pm 0.002$ & $0.008 \pm 0.005$ & 50.00 \\
Porifera & 7 & 0.0000 & 0.0758 & $0.001 \pm 0.004$ & $0.043 \pm 0.009$ & \\
\hline
\end{tabular}




\subsubsection{2. $\quad$ Sampling}

It is appropriate to accept evidence of no genetic structure only when appropriate molecular markers are employed, when the sample size is large enough, and when the location of sampled populations encompasses the full extent of the known distribution of the taxon in question. In practice, these criteria are rarely met in most deep-sea studies. In the collected data, the mean and median sample sizes were 24.63 and 16.00 individuals per population, respectively (Figure 2.8). Interestingly, 84 of 216 records $(38.89 \%)$ that revealed a genetic differentiation were based on less than 10 individuals per population. Sample size as an independent variable did not explain significant variation in either $F_{S T}$ minimum or $F_{S T}$ maximum values (Table 2.5), and nor did it explain significant variation in $\Phi_{S T}$ minimum although it did explain significant variation in $\Phi_{S T}$ maximum values (Table 2.6).

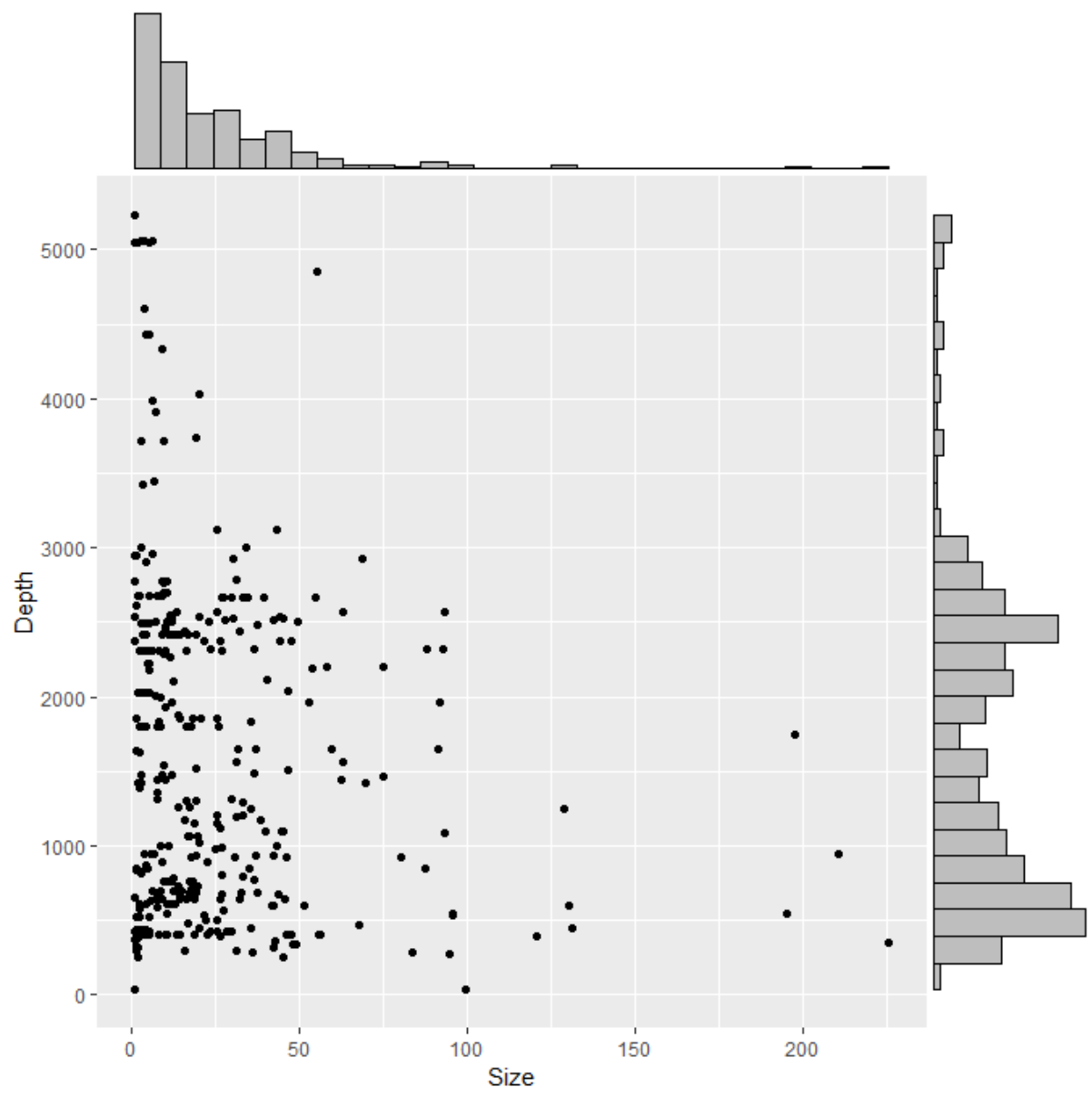

Figure 2.8 Average sample size with depth distribution of reviewed studies

Histogram on top is density of the average sample size, and histogram on the right is density of the average depth. 
For deep-sea genetic studies, samples from different depth collections were important for barrier detection. In the reviewed studies, a greater depth range of sampling resulted in a greater detection rate of genetic differentiation, i.e., depth range is strongly associated with an ability to detect a barrier to gene flow (Table 2.8). The GLM analysis showed that the records of detected barriers had a greater depth range than undetected records $(\mathrm{p}<0.01)$ (Table 2.8). In addition, the number of bio geographic provinces sampled was also significantly associated with barrier detection $(\mathrm{p}<0.01)$, that is, more provinces sampled the greater the chance to detect one or more genetic barriers (Table 2.8). This suggests that genetic barriers may be associated with province boundaries (rather than with distance per se because, as noted previously, distance did not explain significant variation in barrier detection).

Table 2.8 Detection of a barrier to gene flow as a function of depth

\begin{tabular}{ccccc}
\hline \multirow{2}{*}{ Depth barriers } & \multicolumn{2}{c}{ Unstandardised (SD) } & \multicolumn{2}{c}{ Standardised (SE) } \\
\cline { 2 - 5 } & Detected & Undetected & Detected & Undetected \\
\hline Min & $741.579 \pm 751.928$ & $615.163 \pm 654.051$ & $734.011 \pm 56.269 * *$ & $826.930 \pm 78.731$ \\
Max & $2051.842 \pm 1154.352$ & $2480.07 \pm 1467.091$ & $2817.380 \pm 109.970 * *$ & $2656.391 \pm 153.869$ \\
Depth Range & $1310.263 \pm 1240.331$ & $1864.907 \pm 1283.325$ & $2083.368 \pm 113.658^{* *}$ & $1829.461 \pm 159.028$ \\
\hline
\end{tabular}

For population genetic studies, a greater sample size and more populations sampled would tend, on average, to provide more information and to provide more spatially explicit detail. However, a major challenge in studying population genetics in the deep sea is to obtain material that can be used for genetic analysis, given the costs and constraints of sampling. This is especially true for microfauna that are extremely small, difficult to separate from sediment, and are typically fixed in formalin which degrades DNA (Chase et al., 1998). The other disappointing aspect of sampling limitation is that most old specimens are not suitable for DNA extraction. For example, Miller et al. (2010) were unable to get sequence data from scleractinians more than 5-6 years old unless the specimens had been ultra-frozen. Similarly, Boschen et al. (2015) were only able to obtain reasonable quality DNA from 150 of $942(\sim 16 \%)$ archived vent mussels. Although sampling has been problematic in the past, new technologies for both sampling, such as new remotely operated vehicles (ROVs) and autonomously underwater vehicles (AUVs), and genetic research such as next-generation sequencing, may eventually help to elucidate patterns of genetic connectivity in the deep sea. 


\subsubsection{Barrier occurrences amongst habitat types}

Amongst different habitat types, the continental slope was a relatively well-studied area $(48.7 \%$ of all studies), and the water column was the least researched area $(3.4 \%)$ (Figure 2.9). The $\chi^{2}$ test results for barrier detection and the sampled areas showed that barrier detection was significantly different amongst the different habitat types $(p<0.01)$, which indicated that the occurrence of genetic difference was at least in part a function of different sampled efforts within the different habitat types. The rate of barrier detection decreased sequentially according to habitat type, from cold seep, water column, hydrothermal vent, slope, seamount and abyssal plain. All studies in the review reported that the high percentage of barriers to gene flow detected in cold seep and water column habitats were caused by biological factors that were presumably evolutionary adaptations to the habitat in question (e.g. Cunha et al., 2012; Laakmann et al., 2012; Cowart et al., 2013; Faure et al., 2015). Because limited research has been conducted in these habitats, it was difficult to draw further conclusions about the mechanisms that may result in isolation for these two open ecosystems.

In the present review, it was found that $66.32 \%$ of cases from hydrothermal vents and $75.00 \%$ of cases from seeps were genetically isolated. However, amongst 109 hydrothermal vent records in this study, only 6 records reported that the thermal system was the main factor that contributed to the isolation. The high variability of environmental conditions at hydrothermal vents (steep thermal gradients and high chemical heterogeneity) and the constant modification of the hydrothermal vent landscape due to the fluid displacement has led to recurrent 'crash and flush' in populations that is likely to promote genetic structure and cause localised low genetic diversity (Faure et al., 2009). In other studies of vents, distance and currents were more important than habitat type in explaining genetic differentiation. However, some vent-associated species seem to have a long larval dispersal duration, which may help to achieve genetic connection between patches of habitat. For instance, Kojima et al. (2003, 2006) found that tube worms, Arcovestia ivanovi and Lamellibrachia juni, had migrated from hydrothermal fields in the Eastern Pacific Rise to those in the back-arc basins in the western Pacific, which indicates that long distance genetic connectivity exists. A similar result was also obtained for other hydrothermal species, including Riftia pachyptila (Bucklin 1988; Black et al., 1994; Coykendall et al., 2011), Bathymodiolus thermophiles (Craddock et al., 1995), and Tevnia jerichonana and Oasisia alvinae (Black et al., 1998). In addition, Austinograea rodriguezensis, Rimicaris kairei, Alviniconcha sp. and populations of 
the scaly-foot gastropod were all reported has having high connectivity and therefore low levels of genetic structure along the central Indian Ocean Ridge (Beedessee et al., 2013).

Seamounts constitute a distinct deep-sea habitat characterised by accelerated currents, exposed hard substrata, and a patchy structure in an otherwise continuous oceanic habitat. In the reviewed studies, seamount as a habitat type does not appear to be important in explaining the presence of barriers to gene flow. Distance is also not reported as being a barrier that isolates different seamount populations for all species. What is important, as noted previously, is depth, as recorded for corals and echinoderms (Miller et al., 2010; Miller et al., 2011; Miller \& Gunasekara, 2016; Ayala et al., 1975; Foltz et al., 2013). Although currents and topography are important physical elements that influence seamount ecosystems (White \& Mohn, 2004), neither appears to impede genetic connectivity and they may even promote it.

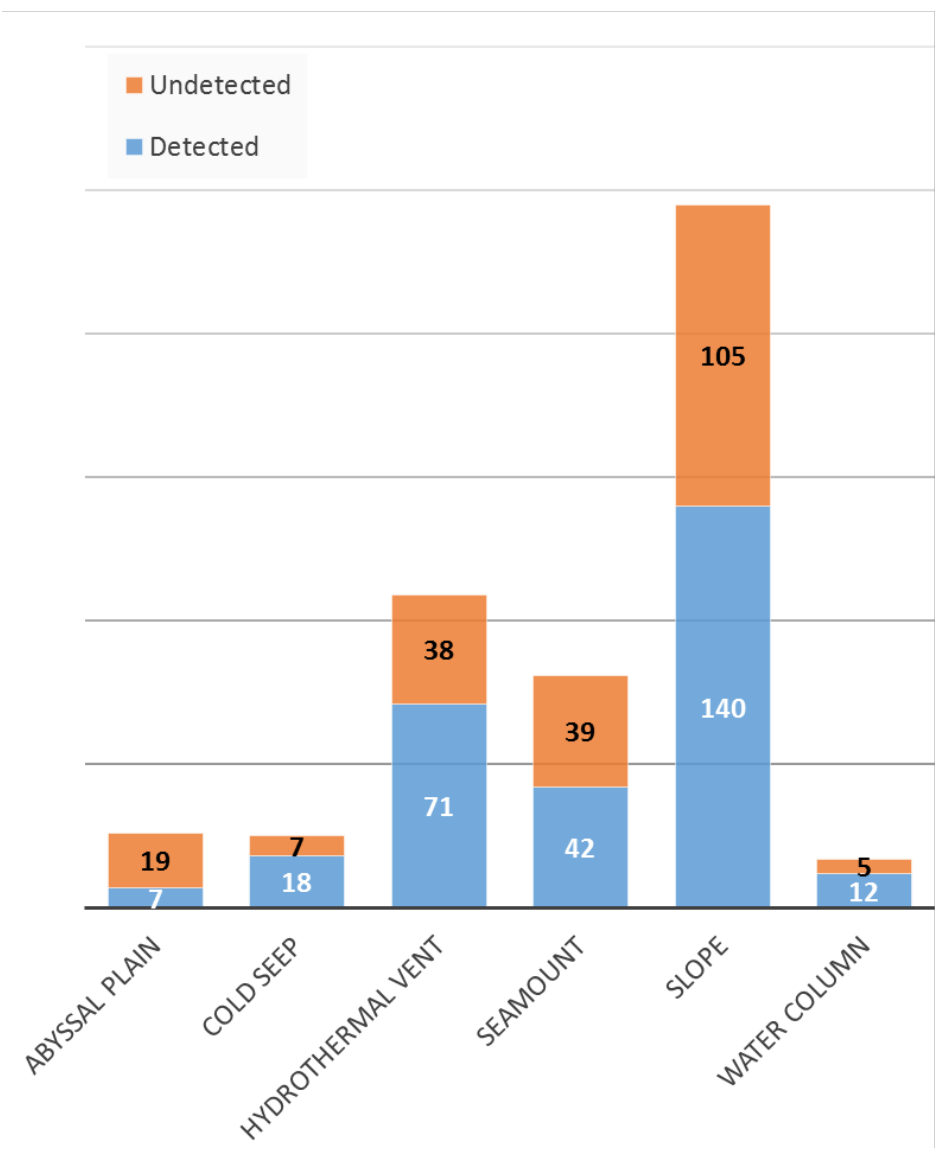

Figure 2.9 Comparisons of the presence of barriers to gene flow as a function of habitat type 


\subsubsection{Barrier occurrence difference between VMEs and non-VMEs}

Both the $\chi^{2}$ test $(\mathrm{p}>0.05)$ and Cramer's coefficient values $(\mathrm{V}>0.15)$ agreed that VMEs and VME indicator taxa were not associated with genetic barrier detection. In other words, barriers to genetic connectivity were reported at similar rates for all habitat types as they were for VME habitats, and also for indicator taxa (such as corals and sponges) that occurred at non-VME sites when compared with such taxa at VME sites (Table 2.9). Only temperature explained significant variation in barrier detection between VME and non-VME sites (Table 2.9), whilst in the comparison of indicator taxa and non-indicator taxa, both distance and topography explained significant variation in barrier detection. In the examined indicator taxa, topography was a genetic barrier amongst different populations, and distance genetically isolated most of VME indicator taxa populations. These results indicate that different habitats have different genetic connectivity patterns, and therefore that conservation and management plans need to be adapted to suit different habitat types.

Table 2.9 Comparisons of barrier detection between VMEs and non-VMEs and between indicator taxa and non-indicator taxa

\begin{tabular}{ccccc}
\hline \multirow{2}{*}{ Variables } & \multicolumn{4}{c}{ VMEs and non-VMEs Indicator taxa and non-indicator taxa } \\
\cline { 2 - 5 } & Cramer's $\mathbf{V} \boldsymbol{\chi}^{\mathbf{2}}$ (p value) & Cramer's V & $\boldsymbol{\chi}^{\mathbf{2}}$ (p value) \\
\hline Barrier detection & 0.010 & 0.829 & 0.060 & 0.180 \\
Distance & 0.117 & 0.254 & 0.212 & 0.039 \\
Depth & 0.012 & 0.895 & 0.172 & 0.068 \\
Current & 0.025 & 0.835 & 0.053 & 0.664 \\
Topography & 0.119 & 0.350 & 0.262 & 0.039 \\
Temperature & 1.000 & 0.003 & - & - \\
Life & 0.001 & 0.994 & 0.160 & 0.845 \\
\hline
\end{tabular}

-, insufficient temperature data were not available.

\subsection{Implications for management}

Understanding patterns of deep-sea genetic connectivity is essential to provide information and recommendations for management and conservation to protect or minimise negative effects from anthropological disturbances in the deep-sea ecosystem. Currently, in the World Database on Protected Areas (WDPA), 175 countries have established marine protected areas of different types, which cover about $12.34 \%\left(44,643,023.23 \mathrm{~km}^{2}\right)$ of the global ocean. This includes $3,345,306.37 \mathrm{~km}^{2}$ of No-Take areas, which cover about $0.92 \%$ of 
the global ocean (WDPA database, http://www.unep-wcmc.org/wdpa). Amongst these protected areas, less than $0.5 \%$ of the global surface has been protected, and most of the areas are located in shallow water (Chape et al., 2003). In the few deep-sea protection areas, all are associated with seamounts and hydrothermal vents, or cold-water corals (see summary in Davies et al., 2007). Based on the aforementioned results and discussions, patterns of genetic connectivity patterns varied amongst different species and spatial scales, and were caused by different factors. Specific conservation strategies should be adopted depending on the VME location and community composition.

\subsubsection{Spatial management}

From the summary results, it was very difficult to draw a clear pattern: the genetic connectivity varied a lot amongst different species, different habitats and at different scales. To address these complex patterns, spatial management would be the optimal solution, which means different management strategies are adapted to address different patterns of genetic connectivity. In a broad context, large spatial scale genetic connectivity should consider oceanic-scale protection (e.g. giant squid), and regional protection should be considered for moderate genetic connectivity (e.g. fish Sebastes mentella), and local protection should be set in place for small-scale structured populations (e.g. antipatharians).

Of those factors that affected genetic connectivity, depth is very important as a genetic barrier for most deep-sea animals, indicating that vertical protection is essential for deep-sea conservation. However, protection sites should not be simply placed by depth, because genetic diversity or differentiation is not simply correlated with depth. Significant differences in barrier detection amongst different habitats indicated that more reserves should place on cold seep and hydrothermal vent sites. Non-benthic protection should be considered for organisms inhabited the water column, because a greater level of genetic isolation was found in this habitat. More factors, such as seafloor topography and oceanic dynamics, included in the design would be considered appropriate.

\subsubsection{Management amongst habitats}

In the deep sea, geological variability (e.g., substratum type, topographic complexity), hydrography (e.g., prevailing current, water density) and their complex interactions form and shape several different habitats (Oldham et al., 2014). These environmentally distinct habitats 
may give rise to different selection regimes for different populations, which indirectly shape genetic differentiation and form barriers to gene flow.

The results of the present study showed that barriers to gene flow were detected in all different habitats. Except for populations on the abyssal plain, more half records in the populations associated with other habitats were revealed genetic structures, and organisms associated with hydrothermal vents and cold seeps exhibited more population genetic differentiation compared to organisms that inhabited other habitats (Figure 2.9). This suggests that the priorities of placing protection areas should consider the different habitat types, and that areas that are more isolated be allocated to higher conservation needs, such as hydrothermal vents and cold seeps. Different habitats are presently facing different levels and types of anthropologic disturbance affects: the continental slope is the most affected at present (Ramirez-Llodra et al., 2011). Although it is the most disturbed, these areas have received the most protection from fisheries (e.g. fisheries closure areas, marine protected areas and no-take marine reserves). However, hydrothermal vents, cold seeps and seamounts are also directly affected by human resource exploitation, such as fishing and the prospect of mining in the very near future. Recently, the Regional Fisheries Management Organizations (RFMOs) and some countries have closed a range of seamounts, hydrothermal vents and slope areas to bottom fisheries (Ramirez-Llodra et al., 2011), but for such decisions, most have not included connectivity information when the closure areas were designed or established. Based on the findings, protection areas in these habitats would lessen human impacts on the deep sea.

\subsubsection{Management of VMEs and VME indicator taxa}

Distance and topography were significantly related to VME indicator taxon genetic connectivity (Table 2.9). When designing protection areas, the distance to neighbourhood protection and bathymetric variables should be preferentially considered. Reasonable distance between protected areas will help to maintain population connectivity, and provide sources for the threatened populations. Because different species have different scales of genetic connectivity, the distance range would be established on a species by species basis. Because all of the VME indicator taxa are benthic, it is not surprising that topography was an important factor in helping to explain genetic barrier detection. This finding indicates that populations inhabiting in or on different topographic features should be considered as different management units. But other biological factors, such as growth rate and ability to 
recover after artificial disturbance, should be included in the design as well, because they are important for management but could not be reflected by the genetic markers.

\subsubsection{Management Units}

The model indicated that there were genetic barriers between deep-sea bio geographic provinces, which suggests that management should be applied at the spatial scale of the provinces. Genetic differentiation was significantly related to number of provinces covered (Figure 2.2, Table 2.4). Studies from both the BY7 (Cocos Plate) and BY9 (Antarctica) provinces reported that currents shaped genetic connectivity. Equatorial currents in BY7 caused a north-south differentiation $(82.22 \%$ of records from this province had a barrier) (e.g. Coykendall et al., 2011; Craddock, Lutz \& Vrijenhoek 1997; Hurtado, Lutz \& Vrijenhoek 2004; Jollivet et al., 1995; Matabos et al., 2008; Plouviez et al., 2009; Shank \& Halanych 2007; Won et al., 2003), whereas the Antarctic Circumpolar Current (ACC) in BY9 (59.10\% of records did not detect a barrier) promoted circular connectivity (e.g. Arango, SolerMembrives \& Miller 2011; Baird, Miller \& Stark 2011; Fevolden \& Schneppenheim 1989; Neal et al., 2014; Pawlowski, Bowser \& Gooday 2007; Raupach et al., 2010; Riehl \& Kaiser 2012; Roterman et al., 2016; Wilson et al., 2007). Whilst it is difficult to provide general suggestions for management or conservation in all provinces, different features of each province might provide a basic idea for management. Biogeographic provinces could be considered as a de facto management unit if not much information is available to help make informed management decisions.

\subsection{Future work}

To obtain a better understanding of genetic connectivity in the deep sea, there are still many gaps that exist in my collected data and analyses. Firstly, data are absent from the Arctic Ocean and most of the Indian, SE Pacific, NW Pacific, and S Atlantic oceans, and few records exist from hadal depths and most of the abyssal depths are also lacking data on genetic connectivity. Secondly, increasing awareness of new species and a lack of information about the reproduction modes and larval dispersal strategies for most species creates difficulties when attempting to explain the connectivity patterns. This, in turn, directly hampers protection design. Thirdly, highly polymorphic genetic markers (i.e., markers that are most informative for gene flow) are still unavailable for most deep-sea species, making it 
difficult to uncover the population genetic structure and barriers to gene flow. Although highly polymorphic co-dominant markers that are ideally suited for deep-sea studies, such as microsatellites and SNPs, can now be generated quickly and reasonably cheaply, most studies are currently still using DNA sequence data for species from the deep sea. These limitations or problems are the most important areas to be addressed in future analyses. In addition, in situ investigations of VME resilience to anthropogenic disturbance and recovery from damage are also required to identify appropriate management units and to aid in the design of protection networks for deep-sea species. 


\section{Appendix}

Table S2.1 Details of publications included in the database

\begin{tabular}{|c|c|c|c|c|c|c|c|c|}
\hline ID & Species & $\begin{array}{l}\text { Depth } \\
\text { cover (m) }\end{array}$ & $\begin{array}{l}\text { Sample } \\
\text { size }\end{array}$ & Provinces Code & $\begin{array}{l}\text { Genetic } \\
\text { Marker }\end{array}$ & $\begin{array}{l}\text { Barrier } \\
\text { Detectio } \\
\mathrm{n}\end{array}$ & Factors & Reference \\
\hline 1 & Abyssorchomene spp & 1013 & 419 & BY3 & Alloenzyme & Yes & Topography & $\begin{array}{l}\text { France } \text { et al., } \\
\text { (1994) }\end{array}$ \\
\hline 2 & Acanella & 3660 & 21 & BY4 & ND6 & No & Limitations & $\begin{array}{l}\text { Thoma et al., } \\
(2009)\end{array}$ \\
\hline 3 & $\begin{array}{l}\text { Acanthaspidia } \\
\text { drygalskii }\end{array}$ & 3433 & 36 & BY9 BY10 & $16 \mathrm{~s}$ & No & Current & $\begin{array}{l}\text { Raupach et al., } \\
\text { (2006) }\end{array}$ \\
\hline 4 & $\begin{array}{l}\text { Acanthephyra } \\
\text { pelagica }\end{array}$ & & 815 & BY4 & SSR & Yes & Distance & $\begin{array}{l}\text { Jorquera- } \\
\text { Paegelow et al., } \\
\text { (2014) }\end{array}$ \\
\hline 5 & Adipicola pacifica & 277 & 128 & BY12 & ND4 & No & $\begin{array}{l}\text { Biological } \\
\text { Factors }\end{array}$ & $\begin{array}{l}\text { Fukasawa et al., } \\
\text { (2015) }\end{array}$ \\
\hline 6 & Aetideidae & 2000 & 17 & BY1 BY9 & COI & No & Current & $\begin{array}{l}\text { Laakmann et al., } \\
\text { (2012) }\end{array}$ \\
\hline 7 & Aetideidae & 2000 & 17 & BY1 BY9 & $18 \mathrm{~s}$ & Yes & $\begin{array}{l}\text { Biological } \\
\text { Factors }\end{array}$ & $\begin{array}{l}\text { Laakmann et al., } \\
\text { (2012) }\end{array}$ \\
\hline 8 & Aetideidae & 2000 & 17 & BY1 BY9 & $28 \mathrm{~s}$ & Yes & $\begin{array}{l}\text { Biological } \\
\text { Factors }\end{array}$ & $\begin{array}{l}\text { Laakmann et al., } \\
\text { (2012) }\end{array}$ \\
\hline 9 & Aetideidae & 2000 & 17 & BY1 BY9 & ITS2 & Yes & $\begin{array}{l}\text { Biological } \\
\text { Factors }\end{array}$ & $\begin{array}{l}\text { Laakmann et al., } \\
\text { (2012) }\end{array}$ \\
\hline 10 & Allopathes robillardi & 0 & 54 & BY11 BY6 BY10 & ITS & Yes & Topography & $\begin{array}{l}\text { Miller et al., } \\
(2010)\end{array}$ \\
\hline 11 & Alvinella caudata & 625 & 176 & BY7 & Alloenzyme & Yes & Temperature & $\begin{array}{l}\text { Jollivet et al., } \\
\text { (1995) }\end{array}$ \\
\hline 12 & Alvinella pompejana & 625 & 185 & BY7 & Alloenzyme & Yes & Temperature & $\begin{array}{l}\text { Jollivet et al., } \\
\text { (1995) }\end{array}$ \\
\hline 13 & Alvinella pompejana & 340 & 246 & BY7 & $\mathrm{COI}$ & Yes & $\begin{array}{l}\text { Biological } \\
\text { Factors \& Ocean } \\
\text { current }\end{array}$ & $\begin{array}{l}\text { Plouviez et al., } \\
\text { (2009) }\end{array}$ \\
\hline 14 & Alvinella pompejana & 826 & 93 & BY7 & $\mathrm{COI}$ & No & $\begin{array}{l}\text { Biological } \\
\text { Factors }\end{array}$ & $\begin{array}{l}\text { Hurtado et al., } \\
\text { (2004) }\end{array}$ \\
\hline 15 & Alvinella pompejana & 346 & 67 & BY7 & $\mathrm{COI}$ & Yes & Current & $\begin{array}{l}\text { Plouviez et al., } \\
(2010)\end{array}$ \\
\hline 16 & Alvinella pompejana & 346 & 17 & BY7 & SAHH & Yes & Current & $\begin{array}{l}\text { Plouviez et al., } \\
(2010)\end{array}$ \\
\hline 17 & Alvinella pompejana & 346 & 20 & BY7 & PGM & Yes & Current & $\begin{array}{l}\text { Plouviez et al., } \\
(2010)\end{array}$ \\
\hline 18 & Alvinella pompejana & 346 & 20 & BY7 & GlobX & Yes & Current & $\begin{array}{l}\text { Plouviez et al., } \\
(2010)\end{array}$ \\
\hline 19 & Alvinella pompejana & 346 & 40 & BY7 & Alloenzyme & Yes & Current & $\begin{array}{l}\text { Plouviez et al., } \\
(2010)\end{array}$ \\
\hline 20 & Alvinella pompejana & 346 & 74 & BY7 & RFLP & Yes & Current & $\begin{array}{l}\text { Plouviez et al., } \\
(2010)\end{array}$ \\
\hline 21 & Alviniconcha & 0 & 44 & BY11 & $\mathrm{COI}$ & No & $\begin{array}{l}\text { Biological } \\
\text { Factors }\end{array}$ & $\begin{array}{l}\text { Beedessee } \text { et al., } \\
(2013)\end{array}$ \\
\hline 22 & $\begin{array}{l}\text { Alviniconcha } \\
\text { hessleri }\end{array}$ & 2130 & 100 & BY12 & ATPS $\beta$ & No & Temperature & $\begin{array}{l}\text { Hidaka et al., } \\
(2015)\end{array}$ \\
\hline 23 & $\begin{array}{l}\text { Alviniconcha } \\
\text { hessleri }\end{array}$ & 2130 & 40 & BY13 & COI & No & Temperature & $\begin{array}{l}\text { Hidaka et al., } \\
\text { (2015) }\end{array}$ \\
\hline 24 & Alviniconcha spp. & 250 & 28 & BY12 & Alloenzyme & Yes & $\begin{array}{l}\text { Biological } \\
\text { Factors }\end{array}$ & $\begin{array}{l}\text { Denis et al., } \\
\text { (1993) }\end{array}$ \\
\hline 25 & $\begin{array}{l}\text { Alvinocaris } \\
\text { dissimilis }\end{array}$ & 0 & 17 & BY12 & COI & No & Topography & $\begin{array}{l}\text { Yahagi et al., } \\
(2015)\end{array}$ \\
\hline 26 & $\begin{array}{l}\text { Alvinocaris } \\
\text { longirostris }\end{array}$ & 723 & 66 & BY12 & $\mathrm{COI}$ & No & Topography & $\begin{array}{l}\text { Yahagi et al., } \\
(2015)\end{array}$ \\
\hline 27 & Alvinocaris sp & 264 & 38 & BY12 & COI & No & Topography & $\begin{array}{l}\text { Yahagi et al., } \\
(2015)\end{array}$ \\
\hline 28 & Anoplopoma fimbria & 414 & 57 & BY3 & COI & No & Limitations & $\begin{array}{l}\text { Tripp-Valdez et } \\
\text { al., (2012) }\end{array}$ \\
\hline 29 & Anoplopoma fimbria & 414 & 183 & BY3 & SSR & Yes & $\begin{array}{l}\text { Biological } \\
\text { Factors }\end{array}$ & $\begin{array}{l}\text { Tripp-Valdez et } \\
\text { al., (2012) }\end{array}$ \\
\hline 30 & Antimora rostrata & 1825 & 393 & BY4 & SSR & No & Limitations & $\begin{array}{l}\text { White et al., } \\
\text { (2011) }\end{array}$ \\
\hline 31 & Aphanopus carbo & 670 & 651 & BY4 & SSR & No & $\begin{array}{l}\text { Biological } \\
\text { Factors }\end{array}$ & $\begin{array}{l}\text { Longmore et al., } \\
\text { (2014) }\end{array}$ \\
\hline 32 & Architeuthis dux & 700 & 43 & $\begin{array}{l}\text { BY13 BY6 BY4 } \\
\text { BY11 BY12 BY2 } \\
\text { BY14 }\end{array}$ & $\begin{array}{l}\text { mtDNA } \\
\text { genome }\end{array}$ & No & $\begin{array}{l}\text { Biological } \\
\text { Factors }\end{array}$ & $\begin{array}{l}\text { Winkelmann et } \\
\text { al., (2013) }\end{array}$ \\
\hline 33 & $\begin{array}{l}\text { Argyropelecus } \\
\text { hemigimnus }\end{array}$ & 550 & 12 & BY4 BY 10 BY 13 & $\mathrm{COI}$ & Yes & Depth \& Distance & $\begin{array}{l}\text { Gordeeva et al., } \\
\text { (2014) }\end{array}$ \\
\hline 34 & $\begin{array}{l}\text { Aristaeomorpha } \\
\text { foliacea }\end{array}$ & & 317 & BY4 BY11 & COI & Yes & $\begin{array}{l}\text { Biological } \\
\text { Factors \& } \\
\text { Topography }\end{array}$ & $\begin{array}{l}\text { Fernándeza et } \\
\text { al., (2013) }\end{array}$ \\
\hline 35 & Aristeus antennatus & 821 & 230 & BY4 & SSR & No & $\begin{array}{l}\text { Biological } \\
\text { Factors }\end{array}$ & $\begin{array}{l}\text { Sacco et al., } \\
(2011)\end{array}$ \\
\hline 36 & Aristeus antennatus & & 175 & BY4 & D-loop & No & $\begin{array}{l}\text { Biological } \\
\text { Factors }\end{array}$ & $\begin{array}{l}\text { Maggio et al., } \\
(2009)\end{array}$ \\
\hline
\end{tabular}




\begin{tabular}{|c|c|c|c|c|c|c|c|c|}
\hline 37 & Aristeus antennatus & & 236 & BY4 & D-loop & No & Limitations & $\begin{array}{l}\text { Lo Brutto et al., } \\
\text { (2012) }\end{array}$ \\
\hline 38 & Aristeus antennatus & & 192 & BY4 & AFLP & No & Limitations & $\begin{array}{l}\text { Lo Brutto et al., } \\
\text { (2012) }\end{array}$ \\
\hline 39 & Aristeus antennatus & 220 & 319 & BY4 & $\begin{array}{l}\mathrm{COI}(500)+1 \\
6 \mathrm{~s}(447)\end{array}$ & No & $\begin{array}{l}\text { Biological } \\
\text { Factors }\end{array}$ & $\begin{array}{l}\text { Marra et al., } \\
\text { (2015) }\end{array}$ \\
\hline 40 & Aristeus antennatus & 1150 & 321 & BY4 & $16 \mathrm{~s}$ & No & Depth & $\begin{array}{l}\text { Sarda et al., } \\
\text { (2010) }\end{array}$ \\
\hline 41 & Aristeus antennatus & & 137 & BY4 & $\begin{array}{l}\text { COI514+16s } \\
547\end{array}$ & No & $\begin{array}{l}\text { Biological } \\
\text { Factors }\end{array}$ & $\begin{array}{l}\text { Roldan et al., } \\
\text { (2009) }\end{array}$ \\
\hline 42 & Aristeus antennatus & 100 & 175 & BY4 & D-loop & No & $\begin{array}{l}\text { Biological } \\
\text { Factors }\end{array}$ & $\begin{array}{l}\text { Maggio et al., } \\
(2009)\end{array}$ \\
\hline 43 & Aristeus antennatus & 821 & 227 & BY4 & SSR & No & $\begin{array}{l}\text { Biological } \\
\text { Factors }\end{array}$ & $\begin{array}{l}\text { Cannas et al., } \\
\text { (2011) }\end{array}$ \\
\hline 44 & Aristeus antennatus & & 497 & BY4 & $\mathrm{COI}$ & Yes & Topography & $\begin{array}{l}\text { Fernández et al., } \\
\text { (2011) }\end{array}$ \\
\hline 45 & Aristeus antennatus & & 506 & BY4 & $16 \mathrm{~s}$ & Yes & Topography & $\begin{array}{l}\text { Fernández et al., } \\
\text { (2011) }\end{array}$ \\
\hline 46 & $\begin{array}{l}\text { Asteroschema } \\
\text { clavigerum }\end{array}$ & 1000 & 91 & BY4 & $16 \mathrm{~s}$ & Yes & Depth & $\begin{array}{l}\text { Cho et al., } \\
(2010)\end{array}$ \\
\hline 47 & $\begin{array}{l}\text { Asteroschema } \\
\text { clavigerum }\end{array}$ & 1000 & 37 & BY4 & $\mathrm{COI}$ & Yes & Depth & $\begin{array}{l}\text { Cho et al., } \\
(2010)\end{array}$ \\
\hline 48 & Atlantoserolis vemae & 1185 & 17 & AB5 & $18 \mathrm{~s}$ & Yes & Topography & $\begin{array}{l}\text { Brandt et al., } \\
\text { (2014) }\end{array}$ \\
\hline 49 & Atlantoserolis vemae & 1185 & 20 & AB5 & $16 \mathrm{~s}$ & Yes & Topography & $\begin{array}{l}\text { Brandt et al., } \\
\text { (2014) }\end{array}$ \\
\hline 50 & Atlantoserolis vemae & 1185 & 17 & AB5 & COI & Yes & Topography & $\begin{array}{l}\text { Brandt et al., } \\
\text { (2014) }\end{array}$ \\
\hline 51 & $\begin{array}{l}\text { Austinograea } \\
\text { rodriguezensis }\end{array}$ & 0 & 36 & BY11 & COI & No & Current & $\begin{array}{l}\text { Beedessee et al., } \\
\text { (2013) }\end{array}$ \\
\hline 52 & $\begin{array}{l}\text { Austrolaenilla } \\
\text { antarctica }\end{array}$ & 2252 & 19 & BY9 & $\mathrm{COI}$ & Yes & $\begin{array}{l}\text { Biological } \\
\text { Factors }\end{array}$ & $\begin{array}{l}\text { Neal et al., } \\
(2014)\end{array}$ \\
\hline 53 & $\begin{array}{l}\text { Austrolaenilla } \\
\text { pelagica }\end{array}$ & 2253 & 19 & BY9 & COI & No & Limitations & $\begin{array}{l}\text { Neal et al., } \\
(2015)\end{array}$ \\
\hline 54 & Bathybembix bairdii & 577 & 21 & BY3 & Alloenzyme & Yes & Depth & $\begin{array}{l}\text { Siebenaller } \text { et } \\
\text { al., (1978) }\end{array}$ \\
\hline 55 & Bathymodiolus & 416 & 120 & BY7 & $\mathrm{COI}$ & Yes & Distance & $\begin{array}{l}\text { Won et al., } \\
(2003)\end{array}$ \\
\hline 56 & Bathymodiolus & 416 & 10 & BY7 & Alloenzyme & Yes & Distance & $\begin{array}{l}\text { Won et al., } \\
(2003)\end{array}$ \\
\hline 57 & Bathymodiolus & 1140 & 22 & BY6 & COI & Yes & Current & $\begin{array}{l}\text { Smith et al., } \\
(2004)\end{array}$ \\
\hline 58 & Bathymodiolus & 1140 & 22 & BY6 & Alloenzyme & Yes & Current & $\begin{array}{l}\text { Smith et al., } \\
\text { (2004) }\end{array}$ \\
\hline 59 & Bathymodiolus & 3310 & 67 & BY4 BY12 & ND4 & No & Topography & $\begin{array}{l}\text { Kyuno et al., } \\
(2009)\end{array}$ \\
\hline 60 & Bathymodiolus & & & BY4 & SNP & Yes & Distance & $\begin{array}{l}\text { Corinna et al., } \\
\text { (2015) }\end{array}$ \\
\hline 61 & $\begin{array}{l}\text { Bathymodiolus } \\
\text { brevior }\end{array}$ & 2413 & 241 & BY6 BY11 BY12 & COI & Yes & Distance & $\begin{array}{l}\text { Breusing et al., } \\
(2015)\end{array}$ \\
\hline 62 & $\begin{array}{l}\text { Bathymodiolus } \\
\text { brevior }\end{array}$ & 2413 & 241 & BY6 BY11 BY12 & ND4 & Yes & Distance & $\begin{array}{l}\text { Breusing et al., } \\
(2015)\end{array}$ \\
\hline 63 & $\begin{array}{l}\text { Bathymodiolus } \\
\text { brevior }\end{array}$ & 2413 & 241 & BY6 BY11 BY12 & Col-1 & No & $\begin{array}{l}\text { Current \& } \\
\text { Biological } \\
\text { Factors }\end{array}$ & $\begin{array}{l}\text { Breusing et al., } \\
(2015)\end{array}$ \\
\hline 64 & $\begin{array}{l}\text { Bathymodiolus } \\
\text { brevior }\end{array}$ & 2414 & 241 & BY6 BY11 BY13 & Val tRNA & No & $\begin{array}{l}\text { Current \& } \\
\text { Biological } \\
\text { Factors }\end{array}$ & $\begin{array}{l}\text { Breusing et al., } \\
(2015)\end{array}$ \\
\hline 65 & $\begin{array}{l}\text { Bathymodiolus } \\
\text { brevior }\end{array}$ & 2414 & 241 & BY6 BY11 BY13 & Met tRNA & No & $\begin{array}{l}\text { Current \& } \\
\text { Biological } \\
\text { Factors }\end{array}$ & $\begin{array}{l}\text { Breusing et al., } \\
\text { (2015) }\end{array}$ \\
\hline 66 & $\begin{array}{l}\text { Bathymodiolus } \\
\text { brevior }\end{array}$ & 2414 & 241 & BY6 BY11 BY13 & EF1 $\alpha$ & No & $\begin{array}{l}\text { Current \& } \\
\text { Biological } \\
\text { Factors }\end{array}$ & $\begin{array}{l}\text { Breusing et al., } \\
(2015)\end{array}$ \\
\hline 67 & $\begin{array}{l}\text { Bathymodiolus } \\
\text { brevior }\end{array}$ & 2414 & 241 & BY6 BY11 BY14 & Cat & Yes & $\begin{array}{l}\text { Current \& } \\
\text { Biological } \\
\text { Factors }\end{array}$ & $\begin{array}{l}\text { Breusing et al., } \\
(2015)\end{array}$ \\
\hline 68 & $\begin{array}{l}\text { Bathymodiolus } \\
\text { brevior }\end{array}$ & 2414 & 241 & BY6 BY11 BY14 & $\mathrm{H} 3$ & No & $\begin{array}{l}\text { Current \& } \\
\text { Biological } \\
\text { Factors }\end{array}$ & $\begin{array}{l}\text { Breusing et al., } \\
\text { (2015) }\end{array}$ \\
\hline 69 & $\begin{array}{l}\text { Bathymodiolus } \\
\text { brevior }\end{array}$ & 2414 & 241 & BY6 BY11 BY14 & Alloenzyme & Yes & Distance & $\begin{array}{l}\text { Breusing et al., } \\
(2015)\end{array}$ \\
\hline 70 & $\begin{array}{l}\text { Bathymodiolus } \\
\text { brooksi }\end{array}$ & 2213 & 67 & BY4 & COI & Yes & $\begin{array}{l}\text { Biological } \\
\text { Factors \& Vent }\end{array}$ & $\begin{array}{l}\text { Faure } \text { et al., } \\
\text { (2015) }\end{array}$ \\
\hline 71 & $\begin{array}{l}\text { Bathymodiolus } \\
\text { brooksi }\end{array}$ & 2213 & 67 & BY4 & ND4 & No & $\begin{array}{l}\text { Biological } \\
\text { Factors \& Vent }\end{array}$ & $\begin{array}{l}\text { Faure } \text { et al., } \\
(2015)\end{array}$ \\
\hline 72 & $\begin{array}{l}\text { Bathymodiolus } \\
\text { childressi }\end{array}$ & 1828 & 99 & BY4 & $\mathrm{COI}$ & Yes & $\begin{array}{l}\text { Biological } \\
\text { Factors \& Vent }\end{array}$ & $\begin{array}{l}\text { Faure } \text { et al., } \\
\text { (2015) }\end{array}$ \\
\hline 73 & $\begin{array}{l}\text { Bathymodiolus } \\
\text { childressi }\end{array}$ & 1828 & 76 & BY4 & ND4 & No & $\begin{array}{l}\text { Biological } \\
\text { Factors \& Vent }\end{array}$ & $\begin{array}{l}\text { Faure } \text { et al., } \\
\text { (2015) }\end{array}$ \\
\hline 74 & $\begin{array}{l}\text { Bathymodiolus } \\
\text { geikotsucola }\end{array}$ & 31 & 40 & BY12 & ND4 & No & $\begin{array}{l}\text { Biological } \\
\text { Factors }\end{array}$ & $\begin{array}{l}\text { Fukasawa et al., } \\
\text { (2015) }\end{array}$ \\
\hline 75 & $\begin{array}{l}\text { Bathymodiolus } \\
\text { heckerae }\end{array}$ & 0 & 32 & BY4 & $\mathrm{COI}$ & Yes & $\begin{array}{l}\text { Biological } \\
\text { Factors \& Vent }\end{array}$ & $\begin{array}{l}\text { Faure et al., } \\
(2015)\end{array}$ \\
\hline 76 & $\begin{array}{l}\text { Bathymodiolus } \\
\text { heckerae }\end{array}$ & 0 & 32 & BY4 & ND4 & No & Limitations & $\begin{array}{l}\text { Faure et al., } \\
\text { (2015) }\end{array}$ \\
\hline
\end{tabular}




\begin{tabular}{|c|c|c|c|c|c|c|c|c|}
\hline 77 & $\begin{array}{l}\text { Bathymodiolus } \\
\text { platifrons }\end{array}$ & 258.7 & 52 & BY12 & $\begin{array}{l}\text { COI+Cytb+ } \\
16 \mathrm{~s}\end{array}$ & No & $\begin{array}{l}\text { Biological } \\
\text { Factors }\end{array}$ & $\begin{array}{l}\text { Shen et al., } \\
(2016)\end{array}$ \\
\hline 78 & $\begin{array}{l}\text { Bathymodiolus } \\
\text { platifrons }\end{array}$ & 280 & 28 & BY12 & SNP & Yes & Temperature & Xu et al., (2016) \\
\hline 79 & $\begin{array}{l}\text { Bathymodiolus sp. } \\
\text { nov. }\end{array}$ & 0 & 5 & BY4 & $\mathrm{COI}$ & Yes & $\begin{array}{l}\text { Biological } \\
\text { Factors \& Vent }\end{array}$ & $\begin{array}{l}\text { Faure et al., } \\
(2015)\end{array}$ \\
\hline 80 & $\begin{array}{l}\text { Bathymodiolus sp. } \\
\text { nov. }\end{array}$ & 0 & 5 & BY4 & ND4 & No & Limitations & $\begin{array}{l}\text { Faure } \text { et al., } \\
\text { (2015) }\end{array}$ \\
\hline 81 & Bathymodiolus spp & 885 & 161 & BY14 & Alloenzyme & Yes & Distance & $\begin{array}{l}\text { Moraga et al., } \\
\text { (1994) }\end{array}$ \\
\hline 82 & $\begin{array}{l}\text { Bathymodiolus } \\
\text { thermophilus }\end{array}$ & 170 & 58 & BY7 & RFLP & No & $\begin{array}{l}\text { Biological } \\
\text { Factors }\end{array}$ & $\begin{array}{l}\text { Craddock et al., } \\
\text { (1995) }\end{array}$ \\
\hline 83 & $\begin{array}{l}\text { Bathymodiolus } \\
\text { thermophilus }\end{array}$ & 170 & 58 & BY7 & Alloenzyme & No & $\begin{array}{l}\text { Biological } \\
\text { Factors }\end{array}$ & $\begin{array}{l}\text { Craddock et al., } \\
\text { (1995) }\end{array}$ \\
\hline 84 & $\begin{array}{l}\text { Bathymodiolus } \\
\text { thermophilus }\end{array}$ & 340 & 309 & BY7 & $\mathrm{COI}$ & Yes & $\begin{array}{l}\text { Biological } \\
\text { Factors \& Ocean } \\
\text { current }\end{array}$ & $\begin{array}{l}\text { Plouviez et al., } \\
\text { (2009) }\end{array}$ \\
\hline 85 & $\begin{array}{l}\text { Bathymodiolus } \\
\text { thermophilus }\end{array}$ & 600 & 346 & BY7 & Alloenzyme & Yes & Distance & $\begin{array}{l}\text { Plouviez et al., } \\
\text { (2013) }\end{array}$ \\
\hline 86 & $\begin{array}{l}\text { Bathymodiolus } \\
\text { thermophilus }\end{array}$ & 600 & 347 & BY7 & $\mathrm{COI}$ & Yes & Depth \& Distance & $\begin{array}{l}\text { Plouviez et al., } \\
\text { (2013) }\end{array}$ \\
\hline 87 & $\begin{array}{l}\text { Bathymodiolus } \\
\text { thermophilus }\end{array}$ & 600 & 83 & BY7 & Sulfo1 & Yes & Depth \& Distance & $\begin{array}{l}\text { Plouviez et al., } \\
\text { (2013) }\end{array}$ \\
\hline 88 & $\begin{array}{l}\text { Bathymodiolus } \\
\text { thermophilus }\end{array}$ & 600 & 97 & BY7 & Lyso & Yes & Depth \& Distance & $\begin{array}{l}\text { Plouviez et al., } \\
\text { (2013) }\end{array}$ \\
\hline 89 & $\begin{array}{l}\text { Bathymodiolus } \\
\text { thermophilus }\end{array}$ & 600 & 94 & BY7 & SAHH & Yes & Depth \& Distance & $\begin{array}{l}\text { Plouviez et al., } \\
\text { (2013) }\end{array}$ \\
\hline 90 & $\begin{array}{l}\text { Bathymodiolus } \\
\text { thermophilus }\end{array}$ & 600 & 45 & BY7 & $\mathrm{EF} 1 \alpha$ & Yes & Depth \& Distance & $\begin{array}{l}\text { Plouviez et al., } \\
\text { (2013) }\end{array}$ \\
\hline 91 & Bathypathes & 3660 & 18 & BY4 & $\mathrm{COI}$ & No & Limitations & $\begin{array}{l}\text { Thoma et al., } \\
\text { (2009) }\end{array}$ \\
\hline 92 & Bathypathes & 3660 & 18 & BY4 & ND2 & No & Limitations & $\begin{array}{l}\text { Thoma et al., } \\
\text { (2009) }\end{array}$ \\
\hline 93 & Bathypathes & 3660 & 18 & BY4 & ND1 & No & Limitations & $\begin{array}{l}\text { Thoma et al., } \\
\text { (2009) }\end{array}$ \\
\hline 94 & Benthodytes typica & 2050 & 86 & BY4 & Alloenzyme & No & $\begin{array}{l}\text { Biological } \\
\text { Factors }\end{array}$ & $\begin{array}{l}\text { Bisol et al., } \\
\text { (1984) }\end{array}$ \\
\hline 95 & Benthogone rosea & 2050 & 51 & BY4 & Alloenzyme & No & $\begin{array}{l}\text { Biological } \\
\text { Factors }\end{array}$ & $\begin{array}{l}\text { Bisol et al., } \\
\text { (1984) }\end{array}$ \\
\hline 96 & $\begin{array}{l}\text { Benthosema } \\
\text { suborbitale }\end{array}$ & 640 & 10 & BY4 BY 10 BY 13 & $\mathrm{COI}$ & Yes & Depth & $\begin{array}{l}\text { Gordeeva et al., } \\
\text { (2014) }\end{array}$ \\
\hline 97 & Beryx decadactylus & & 305 & BY4 & D-loop & No & $\begin{array}{l}\text { Biological } \\
\text { Factors }\end{array}$ & $\begin{array}{l}\text { Fries et al., } \\
\text { (2011) }\end{array}$ \\
\hline 98 & Beryx splendens & & 204 & $\begin{array}{l}\text { BY4 BY11 BY6 } \\
\text { BY10 BY12 }\end{array}$ & Cytb & Yes & Current & $\begin{array}{l}\text { Lévy-Hartmann, } \\
\text { et al., (2011) }\end{array}$ \\
\hline 99 & Beryx splendens & 596 & 238 & BY12 & Cytb & Yes & Distance & $\begin{array}{l}\text { Hoarau et al., } \\
(2000)\end{array}$ \\
\hline 100 & $\begin{array}{l}\text { Betamorpha } \\
\text { fusiformis }\end{array}$ & 3864 & 50 & BY9 AB6 & $16 \mathrm{~s}$ & Yes & Topography & $\begin{array}{l}\text { Raupach et al., } \\
(2007)\end{array}$ \\
\hline 101 & $\begin{array}{l}\text { Betamorpha } \\
\text { fusiformis }\end{array}$ & 3864 & 50 & BY9 AB6 & $18 \mathrm{~s}$ & No & Limitations & $\begin{array}{l}\text { Raupach et al., } \\
\text { (2007) }\end{array}$ \\
\hline 102 & Bolinichthys indicus & 625 & 10 & BY4 BY 10 BY13 & $\mathrm{COI}$ & Yes & Depth & $\begin{array}{l}\text { Gordeeva et al., } \\
\text { (2014) }\end{array}$ \\
\hline 103 & $\begin{array}{l}\text { Branchipolynoe } \\
\text { symmytilida }\end{array}$ & 340 & 207 & BY7 & $\mathrm{COI}$ & Yes & $\begin{array}{l}\text { Biological } \\
\text { Factors \& Ocean } \\
\text { current }\end{array}$ & $\begin{array}{l}\text { Plouviez et al., } \\
\text { (2009) }\end{array}$ \\
\hline 104 & $\begin{array}{l}\text { Branchipolynoe } \\
\text { symmytilida }\end{array}$ & 826 & 61 & BY7 & $\mathrm{COI}$ & No & $\begin{array}{l}\text { Biological } \\
\text { Factors }\end{array}$ & $\begin{array}{l}\text { Hurtado et al., } \\
\text { (2004) }\end{array}$ \\
\hline 105 & Brosme brosme & 900 & 764 & BY2 BY4 & SSR & Yes & $\begin{array}{l}\text { Depth \& } \\
\text { Biological } \\
\text { Factors }\end{array}$ & $\begin{array}{l}\text { Knutsen et al., } \\
(2009)\end{array}$ \\
\hline 106 & $\begin{array}{l}\text { Caecosagitta } \\
\text { macrocephala }\end{array}$ & 3999 & 52 & BY3 BY13 & $\mathrm{COI}$ & Yes & Depth \& Current & $\begin{array}{l}\text { Miyamoto et al., } \\
(2010)\end{array}$ \\
\hline 107 & $\begin{array}{l}\text { Caecosagitta } \\
\text { macrocephala }\end{array}$ & 3999 & 52 & BY3 BY13 & ITS1 & Yes & Depth \& Current & $\begin{array}{l}\text { Miyamoto et al., } \\
(2010)\end{array}$ \\
\hline 108 & $\begin{array}{l}\text { Calanus } \\
\text { finmarchicus }\end{array}$ & & 182 & BY2 BY1 & $16 \mathrm{~s}$ & Yes & $\begin{array}{l}\text { Biological } \\
\text { Factors }\end{array}$ & $\begin{array}{l}\text { Bucklin et al., } \\
\text { (1996) }\end{array}$ \\
\hline 109 & $\begin{array}{l}\text { Calanus } \\
\text { finmarchicus }\end{array}$ & & 92 & BY2 BY1 & $\mathrm{COI}$ & Yes & Current & $\begin{array}{l}\text { Bucklin et al., } \\
(2000)\end{array}$ \\
\hline 110 & $\begin{array}{l}\text { Calanus } \\
\text { finmarchicus }\end{array}$ & & 83 & BY2 BY1 & PGI & Yes & Current & $\begin{array}{l}\text { Bucklin et al., } \\
(2000)\end{array}$ \\
\hline 111 & Callogorgia a delta & 415 & 113 & BY4 & SSR & Yes & Depth & $\begin{array}{l}\text { Quattrini et al., } \\
\text { (2014) }\end{array}$ \\
\hline 112 & $\begin{array}{l}\text { Carcharhinus } \\
\text { acronotus }\end{array}$ & 0 & 257 & BY4 & D-loop & Yes & $\begin{array}{l}\text { Current \& } \\
\text { Topography }\end{array}$ & $\begin{array}{l}\text { Portnoy et al., } \\
(2014)\end{array}$ \\
\hline 113 & $\begin{array}{l}\text { Carcharhinus } \\
\text { acronotus }\end{array}$ & 0 & 650 & BY4 & SSR & Yes & $\begin{array}{l}\text { Current \& } \\
\text { Topography }\end{array}$ & $\begin{array}{l}\text { Portnoy et al., } \\
\text { (2014) }\end{array}$ \\
\hline 114 & $\begin{array}{l}\text { Centroscymnus } \\
\text { coelolepis }\end{array}$ & 1825 & 155 & BY4 BY10 BY11 & D-loop & Yes & Topography & $\begin{array}{l}\text { Catarino et al., } \\
(2015)\end{array}$ \\
\hline 115 & $\begin{array}{l}\text { Centroscymnus } \\
\text { coelolepis }\end{array}$ & 1825 & 503 & BY4 BY10 BY11 & SSR & Yes & Topography & $\begin{array}{l}\text { Catarino et al., } \\
(2015)\end{array}$ \\
\hline 116 & $\begin{array}{l}\text { Centroscymnus } \\
\text { crepidater }\end{array}$ & 550 & 92 & BY6 BY4 & D-loop & Yes & Current & $\begin{array}{l}\text { Cunha et al., } \\
\text { (2012) }\end{array}$ \\
\hline 117 & $\begin{array}{l}\text { Centroscymnus } \\
\text { crepidater }\end{array}$ & 550 & 61 & BY6 BY4 & $\mathrm{COI}$ & Yes & Current & $\begin{array}{l}\text { Cunha et al., } \\
(2012)\end{array}$ \\
\hline
\end{tabular}




\begin{tabular}{|c|c|c|c|c|c|c|c|c|}
\hline 118 & $\begin{array}{l}\text { Centroscymnus } \\
\text { crepidater }\end{array}$ & 550 & 160 & BY6 BY4 & SSR & Yes & Current & $\begin{array}{l}\text { Cunha et al., } \\
\text { (2012) }\end{array}$ \\
\hline 119 & $\begin{array}{l}\text { Ceratoscopelus } \\
\text { warmingii }\end{array}$ & 725 & 20 & BY4 BY 10 BY 13 & $\mathrm{COI}$ & Yes & Depth \& Distance & $\begin{array}{l}\text { Gordeeva et al., } \\
\text { (2014) }\end{array}$ \\
\hline 120 & $\begin{array}{l}\text { cf. Ampelisca } \\
\text { chiltoni }\end{array}$ & 1000 & 28 & BY6 & $\mathrm{COI}$ & Yes & Topography & $\begin{array}{l}\text { Knox et al., } \\
(2012)\end{array}$ \\
\hline 121 & $\begin{array}{l}\text { cf. Oediceroides } \\
\text { apicalis }\end{array}$ & 1000 & 25 & BY6 & $\mathrm{COI}$ & Yes & Topography & $\begin{array}{l}\text { Knox et al., } \\
(2012)\end{array}$ \\
\hline 122 & Chaceon notialis & 750 & 35 & BY13 & $16 \mathrm{~s}$ & No & Limitations & $\begin{array}{l}\text { Mantelatto et al., } \\
\text { (2014) }\end{array}$ \\
\hline 123 & $\begin{array}{l}\text { Chaceon } \\
\text { quinquedens }\end{array}$ & 616 & 37 & BY4 & $16 \mathrm{~s}$ & Yes & $\begin{array}{l}\text { Biological } \\
\text { Factors }\end{array}$ & $\begin{array}{l}\text { Weinberg et al., } \\
\text { (2003) }\end{array}$ \\
\hline 124 & $\begin{array}{l}\text { Chaceon } \\
\text { quinquedens }\end{array}$ & 616 & 15 & BY4 & ITS & Yes & $\begin{array}{l}\text { Biological } \\
\text { Factors }\end{array}$ & $\begin{array}{l}\text { Weinberg et al., } \\
\text { (2003) }\end{array}$ \\
\hline 125 & Chaceon ramosae & 750 & 35 & BY13 & $\mathrm{COI}$ & No & Limitations & $\begin{array}{l}\text { Mantelatto et al., } \\
\text { (2014) }\end{array}$ \\
\hline 126 & Chelator aequabilis & 368 & 3 & BY13 & $16 \mathrm{~s}$ & No & Limitations & $\begin{array}{l}\text { Brix et al., } \\
(2015)\end{array}$ \\
\hline 127 & Chelator aequabilis & 368 & 4 & BY13 & $\mathrm{COI}$ & No & Limitations & $\begin{array}{l}\text { Brix et al., } \\
(2015)\end{array}$ \\
\hline 128 & Chelator aequabilis & 368 & 2 & BY13 & $18 \mathrm{~s}$ & No & Limitations & $\begin{array}{l}\text { Brix et al., } \\
(2015)\end{array}$ \\
\hline 129 & Chelator insignis & 2537 & 47 & BY2 & $\mathrm{COI}$ & Yes & Depth & $\begin{array}{l}\text { Brix et al., } \\
(2014)\end{array}$ \\
\hline 130 & Chelator insignis & 2537 & 36 & BY2 & $16 \mathrm{~s}$ & Yes & Depth & $\begin{array}{l}\text { Brix et al., } \\
(2014)\end{array}$ \\
\hline 131 & Chelator insignis & 2537 & 11 & BY2 & $18 \mathrm{~s}$ & Yes & Depth & $\begin{array}{l}\text { Brix et al., } \\
(2014)\end{array}$ \\
\hline 132 & Chelator insignis & 2563 & 3 & BY13 & $\mathrm{COI}$ & No & Limitations & $\begin{array}{l}\text { Brix et al., } \\
(2015)\end{array}$ \\
\hline 133 & Chelator insignis & 2563 & 2 & BY13 & $16 \mathrm{~S}$ & No & Limitations & $\begin{array}{l}\text { Brix et al., } \\
(2015)\end{array}$ \\
\hline 134 & Chelator rugosus & 1 & 6 & BY13 & $\mathrm{COI}$ & No & Limitations & $\begin{array}{l}\text { Brix et al., } \\
(2015)\end{array}$ \\
\hline 135 & Chelator rugosus & 1 & 4 & BY13 & $16 \mathrm{~S}$ & No & Limitations & $\begin{array}{l}\text { Brix et al., } \\
(2015)\end{array}$ \\
\hline 136 & Chelator rugosus & 1 & 3 & BY13 & $18 \mathrm{~S}$ & No & Limitations & $\begin{array}{l}\text { Brix et al., } \\
(2015)\end{array}$ \\
\hline 137 & $\begin{array}{l}\text { Chorismus } \\
\text { antarcticus }\end{array}$ & 1968 & 178 & BY9 & $\mathrm{COI}$ & No & $\begin{array}{l}\text { Biological } \\
\text { Factors }\end{array}$ & $\begin{array}{l}\text { Raupach et al., } \\
(2010)\end{array}$ \\
\hline 138 & $\begin{array}{l}\text { Chorismus } \\
\text { antarcticus }\end{array}$ & 1968 & 178 & BY9 & $16 \mathrm{~s}$ & No & Limitations & $\begin{array}{l}\text { Raupach et al., } \\
(2010)\end{array}$ \\
\hline 139 & $\begin{array}{l}\text { Chorismus } \\
\text { antarcticus }\end{array}$ & 1968 & 178 & BY9 & $28 \mathrm{~s}$ & No & Limitations & $\begin{array}{l}\text { Raupach et al., } \\
(2010)\end{array}$ \\
\hline 140 & $\begin{array}{l}\text { Chorismus } \\
\text { antarcticus }\end{array}$ & 1968 & 178 & BY9 & $16 \mathrm{~s}$ & No & Limitations & $\begin{array}{l}\text { Raupach et al., } \\
(2010)\end{array}$ \\
\hline 141 & $\begin{array}{l}\text { Chorismus } \\
\text { antarcticus }\end{array}$ & 1968 & 178 & BY9 & $28 \mathrm{~s}$ & No & Limitations & $\begin{array}{l}\text { Raupach et al., } \\
(2010)\end{array}$ \\
\hline 142 & Chorocaris sp. 2 & 650 & 179 & BY12 & $\mathrm{COI}$ & Yes & Topography & $\begin{array}{l}\text { Thaler et al., } \\
\text { (2014) }\end{array}$ \\
\hline 143 & Chorocaris sp. 2 & 650 & 273 & BY12 & SSR & Yes & Topography & $\begin{array}{l}\text { Thaler } \text { et al., } \\
\text { (2014) }\end{array}$ \\
\hline 144 & Chrysogorgia & 3660 & 24 & BY4 & MutS & No & Limitations & $\begin{array}{l}\text { Thoma et al., } \\
\text { (2009) }\end{array}$ \\
\hline 145 & $\begin{array}{l}\text { Chrysomallon } \\
\text { squamiferum }\end{array}$ & 0 & 93 & BY11 & $\mathrm{COI}$ & Yes & $\begin{array}{l}\text { Biological } \\
\text { Factors }\end{array}$ & $\begin{array}{l}\text { Chen et al., } \\
\text { (2015) }\end{array}$ \\
\hline 146 & $\begin{array}{l}\text { Cibicides } \\
\text { wuellerstorfi }\end{array}$ & 4403 & 37 & AB2 AB6 & $12 \mathrm{~s}$ & No & $\begin{array}{l}\text { Biological } \\
\text { Factors }\end{array}$ & $\begin{array}{l}\text { Pawlowski et } \\
\text { al., (2007) }\end{array}$ \\
\hline 147 & $\begin{array}{l}\text { Cibicides } \\
\text { wuellerstorfi }\end{array}$ & 4403 & 37 & AB2 AB6 & ITS & No & $\begin{array}{l}\text { Biological } \\
\text { Factors }\end{array}$ & $\begin{array}{l}\text { Pawlowski et } \\
\text { al., (2007) }\end{array}$ \\
\hline 148 & $\begin{array}{l}\text { Cibicides } \\
\text { wuellerstorfi }\end{array}$ & 4403 & 12 & BY9 BY10 & ITS & Yes & $\begin{array}{l}\text { Biological } \\
\text { Factors }\end{array}$ & $\begin{array}{l}\text { Brandt et al., } \\
\text { (2007) }\end{array}$ \\
\hline 149 & $\begin{array}{l}\text { Cibicides } \\
\text { wuellerstorfi }\end{array}$ & 808 & 23 & BY7 BY4 & $12 \mathrm{~s}$ & No & Limitations & $\begin{array}{l}\text { Burkett et al., } \\
(2014)\end{array}$ \\
\hline 150 & $\begin{array}{l}\text { Clencharia } \\
\text { abyssorum }\end{array}$ & 2106 & 43 & BY2 AB2 & $16 \mathrm{~s}$ & No & Limitations & $\begin{array}{l}\text { Etter } \text { et al., } \\
\text { (2007) }\end{array}$ \\
\hline 151 & Corallium lauuense & 190 & 134 & BY14 & SSR & Yes & Current & $\begin{array}{l}\text { Baco et al., } \\
(2005)\end{array}$ \\
\hline 152 & Corallium rubrum & 20 & 2 & BY4 & Alloenzyme & Yes & $\begin{array}{l}\text { Biological } \\
\text { Factors }\end{array}$ & $\begin{array}{l}\text { Abbiati et al., } \\
\text { (1993) }\end{array}$ \\
\hline 153 & Corallium rubrum & 234 & 12 & BY4 & SSR & Yes & Depth & $\begin{array}{l}\text { Costantini et al., } \\
\text { (2009) }\end{array}$ \\
\hline 154 & Corallium rubrum & 234 & 12 & BY4 & MutS & Yes & Depth & $\begin{array}{l}\text { Costantini et al., } \\
\text { (2009) }\end{array}$ \\
\hline 155 & Corallium rubrum & 234 & 12 & BY4 & ITS1 & Yes & Depth & $\begin{array}{l}\text { Costantini et al., } \\
(2009)\end{array}$ \\
\hline 156 & Corallium rubrum & 20 & 398 & BY4 & SSR & Yes & $\begin{array}{l}\text { Biological } \\
\text { Factors }\end{array}$ & $\begin{array}{l}\text { Costantini et al., } \\
\text { (2007) }\end{array}$ \\
\hline 157 & $\begin{array}{l}\text { Coryphaenoides } \\
\text { armatus }\end{array}$ & 3479 & 210 & BY4 BY11 & SSR & Yes & $\begin{array}{l}\text { Biological } \\
\text { Factors }\end{array}$ & $\begin{array}{l}\text { Ritchie et al., } \\
\text { (2013) }\end{array}$ \\
\hline 158 & $\begin{array}{l}\text { Coryphaenoides } \\
\text { armatus }\end{array}$ & 750 & 10 & $\begin{array}{l}\text { BY4 BY7 BY2 } \\
\text { BY3 BY8 }\end{array}$ & Alloenzyme & No & $\begin{array}{l}\text { Biological } \\
\text { Factors }\end{array}$ & $\begin{array}{l}\text { Wilson et al., } \\
\text { (1984) }\end{array}$ \\
\hline 159 & $\begin{array}{l}\text { Coryphaenoides } \\
\text { rupestris }\end{array}$ & 1033 & 417 & BY4 & SSR & Yes & Depth \& Distance & $\begin{array}{l}\text { White et al., } \\
(2010)\end{array}$ \\
\hline 160 & $\begin{array}{l}\text { Coryphaenoides } \\
\text { yaquinae }\end{array}$ & 2400 & 11 & BY7 BY3 & Alloenzyme & No & $\begin{array}{l}\text { Biological } \\
\text { Factors }\end{array}$ & $\begin{array}{l}\text { Wilson et al., } \\
\text { (1983) }\end{array}$ \\
\hline
\end{tabular}




\begin{tabular}{|c|c|c|c|c|c|c|c|c|}
\hline 161 & $\begin{array}{l}\text { Desmophyllum } \\
\text { dianthus }\end{array}$ & 826 & 112 & BY4 & ITS & Yes & Depth \& Distance & $\begin{array}{l}\text { Addamo et al., } \\
(2012)\end{array}$ \\
\hline 162 & $\begin{array}{l}\text { Desmophyllum } \\
\text { dianthus }\end{array}$ & 826 & 98 & BY4 & $28 \mathrm{~s}$ & No & $\begin{array}{l}\text { Biological } \\
\text { Factors }\end{array}$ & $\begin{array}{l}\text { Addamo et al., } \\
(2012)\end{array}$ \\
\hline 163 & $\begin{array}{l}\text { Desmophyllum } \\
\text { dianthus }\end{array}$ & 826 & 57 & BY4 & $16 \mathrm{~s}$ & No & $\begin{array}{l}\text { Biological } \\
\text { Factors }\end{array}$ & $\begin{array}{l}\text { Addamo et al., } \\
\text { (2012) }\end{array}$ \\
\hline 164 & $\begin{array}{l}\text { Desmophyllum } \\
\text { dianthus }\end{array}$ & 826 & 51 & BY4 & COI & No & $\begin{array}{l}\text { Biological } \\
\text { Factors }\end{array}$ & $\begin{array}{l}\text { Addamo et al., } \\
\text { (2012) }\end{array}$ \\
\hline 165 & $\begin{array}{l}\text { Desmophyllum } \\
\text { dianthus }\end{array}$ & 700 & 58 & BY11 BY6 BY10 & ITS & Yes & $\begin{array}{l}\text { Depth \& } \\
\text { Topography }\end{array}$ & $\begin{array}{l}\text { Miller et al., } \\
(2010)\end{array}$ \\
\hline 166 & $\begin{array}{l}\text { Desmophyllum } \\
\text { dianthus }\end{array}$ & 700 & 30 & BY11 BY6 BY10 & D-loop & Yes & $\begin{array}{l}\text { Depth \& } \\
\text { Topography }\end{array}$ & $\begin{array}{l}\text { Miller et al., } \\
(2010)\end{array}$ \\
\hline 167 & $\begin{array}{l}\text { Desmophyllum } \\
\text { dianthus }\end{array}$ & 1400 & 162 & BY6 BY8 BY10 & D-loop & Yes & Depth & $\begin{array}{l}\text { Miller et al., } \\
(2011)\end{array}$ \\
\hline 168 & $\begin{array}{l}\text { Desmophyllum } \\
\text { dianthus }\end{array}$ & 1400 & 162 & BY6 BY8 BY10 & ITS & Yes & Depth & $\begin{array}{l}\text { Miller et al., } \\
\text { (2011) }\end{array}$ \\
\hline 169 & $\begin{array}{l}\text { Desmophyllum } \\
\text { dianthus }\end{array}$ & 1400 & 162 & BY6 BY8 BY10 & $16 \mathrm{~s}$ & Yes & Depth & $\begin{array}{l}\text { Miller et al., } \\
\text { (2011) }\end{array}$ \\
\hline 170 & $\begin{array}{l}\text { Desmophyllum } \\
\text { dianthus }\end{array}$ & 1906 & 313 & BY10 & SSR & Yes & Depth & $\begin{array}{l}\text { Miller et al., } \\
\text { (2016) }\end{array}$ \\
\hline 171 & Diaphus mollis & 565 & 12 & BY4 BY 10 BY13 & COI & Yes & Depth & $\begin{array}{l}\text { Gordeeva et al., } \\
\text { (2014) }\end{array}$ \\
\hline 172 & $\begin{array}{l}\text { Diogenichthys } \\
\text { atlanticus }\end{array}$ & 550 & 11 & BY4 BY 10 BY 13 & COI & Yes & Depth \& Distance & $\begin{array}{l}\text { Gordeeva et al., } \\
\text { (2014) }\end{array}$ \\
\hline 173 & $\begin{array}{l}\text { Diplopteraster } \\
\text { multipes }\end{array}$ & 1800 & 31 & BY3 & Alloenzyme & No & Limitations & $\begin{array}{l}\text { Ayala et al., } \\
(1975)\end{array}$ \\
\hline 174 & $\begin{array}{l}\text { Dissostichus } \\
\text { eleginoides }\end{array}$ & 1800 & 450 & BY13 BY10 & SSR & Yes & Current & $\begin{array}{l}\text { Shaw et al., } \\
(2004)\end{array}$ \\
\hline 175 & $\begin{array}{l}\text { Dissostichus } \\
\text { eleginoides }\end{array}$ & 1800 & 396 & BY13 BY10 & ND2 & Yes & Current & $\begin{array}{l}\text { Shaw et al., } \\
(2004)\end{array}$ \\
\hline 176 & $\begin{array}{l}\text { Dissostichus } \\
\text { eleginoides }\end{array}$ & 1800 & 446 & BY13 BY10 & D-loop & Yes & Current & $\begin{array}{l}\text { Shaw et al., } \\
\text { (2004) }\end{array}$ \\
\hline 177 & $\begin{array}{l}\text { Dissostichus } \\
\text { eleginoides }\end{array}$ & & 151 & BY10 & $12 \mathrm{~s}$ & Yes & $\begin{array}{l}\text { Distance \&Depth } \\
\& \text { Topography }\end{array}$ & $\begin{array}{l}\text { Rogers et al., } \\
(2006)\end{array}$ \\
\hline 178 & $\begin{array}{l}\text { Dissostichus } \\
\text { eleginoides }\end{array}$ & & 151 & BY10 & $16 \mathrm{~s}$ & No & Limitations & $\begin{array}{l}\text { Rogers et al., } \\
(2006)\end{array}$ \\
\hline 179 & $\begin{array}{l}\text { Dissostichus } \\
\text { eleginoides }\end{array}$ & & 274 & BY10 & SSR & Yes & $\begin{array}{l}\text { Distance \&Depth } \\
\text { \& Topography }\end{array}$ & $\begin{array}{l}\text { Rogers et al., } \\
(2006)\end{array}$ \\
\hline 180 & $\begin{array}{l}\text { Enallopsammia } \\
\text { rostrata }\end{array}$ & 0 & 75 & BY11 BY6 BY10 & ITS & Yes & Topography & $\begin{array}{l}\text { Miller et al., } \\
(2010)\end{array}$ \\
\hline 181 & $\begin{array}{l}\text { Enallopsammia } \\
\text { rostrata }\end{array}$ & 0 & 90 & BY11 BY6 BY10 & D-loop & Yes & Topography & $\begin{array}{l}\text { Miller et al., } \\
(2010)\end{array}$ \\
\hline 182 & Ennucula similis & 1650 & 53 & BY2 AB2 & $16 \mathrm{~s}$ & Yes & Depth & $\begin{array}{l}\text { Etter et al., } \\
(2007)\end{array}$ \\
\hline 183 & Epistominella exigua & 4403 & 37 & AB2 AB6 & $12 \mathrm{~s}$ & Yes & Depth & $\begin{array}{l}\text { Pawlowski et } \\
\text { al., (2007) }\end{array}$ \\
\hline 184 & Epistominella exigua & 4403 & 37 & AB2 AB6 & ITS & Yes & Depth & $\begin{array}{l}\text { Pawlowski et } \\
\text { al., (2007) }\end{array}$ \\
\hline 185 & Epistominella exigua & 4403 & 42 & BY9 BY10 & ITS & Yes & $\begin{array}{l}\text { Biological } \\
\text { Factors }\end{array}$ & $\begin{array}{l}\text { Brandt et al., } \\
\text { (2007) }\end{array}$ \\
\hline 186 & Epistominella exigua & 2580 & 23 & $\begin{array}{l}\text { BY12 BY2 BY4 } \\
\text { BY13 }\end{array}$ & $12 \mathrm{~s}$ & No & $\begin{array}{l}\text { Biological } \\
\text { Factors }\end{array}$ & $\begin{array}{l}\text { Lecroq et al., } \\
(2009)\end{array}$ \\
\hline 187 & Epistominella exigua & 2580 & 118 & $\begin{array}{l}\text { BY12 BY2 BY4 } \\
\text { BY13 }\end{array}$ & ITS & No & $\begin{array}{l}\text { Biological } \\
\text { Factors }\end{array}$ & $\begin{array}{l}\text { Lecroq et al., } \\
(2009)\end{array}$ \\
\hline 188 & Epistominella vitrea & 4773 & 7 & BY9 & $12 \mathrm{~s}$ & No & Limitations & $\begin{array}{l}\text { Pawlowski et } \\
\text { al., (2007) }\end{array}$ \\
\hline 189 & Epistominella vitrea & 4773 & 9 & BY9 & ITS & No & Limitations & $\begin{array}{l}\text { Pawlowski et } \\
\text { al., (2007) }\end{array}$ \\
\hline 190 & Escarpia laminata & 1752 & 17 & BY4 & COI & Yes & Distance & $\begin{array}{l}\text { Cowart et al., } \\
(2013)\end{array}$ \\
\hline 191 & Escarpia laminata & 1752 & 16 & BY4 & $16 \mathrm{~s}$ & Yes & Distance & $\begin{array}{l}\text { Cowart et al., } \\
\text { (2013) }\end{array}$ \\
\hline 192 & Escarpia laminata & 1752 & 17 & BY4 & Cytb & Yes & Distance & $\begin{array}{l}\text { Cowart et al., } \\
(2013)\end{array}$ \\
\hline 193 & Escarpia laminata & 1752 & 17 & BY4 & $\begin{array}{l}\text { Haemoglobi } \\
\text { n subunit B2 }\end{array}$ & Yes & Distance & $\begin{array}{l}\text { Cowart et al., } \\
\text { (2013) }\end{array}$ \\
\hline 194 & Escarpia laminata & 1752 & 129 & BY4 & SSR & Yes & Distance & $\begin{array}{l}\text { Cowart et al., } \\
\text { (2013) }\end{array}$ \\
\hline 195 & $\begin{array}{l}\text { Escarpia } \\
\text { southwardae }\end{array}$ & 1752 & 17 & BY4 & COI & Yes & Distance & $\begin{array}{l}\text { Cowart et al., } \\
\text { (2013) }\end{array}$ \\
\hline 196 & $\begin{array}{l}\text { Escarpia } \\
\text { southwardae }\end{array}$ & 1752 & 19 & BY4 & $16 \mathrm{~s}$ & Yes & Distance & $\begin{array}{l}\text { Cowart et al., } \\
\text { (2013) }\end{array}$ \\
\hline 197 & $\begin{array}{l}\text { Escarpia } \\
\text { southwardae }\end{array}$ & 1752 & 12 & BY4 & Cytb & Yes & Distance & $\begin{array}{l}\text { Cowart et al., } \\
\text { (2013) }\end{array}$ \\
\hline 198 & $\begin{array}{l}\text { Escarpia } \\
\text { southwardae }\end{array}$ & 1752 & 16 & BY4 & $\begin{array}{l}\text { Haemoglobi } \\
\text { n subunit B2 }\end{array}$ & Yes & Distance & $\begin{array}{l}\text { Cowart et al., } \\
\text { (2013) }\end{array}$ \\
\hline 199 & $\begin{array}{l}\text { Escarpia } \\
\text { southwardae }\end{array}$ & 1752 & 80 & BY4 & SSR & Yes & Temperature & $\begin{array}{l}\text { Cowart et al., } \\
\text { (2013) }\end{array}$ \\
\hline 200 & Escarpia spicata & 1752 & 8 & BY4 & COI & Yes & Distance & $\begin{array}{l}\text { Cowart et al., } \\
\text { (2013) }\end{array}$ \\
\hline 201 & Escarpia spicata & 1752 & 10 & BY4 & $16 \mathrm{~s}$ & Yes & Distance & $\begin{array}{l}\text { Cowart et al., } \\
(2013)\end{array}$ \\
\hline 202 & Escarpia spicata & 1752 & 10 & BY4 & Cytb & Yes & Distance & $\begin{array}{l}\text { Cowart et al., } \\
(2013)\end{array}$ \\
\hline 203 & Escarpia spicata & 1752 & 10 & BY4 & $\begin{array}{l}\text { Haemoglobi } \\
\text { n subunit B2 }\end{array}$ & Yes & Distance & $\begin{array}{l}\text { Cowart et al., } \\
(2013)\end{array}$ \\
\hline
\end{tabular}




\begin{tabular}{|c|c|c|c|c|c|c|c|c|}
\hline 204 & Etelis "marshi" & 290 & 770 & BY14 & Cytb & No & $\begin{array}{l}\text { Biological } \\
\text { Factors }\end{array}$ & $\begin{array}{l}\text { Andrews } \text { et al., } \\
(2014)\end{array}$ \\
\hline 205 & Etelis "marshi" & 290 & 770 & BY14 & SSR & No & $\begin{array}{l}\text { Biological } \\
\text { Factors }\end{array}$ & $\begin{array}{l}\text { Andrews et al., } \\
(2014)\end{array}$ \\
\hline 206 & Etelis carbunculus & & 90 & BY12 & D-loop & No & $\begin{array}{l}\text { Biological } \\
\text { Factors \& } \\
\text { Limitations }\end{array}$ & $\begin{array}{l}\text { Loeun et al., } \\
\text { (2014) }\end{array}$ \\
\hline 207 & Etelis coruscans & 290 & 787 & BY14 & Cytb & No & $\begin{array}{l}\text { Biological } \\
\text { Factors }\end{array}$ & $\begin{array}{l}\text { Andrews et al., } \\
\text { (2014) }\end{array}$ \\
\hline 208 & Etelis coruscans & 290 & 787 & BY14 & SSR & No & $\begin{array}{l}\text { Biological } \\
\text { Factors }\end{array}$ & $\begin{array}{l}\text { Andrews et al., } \\
\text { (2014) }\end{array}$ \\
\hline 209 & Etelis coruscans & & 90 & BY12 & D-loop & No & $\begin{array}{l}\text { Biological } \\
\text { Factors \& } \\
\text { Limitations }\end{array}$ & $\begin{array}{l}\text { Loeun et al., } \\
\text { (2014) }\end{array}$ \\
\hline 210 & Euchaetidae & 2000 & 22 & BY1 BY9 & COI & No & Current & $\begin{array}{l}\text { Laakmann et al., } \\
(2012)\end{array}$ \\
\hline 211 & Euchaetidae & 2000 & 22 & BY1 BY9 & $18 \mathrm{~s}$ & Yes & $\begin{array}{l}\text { Biological } \\
\text { Factors }\end{array}$ & $\begin{array}{l}\text { Laakmann et al., } \\
\text { (2012) }\end{array}$ \\
\hline 212 & Euchaetidae & 2000 & 22 & BY1 BY9 & $28 \mathrm{~s}$ & Yes & $\begin{array}{l}\text { Biological } \\
\text { Factors }\end{array}$ & $\begin{array}{l}\text { Laakmann et al., } \\
\text { (2012) }\end{array}$ \\
\hline 213 & Euchaetidae & 2000 & 22 & BY1 BY9 & ITS2 & Yes & $\begin{array}{l}\text { Biological } \\
\text { Factors }\end{array}$ & $\begin{array}{l}\text { Laakmann et al., } \\
\text { (2012) }\end{array}$ \\
\hline 214 & Eulepetopsis vitrea & 115 & 559 & BY7 & Alloenzyme & Yes & Limitations & $\begin{array}{l}\text { Craddock et al., } \\
\text { (1997) }\end{array}$ \\
\hline 215 & Eulepetopsis vitrea & 340 & 297 & BY7 & COI & Yes & $\begin{array}{l}\text { Biological } \\
\text { Factors \& Ocean } \\
\text { current }\end{array}$ & $\begin{array}{l}\text { Plouviez et al., } \\
\text { (2009) }\end{array}$ \\
\hline 216 & Eumunida annulosa & 1348 & 34 & BY12 & COI & No & Limitations & $\begin{array}{l}\text { Samadi et al., } \\
(2006)\end{array}$ \\
\hline 217 & $\begin{array}{l}\text { Eumunida } \\
\text { sternomaculata }\end{array}$ & 1348 & 20 & BY12 & COI & No & Limitations & $\begin{array}{l}\text { Samadi et al., } \\
(2006)\end{array}$ \\
\hline 218 & Euphausia superba & & 880 & BY9 & Alloenzyme & No & $\begin{array}{l}\text { Biological } \\
\text { Factors }\end{array}$ & $\begin{array}{l}\text { Fevolden et al., } \\
\text { (1989) }\end{array}$ \\
\hline 219 & Euphausia superba & 2500 & 1185 & BY9 BY 10 & Alloenzyme & No & Current & $\begin{array}{l}\text { MacDonald et } \\
\text { al., (1986) }\end{array}$ \\
\hline 220 & Euphausia superba & & 249 & BY9 & ND1 & Yes & Current & $\begin{array}{l}\text { Zane et al., } \\
\text { (1998) }\end{array}$ \\
\hline 221 & Euphausia superba & 910 & 585 & $\begin{array}{l}\text { BY13 } \\
\text { BY1 }\end{array}$ & SNP & No & $\begin{array}{l}\text { Biological } \\
\text { Factors }\end{array}$ & $\begin{array}{l}\text { Batta-Lonaa et } \\
\text { al., (2011) }\end{array}$ \\
\hline 222 & Eurythenes gryllus & 4648 & 95 & $\begin{array}{l}\text { BY1 BY4 AB2 } \\
\text { AB12 } \\
\text { AB6 AB3 AB5 }\end{array}$ & $16 \mathrm{~s}$ & Yes & Depth & $\begin{array}{l}\text { France } \text { et al., } \\
\text { (1996) }\end{array}$ \\
\hline 223 & Eurythenes gryllus & 3854 & 47 & $\begin{array}{l}\text { AB2 BY4 BY14 } \\
\text { AB11 AB12 BY1 } \\
\text { BY9 BY10 }\end{array}$ & COI & Yes & Depth & $\begin{array}{l}\text { Havermans et } \\
\text { al., (2013) }\end{array}$ \\
\hline 224 & Eurythenes gryllus & 3855 & 47 & $\begin{array}{l}\text { AB6 AB3 AB5 } \\
\text { AB2 BY4 BY14 } \\
\text { AB11 AB12 BY1 } \\
\text { BY9 BY10 }\end{array}$ & $16 \mathrm{~s}$ & Yes & Depth & $\begin{array}{l}\text { Havermans et } \\
\text { al., (2013) }\end{array}$ \\
\hline 225 & Eurythenes gryllus & 3856 & 47 & $\begin{array}{l}\text { AB6 AB3 AB5 } \\
\text { AB2 BY4 BY14 } \\
\text { AB11 AB12 BY1 } \\
\text { BY9 BY10 }\end{array}$ & $28 \mathrm{~s}$ & Yes & Depth & $\begin{array}{l}\text { Havermans et } \\
\text { al., (2013) }\end{array}$ \\
\hline 226 & Eurythenes gryllus & 0 & 107 & BY14 & Alloenzyme & Yes & Topography & $\begin{array}{l}\text { Bucklin et al., } \\
\text { (1987) }\end{array}$ \\
\hline 227 & Eusirus giganteus & 636 & 52 & BY9 & COI & Yes & $\begin{array}{l}\text { Biological } \\
\text { Factors \& } \\
\text { Limitations }\end{array}$ & $\begin{array}{l}\text { Baird et al., } \\
\text { (2011) }\end{array}$ \\
\hline 228 & Eusirus giganteus & 636 & 57 & BY9 & Cytb & Yes & $\begin{array}{l}\text { Biological } \\
\text { Factors \& } \\
\text { Limitations }\end{array}$ & $\begin{array}{l}\text { Baird et al., } \\
(2011)\end{array}$ \\
\hline 229 & Eusirus giganteus & 636 & 21 & BY9 & ITS2 & No & $\begin{array}{l}\text { Biological } \\
\text { Factors \& } \\
\text { Limitations }\end{array}$ & $\begin{array}{l}\text { Baird et al., } \\
(2011)\end{array}$ \\
\hline 230 & Eusirus perdentatus & 635 & 65 & BY9 & COI & Yes & $\begin{array}{l}\text { Biological } \\
\text { Factors \& } \\
\text { Limitations }\end{array}$ & $\begin{array}{l}\text { Baird et al., } \\
(2011)\end{array}$ \\
\hline 231 & Eusirus perdentatus & 635 & 52 & BY9 & Cytb & Yes & $\begin{array}{l}\text { Biological } \\
\text { Factors \& } \\
\text { Limitations }\end{array}$ & $\begin{array}{l}\text { Baird et al., } \\
\text { (2011) }\end{array}$ \\
\hline 232 & Eusirus perdentatus & 635 & 12 & BY9 & ITS2 & No & $\begin{array}{l}\text { Biological } \\
\text { Factors \& } \\
\text { Limitations }\end{array}$ & $\begin{array}{l}\text { Baird et al., } \\
\text { (2011) }\end{array}$ \\
\hline 233 & $\begin{array}{l}\text { Frigidoalvania } \\
\text { brychia }\end{array}$ & 645 & 63 & BY4 & $16 \mathrm{~s}$ & Yes & Depth & $\begin{array}{l}\text { Quattro et al., } \\
\text { (2001) }\end{array}$ \\
\hline 234 & Gigantidas gladius & 693 & 150 & BY6 & COI & No & Limitations & $\begin{array}{l}\text { Boschen et al., } \\
\text { (2015) }\end{array}$ \\
\hline 235 & $\begin{array}{l}\text { Gigantopelta } \\
\text { chessoia }\end{array}$ & 250 & 84 & BY9 & COI & No & $\begin{array}{l}\text { Biological } \\
\text { Factors }\end{array}$ & $\begin{array}{l}\text { Roterman et al., } \\
(2016)\end{array}$ \\
\hline 236 & $\begin{array}{l}\text { Gigantopelta } \\
\text { chessoia }\end{array}$ & 250 & 84 & BY9 & SSR & No & $\begin{array}{l}\text { Biological } \\
\text { Factors }\end{array}$ & $\begin{array}{l}\text { Roterman et al., } \\
(2016)\end{array}$ \\
\hline 237 & $\begin{array}{l}\text { Glycera cf } \\
\text { kerguelensis }\end{array}$ & 3237 & 38 & BY13 & COI & Yes & Depth & $\begin{array}{l}\text { Schuller et al., } \\
\text { (2011) }\end{array}$ \\
\hline 238 & $\begin{array}{l}\text { Glycera cf } \\
\text { kerguelensis }\end{array}$ & 3237 & 11 & BY13 & $28 \mathrm{~s}$ & Yes & Depth & $\begin{array}{l}\text { Schuller et al., } \\
(2011)\end{array}$ \\
\hline
\end{tabular}




\begin{tabular}{|c|c|c|c|c|c|c|c|c|}
\hline 239 & $\begin{array}{l}\text { Goniocorella } \\
\text { dumosa }\end{array}$ & 1072 & 133 & BY6 BY10 & ITS & Yes & Depth & $\begin{array}{l}\text { Zeng et al., } \\
(2016)\end{array}$ \\
\hline 240 & $\begin{array}{l}\text { Goniocorella } \\
\text { dumosa }\end{array}$ & 1072 & 70 & BY6 BY10 & D-loop & No & Limitations & $\begin{array}{l}\text { Zeng et al., } \\
\text { (2016) }\end{array}$ \\
\hline 241 & $\begin{array}{l}\text { Goniocorella } \\
\text { dumosa }\end{array}$ & 1072 & 78 & BY6 BY10 & SSR & Yes & Depth & $\begin{array}{l}\text { Zeng et al., } \\
(2016)\end{array}$ \\
\hline 242 & $\begin{array}{l}\text { Helicolenus } \\
\text { dactylopterus }\end{array}$ & 800 & 212 & BY4 & Cytb & Yes & Current & $\begin{array}{l}\text { Aboim et al., } \\
(2005)\end{array}$ \\
\hline 243 & $\begin{array}{l}\text { Helicolenus } \\
\text { dactylopterus }\end{array}$ & 800 & 208 & BY4 & D-loop & Yes & Current & $\begin{array}{l}\text { Aboim et al., } \\
(2005)\end{array}$ \\
\hline 244 & Hesiolyra bergi & 340 & 235 & BY7 & $\mathrm{COI}$ & No & $\begin{array}{l}\text { Biological } \\
\text { Factors }\end{array}$ & $\begin{array}{l}\text { Plouviez et al., } \\
(2009)\end{array}$ \\
\hline 245 & $\begin{array}{l}\text { Hexadella } \\
\text { dedritifera }\end{array}$ & 673 & 30 & BY4 & $\mathrm{COI}$ & Yes & Current & $\begin{array}{l}\text { Reveillaud et al., } \\
\text { (2010) }\end{array}$ \\
\hline 246 & $\begin{array}{l}\text { Hexadella } \\
\text { dedritifera }\end{array}$ & 674 & 21 & BY4 & $28 \mathrm{~s}$ & Yes & Current & $\begin{array}{l}\text { Reveillaud et al., } \\
\text { (2010) }\end{array}$ \\
\hline 247 & $\begin{array}{l}\text { Hexadella } \\
\text { dedritifera }\end{array}$ & 675 & 20 & BY4 & ATPs Intron & Yes & Current & $\begin{array}{l}\text { Reveillaud et al., } \\
(2010)\end{array}$ \\
\hline 248 & $\begin{array}{l}\text { Hippasteria } \\
\text { phrygiana }\end{array}$ & 557 & 140 & $\begin{array}{l}\text { BY3 BY4 BY6 } \\
\text { BY8 BY10 BY11 } \\
\text { BY12 BY13 }\end{array}$ & $\mathrm{COI}$ & Yes & $\begin{array}{l}\text { Biological } \\
\text { Factors }\end{array}$ & $\begin{array}{l}\text { Foltz et al., } \\
(2013)\end{array}$ \\
\hline 249 & $\begin{array}{l}\text { Hippasteria } \\
\text { phrygiana }\end{array}$ & 557 & 250 & $\begin{array}{l}\text { BY3 BY4 BY6 } \\
\text { BY8 BY10 BY11 } \\
\text { BY12 BY13 }\end{array}$ & ATPS $\alpha$ & No & Distance & $\begin{array}{l}\text { Foltz et al., } \\
(2013)\end{array}$ \\
\hline 250 & $\begin{array}{l}\text { Holothuria } \\
\text { (Holothuria) } \\
\text { mammata }\end{array}$ & & 177 & BY4 & $16 \mathrm{~s}$ & Yes & Current & $\begin{array}{l}\text { Borrero-Pérez et } \\
\text { al., (2011) }\end{array}$ \\
\hline 251 & $\begin{array}{l}\text { Holothuria } \\
\text { (Holothuria) } \\
\text { mammata }\end{array}$ & & 177 & BY4 & $\mathrm{COI}$ & Yes & Current & $\begin{array}{l}\text { Borrero-Pérez et } \\
\text { al., (2011) }\end{array}$ \\
\hline 252 & $\begin{array}{l}\text { Hoplostethus } \\
\text { atlanticus }\end{array}$ & & 294 & BY4 BY13 & SSR & No & $\begin{array}{l}\text { Biological } \\
\text { Factors }\end{array}$ & $\begin{array}{l}\text { White et al., } \\
(2009)\end{array}$ \\
\hline 253 & $\begin{array}{l}\text { Hoplostethus } \\
\text { atlanticus }\end{array}$ & 1000 & 388 & BY4 & SSR & Yes & Topography & $\begin{array}{l}\text { Carlsson et al., } \\
\text { (2011) }\end{array}$ \\
\hline 254 & $\begin{array}{l}\text { Hoplostethus } \\
\text { atlanticus }\end{array}$ & & 546 & $\begin{array}{l}\text { BY4 BY8 BY11 } \\
\text { BY6 BY10 BY13 }\end{array}$ & $\mathrm{COI}$ & No & $\begin{array}{l}\text { Biological } \\
\text { Factors }\end{array}$ & $\begin{array}{l}\text { Varela et al., } \\
\text { (2012) }\end{array}$ \\
\hline 255 & $\begin{array}{l}\text { Hoplostethus } \\
\text { atlanticus }\end{array}$ & & 546 & $\begin{array}{l}\text { BY4 BY8 BY11 } \\
\text { BY6 BY10 BY13 }\end{array}$ & Cytb & No & $\begin{array}{l}\text { Biological } \\
\text { Factors }\end{array}$ & $\begin{array}{l}\text { Varela et al., } \\
\text { (2012) }\end{array}$ \\
\hline 256 & $\begin{array}{l}\text { Hoplostethus } \\
\text { atlanticus }\end{array}$ & 1730 & 95 & BY6 BY10 & Cytb & No & $\begin{array}{l}\text { Biological } \\
\text { Factors }\end{array}$ & $\begin{array}{l}\text { Varela et al., } \\
\text { (2013) }\end{array}$ \\
\hline 257 & $\begin{array}{l}\text { Hoplostethus } \\
\text { atlanticus }\end{array}$ & 1730 & 546 & BY6 BY 10 & $\mathrm{COI}$ & No & $\begin{array}{l}\text { Biological } \\
\text { Factors }\end{array}$ & $\begin{array}{l}\text { Varela et al., } \\
\text { (2013) }\end{array}$ \\
\hline 258 & $\begin{array}{l}\text { Hoplostethus } \\
\text { atlanticus }\end{array}$ & 1730 & 812 & BY6 BY 10 & SSR & No & $\begin{array}{l}\text { Biological } \\
\text { Factors }\end{array}$ & $\begin{array}{l}\text { Varela et al., } \\
\text { (2013) }\end{array}$ \\
\hline 259 & $\begin{array}{l}\text { Hoplostethus } \\
\text { atlanticus }\end{array}$ & & 246 & BY6 BY10 & RFLP & Yes & Distance & $\begin{array}{l}\text { Smith et al., } \\
\text { (1996) }\end{array}$ \\
\hline 260 & $\begin{array}{l}\text { Hyalinoecia } \\
\text { longibranchiata }\end{array}$ & 326 & 61 & BY6 & $\mathrm{COI}$ & Yes & Current & $\begin{array}{l}\text { Bors et al., } \\
(2012)\end{array}$ \\
\hline 261 & $\begin{array}{l}\text { Hyalinoecia } \\
\text { longibranchiata }\end{array}$ & 326 & 61 & BY6 & $16 \mathrm{~S}$ & Yes & Current & $\begin{array}{l}\text { Bors et al., } \\
(2012)\end{array}$ \\
\hline 262 & $\begin{array}{l}\text { Hyperoglyphe } \\
\text { antarctica }\end{array}$ & & 680 & BY6 BY10 & Alloenzyme & No & Limitations & $\begin{array}{l}\text { Bolch et al., } \\
\text { (1993) }\end{array}$ \\
\hline 263 & Ifremeria nautilei & 350 & 52 & BY12 & $\mathrm{COI}$ & Yes & Distance & $\begin{array}{l}\text { Kojima et al., } \\
(2000)\end{array}$ \\
\hline 264 & Ifremeria nautilei & 1320 & 158 & BY12 & $\mathrm{COI}$ & Yes & Topography & $\begin{array}{l}\text { Thaler et al., } \\
\text { (2011) }\end{array}$ \\
\hline 265 & Ifremeria nautilei & 1320 & 275 & BY12 & SSR & Yes & Topography & $\begin{array}{l}\text { Thaler et al., } \\
\text { (2011) }\end{array}$ \\
\hline 266 & Iridogorgia & 3660 & 15 & BY4 & MutS & No & Limitations & $\begin{array}{l}\text { Thoma et al., } \\
\text { (2009) }\end{array}$ \\
\hline 267 & Jasus paulensis & & 193 & BY13 & COII & No & Current & $\begin{array}{l}\text { von der Heyden } \\
\text { et al., (2007) }\end{array}$ \\
\hline 268 & Keratoisidinae & 2910 & 66 & BY6 BY10 & $16 \mathrm{~s}$ & No & $\begin{array}{l}\text { Biological } \\
\text { Factors \& } \\
\text { Limitations }\end{array}$ & $\begin{array}{l}\text { Smith et al., } \\
\text { (2004) }\end{array}$ \\
\hline 269 & Keratoisidinae & 2910 & 66 & BY6 BY10 & NCR & No & $\begin{array}{l}\text { Biological } \\
\text { Factors \& } \\
\text { Limitations }\end{array}$ & $\begin{array}{l}\text { Smith et al., } \\
\text { (2004) }\end{array}$ \\
\hline 270 & Kiwa tyleri & 239 & 90 & BY9 & $\mathrm{COI}$ & No & Temperature & $\begin{array}{l}\text { Roterman et al., } \\
(2016)\end{array}$ \\
\hline 271 & Kiwa tyleri & 239 & 91 & BY9 & SSR & No & Temperature & $\begin{array}{l}\text { Roterman et al., } \\
(2016)\end{array}$ \\
\hline 272 & Lamellibrachia juni & 1337 & 34 & BY6 BY12 & $\mathrm{COI}$ & Yes & Topography & $\begin{array}{l}\text { Kojima et al., } \\
\text { (2006) }\end{array}$ \\
\hline 273 & Lampadena chavesi & 725 & 5 & BY4 BY 10 BY 13 & $\mathrm{COI}$ & Yes & Depth & $\begin{array}{l}\text { Gordeeva et al., } \\
\text { (2014) }\end{array}$ \\
\hline 274 & $\begin{array}{l}\text { Lampadena } \\
\text { speculigera }\end{array}$ & 850 & 3 & BY4 BY 10 BY 13 & $\mathrm{COI}$ & Yes & Depth \& Distance & $\begin{array}{l}\text { Gordeeva et al., } \\
\text { (2014) }\end{array}$ \\
\hline 275 & Ledella ultima & 1056 & 81 & BY2 AB2 & $16 \mathrm{~s}$ & No & Limitations & $\begin{array}{l}\text { Etter et al., } \\
(2007)\end{array}$ \\
\hline 276 & Ledella ultima & 2581 & 176 & $\begin{array}{l}\text { AB5 AB2 AB4 } \\
\text { BY4 }\end{array}$ & $16 \mathrm{~s}$ & Yes & Topography & $\begin{array}{l}\text { Etter } \text { et al., } \\
\text { (2011) }\end{array}$ \\
\hline 277 & $\begin{array}{l}\text { Lepetodrilus } \\
\text { elevatus }\end{array}$ & 115 & 251 & BY7 & Alloenzyme & No & Limitations & $\begin{array}{l}\text { Craddock et al., } \\
\text { (1997) }\end{array}$ \\
\hline 278 & $\begin{array}{l}\text { Lepetodrilus } \\
\text { elevatus }\end{array}$ & & 330 & BY7 & Alloenzyme & Yes & $\begin{array}{l}\text { Biological } \\
\text { Factors }\end{array}$ & $\begin{array}{l}\text { Matabos et al., } \\
(2007)\end{array}$ \\
\hline
\end{tabular}




\begin{tabular}{|c|c|c|c|c|c|c|c|c|}
\hline 279 & $\begin{array}{l}\text { Lepetodrilus } \\
\text { elevatus }\end{array}$ & & 77 & BY7 & $\mathrm{COI}$ & Yes & $\begin{array}{l}\text { Biological } \\
\text { Factors }\end{array}$ & $\begin{array}{l}\text { Matabos et al., } \\
(2007)\end{array}$ \\
\hline 280 & $\begin{array}{l}\text { Lepetodrilus } \\
\text { elevatus }\end{array}$ & & 334 & BY7 & Alloenzyme & Yes & $\begin{array}{l}\text { Biological } \\
\text { Factors }\end{array}$ & $\begin{array}{l}\text { Matabos et al., } \\
(2007)\end{array}$ \\
\hline 281 & $\begin{array}{l}\text { Lepetodrilus } \\
\text { elevatus }\end{array}$ & 340 & 439 & BY7 & $\mathrm{COI}$ & Yes & $\begin{array}{l}\text { Biological } \\
\text { Factors \& Ocean } \\
\text { current }\end{array}$ & $\begin{array}{l}\text { Plouviez et al., } \\
\text { (2009) }\end{array}$ \\
\hline 282 & $\begin{array}{l}\text { Lepetodrilus } \\
\text { elevatus }\end{array}$ & 1703 & 157 & BY3 & $\mathrm{COI}$ & Yes & Topography & $\begin{array}{l}\text { Johnson et al., } \\
(2006)\end{array}$ \\
\hline 283 & $\begin{array}{l}\text { Lepetodrilus } \\
\text { elevatus }\end{array}$ & 1703 & 157 & BY3 & PGM-I & Yes & Topography & $\begin{array}{l}\text { Johnson et al., } \\
\text { (2006) }\end{array}$ \\
\hline 284 & $\begin{array}{l}\text { Lepetodrilus } \\
\text { elevatus }\end{array}$ & 1703 & 157 & BY3 & Alloenzyme & Yes & Topography & $\begin{array}{l}\text { Johnson et al., } \\
\text { (2006) }\end{array}$ \\
\hline 285 & $\begin{array}{l}\text { Lepetodrilus } \\
\text { galriftensis }\end{array}$ & 115 & 40 & BY7 & Alloenzyme & No & Limitations & $\begin{array}{l}\text { Craddock et al., } \\
\text { (1997) }\end{array}$ \\
\hline 286 & Lepetodrilus nux & 950 & 154 & BY12 & $\mathrm{COI}$ & Yes & $\begin{array}{l}\text { Biological } \\
\text { Factors \& Vent \& } \\
\text { Current }\end{array}$ & $\begin{array}{l}\text { Nakamura et al., } \\
\text { (2014) }\end{array}$ \\
\hline 287 & Lepetodrilus ovalis & 340 & 187 & BY7 & $\mathrm{COI}$ & Yes & $\begin{array}{l}\text { Biological } \\
\text { Factors \& Ocean } \\
\text { current }\end{array}$ & $\begin{array}{l}\text { Plouviez et al., } \\
(2009)\end{array}$ \\
\hline 288 & $\begin{array}{l}\text { Lepetodrilus } \\
\text { pustulosus }\end{array}$ & 115 & 128 & BY7 & Alloenzyme & Yes & Limitations & $\begin{array}{l}\text { Craddock et al., } \\
\text { (1997) }\end{array}$ \\
\hline 289 & Lepetodrilus sp. & 1211 & 140 & BY9 & $\mathrm{COI}$ & Yes & Current & $\begin{array}{l}\text { Roterman et al., } \\
\text { (2016) }\end{array}$ \\
\hline 290 & Lepetodrilus sp. & 1211 & 140 & BY9 & SSR & Yes & Current & $\begin{array}{l}\text { Roterman et al., } \\
(2016)\end{array}$ \\
\hline 291 & $\begin{array}{l}\text { Liocarcinus } \\
\text { depurator }\end{array}$ & 750 & 115 & BY4 & $\mathrm{COI}$ & Yes & Topography & $\begin{array}{l}\text { García- } \\
\text { Merchána et al., } \\
\text { (2012) }\end{array}$ \\
\hline 292 & Lophelia pertusa & & 190 & BY4 & SSR & Yes & Distance & $\begin{array}{l}\text { Morrison et al., } \\
\text { (2008) }\end{array}$ \\
\hline 293 & Lophelia pertusa & 1392 & 401 & BY4 & SSR & Yes & $\begin{array}{l}\text { Current \& } \\
\text { Distance }\end{array}$ & $\begin{array}{l}\text { Morrison et al., } \\
(2011)\end{array}$ \\
\hline 294 & Lophelia pertusa & & 77 & BY4 & ITS & Yes & $\begin{array}{l}\text { Biological } \\
\text { Factors }\end{array}$ & $\begin{array}{l}\text { Le Goff-Vitry et } \\
\text { al., (2004) }\end{array}$ \\
\hline 295 & Lophelia pertusa & & 388 & BY4 & SSR & Yes & $\begin{array}{l}\text { Biological } \\
\text { Factors }\end{array}$ & $\begin{array}{l}\text { Le Goff-Vitry et } \\
\text { al., (2004) }\end{array}$ \\
\hline 296 & Lophelia pertusa & & 142 & BY4 & SSR & Yes & $\begin{array}{l}\text { Biological } \\
\text { Factors }\end{array}$ & $\begin{array}{l}\text { Dahl et al., } \\
(2012)\end{array}$ \\
\hline 297 & Lophelia pertusa & 515 & 10 & BY4 & ITS2 & No & Limitations & $\begin{array}{l}\text { Flot et al., } \\
(2013)\end{array}$ \\
\hline 298 & Lophelia pertusa & 515 & 2 & BY4 & $\begin{array}{l}\text { mtDNA } \\
\text { genome }\end{array}$ & No & Limitations & $\begin{array}{l}\text { Flot et al., } \\
(2013)\end{array}$ \\
\hline 299 & Lycodes japonicus & 103 & 53 & BY3 & COI & No & $\begin{array}{l}\text { Biological } \\
\text { Factors }\end{array}$ & $\begin{array}{l}\text { Sakuma et al., } \\
(2015)\end{array}$ \\
\hline 300 & Lycodes japonicus & 103 & 53 & BY3 & Cytb & No & $\begin{array}{l}\text { Biological } \\
\text { Factors }\end{array}$ & $\begin{array}{l}\text { Sakuma et al., } \\
\text { (2015) }\end{array}$ \\
\hline 301 & Lycodes matsubarai & 189 & 90 & BY3 BY12 & D-loop & No & Limitations & $\begin{array}{l}\text { Sakuma et al., } \\
\text { (2014) }\end{array}$ \\
\hline 302 & Lycodes matsubarai & 189 & 90 & BY3 BY12 & Cytb & Yes & $\begin{array}{l}\text { Biological } \\
\text { Factors \& } \\
\text { Topography }\end{array}$ & $\begin{array}{l}\text { Sakuma et al., } \\
(2014)\end{array}$ \\
\hline 303 & Lycodes ocellatus & 102 & 37 & BY3 & $\mathrm{COI}$ & No & $\begin{array}{l}\text { Biological } \\
\text { Factors }\end{array}$ & $\begin{array}{l}\text { Sakuma et al., } \\
(2015)\end{array}$ \\
\hline 304 & Lycodes ocellatus & 102 & 37 & BY3 & Cytb & No & $\begin{array}{l}\text { Biological } \\
\text { Factors }\end{array}$ & $\begin{array}{l}\text { Sakuma et al., } \\
(2015)\end{array}$ \\
\hline 305 & $\begin{array}{l}\text { Macropipus } \\
\text { tuberculatus }\end{array}$ & 750 & 115 & BY4 & $\mathrm{COI}$ & Yes & Topography & $\begin{array}{l}\text { García- } \\
\text { Merchána et al., } \\
\text { (2012) }\end{array}$ \\
\hline 306 & Macroscapha spp. & 2809 & 219 & BY9 & $\mathrm{COI}$ & Yes & Depth \& Distance & $\begin{array}{l}\text { Brandãoa et al., } \\
\text { (2010) }\end{array}$ \\
\hline 307 & Macroscapha spp. & 2809 & 219 & BY9 & ITS & Yes & Depth \& Distance & $\begin{array}{l}\text { Brandãoa et al., } \\
(2010)\end{array}$ \\
\hline 308 & Macrostylis roaldi & 937 & 22 & BY9 & $\mathrm{COI}$ & No & Depth & $\begin{array}{l}\text { Riehl et al., } \\
\text { (2012) }\end{array}$ \\
\hline 309 & Macrostylis roaldi & 937 & 39 & BY9 & $12 \mathrm{~s}$ & No & Depth & $\begin{array}{l}\text { Riehl et al., } \\
\text { (2012) }\end{array}$ \\
\hline 310 & Macrostylis roaldi & 937 & 35 & BY9 & $16 \mathrm{~s}$ & No & Depth & $\begin{array}{l}\text { Riehl et al., } \\
\text { (2012) }\end{array}$ \\
\hline 311 & $\begin{array}{l}\text { Macruronus } \\
\text { novaezelandiae }\end{array}$ & & 105 & BY6 BY 10 & RFLP & No & $\begin{array}{l}\text { Biological } \\
\text { Factors }\end{array}$ & $\begin{array}{l}\text { Smith et al., } \\
\text { (1996) }\end{array}$ \\
\hline 312 & Madrepora oculata & 0 & 42 & BY11 BY6 BY10 & $16 \mathrm{~s}$ & No & Limitations & $\begin{array}{l}\text { Miller et al., } \\
(2010)\end{array}$ \\
\hline 313 & Madrepora oculata & 1301 & 74 & BY6 BY10 & ITS & Yes & Depth & $\begin{array}{l}\text { Zeng et al., } \\
\text { (2016) }\end{array}$ \\
\hline 314 & Madrepora oculata & 1301 & 134 & BY6 BY 10 & SSR & No & Limitations & $\begin{array}{l}\text { Zeng et al., } \\
\text { (2016) }\end{array}$ \\
\hline 315 & Metallogorgia & 3660 & 39 & BY4 & MutS & No & Limitations & $\begin{array}{l}\text { Thoma et al., } \\
\text { (2009) }\end{array}$ \\
\hline 316 & $\begin{array}{l}\text { Microstomus } \\
\text { pacificus }\end{array}$ & & 110 & BY3 & D-loop & Yes & $\begin{array}{l}\text { Biological } \\
\text { Factors }\end{array}$ & $\begin{array}{l}\text { Stepien et al., } \\
\text { (1999) }\end{array}$ \\
\hline 317 & Moroteuthis ingens & 1355 & 73 & BY9 BY10 & RAPD & No & $\begin{array}{l}\text { Biological } \\
\text { Factors }\end{array}$ & $\begin{array}{l}\text { Sands et al., } \\
(2003)\end{array}$ \\
\hline 318 & Munida acantha & 1348 & 21 & BY12 & $\mathrm{COI}$ & No & Limitations & $\begin{array}{l}\text { Samadi et al., } \\
(2006)\end{array}$ \\
\hline
\end{tabular}




\begin{tabular}{|c|c|c|c|c|c|c|c|c|}
\hline 319 & Munida acantha & 1348 & 35 & BY12 & $\mathrm{COI}$ & No & Limitations & $\begin{array}{l}\text { Samadi et al., } \\
\text { (2006) }\end{array}$ \\
\hline 320 & Munida gracilis & 325 & 52 & BY6 & $\mathrm{COI}$ & No & $\begin{array}{l}\text { Biological } \\
\text { Factors }\end{array}$ & $\begin{array}{l}\text { Bors et al., } \\
(2012)\end{array}$ \\
\hline 321 & Munida intermedia & 750 & 113 & BY4 & $\mathrm{COI}$ & No & Current & $\begin{array}{l}\text { García- } \\
\text { Merchána et al., } \\
\text { (2012) }\end{array}$ \\
\hline 322 & Munida thoe & 1348 & 35 & BY12 & $\mathrm{COI}$ & No & Limitations & $\begin{array}{l}\text { Samadi et al., } \\
\text { (2006) }\end{array}$ \\
\hline 323 & Munida zebra & 1348 & 36 & BY12 & $\mathrm{COI}$ & No & Limitations & $\begin{array}{l}\text { Samadi et al., } \\
(2006)\end{array}$ \\
\hline 324 & Munidopsis lauensis & 650 & 111 & BY12 & $\mathrm{COI}$ & Yes & Topography & $\begin{array}{l}\text { Thaler et al., } \\
\text { (2014) }\end{array}$ \\
\hline 325 & Munidopsis lauensis & 650 & 95 & BY12 & SSR & Yes & Topography & $\begin{array}{l}\text { Thaler et al., } \\
\text { (2014) }\end{array}$ \\
\hline 326 & Munidopsis scobina & 100 & 421 & BY11 & Alloenzyme & Yes & $\begin{array}{l}\text { Distance \& } \\
\text { Biological } \\
\text { Factors }\end{array}$ & $\begin{array}{l}\text { Creasey et al., } \\
(2000)\end{array}$ \\
\hline 327 & $\begin{array}{l}\text { Myxoderma } \\
\text { sacculatum }\end{array}$ & 1800 & 31 & BY3 & Alloenzyme & No & Limitations & $\begin{array}{l}\text { Ayala et al., } \\
\text { (1975) }\end{array}$ \\
\hline 328 & Narella & 3056 & 41 & BY3 BY14 & MutS & Yes & Depth & $\begin{array}{l}\text { Baco et al., } \\
(2012)\end{array}$ \\
\hline 329 & Narella & 3056 & 41 & BY3 BY14 & ND2 & Yes & Depth \& Distance & $\begin{array}{l}\text { Baco et al., } \\
(2012)\end{array}$ \\
\hline 330 & Narella & 3056 & 41 & BY3 BY14 & ND6 & Yes & Depth & $\begin{array}{l}\text { Baco et al., } \\
(2012)\end{array}$ \\
\hline 331 & Narella & 3056 & 41 & BY3 BY14 & NCR1 & Yes & Depth \& Distance & $\begin{array}{l}\text { Baco et al., } \\
(2012)\end{array}$ \\
\hline 332 & Narella & 3056 & 41 & BY3 BY14 & $\mathrm{COI}$ & Yes & Depth & $\begin{array}{l}\text { Baco et al., } \\
\text { (2012) }\end{array}$ \\
\hline 333 & $\begin{array}{l}\text { Nassaria } \\
\text { problematica }\end{array}$ & 1348 & 24 & BY12 & $\mathrm{COI}$ & Yes & $\begin{array}{l}\text { Biological } \\
\text { Factors }\end{array}$ & $\begin{array}{l}\text { Samadi et al., } \\
(2006)\end{array}$ \\
\hline 334 & $\begin{array}{l}\text { Nearchaster } \\
\text { aciculosus }\end{array}$ & 1800 & 31 & BY3 & Alloenzyme & No & Limitations & $\begin{array}{l}\text { Ayala et al., } \\
(1975)\end{array}$ \\
\hline 335 & $\begin{array}{l}\text { Nematocarcinus } \\
\text { lanceopes }\end{array}$ & 1968 & 187 & BY9 & $\mathrm{COI}$ & No & $\begin{array}{l}\text { Biological } \\
\text { Factors }\end{array}$ & $\begin{array}{l}\text { Raupach et al., } \\
(2010)\end{array}$ \\
\hline 336 & $\begin{array}{l}\text { Nematocarcinus } \\
\text { lanceopes }\end{array}$ & 1968 & 187 & BY9 & $16 \mathrm{~s}$ & No & Limitations & $\begin{array}{l}\text { Raupach et al., } \\
(2010)\end{array}$ \\
\hline 337 & $\begin{array}{l}\text { Nematocarcinus } \\
\text { lanceopes }\end{array}$ & 1968 & 187 & BY9 & $28 \mathrm{~s}$ & No & Limitations & $\begin{array}{l}\text { Raupach et al., } \\
(2010)\end{array}$ \\
\hline 338 & $\begin{array}{l}\text { Nematocarcinus } \\
\text { lanceopes }\end{array}$ & 1968 & 187 & BY9 & $16 \mathrm{~s}$ & No & Limitations & $\begin{array}{l}\text { Raupach et al., } \\
(2010)\end{array}$ \\
\hline 339 & $\begin{array}{l}\text { Nematocarcinus } \\
\text { lanceopes }\end{array}$ & 1968 & 187 & BY9 & $28 \mathrm{~s}$ & No & Limitations & $\begin{array}{l}\text { Raupach et al., } \\
(2010)\end{array}$ \\
\hline 340 & $\begin{array}{l}\text { Neoaulaxinia } \\
\text { persicum }\end{array}$ & 660.5 & 34 & BY6 BY10 & $\mathrm{COI}$ & No & Limitations & $\begin{array}{l}\text { Zeng et al., } \\
(2015)\end{array}$ \\
\hline 341 & $\begin{array}{l}\text { Neoaulaxinia } \\
\text { persicum }\end{array}$ & 660.5 & 12 & BY6 BY10 & $12 \mathrm{~s}$ & No & Limitations & $\begin{array}{l}\text { Zeng et al., } \\
(2015)\end{array}$ \\
\hline 342 & $\begin{array}{l}\text { Notoscopelus } \\
\text { resplendens }\end{array}$ & 700 & 9 & BY4 BY 10 BY13 & $\mathrm{COI}$ & Yes & Depth \& Distance & $\begin{array}{l}\text { Gordeeva et al., } \\
\text { (2014) }\end{array}$ \\
\hline 343 & Nucula atacellana & 2732 & 91 & BY2 AB2 & $16 \mathrm{~s}$ & Yes & Depth & $\begin{array}{l}\text { Etter et al., } \\
(2007)\end{array}$ \\
\hline 344 & Nucula atacellana & 2808 & 130 & BY4 BY13 & $16 \mathrm{~s}$ & Yes & Depth & $\begin{array}{l}\text { Zardus et al., } \\
\text { (2006) }\end{array}$ \\
\hline 345 & Nucula atacellana & 2700 & 89 & BY4 & $16 \mathrm{~s}$ & Yes & Depth \& Distance & $\begin{array}{l}\text { Chase et al., } \\
\text { (1998) }\end{array}$ \\
\hline 346 & Nucula atacellana & 2865 & 188 & BY4 BY13 & $\mathrm{COI}$ & Yes & Depth & $\begin{array}{l}\text { Jennings et al., } \\
\text { (2014) }\end{array}$ \\
\hline 347 & Nucula atacellana & 2865 & 188 & BY4 BY13 & $16 \mathrm{~s}$ & Yes & Depth & $\begin{array}{l}\text { Jennings et al., } \\
(2014)\end{array}$ \\
\hline 348 & Nucula atacellana & 2865 & 188 & BY4 BY13 & CAL & Yes & Depth & $\begin{array}{l}\text { Jennings et al., } \\
\text { (2014) }\end{array}$ \\
\hline 349 & Nucula atacellana & 2200 & 93 & BY4 & $\mathrm{COI}$ & Yes & Depth & $\begin{array}{l}\text { Jennings et al., } \\
\text { (2013) }\end{array}$ \\
\hline 350 & Nucula atacellana & 2200 & 93 & BY4 & CAL & Yes & Depth & $\begin{array}{l}\text { Jennings et al., } \\
\text { (2013) }\end{array}$ \\
\hline 351 & Nucula atacellana & 2200 & 84 & BY4 & MAC & Yes & Depth & $\begin{array}{l}\text { Jennings et al., } \\
(2013)\end{array}$ \\
\hline 352 & Nucula atacellana & 2200 & 91 & BY4 & DAC3 & Yes & Depth & $\begin{array}{l}\text { Jennings et al., } \\
(2013)\end{array}$ \\
\hline 353 & Nucula atacellana & 2200 & 91 & BY4 & DAC6 & Yes & Depth & $\begin{array}{l}\text { Jennings et al., } \\
(2013)\end{array}$ \\
\hline 354 & Nymphon australe & 1032 & 131 & BY9 & $\mathrm{COI}$ & Yes & $\begin{array}{l}\text { Biological } \\
\text { Factors }\end{array}$ & $\begin{array}{l}\text { Arangoa et al., } \\
(2010)\end{array}$ \\
\hline 355 & Nymphon australe & 1032 & 81 & BY9 & $16 \mathrm{~s}$ & No & $\begin{array}{l}\text { Biological } \\
\text { Factors }\end{array}$ & $\begin{array}{l}\text { Arangoa et al., } \\
(2010)\end{array}$ \\
\hline 356 & Oasisia alvinae & 747 & 4 & BY3 BY7 & Alloenzyme & No & $\begin{array}{l}\text { Distance \& } \\
\text { Biological } \\
\text { Factors }\end{array}$ & $\begin{array}{l}\text { Black et al., } \\
\text { (1998) }\end{array}$ \\
\hline 357 & Oasisia alvinae & 747 & 8 & BY3 BY7 & $\mathrm{COI}$ & No & $\begin{array}{l}\text { Distance \& } \\
\text { Biological } \\
\text { Factors }\end{array}$ & $\begin{array}{l}\text { Black et al., } \\
\text { (1998) }\end{array}$ \\
\hline 358 & Oasisia alvinae & 826 & 43 & BY7 & $\mathrm{COI}$ & Yes & Topography & $\begin{array}{l}\text { Hurtado et al., } \\
(2004)\end{array}$ \\
\hline 359 & $\begin{array}{l}\text { Ophiacantha } \\
\text { vivipara }\end{array}$ & 3300 & 72 & BY9 & $\mathrm{COI}$ & Yes & $\begin{array}{l}\text { Depth \& } \\
\text { Temperature }\end{array}$ & $\begin{array}{l}\text { O'Hara et al., } \\
(2014)\end{array}$ \\
\hline
\end{tabular}




\begin{tabular}{|c|c|c|c|c|c|c|c|c|}
\hline 360 & Ophiactis abyssicola & 1627 & 160 & BY4 BY11 BY6 & COI & Yes & $\begin{array}{l}\text { Depth \& Distance } \\
\& \text { Temperature }\end{array}$ & $\begin{array}{l}\text { O'Hara et al., } \\
(2014)\end{array}$ \\
\hline 361 & Ophiocreas oedipus & 1000 & 25 & BY4 & $16 \mathrm{~s}$ & Yes & Depth \& Distance & $\begin{array}{l}\text { Cho et al., } \\
(2010)\end{array}$ \\
\hline 362 & Ophiocreas oedipus & 1000 & 25 & BY4 & $\mathrm{COI}$ & Yes & Depth \& Distance & $\begin{array}{l}\text { Cho et al., } \\
(2010)\end{array}$ \\
\hline 363 & $\begin{array}{l}\text { Ophiomusium } \\
\text { lymani }\end{array}$ & 792 & 75 & BY4 & Alloenzyme & No & Limitations & $\begin{array}{l}\text { Hensley et al., } \\
\text { (1995) }\end{array}$ \\
\hline 364 & $\begin{array}{l}\text { Ophiomusium } \\
\text { lymani }\end{array}$ & 1000 & 233 & BY3 & Alloenzyme & No & Distance & $\begin{array}{l}\text { Doyle et al., } \\
\text { (1972) }\end{array}$ \\
\hline 365 & $\begin{array}{l}\text { Ophiomusium } \\
\text { lymani }\end{array}$ & 0 & 257 & BY3 & Alloenzyme & No & Limitations & $\begin{array}{l}\text { Ayala et al., } \\
\text { (1974) }\end{array}$ \\
\hline 366 & Ophiomyxa vivipara & 3300 & 102 & BY6 BY11 & $\mathrm{COI}$ & Yes & $\begin{array}{l}\text { Depth \& } \\
\text { Temperature }\end{array}$ & $\begin{array}{l}\text { O'Hara et al., } \\
\text { (2014) }\end{array}$ \\
\hline 367 & $\begin{array}{l}\text { Ophioplinthaca } \\
\text { abyssalis }\end{array}$ & 1000 & 113 & BY4 & $16 \mathrm{~s}$ & Yes & Topography & $\begin{array}{l}\text { Cho et al., } \\
(2010)\end{array}$ \\
\hline 368 & $\begin{array}{l}\text { Ophioplinthaca } \\
\text { chelys }\end{array}$ & 1000 & 68 & BY4 & $16 \mathrm{~s}$ & Yes & Topography & $\begin{array}{l}\text { Cho et al., } \\
(2010)\end{array}$ \\
\hline 369 & Ophiothrix aristulata & 3300 & 82 & BY6 & $\mathrm{COI}$ & Yes & $\begin{array}{l}\text { Depth \& } \\
\text { Temperature }\end{array}$ & $\begin{array}{l}\text { O'Hara et al., } \\
(2014)\end{array}$ \\
\hline 370 & Ophiura ooplax & 3300 & 73 & BY6 & COI & Yes & $\begin{array}{l}\text { Depth \& } \\
\text { Temperature }\end{array}$ & $\begin{array}{l}\text { O'Hara et al., } \\
\text { (2014) }\end{array}$ \\
\hline 371 & $\begin{array}{l}\text { Oridorsalis } \\
\text { umbonatus }\end{array}$ & 4403 & 37 & AB2 AB6 & $12 \mathrm{~s}$ & Yes & Distance & $\begin{array}{l}\text { Pawlowski et } \\
\text { al., (2007) }\end{array}$ \\
\hline 372 & $\begin{array}{l}\text { Oridorsalis } \\
\text { umbonatus }\end{array}$ & 4403 & 37 & AB2 AB6 & ITS & Yes & Distance & $\begin{array}{l}\text { Pawlowski et } \\
\text { al., (2007) }\end{array}$ \\
\hline 373 & $\begin{array}{l}\text { Oridorsalis } \\
\text { umbonatus }\end{array}$ & 4403 & 18 & BY9 BY10 & ITS & Yes & $\begin{array}{l}\text { Biological } \\
\text { Factors }\end{array}$ & $\begin{array}{l}\text { Brandt et al., } \\
\text { (2007) }\end{array}$ \\
\hline 374 & Ostracoda & 4403 & 21 & BY9 & Sequencing & Yes & Limitations & $\begin{array}{l}\text { Brandt et al., } \\
(2007)\end{array}$ \\
\hline 375 & Ostracoda & 4403 & 21 & BY9 & Sequencing & Yes & Limitations & $\begin{array}{l}\text { Brandt et al., } \\
(2007)\end{array}$ \\
\hline 376 & Ostracoda & 4403 & 21 & BY9 & $12 \mathrm{~s}$ & Yes & Limitations & $\begin{array}{l}\text { Brandt et al., } \\
(2007)\end{array}$ \\
\hline 377 & Pagellus bogaraveo & 700 & 19 & BY4 & Cytb & No & Limitations & $\begin{array}{l}\text { Stockley et al., } \\
(2005)\end{array}$ \\
\hline 378 & Pagellus bogaraveo & 700 & 140 & BY4 & D-loop & Yes & $\begin{array}{l}\text { Biological } \\
\text { Factors }\end{array}$ & $\begin{array}{l}\text { Stockley et al., } \\
(2005)\end{array}$ \\
\hline 379 & Pagellus bogaraveo & 700 & 350 & BY4 & SSR & Yes & $\begin{array}{l}\text { Biological } \\
\text { Factors }\end{array}$ & $\begin{array}{l}\text { Stockley et al., } \\
(2005)\end{array}$ \\
\hline 380 & Pagellus bogaraveo & & 123 & BY4 & SSR & No & $\begin{array}{l}\text { Biological } \\
\text { Factors }\end{array}$ & $\begin{array}{l}\text { Piñera et al., } \\
\text { (2007) }\end{array}$ \\
\hline 381 & Pagurus alatus & 750 & 93 & BY4 & $\mathrm{COI}$ & No & Distance & $\begin{array}{l}\text { García- } \\
\text { Merchána et al., } \\
\text { (2012) }\end{array}$ \\
\hline 382 & Pagurus excavatus & 750 & 114 & BY4 & $\mathrm{COI}$ & No & Distance & $\begin{array}{l}\text { García- } \\
\text { Merchána et al., } \\
\text { (2012) }\end{array}$ \\
\hline 383 & Pandalus borealis & 500 & 2100 & BY1 BY2 BY4 & SSR & Yes & Temperature & $\begin{array}{l}\text { Jorde } \text { et al., } \\
\text { (2015) }\end{array}$ \\
\hline 384 & Pandalus borealis & 450 & 1893 & BY1 & SSR & No & Current & $\begin{array}{l}\text { Knutsen et al., } \\
(2014)\end{array}$ \\
\hline 385 & Pandalus borealis & 564 & 3865 & BY4 & Alloenzyme & Yes & $\begin{array}{l}\text { Biological } \\
\text { Factors }\end{array}$ & $\begin{array}{l}\text { Drengstig et al., } \\
(2000)\end{array}$ \\
\hline 386 & Paragorgia arborea & 1480 & 92 & $\begin{array}{l}\text { BY4 BY3 BY12 } \\
\text { BY6 BY8 BY10 }\end{array}$ & $\begin{array}{l}\text { mtDNA } \\
\text { genome }\end{array}$ & Yes & Distance & $\begin{array}{l}\text { Herrera et al., } \\
(2012)\end{array}$ \\
\hline 387 & Paragorgia arborea & 1480 & 48 & $\begin{array}{l}\text { BY4 BY3 BY12 } \\
\text { BY6 BY8 BY10 }\end{array}$ & ITS2 & Yes & Distance & $\begin{array}{l}\text { Herrera et al., } \\
\text { (2012) }\end{array}$ \\
\hline 388 & Paralvinella grasslei & 625 & 182 & BY7 & Alloenzyme & No & $\begin{array}{l}\text { Biological } \\
\text { Factors }\end{array}$ & $\begin{array}{l}\text { Jollivet et al., } \\
\text { (1995) }\end{array}$ \\
\hline 389 & Paramuricea & 3660 & 85 & BY4 & $\mathrm{COI}$ & No & Limitations & $\begin{array}{l}\text { Thoma et al., } \\
(2009)\end{array}$ \\
\hline 390 & Paramuricea & 3660 & 85 & BY4 & ND1 & No & Limitations & $\begin{array}{l}\text { Thoma et al., } \\
\text { (2009) }\end{array}$ \\
\hline 391 & Paramuricea & 3660 & 85 & BY4 & ND2 & No & Limitations & $\begin{array}{l}\text { Thoma et al., } \\
\text { (2009) }\end{array}$ \\
\hline 392 & Parantipathes & 3660 & 32 & BY4 & ND2 & No & Limitations & $\begin{array}{l}\text { Thoma et al., } \\
\text { (2009) }\end{array}$ \\
\hline 393 & Parantipathes & 3660 & 32 & BY4 & ND1 & No & Limitations & $\begin{array}{l}\text { Thoma et al., } \\
\text { (2009) }\end{array}$ \\
\hline 394 & Parantipathes & 3660 & 32 & BY4 & COI & No & Limitations & $\begin{array}{l}\text { Thoma et al., } \\
\text { (2009) }\end{array}$ \\
\hline 395 & $\begin{array}{l}\text { Parapenaeus } \\
\text { longirostris }\end{array}$ & 750 & 101 & BY4 & $\mathrm{COI}$ & No & Distance & $\begin{array}{l}\text { García- } \\
\text { Merchána et al., } \\
\text { (2012) }\end{array}$ \\
\hline 396 & $\begin{array}{l}\text { Parapenaeus } \\
\text { longirostris }\end{array}$ & & 240 & BY4 & AFLP & Yes & Distance & $\begin{array}{l}\text { Brutto et al., } \\
\text { (2013) }\end{array}$ \\
\hline 397 & $\begin{array}{l}\text { Parapenaeus } \\
\text { longirostris }\end{array}$ & & 107 & BY4 & D-loop & Yes & Distance & $\begin{array}{l}\text { Brutto et al., } \\
\text { (2013) }\end{array}$ \\
\hline 398 & $\begin{array}{l}\text { Parbolasia } \\
\text { corrugatus }\end{array}$ & & 115 & BY9 & Alloenzyme & Yes & $\begin{array}{l}\text { Biological } \\
\text { Factors \& Ocean } \\
\text { current }\end{array}$ & $\begin{array}{l}\text { Rogers et al., } \\
(1998)\end{array}$ \\
\hline 399 & $\begin{array}{l}\text { Pareledone } \\
\text { aequipapillae }\end{array}$ & & 350 & BY9 & $\mathrm{COI}$ & Yes & Current & $\begin{array}{l}\text { Allcock et al., } \\
(2010)\end{array}$ \\
\hline 400 & Pareledone turqueti & 1091 & 82 & BY9 & Alloenzyme & Yes & Depth & $\begin{array}{l}\text { Allcock et al., } \\
\text { (1997) }\end{array}$ \\
\hline
\end{tabular}




\begin{tabular}{|c|c|c|c|c|c|c|c|c|}
\hline 401 & Parvochelus russus & 0 & 5 & BY13 & $\mathrm{COI}$ & No & Limitations & $\begin{array}{l}\text { Brix et al., } \\
(2015)\end{array}$ \\
\hline 402 & Parvochelus russus & 0 & 2 & BY13 & $16 \mathrm{~S}$ & No & Limitations & $\begin{array}{l}\text { Brix et al., } \\
(2015)\end{array}$ \\
\hline 403 & Parvochelus russus & 0 & 1 & BY13 & $18 \mathrm{~S}$ & No & Limitations & $\begin{array}{l}\text { Brix et al., } \\
(2015)\end{array}$ \\
\hline 404 & $\begin{array}{l}\text { Penares } \\
\text { palmatoclada }\end{array}$ & 1055 & 22 & BY6 BY10 & $\mathrm{COI}$ & No & Limitations & $\begin{array}{l}\text { Zeng et al., } \\
\text { (2015) }\end{array}$ \\
\hline 405 & $\begin{array}{l}\text { Penares } \\
\text { palmatoclada }\end{array}$ & 1055 & 18 & BY6 BY10 & $12 \mathrm{~s}$ & No & Limitations & $\begin{array}{l}\text { Zeng et al., } \\
\text { (2015) }\end{array}$ \\
\hline 406 & Phoxocephalidae & 1000 & 297 & BY6 & $\mathrm{COI}$ & Yes & $\begin{array}{l}\text { Biological } \\
\text { Factors }\end{array}$ & $\begin{array}{l}\text { Knox et al., } \\
(2012)\end{array}$ \\
\hline 407 & Phoxocephalidae sp & 1000 & 12 & BY6 & $\mathrm{COI}$ & Yes & Topography & $\begin{array}{l}\text { Knox et al., } \\
(2012)\end{array}$ \\
\hline 408 & Pleraster jordani & 1800 & 31 & BY3 & Alloenzyme & No & Limitations & $\begin{array}{l}\text { Ayala et al., } \\
\text { (1975) }\end{array}$ \\
\hline 409 & Pleroma menoui & 734 & 19 & BY6 BY10 & $\mathrm{COI}$ & No & Limitations & $\begin{array}{l}\text { Zeng et al., } \\
\text { (2015) }\end{array}$ \\
\hline 410 & $\begin{array}{l}\text { Plesionika } \\
\text { heterocarpus }\end{array}$ & 750 & 118 & BY4 & $\mathrm{COI}$ & No & Distance & $\begin{array}{l}\text { García- } \\
\text { Merchána et al., } \\
\text { (2012) }\end{array}$ \\
\hline 411 & $\begin{array}{l}\text { Pocillopora } \\
\text { damicornis }\end{array}$ & & 16 & BY14 & CALM & No & Limitations & $\begin{array}{l}\text { Flot et al., } \\
(2008)\end{array}$ \\
\hline 412 & $\begin{array}{l}\text { Pocillopora } \\
\text { damicornis }\end{array}$ & & 16 & BY14 & $\mathrm{EF} 1 \alpha$ & No & Limitations & $\begin{array}{l}\text { Flot et al., } \\
(2008)\end{array}$ \\
\hline 413 & $\begin{array}{l}\text { Pocillopora } \\
\text { damicornis }\end{array}$ & & 16 & BY14 & ATPS $\beta$ & No & Limitations & $\begin{array}{l}\text { Flot et al., } \\
\text { (2008) }\end{array}$ \\
\hline 414 & Pocillopora ligulata & & 6 & BY14 & ITS2 & No & Limitations & $\begin{array}{l}\text { Flot et al., } \\
(2008)\end{array}$ \\
\hline 415 & $\begin{array}{l}\text { Pocillopora } \\
\text { molokensis }\end{array}$ & & 5 & BY14 & D-loop & No & Limitations & $\begin{array}{l}\text { Flot et al., } \\
(2008)\end{array}$ \\
\hline 416 & $\begin{array}{l}\text { Pocillopora } \\
\text { molokensis }\end{array}$ & & 5 & BY14 & ORF & No & Limitations & $\begin{array}{l}\text { Flot et al., } \\
(2008)\end{array}$ \\
\hline 417 & $\begin{array}{l}\text { Poecillastra } \\
\text { laminaris }\end{array}$ & 1385 & 54 & BY6 BY10 & $\mathrm{COI}$ & Yes & Current & $\begin{array}{l}\text { Zeng et al., } \\
\text { (2015) }\end{array}$ \\
\hline 418 & $\begin{array}{l}\text { Poecillastra } \\
\text { laminaris }\end{array}$ & 1385 & 49 & BY6 BY10 & Cytb & Yes & Current & $\begin{array}{l}\text { Zeng et al., } \\
(2015)\end{array}$ \\
\hline 419 & $\begin{array}{l}\text { Polyprion } \\
\text { americanus }\end{array}$ & 560 & 422 & BY4 & SSR & Yes & $\begin{array}{l}\text { Temperature \& } \\
\text { Current }\end{array}$ & $\begin{array}{l}\text { Ball et al., } \\
(2000)\end{array}$ \\
\hline 420 & $\begin{array}{l}\text { Polyprion } \\
\text { americanus }\end{array}$ & 200 & 179 & BY4 BY6 BY13 & ND1 & No & $\begin{array}{l}\text { Biological } \\
\text { Factors }\end{array}$ & $\begin{array}{l}\text { Sedberry et al., } \\
\text { (1996) }\end{array}$ \\
\hline 421 & $\begin{array}{l}\text { Promachocrinus } \\
\text { kerguelensis }\end{array}$ & 1054 & 37 & BY9 & $\mathrm{COI}$ & No & $\begin{array}{l}\text { Biological } \\
\text { Factors }\end{array}$ & $\begin{array}{l}\text { Wilson et al., } \\
\text { (2007) }\end{array}$ \\
\hline 422 & $\begin{array}{l}\text { Promachocrinus } \\
\text { kerguelensis }\end{array}$ & 1054 & 37 & BY9 & Cytb & No & $\begin{array}{l}\text { Biological } \\
\text { Factors }\end{array}$ & $\begin{array}{l}\text { Wilson et al., } \\
\text { (2007) }\end{array}$ \\
\hline 423 & $\begin{array}{l}\text { Pseudopentaceros } \\
\text { wheeleri }\end{array}$ & & 10 & BY14 & $\mathrm{COI}$ & No & Topography & $\begin{array}{l}\text { Martin et al., } \\
\text { (1992) }\end{array}$ \\
\hline 424 & $\begin{array}{l}\text { Pseudopentaceros } \\
\text { wheeleri }\end{array}$ & & 17 & BY14 & RFLP & No & Topography & $\begin{array}{l}\text { Martin et al., } \\
\text { (1992) }\end{array}$ \\
\hline 425 & Riftia pachyptila & 610 & 235 & BY3 BY7 & Alloenzyme & Yes & Distance & $\begin{array}{l}\text { Black et al., } \\
\text { (1994) }\end{array}$ \\
\hline 426 & Riftia pachyptila & & 100 & BY7 & Alloenzyme & Yes & $\begin{array}{l}\text { Biological } \\
\text { Factors }\end{array}$ & $\begin{array}{l}\text { Bucklin et al., } \\
\text { (1988) }\end{array}$ \\
\hline 427 & Riftia pachyptila & 754 & 352 & BY7 & Rpt 46.1 & Yes & Distance & $\begin{array}{l}\text { Coykendall et } \\
\text { al., (2011) }\end{array}$ \\
\hline 428 & Riftia pachyptila & 754 & 380 & BY7 & Rpt 84.1 & Yes & Distance & $\begin{array}{l}\text { Coykendall et } \\
\text { al., (2011) }\end{array}$ \\
\hline 429 & Riftia pachyptila & 754 & 171 & BY7 & Cytb & Yes & Distance & $\begin{array}{l}\text { Coykendall et } \\
\text { al., (2011) }\end{array}$ \\
\hline 430 & Riftia pachyptila & 754 & 10 & BY7 & ATPS $\alpha$ & Yes & Distance & $\begin{array}{l}\text { Coykendall et } \\
\text { al., (2011) }\end{array}$ \\
\hline 431 & Riftia pachyptila & 826 & 135 & BY7 & $\mathrm{COI}$ & Yes & Topography & $\begin{array}{l}\text { Hurtado et al., } \\
\text { (2004) }\end{array}$ \\
\hline 432 & Riftia pachyptila & 618 & 29 & BY7 & AFLP & Yes & $\begin{array}{l}\text { Biological } \\
\text { Factors }\end{array}$ & $\begin{array}{l}\text { Shank et al., } \\
\text { (2007) }\end{array}$ \\
\hline 433 & Rimicaris exoculata & & 312 & BY4 & Alloenzyme & No & $\begin{array}{l}\text { Biological } \\
\text { Factors }\end{array}$ & $\begin{array}{l}\text { Creasey et al., } \\
\text { (1996) }\end{array}$ \\
\hline 434 & Rimicaris exoculata & 1300 & 12 & BY4 & $\mathrm{COI}$ & No & Limitations & $\begin{array}{l}\text { Petersen et al., } \\
(2010)\end{array}$ \\
\hline 435 & Rimicaris exoculata & 1300 & 12 & BY4 & Cytb & No & Limitations & $\begin{array}{l}\text { Petersen et al., } \\
(2010)\end{array}$ \\
\hline 436 & Rimicaris exoculata & 1450 & 152 & BY4 & $\mathrm{COI}$ & No & $\begin{array}{l}\text { Biological } \\
\text { Factors }\end{array}$ & $\begin{array}{l}\text { Teixeira } \text { et al., } \\
\text { (2011) }\end{array}$ \\
\hline 437 & Rimicaris exoculata & 1450 & 687 & BY4 & SSR & No & $\begin{array}{l}\text { Biological } \\
\text { Factors }\end{array}$ & $\begin{array}{l}\text { Teixeira et al., } \\
\text { (2012) }\end{array}$ \\
\hline 438 & Rimicaris kairei & 0 & 54 & BY11 & $\mathrm{COI}$ & No & $\begin{array}{l}\text { Biological } \\
\text { Factors }\end{array}$ & $\begin{array}{l}\text { Beedessee et al., } \\
\text { (2013) }\end{array}$ \\
\hline 439 & Sassia remensa & 1348 & 24 & BY12 & $\mathrm{COI}$ & No & $\begin{array}{l}\text { Biological } \\
\text { Factors }\end{array}$ & $\begin{array}{l}\text { Samadi et al., } \\
\text { (2006) }\end{array}$ \\
\hline 440 & $\begin{array}{l}\text { Scaphander } \\
\text { bathymophilus }\end{array}$ & 4400 & 68 & BY4 & COI & Yes & Topography & $\begin{array}{l}\text { Eilertsen et al., } \\
\text { (2015) }\end{array}$ \\
\hline 441 & $\begin{array}{l}\text { Scaphander } \\
\text { bathymophilus }\end{array}$ & 4401 & 68 & BY4 & $16 \mathrm{~s}$ & Yes & Topography & $\begin{array}{l}\text { Eilertsen et al., } \\
(2015)\end{array}$ \\
\hline 442 & $\begin{array}{l}\text { Scaphander } \\
\text { lignarius }\end{array}$ & 784 & 112 & BY4 & $\mathrm{COI}$ & No & Limitations & $\begin{array}{l}\text { Eilertsen et al., } \\
\text { (2015) }\end{array}$ \\
\hline
\end{tabular}




\begin{tabular}{|c|c|c|c|c|c|c|c|c|}
\hline 443 & $\begin{array}{l}\text { Scaphander } \\
\text { lignarius }\end{array}$ & 784 & 111 & BY4 & $16 \mathrm{~s}$ & No & Limitations & $\begin{array}{l}\text { Eilertsen } \text { et al., } \\
(2015)\end{array}$ \\
\hline 444 & $\begin{array}{l}\text { Scaphander } \\
\text { lignarius }\end{array}$ & 784 & 92 & BY4 & $28 \mathrm{~s}$ & No & Limitations & $\begin{array}{l}\text { Eilertsen } \text { et al., } \\
(2015)\end{array}$ \\
\hline 445 & Scaphander nobilis & 2800 & 9 & BY4 & $16 \mathrm{~s}$ & No & $\begin{array}{l}\text { Biological } \\
\text { Factors }\end{array}$ & $\begin{array}{l}\text { Eilertsen et al., } \\
(2015)\end{array}$ \\
\hline 446 & $\begin{array}{l}\text { Scaphander } \\
\text { punctostriatus }\end{array}$ & 2530 & 150 & BY4 & COI & Yes & $\begin{array}{l}\text { Biological } \\
\text { Factors }\end{array}$ & $\begin{array}{l}\text { Eilertsen et al., } \\
(2015)\end{array}$ \\
\hline 447 & $\begin{array}{l}\text { Scaphander } \\
\text { punctostriatus }\end{array}$ & 2530 & 150 & BY4 & $16 \mathrm{~s}$ & Yes & $\begin{array}{l}\text { Biological } \\
\text { Factors }\end{array}$ & $\begin{array}{l}\text { Eilertsen et al., } \\
(2015)\end{array}$ \\
\hline 448 & $\begin{array}{l}\text { Scaphander } \\
\text { punctostriatus }\end{array}$ & 2530 & 150 & BY4 & $28 \mathrm{~s}$ & No & $\begin{array}{l}\text { Biological } \\
\text { Factors }\end{array}$ & $\begin{array}{l}\text { Eilertsen } \text { et al., } \\
\text { (2015) }\end{array}$ \\
\hline 449 & Scaphander watsoni & 400 & 32 & BY4 & COI & No & $\begin{array}{l}\text { Biological } \\
\text { Factors }\end{array}$ & $\begin{array}{l}\text { Eilertsen et al., } \\
(2015)\end{array}$ \\
\hline 450 & Scaphander watsoni & 400 & 32 & BY4 & $16 \mathrm{~s}$ & No & $\begin{array}{l}\text { Biological } \\
\text { Factors }\end{array}$ & $\begin{array}{l}\text { Eilertsen et al., } \\
\text { (2015) }\end{array}$ \\
\hline 451 & Scaphander watsoni & 400 & 32 & BY4 & $28 \mathrm{~s}$ & Yes & $\begin{array}{l}\text { Biological } \\
\text { Factors }\end{array}$ & $\begin{array}{l}\text { Eilertsen et al., } \\
\text { (2015) }\end{array}$ \\
\hline 452 & $\begin{array}{l}\text { Scyliorhinus } \\
\text { canicula }\end{array}$ & 446 & 431 & BY4 & COI & Yes & Topography & $\begin{array}{l}\text { Kousteni et al., } \\
(2015)\end{array}$ \\
\hline 453 & $\begin{array}{l}\text { Scyliorhinus } \\
\text { canicula }\end{array}$ & 446 & 834 & BY4 & SSR & Yes & Topography & $\begin{array}{l}\text { Kousteni et al., } \\
(2015)\end{array}$ \\
\hline 454 & Sebastes fasciatus & 295 & 596 & BY4 & SSR & Yes & Limitations & $\begin{array}{l}\text { Valentin et al., } \\
\text { (2014) }\end{array}$ \\
\hline 455 & Sebastes marinus & & 130 & BY2 & ND3 & Yes & Depth & $\begin{array}{l}\text { Schmidt et al., } \\
\text { (2005) }\end{array}$ \\
\hline 456 & Sebastes mentella & 700 & 2931 & BY2 & Cytb & Yes & Depth & $\begin{array}{l}\text { Ingimarsdóttir et } \\
\text { al., (2008) }\end{array}$ \\
\hline 457 & Sebastes mentella & & 98 & BY2 & SSR & Yes & Depth & $\begin{array}{l}\text { Pampoulie et al., } \\
\text { (2008) }\end{array}$ \\
\hline 458 & Sebastes mentella & & 130 & BY2 & SSR & Yes & $\begin{array}{l}\text { Biological } \\
\text { Factors }\end{array}$ & $\begin{array}{l}\text { Schmidt et al., } \\
(2005)\end{array}$ \\
\hline 459 & Sebastes mentella & 659 & 1763 & BY2 & Alloenzyme & Yes & Depth & $\begin{array}{l}\text { Daníelsdóttir et } \\
\text { al., (2008) }\end{array}$ \\
\hline 460 & Sebastes mentella & 295 & 495 & BY4 & SSR & Yes & Depth & $\begin{array}{l}\text { Valentin et al., } \\
(2014)\end{array}$ \\
\hline 461 & Sebastes mentella & & 4 & BY2 & $16 \mathrm{~s}$ & No & Limitations & $\begin{array}{l}\text { Sundt et al., } \\
\text { (1998) }\end{array}$ \\
\hline 462 & Sebastes mentella & 580 & 1946 & BY2 BY1 & Alloenzyme & Yes & Depth & $\begin{array}{l}\text { Johansen et al., } \\
(2000)\end{array}$ \\
\hline 463 & Sebastes mentella & & 1901 & BY2 & SSR & Yes & Depth & $\begin{array}{l}\text { Stefánsson et al., } \\
(2009)\end{array}$ \\
\hline 464 & Sebastes mentella & 502 & 1240 & BY2 & SSR & Yes & Depth & $\begin{array}{l}\text { Stefánsson et al., } \\
(2009)\end{array}$ \\
\hline 465 & Sebastes mentella & & 973 & BY2 BY4 & SSR & Yes & Current & $\begin{array}{l}\text { Roques et al., } \\
(2002)\end{array}$ \\
\hline 466 & $\begin{array}{l}\text { Sebastolobus } \\
\text { macrochir }\end{array}$ & 781 & 66 & BY12 & D-loop & No & $\begin{array}{l}\text { Biological } \\
\text { Factors }\end{array}$ & $\begin{array}{l}\text { Sakaguchi et al., } \\
\text { (2014) }\end{array}$ \\
\hline 467 & Shinkaia crosnieri & 377.7 & 64 & BY12 & $\begin{array}{l}\mathrm{COI}+\mathrm{Cytb}+ \\
16 \mathrm{~s}\end{array}$ & Yes & Temperature & $\begin{array}{l}\text { Shen et al., } \\
(2016)\end{array}$ \\
\hline 468 & Shinkaia crosnieri & 355 & 66 & BY12 & COI & Yes & Temperature & $\begin{array}{l}\text { Yang et al., } \\
(2016)\end{array}$ \\
\hline 469 & Shinkaia crosnieri & 355 & 76 & BY12 & ANT & No & Limitations & $\begin{array}{l}\text { Yang et al., } \\
(2016)\end{array}$ \\
\hline 470 & $\begin{array}{l}\text { Shinkaicaris } \\
\text { leurokolos }\end{array}$ & 1071 & 53 & BY12 & COI & No & Topography & $\begin{array}{l}\text { Yahagi et al., } \\
(2015)\end{array}$ \\
\hline 471 & $\begin{array}{l}\text { Sicyopus } \\
\text { commensalis }\end{array}$ & 0 & 55 & BY4 & Alloenzyme & No & $\begin{array}{l}\text { Biological } \\
\text { Factors }\end{array}$ & $\begin{array}{l}\text { Bronsdon et al., } \\
\text { (1997) }\end{array}$ \\
\hline 472 & Sigmops elongatus & 701 & 7 & BY4 BY 10 BY 13 & COI & Yes & Depth & $\begin{array}{l}\text { Gordeeva et al., } \\
\text { (2014) }\end{array}$ \\
\hline 473 & $\begin{array}{l}\text { Solenosmilia } \\
\text { variabilis }\end{array}$ & 0 & 33 & BY11 BY6 BY10 & $16 \mathrm{~s}$ & No & Limitations & $\begin{array}{l}\text { Miller et al., } \\
(2010)\end{array}$ \\
\hline 474 & $\begin{array}{l}\text { Solenosmilia } \\
\text { variabilis }\end{array}$ & 0 & 12 & BY11 BY6 BY10 & D-loop & No & $\begin{array}{l}\text { Biological } \\
\text { Factors }\end{array}$ & $\begin{array}{l}\text { Miller et al., } \\
(2010)\end{array}$ \\
\hline 475 & $\begin{array}{l}\text { Solenosmilia } \\
\text { variabilis }\end{array}$ & 1483 & 186 & BY6 BY10 & ITS & No & Limitations & $\begin{array}{l}\text { Zeng et al., } \\
(2016)\end{array}$ \\
\hline 476 & $\begin{array}{l}\text { Solenosmilia } \\
\text { variabilis }\end{array}$ & 308 & 568 & BY10 & SSR & Yes & $\begin{array}{l}\text { Biological } \\
\text { Factors }\end{array}$ & $\begin{array}{l}\text { Miller et al., } \\
(2016)\end{array}$ \\
\hline 477 & $\begin{array}{l}\text { Solenosmilia } \\
\text { variabilis }\end{array}$ & 1483 & 139 & BY6 BY10 & D-loop & No & Limitations & $\begin{array}{l}\text { Zeng et al., } \\
\text { (2016) }\end{array}$ \\
\hline 478 & $\begin{array}{l}\text { Solenosmilia } \\
\text { variabilis }\end{array}$ & 1483 & 198 & BY6 BY10 & SSR & Yes & Distance & $\begin{array}{l}\text { Zeng et al., } \\
\text { (2016) }\end{array}$ \\
\hline 479 & $\begin{array}{l}\text { Stephanauge } \\
\text { inornata }\end{array}$ & 0 & 75 & BY4 & Alloenzyme & No & $\begin{array}{l}\text { Biological } \\
\text { Factors }\end{array}$ & $\begin{array}{l}\text { Bronsdon et al., } \\
\text { (1997) }\end{array}$ \\
\hline 480 & $\begin{array}{l}\text { Stephanocyathus } \\
\text { spiniger }\end{array}$ & 0 & 18 & BY11 BY6 BY10 & ITS & No & Current & $\begin{array}{l}\text { Miller et al., } \\
(2010)\end{array}$ \\
\hline 481 & $\begin{array}{l}\text { Stephanocyathus } \\
\text { spiniger }\end{array}$ & 0 & 5 & BY11 BY6 BY10 & $16 \mathrm{~s}$ & No & Limitations & $\begin{array}{l}\text { Miller et al., } \\
\text { (2010) }\end{array}$ \\
\hline 482 & Stereomastis suhmi & 2620 & 14 & BY10 \& BY13 & COI & No & Current & $\begin{array}{l}\text { Farias et al., } \\
(2015)\end{array}$ \\
\hline 483 & Sternoptyx diaphana & 300 & 11 & BY4 BY 10 BY 13 & COI & Yes & Distance & $\begin{array}{l}\text { Gordeeva et al., } \\
\text { (2014) }\end{array}$ \\
\hline 484 & $\begin{array}{l}\text { Stichopathes } \\
\text { filiformis }\end{array}$ & 0 & 17 & BY11 BY6 BY10 & ITS & No & Limitations & $\begin{array}{l}\text { Miller et al., } \\
(2010)\end{array}$ \\
\hline 485 & $\begin{array}{l}\text { Stichopathes } \\
\text { variabilis }\end{array}$ & 0 & 41 & BY11 BY6 BY10 & $16 \mathrm{~s}$ & Yes & Topography & $\begin{array}{l}\text { Miller et al., } \\
(2010)\end{array}$ \\
\hline
\end{tabular}




\begin{tabular}{|c|c|c|c|c|c|c|c|c|}
\hline 486 & $\begin{array}{l}\text { Stichopathes } \\
\text { variabilis }\end{array}$ & 0 & 57 & BY11 BY6 BY10 & ITS & Yes & Topography & $\begin{array}{l}\text { Miller et al., } \\
(2010)\end{array}$ \\
\hline 487 & Terebellides gingko & 5152.5 & 15 & BY13 & $16 \mathrm{~s}$ & No & $\begin{array}{l}\text { Biological } \\
\text { Factors }\end{array}$ & $\begin{array}{l}\text { Schuller et al., } \\
\text { (2012) }\end{array}$ \\
\hline 488 & Tevnia jerichonana & 747 & 4 & BY3 BY7 & Alloenzyme & No & $\begin{array}{l}\text { Distance \& } \\
\text { Biological } \\
\text { Factors }\end{array}$ & $\begin{array}{l}\text { Black et al., } \\
\text { (1998) }\end{array}$ \\
\hline 489 & Tevnia jerichonana & 747 & 8 & BY3 BY7 & $\mathrm{COI}$ & No & $\begin{array}{l}\text { Distance \& } \\
\text { Biological } \\
\text { Factors }\end{array}$ & $\begin{array}{l}\text { Black et al., } \\
\text { (1998) }\end{array}$ \\
\hline 490 & Tevnia jerichonana & 512 & 24 & BY5 BY7 & COI & Yes & Distance & $\begin{array}{l}\text { Zhang et al., } \\
\text { (2015) }\end{array}$ \\
\hline 491 & Tevnia jerichonana & 512 & 27 & BY5 BY7 & Cytb & Yes & Distance & $\begin{array}{l}\text { Zhang et al., } \\
\text { (2015) }\end{array}$ \\
\hline 492 & Tevnia jerichonana & 512 & 38 & BY5 BY7 & HSP & Yes & Distance & $\begin{array}{l}\text { Zhang et al., } \\
\text { (2015) }\end{array}$ \\
\hline 493 & Tevnia jerichonana & 512 & 20 & BY5 BY7 & ATPS $\alpha$ & No & Limitations & $\begin{array}{l}\text { Zhang et al., } \\
(2015)\end{array}$ \\
\hline 494 & Tevnia jerichonana & 826 & 114 & BY7 & $\mathrm{COI}$ & Yes & Topography & $\begin{array}{l}\text { Hurtado et al., } \\
\text { (2004) }\end{array}$ \\
\hline 495 & Tokoprymno maia & 1350 & 80 & BY6 BY9 BY10 & ITS2+28s & Yes & Current & $\begin{array}{l}\text { Dueñas et al., } \\
\text { (2016) }\end{array}$ \\
\hline 496 & $\begin{array}{l}\text { Valenciennellus } \\
\text { tripunctulatus }\end{array}$ & 200 & 11 & BY4 BY 10 BY 13 & COI & No & Current & $\begin{array}{l}\text { Gordeeva et al., } \\
\text { (2014) }\end{array}$ \\
\hline 497 & Ventiella sulfuris & 180 & 310 & BY7 & Alloenzyme & Yes & Topography & $\begin{array}{l}\text { France et al., } \\
\text { (1992) }\end{array}$ \\
\hline 498 & Vestimentiferan & 3118 & 37 & BY14 BY5 & $\mathrm{COI}$ & Yes & Depth & $\begin{array}{l}\text { Mcmullin et al., } \\
(2003)\end{array}$ \\
\hline 499 & Vestimentiferan & 3118 & 37 & BY14 BY6 & $16 \mathrm{~s}$ & Yes & Depth & $\begin{array}{l}\text { Mcmullin et al., } \\
\text { (2003) }\end{array}$ \\
\hline 500 & $\begin{array}{l}\text { Vinciguerria } \\
\text { nimbaria }\end{array}$ & 300 & 10 & BY4 BY 10 BY 13 & $\mathrm{COI}$ & Yes & Depth & $\begin{array}{l}\text { Gordeeva et al., } \\
\text { (2014) }\end{array}$ \\
\hline 501 & Vulcanolepas & 2226 & 96 & BY11 & SNP & Yes & Current & $\begin{array}{l}\text { Herrera et al., } \\
(2015)\end{array}$ \\
\hline 502 & Zoroaster fulgens & 3556 & 25 & BY4 & $16 \mathrm{~s}$ & Yes & Depth & $\begin{array}{l}\text { Howell et al., } \\
(2004)\end{array}$ \\
\hline 503 & Zoroaster fulgens & 3556 & 19 & BY4 & COI & Yes & Depth & $\begin{array}{l}\text { Howell et al., } \\
\text { (2004) }\end{array}$ \\
\hline
\end{tabular}



Chapter 3. Data mining from Poecillastra laminaris

(Demosponge) 454 sequencing data 


\subsection{Introduction}

Poecillastra laminaris (Sollas) is a common demosponge found in the New Zealand region, and is widely distributed on seamounts and other habitats from 100 to $1500 \mathrm{~m}$ water depth (van Soest et al., 2005). Extensive deep-sea trawl fisheries occur on seamounts throughout the New Zealand Exclusive Economic Zone (EEZ) (e.g., Clark \& O'Driscoll, 2003), and benthic fauna such as demosponges are known to be vulnerable to damage from such fishing activities (e.g., Clark \& Tittensor, 2010). Because this species, like other structure-forming sponges, may provide shelter and habitat (e.g., places for reproduction) for other species (Murillo et al., 2012), it can be considered as an indicator taxon for Vulnerable Marine Ecosystems (VMEs) in the South Pacific Ocean (Parker et al., 2009). Knowledge of population differentiation and connectivity of sponge species in the South Pacific is generally lacking, despite the recognition that such information is useful for spatial management planning (Almany et al., 2009). As such, knowledge about the population genetic structure of Poecillastra laminaris has the potential to contribute to improved spatial management measures, including the identification or re-design of marine protection areas (MPAs) in the New Zealand EEZ and the wider South Pacific.

Next-generation sequencing (NGS), also known as high-throughput sequencing, is now very popular in genetic studies and allows researchers to sequence DNA and RNA much more quickly and cheaply than the previously used Sanger sequencing. However, the power of discovering genetic markers in deep-sea animals, and especially in deep-sea sponges, is still in its infancy and remains a challenge (Sharma et al., 2012). In this study, to develop suitable markers for Poecillastra laminaris, next generation sequencing was utilised to obtain genomic information and also to generate new genetic markers for population studies.

\subsection{Materials and methods}

\subsubsection{Samples, DNA extractions and pyrosequencing}

The Poecillastra laminaris used in this study, NIWA ID 86172, was collected on 18 October 2012 during cruise TAN1213, from a seamount on the Colville Ridge, New Zealand (30.177 S, $179.743 \mathrm{E})$, between 387 and $422 \mathrm{~m}$ depth. After collection, the specimen was kept in absolute ethanol at the National Institute of Water and Atmospheric Research (NIWA) invertebrate collection. DNA from this individual was extracted using the guanidinium 
thiocyanate method (Boom et al., 1990), and was then quantified by Nanodrop 1000 (Thermo Scientific) and $0.5 \%$ agar electrophoresis on $80 \mathrm{~V}$ for $2 \mathrm{~h}$. Before 454 GS-FLX Pyrosequencing, a genomic DNA barcoded library was constructed (Macrogen Inc., Korea). The 454 GS-FLX Titanium platform was utilised to sequence the library (Macrogen Inc., Korea).

\subsubsection{Microsatellite discovery and characterisation}

MSATCOMMANDER 0.8 was used to identify microsatellite sequences and to design primers for genetic analysis. Microsatellites, also called simple sequence repeats (SSR), with a minimum of 5 repeats for di-, tri-, tetra-, penta-, and hexanucleotides were selected, and MSATCOMMANDER with default settings, except for the minimum and maximum PCR product size (set to $100-400 \mathrm{bp}$ ), was used to design PCR primers. After primers were chosen with similar annealing temperatures and low penalty scores for both forward and reverse primers, the top 50 primer pairs were used for further PCR amplification test.

The PCR successfully-amplified loci were chosen to genotype to test for their levels of polymorphism. M13 (fluorescent labelling) was used as an economic tool to test the polymorphism of PCR-succeeded candidate loci, and the forward primers of these loci were subsequently tagged with M13 sequences (Schuelke, 2000). To test whether these loci were the polymorphic, 30 individuals were selected to conduct the PCR amplification. PCR amplifications were carried out in $30 \mu \mathrm{l}$ reactions consisting of $15 \mu \mathrm{l}$ PCR Red Mixture (MyTaq $^{\mathrm{TM}}$ Red Mix), $0.5 \mu 1$ of forward primer and labelled M13, $1 \mu 1$ of reverse primer and genomic DNA, and $12 \mu 1$ of water. The thermal cycler was programmed for touchdown PCR, in which denaturation at $94{ }^{\circ} \mathrm{C}$ for $5 \mathrm{~min}$ was followed by 20 cycles of three $45 \mathrm{~s}$ steps: denaturation at $94^{\circ} \mathrm{C}$, annealing at $65-55^{\circ} \mathrm{C}$ and extension at $72{ }^{\circ} \mathrm{C}$; the initial annealing temperature of $65^{\circ} \mathrm{C}$ was reduced by $0.5^{\circ} \mathrm{C}$ every cycle to reach $55^{\circ} \mathrm{C}$, and then followed by 10 cycles of annealing at $55{ }^{\circ} \mathrm{C}$. After the first 30 cycles, another 8 cycles of annealing at $53{ }^{\circ} \mathrm{C}$ were employed to amplify the M13. The PCR ended with an extra extension of $30 \mathrm{~min}$ at $72{ }^{\circ} \mathrm{C}$.

Fluorescently labelled PCR products were run on an ABI 3730 DNA Sequencer (Macrogen Inc., Korea), scored using the GeneScan-500 (LIZ) size standard, and analysed with GeneMarker software (GeneMarker, USA). Allelic frequencies, number and size of alleles, and observed and expected heterozygosities were estimated in GenAlEx 6.5 (Peakall 
\& Smouse, 2012). Departures from Hardy-Weinberg equilibrium (HWE) and linkage disequilibrium (LD) were tested in GENEPOP on the web v4.0 with default settings. Polymorphic information content (PIC) was calculated using CERVUS 3.0 (Marshall et al., 1998).

\subsubsection{Mitochondrial genome assembly and marker design}

Using a DNA alignment methodology similar to that described by Elbrecht et al. (2015), the mitogenome of Geodia neptuni (GenBank No. NC_006990, Lavrov et al., 2005) was used as a reference to identify sequences of mitochondrial origin in the quality-trimmed 454 data using the discontinuous megablast algorithm BLASTn within Geneious R7 (max evalues $=1 \mathrm{e}-20)$ (Drummond et al., 2011). After extracting the relevant reads, the genome was assembled by mapping to the reference mitogenome of $G$. neptuni using Geneious software. The first draft genome was subsequently used as a search query to identify additional reads in the 454 read database. The mitogenome was annotated for coding DNA sequence (CDS), tRNA and rRNA by comparison with all available Demospongiae mitogenomes in GenBank $(\mathrm{n}=8)$. Thereafter, the ORFs finder and Annotation (Invertebrate Mitochondrial) functions in Geneious were used to check the position and length of each CDSs.

After assembled genome, the genes, Cytochrome $c$ oxidase subunit I (COI), Cytochrome b (Cytb) and 12 Ribosomal RNA (12S), were selected to design specific primers in Geneious (v 7.0). To obtain cross-ampilified primers for other study sponges, the primers binding areas were restricted to the shared sequences in three genes between G. neptuni and Poecillastra laminaris mitogenomes. Three different pairs of primers for each gene were designed, and three Poecillastra laminaris were used to test the primers performance (PCR amplification and polymorphism).

\subsection{Results}

\subsubsection{Next Generation Sequencing}

For 454 pyrosequencing of Poecillastra laminaris genomic DNA, the total number of base pairs was $171,248,552$ with 257,561 reads and an average sequence length of $665 \mathrm{bp}$. Because there is no published genome information for closely related species, it was difficult 
to estimate the coverage of this sequencing data, and it is impossible to compare the distribution patterns of microsatellites.

\subsubsection{Mitogenome assembly}

After assembly, the complete mitogenome of Poecillastra laminaris was $18,413 \mathrm{bp}$ in length. The final assembly contained 530 reads from the 454 pyrosequencing data base, with a minimum and maximum coverage of 7 and 46 sequences, respectively, and an average sequence coverage of 22.7 ( $\mathrm{SD}=6.1$ ). The mitochondrial genome included 14 protein-coding genes, 2 ribosomal RNA genes and 24 transfer RNA genes. The base composition of the genome was A (29.1\%), T (35.2\%), C (14.0\%) and G (21.7\%) with a GC content of $35.7 \%$ (Table 3.1). The annotated mitogenome is available online in NCBI database (GenBank accession number KM362735).

Table 3.1 Characterisation of the mitochondrial genome of Poecillastra laminaris

\begin{tabular}{|c|c|c|c|c|c|c|c|c|c|}
\hline Name & Location5'-3' & Size & Start & Stop & Name & Location5'-3' & Size & Start & Stop \\
\hline$r n l r R N A$ & $32-2449$ & 2418 & & & nad3 & 9442-9798 & 357 & ATG & TAG \\
\hline COII & 2474-3232 & 759 & ATG & TAA & $\operatorname{trn} R(u c g) t R N A$ & 9811-9881 & 71 & & \\
\hline $\operatorname{trnK}($ uuu $) \operatorname{tRNA}$ & $3268-3340$ & 73 & & & $\operatorname{nad} 4 \mathrm{~L}$ & $9882-10,211$ & 301 & GTG & TAA \\
\hline ATP8 & $3342-3533$ & 192 & ATG & TAA & COI & $10,199-11,761$ & 1563 & GTG & TAA \\
\hline ATP6 & $3533-4267$ & 735 & ATG & TAA & $\operatorname{trn} S(u g a) t R N A$ & $11,795-11,879$ & 75 & & \\
\hline $\operatorname{trn} R(u c u) t R N A$ & $4289-4361$ & 73 & & & $\operatorname{trnI}(\mathrm{cau}) \operatorname{tRNA}$ & $11,915-11,987$ & 72 & & \\
\hline COIII & $4380-5168$ & 789 & ATG & TAG & $\operatorname{trn} D(g u c) t R N A$ & $12,001-12,072$ & 72 & & \\
\hline $\operatorname{trn} Q$ (uug) $t R N A$ & $5192-5264$ & 73 & & & $\operatorname{trn} C(g c a) t R N A$ & $12,073-12,144$ & 72 & & \\
\hline $\operatorname{trn} W(u c a) t R N A$ & $5283-5353$ & 71 & & & nadl & $12,165-13,148$ & 984 & ATG & TAG \\
\hline $\operatorname{trn} N(\mathrm{guu}) \operatorname{tRNA}$ & $5371-5442$ & 72 & & & $\operatorname{trn} L($ uaa) $\operatorname{tRNA}$ & $13,181-13,261$ & 81 & & \\
\hline $\operatorname{trnL}$ (uag) $t R N A$ & $5443-5516$ & 74 & & & $\operatorname{trnI}(\mathrm{gau}) t R N A$ & $13,286-13,359$ & 74 & & \\
\hline$C y t b$ & 5519-6664 & 1146 & GTG & TAA & $\operatorname{trn} Y(g u a) t R N A$ & $13,409-13,481$ & 73 & & \\
\hline $\operatorname{trn} T(u g u) t R N A$ & $6675-6747$ & 73 & & & $\operatorname{trnM}(\mathrm{cau}) \mathrm{t} R N A$ & $13,489-13,559$ & 71 & & \\
\hline$A T P 9$ & $6788-7024$ & 237 & ATG & TAA & nad2 & $13,590-14,987$ & 1398 & ATG & TAA \\
\hline $\operatorname{trn} S(g c u) t R N A$ & $7062-7146$ & 85 & & & nad5 & $14,991-16,835$ & 1845 & ATG & TAG \\
\hline $\operatorname{trn} P($ ugg) $t R N A$ & $7176-7248$ & 73 & & & $\operatorname{trn} A(u g c) t R N A$ & $16,826-16,898$ & 73 & & \\
\hline nad4 & $7270-8697$ & 1428 & ATG & TAG & $\operatorname{trnF}(\mathrm{gaa}) \operatorname{tRNA}$ & $16,917-16,989$ & 73 & & \\
\hline $\operatorname{trnH}(g u g) t R N A$ & 8703-8774 & 72 & & & rns $r R N A$ & $17,020-18,254$ & 1235 & & \\
\hline $\operatorname{trn} E(u u c) t R N A$ & $8802-8873$ & 72 & & & $\operatorname{trn} G(u c c) t R N A$ & $18,255-18,326$ & 72 & & \\
\hline nad6 & $8876-9433$ & 558 & GTG & TAA & $\operatorname{trn} V(u a c) t R N A$ & $18,341-18,413$ & 73 & & \\
\hline
\end{tabular}

The complete mitochondrial genome of Poecillastra laminaris (18,413 bp) was $2.2 \%$ longer than the genome of $G$. neptuni $(18,020 \mathrm{bp})$, mainly due to insertions in non-coding regions. There were however, 6 insertions in coding regions, including $12 \mathrm{bp}$ and $21 \mathrm{bp}$ in the 
nad5, 9 bp in the nad4L, 9 bp in the nad4, 18 bp in the COII and 10 bp in the ATP9 genes. The number and order of predicted genes in the Poecillastra laminaris mitogenome were identical according to G. neptuni, and the pairwise sequence identity was $92.0 \%$.

In three designed markers, all genes were successfully amplified in Poecillastra laminaris (details see Table 3.2). Furthermore, these three pairs of primers were used to amplify in Penares sp., Pleroma menoui and Neoaulaxinia persicum, and only COI was amplified in all other three species. The Cytb was failed to obtain PCR products from other three species, but $12 S$ was succeeded to amplify in Penares sp. and $N$. persicum. These three markers were used in following chapters to investigate genetic connectivity patterns of sponges and reveal the environmental factors that explained genetic structures.

Table 3.2 Details of PCR primers and conditions used to amplify three DNA regions

\begin{tabular}{ccccc}
\hline DNA regions & Primer names & Primers 5'-3, & $\begin{array}{c}\text { Annealing } \\
\text { temperature }\left({ }^{\circ} \mathbf{C}\right)\end{array}$ & $\begin{array}{c}\text { Trimmed size } \\
\text { (bp) }\end{array}$ \\
\hline \multirow{2}{*}{$12 S$} & Rnl F & GACACAGGGATGTCGCAGAA & 50 & $\sim 700$ \\
& Rnl R & TGACGGGCGATTTGTACGAA & & $400-470$ \\
\multirow{2}{*}{$C O I$} & $19 \mathrm{~F}$ & TGGTGCTTTTTCGGGGATGA & 50 & 402 \\
& $570 \mathrm{R}$ & ATCGCACCAGCCAATACAGG & & \multirow{2}{*}{50} \\
\multirow{2}{*}{$C y t b$} & $C y t b \mathrm{~F}$ & CGCTAATGGGGCGTCTATGT & & \\
\hline
\end{tabular}

\subsubsection{Microsatellite distribution}

In total, 48,938 putative microsatellites were discovered in the Poecillastra laminaris DNA sequence data, of which the di-, tri-, tetra-, penta- and hexanucleotide repeats numbered 46,254 (94.51\%), 640 (1.31\%), 1,764 (3.61\%), $110(0.22 \%)$ and 170 (0.35\%), respectively. The abundance of dinucleotide repeats was much higher than other repeat types, with a dominance of AC repeats (89.45\% in dinucleotide repeats).

\subsubsection{Microsatellite characterisation}

After primary exclusion of PCR-failed and monomorphic loci, only 10 polymorphic loci were amplified in most specimens. These 10 microsatellites were highly polymorphic, with 10 to 27 alleles per locus (mean \pm SD of $16.2 \pm 5.1$ ). Expected and observed heterozygosities of those loci were 0.786-0.952 and 0.550-0.926, respectively. Except PNL8, the other 9 loci showed significant deviations from Hardy-Weinberg Equilibrium; significant linkage disequilibrium was only observed between PLN21 and PLN48 $(P=0.0465)$. 
Sequences containing microsatellites identified for Poecillastra laminaris have been deposited in GenBank (accession numbers in Table 3.3 ).

Table 3.3 Characterisation of 10 microsatellites for Poecillastra laminaris

\begin{tabular}{|c|c|c|c|c|c|c|c|c|}
\hline Locus & Primer sequences (5'-3') & Motif & $\mathbf{N}$ & $\begin{array}{c}\text { Size } \\
\text { range }\end{array}$ & $\mathbf{N a}$ & Ho & He & $\begin{array}{l}\text { GenBank } \\
\text { No. }\end{array}$ \\
\hline PLN4 & $\begin{array}{l}\text { F: TGT TAG CGC CTG TGT TGA AC } \\
\text { R: TGC CCG GAG AGT TAT TGT GG }\end{array}$ & $\begin{array}{l}{[\mathrm{ACAG}] 7} \\
\ldots[\mathrm{ACAG} \\
] 17\end{array}$ & 23 & $124-244$ & 21 & 0.783 & 0.936 & KT372680 \\
\hline PLN7 & $\begin{array}{l}\text { F: CAG TGG ATG TGC ATT GTG GG } \\
\text { R: TAC ACA TGC CCT GGT CTC TG }\end{array}$ & $\begin{array}{l}{[\mathrm{ACAT}] 6} \\
\ldots[\mathrm{ACAT} \\
] 6\end{array}$ & 20 & $392-488$ & 16 & 0.550 & 0.920 & KT372681 \\
\hline PLN8 & $\begin{array}{l}\text { F: GCA AAC GGT TTC CCT TGT TG } \\
\text { R: CTG TGT CCA CCT GCA ATA CG }\end{array}$ & [ACAT]7 & 21 & $414-478$ & 16 & 0.857 & 0.913 & KT372682 \\
\hline PLN17 & $\begin{array}{l}\text { F: CTC TCT TTG TCC TCT CCG GG } \\
\text { R: AGG ATG CCG ACC CAT TCT AC }\end{array}$ & $\begin{array}{l}{[\mathrm{AG}] 8 \ldots[} \\
\mathrm{AG}] 10\end{array}$ & 24 & $267-315$ & 13 & 0.917 & 0.880 & KT372683 \\
\hline PLN21 & $\begin{array}{l}\text { F: TGA AGC CCT ACA GTG GAC AG } \\
\text { R: CTG CCA ACC GTA CCA AGA AC }\end{array}$ & $\begin{array}{c}{[\mathrm{AGG}] 12} \\
\ldots[\mathrm{AGG}] \\
8\end{array}$ & 27 & $262-358$ & 19 & 0.556 & 0.921 & KT372684 \\
\hline PLN26 & $\begin{array}{l}\text { F: TGG TCA CTT GAG ATC GAG CC } \\
\text { R: CAG GTG AAC TCA ATG GCT GC }\end{array}$ & {$[\mathrm{AT}] 11$} & 27 & $126-212$ & 15 & 0.926 & 0.824 & KT372685 \\
\hline PLN27 & $\begin{array}{l}\text { F: GAT GCA GCA CAA GAC CTT CC } \\
\text { R: TCC ATT CCT CAG TAG CTC GC }\end{array}$ & {$[\mathrm{AT}] 11$} & 26 & $336-376$ & 10 & 0.692 & 0.786 & KT372686 \\
\hline PLN34 & $\begin{array}{l}\text { F: CAT GAC AGT TGT GCT CAC CC } \\
\text { R: ATC AAT CAC CTG AGG ACC CG } \\
\text { F: CAT CCA TAT CTG TCG CAC GC }\end{array}$ & {$[\mathrm{AT}] 13$} & 34 & $172-238$ & 15 & 0.735 & 0.817 & KT372687 \\
\hline PLN39 & $\begin{array}{l}\text { R: CAG GAG AGG AGG TCA TCA } \\
\text { CG } \\
\text { F: CAA GAA CCA ACA ACT CCG }\end{array}$ & {$[\mathrm{AT}] 15$} & 28 & $151-285$ & 27 & 0.786 & 0.952 & KT372688 \\
\hline PLN48 & $\begin{array}{l}\text { GG } \\
\text { R: TCG CAT TAA TTT CCG ACC GC }\end{array}$ & {$[\mathrm{CG}] 8$} & 29 & $311-359$ & 10 & 0.172 & 0.788 & КT372689 \\
\hline
\end{tabular}

$\mathrm{N}$ successfully scored individuals, Na No. of different alleles, Ho observed heterozygosity, and He expected heterozygosity.

\subsection{Discussion and conclusion}

Testing showed that all loci exhibited significant heterozygote deficits except PLN17 and PLN26, explaining the fact that most loci deviated from Hardy-Weinberg Equilibrium. Details about whether these deviations result from technical artefacts of the markers themselves (e.g. null alleles) and/or with the examined population were analysed discussed in Chapter 5.

These microsatellite markers are now being applied to multiple populations of Poecillastra laminaris sampled from the New Zealand EEZ to answer questions about genetic structure and barriers to gene flow which will inform current initiatives to develop options for the increased protection of VMEs in the South Pacific Ocean. 

Chapter 4. Data mining from Solenosmilia variabilis (Scleractinia) Next Generation Sequencing data 


\subsection{Introduction}

Due to limited research, the deep-sea ecosystems on the planet remain poorly understood. Invisible geographic and oceanographic barriers bring a challenge for explaining population differentiation and connectivity on different scales. Although there has been rapid growth in developing new and highly informative marker types, sampling constraints and lack of reference genomes have often limited the study of evolutionary questions and ability to address conservation issues (Sharma et al., 2012).

Stony coral Solenosmilia variabilis is included in the family Caryophylliidae, within the Scleractinia. It has a cosmopolitan distribution and occurs in the upper bathyal zone (151$2165 \mathrm{~m}$ from WoRMS and NIWA Invertebrate Collection database). S. variabilis is a branching, matrix-forming stony coral that builds up a similar (repeating) and complex 3dimensional structure that forms ecological niches for a large diversity of associated microand macrofauna (Rogers 1999; Henry \& Roberts 2007; Althaus et al., 2009; Rogers et al., 2014). Stony coral reef habitat is fragile and easily damaged by near-bottom trawling (Clark \& Rowden, 2009), and the coral is potentially vulnerable to the effects of climate change and ocean acidification as well (Fallon et al., 2014). Owing to its important ecological function, this coral is an indicator for Vulnerable Marine Ecosystem (VME) in the South Pacific (Parker et al., 2009). Unfortunately, the current knowledge of deep-sea species is limited: reproduction, larval dispersal and recruitment information for $S$. variabilis are still not wellstudied. Using genetic tools to understandpatterns of population connectivity of this species will help to provide valuable information for deep-sea conservation.

Despite its importance, no DNA markers have yet been developed for this species. As reported in a previous study (Addamo et al., 2015), microsatellites have been developed in less than 3\% of Scleractinian species. These limited genetic resources for Scleractinia have impeded population genetic studies, especially for deep-sea stony corals. However, recent publications reported that Scleractinia showed a degree of microsatellite polymorphism that is transferable, with high levels of polymorphism detected in one species also being found at the corresponding locus of another species for many loci across genus, family and order level (Nakajima et al., 2009; Wang et al., 2009; Tang et al., 2010; Casado-Amezua et al., 2011; 2012; Davies et al., 2013; Shinzato et al., 2014; Zilberberg et al., 2014; Montoya-Maya et al., 2014; Addamo et al., 2015). Microsatellite transferability (cross-species amplification) amongst related species is allowed for by the homologous nature of the DNA sequence in the microsatellite flanking regions. Therefore, transferability can be a very important factor in 
facilitating the use of microsatellites because it reduces costs when fresh samples are difficult to obtain or reference genomes are not available.

Next-generation sequencing (NGS), also known as high-throughput sequencing, has become very popular in genetic studies after its development in the mid to late 1990s. With ongoing technological development, it allows researchers to sequence DNA and RNA much more quickly and cheaply than the previously used Sanger sequencing, and as such has revolutionised the study of genomics and molecular biology. There are a number of distinctive modern sequencing technologies, including Illumina (Solexa), Roche 454, Ion torrent (Proton / PGM) and SOLiD sequencing (Liu et al., 2012). Roche 454 sequencing, also known as pyrosequencing, as used in this study, produces long reads with high accuracy and relatively low cost, and the longer reads are beneficial for genome assembly and microsatellite primer design. Illumina (sequencing by synthesis), which is the sequencing method for the restriction-site-associated DNA (RAD) sequencing data, generates shorter reads but the depth of sequence coverage is much greater than for 454 sequencing. Theoretically, 454 sequencing data with longer reads is better to develop microsatellites, and RAD-sequencing data with higher depth has advantages for discovering SNPs. However, the power of these methodologies to discover markers in non-model species (i.e. with no

reference genome), and especially in taxa from the deep-sea benthos, remains largely unexplored and is a challenge (Sharma et al., 2012).

In this study, to develop suitable markers for $S$. variabilis, next generation sequencing was utilised to obtain genomic information. Markers were developed to assess genetic connectivity and to identify barriers to gene flow for S. variabilis for use in the context of increased protection of New Zealand's VMEs, and cross-amplication in other common stony coral in New Zealand (Goniocorella dumosa, Desmophyllum dianthus and Madrepora oculata) were also tested, and cross-amplifications were compared between species. These developed markers and cross-amplifiction results were considered and utilized in genetic connectivity assessment if feasible.

\subsection{Materials and methods}

\subsubsection{Samples, DNA extraction and pyrosequencing}

Three live S. variabilis (NIWA ID number 94287 (37.707 S, 169.015 W, $1244 \mathrm{~m}$ ); 94550 (41.581 S, $164.255 \mathrm{~W}, 1223 \mathrm{~m}) ; 94590$ (41.365 S, $164.419 \mathrm{~W}, 1220 \mathrm{~m})$ ) were 
collected from the Louisville Seamount Chain off NE New Zealand during cruise TAN1402 in February, 2014. Specimens were initially preserved in absolute ethanol and stored at $80{ }^{\circ} \mathrm{C}$ on board, and then transferred from NIWA to the VUW laboratory under permit from Biosecurity New Zealand. Genomic DNA (gDNA) for pyrosequencing was extracted using the phenol-chloroform method. After extraction, DNA of two species was quantified by Nanodrop 1000 (Thermo Scientific) and 0.5\% agar electrophoresis at $80 \mathrm{~V}$ for $2 \mathrm{~h}$. Before 454 GS-FLX pyrosequencing, the three genomic DNA samples were barcoded as three separate libraries (barcoding and libraries construction were conducted by Macrogen Inc., Korea). The 454 GS-FLX Titanium platform was utilised to sequence these libraries (Macrogen Inc., Korea).

\subsubsection{Mitochondrial genome assembly and marker design}

Following (Elbrecht et al., 2013) the mitogenome of Lophelia pertusa (GenBank No. KC875349 (Flot et al., 2013), with the highest pairwise identity at the COI region, was used to identify DNA sequences and extract all mitogenome reads from the $S$. variabilis libraries using the discontinuous megablast algorithm BLASTn in the Geneious software package (max e-values 1e-20) (Drummond et al., 2013). Extracted reads were assembled by mapping to the reference mitogenome of L. pertusa. The first draft genome was manually checked and then used as a search query to identify additional reads in the 454 read database. The mitogenome was annotated for coding DNA sequence (CDS), tRNA and rRNA by comparison with all available scleractinian mitogenomes in GenBank $(n=30)$. The ORFs finder and Annotation (Invertebrate Mitochondrial) functions in Geneious were used to check the position, start and stop codons and length of each CDS. The same method was applied to construct three mitochondrial genomes from the three libraries but using the mitogenome constructed above as the reference. After assembling the mitogenomes from three libraries, multiple alignment (ClustalW) was utilised to compare differences amongst the three mitogenomes in Geneious.

To develop the polymorphic sequencing markers, the two assembled mitogenomes were aligned to identify the unidentical sites. Candidate genes which the sequences were different from each other in two mitogenomes were selected to design primers in Geneious (v7.0). For ITS marker, the avaible ITS sequences Scleractinia were used to extract ITS reads in 454 data and extracted reads were assembled with the same setting as described above and 
multiple alignment was completed by ClustalW in Geneious (v7). The primers were designed based on the alignment with default settings.

\subsubsection{Microsatellite discovery and characterisation}

MSATCOMMANDER 0.8 was used to isolate microsatellites and design primers for genetic analysis on all library data, respectively. Microsatellites, also called simple sequence repeats (SSR), with a minimum of 5 repeats for di-, tri-, tetra-, penta-, and hexanucleotides were selected, and MSATCOMMANDER with default settings, except for the minimum and maximum PCR product size (set to 100-400 bp), was used to design PCR primers for $S$. variabilis.

To identify polymorphic microsatellites directly in S. variabilis, Microsoft Excel (v 2013) was used to identify the locus which are shared same motif (exactly same repeated motif and primers pair) but having a different number of repeats amongst different libraries. This is to waive the experimental test of whether the locus was polymorphic, and the only experiment is to verify PCR amplification. After successfully-amplified, the selected microsatellites were subsequently characterised by genotyping.

The gDNA of 3 specimens (collected off New Zealand in the SW Pacific Ocean) were initially used to test for successful PCR amplification by visualising amplified products on $2 \%$ agarose gels. Primers that successfully amplified a product (putative microsatellite locus) were allocated to different multiplex systems by Multiplex Manager 1.0 (Holleley \& Geerts, 2009).

The forward primers from each primer pair were fluorescently $5^{\prime}$ end labelled with 6FAM, NED, VIC or PET following the results of the multiplex. For genotyping, PCRs were carried out in a total volume of $40 \mu 1$ with $20 \mu 1$ PCR Red Mixture MyTaq ${ }^{\text {TM }}$ Red Mix), $1 \mu 1$ forward and reverse primers, $2 \mu \mathrm{l}$ of template DNA and topped up with dd $\mathrm{H}_{2} \mathrm{O}$. PCR amplifications were performed with an initial denaturing step of $94{ }^{\circ} \mathrm{C}$ for $10 \mathrm{~min}$, followed by 32 cycles of $30 \mathrm{~s}$ at $94{ }^{\circ} \mathrm{C}, 45 \mathrm{~s}$ annealing at $55^{\circ} \mathrm{C}$, and $45 \mathrm{~s}$ extension at $72{ }^{\circ} \mathrm{C}$, and a final extension of $30 \mathrm{~min}$ at $72{ }^{\circ} \mathrm{C}$.

Nine microsatellite loci of $S$. variabilis from Miller \& Gunasekera (2016) were also introduced in this study, and the amplification process of these 9 loci followed the method described above. The same PCR conditions for the newly developed and the introduced microsatellites were employed for cross-species amplification in three other stony corals 
(most-common stony corals in New Zealand deep-sea coral reef), G. dumosa, D. dianthus and M. oculata.

All microsatellites characterisation in S. variabilis, G. dumosa, D. dianthus and $M$. oculata were based on randomly selected 30 individuals for each species. Fluorescently labelled PCR products were run on an ABI 3730 DNA Sequencer (Macrogen Inc., Korea), scored using the GeneScan-500 (LIZ) size standard, and analysed with GeneMarker software (GeneMarker, USA). Allelic frequencies, number and size of alleles, and observed and expected heterozygosities were estimated in GenAlEx 6.5 (Peakall \& Smouse, 2012). Departures from Hardy-Weinberg equilibrium (HWE) and linkage disequilibrium (LD) were tested in GENEPOP on the web v4.0 with default settings. Polymorphic information content (PIC) was calculated using CERVUS 3.0 (Marshall et al., 1998).

\subsubsection{Single nucleotide polymorphisms (SNPs) discovery}

Three 454 sequencing datasets from the present study and a RAD sequencing dataset from a collaborator's lab (Karen Miller, AIMS, Australia) were combined to develop SNPs for $S$. variabilis population genetics studies. Three RAD samples were pooled as one sample for subsequent analysis due to the lack of RAD index information. To combine 454 and RAD sequencing data, all four datasets were mapped to a reference genome. Because there is no reference genome for S. variabilis the only available Scleractinian genome in NCBI, that for Acropora digitifera, was employed. After data filtering, the clean data from the four datasets were mapped onto the A. digitifera genome for data alignment. BWA (0.7.12), with the number of threads (-t) was 4 and minimum seed length (-k) was 32 in BWA-MEM algorithm, were took for reads mapping to gain the initial alignment result, and (Li \& Durbin, 2009). SAMtools (0.1.18) was used for sorting, merging and PCR duplication based on the initial alignment result (Li et al., 2009). Due to the low mapping rate, a lower standard for SNP detection and filtering were considered, and the SNP and INDEL calling, filtering details were alignment score $\geq 10$ and variation quality score $\geq 10$. 


\subsubsection{Statistical analysis}

\subsubsection{Microsatellites}

To detect whether different sequencing approaches caused markers detection issues, after calculation of the percentage of each type of motif, hierarchical cluster analysis (cluster method used between-group linkage) was employed to reveal the similarity of microsatellite distributions. Additionally, a $\chi^{2}$ test was used to investigate the distributions difference of microsatellites amongst different sequencing methods in the software package IBM SPSS Statistics (v 22.0).

The cross amplification was investigated amongst four species, and whether any differences between the amplifications amongst different spcies (D. dianthus, G. dumosa, M. oculata and $S$. variabilis) was analysised by two different mulitivariances anslyses, Structure (Bayesian) and Discriminant Analysis of Principal Components (DAPC). The software package Structure (v 2.3.4, K from 1 to 5 and the length of burnin period and number of MCMC reps after burnin were 5,000 and 10,000, respectively), and DAPC analysis was completed in $\mathrm{R}$ projected by Package 'adgenet'. Both analyses were run with all 27 microsatellite locus data and then with data from 12 loci that amplified in all four species, including B106, C107, D5, B1, B123, SVA1, SVA3, SVA2, SVA4, SVA12, SVA13 and SVA11. Structure Harvester (http://taylor0.biology.ucla.edu/structureHarvester/)was used to estimate the K value. The wilcoxon signed-rank test by IBM SPSS Statistics (v 22.0) was employed to investigate the cross-amplification differences in previous studies.

\subsubsection{2. $\quad$ SNPs}

Considering the different types of sequencing data (genomic assembled data for $A$. digitifera, 454 datasets for 94287, 94550 and 94590 and RAD-sequencing dataset for L008), and the composition percentage of each type of SNP was calculated. These new data were used for $\chi^{2}$ testing and hierarchical cluster analysis to reveal the relations by SPSS (v 22.0). A Venn diagram, made using the package "VennDiagram" in R, was used to examine the SNPs distributional relationships amongst the different taxa. After transforming into numeric variants the SNPs that were detected in the 5 species-specific datasets, Structure (v 2. 3. 4) was used to reveal the differentiations of those SNPs amongst taxa. 


\subsection{Results and Discussion}

\subsubsection{Next Generation Sequencing}

In S. variabilis, the average length of sequences from each of the three libraries varied from 607 to $727 \mathrm{bp}$, and total compiled library length ranged from 17,479,912 to $239,596,471 \mathrm{bp}$. This accounts for approximately 3.97 to $54.40 \%$ of the genome, assuming a genome size of $420 \mathrm{Mb}$ (estimated from the size of Acropora digitifera (Shinzato et al., 2011). In Rad-seq, the mean length of sequence reads was $151 \mathrm{bp}$ with a total sequence length of $478,110,330 \mathrm{bp}$. After mapping to the A. digitifera genome, the successful mapping rate of the 454 data was about $20 \%$, but the RAD-seq data was only $13.6 \%$ (Table 4.1 and Table 4.2). The alignment results showed that the mapping rate for each sample was low, and this may be due to the great difference between the reference and the sequenced samples. Given the data were mapped to the reference genome of $A$. digitifera, but the annotation file of the reference genome is not available, and the sequence could not be therefore annotated except mitochondrial sequences.

The choice of a sequencing strategy must take into account the research goals, ability to store and analyse data, the ongoing changes in performance parameters, and the cost of NGS platforms (Kumar et al., 2012). Developing suitable genetic markers for S. variabilis to reveal population genetic structuring is the main target. Currently few genetic marker resources exist for $S$. variabilis, wherein 454 pyrosequencing and Rad-sequencing in the present study were shown to be excellent methods for large scale prediction of molecular markers for future genetic studies in non-model organisms. There are many different aspects of these two next generation sequencing methods, but only mitochondrial genome assemble, microsatellite development and single nucleotide polymorphism discovery were compared in this study. In mitochondrial genome assembly, 454 sequencing provided better performance than Rad-sequencing, with a higher coverage and greater depth. Although Rad-sequencing data produced many more microsatellites, the percentage of useful microsatellites was much lower than that derived from the 454 sequencing data. However, Rad-sequencing, as the main stream of developing SNP markers, was much more powerful than 454 sequencing in SNP identifications. The Rad-sequencing generated more SNPs in total and also a greater number of unique SNPs. Both sequencing methods have had a lot of successful examples of SNPs discovery and utilisations in genetic studies (e.g. Emerson et al., 2010; Hohenlohe et al., 
2010). But one doubt about using 454 sequencing to identify SNPs is whether the depth of sequences is sufficient to exclude involute ones, because deep sequence coverage leading to dense and confident SNPs. In the present study, the average coverage depth of sequencing per SNP in 454 sequencing was not significantly different from RAD sequencing using $\chi^{2}$ test. Additionally, mapping to reference genomes reduced the risk of invalid SNP discovery, although the mapping ratio was low.

Table 4.1 Summary information for sequencing data for Solenosmilia variabilis

\begin{tabular}{ccccc}
\hline Sample ID & Sequencing Method & Source & Total Reads & Average length (bp) \\
\hline L008 & Rad-seq & Australia & $3,635,820$ & 151.0 \\
94550 & 454 & New Zealand & 242,903 & 713.1 \\
94590 & 454 & New Zealand & 19,610 & 607.5 \\
94287 & 454 & New Zealand & 218,657 & 727.3 \\
\hline
\end{tabular}

Table 4.2 Summary information for sample alignment mapping to Acropora digitifera

\begin{tabular}{cccc}
\hline Sample ID & Total bases & Mapped bases & Mapping Rate (\%) \\
\hline L008 & $478,110,330$ & $65,043,497$ & 13.60 \\
94550 & $239,596,471$ & $48,869,961$ & 20.40 \\
94590 & $17,479,912$ & $3,555,465$ & 20.34 \\
94287 & $221,635,258$ & $44,454,467$ & 20.06 \\
\hline
\end{tabular}

\subsubsection{Mitogenome assembly and marker development}

The first assembled draft contained 506 reads from three pooled databases, with good overall coverage (minimum coverage was 7 sequences, maximum coverage was 38 sequences, average coverage was $22.0(\mathrm{SD}=6.8)$ ). Having assembled all relevant reads from each library, two $S$. variabilis mitogenomes were successfully obtained, one from a coral from Anvil Seamount and one from an individual collected from Valerie Guyot. The two mitogenomes were almost identical, the length of each being 15,968 bp. Both mitochondrial genomes included 13 protein-coding genes, 2 ribosomal RNA genes, 2 transfer RNA genes and a $D$ loop region (Table 4.3). The base composition of the genome was A (24.2\%), T (41.1\%), C $(13.7 \%)$ and $\mathrm{G}(21.0 \%)$. There were 5 nucleotide differences $(0.03 \%)$ between the two mitogenomes, including synonymous substitutions in the genes nad1, nad4L and nad2 (at $1276 \mathrm{bp}, 6596 \mathrm{bp}$ and 10,502 bp, respectively) and two $1 \mathrm{bp}$ differences (G/T transversions at 8701 and $8768 \mathrm{bp}$ ) in the $D$-loop region. The annotated mitogenomes are available online in the NCBI database (GenBank accession numbers KM609293 and KM609294). 
The full mitochondrial genome of $S$. variabilis, at 15,968 bp, was slightly shorter than that of a branching habitat-forming species not found in the New Zealand region, L. pertusa $(16,149 \mathrm{bp})$. The small difference between the species is primarily due to a $186 \mathrm{bp}$ deletion in the $D$-loop region of $S$. variabilis. The number and order of the predicted genes in the $S$. variabilis mitogenome were identical to L. pertusa, and the pairwise sequence identity between the two taxa was $97.5 \%$.

After successful assembly of the mitogenome of the New Zealand specimens, 1593 reads of Rad-sequencing data of an Australian specimen were mapped to the assembled genome with coverage from 0 to 112 -fold $(12.20 \pm 13.00$ (Mean $\pm \mathrm{SD}$ )), but they failed to assemble another complete genome (coverage was 98.3\%). Pairwise identity between mapped sequences and a reference genome (above assembled genome) was 99.20\%, which indicated that the mitochondrial genomes were much closer within species $(S$. variabilis) than between species (S. variabilis - L. pertusa).

Although unable to assemble the full mitogenome of the third New Zealand specimen, there was no differences in the D-loop region between the two specimens from Valerie Guyot, suggesting that genetic variation in the mitochondrial genome is low. Similarly, limited genetic differences in the mitogenomes of two L. pertusa collected from the Mediterranean and Barents seas have been reported, which differed only by a single non-synonymous substitution (Flot et al., 2013). The variation information of the mitogenome will be beneficial for future phylogenetic and phylogeographic studies of Scleractinia, particularly for $S$. variabilis, and useful where there may be some confusion with specimen identification.

Table 4.3 Characterisation of the mitochondrial genome of Solenosmilia variabilis

\begin{tabular}{cccccccccc}
\hline Name & Location5'-3' & Size (bp) & Start & Stop & Name & Location5'-3' & Size (bp) & Start & Stop \\
\hline nad5 & $1-8295$ & 1836 & ATG & TAA & D-loop & $8296-8818$ & 523 & & \\
nad5 intron & $715-7173$ & 6459 & & & Cytb & $8819-9979$ & 1161 & ATG & TAG \\
nad1 & $824-1771$ & 948 & ATG & TAA & nad2 & $9975-11291$ & 1317 & ATT & TAA \\
ATP6 & $1824-2522$ & 699 & ATG & TAA & nad6 & $11293-11847$ & 555 & GTG & TAA \\
nad4 & $2522-3967$ & 1446 & ATG & TAG & $t R N A-T r p$ & $12250-12319$ & 70 & & \\
$12 S$ rRNA & $3968-4991$ & 1023 & & & ATP8 & $12323-12490$ & 168 & ATG & TAA \\
COIII & $4992-5771$ & 780 & ATG & TAG & COI & $12480-14045$ & 1566 & ATG & TAA \\
COII & $5759-6484$ & 726 & GTG & TAA & $t R N A-M e t$ & $14041-14111$ & 71 & & \\
nad4L & $6474-6773$ & 300 & ATG & TAA & $16 S r R N A$ & $14112-15968$ & 1857 & & \\
nad3 & $6775-7119$ & 345 & ATG & TAA & & & & & \\
\hline
\end{tabular}

High similarities in mitochondrial genes of Scleractinia had been proved in this study, and a slow evolutionary rate in mitogenome would explain this (Shearer et al., 2002). The 
low variation between individuals and species indicated that mitochondrial genes might not be suitable for $S$. variabilis population genetic studies.

After compared the alignments, D-loop, as the most variable area in mitogenome, was selected to design primers. Details of ITS and D-loop primers were present in Table 4.4. After testing in D. dianthus, G. dumosa, M. oculata and S. variabilis, ITS genes were successfully amplified in all species and D-loop was only amplified in G. dumosa and S. variabilis. These two markers were used in following chapters to investigate genetic connectivity patterns of corals and reveal the environmental factors that explained genetic structures.

Table 4.4 Details of PCR primers and conditions used to amplify two DNA regions

\begin{tabular}{ccccc}
\hline DNA regions & Primer names & Primers 5'-3' & $\begin{array}{c}\text { Annealing } \\
\text { temperature }\left({ }^{\circ} \mathbf{C}\right)\end{array}$ & $\begin{array}{c}\text { Trimmed } \\
\text { size (bp) }\end{array}$ \\
\hline \multirow{2}{*}{ ITS } & $348 \mathrm{~F}$ & TTGACGGTGGATCTCTTGGC & 50 & $\sim 400$ \\
& $854 \mathrm{R}$ & CAAATTTGAGCTGGTCCCGC & & \\
D-loop & $74 \mathrm{~F}$ & CCCAGGGGCCTTGTTCAATTTCTA & 50 & $\sim 400$ \\
\hline
\end{tabular}

\subsubsection{Microsatellite distribution}

After searching, MSATCOMMANDER identified totals of 45,389, 17,289, 19,906 and 6,236 microsatellites from specimens L008, 94287, 94550 and 94590, respectively. Because the results for 94590 were much less than other two New Zealand samples, 94590 was excluded from microsatellite distribution comparisons. In the 94287 and 94550 datasets, $37.99 \%$ of microsatellites were dinucleotide, $29.88 \%$ trinucleotide, $24.80 \%$ tetranucleotide, $3.05 \%$ pentanucleotide and $4.28 \%$ hexanucleotide repeats. $\chi^{2}$ testing showed that there were no significant differences between the numbers of different repeat motif types for samples 94287 and 94550 (Table 4.5) ( $\mathrm{p}>0.05$ ). Also, the $\chi^{2}$ test showed the distributions between the 454 and RAD sequencing methodologies were not significantly different $(p>0.05)$. Dinucleotide repeats were the most common type in all datasets except that for 94590, with penta- and hexanucleotide repeats being present at much lower frequencies. Although compositions of trinucleotide and tetranucleotide varied a lot amongst different species, the distributions of microsatellites showed a taxonomically related pattern, with $D$. dianthus and S. variabilis grouped together (they are in the same family, Figure 4.1). The placement of sample 94590 as an outlier is related to the reduced genome coverage of this sample. 
To develop useful microsatellites, sequences containing $>50$-bp sequence on both sides of the microsatellite repeat were considered sufficient for primer design. After this filter, percetnages of those microsatellites were $11.73 \%, 73.61 \%, 32.64 \%$ and $73.58 \%$ in L008, 94550, 94590 and 94287, respectively. It is also worth noting that tetranucleotide and trinucleotide repeat compositions were different in different sequencing methods, with tetranucleotide repeats having a higher proportion than triplet repeats in Rad-sequences, but 454 sequencing was the opposite (Table 4.5). A previous study has noted that tetranucleotide repeats occur at a lower proportion in invertebrate genomes than triplet repeats (Tóth et al., 2000). If true, then this might imply that 454 sequencing was more accurate than Radsequencing. Present study also provided evidences that different sequencing methods were likely to cause sequences abundant and distribution basis, but the difference caused by different sequencing methods was still within species level if no sequencing-libraries construction issues.

Table 4.5 Microsatellite distributions (\%) information for three species

\begin{tabular}{ccccccccc}
\hline \multirow{2}{*}{ Repeats } & \multirow{2}{*}{$\begin{array}{c}\text { Acropora } \\
\text { digitifera }\end{array}$} & \multirow{2}{*}{$\begin{array}{c}\text { Desmophyllu } \\
\boldsymbol{m}\end{array}$} & dianthus & $\boldsymbol{5}$ & \multicolumn{5}{c}{ Solenosmilia variabilis } \\
\cline { 5 - 8 } & & Average & $\mathbf{9 4 2 8 7}$ & $\mathbf{9 4 5 5 0}$ & $\mathbf{9 4 5 9 0}$ & L008 \\
\hline Dinucleotide & 42.33 & 43.31 & 37.99 & 38.34 & 37.64 & 23.00 & 35.65 \\
Trinucleotide & 48.45 & 9.19 & 29.88 & 29.78 & 29.99 & 55.18 & 24.60 \\
Tetranucleotide & 7.17 & 35.43 & 24.80 & 25.74 & 23.86 & 12.99 & 25.18 \\
Pentanucleotide & 1.21 & 9.68 & 3.05 & 3.09 & 3.01 & 2.28 & 2.81 \\
Hexanucleotide & 0.84 & 2.38 & 4.28 & 3.06 & 5.50 & 6.56 & 11.76 \\
\hline
\end{tabular}

* Data from Addamo et al. (2015). Average was the mean of 94278 and 94550.

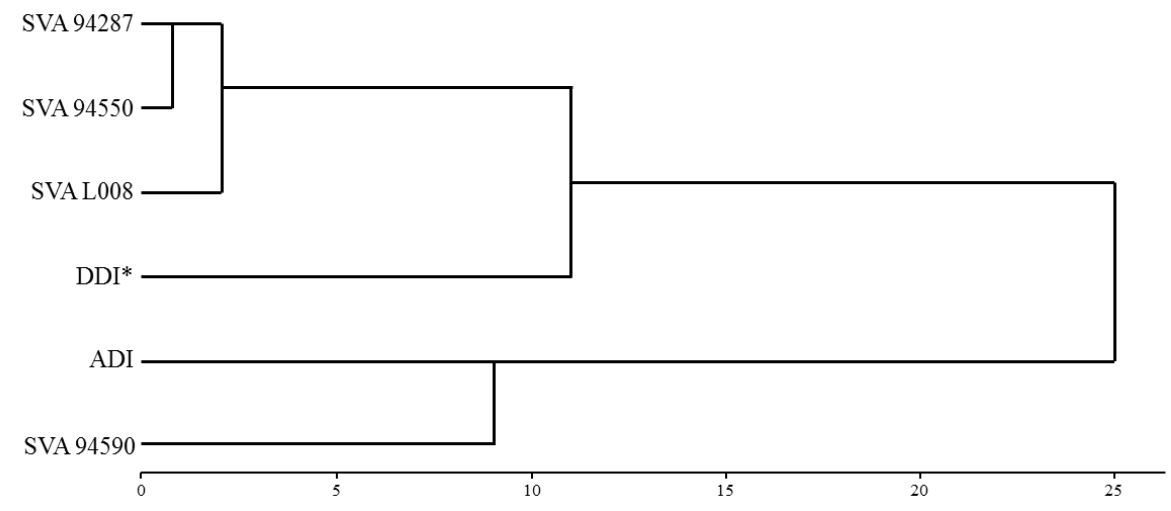

Figure 4.1 Dendrogram of sample similarity based on microsatellite variation amongst three corals

* Data were obtained from Addamo et al. (2015); Distance interval was Squared Euclidean distance. *Private alleles were calculated amongst Goniocorella dumosa, Desmophyllum dianthus, Madrepora oculata, Solenosmilia variabilis. 


\subsubsection{Microsatellite characterisation}

After comparison of primer pairs and motifs, 22 potential polymorphic microsatellites were identified. After primary amplification, 2 homozygote loci and 2 failures were excluded from further characterisations (Table 4.6). Locus SVA20 amplified more than 2 alleles, and it was therefore excluded from scoring for further analysis. In those 27 microsatellites (including 9 loci from Miller \& Gunasekera (2016)), polymorphism information content (PIC) estimates showed that 23 loci were reasonably to highly informative (PIC > 0.5) (Table 4.7). Observed $(\mathrm{Ho})$ and expected $(\mathrm{He})$ heterozygosities averaged 0.724 and 0.731 , respectively. Tests for linkage disequilibrium (LD) yielded 56 (18.98\%) significant P values out of 295 pairwise comparisons $(\mathrm{p}<0.05)$, and only SVA10 and SVA14 were totally unlinked from other markers. 17 markers exhibited significant deviations from HWE (Table 4.7).

Table 4.6 Primer information for Solenosmilia variabilis microsatellites

\begin{tabular}{|c|c|c|c|}
\hline Name & Forward & Reward & Motif \\
\hline SVA1 & ACTTGCACGAGAAGCTTTGG & CAGAGTCCCAGTACAAAGTCG & [AAAC] 6 \\
\hline SVA2 & TTTGCCTACATGACAACCTGC & TGCGTGAGTCCAGAGATCAG & {$[\mathrm{AAAC}] 15$} \\
\hline SVA3 & GACAGCAGGAACGTTACAGC & ATGGATTCGTCGTGTTCCTC & {$[\mathrm{AAC}] 9$} \\
\hline SVA4 & GGTAGTGGAGGTAGGCATCG & GCCGCTAATGAACAGGATCG & {$[\mathrm{AAC}] 9$} \\
\hline SVA5 & GGTGAGCAAGGCCATACAAC & TGTCCCGTATGCCTTGTTTG & {$[\mathrm{AAC}] 9$} \\
\hline SVA7 & ATGGTACCGTAAACTGCCGC & CAGCTCTCСTCTGGTATGCC & [AAT] 12 \\
\hline SVA8 & GGCCGTGGTTATAGATCACAG & CCAAACTCGCCTGATATGCC & {$[$ AATAC] 23} \\
\hline SVA10 & TGGTGGAAAGCAAGTCATGG & TTGGCCATGTCTGCTCAAAG & [AATC]11 \\
\hline SVA11 & AGGAGACGACACTTACGCTG & GCGATCCTGTTCCAACGTTC & [AATG]11 \\
\hline SVA12 & TCTGGTCACCTGGCTAATGG & TGTAGACAGCCATACAATCCG & {$[\mathrm{AATG}] 10$} \\
\hline SVA13 & GCAGGCAGTCAGTTAGTTCG & CTGACAACACAAGGCACCTG & [ACAG]6 \\
\hline SVA14 & TTGTAACCAGGCTCAACTCG & GAACGTTTCGCTGTCTTCATG & [ACATC] 27 \\
\hline SVA15 & ACTAGAGCCCAGTCTGTTCG & TACTGCTCTGTGTGGTTCGG & {$[\mathrm{AG}] 11$} \\
\hline SVA16 & TATTCCCTCCGTGCCAGTAC & GAGTCCAACACGCTAACCAC & {$[\mathrm{AG}] 13$} \\
\hline SVA17 & AAATTCTGTATGCCTCGCGG & AGTTTGTTGGAAATAAGGCGTG & {$[\mathrm{AT}] 8$} \\
\hline SVA18 & AGCCATGTTGTTTCGAGGTC & TGCATCTGAAGCCCTGTCAG & {$[\mathrm{AT}] 9$} \\
\hline SVA19 & ACTGCTACCGTTCCTTGAGG & AATGCAACGATGGTATAGGCC & {$[$ ATC $] 15$} \\
\hline SVA20 & GGGACGCTTATTTGGTCTAGC & AAACGCCATCAACACAGAGG & {$[\mathrm{ATC}] 17$} \\
\hline
\end{tabular}

In this study, 27 primer pairs produced reliable PCR amplicons that were scorable with the number of alleles (NA) per locus varying from 3 to 22 (average 10.96) (Table 4.7). The NAs found in the present study were similar to those reported in Lophelia pertusa (12.6 Le Goff \& Rogers, 2002) and 17.92 (Morrison et al., 2008), but higher than D. dianthus (9.13 - Addamo et al., 2015), Seriatopora hystrix (8.8 - Underwood et al., 2006), and Acropora millepora (8.9 - van Oppen et al., 2007). Amongst examined loci, a high proportion of 
markers not in Hardy-Weinberg equilibrium in every species might explain poor primer annealing (Kwok et al., 1990), differential amplification of size-variant alleles (Wattier et al., 1998), or poor quality DNA causing PCR failure (Gagneux et al., 1997). On the other hand, the Wahlund effect, inbreeding, asexual reproduction and the complex genetic structure of $S$. variabilis have possibly contributed to the departure from equilibrium (Chakraborty et al., 1992).

Table 4.7 Characterisation of 27 microsatellite loci for Solenosmilia variabilis

\begin{tabular}{ccccccccc}
\hline Loci & Range & No & Private* & success & PIC & Ho & He & HW Equilibrium \\
\hline B106 & $253-268$ & 6 & 4 & 33 & 0.547 & 0.970 & 0.621 & $* * *$ \\
C107 & $201-237$ & 9 & 3 & 33 & 0.718 & 0.879 & 0.747 & ns \\
D5 & $166-257$ & 12 & 4 & 33 & 0.799 & 0.727 & 0.817 & $*$ \\
B1 & $119-183$ & 14 & 12 & 33 & 0.807 & 0.758 & 0.821 & $*$ \\
B116 & $227-240$ & 6 & 4 & 33 & 0.624 & 0.697 & 0.684 & ns \\
D103 & $197-206$ & 4 & 1 & 33 & 0.444 & 0.394 & 0.479 & ns \\
B123 & $159-185$ & 5 & 2 & 33 & 0.337 & 0.242 & 0.360 & $* *$ \\
D213 & $105-180$ & 11 & 5 & 33 & 0.652 & 1.000 & 0.699 & $* * *$ \\
C4 & $203-227$ & 3 & 0 & 33 & 0.111 & 0.121 & 0.115 & ns \\
SVA1 & $209-257$ & 9 & 4 & 33 & 0.539 & 0.545 & 0.564 & $* * *$ \\
SVA2 & $270-304$ & 12 & 5 & 32 & 0.847 & 0.563 & 0.862 & $* * *$ \\
SVA3 & $395-418$ & 10 & 2 & 33 & 0.730 & 0.515 & 0.766 & $* * *$ \\
SVA4 & $261-312$ & 15 & 5 & 33 & 0.820 & 0.909 & 0.837 & ns \\
SVA5 & $197-345$ & 15 & 14 & 33 & 0.870 & 1.000 & 0.881 & $* * *$ \\
SVA7 & $341-398$ & 11 & 4 & 33 & 0.821 & 1.000 & 0.841 & ns \\
SVA8 & $260-383$ & 15 & 12 & 32 & 0.838 & 0.594 & 0.850 & $* * *$ \\
SVA10 & $404-472$ & 13 & 6 & 33 & 0.879 & 1.000 & 0.889 & ns \\
SVA11 & $380-484$ & 16 & 13 & 33 & 0.757 & 1.000 & 0.782 & $* * *$ \\
SVA12 & $357-453$ & 15 & 12 & 33 & 0.763 & 0.455 & 0.786 & $* * *$ \\
SVA13 & $185-266$ & 10 & 3 & 33 & 0.683 & 0.848 & 0.702 & ns \\
SVA14 & $284-418$ & 22 & 19 & 33 & 0.939 & 0.818 & 0.942 & $* *$ \\
SVA15 & $197-221$ & 10 & 4 & 33 & 0.679 & 1.000 & 0.720 & $*$ \\
SVA16 & $336-370$ & 10 & 4 & 33 & 0.633 & 0.545 & 0.660 & $* * *$ \\
SVA17 & $329-403$ & 10 & 10 & 8 & 0.854 & 0.750 & 0.867 & ns \\
SVA18 & $316-390$ & 13 & 4 & 33 & 0.823 & 0.697 & 0.839 & $* *$ \\
SVA19 & $338-437$ & 9 & 6 & 5 & 0.868 & 0.800 & 0.880 & ns \\
SVA20 & & & & & Multicopies & & \\
\hline & & & & & & & $* n$ \\
\hline
\end{tabular}

Ho, Observed Heterozygosity; He, Expected Heterozygosity; ns, not significant; ${ }^{*} \mathrm{p}<0.05 ;{ }^{* *} \mathrm{p}<0.01 ; * * * \mathrm{p}<$ 0.001. *Private alleles were calculated amongst Goniocorella dumosa, Desmophyllum dianthus, Madrepora oculata, Solenosmilia variabilis. Success - number of individuals for which successful PCR amplifications were obtained ( $\mathrm{n}=30$ individuals tested) 


\subsubsection{Microsatellite transferability}

To develop population genetic markers for other corals, especially for G. dumosa, these 27 microsatellites were tested for their transferability in three other closely related taxa, G. dumosa, D. dianthus and M. oculata. The details of locus-specific cross amplification in the different species was as follows.

Table 4.8 Cross-amplification information of 27 loci in Goniocorella dumosa

\begin{tabular}{|c|c|c|c|c|c|c|c|c|}
\hline Loci & Range & No & Private* & success & PIC & Ho & $\mathrm{He}$ & HW Equilibrium \\
\hline B106 & $241-256$ & 5 & 3 & 29 & 0.625 & 0.690 & 0.673 & $* *$ \\
\hline $\mathrm{C} 107$ & $177-234$ & 9 & 2 & 29 & 0.712 & 0.517 & 0.749 & $* *$ \\
\hline D5 & $177-298$ & 17 & 9 & 29 & 0.869 & 0.724 & 0.878 & $* * *$ \\
\hline B1 & $104-206$ & 6 & 5 & 29 & 0.508 & 1.000 & 0.589 & $*$ \\
\hline B116 & $237-262$ & 6 & 5 & 15 & 0.732 & 0.800 & 0.769 & $* *$ \\
\hline D103 & $173-203$ & 5 & 2 & 20 & 0.686 & 0.700 & 0.734 & $* * *$ \\
\hline B123 & $158-194$ & 7 & 3 & 29 & 0.670 & 0.483 & 0.716 & $* * *$ \\
\hline $\mathrm{D} 213$ & $105-216$ & 10 & 4 & 27 & 0.801 & 0.815 & 0.822 & $* * *$ \\
\hline $\mathrm{C} 4$ & $106-239$ & 9 & 8 & 24 & 0.796 & 0.583 & 0.818 & $* * *$ \\
\hline SVA1 & $221-249$ & 7 & 1 & 26 & 0.656 & 0.346 & 0.643 & $* * *$ \\
\hline SVA2 & $236-336$ & 7 & 3 & 23 & 0.625 & 0.435 & 0.612 & $* *$ \\
\hline SVA3 & $302-431$ & 16 & 10 & 23 & 0.835 & 0.565 & 0.847 & $* * *$ \\
\hline SVA4 & $201-300$ & 7 & 2 & 23 & 0.818 & 0.462 & 0.787 & ns \\
\hline SVA5 & $187-250$ & 9 & 9 & 10 & 0.753 & 0.400 & 0.715 & $* *$ \\
\hline SVA7 & $311-488$ & 18 & 13 & 25 & 0.787 & 0.440 & 0.797 & $* * *$ \\
\hline SVA8 & $200-320$ & 10 & 7 & 16 & 0.803 & 0.688 & 0.820 & $* * *$ \\
\hline SVA10 & $400-472$ & 9 & 2 & 25 & 0.796 & 0.840 & 0.817 & ns \\
\hline SVA11 & \multicolumn{5}{|c|}{ Monomorphic } & 1.000 & 0.500 & $* * *$ \\
\hline SVA12 & $301-427$ & 9 & 9 & 16 & 0.787 & 0.625 & 0.811 & $* * *$ \\
\hline SVA13 & $218-251$ & 8 & 1 & 28 & 0.724 & 0.964 & 0.756 & $* *$ \\
\hline SVA14 & $360-492$ & 6 & 3 & 18 & 0.603 & 0.500 & 0.664 & $* *$ \\
\hline SVA15 & $183-249$ & 11 & 8 & 23 & 0.793 & 0.609 & 0.816 & $* * *$ \\
\hline SVA16 & $336-380$ & 7 & 3 & 10 & 0.714 & 0.400 & 0.740 & $*$ \\
\hline SVA17 & \multicolumn{8}{|c|}{ Monomorphic \& Homozygotes } \\
\hline SVA18 & $316-400$ & 14 & 5 & 21 & 0.807 & 0.667 & 0.828 & ns \\
\hline SVA19 & \multicolumn{5}{|c|}{ Monomorphic } & 1.000 & 0.500 & $* * *$ \\
\hline SVA20 & $359-440$ & 5 & 5 & 14 & 0.760 & 0.500 & 0.793 & $* * *$ \\
\hline
\end{tabular}

Ho, Observed heterozygosity; He, Expected heterozygosity; ns, not significant; ${ }^{*} \mathrm{p}<0.05 ;{ }^{* *} \mathrm{p}<0.01 ;{ }^{* * *} \mathrm{p}<$ 0.001. *Private alleles were calculated amongst Goniocorella dumosa, Desmophyllum dianthus, Madrepora oculata, Solenosmilia variabilis. Success - number of individuals for which successful PCR amplifications were obtained ( $\mathrm{n}=29$ individuals tested) 


\subsubsection{Goniocorella dumosa}

In G. dumosa, all 27 loci were successfully amplified, but SVA11, SVA17 and SVA19 were monomorphic. The $N A$ of 24 polymorphic microsatellite loci varied from 5 to 18, with an average of 7.64 (Table 4.8). PIC estimates showed all locus were highly informative. $H o$ and $H e$ were 0.630 and 0.748 , respectively. LD generated 78 (29.21\%) significant $\mathrm{P}$ values out of 267 pairwise comparisons $(\mathrm{p}<0.05)$, and none of them were totally unlinked from other markers. Only three markers showed no significant deviation from HWE (Table 4.8).

\subsubsection{Desmophyllum dianthus}

21 of 28 loci were polymorphic and produced 3-16 alleles per locus with an average of 7.64 in D. dianthus (Table 4.9). Loci D103, D213, SVA8, SVA10 and SVA20 were unsuccessful amplifications and locus B116 was monomorphic. Lower values of $\mathrm{Ho}$ and $\mathrm{He}$ were found in $D$. dianthus than $S$. variabilis, which were 0.460 and 0.681 , respectively. PIC levels? Following testing for LD, only $5(2.87 \%)$ significant $\mathrm{P}$ values were found in 174 pairwise comparisons $(\mathrm{p}<0.05)$, and only B1 \& SVA3, SVA5 \& SVA7, D5 \& SVA18, SVA4 \& SVA13 and C4 \& SVA16 formed linkage pairs. Eight loci exhibited significant deviations from HWE (Table 4.9).

\subsubsection{Madrepora oculata}

Less than half (13 of 27 loci) of locus were successfully amplified and scored with $N A$ varying from 3 to 9, with an average of 4.6 in M. oculata (Table 4.10). The average values of $H o$ and $H e$ were 0.709 and 0.609 , which were similar to S. variabilis. LD generated 3 (5.56\%) significant $\mathrm{P}$ values out of 54 pairwise comparisons $(\mathrm{p}<0.05)$, and all loci were totally unlinked from each other, except C107 \& D5, C107 \& SVA13 and D5 \& SVA13. Only 2 markers exhibited significant deviations from HWE $(\mathrm{p}<0.01)$ (Table 4.10). 
Table 4.9 Cross-amplification information of 27 loci in Desmophyllum dianthus

\begin{tabular}{|c|c|c|c|c|c|c|c|c|}
\hline Loci & Range & No & Private* & success & PIC & Ho & He & HW Equilibrium \\
\hline B106 & $247-274$ & 10 & 6 & 22 & 0.856 & 0.909 & 0.870 & ns \\
\hline $\mathrm{C} 107$ & $197-239$ & 9 & 9 & 22 & 0.787 & 0.556 & 0.809 & ns \\
\hline D5 & $219-263$ & 11 & 11 & 19 & 0.783 & 0.652 & 0.802 & ns \\
\hline B1 & $119-149$ & 10 & 9 & 14 & 0.778 & 0.600 & 0.801 & ns \\
\hline B116 & \multicolumn{8}{|c|}{ Monomorphic \& Homozygotes } \\
\hline D103 & \multicolumn{8}{|c|}{ No amplification } \\
\hline B123 & $169-177$ & 3 & 2 & 12 & 0.545 & 0.000 & 0.500 & $* *$ \\
\hline D213 & \multicolumn{8}{|c|}{ No amplifications } \\
\hline $\mathrm{C} 4$ & $185-227$ & 4 & 3 & 21 & 0.182 & 0.190 & 0.178 & ns \\
\hline SVA1 & $175-223$ & 3 & 3 & 4 & 0.714 & 0.000 & 0.625 & $*$ \\
\hline SVA2 & $268-292$ & 6 & 1 & 21 & 0.735 & 0.571 & 0.771 & $* *$ \\
\hline SVA3 & $385-421$ & 12 & 10 & 22 & 0.859 & 0.636 & 0.872 & ns \\
\hline SVA4 & $279-342$ & 12 & 9 & 15 & 0.852 & 0.667 & 0.864 & ns \\
\hline SVA5 & $320-341$ & 5 & 4 & 9 & 0.589 & 0.222 & 0.630 & $* *$ \\
\hline SVA7 & $365-380$ & 6 & 0 & 15 & 0.685 & 0.533 & 0.662 & ns \\
\hline SVA8 & \multicolumn{8}{|c|}{ No amplification } \\
\hline SVA10 & \multicolumn{8}{|c|}{ No amplification } \\
\hline SVA11 & $338-498$ & 9 & 9 & 23 & 0.371 & 0.304 & 0.380 & $*$ \\
\hline SVA12 & $361-392$ & 5 & 4 & 16 & 0.708 & 0.250 & 0.752 & $* * *$ \\
\hline SVA13 & $206-281$ & 16 & 6 & 23 & 0.83 & 0.696 & 0.840 & ns \\
\hline SVA14 & $157-298$ & 12 & 12 & 15 & 0.849 & 0.467 & 0.860 & $* *$ \\
\hline SVA15 & $199-245$ & 8 & 5 & 10 & 0.832 & 0.700 & 0.850 & ns \\
\hline SVA16 & $330-342$ & 6 & 3 & 23 & 0.706 & 0.348 & 0.749 & $* * *$ \\
\hline SVA17 & $353-387$ & 3 & 3 & 12 & 0.150 & 0.167 & 0.156 & ns \\
\hline SVA18 & $379-383$ & 3 & 3 & 20 & 0.371 & 0.300 & 0.454 & ns \\
\hline SVA19 & $314-389$ & 15 & 13 & 18 & 0.906 & 0.889 & 0.881 & ns \\
\hline SVA20 & \multicolumn{8}{|c|}{ No amplification } \\
\hline
\end{tabular}

Ho, Observed heterozygosity; He, Expected heterozygosity; ns, not significant; ${ }^{*} \mathrm{p}<0.05 ; * * \mathrm{p}<0.01 ; * * * \mathrm{p}<$ 0.001. *Private alleles were calculated amongst Goniocorella dumosa, Desmophyllum dianthus, Madrepora oculata, Solenosmilia variabilis. Success - number of individuals for which successful PCR amplifications were obtained ( $\mathrm{n}=23$ individuals tested) 
Table 4.10 Cross-amplification information of 27 loci in Madrepora oculata

\begin{tabular}{|c|c|c|c|c|c|c|c|c|}
\hline Loci & Range & No & Private* & success & PIC & Ho & $\mathrm{He}$ & HW Equilibrium \\
\hline B106 & $220-256$ & 9 & 7 & 16 & 0.683 & 1.000 & 0.723 & ns \\
\hline $\mathrm{C} 107$ & $198-243$ & 7 & 3 & 16 & 0.712 & 0.563 & 0.750 & ns \\
\hline D5 & $210-272$ & 6 & 6 & 12 & 0.643 & 0.833 & 0.681 & ns \\
\hline B1 & & & Monomorph & & & 1.000 & 0.500 & $* *$ \\
\hline B116 & \multicolumn{8}{|c|}{ No amplification } \\
\hline D103 & \multicolumn{8}{|c|}{ No amplification } \\
\hline B123 & $168-198$ & 5 & 3 & 5 & 0.701 & 0.400 & 0.740 & ns \\
\hline D213 & $105-144$ & 3 & 0 & 7 & 0.465 & 0.857 & 0.561 & ns \\
\hline $\mathrm{C} 4$ & \multicolumn{8}{|c|}{ No amplification } \\
\hline SVA1 & $225-237$ & 3 & 0 & 7 & 0.325 & 0.286 & 0.357 & ns \\
\hline SVA2 & $276-292$ & 3 & 0 & 5 & 0.410 & 0.200 & 0.460 & ns \\
\hline SVA3 & $344-416$ & 7 & 1 & 6 & 0.765 & 1.000 & 0.792 & ns \\
\hline SVA4 & $248-288$ & 3 & 2 & 2 & 0.555 & 0.500 & 0.625 & ns \\
\hline SVA5 & \multicolumn{8}{|c|}{ No amplification } \\
\hline SVA7 & \multicolumn{8}{|c|}{ No amplification } \\
\hline SVA8 & \multicolumn{8}{|c|}{ No amplification } \\
\hline SVA10 & $380-452$ & 5 & 1 & 6 & 0.644 & 1.000 & 0.694 & ns \\
\hline SVA11 & $420-448$ & 3 & 1 & 14 & 0.424 & 1.000 & 0.533 & $* *$ \\
\hline SVA12 & $421-437$ & 3 & 0 & 3 & 0.593 & 0.667 & 0.667 & ns \\
\hline SVA13 & $221-230$ & 3 & 0 & 16 & 0.371 & 0.625 & 0.447 & ns \\
\hline SVA14 & \multicolumn{8}{|c|}{ No amplification } \\
\hline SVA15 & \multicolumn{8}{|c|}{ No amplification } \\
\hline SVA16 & \multicolumn{8}{|c|}{ No amplification } \\
\hline SVA17 & \multicolumn{8}{|c|}{ No amplification } \\
\hline SVA18 & \multicolumn{8}{|c|}{ No amplification } \\
\hline SVA19 & \multicolumn{8}{|c|}{ No amplification } \\
\hline SVA20 & \multicolumn{8}{|c|}{ No amplification } \\
\hline
\end{tabular}

Ho, Observed heterozygosity; He, Expected heterozygosity; ns, not significant; * $\mathrm{p}<0.05 ; * * \mathrm{p}<0.01 ; * * * \mathrm{p}<$ 0.001. *Private alleles were calculated amongst Goniocorella dumosa, Desmophyllum dianthus, Madrepora oculata, Solenosmilia variabilis. Success - number of individuals for which successful PCR amplifications were obtained $(\mathrm{n}=16$ individuals tested $)$

\subsubsection{Differences in cross-species amplifications}

Not all of the 27 microsatellite loci worked in every species, and the amplification rate decreased from $G$. dumosa $(100 \%)$, to D. dianthus $(81.48 \%)$ and on to M. oculata $(51.85 \%)$, in accordance with the increasing genetic divergence amongst the species (Primmer \& Merila, 2002). The successfully-amplified ratio in this study also showed that the cross-amplification is closed related to taxonomical and morphological relations. The lowest was found in M. oculata, which is in the family Oculinidae. Although G. dumosa, D. dianthus and S. variabilis belong to the Caryophylliidae, G. dumosa and S. variabilis 
(branching and bushy colonies) are more morphologically similar than they are to D. dianthus (cup-shape and solitary colonies). This might explain the higher success rate of successful cross amplification in $G$. dumosa than $D$. dianthus. Only 12 loci were successfully amplified in all four species, and the allele ranges of each locus were very similar amongst species. Both Structure and DAPC analysis (Figure 4.2 and Figure 4.3) agreed that either all loci (27) or the loci (12) amplified in all species indicated that there were four groups $(K=4)$.

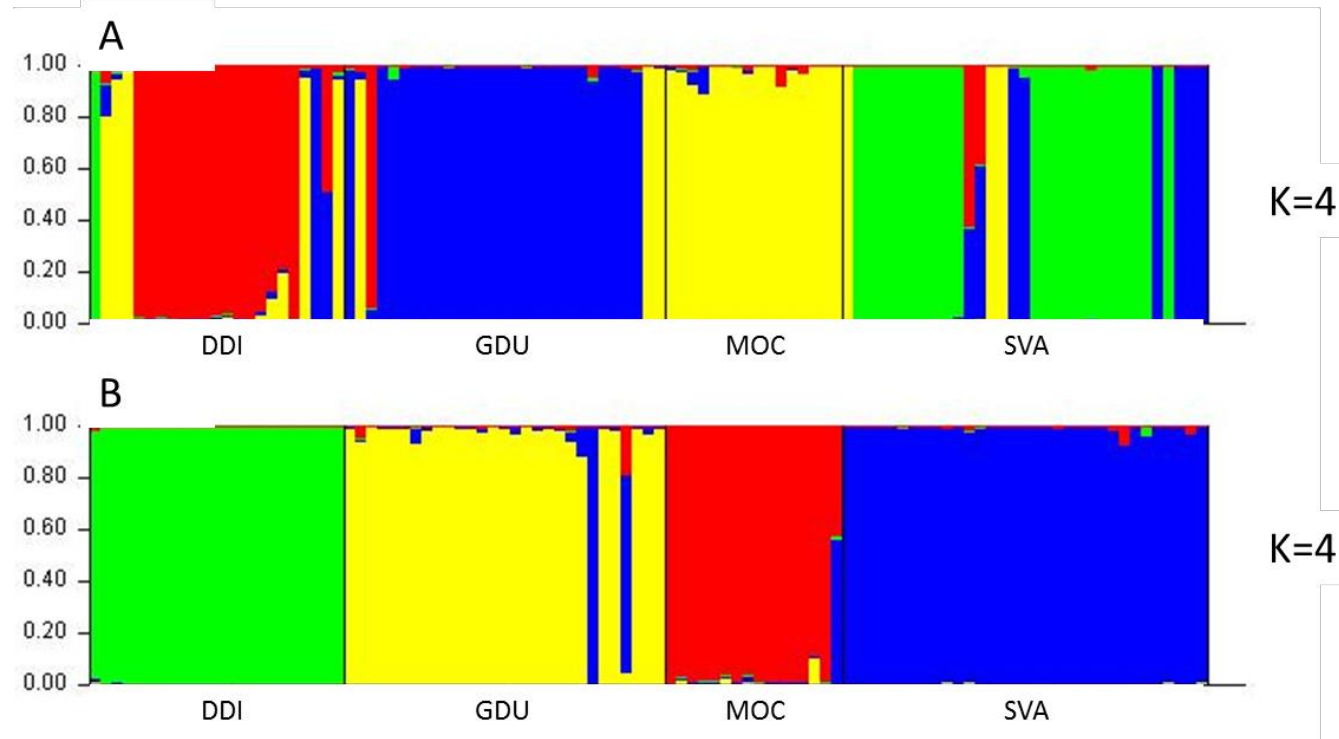

Figure 4.2 Structure analyses of all loci (A) and 12 loci that amplified for all four species (B)

GDU, Goniocorella dumosa; DDI, Desmophyllum dianthus; MOC, Madrepora oculata and SVA, Solenosmilia variabilis.

Here, 153 microsatellite loci from ten publications including this study, all of which have reported cross-amplification in Scleractinia, have been summarized in Table S4.1. From these microsatellites, over half of the loci could amplify in at least two different species of the same genus. The proportion of loci that cross-amplified decreased as taxonomic level increased. Trinucleotide repeats are the most common microsatellite motifs that could amplify in other species, and analysis of G/C (\%) content showed that most cross-amplified loci were composed of A/T rich repeats (Figure 4.4). A Wilcoxon signed-rank test showed that NA, range and He did not elicit a statistically significant change in cross amplification between the species that microsatellites developed from and the species that the microsatellites could cross amplify $(Z=-1.871, p=0.061 ; Z=-0.022, p=0.983 ; Z=-1.454$, $\mathrm{p}=0.146$, respectively), but fragment size range (low and upper boundary) and Ho were significantly different between the two species $(Z=-2.261, p=0.024 ; Z=-1.987, p=0.047$; $\mathrm{Z}=-4.811, \mathrm{p}<0.001)$. This result meant that the differences of the same loci in different 
species were distinguished between the species even sharing some alleles. In the present study, structure and DAPC were revealed a relatively clear separation amongst species, although a few misidentified in Structure analysis (Figure 4.2) and DAPC complot (Figure 4.3) were because the PCR amplification failures.
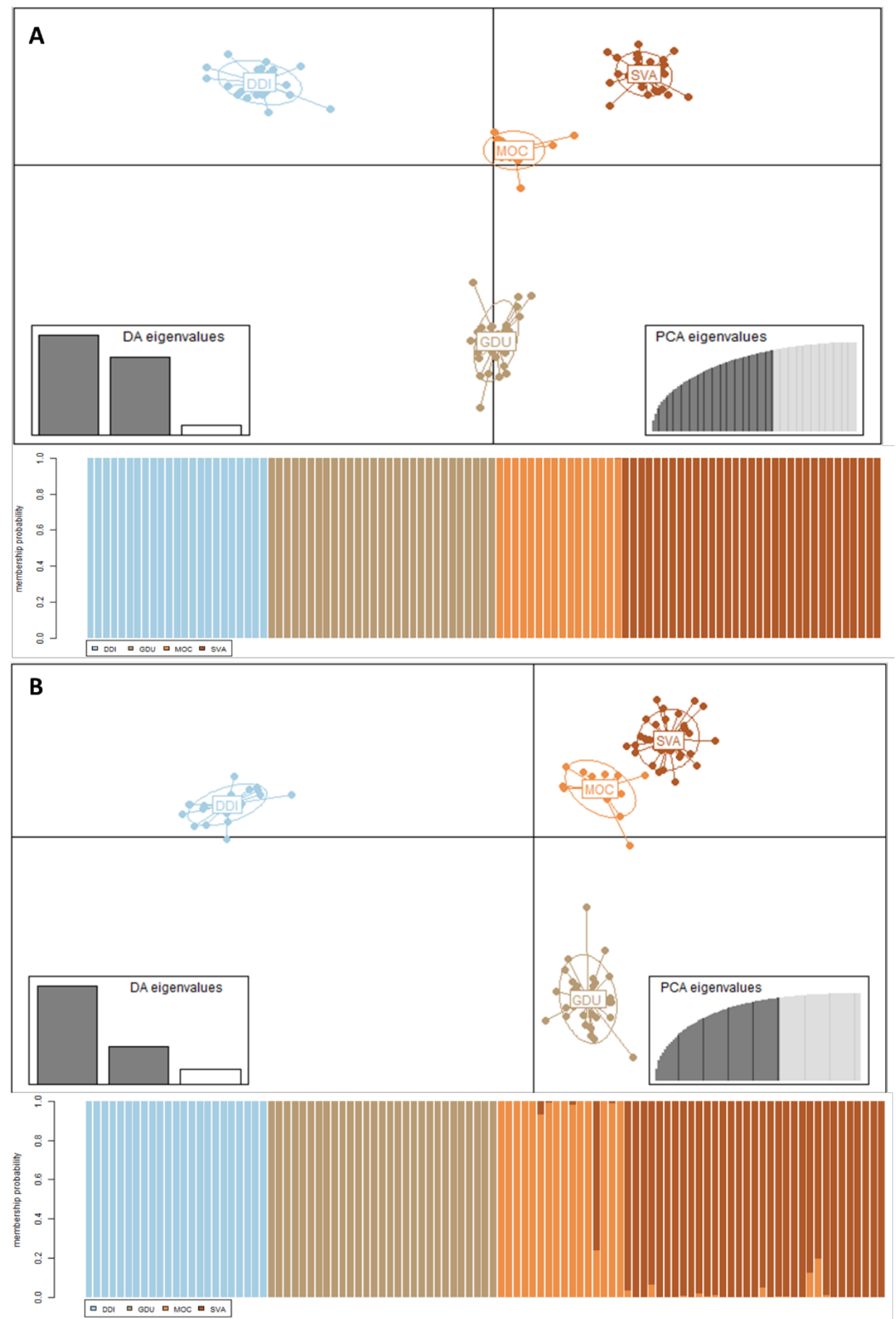

Figure 4.3 DAPC scatterplot (above) and complot (below) of all loci (A, 27 loci) and part (B, 12 loci) amongst four species

GDU, Goniocorella dumosa; DDI, Desmophyllum dianthus; MOC, Madrepora oculata and SVA, Solenosmilia variabilis. Both charts (A and B) were based on 60 principal components. 
A

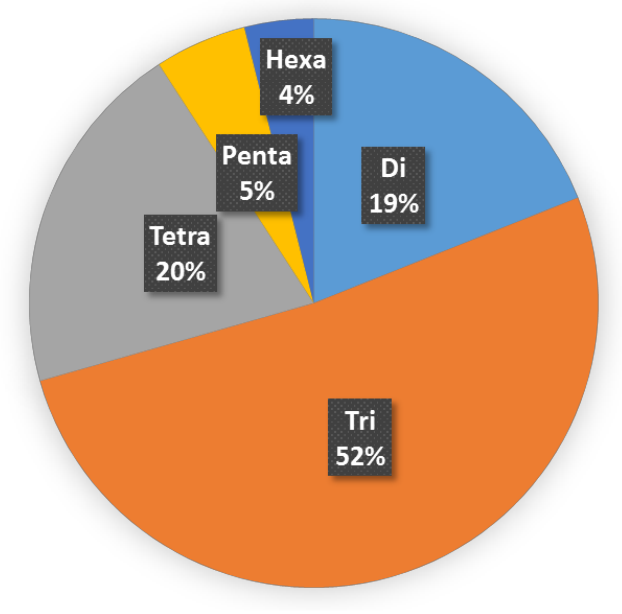

B

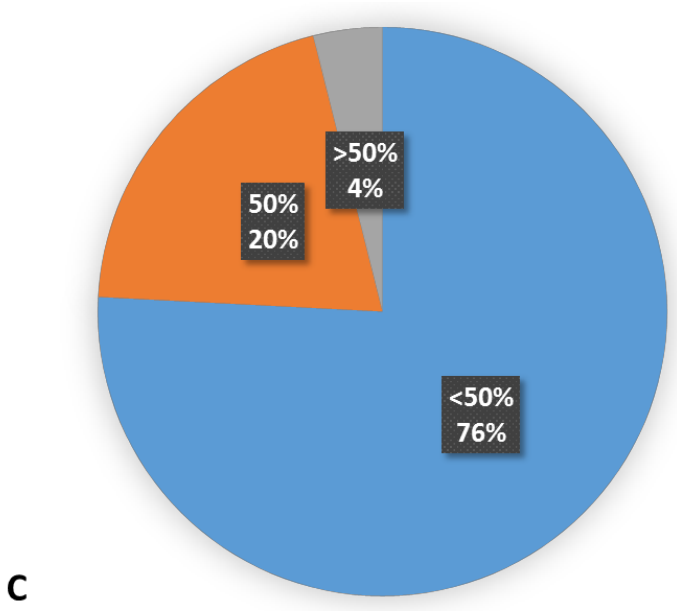

Figure 4.4 Distribution features of cross-amplified microsatellites of motifs (A), transferability level (B), G/C content (C)

Di, Tri, Tetra, Penta and Hexa were Dinucleotide, Trinucleotide, Tetranucleotide, Pentanucleotide and Hexanucleotide repeat motifs. Order, Family and Genus represent transferability in different order, family and genus.

\subsubsection{Single nucleotide polymorphisms (SNPs)}

After filtering the datasets, 102,678 reads were generated, and 62655, 8618, 63643 and 152023 SNPs were found, with average depth of 51.27, 43.21, 3.38 and 38.84, in samples L008, 94550, 94590 and 94287, respectively. A total of 846 insertions or deletions (indels) were discovered, and 715, 677, 221 and 711 were discovered in L008, 94550, 94590 and 94287, respectively (Table 4.11). 
Table 4.11 SNPs distributions amongst different samples

\begin{tabular}{|c|c|c|c|c|c|c|}
\hline \multirow[b]{2}{*}{ SNPs } & & \multirow{2}{*}{$\begin{array}{c}\text { Acropora digitifera } \\
\mathrm{D}=1\end{array}$} & \multicolumn{4}{|c|}{ Solenosmilia variabilis } \\
\hline & & & $\begin{array}{c}\text { L008 } \\
D=51.27 \pm 66.08\end{array}$ & $\begin{array}{c}94550 \\
D=43.21 \pm 59.50\end{array}$ & $\begin{array}{c}94590 \\
D=3.38 \pm 4.91\end{array}$ & $\begin{array}{c}94287 \\
D=38.84 \pm 54.62\end{array}$ \\
\hline \multirow{4}{*}{ Homogeneity } & A & 41419 & 24342 & 14592 & 2087 & 14954 \\
\hline & $\mathrm{C}$ & 34770 & 23421 & 14695 & 2132 & 14962 \\
\hline & G & 34129 & 23423 & 14646 & 2015 & 14803 \\
\hline & $\mathrm{T}$ & 41705 & 24491 & 14849 & 1984 & 15079 \\
\hline \multirow{2}{*}{ Transition* } & A-G & & 2127 & 1168 & 110 & 1217 \\
\hline & $\mathrm{C}-\mathrm{T}$ & & 2190 & 1215 & 132 & 1180 \\
\hline \multirow{4}{*}{ Transversion* } & A-C & & 650 & 369 & 52 & 364 \\
\hline & G-C & & 459 & 282 & 23 & 254 \\
\hline & G-T & & 723 & 348 & 29 & 364 \\
\hline & $\mathrm{T}-\mathrm{A}$ & & 852 & 491 & 54 & 466 \\
\hline Indel & & 846 & 715 & 677 & 221 & 711 \\
\hline Total & & 152869 & 103393 & 63332 & 8839 & 64354 \\
\hline
\end{tabular}

D means sequences depth (Mean \pm SD). * Transition and Transversion in L008 are including within heterozygosity a genome and within a population, Transition and Transversion in 94550,94590 and 94287 are heterozygosity within the genome.

A Venn diagram showed the relation and distribution of the SNPs that excluded indels amongst the four $S$. variabilis samples. In total, 64,352 (62.67\%), 20,888 (33.34\%), 1,708 (19.82\%) and 20,554 (32.30\%) unique loci were found in samples L008, 94550, 94590 and 94287, respectively (Figure 4.5). Only 1,983 SNPs were identical amongst S. variabilis samples but different from $A$. digitifera, which were small proportion in L008 (1.93\%), $94550(3.17 \%)$ and 94287 (3.12\%). Hierarchical cluster analysis provided a further illustration of the relationship of SNPs amongst samples (Figure 4.6). Samples 94287 and 94550 firstly joined together, and then grouped with L008. The limited coverage of sample 94590 might explain why it grouped with $A$. digitifera rather than with the other three $S$. variabilis samples. However, STRUCTURE analysis of SNPs also failed to tell the difference between species which was same as microsatellites (Figure 4.7). This might be related to the methods used to develop these SNPs, because these SNPs were from the cross-genus shared sequences, where might be very conserved. The common SNPs between or amongst samples might cause this blurring of species boundaries by the SNPs markers, and a further examination of those loci was expected to solve this problem. 


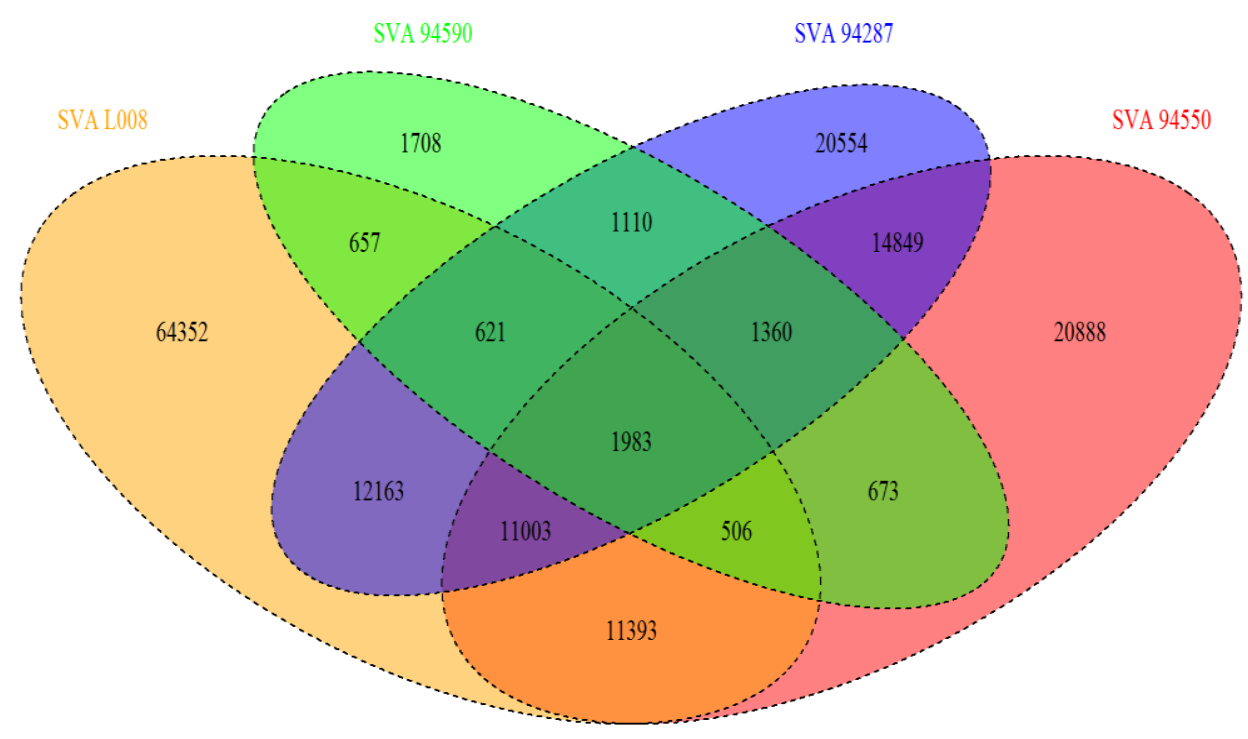

Figure 4.5 Venn diagram of SNPs in four Solenosmilia variabilis samples

Distance interval was Squared Euclidean distance. SVA, Solenosmilia variabilis (94287, 94550 and 94590 were 454 sequencing, and L008 was Illumina sequencing), and ADI, Acropora digitifera.

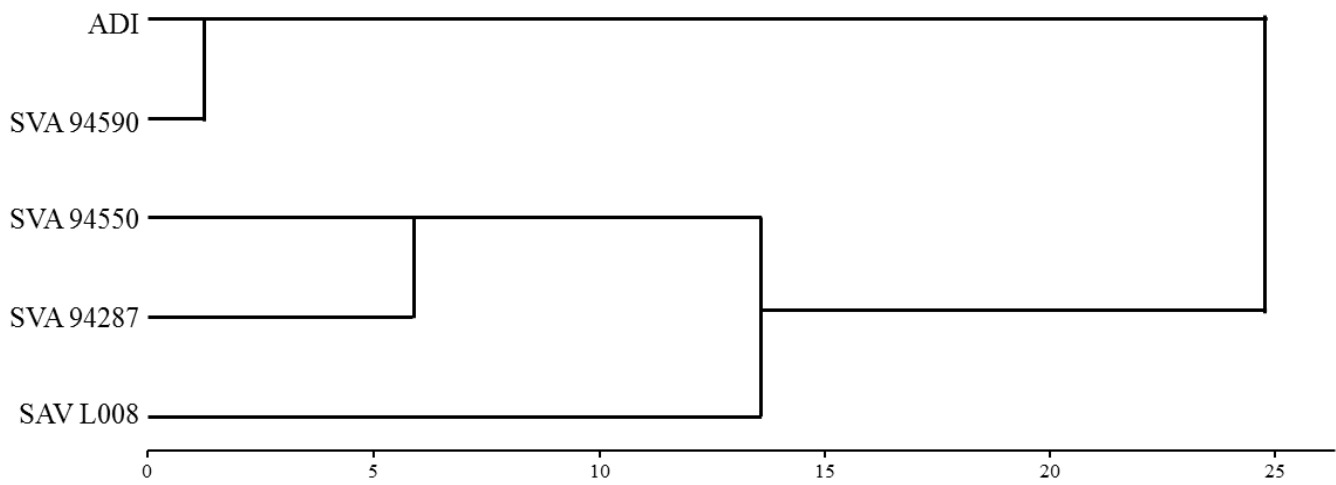

Figure 4.6 Dendrogram of 5 samples revealed by SNPs that were detected in all datasets

Distance interval was Squared Euclidean distance. SVA, Solenosmilia variabilis (94287, 94550 and 94590 were 454 sequencing, and L008 was Illumina sequencing), and ADI, Acropora digitifera. 


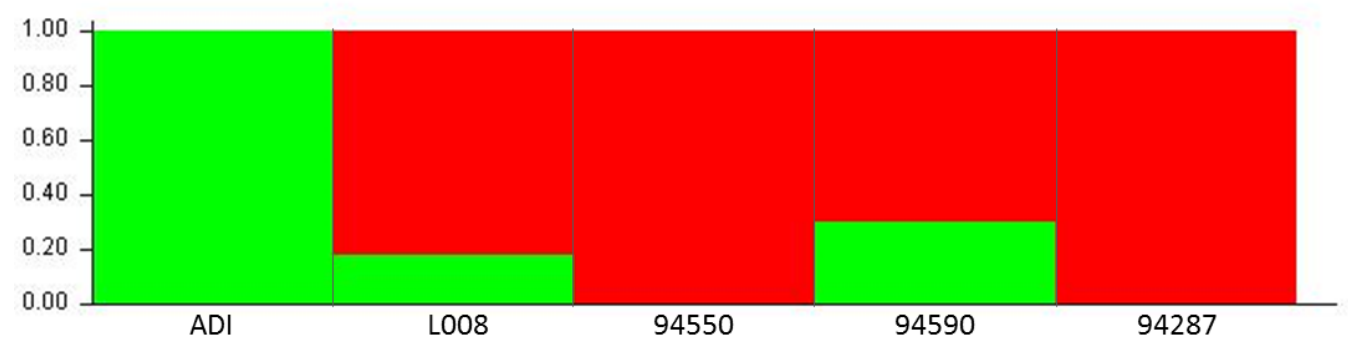

Figure 4.7 Structures results of sample coral samples based on SNPs $(K=2)$

SVA, Solenosmilia variabilis (94287, 94550 and 94590 were 454 sequencing, and L008 was Illumina sequencing), and ADI, Acropora digitifera.

\subsection{Conclusion}

Three marker types, mitochondrial genes from full mitogenome sequence, microsatellites and SNPs, were generated from next generation sequencing data for $S$. variabilis. Mitochondrial genes have slow evolutionary rates and are relatively conserved, making them not particularly suitable for population genetic studies in stony corals. An exception here is the non-coding mtDNA D-loop region. Microsatellites are informative markers that may be useful in further studies, and their cross-amplification showed high and informative levels of polymorphism in other stony coral species. These markers are good candidates for the genetic studies, and performance and details information, such as deviation from HBW and neutrality of the markers, were discussed in Chapter 6 when applying their to test different populations. SNPs are supposed to be good markers for population studies. A further selection of SNPs needs to be examined, and then potential loci could be applied for genetic studies of single taxa. 


\section{Appendix}

Table S4.1 Information on the cross-amplification of microsatellite loci in Scleractinia

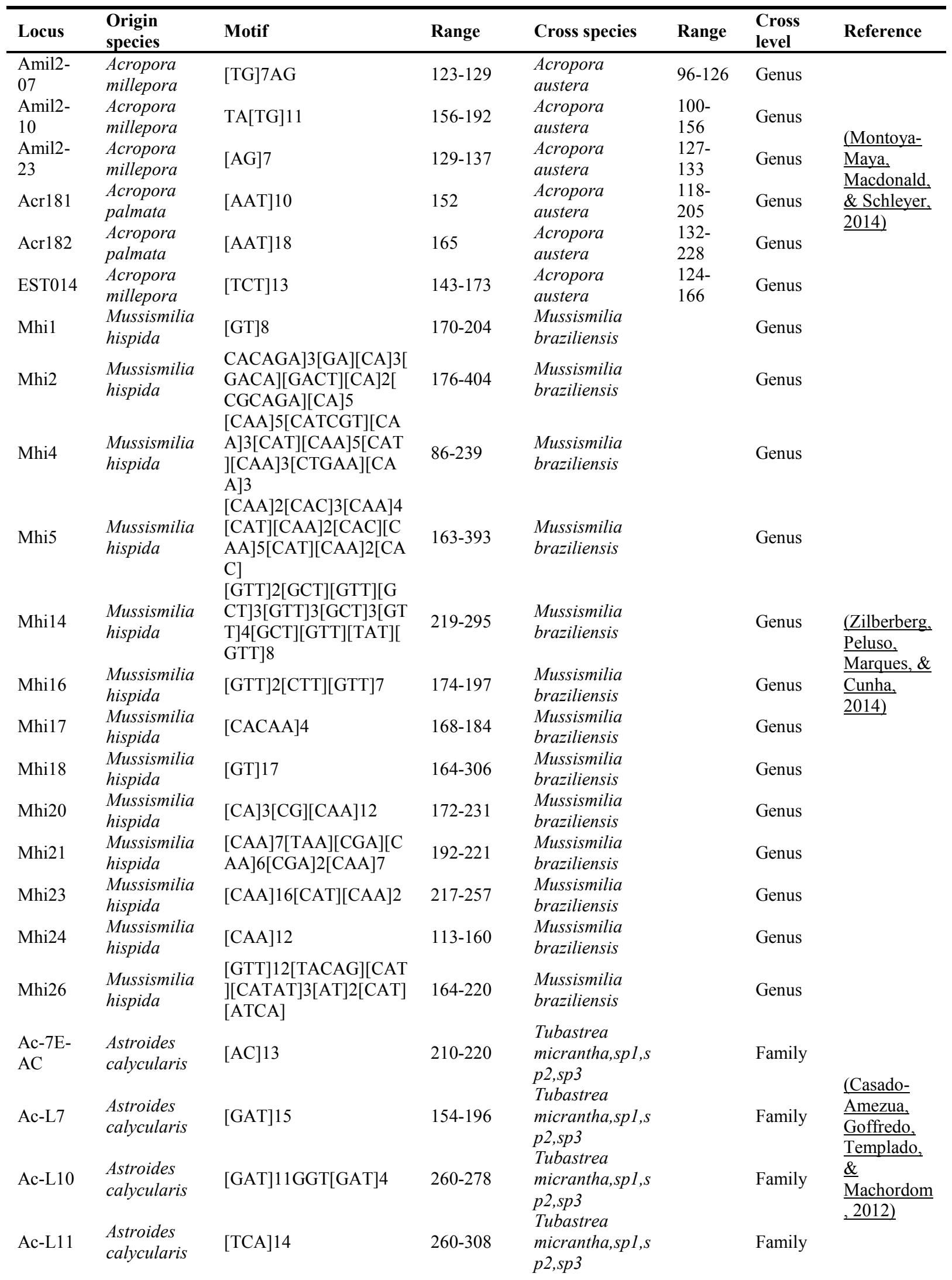




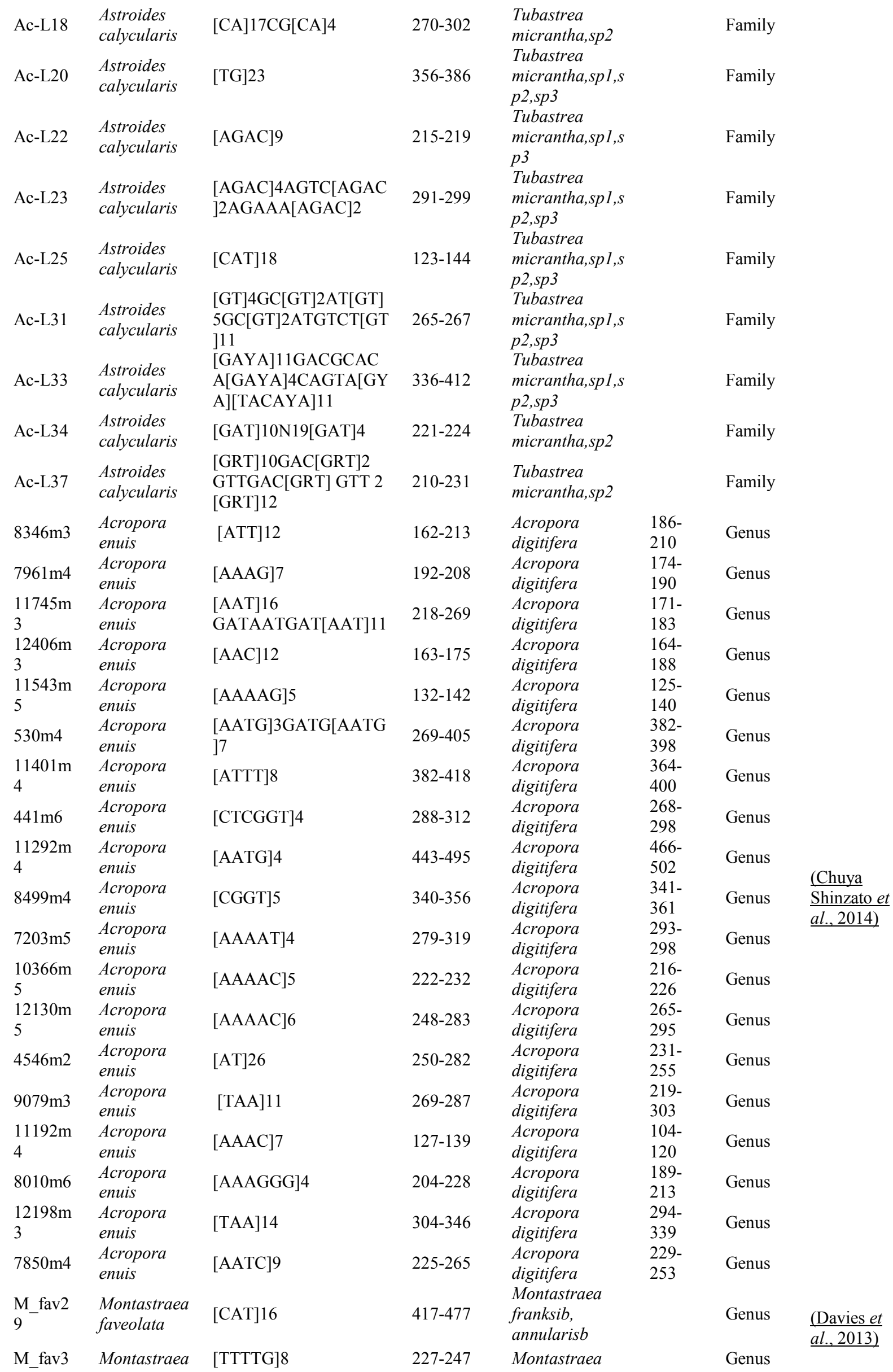




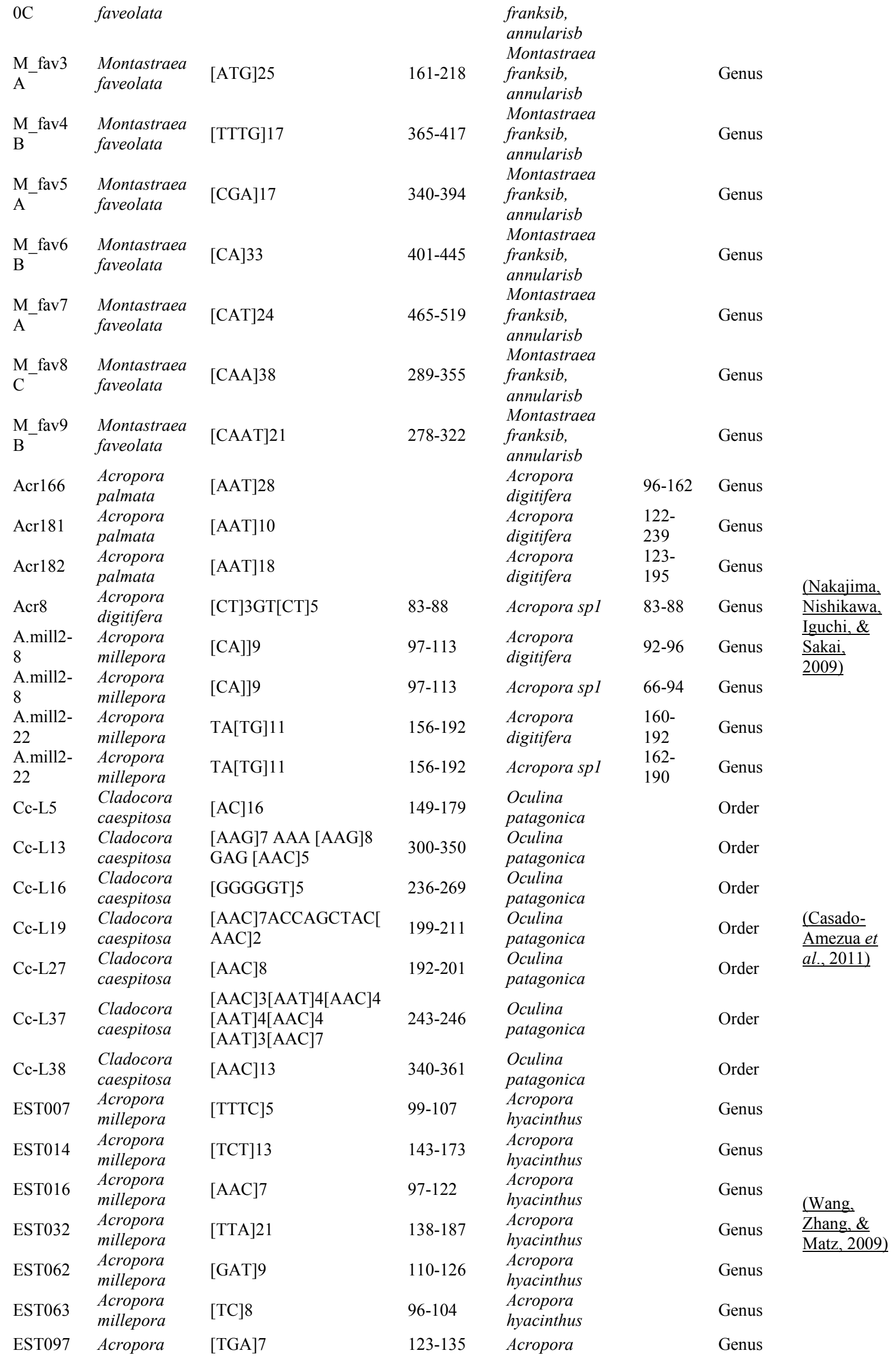




\begin{tabular}{|c|c|c|c|c|c|c|c|}
\hline & millepora & & & hyacinthus & & & \\
\hline EST098 & $\begin{array}{l}\text { Acropora } \\
\text { millepora }\end{array}$ & [TG]12 & $98-118$ & $\begin{array}{l}\text { Acropora } \\
\text { hyacinthus }\end{array}$ & & Genus & \\
\hline EST121 & $\begin{array}{l}\text { Acropora } \\
\text { millepora }\end{array}$ & [ATGCCG]4 & $100-112$ & $\begin{array}{l}\text { Acropora } \\
\text { hyacinthus }\end{array}$ & & Genus & \\
\hline EST122 & $\begin{array}{l}\text { Acropora } \\
\text { millepora }\end{array}$ & [TTA] 18 & $99-141$ & $\begin{array}{l}\text { Acropora } \\
\text { hyacinthus }\end{array}$ & & Genus & \\
\hline EST149 & $\begin{array}{l}\text { Acropora } \\
\text { millepora }\end{array}$ & [GAT]9 & $118-130$ & $\begin{array}{l}\text { Acropora } \\
\text { hyacinthus }\end{array}$ & & Genus & \\
\hline EST181 & $\begin{array}{l}\text { Acropora } \\
\text { millepora }\end{array}$ & {$[\mathrm{ATG}] 10$} & $145-157$ & $\begin{array}{l}\text { Acropora } \\
\text { hyacinthus }\end{array}$ & & Genus & \\
\hline EST196 & $\begin{array}{l}\text { Acropora } \\
\text { millepora }\end{array}$ & [TAA]9 & $117-145$ & $\begin{array}{l}\text { Acropora } \\
\text { hyacinthus }\end{array}$ & & Genus & \\
\hline EST245 & $\begin{array}{l}\text { Acropora } \\
\text { millepora }\end{array}$ & {$[\mathrm{CA}] 10$} & $115-124$ & $\begin{array}{l}\text { Acropora } \\
\text { hyacinthus }\end{array}$ & & Genus & \\
\hline EST254 & $\begin{array}{l}\text { Acropora } \\
\text { millepora }\end{array}$ & {$[\mathrm{CA}] 12$} & $86-100$ & $\begin{array}{l}\text { Acropora } \\
\text { hyacinthus }\end{array}$ & & Genus & \\
\hline $\begin{array}{l}\text { WGS05 } \\
1\end{array}$ & $\begin{array}{l}\text { Acropora } \\
\text { millepora }\end{array}$ & {$[$ GATA $] 8$} & $151-216$ & $\begin{array}{l}\text { Acropora } \\
\text { hyacinthus }\end{array}$ & & Genus & \\
\hline $\begin{array}{l}\text { WGS09 } \\
2\end{array}$ & $\begin{array}{l}\text { Acropora } \\
\text { millepora }\end{array}$ & {$[\mathrm{ATT}] 12$} & $111-200$ & $\begin{array}{l}\text { Acropora } \\
\text { hyacinthus }\end{array}$ & & Genus & \\
\hline $\begin{array}{l}\text { WGS10 } \\
1\end{array}$ & $\begin{array}{l}\text { Acropora } \\
\text { millepora }\end{array}$ & {$[\mathrm{TTA}] 14[\mathrm{GTA}] 10$} & $134-190$ & $\begin{array}{l}\text { Acropora } \\
\text { hyacinthus }\end{array}$ & & Genus & \\
\hline $\begin{array}{l}\text { WGS11 } \\
2\end{array}$ & $\begin{array}{l}\text { Acropora } \\
\text { millepora }\end{array}$ & [AAT]9 & $166-184$ & $\begin{array}{l}\text { Acropora } \\
\text { hyacinthus }\end{array}$ & & Genus & \\
\hline $\begin{array}{l}\text { WGS13 } \\
4\end{array}$ & $\begin{array}{l}\text { Acropora } \\
\text { millepora }\end{array}$ & [GATA]6 & $105-133$ & $\begin{array}{l}\text { Acropora } \\
\text { hyacinthus }\end{array}$ & & Genus & \\
\hline $\begin{array}{l}\text { WGS15 } \\
2\end{array}$ & $\begin{array}{l}\text { Acropora } \\
\text { millepora }\end{array}$ & {$[\mathrm{AT}] 9$} & $87-110$ & $\begin{array}{l}\text { Acropora } \\
\text { hyacinthus }\end{array}$ & & Genus & \\
\hline $\begin{array}{l}\text { WGS15 } \\
3\end{array}$ & $\begin{array}{l}\text { Acropora } \\
\text { millepora }\end{array}$ & [AATC]7 & $106-126$ & $\begin{array}{l}\text { Acropora } \\
\text { hyacinthus }\end{array}$ & & Genus & \\
\hline $\begin{array}{l}\text { WGS18 } \\
9\end{array}$ & $\begin{array}{l}\text { Acropora } \\
\text { millepora }\end{array}$ & [ATCT]7 & $158-194$ & $\begin{array}{l}\text { Acropora } \\
\text { hyacinthus }\end{array}$ & & Genus & \\
\hline $\begin{array}{l}\text { WGS19 } \\
6\end{array}$ & $\begin{array}{l}\text { Acropora } \\
\text { millepora }\end{array}$ & [ATAC]6 & $128-234$ & $\begin{array}{l}\text { Acropora } \\
\text { hyacinthus }\end{array}$ & & Genus & \\
\hline $\begin{array}{l}\text { WGS21 } \\
1\end{array}$ & $\begin{array}{l}\text { Acropora } \\
\text { millepora }\end{array}$ & [TAA] 8 & 181-199 & $\begin{array}{l}\text { Acropora } \\
\text { hyacinthus }\end{array}$ & & Genus & \\
\hline Acr181 & $\begin{array}{l}\text { Acropora } \\
\text { palmata }\end{array}$ & {$[\mathrm{AAT}] 10$} & & $\begin{array}{l}\text { Acropora } \\
\text { muricata }\end{array}$ & $\begin{array}{l}125- \\
197\end{array}$ & Genus & \\
\hline Acr182 & $\begin{array}{l}\text { Acropora } \\
\text { palmata }\end{array}$ & {$[\mathrm{AAT}] 18$} & & $\begin{array}{l}\text { Acropora } \\
\text { muricata }\end{array}$ & $\begin{array}{l}129- \\
183\end{array}$ & Genus & \\
\hline Acr 180 & $\begin{array}{l}\text { Acropora } \\
\text { palmata }\end{array}$ & {$[\mathrm{AAT}] 19$} & & $\begin{array}{l}\text { Acropora } \\
\text { muricata }\end{array}$ & $92-158$ & Genus & \\
\hline Acr207 & $\begin{array}{l}\text { Acropora } \\
\text { palmata }\end{array}$ & {$[\mathrm{AAT}] 20$} & & $\begin{array}{l}\text { Acropora } \\
\text { muricata }\end{array}$ & $\begin{array}{l}101- \\
188\end{array}$ & Genus & \\
\hline Acr166 & $\begin{array}{l}\text { Acropora } \\
\text { palmata }\end{array}$ & {$[\mathrm{AAT}] 28$} & & $\begin{array}{l}\text { Acropora } \\
\text { muricata }\end{array}$ & $87-162$ & Genus & \\
\hline Acr45 & $\begin{array}{l}\text { Acropora } \\
\text { muricata }\end{array}$ & {$[\mathrm{CT}] 17$} & & $\begin{array}{l}\text { Acropora } \\
\text { digitifera }\end{array}$ & & Genus & \\
\hline Acr $1-60$ & $\begin{array}{l}\text { Acropora } \\
\text { muricata }\end{array}$ & {$[\mathrm{GT}] 10$} & $182-213$ & $\begin{array}{l}\text { Acropora } \\
\text { digitifera }\end{array}$ & $\begin{array}{l}171- \\
233\end{array}$ & Genus & (Tang, Wei, \\
\hline Acr53 & $\begin{array}{l}\text { Acropora } \\
\text { muricata }\end{array}$ & {$[\mathrm{GT}] 10$} & $159-171$ & $\begin{array}{l}\text { Acropora } \\
\text { digitifera }\end{array}$ & $\begin{array}{l}161- \\
193\end{array}$ & Genus & $\begin{array}{l}\text { Chen, } \\
\text { Wallace, \& }\end{array}$ \\
\hline Acr3-27 & $\begin{array}{l}\text { Acropora } \\
\text { muricata }\end{array}$ & {$[\mathrm{GT}] 13$} & & $\begin{array}{l}\text { Acropora } \\
\text { digitifera }\end{array}$ & & Genus & Chen, 2010) \\
\hline Acr1-4 & $\begin{array}{l}\text { Acropora } \\
\text { muricata }\end{array}$ & [GT]20 & $137-201$ & $\begin{array}{l}\text { Acropora } \\
\text { digitifera }\end{array}$ & $\begin{array}{l}129- \\
243\end{array}$ & Genus & \\
\hline Acr181 & $\begin{array}{l}\text { Acropora } \\
\text { palmata }\end{array}$ & {$[\mathrm{AAT}] 10$} & & $\begin{array}{l}\text { Acropora } \\
\text { humilis }\end{array}$ & & Genus & \\
\hline Acr 182 & $\begin{array}{l}\text { Acropora } \\
\text { palmata }\end{array}$ & {$[\mathrm{AAT}] 18$} & & $\begin{array}{l}\text { Acropora } \\
\text { humilis }\end{array}$ & & Genus & \\
\hline Acr 180 & $\begin{array}{l}\text { Acropora } \\
\text { palmata }\end{array}$ & {$[\mathrm{AAT}] 19$} & & $\begin{array}{l}\text { Acropora } \\
\text { humilis }\end{array}$ & & Genus & \\
\hline Acr207 & $\begin{array}{l}\text { Acropora } \\
\text { palmata }\end{array}$ & {$[\mathrm{AAT}] 20$} & & $\begin{array}{l}\text { Acropora } \\
\text { humilis }\end{array}$ & & Genus & \\
\hline Acr166 & $\begin{array}{l}\text { Acropora } \\
\text { palmata }\end{array}$ & {$[\mathrm{AAT}] 28$} & & $\begin{array}{l}\text { Acropora } \\
\text { humilis }\end{array}$ & & Genus & \\
\hline
\end{tabular}




\begin{tabular}{|c|c|c|c|c|c|c|c|}
\hline Acr $1-60$ & $\begin{array}{l}\text { Acropora } \\
\text { muricata }\end{array}$ & {$[\mathrm{GT}] 10$} & $182-213$ & $\begin{array}{l}\text { Acropora } \\
\text { humilis }\end{array}$ & & Genus & \\
\hline Acr53 & $\begin{array}{l}\text { Acropora } \\
\text { muricata }\end{array}$ & {$[\mathrm{GT}] 10$} & 159-171 & $\begin{array}{l}\text { Acropora } \\
\text { humilis }\end{array}$ & & Genus & \\
\hline Acr1-4 & $\begin{array}{l}\text { Acropora } \\
\text { muricata }\end{array}$ & {$[\mathrm{GT}] 20$} & $137-201$ & $\begin{array}{l}\text { Acropora } \\
\text { humilis }\end{array}$ & & Genus & \\
\hline Acr181 & $\begin{array}{l}\text { Acropora } \\
\text { palmata }\end{array}$ & [AAT]10 & & $\begin{array}{l}\text { Acropora } \\
\text { hyacinthus }\end{array}$ & & Genus & \\
\hline Acr182 & $\begin{array}{l}\text { Acropora } \\
\text { palmata }\end{array}$ & [AAT]18 & & $\begin{array}{l}\text { Acropora } \\
\text { hyacinthus }\end{array}$ & & Genus & \\
\hline Acr 180 & $\begin{array}{l}\text { Acropora } \\
\text { palmata }\end{array}$ & [AAT]19 & & $\begin{array}{l}\text { Acropora } \\
\text { hyacinthus }\end{array}$ & & Genus & \\
\hline Acr207 & $\begin{array}{l}\text { Acropora } \\
\text { palmata }\end{array}$ & [AAT] 20 & & $\begin{array}{l}\text { Acropora } \\
\text { hyacinthus }\end{array}$ & & Genus & \\
\hline Acr166 & $\begin{array}{l}\text { Acropora } \\
\text { palmata }\end{array}$ & [AAT]28 & & $\begin{array}{l}\text { Acropora } \\
\text { hyacinthus }\end{array}$ & & Genus & \\
\hline Acr45 & $\begin{array}{l}\text { Acropora } \\
\text { muricata }\end{array}$ & {$[\mathrm{CT}] 17$} & & $\begin{array}{l}\text { Acropora } \\
\text { hyacinthus }\end{array}$ & & Genus & \\
\hline Acr1-60 & $\begin{array}{l}\text { Acropora } \\
\text { muricata }\end{array}$ & {$[\mathrm{GT}] 10$} & $182-213$ & $\begin{array}{l}\text { Acropora } \\
\text { hyacinthus }\end{array}$ & & Genus & \\
\hline Acr53 & $\begin{array}{l}\text { Acropora } \\
\text { muricata }\end{array}$ & {$[\mathrm{GT}] 10$} & 159-171 & $\begin{array}{l}\text { Acropora } \\
\text { hyacinthus }\end{array}$ & & Genus & \\
\hline Acr3-27 & $\begin{array}{l}\text { Acropora } \\
\text { muricata }\end{array}$ & {$[\mathrm{GT}] 13$} & & $\begin{array}{l}\text { Acropora } \\
\text { hyacinthus }\end{array}$ & & Genus & \\
\hline Acr1-4 & $\begin{array}{l}\text { Acropora } \\
\text { muricata }\end{array}$ & {$[\mathrm{GT}] 20$} & $137-201$ & $\begin{array}{l}\text { Acropora } \\
\text { hyacinthus }\end{array}$ & & Genus & \\
\hline Acr181 & $\begin{array}{l}\text { Acropora } \\
\text { palmata }\end{array}$ & [AAT] 10 & & Acropora valida & & Genus & \\
\hline Acr182 & $\begin{array}{l}\text { Acropora } \\
\text { palmata }\end{array}$ & [AAT]18 & & Acropora valida & & Genus & \\
\hline Acr180 & $\begin{array}{l}\text { Acropora } \\
\text { palmata }\end{array}$ & [AAT]19 & & Acropora valida & & Genus & \\
\hline Acr207 & $\begin{array}{l}\text { Acropora } \\
\text { palmata }\end{array}$ & [AAT] 20 & & Acropora valida & & Genus & \\
\hline Acr166 & $\begin{array}{l}\text { Acropora } \\
\text { palmata }\end{array}$ & [AAT] 28 & & Acropora valida & & Genus & \\
\hline Acr1-60 & $\begin{array}{l}\text { Acropora } \\
\text { muricata }\end{array}$ & {$[\mathrm{GT}] 10$} & $182-213$ & Acropora valida & & Genus & \\
\hline Acr53 & $\begin{array}{l}\text { Acropora } \\
\text { muricata }\end{array}$ & {$[\mathrm{GT}] 10$} & $159-171$ & Acropora valida & & Genus & \\
\hline Acr1-4 & $\begin{array}{l}\text { Acropora } \\
\text { muricata }\end{array}$ & {$[\mathrm{GT}] 20$} & $137-201$ & Acropora valida & & Genus & \\
\hline Acr181 & $\begin{array}{l}\text { Acropora } \\
\text { palmata }\end{array}$ & [AAT] 10 & & $\begin{array}{l}\text { Acropora } \\
\text { palmata }\end{array}$ & & Genus & \\
\hline Acr181 & $\begin{array}{l}\text { Acropora } \\
\text { palmata }\end{array}$ & [AAT]10 & & Acropora sp1 & $\begin{array}{l}146- \\
234\end{array}$ & Genus & \\
\hline Acr182 & $\begin{array}{l}\text { Acropora } \\
\text { palmata }\end{array}$ & {$[\mathrm{AAT}] 18$} & & $\begin{array}{l}\text { Acropora } \\
\text { palmata }\end{array}$ & & Genus & \\
\hline Acr182 & $\begin{array}{l}\text { Acropora } \\
\text { palmata }\end{array}$ & [AAT]18 & & Acropora sp1 & $\begin{array}{l}137- \\
208\end{array}$ & Genus & \\
\hline Acr 180 & $\begin{array}{l}\text { Acropora } \\
\text { palmata }\end{array}$ & [AAT]19 & & $\begin{array}{l}\text { Acropora } \\
\text { palmata }\end{array}$ & & Genus & \\
\hline Acr180 & $\begin{array}{l}\text { Acropora } \\
\text { palmata }\end{array}$ & [AAT]19 & & $\begin{array}{l}\text { Acropora } \\
\text { digitifera }\end{array}$ & $86-167$ & Genus & \\
\hline Acr207 & $\begin{array}{l}\text { Acropora } \\
\text { palmata }\end{array}$ & [AAT] 20 & & $\begin{array}{l}\text { Acropora } \\
\text { palmata }\end{array}$ & & Genus & \\
\hline Acr207 & $\begin{array}{l}\text { Acropora } \\
\text { palmata }\end{array}$ & {$[\mathrm{AAT}] 20$} & & $\begin{array}{l}\text { Acropora } \\
\text { digitifera }\end{array}$ & $\begin{array}{l}110- \\
188\end{array}$ & Genus & \\
\hline Acr166 & $\begin{array}{l}\text { Acropora } \\
\text { palmata }\end{array}$ & [AAT] 28 & & $\begin{array}{l}\text { Acropora } \\
\text { palmata }\end{array}$ & & Genus & \\
\hline Acr166 & $\begin{array}{l}\text { Acropora } \\
\text { palmata }\end{array}$ & {$[\mathrm{AAT}] 28$} & & Acropora spl & $\begin{array}{l}124- \\
157\end{array}$ & Genus & \\
\hline DdL100 & $\begin{array}{l}\text { Desmophyll } \\
\text { um dianthus }\end{array}$ & [TCA]9 & $128-140$ & Scleractinia & & Order & (Addamo et \\
\hline DdL102 & $\begin{array}{l}\text { Desmophyll } \\
\text { um dianthus }\end{array}$ & [TGT]6 & $123-152$ & Scleractinia & & Order & al., 2015) \\
\hline
\end{tabular}




\begin{tabular}{|c|c|c|c|c|c|}
\hline $\begin{array}{l}\text { DdL107 } \\
*\end{array}$ & $\begin{array}{l}\text { Desmophyll } \\
\text { um dianthus }\end{array}$ & [TTG]7 & $143-179$ & Scleractinia & Order \\
\hline DdL109 & $\begin{array}{l}\text { Desmophyll } \\
\text { um dianthus }\end{array}$ & [TGT]6 & $128-140$ & Scleractinia & Order \\
\hline DdL13 & $\begin{array}{l}\text { Desmophyll } \\
\text { um dianthus }\end{array}$ & {$[\mathrm{GTT}] 15$} & $86-122$ & Scleractinia & Order \\
\hline DdL16 & $\begin{array}{l}\text { Desmophyll } \\
\text { um dianthus }\end{array}$ & {$[\mathrm{CAA}] 11$} & $104-161$ & Scleractinia & Order \\
\hline DdL22* & $\begin{array}{l}\text { Desmophyll } \\
\text { um dianthus }\end{array}$ & {$[\mathrm{ACA}] 13$} & $119-168$ & Scleractinia & Order \\
\hline DdL24 & $\begin{array}{l}\text { Desmophyll } \\
\text { um dianthus }\end{array}$ & [TGTT]10 & $162-212$ & Scleractinia & Order \\
\hline DdL34 & $\begin{array}{l}\text { Desmophyll } \\
\text { um dianthus }\end{array}$ & [GAT]14 & $176-202$ & Scleractinia & Order \\
\hline DdL41 & $\begin{array}{l}\text { Desmophyll } \\
\text { um dianthus }\end{array}$ & [GAT]11 & $150-183$ & Scleractinia & Order \\
\hline DdL50 & $\begin{array}{l}\text { Desmophyll } \\
\text { um dianthus }\end{array}$ & [TTG]11[CTGTTG]3 & $194-281$ & Scleractinia & Order \\
\hline DdL56 & $\begin{array}{l}\text { Desmophyll } \\
\text { um dianthus }\end{array}$ & [GTTT] 8 & $116-148$ & Scleractinia & Order \\
\hline DdL57 & $\begin{array}{l}\text { Desmophyll } \\
\text { um dianthus }\end{array}$ & [TTAC]5 & $117-137$ & Scleractinia & Order \\
\hline DdL58* & $\begin{array}{l}\text { Desmophyll } \\
\text { um dianthus }\end{array}$ & [TCTT]6 & $105-153$ & Scleractinia & Order \\
\hline DdL7* & $\begin{array}{l}\text { Desmophyll } \\
\text { um dianthus }\end{array}$ & {$[\mathrm{CAA}] \mathrm{CAG}[\mathrm{CAA}] 10$} & $214-281$ & Scleractinia & Order \\
\hline DdL73 & $\begin{array}{l}\text { Desmophyll } \\
\text { um dianthus }\end{array}$ & [TTTG]7 & $158-178$ & Scleractinia & Order \\
\hline DdL82 & $\begin{array}{l}\text { Desmophyll } \\
\text { um dianthus }\end{array}$ & GTT]12 & $116-134$ & Scleractinia & Order \\
\hline DdL83 & $\begin{array}{l}\text { Desmophyll } \\
\text { um dianthus }\end{array}$ & {$[\mathrm{TGT}] 8[\mathrm{GGT}] 3$} & $230-260$ & Scleractinia & Order \\
\hline DdL84* & $\begin{array}{l}\text { Desmophyll } \\
\text { um dianthus }\end{array}$ & [CAA]6CAGCAA & $135-210$ & Scleractinia & Order \\
\hline DdL86 & $\begin{array}{l}\text { Desmophyll } \\
\text { um dianthus }\end{array}$ & {$[\mathrm{CAA}] 7$} & $146-152$ & Scleractinia & Order \\
\hline DdL90 & $\begin{array}{l}\text { Desmophyll } \\
\text { um dianthus }\end{array}$ & [ATC]5GTC[ATC]GTC & $104-1119$ & Scleractinia & Order \\
\hline DdL91 & $\begin{array}{l}\text { Desmophyll } \\
\text { um dianthus }\end{array}$ & [GGT]9 & $205-223$ & Scleractinia & Order \\
\hline DdL96 & $\begin{array}{l}\text { Desmophyll } \\
\text { um dianthus }\end{array}$ & {$[\mathrm{AAC}] 7$} & $102-156$ & Scleractinia & Order \\
\hline DdL98 & $\begin{array}{l}\text { Desmophyll } \\
\text { um dianthus }\end{array}$ & [TCA] 8 & $135-150$ & Scleractinia & Order \\
\hline
\end{tabular}


Chapter 5. Patterns of genetic connectivity amongst four deep-sea demosponges in the New Zealand region: implications for the protection of vulnerable marine ecosystems 


\subsection{Introduction}

Larval dispersal and genetic connectivity are poorly understood in the deep sea, despite the fact that they shape patterns of biodiversity and biogeography, and may provide insights to help guide the protection of rare, threatened and endangered species (Hilário et al., 2015; Baco et al., 2016). Analysis of genetic variation and quantification of the extent of genetic connectivity for multiple taxa can be used to plan and evaluate the suitability of the location, size and spacing of marine protected areas (MPAs) and networks (reviewed by Palumbi, 2003; Lotterhos et al., 2014; van Der Meer et al., 2014; Jackson et al., 2015; Truelove et al., 2015). Whilst the identification of source and sink populations, as well as genetic hotspots, has long been achievable in shallow-water biota, this has not been the case for the deep sea. In general, this is because the high cost and technical challenge of sampling in the deep sea make it difficult to acquire specimens at large spatial scales, and across a wide range of depths (Hilário et al., 2015). In addition, whilst analysis of existing (e.g. archived) material may provide opportunities for the examination of genetic variation and population genetic structure, much of the material presently held in many collections is unlikely to be suitable for such work (Boschen et al., 2015). However, given the ongoing anthropogenic threats to the deep sea that include fishing, dumping, oil and gas exploration and potential deep-sea mining (Ramirez-Llodra et al., 2010), there is an increasing need to better understand patterns of population connectivity amongst vulnerable marine ecosystems (VMEs) so that protection can be put in place.

The relatively recent concept of VMEs has presented major challenges for the protection of deep-sea areas. VMEs are defined as "assemblages of marine benthic organisms which are susceptible to anthropogenic disturbance, especially that arising from the impact of fishing gear used in bottom fishing" (FAO, 2009). The protection of VMEs is called for in resolutions of the United Nations General Assembly (UNGA), and such protection is the responsibility of nation states within their Exclusive Economic Zones (EEZs) as well as bodies that manage resource use in the area beyond national jurisdiction (ABNJ) (e.g. Regional Fisheries Management Organisations, RFMOs) (Ardron et al., 2013). VMEs are typically characterised by taxa that are fragile and susceptible to damage (in particular from fishing gear), have high longevity and slow growth rates, and have a limited ability (or complete inability) to recover from major disturbance events (FAO, 2009). Such indicator taxa have been identified for particular regions (e.g. Parker et al., 2009 for the South Pacific Ocean; Parker \& Bowden, 2010 for Antarctica). VMEs are often associated with seamounts, 
which can be sites of intense fishing activity because some fish species aggregate around seamounts for breeding or feeding (Clark \& Rowden, 2009). A variety of different measures have been enacted to help protect VMEs, including spatial and temporal fishing gear restrictions, move-on rules, as well as areas closed to fishing, but the efficacy of these protection measures has been questioned (Auster et al., 2011; Clark \& Dunn, 2012; Penney \& Guinotte, 2013). If VMEs are to be protected adequately by spatial closures then further research is required to improve knowledge of the distribution of VME indicator taxa, to better understand how such VMEs are connected in terms of sources and sinks of larval recruits, and to quantify the spatial scale(s) at which genetic populations are structured.

Sponges are considered to be VME indicator taxa in the South Pacific Ocean (Parker et al., 2009), but studies of their distribution, life history and population connectivity are rare. In shallow-water sponges, genetic structure has been observed in most studies, and across a range of sponges at different spatial scales using different markers. This structure has been attributed to hydrodynamic forces and limited dispersal capabilities (Duran et al., 2004abc; Whalan et al., 2005; Bentlage \& Wörheide, 2007; Whalan et al., 2008a; Blanquer et al., 2009; López-Legentil \& Pawlik; 2009; Blanquer \& Uriz, 2010; DeBiasse et al., 2010; Xavier et al., 2010; Dailianis et al., 2011; Guardiola et al., 2012). This small-scale structure may be explained by limited dispersal ability, because for most sponges the planktonic larvae settle within a few days (Maldonado \& Bergquist, 2002). This information from shallow-water sponges may provide some indication of the genetic structure of deep-sea sponges. However, the environment is very different between shallow and deep water (Watling et al., 2013), and these differences are expected to shape different patterns of population genetic structure. For instance, previous studies have pointed out that lower temperatures increase larval duration and dispersal capabilities (Whalan et al., 2008b), and this longer larval duration may assist sponges to achieve large-scale population connectivity in the deep sea (Hilário et al., 2015). Therefore, information from shallow-water sponges is probably of limited value, and studies of genetic structure and connectivity of deep-sea species are required.

Whilst genetic differentiation amongst many species in New Zealand's coastal environment is well documented (Gardner et al., 2010), patterns of population genetic structure of deep-sea taxa are largely unknown. To date, studies have not revealed any consistent patterns of genetic connectivity or structuring across a range of deep-sea VME indicator taxa in the New Zealand region. A study of bamboo corals (Keratoisidinae) reported common mitochondrial haplotypes occurring over a wide geographical range $\left(27^{\circ} \mathrm{S}-50{ }^{\circ} \mathrm{S}\right)$ around New Zealand and no indication of genetic barriers to dispersal (Smith et al., 2004a). 
Three species of deep-sea corals showed evidence of genetic subdivision on seamounts and slopes within the Australia-New Zealand region, but a further six coral species exhibited either low genetic diversity or no evidence of genetic structure at scales of tens to hundreds of kilometres (Miller et al., 2010). In contrast, a later and broader study of the stony coral Desmophyllum dianthus, reported regional genetic differentiation as well as genetic differences amongst populations with depth across the region (Miller et al., 2011). Along the Kermadec Volcanic Arc (north of New Zealand), Smith et al. (2004b) reported heterogeneity in allozyme gene frequencies between vent mussels collected from two seamounts only 50 $\mathrm{km}$ apart. However, high levels of gene flow in the region amongst sites $10 \mathrm{~s}$ to $100 \mathrm{~s} \mathrm{~km}$ apart and limited genetic structure were reported by a later study of the same vent mussel based on COI variation (Boschen et al., 2015). The role of currents as either promoters of, or barriers to, gene flow is likely to be region-specific and requires much more consideration at a variety of different spatial and temporal scales. For example, in waters south of New Zealand, the Antarctic Circumpolar Current is believed to act as an oceanographic barrier to gene flow of deep-sea octocorals, separating populations to the north (around New Zealand and Tasmania) and south (Antarctica) at approximately $55^{\circ} \mathrm{S}$ on the Macquarie Ridge (Dueñas et al., 2016). In addition, deep water (1000 m depth) currents flow from north and south off New Zealand's east coast and meet along the Chatham Rise (Chiswell et al., 2015), and this might act as a barrier to larval dispersal in some regions but also act to promote mixing and thereby enhance diversity in others.

As noted by Miller et al. (2010) and Boschen et al. (2015), in the New Zealand region, understanding of the degree of isolation of deep-sea populations with respect to gene flow and connectivity is important for management planning, and the design of a network of marine protected areas. Whilst deep-sea genetic studies in the New Zealand region span a range of VME indicator taxa, the spatial coverage is patchy and therefore difficult to interpret holistically in terms of management recommendations for protection. In the present study, mitochondrial DNA sequence variation was examined to assess genetic connectivity amongst populations of four deep-sea demosponge species in the New Zealand region to provide information for management of VMEs in the South Pacific region. 


\subsection{Materials and methods}

\subsubsection{Samples}

Ten sponges (Poecillastra laminaris, Penares sp., Pleroma menoui, Neoaulaxinia persicum, Ecionemia novaezealandiae, Hyalascus maui, Geodia vestigifera, Herengeria vasiformis, Reidispongia coerulea, Neoschrammeniella fulvodesmus) were selected as potential candidates for study, based on their adherence to VME criteria (FAO, 2009), wide distribution in the New Zealand region, and the availability of samples. After initial assessment of the available specimens for these ten species in the NIWA Invertebrate Collection, and the results of preliminary screening analysis, samples from four demosponges (Neoaulaxinia persicum, Penares sp., Pleroma menoui and Poecillastra laminaris) were analysed. The total number of samples across all four species was 117 (Table 5.1), and most were collected from within the New Zealand EEZ (Figure 5.1). The majority of specimens were from seamount and slope habitats from 122-1507 m water depth: most were preserved in ethanol, with the rest dry-preserved. Due to patchy deep-sea sampling and the different distributions of the species, samples for all species were not available throughout the study region. Penares sp. and Poecillastra laminaris samples were found in both biogeographic provinces, but $N$. persicum and P. menoui samples were not collected from the waters south of New Zealand. The full distributions of the latter two species are still unknown, but they have not yet been found in the southern province and are currently considered species with a northern restricted distribution.

In the New Zealand region, the deep sea is comprised of various bathymetries, topographies and hydrodynamics (Snelder et al., 2007). In the present study, because sampling at each site recovered only one individual sponge, all specimens within $1^{\circ}$ of latitude and longitude were grouped into one population: N. persicum, P. palmatoclada, $P$. menoui and Poecillastra laminaris were assigned into 8, 7, 8 and 19 populations for plotting of haplotype distributions, respectively. Samples for all species were categorised into $100 \mathrm{~m}$ depth range intervals based on their depth of collection.

Within the New Zealand region, two deep ocean floor biogeographic provinces are presently recognised, a northern (BY6) and a southern (BY10) province (Figure 5.1), each with its own characteristics (e.g., bottom water temperature and particulate organic carbon (POC) flux) (Watling et al., 2013). To investigate genetic differentiation amongst 
populations, a hierarchical testing framework based on features (provinces, regions, geomorphic characters) at different spatial scales (Figure 5.1) was employed. First, samples from all 4 species were assigned to the northern and southern biogeographic provinces following Watling et al. (2013) with a boundary at $45^{\circ} \mathrm{S}$. Second, samples were divided into northern (north of the Chatham Rise), central (the Chatham Rise) and southern (south of the Chatham Rise) regions with boundaries at latitudes $42{ }^{\circ} \mathrm{S}$ and $45^{\circ} \mathrm{S}$. For $N$. persicum and $P$. menoui, a further division at latitude $34{ }^{\circ} \mathrm{S}$ was to separate the species into an upper (Fiji Basin) and lower (New Zealand EEZ) group in the northern province. Third, samples were grouped based on geomorphic features, including the Fiji Basin, Kermadec Ridge, Challenger Plateau, Hikurangi Margin, Chatham Rise, Campbell Plateau and Macquarie Ridge. Full collection details are summarised in Table S5.1.

Table 5.1 Number of partial sequences plus length of sequence (bp) for three mitochondrial genes

\begin{tabular}{lcccccc}
\hline \multirow{2}{*}{ Species } & \multicolumn{2}{c}{ COI } & \multicolumn{2}{c}{ 12S } & \multicolumn{2}{c}{ Cytb } \\
\cline { 2 - 7 } & $\mathbf{n}$ & length & $\mathbf{n}$ & length & $\mathbf{n}$ & length \\
\hline Poecillastra laminaris & 54 & 402 & & & 49 & 402 \\
Penares sp. & 22 & 423 & 18 & 694 & & \\
Neoaulaxinia persicum & 34 & 462 & 12 & 704 & & \\
Pleroma menoui & 19 & 431 & & & & \\
\hline
\end{tabular}

\subsubsection{Molecular methods}

Total DNA was recovered using a guanidine thiocyanate extraction methodology (Boom et al., 1990) and DNA was stored at $4{ }^{\circ} \mathrm{C}$ in $0.1 \mathrm{X}$ TE buffer. To identify informative population genetic markers in demosponges, three mitochondrial DNA regions with different rates of mutation, the ribosomal subunit (12S), cytochrome oxidase subunit I (COI) and cytochrome $b(C y t b)$, were used to estimate genetic connectivity and population genetic differentiation. Sponge-specific primers for $12 S, C O I$ and $C y t b$ were and designed and utilised to amplify these genes in this study; primers and annealing temperatures used for each species are summarised in (Chapter 3). PCR reactions $(30 \mu \mathrm{l})$ for all three genes contained $1 \mu \mathrm{l}$ DNA template, $15 \mu 12 \times$ MyTaq $^{\mathrm{TM}}$ Mix (Bioline, UK), $1 \mu \mathrm{l}$ of each primer (Macrogen, Korea), and $12 \mu \mathrm{l} \mathrm{ddH}_{2} \mathrm{O}$. The thermal cycling profile consisted of an initial denaturation step at $94{ }^{\circ} \mathrm{C}$ for $5 \mathrm{~min}$, then 32 cycles of a three-step program $\left(95^{\circ} \mathrm{C}\right.$ for $30 \mathrm{~s}$, $50{ }^{\circ} \mathrm{C}$ for $30 \mathrm{~s}$ and $72{ }^{\circ} \mathrm{C}$ for $45 \mathrm{~s}$ ), with a 20 -min final extension at $72{ }^{\circ} \mathrm{C}$. PCR products were sent to Macrogen (Macrogen Inc, Korea) for purification and sequencing on an ABI3730 sequencer. Total species-specific sample size was large enough for only 
Poecillastra laminaris to permit assessment of nuclear DNA variation at 10 polymorphic microsatellite loci (described in Chapter 3).

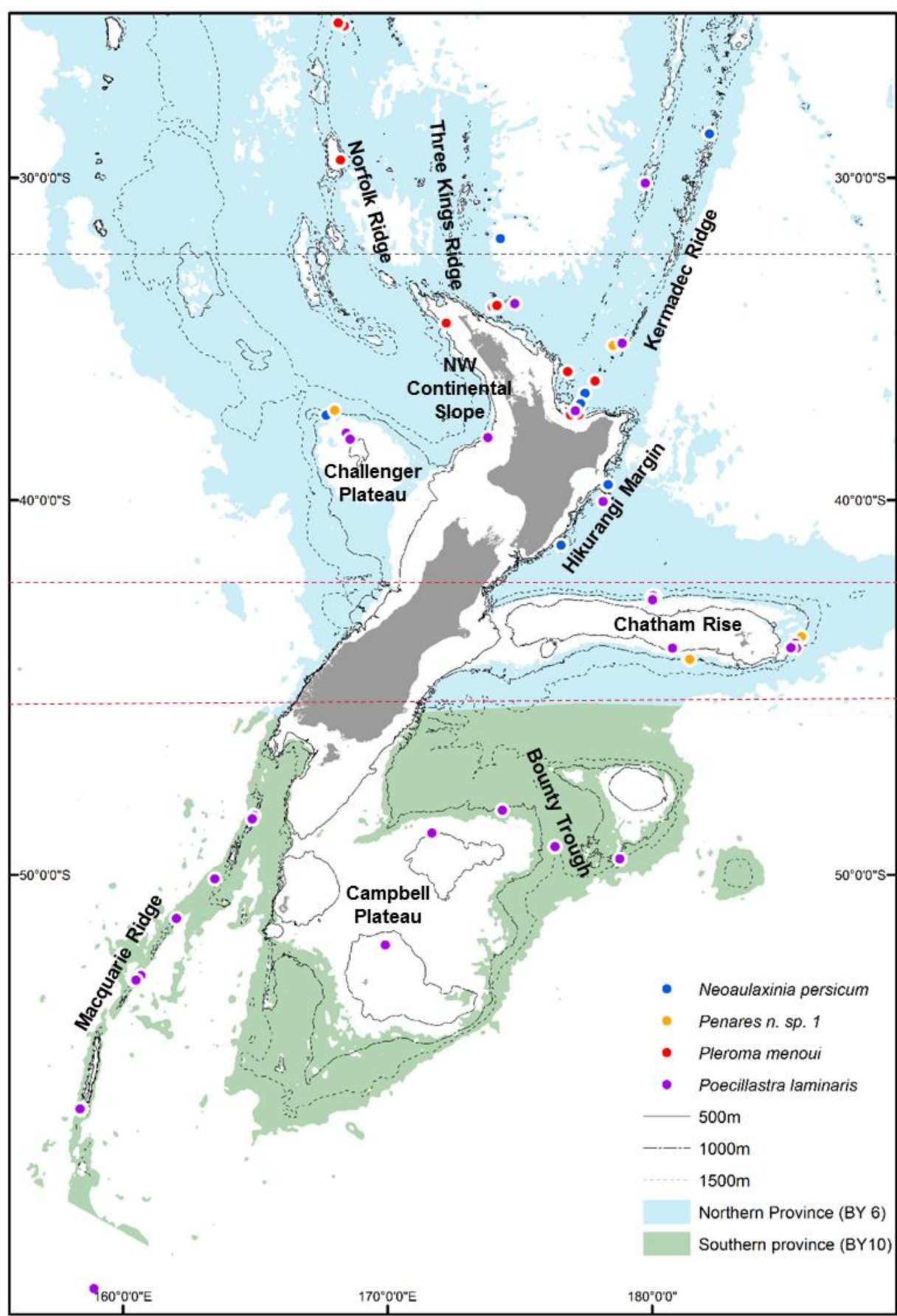

Figure 5.1 Map showing the locations of samples for the study species.

Hierarchical testing of regional genetic differentiation is employed: (1) blue (northern) and green (southern) backgrounds represent the two biogeographic lower bathyal provinces (BY6 and BY10) of Watling et al. (2013) in the New Zealand region; (2) red dashed lines at $42^{\circ} \mathrm{S}$ and $45^{\circ} \mathrm{S}$ indicate boundaries between north $\left(<42^{\circ} \mathrm{S}\right)$, central $\left(42^{\circ} \mathrm{S}\right.$ to $\left.45^{\circ} \mathrm{S}\right)$ and south $\left(>45^{\circ} \mathrm{S}\right)$ regions for Poecillastra laminaris and Penares sp. and black dashed line at $32^{\circ} \mathrm{S}$ shows boundary between the north and south regions for Neoaulaxinia persicum and Pleroma menoui; (3) major geomorphic features are named on the map. 


\subsubsection{Data analysis}

All sequences were checked and edited using Geneious (v 7, Biomatters Ltd, New Zealand). Sequences with HQ values (percentage of untrimmed bases in a sequence that is high quality) of $<80 \%$ were discarded. Multiple sequences were aligned using the plugin ClustalW Alignment (Gap Open Cost=100 and Gap Extend Cost=10) in Geneious, and then all alignments were viewed by eye. Neighbour-Joining (NJ) phylogenetic trees were constructed with default settings and highest similarity sequence in NCBI as an out-group by using the Tree Builder option in Geneious v7. For all populations with two or more individuals' intra-specific genetic diversity was evaluated by computing the number of haplotypes, the number of polymorphic sites, haplotypic diversity $(h)$, and nucleotide diversity $(\pi)$. A $t$-test was used to compare the difference of haplotypes diversity between markers.

AMOVA of sequence variation between/amongst regions (northern versus southern biogeographic provinces; northern versus central versus southern regions; amongst geomorphic features) and amongst populations was tested. Because small sample size will influence the accuracy of the results, AMOVA amongst populations on geomorphic features was employed only when population-specific sample sizes were larger than five. The AMOVA of sequence variation amongst populations on geomorphic feature with sample sizes $>5$ showed the same pattern of results as when sample szies were less than five. Therefore, to maximise information return the AMOVA results from all geomorphic feature populations are presented, but conclusions about these population were only drawn when there was general agreement between the two different approaches based on sample size.

Mismatch Distribution, Tajima's D (Tajima, 1989) and the Ramos-Onsins-Rozas Test (R2) of Neutrality were analysed using the R-package software program PEGAS v0.6 (Paradis, 2010). Pairwise comparisons of population differentiation were performed in Arlequin and significance values estimated after 1000 permutations (Excoffier \& Lischer, 2010). Between-province or within-province $\Phi_{S T}$ statistics were calculated to test for genetic differentiation amongst populations. If significant differentiation amongst populations was detected, the location of the genetic discontinuity was identified using Barrier v2.2 (Manni et al., 2004). SAMOVA, using SPADS V1.0 (Dellicour \& Mardulyn, 2014), was used to detect the number of potential populations per species with non-significant variant components identified amongst populations in AMOVA. 
In order to visualise the spatial patterns of genetic variation for each marker, the specimens were colour-coded according to haplotype, their geographic coordinates of collection were plotted using ArcGIS (ESRI, USA), and their collection depth was plotted on an X-Y scatter plot. To assess the amount of variability in the populations of each species that was represented in the samples, haplotype accumulation curves were generated (Gotelli \& Colwell, 2001) by calculating estimates of the mean and variance for the number of accumulated haplotypes through 1000 random permutations, using the R package SPIDER v1.1 (Brown et al., 2012). The Mantel test (Mantel, 1967) was employed to test for isolationby-depth by comparing the matrix of $\Phi_{S T}$ values to the matrix of depth (m) values and for isolation-by-distance by comparing the matrix of $\Phi_{S T}$ values to the matrix of shortest actual distances $(\mathrm{km})$ between pairs of sites (Isolation by Distance, web service Jensen et al., 2005).

The program MIGRATE (Beerli \& Felsenstein, 1999) was used to estimate $\theta$ (theta, the effective population size $\times$ the mutation rate per site), and migration $(\mathrm{M})$ under the sequence model. A full migration matrix model was employed as the migration estimating model, and each Markov chain Monte Carlo (MCMC) run consisted of 10 short chains (sampling 50,000 trees) and 1 long chain (sampling 500,000 trees) with a burn-in period of 10,000 trees. All runs were repeated five times to verify consistency of results, and the data presented are the average of the five runs.

For microsatellite data analysis, allelic frequencies, number of alleles, departures from Hardy-Weinberg equilibrium (HWE), linkage disequilibrium (LD) and observed and expected heterozygosities were estimated in GenAlEx (v 6.5). Arlequin was used to estimate Weir and Cockerham's (1984) unbiased estimator of Wright's F statistic $\left(F_{S T}\right)$. MicroChecker (v2.2.1, van Oosterhout et al., 2004) was used to identify possible genotyping errors such as stuttering, large allele dropout, and null alleles within the data set by performing 10,000 randomisations (threshold was set at 0.10 then raised to 0.15 when insufficient loci were present). Loci under selection and/or with null alleles were removed and a second (neutral loci) data set was created. Both the full ( $\mathrm{n}=10$ loci) and the reduced ( $\mathrm{n}=4$ loci) data sets were tested for population genetic differentiation using Arlequin (AMOVA, 10,000 permutations) for differentiation between provinces and amongst regions. The Mantel test (GenAlEx) was employed to test for isolation-by-depth by comparing the matrix of Nei's unbiased genetic distance values to the matrix of depth $(\mathrm{m})$ values and isolation-by-distance by comparing the matrix of Nei's unbiased genetic distance values to the matrix of shortest actual distances $(\mathrm{km})$ between pairs of sites. 


\subsection{Results}

After trimming and checking, $\mathrm{COI}$ sequence data were obtained for all 4 species, $12 \mathrm{~S}$ sequence data for two species, and Cytb sequence data for one species (Table 5.2). Overall haploptypic diversity for all populations for COI was greater than that for $12 S$ of $P$. palmatoclada, $12 S$ of $N$. persicum or Cytb of Poecillastra laminaris, but $t$-test showed that none reached significance $(\mathrm{p}>0.05)$ (Table 5.3 -Table 5.5).

Table 5.2 Number of partial sequences (n) plus length of sequence (bp) for three mitochondrial genes for each of four sponge species

\begin{tabular}{ccccc}
\hline Genes & Species & $\mathbf{n}$ & Trimmed length (bp) & NCBI Accession ID \\
\hline \multirow{4}{*}{ COI } & Poecillastra laminaris & 54 & 402 & Hap_1-18: KX685272-KX685289 \\
& Penares sp. & 16 & 423 & Hap_1-5: KX685302-KX685306 \\
& Neoaulaxinia persicum & 30 & 462 & Hap_1-7: KX685290-KX685296 \\
\multirow{2}{*}{ CytB } & Pleroma menoui & 16 & 431 & Hap_1-5: KX685297-KX685301 \\
\multirow{2}{*}{$12 S$} & Poecillastra laminaris & 49 & 402 & Hap_1-18: KX685307-KX685324 \\
& Penares sp. & 14 & 694 & Hap_1-2: KX685325-KX685326 \\
& Neoaulaxinia persicum & 11 & 704 & Hap_1-2: KX685327-KX685328 \\
\hline
\end{tabular}

Table 5.3 Nucleotide diversity statistics for Poecillastra laminaris at three different spatial scales

\begin{tabular}{cccccccc}
\hline \multirow{2}{*}{ Grouping } & \multicolumn{3}{c}{ COI } & \multicolumn{4}{c}{ Cytb } \\
\cline { 3 - 8 } Province & Northern & 34 & 0.848 & 0.0145 & 30 & 0.800 & 0.0120 \\
& Southern & 19 & 0.749 & 0.0156 & 19 & 0.811 & 0.0152 \\
\hline \multirow{3}{*}{ Region } & North & 15 & 0.619 & 0.0037 & 14 & 0.681 & 0.0054 \\
& Central & 19 & 0.895 & 0.0185 & 17 & 0.721 & 0.0142 \\
& South & 19 & 0.749 & 0.0156 & 19 & 0.811 & 0.0152 \\
\hline \multirow{3}{*}{ Geomorphic feature } & Kermadec Ridge & 9 & 0.556 & 0.0021 & 9 & 0.583 & 0.0017 \\
& Challenger Plateau & 2 & 0 & 0 & 2 & 0 & 0 \\
& Hikurangi Margin & 2 & 1 & 0.0075 & 2 & 1 & 0.0100 \\
& Chatham Rise & 19 & 0.895 & 0.0185 & 17 & 0.721 & 0.0142 \\
& Bounty Trough & 2 & 1 & 0.0473 & 2 & 1 & 0.0274 \\
& Campbell Plateau & 3 & 0 & 0 & 3 & 0 & 0 \\
& Macquarie Ridge & 13 & 0.628 & 0.0121 & 14 & 0.758 & 0.0137 \\
\hline & & 53 & 0.893 & 0.0185 & 49 & 0.889 & 0.0152
\end{tabular}

$\mathrm{n}$ is the number of sequences for analysis from the site, $\mathrm{s}$ is the number of polymorphic nucleotide sites in the sequence, $\mathrm{h}$ is the number of haplotypes represented at the site, $\mathrm{Hd}$ is haplotype diversity, and $\pi$ is nucleotide diversity. 


\subsubsection{Geographic distribution of haplotypes}

In total, 11 unique COI haplotypes were observed for Poecillastra laminaris in the northern province, only 4 haplotypes were restricted to the southern province, and 8 haplotypes were restricted to individuals (Figure 5.2A). Hap 1 was the numerically dominant COI haplotype, with all occurrences except one individual (in S17) occurring in the north. For $C y t b, 9$ haplotypes were only distributed in the northern province and 7 haplotypes were only found in the southern province. Hap 5 was the numerically most abundant haplotype and it was only found in the northern province.

Table 5.4 Nucleotide diversity statistics for Penares sp. at three different spatial scales

\begin{tabular}{|c|c|c|c|c|c|c|c|}
\hline \multirow{2}{*}{\multicolumn{2}{|c|}{ Grouping }} & \multicolumn{2}{|c|}{$\mathrm{COI}$} & \multicolumn{4}{|c|}{$12 S$} \\
\hline & & $\mathbf{n}$ & Hd & $\pi$ & $\mathbf{n}$ & Hd & $\pi$ \\
\hline \multirow{2}{*}{ Province } & Northern & 14 & 0.396 & 0.0025 & 12 & 0.167 & 0.0002 \\
\hline & Southern & 2 & 1 & 0.0047 & 2 & 0 & 0 \\
\hline \multirow{3}{*}{ Region } & North & 7 & 0.524 & 0.0041 & 6 & 0.333 & 0.0005 \\
\hline & Central & 7 & 0.286 & 0.0007 & 6 & 0 & 0 \\
\hline & South & 2 & 1 & 0.0047 & 2 & 0 & 0 \\
\hline \multirow{5}{*}{ Geomorphic feature } & Kermadec Ridge & 5 & 0.7 & 0.0052 & 4 & 0.5 & 0.0004 \\
\hline & Challenger Plateau & 1 & - & - & 1 & - & - \\
\hline & Hikurangi Margin & 1 & - & - & 1 & - & - \\
\hline & Chatham Rise & 7 & 0.286 & 0.0007 & 6 & 0 & 0 \\
\hline & Macquarie Ridge & 2 & 1 & 0.0047 & 2 & 0 & 0 \\
\hline \multicolumn{2}{|c|}{ Total } & 30 & 0.411 & 0.0025 & 26 & 0.148 & 0.0002 \\
\hline
\end{tabular}

$\mathrm{n}$ is the number of sequences for analysis from the site, $\mathrm{s}$ is the number of polymorphic nucleotide sites in the sequence, $\mathrm{h}$ is the number of haplotypes represented at the site, $\mathrm{Hd}$ is haplotype diversity, and $\pi$ is nucleotide diversity.

A numerically dominant COI haplotype (Hap1) was observed for Penares sp. across the northern and southern provinces: other haplotypes were only found in single individuals (Figure 5.2B). For 12S, Hap1 was shared across both provinces, and other haplotypes were only found in single individuals. There was no support for the hypothesis of geographic genetic structure by province for haplotypic diversity distributions of $\mathrm{COI}$ or $12 \mathrm{~S}$ for this species.

Eleven COI haplotypes for $N$. persicum were found within the northern province, but only Hap1 was widely spread across this province. There was limited evidence of $12 \mathrm{~S}$ haplotypic diversity within the northern province. The haplotypic distributions of $C O I$ and $12 S$ did not reveal patterns of spatial genetic differentiation (Figure 5.2C). 
Only five COI haplotypes of Pleroma menoui were found in the northern province: Hap2 was distributed across the region (Figure 5.2D). There was limited evidence to suggest the presence of spatial genetic structure.

\subsubsection{Population genetic diversity}

For Poecillastra laminaris the COI haplotypic diversity of the northern province was greater than in the southern province, whereas nucleotide diversity was lower. The middle region was the highest haplotypic and nucleotide diversity amongst three regions. Testing of differences in haplotypic diversity between geomorphic features, the highest haplotypic diversity was found in Chatham Rise, but the highest nucleotide diversity was in Campbell Plateau. For Cytb, both haplotypic and nucleotide diversity were greater in the southern than in the northern province, and the haplotypic and nucleotide diversity of south region was higher than other two regions. The greatest haplotypic and nucleotide diversity values of $C y t b$ were all found in Macquarie amongst the geomorphic groups (Figure 5.2A and Table 5.3).

Table 5.5 Nucleotide diversity statistics for Neoaulaxinia persicum and Pleroma menoui at two different spatial scales

\begin{tabular}{|c|c|c|c|c|c|c|c|c|c|c|}
\hline \multirow{3}{*}{\multicolumn{2}{|c|}{ Grouping }} & \multicolumn{6}{|c|}{ Neoaulaxinia persicum } & \multirow{2}{*}{\multicolumn{3}{|c|}{$\frac{\text { Pleroma menoui }}{\text { COI }}$}} \\
\hline & & \multicolumn{3}{|c|}{$\mathrm{COI}$} & \multicolumn{3}{|c|}{$12 S$} & & & \\
\hline & & $\mathrm{n}$ & $\mathrm{Hd}$ & $\pi$ & $\mathrm{n}$ & Hd & $\pi$ & $\mathrm{n}$ & $\mathrm{Hd}$ & $\pi$ \\
\hline \multirow{2}{*}{ Region } & North & 2 & 0 & 0 & 1 & 0 & 0 & 7 & 0.524 & 0.002 \\
\hline & South & 28 & 0.537 & 0.0019 & 10 & 0 & 0 & 9 & 0.417 & 0.0026 \\
\hline \multirow{7}{*}{ Geomorphic feature } & Norfolk Ridge & - & - & - & - & - & - & 4 & 0.833 & 0.0035 \\
\hline & Kermadec Ridge & 7 & 0.288 & 0.0006 & 4 & 0.5 & 0.02547 & 7 & 0.286 & 0.0007 \\
\hline & Three Kings Ridge & 12 & 0.318 & 0.0011 & 4 & 0 & 0 & 2 & 0 & 0 \\
\hline & NE Continental Slope & - & - & - & - & - & - & 3 & 0.667 & 0.0062 \\
\hline & Challenger Plateau & 1 & - & - & - & - & - & - & - & - \\
\hline & Hikurangi Margin & 2 & 0 & 0 & 1 & - & - & - & - & - \\
\hline & Chatham Rise & 8 & 0.75 & 0.003 & 2 & 1 & 0 & - & - & - \\
\hline
\end{tabular}

$\mathrm{n}$ is the number of sequences for analysis from the site, $\mathrm{s}$ is the number of polymorphic nucleotide sites in the sequence, $\mathrm{h}$ is the number of haplotypes represented at the site, Hd is haplotype diversity, and $\pi$ is nucleotide diversity. 


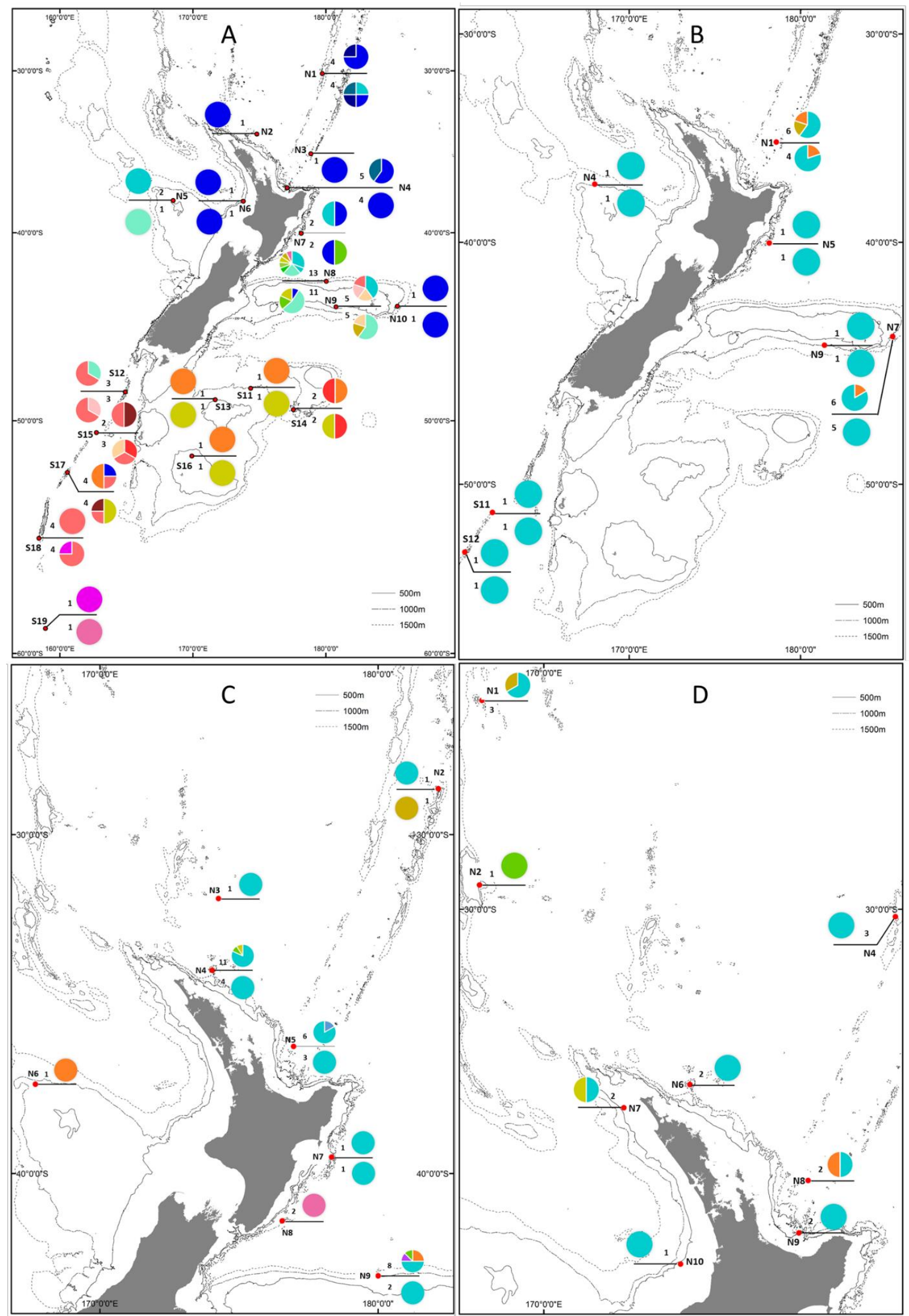

Figure 5.2 Haplotype map for Poecillastra laminaris COI (above line) and Cytb (below line) (A), Penares sp. COI (above line) and $12 S$ (below line) (B), Neoaulaxinia persicum COI (above line) and $12 S$ (below line) (C), Pleroma menoui COI (D) 
For Penares sp. the COI nucleotide diversity of the southern province was greater than that of the northern province. For the three regions the south had the highest values, mainly contributed by samples from Macquarie Ridge. For $12 S$, nucleotide diversity was greater in the northern than the southern province. The north region had the greatest nucleotide diversity amongst the regions, which was closely related to high diversity associated with populations from the Kermadec Ridge (Figure 5.2B and Table 5.4)

For $N$. persicum the COI haplotypic and nucleotide diversity values were greater in the south than north region: populations on the Three Kings Ridge had the highest nucleotide and Chatham Rise populations had the highest haplotype diversity values. For $12 S$ the haplotype number, the number of polymorphic sites, haplotype diversity and nucleotide diversity values could only be calculated for the south region. The Kermadec Ridge population had the highest nucleotide and haplotypic diversity (Figure 5.2C and Table 5.5).

For Pleroma menoui, the highest COI nucleotide diversity was in populations from the Norfolk Ridge, and the nucleotide diversity in the north was greater than in the south region. The haplotypic diversity for Pleroma menoui followed the same pattern (Figure 5.2D and Table 5.5).

\subsubsection{Population genetic structure}

Amongst the Poecillastra laminaris populations, $\Phi_{S T}$ values for both $C O I$ and $C y t b$ were highly variable (Tables S5.2-5.5), but were often based on small sample sizes (sometimes 2 individuals per population). The AMOVA of genetic structure across Poecillastra laminaris populations (Table 5.6) revealed significant differentiation between populations of the northern and southern provinces, amongst the three regions and also amongst the different geomorphic features for both COI and Cytb $(\mathrm{p}<0.01)$. Except for a weak significant result for in $C y t b$, populations within the provinces, regions or geomorphic features were not statistically significantly different $(p>0.05)$, whereas significant variation existed within all populations $(\mathrm{p}<0.01)$. Whilst this non-significant differentiation amongst populations might result from an under-estimated number of populations, the SAMOVA based on all $C O I$ and $C y t b$ sequences revealed that the potential number of populations was 18 (Figure S5.1), which was very close to the actual number of sampling sites (19), although 8 sites (N2, N3, N6, N10, S11, S13, S16 and S19) had only one sequence present for one or the other marker. The NJ phylogenetic trees revealed evidence of geographic structure for the Poecillastra laminaris populations (Figure S5.2). Whilst not all sequences were grouped 
according to sampling location, most were, providing support for a north and south differentiation.

Table 5.6 Hierarchical AMOVA results for Poecillastra laminaris when tested for genetic variation at mitochondrial genes

\begin{tabular}{ccccccc}
\hline Source of variation & \multicolumn{3}{c}{ COI } & \multicolumn{3}{c}{ Cytb } \\
\cline { 2 - 7 } & df & ss & $\mathbf{\% V}$ & df & ss & \%V \\
\hline Between provinces & 1 & 40.201 & $32.34^{* *}$ & 1 & 22.481 & $21.9^{* *}$ \\
Within provinces between populations & 16 & 60.618 & 9.53 & 16 & 54.323 & 11.87 \\
Within populations & 35 & 92.068 & $58.13^{* *}$ & 33 & 76 & $66.23^{* *}$ \\
Total & 52 & 192.887 & & 50 & 152.804 & \\
\hline Amongst regions & 2 & 59.329 & $36.17^{* *}$ & 2 & 35.06 & $25.42^{* *}$ \\
Within regions between populations & 15 & 41.49 & 1.32 & 15 & 41.744 & $5.80^{*}$ \\
Within populations & 35 & 92.068 & $62.51^{* *}$ & 33 & 76 & $68.7^{* *}$ \\
Total & 52 & 192.887 & & 50 & 152.804 & \\
\hline Within geomorphic features between populations & 4 & 68.37 & $38.58^{* *}$ & 4 & 50.037 & $34.53^{* *}$ \\
Within populations & 11 & 26.702 & -2.15 & 12 & 24.596 & -3.03 \\
Total & 34 & 90.568 & $63.58^{* *}$ & 32 & 74 & $68.50^{* *}$
\end{tabular}

Significant values of $\mathrm{p}<0.05$ are marked as $*$, and $\mathrm{p}<0.01$ are marked as **.

Table 5.7 Hierarchical AMOVA results for Penares sp. when tested for genetic variation at mitochondrial genes

\begin{tabular}{|c|c|c|c|c|c|c|}
\hline \multirow{2}{*}{ Source of variation } & \multicolumn{3}{|c|}{$\mathrm{COI}$} & \multicolumn{3}{|c|}{$12 S$} \\
\hline & df & ss & $\% \mathrm{~V}$ & df & ss & $\% \mathbf{V}$ \\
\hline Between provinces & 1 & 0.768 & 10.53 & 1 & 0.012 & -22.35 \\
\hline Within provinces between populations & 3 & 1.6 & -0.01 & 3 & 0.167 & -18.82 \\
\hline Within populations & 11 & 6.257 & 91.47 & 9 & 0.75 & 141.18 \\
\hline Total & 15 & 8.625 & & 13 & 0.929 & \\
\hline Amongst regions & 2 & 1.625 & 18.14 & 2 & 0.095 & 32.24 \\
\hline Within regions between populations & 4 & 1.767 & 16.08 & 4 & 0.083 & -89.32 \\
\hline Within populations & 9 & 5.233 & 97.94 & 7 & 0.75 & 157.08 \\
\hline Total & 15 & 8.625 & & 13 & 0.929 & \\
\hline Amongst geomorphic features & 4 & 2.368 & 8.06 & 4 & 0.179 & 60.04 \\
\hline Within geomorphic features between populations & 2 & 1.024 & -8.89 & 2 & 0 & -119.88 \\
\hline Within populations & 9 & 5.233 & 100.83 & 7 & 0.75 & 159.85 \\
\hline Total & 15 & 8.625 & & 13 & 0.929 & \\
\hline
\end{tabular}

Significant values of $\mathrm{p}<0.05$ are marked as *, and $\mathrm{p}<0.01$ are marked as **.

In contrast, analysis of $\Phi_{S T}$ values revealed no genetic structure within each of the Penares sp., N. persicum and Pleroma menoui samples. Species-specific pairwise population $\Phi_{S T}$ values were all below 0.1 with no statistically significant differences between pairs of populations. AMOVA revealed no significant $(\mathrm{p}>0.05)$ hierarchical population genetic 
structure for Penares sp. and P. menoui across the sampled area (Table 5.7 and Table 5.8). Similarly, NJ phylogenetic trees and haplotypes distribution did not support geographic structure between and within provinces (Figures S5.3-5.6).

Table 5.8 Hierarchical AMOVA results for Neoaulaxinia persicum and Pleroma menoui when tested for genetic variation at the $\mathrm{COI}$ mitochondrial gene

\begin{tabular}{cccccccc}
\hline \multirow{2}{*}{ Source of variation } & \multicolumn{3}{c}{ Neoaulaxinia persicum } & \multicolumn{3}{c}{ Pleroma menoui } \\
\cline { 2 - 7 } & $\mathbf{d f}$ & $\mathbf{s s}$ & $\mathbf{\% V}$ & $\mathbf{d f}$ & \multicolumn{1}{c}{$\mathbf{s s}$} & $\mathbf{\% V}$ \\
\hline Between regions & 1 & 0.133 & -25.68 & 1 & 0.484 & -5.51 \\
Within regions between populations & 6 & 3.564 & 17.05 & 6 & 3.849 & 25.99 \\
Within populations & 22 & 8.436 & 108.63 & 8 & 3.167 & 79.53 \\
Total & 29 & 12.133 & & 15 & 7.5 & & -6.04 \\
Amongst geomorphic features & 3 & 1.44 & -18.41 & 3 & 1.726 & 27.45 \\
Within geomorphic features between populations & 4 & 2.258 & 27.47 & 4 & 2.607 & 78.59 \\
Within populations & 22 & 8.436 & 90.94 & 8 & 3.167 & 7.5 & \\
\hline Total & 29 & 12.133 & & 15 & &
\end{tabular}

Significant values of $\mathrm{p}<0.05$ are marked as $*$, and $\mathrm{p}<0.01$ are marked as **. AMOVA analysis for $12 \mathrm{~S}$ in Neoaulaxinia persicum was not possible.

\subsubsection{Isolation by distance and depth}

The seven tests across all 4 species revealed no significant isolation by depth relationship, but one significant isolation by distance relationship, which was for COI of Poecillastra laminaris (Table 5.9).

Table 5.9 Mantel tests of isolation by depth and isolation by distance amongst different markers and species

\begin{tabular}{|c|c|c|c|c|c|c|c|c|}
\hline \multirow[b]{2}{*}{ Species } & \multicolumn{4}{|c|}{ Depth } & \multicolumn{4}{|c|}{ Distance } \\
\hline & COI & Cytb & $12 S$ & $\begin{array}{c}\text { Microsate } \\
\text { llites }\end{array}$ & $\mathrm{COI}$ & Cytb & $12 S$ & $\begin{array}{c}\text { Microsate } \\
\text { llites }\end{array}$ \\
\hline $\begin{array}{c}\text { Poecillastra } \\
\text { laminaris }\end{array}$ & $\begin{array}{c}0.025 \\
(0.212)\end{array}$ & $\begin{array}{c}0.121 \\
(0.052)\end{array}$ & & $\begin{array}{c}0.009 \\
(0.400)\end{array}$ & $\begin{array}{c}0.411 \\
(<0.001)\end{array}$ & $\begin{array}{c}0.403 \\
(1.000)\end{array}$ & & $\begin{array}{l}-0.019 \\
(0.400)\end{array}$ \\
\hline Penares sp. & $\begin{array}{c}0.169 \\
(0.060)\end{array}$ & & $\begin{array}{c}0.114 \\
(0.240)\end{array}$ & & $\begin{array}{c}0.103 \\
(0.280)\end{array}$ & & $\begin{array}{l}-0.092 \\
(0.610)\end{array}$ & \\
\hline $\begin{array}{l}\text { Neoaulaxinia } \\
\text { persicum }\end{array}$ & $\begin{array}{l}-0.049 \\
(0.039)\end{array}$ & & $\begin{array}{c}-0.202 \\
(0.170)\end{array}$ & & $\begin{array}{c}0.156 \\
(0.060)\end{array}$ & & $\begin{array}{c}0.554 \\
(0.070)\end{array}$ & \\
\hline Pleroma menoui & $\begin{array}{c}0.001 \\
(0.460)\end{array}$ & & & & $\begin{array}{l}-0.032 \\
(0.520)\end{array}$ & & & \\
\hline
\end{tabular}

Data are shown as $\mathrm{R}^{2}$ ( $\mathrm{p}$ value). Numbers in bold indicate significant correlations. 


\subsubsection{Tajima's D and R2 tests of neutrality and mismatch distribution}

Tajima's D was negative for all four species for all mtDNA markers, but only statistically significant $(\mathrm{p}<0.05)$ in populations of $N$. persicum $(C O I$ and $12 S), P$. menoui (COI) and Penares sp. (12S). The Ramos-Onsins-Rozas test of neutrality revealed that all R2 values were low and not statistically significant (Table S5.6). The mismatch distribution analyses showed no evidence of rapid population expansion and indicated that the empirical and stable expectations were not in agreement for all four species (Figures S5.7-5.9), which implied that the different species had different population histories. Also, such negative values in the absence of evidence for rapid expansion may signify an excess of low frequency polymorphisms of the mtDNA markers of P. palmatoclada, N. persicum and P. menoui.

\subsubsection{Genetic barriers}

Genetic barriers to gene flow between/amongst populations of Poecillastra laminaris based on the COI pairwise population $\Phi_{S T}$ values and geographical coordinates were predicted to occur around the north of the North Island and the south of the South Island. Based on the $C y t b \Phi_{S T}$ values the analysis indicated that barriers may also exist to the west and north of the North Island (Figure S5.10).

\subsubsection{Larval migration}

Because of small sample sizes, only Poecillastra laminaris had enough datasets to predict the larval migrations amongst populations. The average $\theta$ values estimated by the MIGRATE software for two loci (COI and $C y t b$ ) data set ranged from 0.0001 (Kermadec Ridge) to 0.0079 (Hikurangi Margin) (Table 5.10). Estimates of per generation immigrants were also variable amongst samples (only migrate values $>1$ are shown in Table 5.10). For northern populations (Kermadec Ridge, Challenger Plateau and Hikurangi Margin) there was no contribution to the southern populations (Campbell Plateau and Macquarie Ridge), but the Macquarie Ridge population contributed immigrants to the Kermadec Ridge and Challenger Plateau populations. The Kermadec Ridge was the only source of immigrants to the Hikurangi Margin population, and the Chatham Rise population (in the central region) contributed to all populations except the Hikurangi Margin and received immigrants from all populations except those in the south (Table 5.10). 


\subsubsection{Microsatellite variation}

The null alleles and selection information of all microsatellites present in Table S5.7. Number of alleles per locus varied from 6 to 16 (average $=12.53$ ), excepted heterozygosity (He) ranged from 0.665 to 0.893 per locus, and significant heterozygote deficiencies (5) slightly outnumbered significant heterozygote excesses (3) (Table S5.8). Diversity index values $(\mathrm{N}, \mathrm{Na}, \mathrm{Ne})$ were greater in the northern than in the southern province, whereas diversity index values were greater in the south region than in the central or north regions (Table S5.8). For the full (10 loci) data set, AMOVA revealed no significant genetic structure amongst the north-central-south regions or between the northern-southern provinces. However, for the neutral data set (4 loci) AMOVA revealed significant genetic structure amongst the north-central-south regions but not between the northern-southern provinces (Table S5.9). At the geomorphic population level, the full data set revealed significant structure amongst population, but the neutral loci data set did not reveal the structure. Examination of $F_{S T}$ values further illustrated that the north region was isolated from the central and south regions (Tables S5.10-11). There was no isolation by distance or by depth patterns observed for microsatellite variation in Poecillastra laminaris.

Table 5.10 Theta and number of effective migrants per generation amongst pooled populations from 6 locations based on $\mathrm{COI}$ and $\mathrm{Cytb}$ sequences for Poecillastra laminaris

\begin{tabular}{|c|c|c|c|c|c|c|}
\hline & To Kermadec & To Challenger T & To Hikurangi & To Chatham & To Campbell & To Macquarie \\
\hline From Kermadec Ridge (North) & 0.0001 & 156.8 & 2360 & 470.37 & 0.00 & 0.00 \\
\hline $\begin{array}{l}\text { From Challenger Plateau } \\
\text { (North) }\end{array}$ & 1010.00 & 0.0027 & 0.00 & 417.08 & 0.00 & 0.00 \\
\hline $\begin{array}{l}\text { From Hikurangi Margin } \\
\text { (North) }\end{array}$ & 22.39 & 0.00 & 0.0079 & 89.57 & 0.00 & 0.00 \\
\hline From Chatham Rise (Central) & 464.27 & 2230.00 & 0.00 & 0.0067 & 650.04 & 557.16 \\
\hline From Campbell Plateau (South) & 0.00 & 0.00 & 0.00 & 0.00 & 0.0063 & 582.17 \\
\hline From Macquarie Ridge (South) & 314.76 & 366.92 & 0.00 & 0.00 & 1420.00 & 0.0042 \\
\hline
\end{tabular}

For immigration occurring between population pairs, the upper row is the receiving population and the left column is the broadcasting population. $\theta$ values are given in the diagonal. Populations were pooled: N1, N3, and N4 are Kermadec Ridge; N5 and N6 are Challenger Plateau; N7 is Hikurangi Margin; N8, N9 and N10 are Chatham Rise; S11, S13, S14 and S16 are Campbell Plateau; S12, S15, S17, S18 and S19 are Macquarie Ridge. 


\subsection{Discussion}

This study is one of only a few to have examined genetic connectivity of deep-sea invertebrate populations in the New Zealand region, and also one of a very limited number of world-wide studies to focus on deep-sea sponge population genetics. The potential reasons for the observed patterns of population genetic structure of the four study demosponges are discussed below, as well the implications of these genetic connectivity patterns for the design of MPAs to protect VMEs in the region. First, the issues relating to sampling and marker selection are considered.

\subsubsection{Sampling effect, marker selection and genetic diversity}

A multi-species approach has been employed to understand the extent of genetic connectivity, to identify the location of putative barriers to gene flow within the New Zealand region, and to identify congruent and incongruent patterns of connectivity and dispersal amongst four demosponges. As noted in previous studies, one major limitation of studies of deep-sea genetic connectivity is often the limited availability of material, both numbers of individuals per population and numbers of populations (Cho \& Shank, 2010; Miller et al., 2010; Boschen et al., 2015). In this study, because the sample sizes for all sponge populations inhabiting different sites were small, this may result in a failure to reveal the full extent of genetic diversity within such populations (Figure S5.11). For example, (1) the sample size was > 30 individuals for only Poecillastra laminaris and N. persicum, (2) the haplotype accumulation analysis showed that all four species did not have enough specimens to recover all expected haplotypic diversity, and (3) SAMOVA results indicated that a better understanding of population structure within the northern and southern provinces required more samples (Figure S5.1). In reality, an understanding of the population genetics of many deep-sea taxa has to be pursued with less than perfect sample sets because of the current management need to protect biodiversity, and particularly VMEs. Because of the recognised need to protect VMEs, studies such as that of Boschen et al. (2015) and the present one attempt to minimise the biases of small sample sizes by using conservative analytical approaches and qualitative interpretations of genetic structure and patterns.

Studies of the population genetic variation of sponges are uncommon, but typically report that the genetic diversity of the mitochondrial genome in demosponges is low and that rates of evolution are slow (Duran et al., 2004; Lavrov et al., 2005; Wörheide, 2006). 
Consistent with these previous findings, $12 S$ in Penares sp. and $N$. persicum exhibited low levels of nucleotide polymorphism (usually $\pi<0.01$ ), whereas the nucleotide diversity of $C O I$ was generally $>0.01$ in all four species, which is much higher than in the above studies. In Poecillastra laminaris, both COI and Cytb exhibited significant genetic differentiation within populations from the New Zealand region even allowing for small sample sizes, which indicates that both markers are appropriate for population genetic research for this species. Geographically widespread haplotypes may possibly be explained by mutation rates that are too low to be sensitive to recent population change events (Smith et al., 2004b). Whilst $12 S$ for Penares sp. and N. persicum and COI for P. menoui are not informative enough to reveal species-specific population structure, sufficient genetic variation within and amongst the four sponge species was observed to reveal discordant patterns of genetic structure of sufficient magnitude to be useful to inform management options.

\subsubsection{Patterns of genetic connectivity}

Several patterns of connectivity and dispersal amongst the four demosponges were revealed by mtDNA markers within and between the two lower bathyal biogeographic provinces in the New Zealand region. Penares sp. exhibited an apparent absence of regional genetic differentiation between the provinces, consistent with results for highly mobile deepsea species, including fish (Bolch et al., 1993; Varela et al., 2012, 2013) and giant squid (Winkelmann et al., 2013). Sponges are sessile and one possible explanation for panmixia is that Penares sp. larvae may have a long dispersal duration which would allow the species to overcome the physical barrier of distance between the two provinces (see section below). In addition, the present day distribution of lithistids probably reflects palaeoenvironmental change around the time of the Eocene, whereas astrophorina such as Poecillastra laminaris and other non-lithistids are not so constrained (Kelly et al., 2003; Kelly, 2003).

Between-province genetic structure was observed for Poecillastra laminaris which is consistent with a putative barrier to gene flow between the northern and southern provinces (indicated by both $C O I$ and $C y t b$ markers). Similar large-scale north-south genetic population structure has also been reported previously in the region for corals (Miller et al., 2010), fish (Smith et al., 1996a; Lévy-Hartmann et al., 2011), and a sea star (Foltz et al., 2013). Within the biogeographic provinces, gene flow was evident in all four sponge species, even at scales of 1000 s of kilometres. Similar levels of gene flow, with little or no evidence of withinprovince structure in the same region, have been observed amongst populations of fish (e.g. 
(Sedberry \& Carlin, 1996; Hoarau \& Borsa, 2000), ophiuroids (e.g., (O’Hara et al., 2014) and crustaceans (Bors et al., 2012). Other studies in the region have revealed evidence of genetic structure at smaller spatial scales within provinces, for example for deep-sea amphipods (Knox et al., 2012) and polychaetes (Bors et al., 2012). SAMOVA results (Figure S1) indicated that there was potentially genetic structure for Poecillastra laminaris within provinces, and barriers analysis of $C O I$ sequences for this species suggests a west-east barrier to population dispersal (Figure S6). However, with limited samples it is not possible to be certain that such within province population structure exists for Poecillastra laminaris.

\subsubsection{Life history and dispersal strategies}

Species-specific differences in reproductive strategies may contribute to differences in patterns of gene flow and genetic structure observed across taxa (e.g. Samadi et al., 2006; Bors et al., 2012). Populations of Poecillastra laminaris exhibited significant genetic structure amongst the geomorphic features, consistent with patterns of genetic structure exhibited by shallow-water sponges. Small-scale genetic structure was detected in Crambe crambe (Duran et al., 2004; Calderón et al., 2007), Phyllospongia lamellose, Phyllospongia alcicornis, Carterospongia flabellifera and Collospongia auris (Benzie et al., 1994), Paraleucilla magna (Guardiola et al., 2012), Scopalina lophyropoda (Blanquer et al., 2009), Xestospongia muta (López-Legentil \& Pawlik, 2008), and Xestospongia testudinaria (Bell et $a l ., 2014$ ), and they reported that asexual reproduction (Calderón et al., 2007), larval dispersal (Blanquer et al., 2009; López-Legentil \& Pawlik, 2008), inbreeding (Duran et al., 2004; Guardiola et al., 2012; Bell et al., 2014), and self-recruitment (Bell et al., 2014) all played important roles in structuring populations. In the present study, strong self-recruitment was observed in Poecillastra laminaris (Figure S5.11), further supporting the suggestion that self-recruitment is an explanation for some patterns of genetic structure detected in sponges. Deep-sea demosponges have a wide-range of reproductive strategies (Witte, 1996; Lemoine et al., 2007), but no studies have determined empirically the reproductive strategies or mode of larval dispersal for the four species examined in the present study. Studies of other demosponges have reported asexual and sexual reproduction, and dispersal by larvae and by floating propagules, to the extent that these markedly different strategies corresponded directly with the different genetic connectivity patterns amongst different species (Maldonado, 2006; Mariani et al., 2006; Lemoine et al., 2007). This limited information 
brought challenges to assessing the importance of reproductive and dispersal strategies on population structure and genetic connectivity.

Nonetheless, results of some of the analyses suggest that the study sponges are likely to have larvae capable of wide dispersal. Haplotype networks for Penares sp., Pleroma menoui and N. persicum revealed rapid population expansion (Slatkin \& Hudson, 1991). Also, statistically significant negative Tajima's D values for all species' populations and widespread distributions of haplotypes in each of the three species also might provide further support for the recent expansion. Mismatch distributions analysis accepted the hypothesis of rapid expansion of these populations in their examined provinces, although such an interpretation must be viewed with caution given the small sample sizes. This recent expansion may explain the panmixia of populations in the examined areas.

\subsubsection{Oceanographic dynamics}

The northern and southern geographical genetic differentiation reported for Poecillastra laminaris, and the within-province pattern of genetic homogeneity reported for Poecillastra laminaris, P. menoui and N. persicum, may be attributable to a relationship between sponge distribution and oceanic current systems. Currents may promote the longdistance transport of larvae, but may also act as potential barriers to gene flow or agents of retention of larvae (Rogers et al., 1998; Zane et al., 1998; Sutton \& Chereskin, 2002; Shaw et al., 2004; Plouviez et al., 2013). In the New Zealand region, the major currents with the greatest potential influence on population connectivity are associated with the Tasman Front, the Subtropical Front and the Subantarctic Front (Figure 5.3). The different currents and frontal systems give rise to different environments in the north and south of the region, and may impede larval dispersal and reduce or perhaps even block gene flow across the Chatham Rise. The haplotypic distribution of Poecillastra laminaris (which was sampled across the entire study region) supports the contention that gene flow into and through the study region could be affected by ocean current dynamics, and indicates that two main population connection routes may exist: (1) A northern route from the northwest of North Island with the southerly flow of the East Auckland Current down the east coast of North Island with the East Cape Current, and then eastwards immediately north of the Chatham Rise and the Subtropical Front, and (2) a southern route from south of South Island northwards with the Southland Current, then eastwards immediately south of Chatham Rise and the Subtropical Front. The unique Poecillastra laminaris haplotypes for COI and Cytb found at the most 
southerly sampling location in the region (Site S19) may indicate that populations in this area are isolated by the Antarctic Circumpolar Current in the Subantarctic Front. Further evidence is needed to support this hypothesis for Poecillastra laminaris, but a recent study of octocorals indicates that this current is capable of separating and structuring octocoral populations, and it acts as a 'soft' barrier (Dueñas et al., 2016). This hypothesis is also supported by studies of the present day versus palaeoenvironmental distribution of some Recent and fossil lithistid sponges (Kelly et al., 2003; Kelly, 2003; Kelly and Buckeridge, 2005).

Amongst deep-sea species found off New Zealand, genetic differentiation between the Challenger Plateau and the Chatham Rise has been found for orange roughy (Smith et al., 1996a), amphipods (Knox et al., 2012) and quill worms (Bors et al., 2012), some of which was attributed to ocean current dynamics. In this study, differentiation was not detected between populations at these two locations, although the mitochondrial marker used may be insensitive to recent population events in sponges (Becerro et al., 2012). More powerful markers, such as microsatellites and SNPs, might be able to clarify the possible existence of genetic barriers within/between provinces.

\subsubsection{Depth and associated environmental gradients}

Depth is an important factor contributing to and explaining patterns of genetic connectivity for benthic fauna, particularly for taxa that are distributed across a wide depth range (McMullin et al., 2003; Brandão et al., 2010a; Miller et al., 2010; Schüller 2011; O'Hara et al., 2014). In the present study, there was no evidence for isolation by depth. However, the small samples sizes will have reduced the power to detect such a relationship. In addition, the deepest specimen was collected from $\sim 1500 \mathrm{~m}$, whilst depth-related distinctions in population structure reported in most previous studies were deeper: $3000 \mathrm{~m}$ for the amphipod Eurythenes gryllus (Havermans et al., 2013), $2500 \mathrm{~m}$ for the bivalve Deminucula atacellana (Chase et al., 1998), and $1700 \mathrm{~m}$ for ophiuroids (O'Hara et al., 2014). However, Miller et al. (2011) reported that the stony coral Desmophyllum dianthus from different depth strata $(<600 \mathrm{~m}, 1000-1500 \mathrm{~m},>1500 \mathrm{~m})$ were strongly differentiated, indicating limited vertical larval dispersal. These authors suggested that this genetic differentiation with depth was consistent with the stratification of different water masses in the region. Further sampling across a greater depth range within the New Zealand region is 
required to test the existence of a relationship between depth and genetic differentiation for the four sponge species examined in the present study.

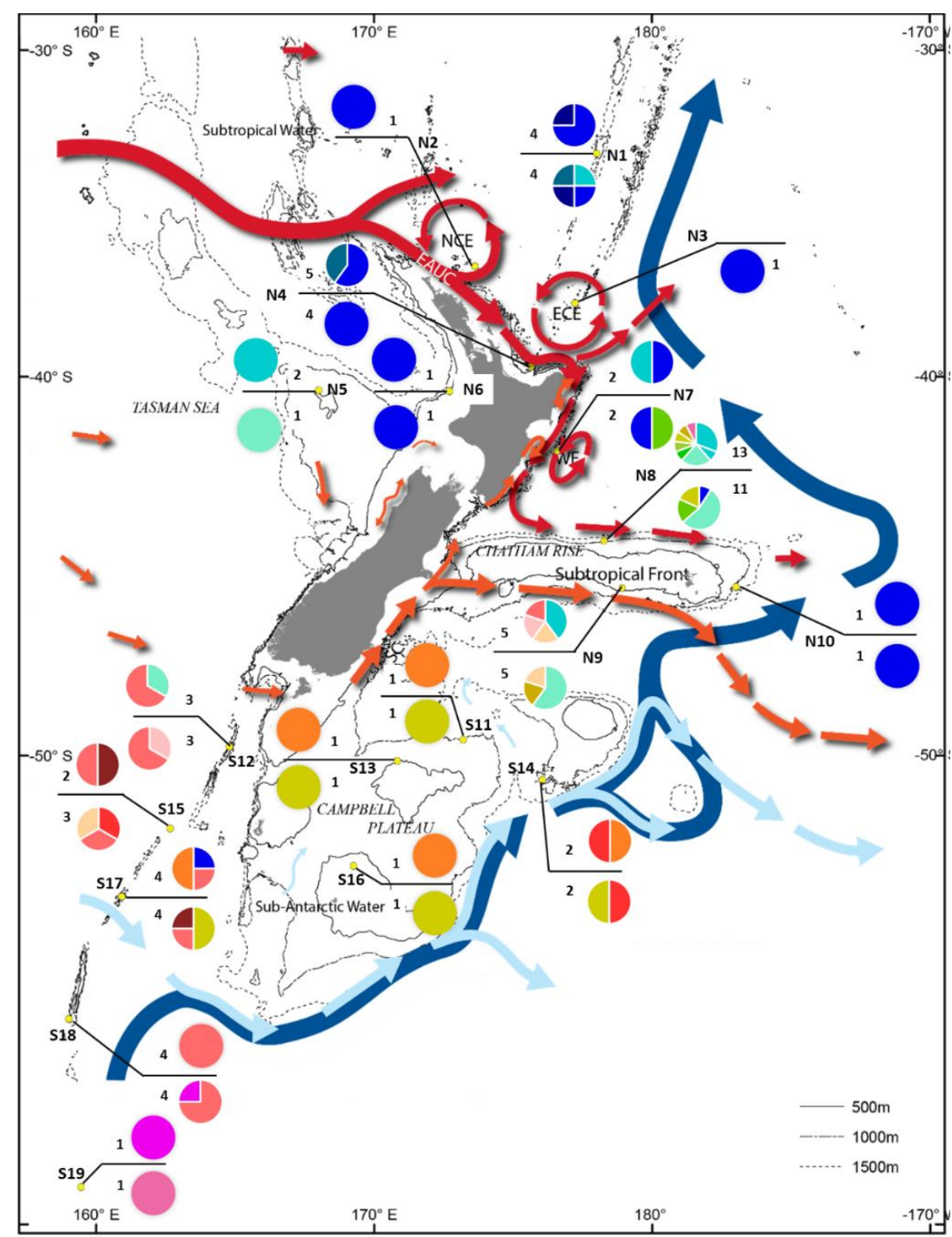

Figure 5.3 Haplotype map for Poecillastra laminaris COI (above line) and Cytb (below line) overlain with a summary of the major currents in the New Zealand region

Pie charts indicate haplotypic composition of each location and numbers indicate the total number of sequences from each location. Note EAUC, East Auckland Currents; NCE, North Cape Eddy; ECE, East Cape Eddy; WE, Wairarapa Eddy. (Leathwick et al., 2008, Bors et al., 2012).

\subsubsection{Conservation and management implications}

In order to protect the seafloor fauna from the effects of bottom trawling, 17 seamount closures and 17 Benthic Protection Areas (BPAs) were established throughout the New 
Zealand EEZ in 2001 and 2007, respectively (Brodie \& Clark, 2002; Helson et al., 2010) (Figure 5.4). These areas protect the benthos and water column fauna up to a height of $50 \mathrm{~m}$ off the substratum, from fishing-related activities. The total area protected amounts to $\sim 1.2$ million $\mathrm{km}^{2}$ of seabed that is $\sim 30 \%$ of New Zealand's EEZ. Amongst the criteria for the selection of these protected areas was that they include fauna vulnerable to disturbance from bottom trawling (i.e. VMEs) and are representative of the benthic fauna and habitats found throughout the EEZ. However, genetic connectivity of the fauna within and between these areas was not initially considered. Since their instigation, only two studies have examined the efficacy of the BPA design, and both have suggested some of the current BPAs could be relocated to provide more effective protection of biodiversity (Leathwick et al., 2008; Bors et al., 2012).

Understanding connectivity amongst areas is fundamental to designing an effective MPA network, or for modifying the existing distribution of MPAs to create a network (Palumbi, 2003). To maintain genetic diversity, protection of genetically distinct populations should also be considered as an integral component of MPA area design (Bors et al., 2012). The results of the present study indicate that populations of Penares sp., Pleroma menoui and $N$. persicum are likely to be well connected amongst the presently protected areas within their distribution. Data for Poecillastra laminaris, the most widely sampled species, provides more useful information for assessing the effectiveness of current protection measures and future MPA design in the EEZ as a proxy for VME indicator species with similar patterns of genetic connectivity (Figure 5.4). Populations associated with the Kermadec Ridge, Challenger Plateau Chatham Rise and the Macquarie Ridge may be the main genetic sources for populations around New Zealand. The Kermadec BPA provides protection for the northern source of genetic population connectivity, and this large BPA has recently been proposed as a full ocean sanctuary (http://www.mfe.govt.nz/marine/kermadec-ocean-sanctuary/questionanswers-kermadec-ocean-sanctuary). The Challenger Plateau is partly covered by a large BPA that will protect, at least in part, the genetic source populations on that part of the Plateau. However, there are no BPAs on the Macquarie Ridge, although two seamount closures to the west and east of the ridge, and a BPA to the south, may provide some protection for the southern source of genetic connectivity. There are protected areas in relatively close proximity to the remainder of the study sites that represent the range of genetic variation observed for Poecillastra laminaris across the region. However, if these areas protect this diversity will depend on whether they provide suitable habitat for VME indicator taxa such as sponges. 


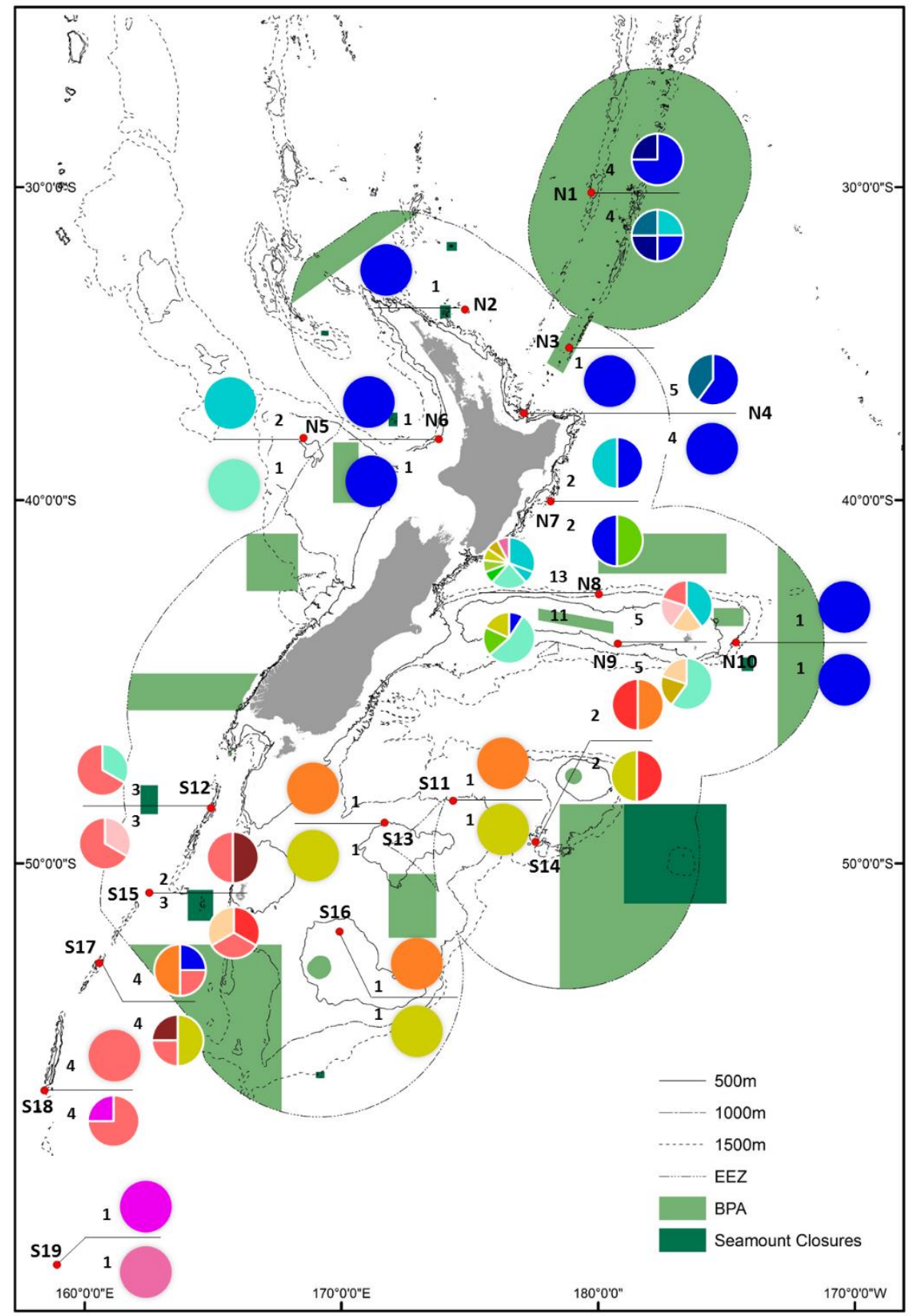

Figure 5.4 Haplotype map for Poecillastra laminaris COI (above line) and Cytb (below line) overlain with protected areas within the New Zealand EEZ

Pie charts indicate haplotypic composition of each location and numbers indicate the total number of sequences from each location.

The findings of the present study indicate that new BPAs on the Chatham Rise should be considered for sponges. The current two BPAs on the Rise cover a very limited depth range along the Rise, and this may not be sufficient to protect the genetic variation across the 
Rise. Whilst there are no population genetic data for Poecillastra laminaris from within these two BPAs, the haplotypic distributions showed that the populations sampled on the Chatham Rise had the highest genetic diversity. This result suggests that further protected areas, or an enlarging of the current BPAs, could be considered to afford greater protection to the benthic biodiversity associated with the Chatham Rise. This was also a finding of Bors et al. (2012) for other benthic taxa studied on the Chatham Rise. The results of the present study, and those of previous genetic connectivity studies around New Zealand (Smith et al., 2004b; Arango et al., 2011; Bors et al., 2012; Knox et al., 2012; Foltz et al., 2013; Boschen et al., 2015; Dueñas et al., 2016), are now beginning to provide information that can be used to improve the design (location, size and spacing) of the BPAs and any other similar protection measures in the EEZ, and demonstrate the need for a flexible spatial management system that can be periodically adjusted to accommodate increased understanding about the connectivity of a range of benthic taxa. 


\section{Appendix}

Table S5.1 Details of collections of four demosponges and haplotypes codes

\begin{tabular}{|c|c|c|c|c|c|c|c|c|}
\hline $\begin{array}{c}\text { NIWA } \\
\text { Accession }\end{array}$ & Date & Latitude & Longitude & $\begin{array}{c}\text { Depth } \\
\text { (m) }\end{array}$ & Taxon & Group & $\mathrm{COI}$ & Cytb/12S \\
\hline 18223 & $6 / 12 / 1989$ & -37.135 & 177.297 & 1050 & Neoaulaxinia persicum & 5 & Hap_3 & \\
\hline 18224 & - & -31.98 & 174.265 & 1178.5 & Neoaulaxinia persicum & 3 & Hap_1 & \\
\hline 18226 & $14 / 04 / 2002$ & -34.118 & 174.15 & 735 & Neoaulaxinia persicum & 5 & Hap_1 & Hap_1 \\
\hline 18227 & $17 / 04 / 2002$ & -34.163 & 173.962 & 786 & Neoaulaxinia persicum & 4 & Hap_1 & Hap_1 \\
\hline 18228 & $17 / 04 / 2002$ & -34.163 & 173.962 & 786 & Neoaulaxinia persicum & 4 & Hap_1 & \\
\hline 18229 & $17 / 04 / 2002$ & -34.163 & 173.962 & 786 & Neoaulaxinia persicum & 4 & Hap_1 & \\
\hline 18230 & $17 / 04 / 2002$ & -34.163 & 173.962 & 786 & Neoaulaxinia persicum & 4 & Hap_5 & Hap_1 \\
\hline 18231 & $17 / 04 / 2002$ & -34.162 & 173.962 & 795 & Neoaulaxinia persicum & 4 & Hap_1 & \\
\hline 18232 & $17 / 04 / 2002$ & -34.162 & 173.962 & 795 & Neoaulaxinia persicum & 4 & Hap_1 & \\
\hline 18233 & $17 / 04 / 2002$ & -34.162 & 173.962 & 795 & Neoaulaxinia persicum & 4 & Hap_1 & Hap_1 \\
\hline 18234 & $18 / 04 / 2002$ & -34.159 & 173.963 & 790 & Neoaulaxinia persicum & 4 & Hap_1 & Hap_1 \\
\hline 18235 & $18 / 04 / 2002$ & -34.164 & 173.964 & 812.5 & Neoaulaxinia persicum & 4 & Hap_1 & \\
\hline 18236 & $18 / 04 / 2002$ & -34.164 & 173.964 & 812.5 & Neoaulaxinia persicum & 4 & Hap_2 & \\
\hline 18238 & $19 / 04 / 2002$ & -34.042 & 174.817 & 836 & Neoaulaxinia persicum & 4 & Hap_1 & \\
\hline 18239 & $16 / 04 / 2001$ & -42.786 & 180.015 & 946.5 & Neoaulaxinia persicum & 9 & Hap_4 & Hap_1 \\
\hline 18241 & $15 / 04 / 2001$ & -42.767 & 180.011 & 816 & Neoaulaxinia persicum & 9 & Hap_5 & \\
\hline 18243 & $18 / 04 / 2001$ & -42.785 & 180.002 & 961 & Neoaulaxinia persicum & 9 & Hap_1 & \\
\hline 18246 & $21 / 04 / 2001$ & -42.761 & 180.007 & 845.5 & Neoaulaxinia persicum & 9 & Hap_6 & Hap_1 \\
\hline 18247 & $15 / 04 / 2001$ & -42.767 & 180.011 & 816 & Neoaulaxinia persicum & 9 & Hap_1 & \\
\hline 18248 & $15 / 04 / 2001$ & -42.767 & 180.011 & 816 & Neoaulaxinia persicum & 9 & Hap_1 & \\
\hline 24705 & $21 / 04 / 2001$ & -42.761 & 180.007 & 845.5 & Neoaulaxinia persicum & 9 & Hap_1 & \\
\hline 25136 & $29 / 05 / 2006$ & -42.755 & 180.006 & 945 & Neoaulaxinia persicum & 9 & Hap_6 & \\
\hline 25613 & $25 / 03 / 1967$ & -37.392 & 177.2 & 602 & Neoaulaxinia persicum & 5 & Hap_1 & Hap_1 \\
\hline 25647 & - & -37.484 & 167.685 & 904 & Neoaulaxinia persicum & 6 & Hap_6 & \\
\hline 25650 & $22 / 03 / 1982$ & -28.55 & 182.167 & 800 & Neoaulaxinia persicum & 2 & Hap_1 & Hap_2 \\
\hline 27033 & $6 / 11 / 2006$ & -39.544 & 178.332 & 817 & Neoaulaxinia persicum & 7 & Hap_1 & Hap_1 \\
\hline 27035 & $10 / 11 / 2006$ & -41.295 & 176.559 & 740.5 & Neoaulaxinia persicum & 8 & Hap_7 & \\
\hline 82328 & $18 / 04 / 2012$ & -36.452 & 177.846 & 1143 & Neoaulaxinia persicum & 5 & Hap_1 & \\
\hline 82712 & $22 / 04 / 2012$ & -36.822 & 177.46 & 903.5 & Neoaulaxinia persicum & 5 & Hap_1 & Hap_1 \\
\hline 82859 & $24 / 04 / 2012$ & -36.45 & 177.84 & 856 & Neoaulaxinia persicum & 5 & Hap_1 & \\
\hline 32057 & $6 / 03 / 2007$ & -40.03 & 178.19 & 659 & Penares sp. & 5 & Hap_1 & Hap_1 \\
\hline 40143 & $9 / 04 / 2008$ & -52.486 & 160.412 & 455 & Penares sp. & 12 & Hap_3 & Hap_1 \\
\hline 50584 & $19 / 12 / 2007$ & -43.85 & 185.643 & 802 & Penares sp. & 7 & Hap_1 & Hap_1 \\
\hline 52637 & $5 / 04 / 2008$ & -51.05 & 162.022 & 443.5 & Penares sp. & 11 & Hap_1 & Hap_1 \\
\hline 53591 & $26 / 06 / 2009$ & -44.148 & 185.308 & 520 & Penares sp. & 7 & Hap_1 & Hap_1 \\
\hline 53776 & $26 / 06 / 2009$ & -44.158 & 185.443 & 585 & Penares sp. & 7 & Hap_1 & Hap_1 \\
\hline 54032 & $27 / 06 / 2009$ & -44.145 & 185.275 & 790.5 & Penares sp. & 7 & Hap_2 & Hap_1 \\
\hline 54053 & $27 / 06 / 2009$ & -44.15 & 185.241 & 564 & Penares sp. & 7 & Hap_1 & Hap_1 \\
\hline 54239 & $28 / 06 / 2009$ & -44.16 & 185.446 & 551.5 & Penares sp. & 7 & Hap_1 & \\
\hline 64513 & $2 / 06 / 2010$ & -35.355 & 178.524 & 1187.5 & Penares sp. & 1 & Hap_4 & Hap_2 \\
\hline 64903 & $7 / 06 / 2010$ & -35.355 & 178.538 & 1305 & Penares sp. & 1 & Hap_1 & Hap_1 \\
\hline 65028 & $8 / 06 / 2010$ & -35.285 & 178.863 & 1260 & Penares sp. & 1 & Hap_5 & Hap_1 \\
\hline 66051 & $24 / 11 / 2009$ & -44.468 & 181.416 & 710 & Penares sp. & 9 & Hap_1 & Hap_1 \\
\hline
\end{tabular}




\begin{tabular}{|c|c|c|c|c|c|c|c|c|}
\hline 66087 & $16 / 07 / 2009$ & -37.333 & 168 & 935.5 & Penares sp. & 4 & Hap_1 & Hap_1 \\
\hline 72415 & $8 / 03 / 2011$ & -35.355 & 178.531 & 1315.5 & Penares sp. & 1 & Hap_1 & \\
\hline 72476 & 9/03/2011 & -35.351 & 178.54 & 1232.5 & Penares sp. & 1 & Hap_1 & Hap_1 \\
\hline 18347 & 18/06/1981 & -34.66 & 172.218 & 270 & Pleroma menoui & 7 & Hap_2 & \\
\hline 18348 & 28/11/1989 & -36.162 & 176.806 & 945 & Pleroma mепоиi & 8 & Hap_4 & \\
\hline 18354 & $17 / 04 / 2002$ & -34.146 & 173.963 & 812.5 & Pleroma menoui & 6 & Hap_2 & \\
\hline 18355 & $14 / 04 / 2002$ & -34.109 & 174.134 & 581 & Pleroma menoui & 6 & Hap_2 & \\
\hline 18522 & 11/08/1999 & -24.88 & 168.363 & 529 & Pleroma menoui & 1 & Hap_2 & \\
\hline 18523 & $11 / 08 / 1999$ & -24.88 & 168.363 & 529 & Pleroma menoui & 1 & Hap_2 & \\
\hline 18524 & 11/08/1999 & -24.778 & 168.139 & 268.5 & Pleroma menoui & 1 & Hap_3 & \\
\hline 25054 & $12 / 11 / 2004$ & -37.472 & 177.224 & 211.5 & Pleroma menoui & 9 & Hap_2 & \\
\hline 25606 & 18/06/1981 & -34.66 & 172.218 & 270 & Pleroma menoui & 7 & Hap_5 & \\
\hline 31160 & $15 / 11 / 2004$ & -37.474 & 176.91 & 252 & Pleroma mепоиi & 9 & Hap_2 & \\
\hline 44566 & 23/07/1975 & -29.413 & 168.22 & 570 & Pleroma menoui & 2 & Hap_1 & \\
\hline 62490 & $4 / 03 / 2011$ & -36.448 & 177.839 & 838 & Pleroma menoui & 8 & Hap_2 & \\
\hline 73762 & $4 / 04 / 2011$ & -38.154 & 173.789 & 294 & Pleroma menoui & 10 & Hap_2 & \\
\hline 86173 & $18 / 10 / 2012$ & -30.178 & 179.74 & 404.5 & Pleroma menoui & 4 & Hap_2 & \\
\hline 86174 & $18 / 10 / 2012$ & -30.178 & 179.74 & 404.5 & Pleroma menoui & 4 & Hap_2 & \\
\hline 86180 & $18 / 10 / 2012$ & -30.178 & 179.74 & 404.5 & Pleroma menoui & 4 & Hap_2 & \\
\hline 24692 & $15 / 04 / 2001$ & -42.767 & 180.011 & 816 & Poecillastra laminaris & 8 & Hap_5 & Hap_7 \\
\hline 24694 & $16 / 04 / 2001$ & -42.786 & 180.015 & 946.5 & Poecillastra laminaris & 8 & Hap_4 & Hap_5 \\
\hline 24701 & $20 / 04 / 2001$ & -42.768 & 180.078 & 922.5 & Poecillastra laminaris & 8 & Hap_4 & Hap_5 \\
\hline 24702 & $21 / 04 / 2001$ & -42.729 & 180.11 & 1030 & Poecillastra laminaris & 8 & Hap_4 & Hap_5 \\
\hline 25102 & $28 / 05 / 2006$ & -42.758 & 180.011 & 805 & Poecillastra laminaris & 8 & Hap_6 & Hap_8 \\
\hline 25103 & $28 / 05 / 2006$ & -42.758 & 180.011 & 805 & Poecillastra laminaris & 8 & Hap_6 & Hap_9 \\
\hline 25105 & $28 / 05 / 2006$ & -42.758 & 180.011 & 805 & Poecillastra laminaris & 8 & Hap_7 & Hap_5 \\
\hline 25106 & $28 / 05 / 2006$ & -42.758 & 180.011 & 805 & Poecillastra laminaris & 8 & Hap_6 & Hap_8 \\
\hline 25193 & $4 / 06 / 2006$ & -42.787 & 180.018 & 998 & Poecillastra laminaris & 8 & Hap_8 & \\
\hline 25653 & $4 / 06 / 2006$ & -42.716 & 180.036 & 1017.5 & Poecillastra laminaris & 8 & Hap_4 & Hap_5 \\
\hline 26995 & $4 / 11 / 2006$ & -40.04 & 178.143 & 738.5 & Poecillastra laminaris & 7 & Hap_4 & Hap_6 \\
\hline 28596 & $29 / 05 / 2007$ & -38.025 & 168.442 & 572.5 & Poecillastra laminaris & 5 & Hap_4 & Hap_5 \\
\hline 31184 & $16 / 11 / 2004$ & -37.334 & 177.08 & 466 & Poecillastra laminaris & 4 & Hap_1 & Hap_1 \\
\hline 31192 & $14 / 11 / 2004$ & -37.355 & 177.102 & 270 & Poecillastra laminaris & 4 & Hap_3 & Hap_1 \\
\hline 31201 & $16 / 11 / 2004$ & -37.352 & 177.099 & 353 & Poecillastra laminaris & 4 & Hap_1 & \\
\hline 39835 & $1 / 04 / 2008$ & -50.098 & 163.471 & 1096.5 & Poecillastra laminaris & 15 & Hap_14 & Hap_13 \\
\hline 40107 & $9 / 04 / 2008$ & -52.486 & 160.412 & 455 & Poecillastra laminaris & 17 & Hap_15 & Hap_9 \\
\hline 40169 & $9 / 04 / 2008$ & -52.498 & 160.491 & 122 & Poecillastra laminaris & 17 & Hap_15 & Hap_9 \\
\hline 40308 & $9 / 04 / 2008$ & -52.398 & 160.663 & 444.5 & Poecillastra laminaris & 17 & Hap_1 & Hap_16 \\
\hline 40406 & $9 / 04 / 2008$ & -52.392 & 160.685 & 515 & Poecillastra laminaris & 17 & Hap_14 & Hap_13 \\
\hline 40734 & $15 / 04 / 2008$ & -55.381 & 158.43 & 570.5 & Poecillastra laminaris & 18 & Hap_14 & Hap_17 \\
\hline 40794 & $15 / 04 / 2008$ & -55.362 & 158.432 & 565.5 & Poecillastra laminaris & 18 & Hap_14 & Hap_13 \\
\hline 40794 & $15 / 04 / 2008$ & -55.362 & 158.432 & 565.5 & Poecillastra laminaris & 18 & Hap_14 & Hap_13 \\
\hline 40872 & $15 / 04 / 2008$ & -55.369 & 158.385 & 539 & Poecillastra laminaris & 18 & Hap_14 & Hap_13 \\
\hline 48103 & $2 / 12 / 2008$ & -48.972 & 171.679 & 526.5 & Poecillastra laminaris & 13 & Hap_15 & Hap_9 \\
\hline 51814 & $19 / 04 / 2002$ & -34.053 & 174.808 & 865 & Poecillastra laminaris & 2 & Hap_1 & \\
\hline 51910 & $21 / 04 / 2001$ & -42.721 & 180.039 & 951 & Poecillastra laminaris & 8 & Hap_9 & \\
\hline 51914 & $21 / 04 / 2001$ & -42.721 & 180.039 & 951 & Poecillastra laminaris & 8 & Hap_10 & Hap_9 \\
\hline 52473 & $3 / 06 / 2007$ & -38.204 & 168.584 & 519 & Poecillastra laminaris & 5 & Hap_4 & Hap_5 \\
\hline 52500 & $16 / 11 / 2004$ & -37.352 & 177.099 & 353 & Poecillastra laminaris & 4 & Hap_3 & Hap_1 \\
\hline
\end{tabular}




\begin{tabular}{|c|c|c|c|c|c|c|c|c|}
\hline 52632 & $5 / 04 / 2008$ & -51.05 & 162.022 & 443.5 & Poecillastra laminaris & 15 & Hap_17 & Hap_15 \\
\hline 52634 & $30 / 03 / 2008$ & -48.535 & 164.948 & 1086 & Poecillastra laminaris & 12 & Hap_5 & Hap_12 \\
\hline 52639 & $19 / 04 / 2008$ & -59.049 & 158.908 & 1507.5 & Poecillastra laminaris & 19 & Hap_18 & Hap_18 \\
\hline 52966 & $4 / 11 / 2006$ & -40.04 & 178.147 & 765 & Poecillastra laminaris & 7 & Hap_1 & Hap_1 \\
\hline 53408 & $22 / 06 / 2009$ & -42.835 & 180.013 & 822.5 & Poecillastra laminaris & 8 & Hap_11 & Hap_5 \\
\hline 53777 & $26 / 06 / 2009$ & -44.158 & 185.443 & 585 & Poecillastra laminaris & 9 & Hap_12 & Hap_10 \\
\hline 53778 & $26 / 06 / 2009$ & -44.158 & 185.443 & 585 & Poecillastra laminaris & 9 & Hap_13 & Hap_5 \\
\hline 53985 & $27 / 06 / 2009$ & -44.149 & 185.307 & 553 & Poecillastra laminaris & 9 & Hap_4 & Hap_5 \\
\hline 54352 & $28 / 06 / 2009$ & -44.03 & 185.409 & 812 & Poecillastra laminaris & 9 & Hap_4 & Hap_5 \\
\hline 58816 & $11 / 10 / 2002$ & -48.403 & 174.341 & 896 & Poecillastra laminaris & 11 & Hap_15 & Hap_9 \\
\hline 62135 & $27 / 06 / 2009$ & -44.15 & 185.241 & 564 & Poecillastra laminaris & 9 & Hap_14 & Hap_11 \\
\hline 65027 & $8 / 06 / 2010$ & -35.284 & 178.864 & 1265 & Poecillastra laminaris & 3 & & Hap_1 \\
\hline 70623 & $30 / 11 / 1998$ & -48.618 & 164.885 & 940 & Poecillastra laminaris & 12 & Hap_14 & Hap_13 \\
\hline 70624 & $30 / 11 / 1998$ & -48.618 & 164.885 & 940 & Poecillastra laminaris & 12 & Hap_14 & Hap_13 \\
\hline 70625 & $17 / 03 / 1978$ & -44.16 & 180.763 & 320 & Poecillastra laminaris & 10 & Hap_1 & Hap_1 \\
\hline 73767 & $4 / 04 / 2011$ & -38.154 & 173.789 & 294 & Poecillastra laminaris & 6 & Hap_1 & Hap_1 \\
\hline 76254 & $14 / 11 / 2004$ & -37.355 & 177.101 & 276.5 & Poecillastra laminaris & 4 & Hap_1 & Hap_1 \\
\hline 76474 & $5 / 09 / 2006$ & -51.68 & 169.914 & 370 & Poecillastra laminaris & 16 & Hap_15 & Hap_9 \\
\hline 76823 & $22 / 04 / 2003$ & -49.612 & 178.776 & 318.5 & Poecillastra laminaris & 14 & Hap_15 & Hap_9 \\
\hline 81658 & $18 / 10 / 2012$ & -30.187 & 179.72 & 410 & Poecillastra laminaris & 1 & Hap_1 & Hap_2 \\
\hline 86091 & $18 / 10 / 2012$ & -30.187 & 179.72 & 410 & Poecillastra laminaris & 1 & Hap_1 & Hap_3 \\
\hline 86172 & $18 / 10 / 2012$ & -30.178 & 179.74 & 404.5 & Poecillastra laminaris & 1 & Hap_2 & \\
\hline 86178 & $18 / 10 / 2012$ & -30.177 & 179.737 & 404 & Poecillastra laminaris & 1 & & Hap_4 \\
\hline 86182 & $18 / 10 / 2012$ & -30.178 & 179.74 & 404.5 & Poecillastra laminaris & 1 & Hap_1 & Hap_1 \\
\hline 86710 & $13 / 11 / 2008$ & -49.308 & 176.328 & 1040 & Poecillastra laminaris & 14 & Hap_16 & Hap_14 \\
\hline
\end{tabular}

Table S5.2 Poecillastra laminaris

Pairwise $\Phi_{S T}$ values for $C O I$ (below diagonal) and $C y t b$ (above diagonal) amongst populations

\begin{tabular}{lccccccccc}
\hline Population & Kermadec & Three Kings & Challenger & NW Slope & Hikurangi & Chatham & Campbell & Bounty & Macquarie \\
\hline Kermadec & & - & $0.891^{* *}$ & -1 & 0.41 & $0.371^{* *}$ & $0.937^{* *}$ & $0.700^{*}$ & $0.400^{* *}$ \\
Three Kings & -0.875 & & - & - & - & - & - & - & - \\
Challenger & $0.780^{*}$ & 1 & & 1 & 0.5 & -0.058 & $1.000^{*}$ & 0.421 & $0.432^{* *}$ \\
NW Slope & -0.875 & 0 & 1 & & -1 & -0.063 & 1 & -0.467 & -0.039 \\
Hikurangi & 0.264 & -1 & 0 & -1 & & -0.012 & 0.848 & 0.063 & 0.154 \\
Chatham & $0.329^{* *}$ & -0.101 & -0.104 & -0.101 & -0.072 & & $0.474^{* *}$ & 0.144 & $0.226^{* *}$ \\
Campbell & $0.95^{* *}$ & 1 & 1 & 1 & 0.919 & 0.469 & & 0.250 & $0.434^{*}$ \\
Bounty & $0.652^{*}$ & -0.727 & 0.136 & -0.727 & 0 & $0.148^{*}$ & 0.25 & & 0.058 \\
Macquarie & $0.650^{* *}$ & 0.444 & $0.502^{*}$ & 0.444 & $0.462^{*}$ & $0.210^{* *}$ & $0.467^{* *}$ & 0.238 & \\
\hline
\end{tabular}

Significant values of $\mathrm{p}<0.05$ are marked as $*$, and $\mathrm{p}<0.01$ are marked as **. - means data is unavailable. 
Table S5.3 Penares sp.

Pairwise $\Phi_{S T}$ values for $\mathrm{COI}$ (below diagonal) and $12 \mathrm{~S}$ (above diagonal) amongst populations

\begin{tabular}{lccccc}
\hline Population & Macquarie & Kermadec & Challenger & Hikurangi & Chatham \\
\hline Macquarie & 0 & -0.263 & 0 & 0 & 0 \\
Kermadec & 0.029 & & -1.000 & -1.000 & 0.111 \\
Challenger & -1.000 & -0.571 & & 0 & 0 \\
Hikurangi & -1.000 & -0.571 & 0 & & 0 \\
Chatham & 0.281 & 0.224 & -1.000 & -1.000 & \\
\hline
\end{tabular}

Significant values of $\mathrm{p}<0.05$ are marked as *, and $\mathrm{p}<0.01$ are marked as **.

Table S5.4 Neoaulaxinia persicum

Pairwise $\Phi_{S T}$ values for $\mathrm{COI}$ (below diagonal) and $12 \mathrm{~S}$ (above diagonal) amongst populations

\begin{tabular}{lccccc}
\hline Population & Kermadec & Three Kings & Chatham & Challenger & Hikurangi \\
\hline Kermadec & 0 & 0 & -0.263 & - & -1 \\
Three Kings & -0.016 & 0 & & - & 0 \\
Chatham & 0.16 & 0.01 & 0 & - & 0 \\
Challenger & 0.867 & 0.76 & 0.143 & 0 & - \\
Hikurangi & 0.273 & 0.052 & -0.262 & 0.333 & 0 \\
\hline
\end{tabular}

Significant values of $\mathrm{p}<0.05$ are marked as *, and $\mathrm{p}<0.01$ are marked as **.

Table S5.5 Pleroma menoui

Pairwise $\Phi_{S T}$ values for $C O I$ amongst populations

\begin{tabular}{lcccc}
\hline Population & Norfolk & Kermadec & Three Kings & NW Slope \\
\hline Norfolk & 0 & & & \\
Kermadec & 0.095 & 0 & & \\
Three Kings & -0.263 & -0.313 & 0 & \\
NW Slope & 0.029 & 0.225 & -0.2 & 0 \\
\hline
\end{tabular}

Significant values of $\mathrm{p}<0.05$ are marked as *, and $\mathrm{p}<0.01$ are marked as **.

Table S5.6 Tajima's (D) and the Ramos-Onsins-Rozas Test of Neutrality (R2) test results for different markers across four different species

\begin{tabular}{lccc}
\hline \multicolumn{1}{c}{ Species } & Marker & D & R2 \\
\hline Poecillastra laminaris & COI & $-0.3025(0.7623)$ & $0.0958(0.4354)$ \\
Poecillastra laminaris & Cytb & $-0.3757(0.7071)$ & $0.0940(0.4180)$ \\
Penares sp. & COI & $-0.7804(0.4352)$ & $0.1117(0.2054)$ \\
Penares sp. & $12 S$ & $-1.1552(0.2480)$ & $0.2575(0.9838)$ \\
Neoaulaxinia persicum & COI & $-0.9194(0.3579)$ & $0.0862(0.1822)$ \\
Neoaulaxinia persicum & $12 S$ & $-3.0332(0.0242)$ & $0.2890(1.0000)$ \\
Pleroma menoui & COI & $-2.1098(0.0349)$ & $0.1326(0.4366)$ \\
\hline
\end{tabular}

Note: Data expressed as value ( $\mathrm{p}$ value) 
Table S5.7 Poecillastra laminaris

Tests for detection of null alleles and selection for all ten microsatellite loci across all samples

\begin{tabular}{lccc}
\hline \multicolumn{1}{c}{ Locus } & NullAlleles? & Oosterhout & Selection? \\
\hline PLN4 & yes & 0.0865 & No \\
PLN7 & yes & 0.1826 & Positive \\
PLN8 & no & 0.0341 & No \\
PLN17 & no & -0.059 & Balancing \\
PLN21 & yes & 0.2173 & Positive \\
PLN26 & no & -0.0791 & No \\
PLN27 & no & 0.0795 & No \\
PLN34 & no & 0.0385 & No \\
PLN39 & yes & 0.0623 & No \\
PLN48 & yes & 0.3459 & No \\
\hline
\end{tabular}

Oosterhout - estimate of frequency of null alleles (from MicroChecker software)

Table S5.8 Microsatellite diversity statistics for Poecillastra laminaris at two different spatial scales

\begin{tabular}{|c|c|c|c|c|c|c|c|c|}
\hline Group & Loci & ong26 & ong34 & ong27 & ong17 & ong8 & Mean & SE \\
\hline \multirow{6}{*}{$\begin{array}{l}\text { Northern } \\
\text { province }\end{array}$} & $\mathrm{N}$ & 19 & 39 & 20 & 31 & 14 & 24.600 & 4.545 \\
\hline & $\mathrm{Na}$ & 11.000 & 14.000 & 6.000 & 16.000 & 13.000 & 12.000 & 1.703 \\
\hline & $\mathrm{Ne}$ & 5.270 & 5.783 & 3.433 & 8.430 & 9.333 & 6.450 & 1.076 \\
\hline & Ho & 0.947 & 0.769 & 0.600 & 0.903 & 1.000 & 0.844 & 0.072 \\
\hline & $\mathrm{He}$ & 0.810 & 0.827 & 0.709 & 0.881 & 0.893 & 0.824 & 0.033 \\
\hline & HWE & $* * *$ & $*$ & $*$ & $* * *$ & $\mathrm{~ns}$ & & \\
\hline \multirow{6}{*}{ North region } & $\mathrm{N}$ & 10 & 18 & 12 & 16 & 8 & 12.800 & 1.855 \\
\hline & $\mathrm{Na}$ & 6.000 & 9.000 & 6.000 & 12.000 & 9.000 & 8.400 & 1.122 \\
\hline & $\mathrm{Ne}$ & 2.985 & 3.600 & 3.600 & 8.393 & 7.529 & 5.222 & 1.132 \\
\hline & Ho & 0.900 & 0.778 & 0.667 & 0.875 & 1.000 & 0.844 & 0.057 \\
\hline & $\mathrm{He}$ & 0.665 & 0.722 & 0.722 & 0.881 & 0.867 & 0.771 & 0.043 \\
\hline & HWE & $*$ & ns & ns & $*$ & ns & & \\
\hline \multirow{6}{*}{ Central region } & $\mathrm{N}$ & 9 & 21 & 8 & 15 & 6 & 11.800 & 2.746 \\
\hline & $\mathrm{Na}$ & 7.000 & 12.000 & 4.000 & 11.000 & 9.000 & 8.600 & 1.435 \\
\hline & $\mathrm{Ne}$ & 5.400 & 6.255 & 3.122 & 6.250 & 8.000 & 5.805 & 0.793 \\
\hline & Ho & 1.000 & 0.762 & 0.500 & 0.933 & 1.000 & 0.839 & 0.095 \\
\hline & $\mathrm{He}$ & 0.815 & 0.840 & 0.680 & 0.840 & 0.875 & 0.810 & 0.034 \\
\hline & HWE & $\mathrm{ns}$ & $*$ & $\mathrm{~ns}$ & $\mathrm{~ns}$ & $\mathrm{~ns}$ & & \\
\hline \multirow{6}{*}{$\begin{array}{l}\text { Southern } \\
\text { province/ } \\
\text { South region }\end{array}$} & $\mathrm{N}$ & 12 & 23 & 8 & 16 & 6 & 13.000 & 3.033 \\
\hline & $\mathrm{Na}$ & 10.000 & 15.000 & 8.000 & 7.000 & 8.000 & 9.600 & 1.435 \\
\hline & $\mathrm{Ne}$ & 7.024 & 7.399 & 6.095 & 4.655 & 7.200 & 6.475 & 0.507 \\
\hline & Но & 1.000 & 0.826 & 0.875 & 0.938 & 0.500 & 0.828 & 0.087 \\
\hline & $\mathrm{He}$ & 0.858 & 0.865 & 0.836 & 0.785 & 0.861 & 0.841 & 0.015 \\
\hline & HWE & ns & $*$ & ns & ns & ns & & \\
\hline
\end{tabular}

$\mathrm{N}=$ number of individuals successfully PCR amplified, $\mathrm{Na}=$ No. of Different Alleles, $\mathrm{Ne}=$ No. of Effective Alleles, Ho = Observed Heterozygosity, HWE=significances of deviation from Hardy-Weinberg equilibrium. $* * *, p<0.001 ; * *, p<0.01$; ${ }^{*} \mathrm{p}<0.05 ; \mathrm{ns}, \mathrm{p}>0.05$. 
Table S5.9 Hierarchical AMOVA results for Poecillastra laminaris when tested for neutral microsatellite variation $(n=4$ loci)

\begin{tabular}{lcccccccc}
\hline \multirow{2}{*}{ Source of variation } & \multicolumn{4}{c}{ All } & \multicolumn{6}{c}{ Neutral } \\
\cline { 2 - 9 } & df & ss & Var. comp. & $\mathbf{\% V}$ & df & ss & Var. comp. & $\% \mathbf{V}$ \\
\hline Between provinces & 1 & 4.66 & 0.000 & 0 & 1 & 1.981 & 0.002 & 0 \\
Amongst individuals & 61 & 314.586 & 1.618 & $46^{* *}$ & 61 & 112.542 & 0.466 & $34^{* *}$ \\
Within individuals & 63 & 121.000 & 1.921 & $54^{* *}$ & 63 & 57.500 & 0.913 & $66^{* *}$ \\
Total & 125 & 440.246 & 3.539 & & 125 & 172.024 & 1.381 & \\
\hline Amongst regions & 2 & 12.312 & 0.029 & 1 & 2 & 5.503 & 0.022 & $2^{*}$ \\
Amongst individuals & 60 & 306.634 & 1.595 & $45^{* *}$ & 60 & 109.021 & 0.452 & $33^{* *}$ \\
Within individuals & 63 & 121.000 & 1.921 & $54^{* *}$ & 63 & 57.500 & 0.913 & $66^{* *}$ \\
Total & 125 & 440.246 & 3.544 & & 125 & 172.024 & 1.387 & \\
\hline Amongst geomorphic populations & 4 & 26.333 & 0.083 & $2^{* *}$ & 4 & 8.032 & 0.012 & 1 \\
Amongst individuals & 51 & 249.836 & 1.521 & $44^{* *}$ & 51 & 89.656 & 0.446 & $34^{* *}$ \\
Within individuals & 56 & 104.000 & 1.857 & 54 & 56 & 48.500 & 0.866 & $65^{* *}$ \\
Total & 111 & 380.170 & 3.461 & & 111 & 146.188 & 1.324 & \\
\hline
\end{tabular}

$*$, ** indicates significant level at $\mathrm{p}<0.05$ and $\mathrm{p}<0.01$, respectively.

Table S5.10 Poecillastra laminaris

Pairwise $F_{S T}$ values for neutral microsatellites amongst north, central and south regions

\begin{tabular}{lccc}
\hline & North & Central & South \\
\hline North & & & \\
Central & $0.029^{* *}$ & & \\
South & $0.023^{* *}$ & 0.002 & \\
\hline
\end{tabular}

** indicates significant level at $\mathrm{p}<0 . \overline{001 \text {. }}$

Table S5.11 Poecillastra laminaris

Pairwise $F_{S T}$ values for all microsatellites amongst geomorphic populations

\begin{tabular}{lccccc}
\hline & Campbell Plateau & Chatham Rise & NE Slope & Kermadec Ridge & Macquarie Ridge \\
\hline Campbell Plateau & & & & & \\
Chatham Rise & -0.024 & & & & \\
NE Slope & -0.005 & 0.003 & & & \\
Kermadec Ridge & $0.143^{*}$ & $0.096^{* *}$ & 0 & & \\
Macquarie Ridge & -0.008 & -0.008 & -0.022 & $0.06^{*}$ & \\
\hline
\end{tabular}

$*, * *$ indicates significant level at $\mathrm{p}<0.05$ and $\mathrm{p}<0.01$, respectively. 


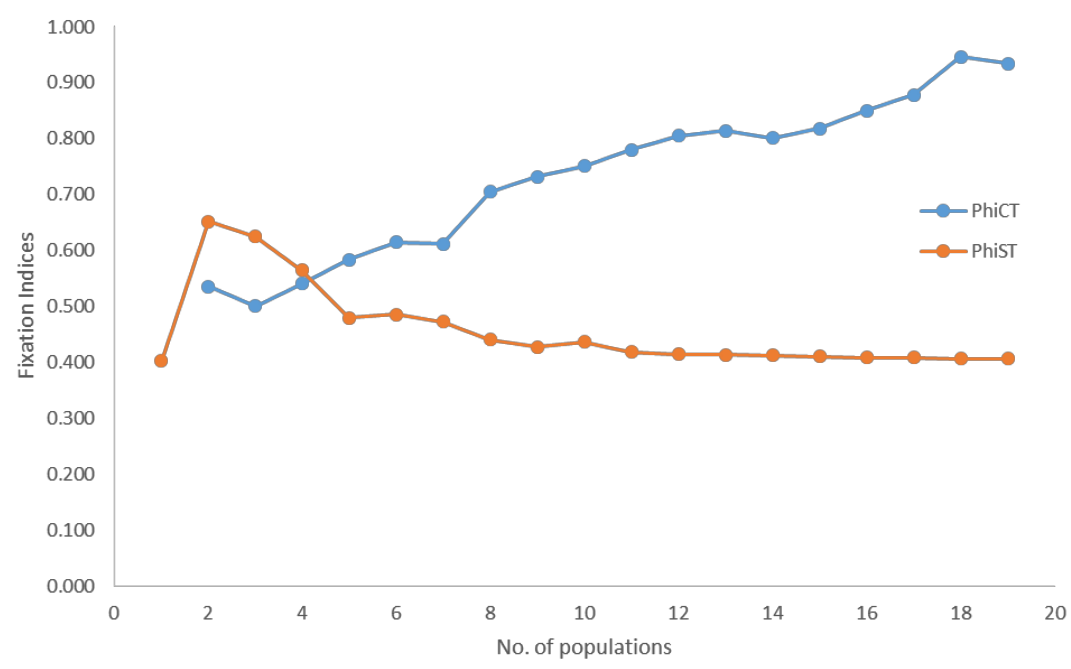

Figure S5.1 Potential number of populations of Poecillastra laminaris based on $C O I$ and $C y t b$ variation

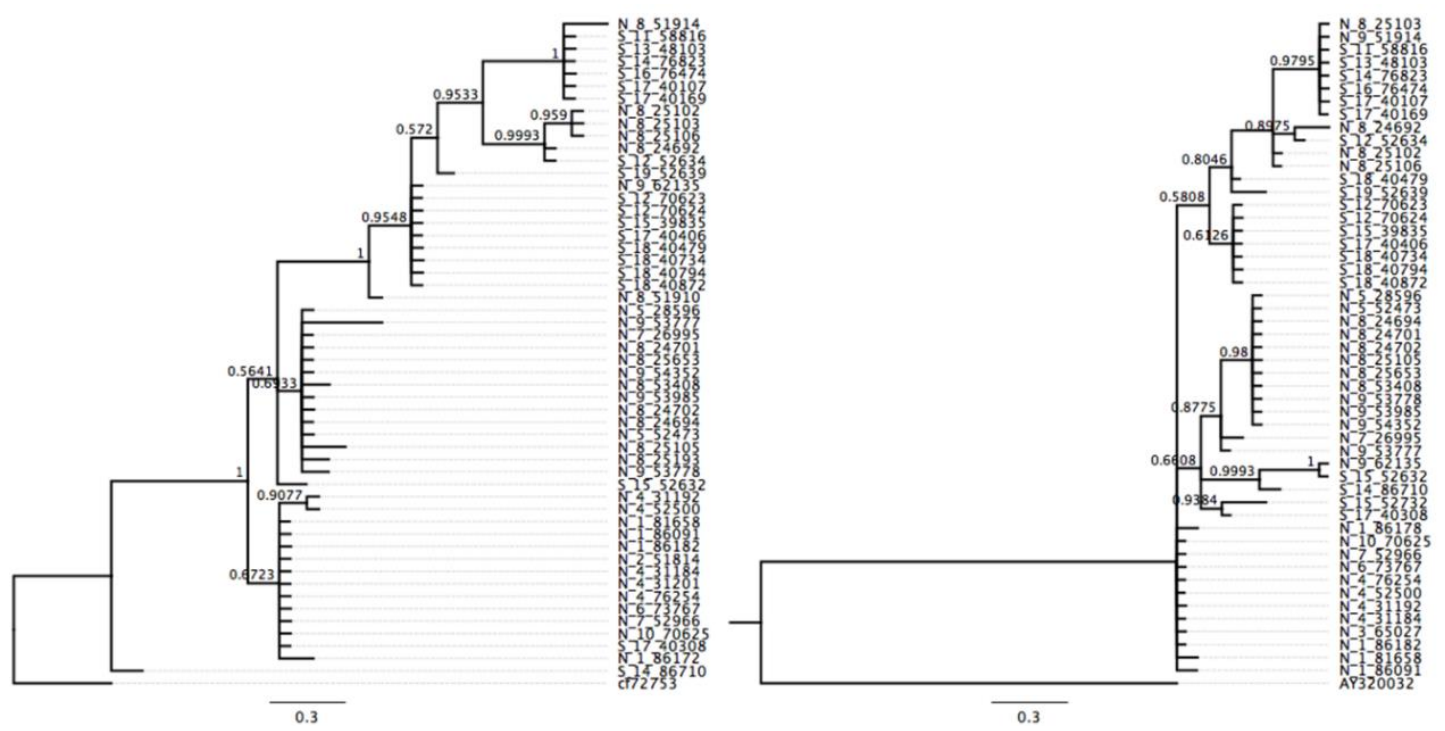

Figure S5.2 NJ phylogenetic tree of $C O I$ (left) and CytB (right) for Poecillastra laminaris

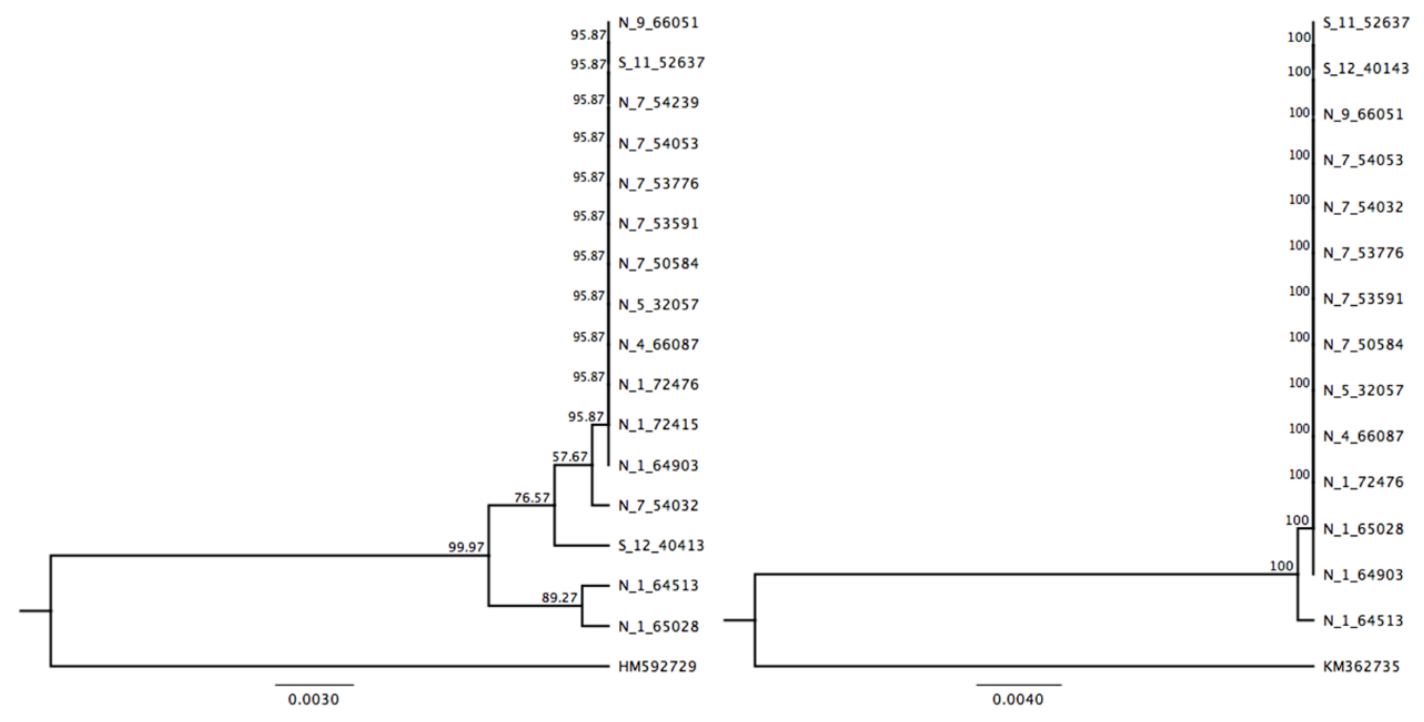

Figure S5.3 NJ phylogenetic tree of $\mathrm{COI}$ (left) and $12 \mathrm{~S}$ (right) for Penares sp. 


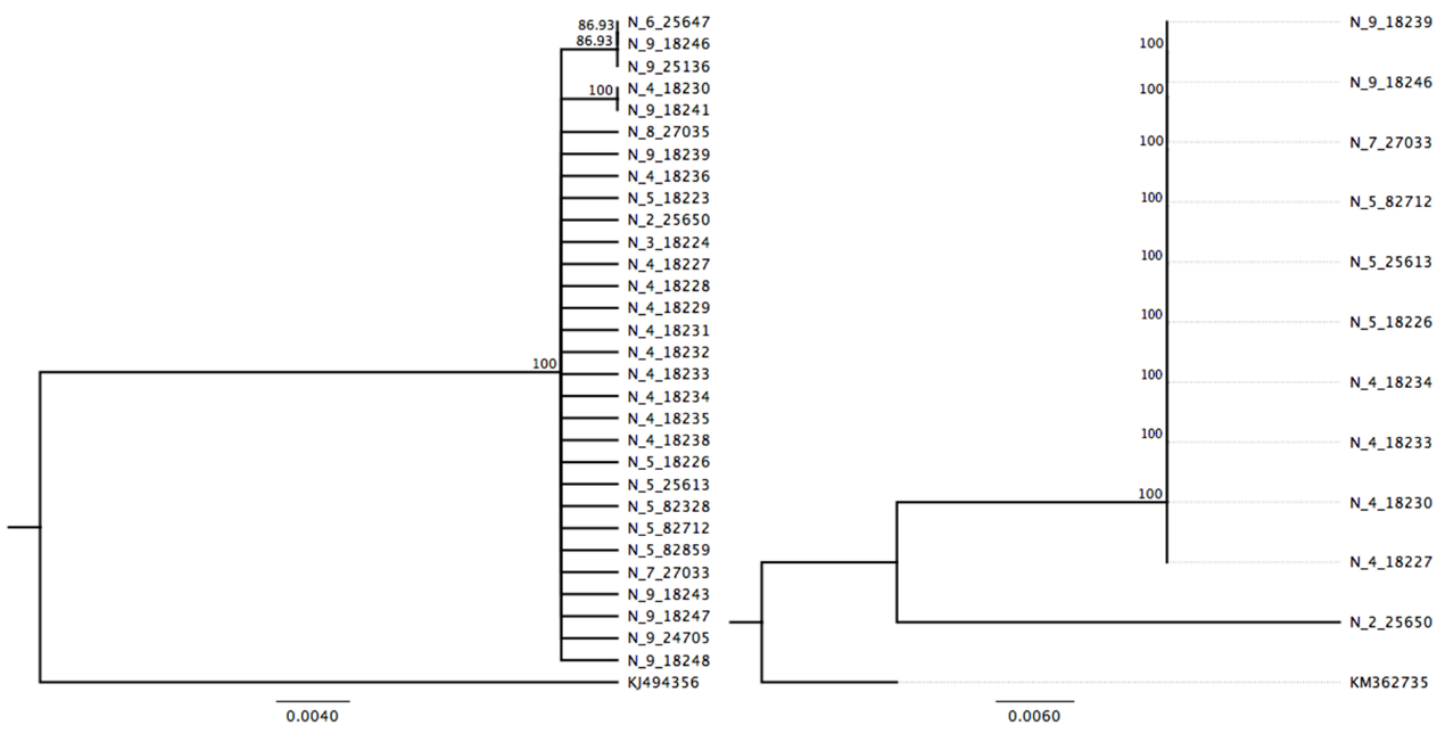

Figure S5.4 NJ phylogenetic tree of COI (left) and $12 S$ (right) for Neoaulaxinia persicum

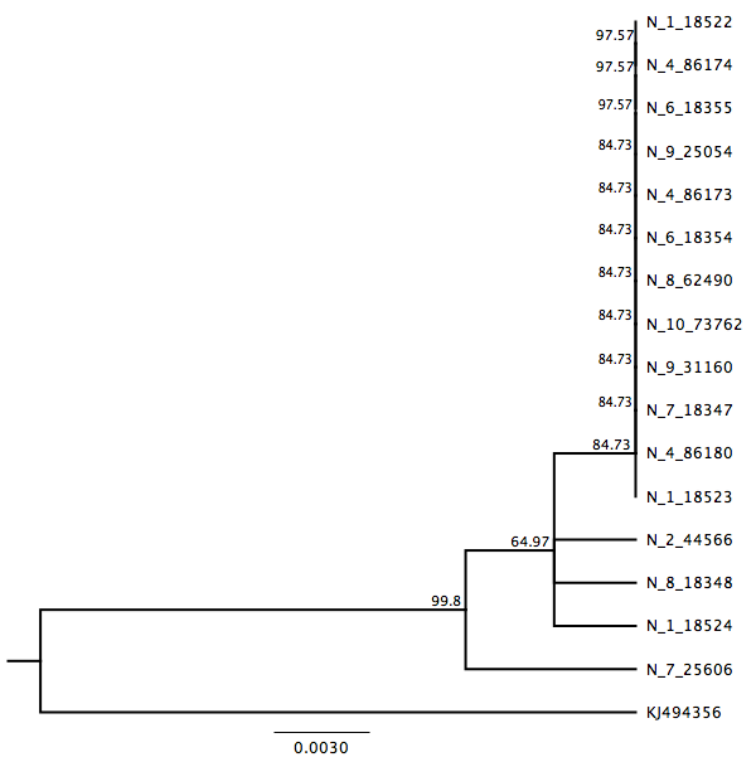

Figure S5.5 NJ phylogenetic tree of COI for Pleroma menoui 

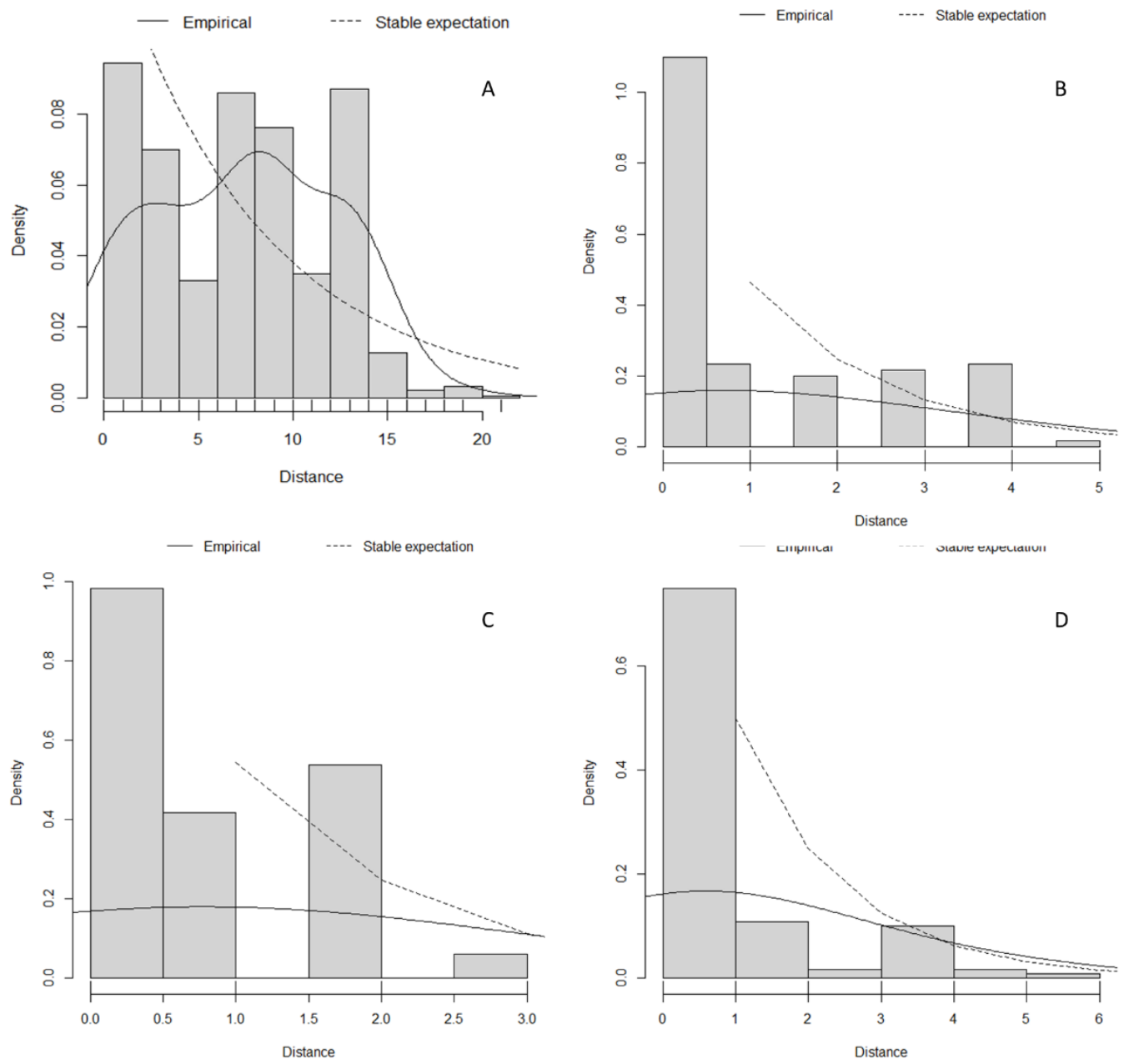

Figure S5.6 Mismatch distribution analysis of COI variation for Poecillastra laminaris (A), Penares sp. (B), Neoaulaxinia persicum (C), and Pleroma menoui (D)

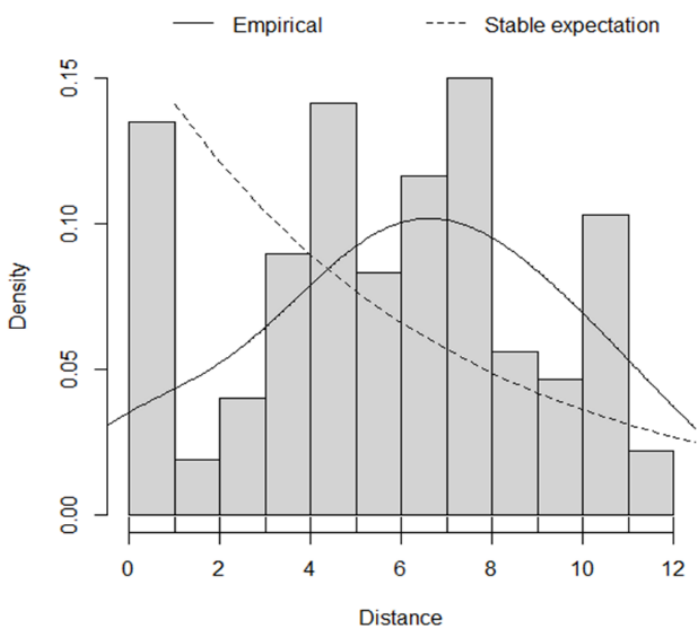

Figure S5.7 Mismatch distribution analysis of Cytb for Poecillastra laminaris 

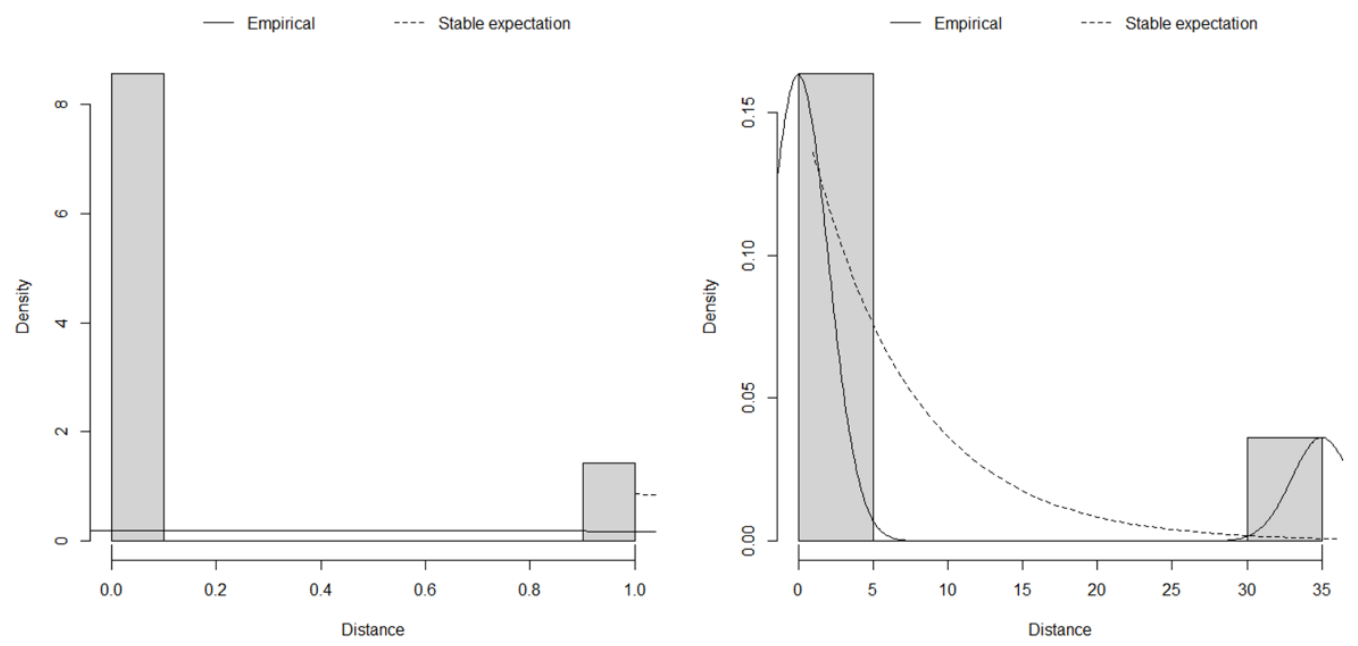

Figure S5.8 Mismatch distribution analysis of $12 S$ for Penares sp. (left) and Neoaulaxinia persicum (right)

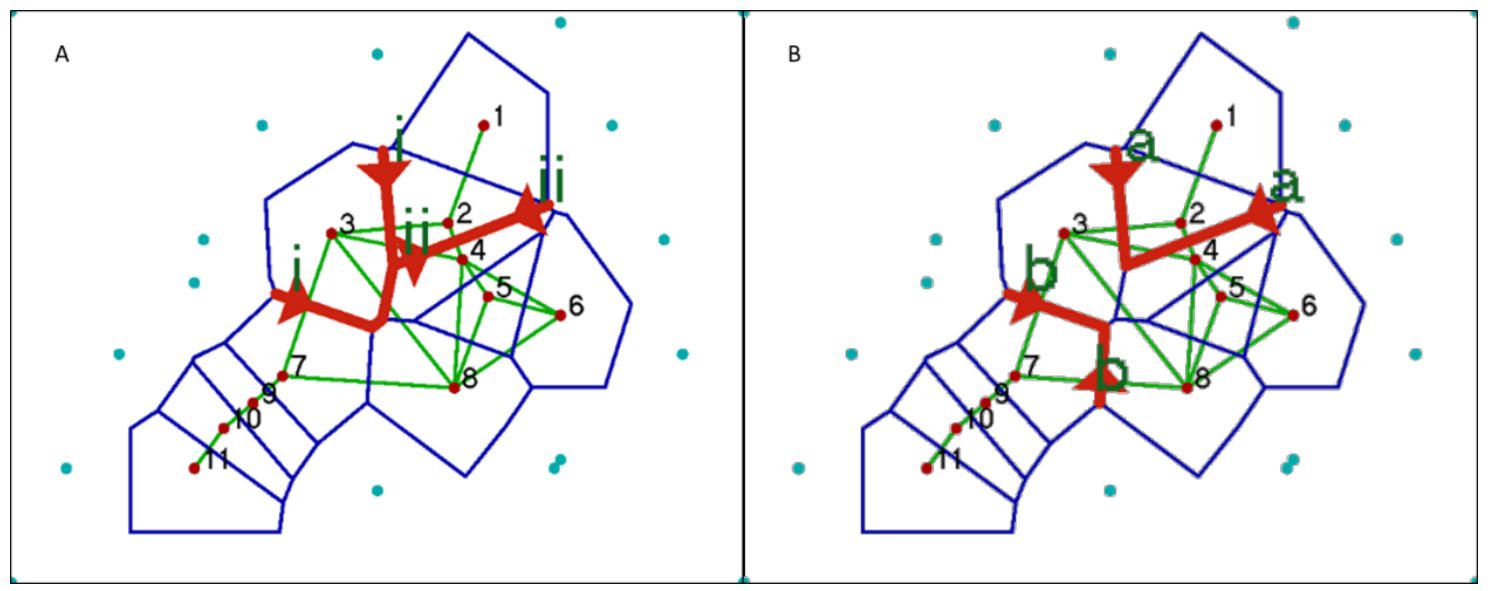

Figure S5.9 Predicted genetic barriers (red lines) for Poecillastra laminaris populations based on COI (A) and Cytb (B) from Barriers software 

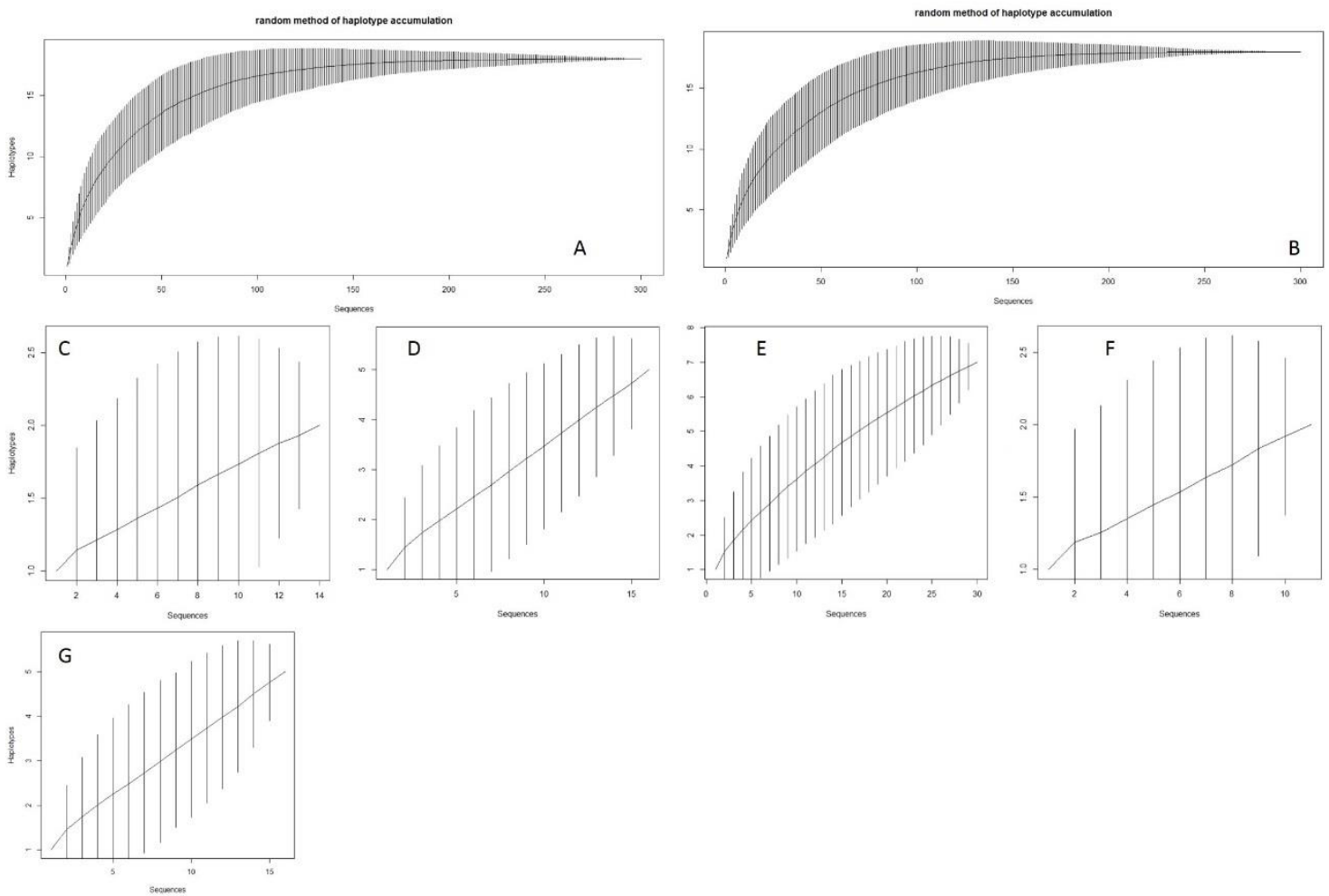

Figure S5.10 Sampling effect test for all species on different markers. A. COI of Poecillastra laminaris, B. Cytb of Poecillastra laminaris, C. COI of Penares sp., D. $12 S$ of Penares sp., E. COI of Neoaulaxinia persicum, F. 12S of Neoaulaxinia persicum, G. COI of Pleroma menoui

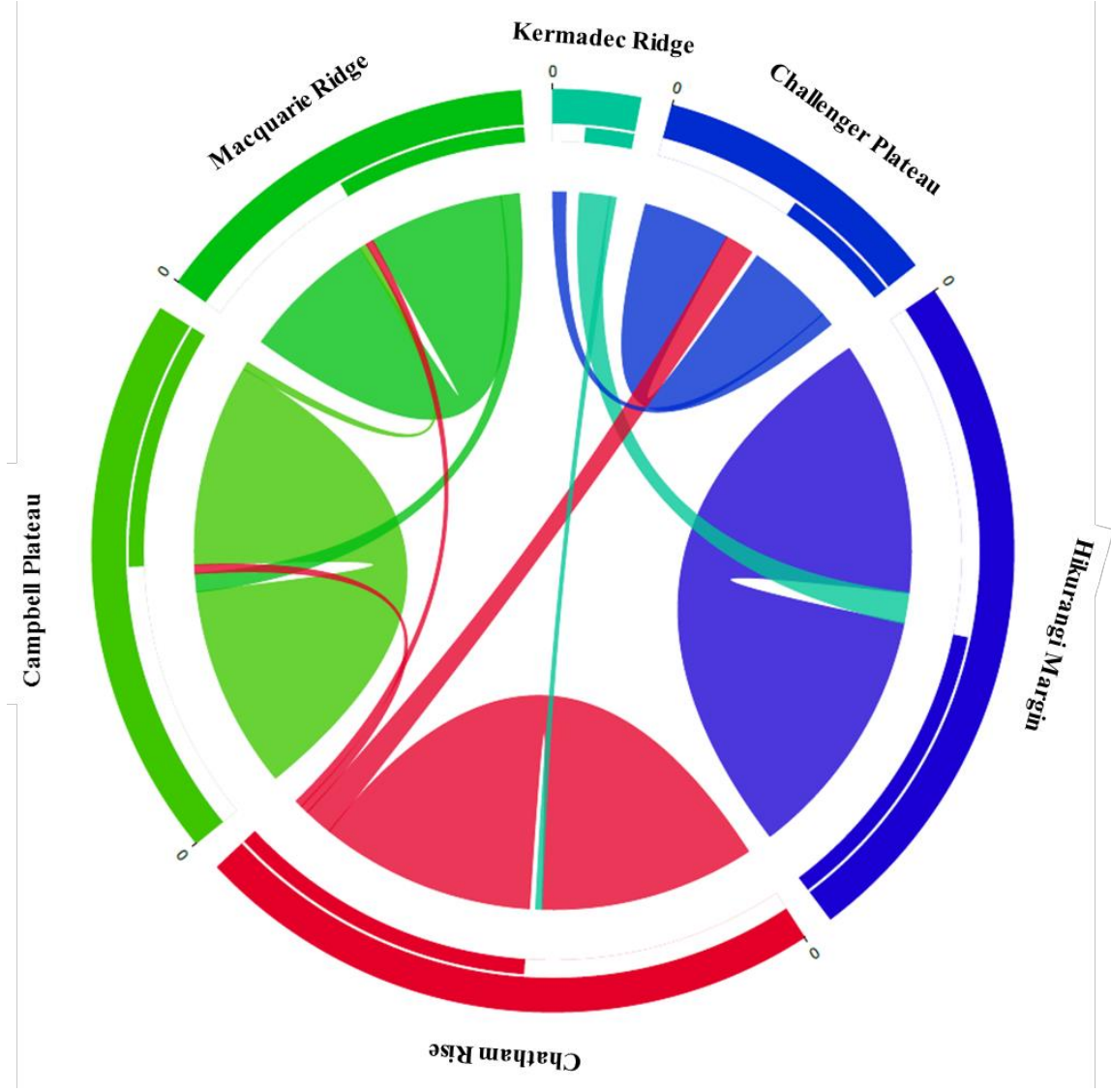

Figure S5.11 Circular plot of migration (gene flow) between and within populations of Poecillastra laminaris on geomorphic features based $C O I$ and $C y t b$ sequences 
Chapter 6. Population structure and genetic connectivity of stony corals (Scleractinia) in the New Zealand region based on analysis of microsatellite and sequence data: implications for conservation and management 


\subsection{Introduction}

Knowledge of the deep sea is still very limited because of the logistical limitations and costs of sampling such a large and inaccessible ecosystem (Ramirez-Llodra et al., 2011). Whilst deep-sea communities and their ecological structures and functions are still being described, they have already faced considerable threats from anthropogenic activities such as fishing, mining, dumping, pollution and climate change (Glover \& Smith, 2003; Roberts et al., 2006; Turley et al., 2007; Ramirez-Llodra et al., 2011; Penney, 2014). Because it is impossible to protect everything in the deep sea (defined here as areas below $200 \mathrm{~m}$ depth), Vulnerable Marine Ecosystems (VMEs) have been selected as a protection priority under United Nations General Assembly Resolutions (61/105, and 59/25). VMEs are habitats and ecosystems characterised by uniqueness or rarity of their species, their significant ecological function, that are easily disturbed by anthropogenic activities and that may exhibit slow or even no recovery from disturbance (FAO, 2009). Based on the FAO characteristics, Parker et al. (2009) identified sponges, anemones, soft corals, sea fans, sea pens, stony corals, black corals, hydro corals, sea lilies and armless seastars as VME indicator taxa for the South Pacific Ocean. The presence of these taxa can be used to identify VMEs that may be vulnerable to impacts from fishing activities in the South Pacific Ocean, and guide management measures designed to protect them (Penny et al., 2009).

If VMEs are to be protected adequately by spatial closures then further research is required to improve knowledge of the distribution of VME indicator taxa, to better understand how such VMEs are connected in terms of sources and sinks of larval recruits, and to quantify the spatial scale(s) at which genetic populations are structured.

Habitat-forming stony corals are considered to be VME indicator taxa (e.g., Parker et al., 2009), and population genetics studies of these corals have reported that structure patterns vary by different species, spatial scale and different markers. Genetic structure in Lophelia pertusa, which is a common and well-studied reef-forming coral in the Northern Hemisphere, was detected at fine-spatial scales using microsatellites (Le Goff-Vitry et al., 2004; Morrison et al., 2008, 2011; Dahl et al., 2012). Populations of Desmophyllum dianthus, which is a cosmopolite cup coral, have been detected to be isolated by depth using different markers (Miller et al., 2010, 2011, 2016; Addamo et al., 2012). Recently, Miller et al. (unpublished) found a fine-scale genetic structure in Solenosmilia variabilis using microsatellites. Studies of other coral species, Enallopsammia rostrata (Miller et al., 2010), Madrepora oculata (Miller et al., 2010), Pocillopora damicornis (Flot et al., 2008), Pocillopora ligulata (Flot et al., 
2008), Pocillopora molokensis (Flot et al., 2008), Solenosmilia variabilis (Miller et al., 2010), and Stephanocyathus spiniger (Miller et al., 2010), have not detected any genetic structure, possibly due to limited samples and invariant genetic markers. Interestingly, the use of mitochondrial markers did not reveal any genetic structure in reef-forming corals such as $E$. rostrata (Miller et al., 2010), L. pertusa (Flot et al., 2013), M. oculata (Miller et al., 2010) and S. variabilis (Miller et al., 2010), and this might indicate that to detect genetic structures in these corals requires more sensitive markers.

In the New Zealand EEZ, the distribution of habitat-forming corals has been evaluated, and three of these species have an affinity for seamount habitat; S. variabilis, $M$. oculata, and Goniocorella dumosa (Tracey et al., 2011). Seamounts are the focus of several deep-sea fisheries in New Zealand waters (Clark \& O'Driscoll, 2003), and both G. dumosa and $S$. variabilis has been recorded in large quantities as bycatch from seamount fisheries (Probert et al., 1997, Anderson \& Clark, 2003). As well as an association with seamounts $G$. dumosa is also associated with phosphorite nodules on soft sediments on the Chatham Rise (Kudrass \& Rad, 1984), which suggests that G. dumosa may be impacted by any future mining of this resource (Dawson, 1984). Unfortunately, knowledge of the mode of larval development of these three corals is still poorly understood, and information about patterns of larval movement and gene flow is sparse.

These data are, however, important for management decisions related to the conservation of corals because if extensive gene exchange exists between populations then the loss of areas of reef may not be damaging to the overall genetic diversity of the species. In this chapter, efforts to elucidate the species-specific patterns of genetic connectivity amongst populations are described. Newly developed microsatellite markers were genotyped and a nuclear gene (internally transcribed spacer, ITS2) and a mitochondrial region (the control region, D-loop) were sequenced for the three VME indicator species, G. dumosa, M. oculata and S. variabilis. A province, region, and geomorphic feature hierarchical framework was employed to test the hypotheses that: (1) the water mass characteristics of two biogeographic provinces (bottom water temperature, salinity, oxygen, and particulate organic matter flux) will influence the north-south population distributions of the three species across the New Zealand region, which in turn will be reflected in a province-scale pattern of genetic structure; (2) current flows from north and south off New Zealand that meet along the Chatham Rise to the east and that form the Sub-tropical Front will act as conduits and barriers (respectively) to larval dispersal, which will be reflected in a north-central-south regionalscale pattern of genetic structure, and higher genetic diversity in the central putative mixing 
region on the Chatham Rise; and (3) hydrodynamic conditions (e.g., current flows, eddies, turbulent mixing) associated with particular topographic features (such as slopes, seamounts, plateau, rises, ridges, troughs, basins) influence the dispersal of larvae, potentially restricting dispersal amongst these features, and thereby generating genetic structure between pairs of populations of corals found on these features. Tests were also conducted to examine the potential influence of geographic distance and depth on genetic population structure. Post hoc analyses were conducted on data to examine patterns of genetic population structure and potential barriers to gene flow independent of the hypothesis testing framework, and to identify potential larval migration patterns. Results of the genetic analyses are interpreted in terms of their significance for management of these VME indicator species around New Zealand and the possible establishment of new offshore marine protected areas.

\subsection{Materials and methods}

\subsubsection{Samples}

Three structure-forming corals (Goniocorella dumosa, Madrepora oculata, Solenosmilia variabilis) were selected based on their adherence to VME criteria, wide distribution in the New Zealand region, and availability of samples. The total number of specimens across all three species was 410 (78 M. oculata, 134 G. dumosa, 198 S. variabilis). Samples were mainly sourced from the NIWA Invertebrate Collection, which archives specimens obtained from a range sampling expeditions since the 1950s. Most specimens were preserved in ethanol, with the rest dry-preserved. Additional samples were collected during a research survey to Louisville Seamount Chain in 2014 (details see Clark et al., 2015). Most of the samples were from within the New Zealand EEZ (Figure 6.1), and the majority of specimens were from seamount and slope habitats from 200-1300 m water depth (range from 198-1805 m).

In the present study, because sampling at any one sampling station recovered only one sample (which may contain one or more specimens), the specimens within $1^{\circ}$ of latitude and longitude were grouped into one preliminary group for plotting the haplotype distributions, and the preliminary groups were further assigned into different populations at different scales that reflect the various bathymetric, topographic and hydrodynamic features that could influence connectivity amongst populations. Specifically, these groups are based on the two lower-bathyal biogeographic provinces that cover the study region (northern and southern 
provinces), three regions delineated by the approximate boundaries of the subtropical front region over the Chatham Rise (north-central-south regions), and on geomorphic features (named features such as seamounts, rises, plateau etc). Due to patchy deep-sea sampling and the different distributions of the species, samples for all species were not available from all locations throughout the region. Meanwhile, to achieve a balance between the validation of results and extracting maximum information content from the specimens, the minimum sample size was set at 4 for population analysis.

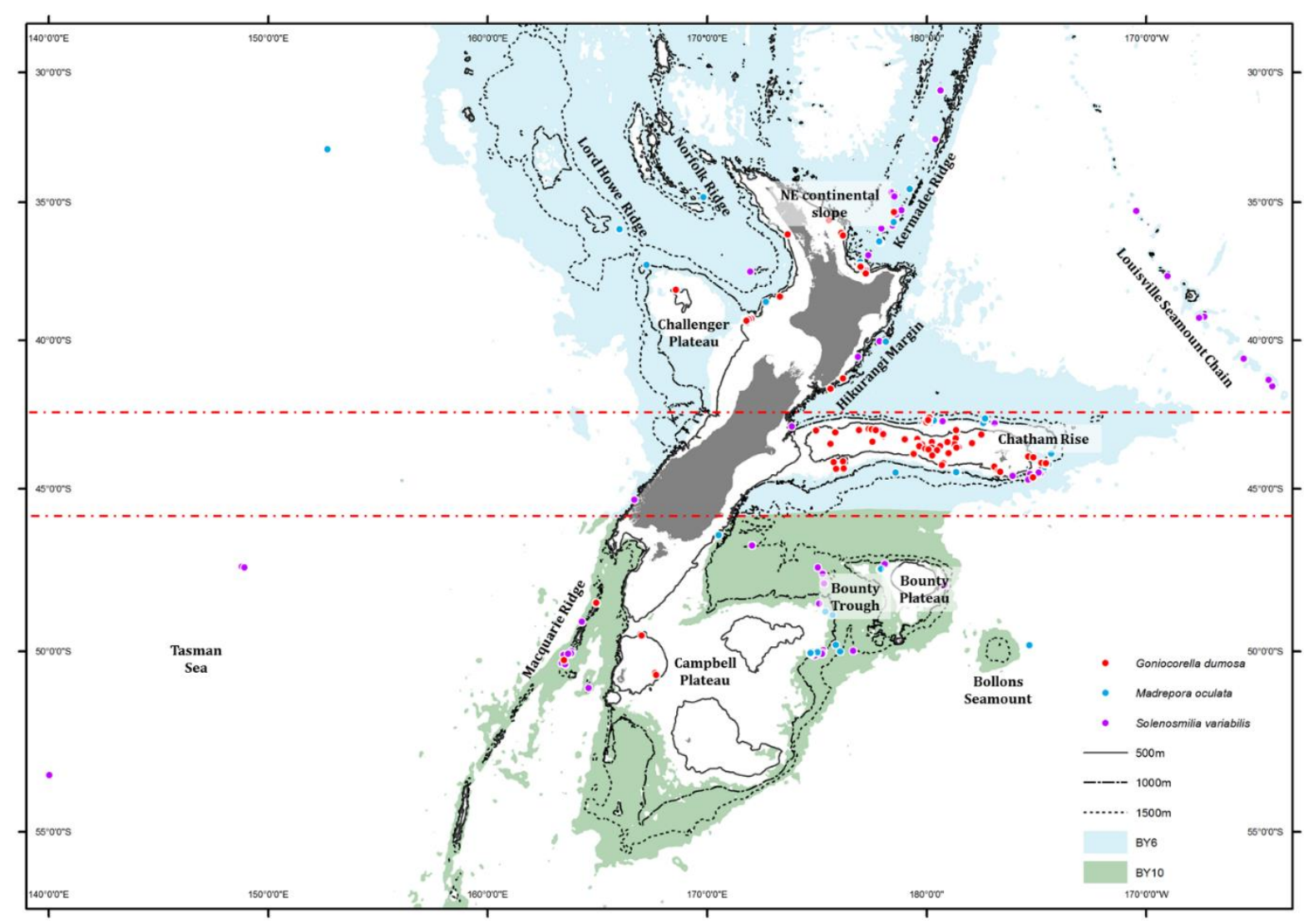

Figure 6.1 Map showing the distribution of samples amongst biogeographic provinces, regions, andgeomorphic features used for the analysis of population structure for three species of deep-sea stony corals

Provinces, BY06 in blue, BY10 in green (Watling et al., 2013), red dashed lines indicate the boundaries for the north-central-south regions, and geomorphic features were labelled with feature names. 


\subsubsection{Molecular methods}

Total DNA was extracted using a guanidinium thiocyanate extraction methodology (Boom et al., 1990) and DNA was stored at $4{ }^{\circ} \mathrm{C}$ in $0.1 \mathrm{X}$ TE buffer. To identify informative population genetic markers in Scleractinia, one mitochondrial DNA region ( $D$-loop, also called the control region) and one nuclear region (partial sequence of the internal transcribed spacer, ITS) were used to estimate genetic connectivity and population genetic differentiation. Coral-specific primers for ITS and D-loop were designed and utilised to amplify these products; primers and annealing temperatures used for all species are summarised in Chapter 4. Only ITS consistently amplified DNA from most specimens for the three species; D-loop failed to amplify in M. oculata due to its absence in the mitochondrial genome of this species (Lin et al., 2012). PCR reactions (30 $\mu$ ) for all three genes contained $1 \mu \mathrm{l}$ DNA template, 15 $\mu \mathrm{l} 2 \times$ MyTaq $^{\mathrm{TM}}$ Mix (Bioline, UK), $1 \mu \mathrm{l}$ of each primer (Macrogen, Korea), and $12 \mu \mathrm{l}$ $\mathrm{ddH}_{2} \mathrm{O}$. The thermal cycling profile consisted of an initial denaturation at $94{ }^{\circ} \mathrm{C}$ for $5 \mathrm{~min}$, then 32 cycles of a three-step programme $\left(95^{\circ} \mathrm{C}\right.$ for $30 \mathrm{~s}, 50{ }^{\circ} \mathrm{C}$ for $30 \mathrm{~s}$ and $72{ }^{\circ} \mathrm{C}$ for $45 \mathrm{~s}$ ), with a 20 -min final extension at $72{ }^{\circ} \mathrm{C}$. PCR products were sent to Macrogen (Macrogen Inc, Korea) for purification and sequencing on an ABI3730 sequencer.

In total, 27 microsatellite loci (described in Chapter 4) were utilised in G. dumosa and $S$. variabilis, and 11 microsatellite loci from a collaborator's laboratory were used for $M$. oculata (see External Contributionss). The forward primers from each primer pair were fluorescently $5^{\prime}$ end labelled with 6-FAM, NED, VIC, or PET for multiplexing (details in Chapter 4). For genotyping, PCRs were carried out in a total volume of $40 \mu$ with $20 \mu 1$ PCR Red Mixture MyTaq ${ }^{\mathrm{TM}}$ Red Mix), $1 \mu$ f forward and reverse primers, $2 \mu 1$ of template DNA and topped up with dd $\mathrm{H}_{2} \mathrm{O}$. PCR amplifications were performed with an initial denaturing step of $94{ }^{\circ} \mathrm{C}$ for $10 \mathrm{~min}$, followed by 32 cycles of $30 \mathrm{~s}$ at $94{ }^{\circ} \mathrm{C}, 45 \mathrm{~s}$ annealing at $55^{\circ} \mathrm{C}$, and $45 \mathrm{~s}$ extension at $72{ }^{\circ} \mathrm{C}$, and a final extension of $30 \mathrm{~min}$ at $72{ }^{\circ} \mathrm{C}$.

Fluorescently labelled PCR products were run on an ABI 3730 DNA Sequencer (Macrogen Inc, Korea), scored using the GeneScan-500 (LIZ) size standard, and analysed with the GeneMarker software (GeneMarker, USA). 


\subsubsection{Data analysis}

\subsubsection{D-loop and ITS}

All sequences were checked and edited using Geneious v7 (Biomatters Ltd, New Zealand). Sequences with HQ values of $<80 \%$ were discarded. Multiple sequences were aligned using the plugin ClustalW Alignment (Gap Open Cost=100 and Gap Extend Cost=10) in Geneious, and then all alignments were viewed by eye. Neighbour-Joining (NJ) phylogenetic trees were constructed with default settings and by using the highest similarity sequence in NCBI as an out-group by using the Tree Builder option in Geneious v7. For all populations with two or more individuals' intra-specific genetic diversity was evaluated by computing the number of haplotypes, the number of polymorphic sites, haplotypic diversity (h), and nucleotide diversity $(\pi)$.

Analyses of molecular variance (AMOVA) between locations (between north and south biogeographic provinces, or amongst northern, central and southern regions, or amongst geomorphic features as appropriate) and amongst populations were tested using the Arlequin software package. Pairwise comparisons of population differentiation were also performed in Arlequin and significance values estimated after 1000 permutations (Excoffier $\&$ Lischer, 2010). Between-location or within-location $\Phi_{S T}$ statistics were calculated to test for genetic differentiation amongst populations.

To visualise the spatial patterns of genetic variation for each marker, the specimens were colour-coded according to haplotype, and their geographic coordinates of collection were plotted using ArcGIS (ESRI, USA). The Mantel test (Mantel, 1967) was employed to test for isolation-by-depth by comparing the matrix of $\Phi_{S T}$ values to the matrix of depth (m) values. This test was also used to assess isolation-by-distance by comparing the matrix of $\Phi_{S T}$ values to the matrix of shortest actual distances $(\mathrm{km})$ between pairs of sites using the software GenAlEx 6.5 (Peakall \& Smouse, 2012).

\subsubsection{Microsatellites}

Micro-Checker 2.2.1 software (van Oosterhout et al., 2004) was used to identify possible genotyping errors such as stuttering, large allele dropout, and null alleles within the data set by performing 10,000 randomisations, where the threshold was set at 0.10 then raised to 0.15 when insufficient loci were present. Loci found to be under selection were removed and a second reduced data set was created. Both the full and the reduced data sets were tested, 
where appropriate, for evidence of population genetic structure and differentiation. Allelic frequencies, number of alleles, departures from Hardy-Weinberg equilibrium (HWE), linkage disequilibrium (LD) and observed and expected heterozygosities were estimated in GenAlEx 6.5. The Arlequin software package was used to estimate Weir and Cockerham's (1984) unbiased estimator of Wright's F statistic $\left(F_{S T}\right)$ and for hierarchical $F_{S T}$ analyses (AMOVA, 10,000 permutations) on the microsatellite data set. If significant differentiation amongst populations was detected, the location of the genetic discontinuity was identified using BARRIER v2.2 (Manni et al., 2004). The Mantel test was employed to test for isolation-by-depth by comparing the matrix of $F_{S T}$ values to the matrix of depth (m) values. This test was also used to test for isolation-by-distance by comparing the matrix of $F_{S T}$ values to the matrix of shortest actual distances $(\mathrm{km})$ between pairs of sites by software GenAlEx 6.5. The software package LOSITAN was employed to test the selected loci for whether they were neutral or under selection (Antao et al., 2008). Additionally, multilocus matches in GenAlex were employed to detect the asexual/colonial relationship between individuals.

Discriminant analysis of principal components (DAPC) (Jombart et al., 2010) was used to identify population structure amongst the microsatellite multi-locus genotypes of all individuals. This method entails no assumptions regarding the cause of structure (i.e., island model versus isolation-by-distance (IBD)). Analysis was implemented in $\mathrm{R} 3.0 .2$ ( $\mathrm{R}$ Development Core Team 2015) using the package "adegenet" 1.4-2 (Jombart, 2008). The optimal number of genetic clusters $(\mathrm{K})$ was chosen when the Bayesian Information Criterion (BIC) values were the lowest. Scatterplots of microsatellite genotypes in relation to discriminant functions were created in "adegenet". Principal components analysis (PCA) in the software package GenAlex was also used to examine population structure.

STRUCTURE (v2.3.4) was employed to infer the number of distinct genetic groups based on Bayesian assignment analysis (Pritchard et al., 2000; Falush et al., 2007). A total run length of 50,000 generations with a burn-in period of 5,000 generations was employed. The models were run in three iterations for $\mathrm{K}$ (number of distinct genetic clusters) values to evaluate likelihood scores and consistency amongst runs (Falush et al., 2007). The optimal number of $\mathrm{K}$ was chosen as the maximum number of clusters based on preliminary analyses, which yielded likelihoods lower than those observed at lower values of $\mathrm{K}$. This number was computed in the online program Structure Harvester (Earl \& vonHoldt, 2012) (available: http://taylor0.biology.ucla.edu/structureHarvester/).

Where any significant $F_{S T}$ or $\Phi_{S T}$ had detected at the scale of geomorphic features, MIGRATE (Beerli \& Felsenstein, 1999; Beerli, 2006) was used to estimate both theta $(\theta)$, 
which is equal to $4 \mathrm{Ne} \mu$ (where $\mathrm{Ne}$ is the long-term effective population size, and $\mu$ is the mutation rate) and $M$, which is equal to the ratio $\mathrm{m} / \mu$ (where $\mathrm{m}$ is the immigration rate). Analyses were performed under the allele model: each Markov chain Monte Carlo (MCMC) run consisted of 10 short chains (sampling 10,000 trees) and three long chains (sampling 100,000 trees) with a burn-in period of 10,000 trees. All runs were repeated five times to verify consistency of results, and the data presented are the average of the five runs. The results were visualised using the circlize package in $\mathrm{R}$ (Gu et al., 2014).

\subsection{Results}

Working on historic (sample age: G. dumosa 1 57 years, average 23 y; M. oculata $1 \sim 55 \mathrm{y}$, average $15 \mathrm{y}$; S. variabilis $1 \sim 50 \mathrm{y}$, average $9 \mathrm{y})$ and also on dry-preserved (31.56\% of G. dumosa and $55.49 \%$ of M. oculata and $58.70 \%$ of $S$. variabilis) samples is a challenge for any genetic study. Before DNA extraction, only $28.09 \%$ of $G$. dumosa, $20.13 \%$ of M. oculata, and $52.62 \%$ of $S$. variabilis passed the initial selection for the following molecular experiments (Table S6.1). After several tests of efficiency of DNA extraction (salt, CTAB, phenol-chloroform, and some commercial kits), the method described above in the previous section was the best performing. Nevertheless, none of the extraction methods worked particularly well for M. oculata. Although the species-specific collections had wide-ranging distributions around the New Zealand EEZ and beyond, DNA sample availability was ultimately limited in some regions for each species.

\subsubsection{Population structure based on ITS and D-loop variation}

After trimming and checking, ITS sequence data were obtained for all 3 species and D-loop sequence data for $G$. dumosa and $S$. variabilis. For ITS, haplotypic diversity was relatively low, with only 6 haplotypes in $83 \mathrm{G}$. dumosa specimens, 4 haplotypes in $40 \mathrm{M}$. oculata and 12 haplotypes in 118 S. variabilis. For D-loop, haplotypic diversity in $S$. variabilis was much lower than that in G. dumosa, with 12 haplotypes in 26 G. dumosa and 4 haplotypes in 141 S. variabilis specimens (Figure S6.1).

\subsubsection{Population genetic diversity}

For G. dumosa, greater ITS haplotypic diversity was found in the southern province than in the northern province, but nucleotide diversity in the northern province was greater 
than in the southern province. D-loop showed that both haplotypic and nucleotide diversity of the southern province was greater than in the northern province. Samples from the Kermadec Ridge had the highest haplotypic and nucleotide diversity for both ITS and D-loop (Table 6.1 and Figure 6.2).

For M. oculata, the ITS haplotypic and nucleotide diversity of the northern province were greater than those of the southern province, and the ITS haplotypic and nucleotide diversity values decreased from the northern to the southern region. The highest haplotypic and nucleotide diversity values were found on the Kermadec Ridge amongst the geomorphic features (Table 6.2 and Figure 6.2).

Table 6.1 ITS and D-loop diversity statistics for Goniocorella dumosa

\begin{tabular}{|c|c|c|c|c|c|c|c|c|c|c|c|}
\hline \multirow{2}{*}{ Populations } & \multicolumn{6}{|c|}{ ITS } & \multicolumn{5}{|c|}{ D-loop } \\
\hline & $\mathbf{N}$ & $\mathbf{n}$ & $\mathbf{s}$ & $\mathbf{h}$ & Hd & $\pi$ & $\mathbf{n}$ & $\mathbf{s}$ & $\mathbf{h}$ & Hd & $\pi$ \\
\hline Northern province & 125 & 80 & 22 & 5 & 0.636 & 0.023 & 26 & 51 & 11 & 0.825 & 0.028 \\
\hline Southern province & 8 & 3 & 1 & 2 & 0.667 & 0.002 & 2 & 25 & 2 & 1.000 & 0.048 \\
\hline North region & 31 & 25 & 21 & 4 & 0.600 & 0.021 & 6 & 30 & 5 & 0.933 & 0.028 \\
\hline Central region & 94 & 55 & 22 & 5 & 0.640 & 0.023 & 20 & 49 & 8 & 0.805 & 0.029 \\
\hline South region & 8 & 3 & 1 & 2 & 0.667 & 0.002 & 2 & 25 & 2 & 1.000 & 0.048 \\
\hline Kermadec Ridge & 14 & 11 & 21 & 4 & 0.709 & 0.026 & 3 & 22 & 2 & 0.667 & 0.028 \\
\hline Northeast continental slope & 7 & 3 & 0 & 1 & 0.000 & 0.000 & & & & & \\
\hline Challenger Plateau & 8 & 8 & 12 & 2 & 0.571 & 0.021 & 2 & 12 & 2 & 1.000 & 0.023 \\
\hline Hikurangi Margin & 2 & 2 & 13 & 2 & 1.000 & 0.040 & 1 & 0 & 1 & 0.000 & 0.000 \\
\hline Chatham Rise & 94 & 55 & 22 & 5 & 0.640 & 0.023 & 20 & 49 & 8 & 0.805 & 0.029 \\
\hline Campbell Plateau & 6 & 2 & 0 & 1 & 0.000 & 0.000 & & & & & \\
\hline Macquarie Ridge & 2 & 1 & 0 & 1 & 0.000 & 0.000 & 1 & 0 & 1 & 0.000 & 0.000 \\
\hline Total & 133 & 83 & 22 & 6 & 0.629 & 0.022 & 70 & 51 & 12 & 0.788 & 0.027 \\
\hline
\end{tabular}

$\mathrm{N}$ is the total number of individuals sampled for a site, $\mathrm{n}$ is the number of sequences for analysis from the site, $\mathrm{s}$ is the number of polymorphic nucleotide sites in the sequence, $\mathrm{h}$ is the number of haplotypes represented at the site, $\mathrm{Hd}$ is haplotype diversity, and $\pi$ is nucleotide diversity.

For S. variabilis ITS and D-loop variation, both the haplotypic and nucleotide diversities of the southern province were greater than those in the northern province. The haplotypic and nucleotide diversity values of ITS and D-loop decreased from the southern to the northern region. Amongst the geomorphic features, the greatest haplotypic and nucleotide diversity values of ITS were found on the Chatham Rise, whereas the greatest haplotypic and nucleotide diversity values of D-loop were found on the Macquarie Ridge (Table 6.3 and Figure 6.3). 
Table 6.2 ITS diversity statistics for Madrepora oculata

\begin{tabular}{ccccccc}
\hline Populations & N & n & s & h & Hd & $\boldsymbol{\pi}$ \\
\hline Northern province & 58 & 26 & 78 & 3 & 0.557 & 0.122 \\
Southern province & 16 & 10 & 1 & 2 & 0.200 & 0.001 \\
\hline North region & 15 & 8 & 78 & 3 & 0.679 & 0.177 \\
Central region & 43 & 16 & 41 & 2 & 0.458 & 0.082 \\
South region & 16 & 10 & 1 & 2 & 0.200 & 0.001 \\
\hline Kermadec Ridge & 7 & 5 & 64 & 2 & 0.600 & 0.168 \\
Challenger Plateau & 5 & 2 & 0 & 1 & 0.000 & 0.000 \\
Norfolk Ridge & 2 & 1 & 0 & 1 & 0.000 & 0.000 \\
Hikurangi Margin & 1 & 1 & 0 & 0 & 0.000 & 0.000 \\
Chatham Rise & 43 & 16 & 41 & 2 & 0.458 & 0.082 \\
Campbell Plateau & 9 & 4 & 0 & 1 & 0.000 & 0.000 \\
Bounty Plateau & 5 & 4 & 1 & 2 & 5.000 & 0.002 \\
Bounty Trough & 2 & 3 & 0 & 1 & 0.000 & 0.000 \\
\hline Total & 74 & 34 & 78 & 4 & 0.476 & 0.095 \\
\hline
\end{tabular}

$\mathrm{N}$ is the total number of individuals sampled for a site, $\mathrm{n}$ is the number of sequences for analysis from the site, $\mathrm{s}$ is the number of polymorphic nucleotide sites in the sequence, $\mathrm{h}$ is the number of haplotypes represented at the site, Hd is haplotype diversity, and $\pi$ is nucleotide diversity.

Table 6.3 ITS and D-loop diversity statistics for Solenosmilia variabilis

\begin{tabular}{|c|c|c|c|c|c|c|c|c|c|c|c|}
\hline \multirow{2}{*}{ Population } & \multicolumn{6}{|c|}{ ITS } & \multicolumn{5}{|c|}{ D-loop } \\
\hline & $\mathbf{N}$ & $\mathbf{n}$ & $\mathbf{s}$ & $\mathbf{h}$ & Hd & $\pi$ & $\mathbf{n}$ & $\mathbf{s}$ & h & Hd & $\pi$ \\
\hline Northern province & 148 & 96 & 155 & 9 & 0.287 & 0.023 & 119 & 4 & 4 & 0.098 & 0.000 \\
\hline Southern province & 36 & 25 & 149 & 5 & 0.420 & 0.055 & 22 & 2 & 2 & 0.173 & 0.001 \\
\hline North region & 56 & 48 & 2 & 3 & 0.121 & 0.000 & 75 & 3 & 3 & 0.079 & 0.000 \\
\hline Central region & 77 & 48 & 155 & 8 & 0.432 & 0.045 & 44 & 3 & 3 & 0.132 & 0.001 \\
\hline South region & 36 & 25 & 149 & 5 & 0.420 & 0.055 & 22 & 2 & 2 & 0.173 & 0.001 \\
\hline Kermadec Ridge & 46 & 32 & 2 & 3 & 0.179 & 0.001 & 49 & 3 & 3 & 0.119 & 0.000 \\
\hline Challenger Plateau & 1 & 1 & 0 & 1 & 0.000 & 0.000 & & & & & \\
\hline Hikurangi Margin & 9 & 2 & 0 & 1 & 0.000 & 0.000 & 8 & 0 & 1 & 0.000 & 0.000 \\
\hline Chatham Rise & 77 & 48 & 155 & 8 & 0.432 & 0.045 & 44 & 3 & 3 & 0.132 & 0.001 \\
\hline Campbell Plateau & 13 & 5 & 0 & 1 & 0.000 & 0.000 & 6 & 0 & 1 & 0.000 & 0.000 \\
\hline Bollons Seamount & 2 & 2 & 0 & 1 & 0.000 & 0.000 & 4 & 0 & 1 & 0.000 & 0.000 \\
\hline Bounty Plateau & 6 & 2 & 18 & 2 & 1.000 & 0.050 & & & & & \\
\hline Bounty Trough & 6 & 1 & 0 & 1 & 0.000 & 0.000 & 2 & 0 & 1 & 0.000 & 0.000 \\
\hline Macquarie Ridge & 19 & 12 & 147 & 4 & 0.636 & 0.103 & 8 & 2 & 2 & 0.429 & 0.002 \\
\hline Tasman Basin & 3 & 2 & 0 & 1 & 0.000 & 0.000 & & & & & \\
\hline Louisville Seamount Chain & 15 & 13 & 0 & 1 & 0.000 & 0.000 & 18 & 0 & 1 & 0.000 & 0.000 \\
\hline Total & 186 & 104 & 156 & 11 & 0.299 & 0.031 & 139 & 3 & 3 & 0.097 & 0.000 \\
\hline
\end{tabular}

$\mathrm{N}$ is the total number of individuals sampled for a site, $\mathrm{n}$ is the number of sequences for analysis from the site, $\mathrm{s}$ is the number of polymorphic nucleotide sites in the sequence, $\mathrm{h}$ is the number of haplotypes represented at the site, Hd is haplotype diversity, and $\pi$ is nucleotide diversity. 


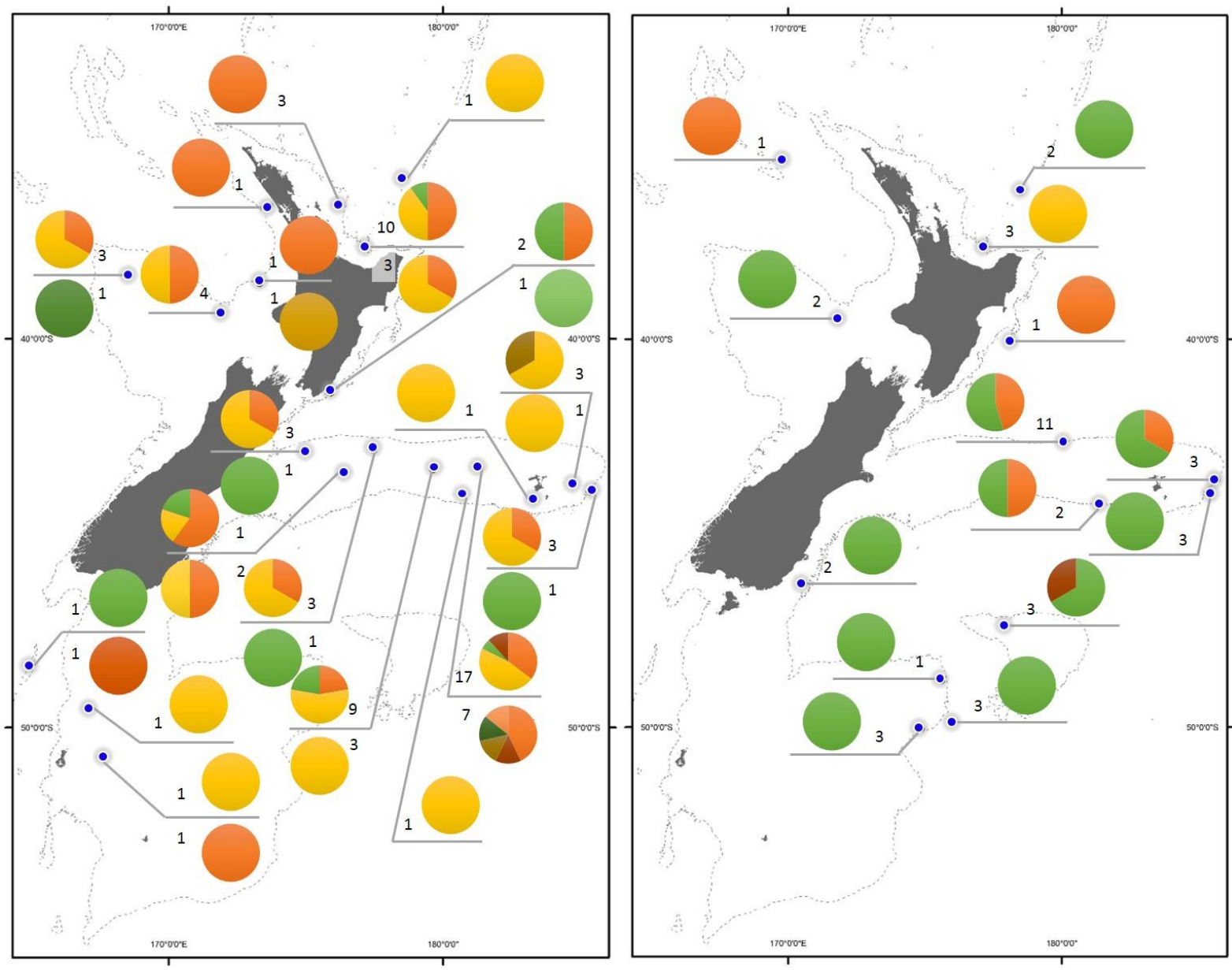

Figure 6.2 Haplotype map for Goniocorella dumosa (left) and Madrepora oculata (right) ITS (above line) and $D$-loop (below line)

Pie charts indicate haplotypic composition of each location and numbers indicate the total number of sequences from each location.

\subsubsection{Geographic distribution of haplotypes}

A single dominant ITS haplotype was observed for each of G. dumosa (Hap1), M. oculata (Hap3) and S. variabilis (Hap2) across the sampled area: other haplotypes were mainly found in a single individual (Figure S6.1). Common haplotypes of D-loop were found in most of the sampled areas for both S. variabilis and G. dumosa. The occurrence of common haplotypes across the whole region for all three species indicated that there was limited geographic genetic structure for haplotypic diversity distributions. 


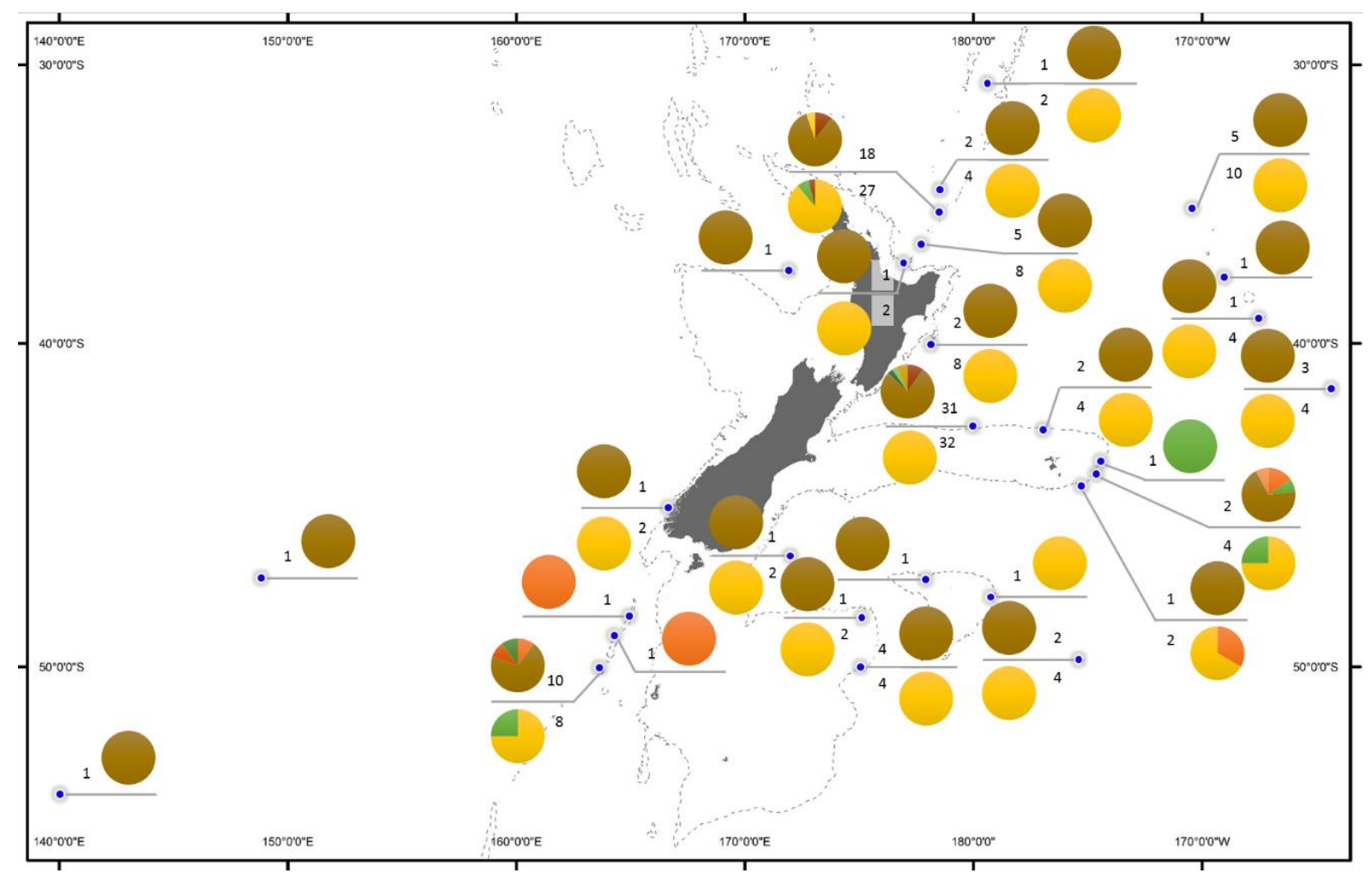

Figure 6.3 Haplotype map for Solenosmilia variabilis ITS (above line) and D-loop (below line)

Pie charts indicate haplotypic composition of each location and numbers indicate the total number of sequences from each location.

\subsubsection{Population genetic structure}

In the three corals, amongst the populations, $\Phi_{S T}$ values for both ITS and D-loop were variable but relatively low. In $G$. dumosa only one significant $\Phi_{S T}$ value was observed, between the Northeast Continental Slope and Chatham Rise populations for ITS. No significant pairwise population $\Phi_{S T}$ values were observed for $M$. oculata for ITS. These pairwise population $\Phi_{S T}$ values were calculated based on small sample sizes $(\sim 2$ individuals/population), and more robust results would be generated from larger sample sizes. However, pairwise population $\Phi_{S T}$ values of $S$. variabilis showed a significant genetic difference between the Macquarie Ridge and Louisville Seamount Chain populations in both the ITS and D-loop markers, and at the ITS marker the Macquarie Ridge population was significantly different from the Kermadec Ridge population. 
Table 6.4 Pairwise $\Phi_{S T}$ values for ITS of Madrepora oculata (above diagonal) and Solenosmilia variabilis (below diagonal) amongst geomorphic feature populations

\begin{tabular}{|c|c|c|c|c|c|c|}
\hline & $\begin{array}{l}\text { Bounty } \\
\text { Plateau }\end{array}$ & $\begin{array}{c}\text { Campbell } \\
\text { Plateau }\end{array}$ & $\begin{array}{c}\text { Chatham } \\
\text { Rise }\end{array}$ & $\begin{array}{c}\text { Kermadec } \\
\text { Ridge }\end{array}$ & $\begin{array}{c}\text { Louisville } \\
\text { Seamount Chain } \\
\end{array}$ & $\begin{array}{c}\text { Macquarie } \\
\text { Ridge }\end{array}$ \\
\hline Bounty Plateau & & 0.063 & 0.148 & 0.447 & - & - \\
\hline Campbell Plateau & - & & 0.175 & 0.5 & - & - \\
\hline Chatham Rise & - & -0.087 & & $0.457 * *$ & - & - \\
\hline Kermadec Ridge & - & -0.094 & 0.019 & & - & - \\
\hline Louisville Seamount Chain & - & 0 & -0.014 & -0.018 & & - \\
\hline Macquarie Ridge & - & -0.018 & 0.032 & $0.223^{*}$ & $0.100^{*}$ & \\
\hline
\end{tabular}

The AMOVA test of genetic structure for both ITS and D-loop across G. dumosa populations (Table 6.5) did not detect any large-scale structure (between northern/southern provinces, or amongst north, centre, south regions, or amongst geomorphic feature populations) $(\mathrm{p}>0.05)$. In contrast, ITS variation revealed significant differentiation amongst the three regions (north, centre, south) for M. oculata $(\mathrm{p}<0.05)$ (Table 6.6). In S. variabilis, both ITS and D-loop variation across the populations (Table 6.7) showed no large-scale structure. The apparent absence of differentiation amongst populations might be caused by low levels of variability across the markers.

Table 6.5 Hierarchical AMOVA results for Goniocorella dumosa when tested for genetic variation at the ITS and D-loop regions

\begin{tabular}{cccccccccc}
\hline \multirow{2}{*}{ Source of variation } & \multicolumn{4}{c}{ ITS } & \multicolumn{4}{c}{ D-loop } \\
\cline { 2 - 10 } & df & ss & Var. comp. & \%V & df & ss & Var. comp. & \%V \\
\hline Between provinces & 1 & 14.472 & 1.329 & 16.77 & 1 & 14.050 & 0.941 & 7.17 \\
Within provinces between populations & 23 & 202.969 & 0.976 & $12.32^{*}$ & 15 & 191.759 & 0.931 & 7.09 \\
Within populations & 58 & 325.872 & 5.618 & $70.91^{*}$ & 11 & 123.833 & 11.258 & 85.74 \\
Total & 82 & 543.313 & 7.923 & & 27 & 329.643 & 13.130 & \\
\hline Amongst regions & 2 & 29.294 & 0.298 & 4.36 & 2 & 18.976 & -0.701 & -5.91 \\
Within provinces between populations & 22 & 188.147 & 0.925 & $13.52^{*}$ & 14 & 186.833 & 1.300 & 10.97 \\
Within populations & 58 & 325.872 & 5.618 & $82.12^{*}$ & 11 & 123.833 & 11.258 & 94.94 \\
Total & 82 & 543.313 & 6.841 & & 27 & 329.643 & 11.857 & \\
\hline Within geomorphic features between populations & 7 & 50.141 & -0.296 & -4.51 & 4 & 47.310 & -0.438 & -3.64 \\
Within populations & 17 & 167.301 & 1.246 & $18.97 *$ & 12 & 158.500 & 1.202 & 10 \\
Total & 58 & 325.872 & 5.618 & $85.54 *$ & 11 & 123.833 & 11.258 & 93.65 \\
\hline Amongst geomorphic features & 82 & 543.313 & 6.582 & & 27 & 326.643 & 12.021 & \\
\hline
\end{tabular}

Significant values of $\mathrm{p}<0.05$ are marked as $*$, and $\mathrm{p}<0.01$ are marked as $* *$. 


\subsubsection{Isolation by distance and by depth}

Amongst the ten tests, there was no statistically significant evidence supporting isolation by distance $(\mathrm{p}>0.05)$, but ITS sequences revealed a significant isolation by depth for M. oculata and G. dumosa (Table 6.8).

Table 6.6 Hierarchical AMOVA results for Madrepora oculata when tested for genetic variation at the ITS and Microsatellites

\begin{tabular}{|c|c|c|c|c|c|c|c|}
\hline \multirow{2}{*}{ Source of variation } & \multicolumn{3}{|c|}{ ITS } & \multicolumn{4}{|c|}{ Microsatellites } \\
\hline & df & Var. comp. & $\% \mathrm{~V}$ & df & ss & Var. comp. & $\% \mathrm{~V}$ \\
\hline Between provinces & 145.818 & 1.304 & 9.25 & 1 & 1.595 & 0.020 & $2.01 * *$ \\
\hline Within provinces between populations & 16312.917 & 6.520 & $46.24 * *$ & 2 & 1.163 & -0.014 & -1.38 \\
\hline Within populations & 21131.778 & 6.255 & $44.50 * *$ & 182 & 183.78 & 1.010 & 99.37 \\
\hline Total & 38490.513 & 14.100 & & 185 & 186.538 & & \\
\hline Amongst regions & 2118.46 & 3.275 & $22.62 *$ & 2 & 2.070 & 0.015 & 1.49 \\
\hline Within provinces between populations & 15240.275 & 4.928 & $34.04 *$ & 1 & 0.688 & -0.017 & -1.68 \\
\hline Within populations & 21131.778 & 6.275 & $43.34 * *$ & 182 & 183.78 & 1.010 & 100.19 \\
\hline Total & 38490.513 & 14.478 & & 185 & 186.538 & 1.008 & \\
\hline Amongst geomorphic features & $\begin{array}{ll}6 & 184.796\end{array}$ & 3.369 & 23.68 & & & & \\
\hline Within geomorphic features between population & s 11173.939 & 4.581 & $32.21 *$ & 3 & 2.758 & -0.002 & -0.24 \\
\hline Within populations & 21131.778 & 6.275 & $44.11 *$ & 182 & 183.780 & 1.01 & 100.24 \\
\hline Total & 38490.513 & 14.225 & & 185 & 186.538 & 1.007 & \\
\hline
\end{tabular}

Significant values of $\mathrm{p}<0.05$ are marked as $*$, and $\mathrm{p}<0.01$ are marked as $* *$.

\subsubsection{Population structure based on microsatellites}

The software MICRO-CHECKER detected null alleles in three corals: loci with high frequencies of null alleles should be theoretically excluded from population genetic analysis. For G. dumosa, M. oculata and S. variabilis, 4 (Table S6.2), 5 (Table S6.3) and 5 (Table S6.4) loci exhibited null alleles, respectively. The software Lositan revealed that 11 loci in $G$. dumosa, 1 locus in M. oculata, and 8 loci in S. variabilis were under balancing selection. In M. oculata, a higher proportion of genotyping failures was detected than for the other two species. Twenty European samples of M. oculata were genotyped to verify the ability of the loci to distinguish between the New Zealand and the European samples (Figure S6.2). To investigate the effects of loci under selection, the AMOVA test and Migrate were run twice, with the neutral only and the all loci dataset. 
Table 6.7 Hierarchical AMOVA results for Solenosmilia variabilis when tested for genetic variation at the ITS and D-loop

\begin{tabular}{cccccccccc}
\hline \multirow{2}{*}{ Source of variation } & \multicolumn{4}{c}{ ITS } & \multicolumn{4}{c}{ D-loop } \\
\cline { 2 - 9 } & df & ss & Var. comp. $\%$ V & df & ss & Var. comp. \%V \\
\hline Between provinces & 1 & 17.656 & 0.345 & 4.88 & 1 & 0.125 & -0.000 & -0.24 \\
Within provinces between populations & 30 & 154.985 & -0.628 & -8.87 & 21 & 2.684 & 0.007 & 6.8 \\
Within populations & 89 & 654.855 & 7.358 & 103.99 & 118 & 10.667 & 0.090 & 91.44 \\
Total & 120 & 827.496 & 7.075 & & 140 & 13.475 & 0.097 & \\
\hline Amongst regions & 2 & 32.042 & 0.407 & 5.84 & 2 & 0.163 & -0.003 & -2.87 \\
Within provinces between populations & 29 & 140.599 & -0.791 & -11.34 & 20 & 2.645 & 0.008 & 8.69 \\
Within populations & 89 & 654.855 & 7.358 & 105.5 & 118 & 10.667 & 0.090 & 94.18 \\
Total & 120 & 827.496 & 6.974 & & 140 & 13.475 & 0.093 & \\
\hline Within geomorphic features between populations & 21 & 108.364 & -0.637 & -9.25 & 14 & 1.945 & 0.010 & 9.99 \\
Within populations & 89 & 654.855 & 7.358 & 106.89 & 118 & 10.667 & 0.090 & 93.83 \\
Total & 10 & 4.277 & 0.162 & 2.35 & 8 & 0.863 & -0.004 & -3.82 \\
\hline
\end{tabular}

Significant values of $\mathrm{p}<0.05$ are marked as $*$, and $\mathrm{p}<0.01$ are marked as $* *$.

Table 6.8 Mantel tests of isolation by distance and isolation by depth amongst different markers in Goniocorella dumosa, Madrepora oculata and Solenosmilia variabilis

\begin{tabular}{ccccccc}
\hline \multirow{2}{*}{ Loci } & \multicolumn{2}{c}{ Goniocorella dumosa } & \multicolumn{2}{c}{ Madrepora oculata } & \multicolumn{2}{c}{ Solenosmilia variabilis } \\
\cline { 2 - 7 } & Distance & Depth & Distance & Depth & Distance & Depth \\
\hline \multirow{2}{*}{ Microsatellites } & 0.0002 & $\mathbf{0 . 0 1 1 5}$ & 0.0024 & 0.0113 & $\mathbf{0 . 0 1 0 8}$ & 0.0017 \\
& $(0.28)$ & $(0.01)$ & $(0.22)$ & $(0.06)$ & $(0.01)$ & $(0.09)$ \\
ITS & 0.0004 & $\mathbf{0 . 0 1 3 2}$ & 0.0015 & $\mathbf{0 . 1 0 2 3}$ & 0.0005 & 0.0047 \\
& $(0.37)$ & $(0.05)$ & $(0.32)$ & $(0.01)$ & $(0.27)$ & $(0.08)$ \\
D-loop & 0.0003 & 0.0022 & NA & NA & 0.0119 & -0.070 \\
& $(0.20)$ & $(0.29)$ & & & $(0.10)$ & $(0.08)$ \\
\hline
\end{tabular}

Expressed as $\mathrm{R}^{2}$ values above and $\mathrm{p}$ values (in brackets) below the diagonal: significant values were bold. NA not available.

\subsubsection{Genotype identity test}

To screen out any genetically identical individuals (clones or fragments of individuals), all genotyped loci were utilised. In S. variabilis (24 loci), only one pair of specimens (NIWA ID 25489, two different fragments) was exactly the same (i.e., shared the same multi-locus genotype) across all amplified loci. These two samples were considered to be from the same colony, and one of them was therefore excluded for population genetic analysis. In $G$. dumosa (20 loci), no specimens with the same multi-locus genotype were 
detected amongst 108 individuals. In M. oculata (11 loci), 2 individuals (NIWA ID 46492 \& 47791) from Campbell Plateau, 2 individuals (ID 4004 \& 4005) from Chatham Rise and 2 individuals (ID 42734 \& 42830) from Chatham Rise were 2-loci identical (the other loci failed to be genotyped), for which it was difficult to distinguish whether the pairs were from the same colony or not. Due to the sample size for M. oculata, those pairs were considered as non-colony individuals, and both of the pairs were kept for the analyses. Except for these 3 pairs, the other 87 were unique genotypes and were considered to be individual specimens.

\subsubsection{Population genetic diversity}

For G. dumosa, the number of alleles per locus varied from 7 to 27 (average $=8.20$ ). Observed heterozygosity $\left(H_{\mathrm{O}}\right)$ ranged from 0.500 to 1.000 per locus and most $H_{\mathrm{O}}$ values were greater in all populations than expected. There were 66 instances of departures from HWE in 140 tests (Table 6.9). For M. oculata, the number of alleles per locus ranged from 5 to 19 (average $=9.96) . H_{\mathrm{O}}$ values ranged from 0.273 to 0.944 per locus, and unlike the other two coral species, were lower in all populations than expected. Approximately two thirds $(68.33 \%)$ of all loci exhibited significant departures from HWE (Table 6.10). For S. variabilis, the number of alleles varied from 7 to 31 (average $=14.42$ ). $H_{\mathrm{O}}$ ranged from 0.211 to 1.000 , and most loci exhibited significant departures from HWE (Table 6.11). 
Table 6.9 Basic population genetic information of microsatellite data at three spatial scales for

Goniocorella dumosa

\begin{tabular}{|c|c|c|c|c|c|c|c|c|c|c|c|}
\hline Population & Locus & B106 & B1 & $\mathrm{C107}$ & D5 & B123 & D213 & SVA13 & $\mathrm{C4}$ & SVA15 & SVA10 \\
\hline \multirow{6}{*}{$\begin{array}{l}\text { Northern } \\
\text { province }\end{array}$} & $\mathrm{N}$ & 104 & 104 & 95 & 91 & 85 & 81 & 73 & 75 & 70 & 63 \\
\hline & $\mathrm{Na}$ & 7 & 7 & 15 & 16 & 12 & 14 & 12 & 15 & 25 & 9 \\
\hline & $\mathrm{Ne}$ & 4.258 & 2.125 & 4.926 & 8.658 & 3.898 & 5.295 & 4.786 & 4.987 & 6.644 & 5.005 \\
\hline & Ho & 0.808 & 0.904 & 0.547 & 0.769 & 0.706 & 0.79 & 0.877 & 0.613 & 0.629 & 0.73 \\
\hline & $\mathrm{He}$ & 0.765 & 0.529 & 0.797 & 0.884 & 0.743 & 0.811 & 0.791 & 0.799 & 0.849 & 0.8 \\
\hline & HWE & $* * *$ & $* * *$ & $* * *$ & $* *$ & $* * *$ & $* * *$ & $* * *$ & $* * *$ & $* * *$ & $* * *$ \\
\hline \multirow{6}{*}{$\begin{array}{l}\text { Southern } \\
\text { Province }\end{array}$} & $\mathrm{N}$ & 4 & 4 & 4 & 4 & 3 & 3 & 3 & 3 & 2 & 2 \\
\hline & $\mathrm{Na}$ & 4 & 3 & 7 & 5 & 3 & 3 & 2 & 4 & 3 & 2 \\
\hline & $\mathrm{Ne}$ & 2.909 & 2.462 & 6.400 & 3.200 & 2.571 & 2.571 & 1.800 & 3.600 & 2.667 & 2.000 \\
\hline & Ho & 0.500 & 1.000 & 1.000 & 0.500 & 1.000 & 1.000 & 0.667 & 1.000 & 1.000 & 0.000 \\
\hline & $\mathrm{He}$ & 0.656 & 0.594 & 0.844 & 0.688 & 0.611 & 0.611 & 0.444 & 0.722 & 0.625 & 0.500 \\
\hline & HWE & ns & $\mathrm{ns}$ & ns & $\mathrm{ns}$ & ns & $\mathrm{ns}$ & ns & ns & ns & ns \\
\hline \multirow{6}{*}{ North Region } & $\mathrm{N}$ & 27 & 27 & 26 & 24 & 25 & 23 & 18 & 23 & 21 & 17 \\
\hline & $\mathrm{Na}$ & 7 & 5 & 10 & 13 & 10 & 10 & 10 & 11 & 17 & 6 \\
\hline & $\mathrm{Ne}$ & 4.942 & 2.236 & 4.012 & 7.024 & 4.209 & 5.264 & 6.353 & 4.301 & 7.056 & 5.352 \\
\hline & Ho & 0.778 & 0.963 & 0.500 & 0.708 & 1.000 & 0.739 & 0.833 & 0.652 & 0.714 & 0.824 \\
\hline & $\mathrm{He}$ & 0.798 & 0.553 & 0.751 & 0.858 & 0.762 & 0.810 & 0.843 & 0.767 & 0.858 & 0.813 \\
\hline & HWE & $*$ & $* *$ & $* * *$ & $\mathrm{~ns}$ & $*$ & $* *$ & $\mathrm{~ns}$ & $\mathrm{~ns}$ & $\mathrm{~ns}$ & $\mathrm{~ns}$ \\
\hline \multirow{6}{*}{$\begin{array}{l}\text { Central } \\
\text { Region }\end{array}$} & $\mathrm{N}$ & 77 & 77 & 69 & 67 & 60 & 58 & 55 & 52 & 49 & 46 \\
\hline & $\mathrm{Na}$ & 6 & 6 & 13 & 16 & 11 & 11 & 10 & 12 & 17 & 8 \\
\hline & $\mathrm{Ne}$ & 3.836 & 2.084 & 4.972 & 8.960 & 3.752 & 4.745 & 4.133 & 5.026 & 6.141 & 4.575 \\
\hline & Ho & 0.818 & 0.883 & 0.565 & 0.791 & 0.583 & 0.810 & 0.891 & 0.596 & 0.592 & 0.696 \\
\hline & $\mathrm{He}$ & 0.739 & 0.520 & 0.799 & 0.888 & 0.733 & 0.789 & 0.758 & 0.801 & 0.837 & 0.781 \\
\hline & HWE & $* * *$ & $* * *$ & $* * *$ & $* * *$ & $* * *$ & $* * *$ & $* * *$ & $* * *$ & $* * *$ & $* * *$ \\
\hline \multirow{6}{*}{ South Region } & $\mathrm{N}$ & 4 & 4 & 4 & 4 & 3 & 3 & 3 & 3 & 2 & 2 \\
\hline & $\mathrm{Na}$ & 4 & 3 & 7 & 5 & 3 & 3 & 2 & 4 & 3 & 2 \\
\hline & $\mathrm{Ne}$ & 2.909 & 2.462 & 6.400 & 3.200 & 2.571 & 2.571 & 1.800 & 3.600 & 2.667 & 2.000 \\
\hline & Ho & 0.500 & 1.000 & 1.000 & 0.500 & 1.000 & 1.000 & 0.667 & 1.000 & 1.000 & 0.000 \\
\hline & $\mathrm{He}$ & 0.656 & 0.594 & 0.844 & 0.688 & 0.611 & 0.611 & 0.444 & 0.722 & 0.625 & 0.500 \\
\hline & HWE & ns & $\mathrm{ns}$ & $\mathrm{ns}$ & ns & ns & ns & ns & ns & ns & ns \\
\hline \multirow{6}{*}{ Chatham Rise } & $\mathrm{N}$ & 77 & 77 & 69 & 67 & 60 & 58 & 55 & 52 & 49 & 46 \\
\hline & $\mathrm{Na}$ & 6 & 6 & 13 & 16 & 11 & 11 & 10 & 12 & 17 & 8 \\
\hline & $\mathrm{Ne}$ & 3.836 & 2.084 & 4.972 & 8.960 & 3.752 & 4.745 & 4.133 & 5.026 & 6.141 & 4.575 \\
\hline & Но & 0.818 & 0.883 & 0.565 & 0.791 & 0.583 & 0.810 & 0.891 & 0.596 & 0.592 & 0.696 \\
\hline & $\mathrm{He}$ & 0.739 & 0.520 & 0.799 & 0.888 & 0.733 & 0.789 & 0.758 & 0.801 & 0.837 & 0.781 \\
\hline & HWE & $* * *$ & $* * *$ & $* * *$ & $* * *$ & $* * *$ & $* * *$ & $* * *$ & $* * *$ & $* * *$ & $* * *$ \\
\hline \multirow{6}{*}{$\begin{array}{l}\text { Kermadec } \\
\text { Ridge }\end{array}$} & $\mathrm{N}$ & 12 & 12 & 12 & 11 & 12 & 12 & 8 & 12 & 9 & 8 \\
\hline & $\mathrm{Na}$ & 6 & 2 & 9 & 9 & 9 & 6 & 5 & 8 & 11 & 6 \\
\hline & $\mathrm{Ne}$ & 4.114 & 1.986 & 3.840 & 4.939 & 4.571 & 4.114 & 3.879 & 4.114 & 8.100 & 4.923 \\
\hline & Ho & 0.750 & 0.917 & 0.500 & 0.455 & 1.000 & 0.750 & 0.750 & 0.750 & 0.889 & 0.750 \\
\hline & $\mathrm{He}$ & 0.757 & 0.497 & 0.740 & 0.798 & 0.781 & 0.757 & 0.742 & 0.757 & 0.877 & 0.797 \\
\hline & HWE & ns & $* *$ & $*$ & $* *$ & ns & $\mathrm{ns}$ & ns & $\mathrm{ns}$ & $\mathrm{ns}$ & ns \\
\hline \multirow{6}{*}{$\begin{array}{c}\text { Challenger } \\
\text { Plateau }\end{array}$} & $\mathrm{N}$ & 7 & 7 & 7 & 7 & 6 & 4 & 5 & 5 & 5 & 5 \\
\hline & $\mathrm{Na}$ & 5 & 4 & 4 & 7 & 5 & 4 & 7 & 7 & 6 & 6 \\
\hline & $\mathrm{Ne}$ & 4.455 & 2.579 & 2.513 & 5.444 & 3.600 & 3.200 & 6.250 & 5.556 & 4.167 & 5.556 \\
\hline & Ho & 0.714 & 1.000 & 0.571 & 1.000 & 1.000 & 0.500 & 1.000 & 0.600 & 0.400 & 1.000 \\
\hline & $\mathrm{He}$ & 0.776 & 0.612 & 0.602 & 0.816 & 0.722 & 0.688 & 0.840 & 0.820 & 0.760 & 0.820 \\
\hline & HWE & $\mathrm{ns}$ & $\mathrm{ns}$ & $\mathrm{ns}$ & $\mathrm{ns}$ & $\mathrm{ns}$ & $\mathrm{ns}$ & $\mathrm{ns}$ & $\mathrm{ns}$ & $*$ & $\mathrm{~ns}$ \\
\hline \multirow{6}{*}{$\begin{array}{c}\mathrm{NE} \\
\text { continental } \\
\text { slope }\end{array}$} & $\mathrm{N}$ & 4 & 4 & 3 & 3 & 4 & 4 & 3 & 4 & 4 & 3 \\
\hline & $\mathrm{Na}$ & 5 & 3 & 4 & 4 & 4 & 6 & 3 & 3 & 7 & 3 \\
\hline & $\mathrm{Ne}$ & 4.000 & 2.462 & 3.600 & 3.000 & 2.909 & 5.333 & 2.571 & 1.684 & 6.400 & 2.571 \\
\hline & Но & 1.000 & 1.000 & 0.333 & 0.667 & 1.000 & 1.000 & 1.000 & 0.500 & 1.000 & 0.667 \\
\hline & $\mathrm{He}$ & 0.750 & 0.594 & 0.722 & 0.667 & 0.656 & 0.813 & 0.611 & 0.406 & 0.844 & 0.611 \\
\hline & HWE & $\mathrm{ns}$ & $\mathrm{ns}$ & $\mathrm{ns}$ & $\mathrm{ns}$ & $\mathrm{ns}$ & $\mathrm{ns}$ & $\mathrm{ns}$ & $\mathrm{ns}$ & $*$ & $\mathrm{~ns}$ \\
\hline
\end{tabular}


Continues

\begin{tabular}{|c|c|c|c|c|c|c|c|c|c|c|c|c|c|}
\hline Population & $\begin{array}{c}\text { Locu } \\
s\end{array}$ & SVA2 & $\begin{array}{c}\text { SVA1 } \\
8\end{array}$ & SVA8 & D103 & SVA1 & B116 & $\begin{array}{c}\text { SVA1 } \\
1\end{array}$ & SVA4 & SVA7 & $\begin{array}{c}\text { SVA2 } \\
0\end{array}$ & Mean & SE \\
\hline \multirow{6}{*}{$\begin{array}{l}\text { Northern } \\
\text { province }\end{array}$} & $\mathrm{N}$ & 66 & 63 & 60 & 64 & 56 & 63 & 54 & 23 & 39 & 34 & 68.15 & 4.841 \\
\hline & $\mathrm{Na}$ & 13 & 22 & 20 & 10 & 11 & 13 & 7 & 16 & 15 & 17 & 13.8 & 1.085 \\
\hline & $\mathrm{Ne}$ & 3.546 & 6.005 & 4.343 & 6.015 & 3.942 & 5.682 & 2.633 & 11.5 & 8.895 & 6.404 & 5.477 & 0.494 \\
\hline & Ho & 0.652 & 0.667 & 0.75 & 0.703 & 0.339 & 0.651 & 1 & 0.913 & 0.641 & 0.824 & 0.726 & 0.033 \\
\hline & $\mathrm{He}$ & 0.718 & 0.833 & 0.77 & 0.834 & 0.746 & 0.824 & 0.62 & 0.913 & 0.888 & 0.844 & 0.788 & 0.02 \\
\hline & HWE & $* * *$ & $* * *$ & $* * *$ & $* * *$ & $* * *$ & $* * *$ & $* * *$ & ns & $* * *$ & ns & & \\
\hline \multirow{6}{*}{$\begin{array}{l}\text { Southern } \\
\text { Province }\end{array}$} & $\mathrm{N}$ & 2 & 2 & 1 & 2 & 1 & 1 & 3 & 0 & 0 & 1 & 2.25 & 0.289 \\
\hline & $\mathrm{Na}$ & 2 & 2 & 2 & 3 & 1 & 2 & 2 & 0 & 0 & 2 & 2.6 & 0.358 \\
\hline & $\mathrm{Ne}$ & 2.000 & 1.600 & 2.000 & 2.667 & 1.000 & 2.000 & 2.000 & 0.000 & 0.000 & 2.000 & 2.272 & 0.298 \\
\hline & Ho & 1.000 & 0.500 & 1.000 & 1.000 & 0.000 & 1.000 & 1.000 & 0.000 & 0.000 & 1.000 & 0.708 & 0.091 \\
\hline & $\mathrm{He}$ & 0.500 & 0.375 & 0.500 & 0.625 & 0.000 & 0.500 & 0.500 & 0.000 & 0.000 & 0.500 & 0.490 & 0.053 \\
\hline & HWE & ns & $\mathrm{ns}$ & ns & $\mathrm{ns}$ & Mono & $\mathrm{ns}$ & ns & Mono & Mono & ns & & \\
\hline \multirow{6}{*}{ North Region } & $\mathrm{N}$ & 18 & 18 & 19 & 20 & 16 & 22 & 9 & 8 & 12 & 15 & 48.75 & 3.754 \\
\hline & $\mathrm{Na}$ & 10 & 12 & 14 & 8 & 10 & 10 & 3 & 9 & 9 & 11 & 11.5 & 0.838 \\
\hline & $\mathrm{Ne}$ & 5.945 & 6.231 & 4.813 & 5.634 & 5.505 & 5.068 & 2.219 & 7.529 & 5.538 & 6.818 & 5.074 & 0.486 \\
\hline & Но & 0.889 & 0.778 & 0.842 & 0.800 & 0.625 & 0.636 & 1.000 & 1.000 & 0.500 & 0.933 & 0.698 & 0.037 \\
\hline & $\mathrm{He}$ & 0.832 & 0.840 & 0.792 & 0.823 & 0.818 & 0.803 & 0.549 & 0.867 & 0.819 & 0.853 & 0.768 & 0.022 \\
\hline & HWE & $* * *$ & $\mathrm{~ns}$ & $\mathrm{~ns}$ & $\mathrm{~ns}$ & ns & $\mathrm{ns}$ & $*$ & ns & $* *$ & $\mathrm{~ns}$ & & \\
\hline \multirow{6}{*}{$\begin{array}{l}\text { Central } \\
\text { Region }\end{array}$} & $\mathrm{N}$ & 48 & 45 & 41 & 44 & 40 & 41 & 45 & 15 & 27 & 19 & 19.4 & 1.238 \\
\hline & $\mathrm{Na}$ & 8 & 19 & 15 & 10 & 9 & 12 & 6 & 15 & 14 & 12 & 9.75 & 0.695 \\
\hline & $\mathrm{Ne}$ & 2.753 & 5.556 & 3.979 & 5.779 & 2.747 & 5.827 & 2.682 & $\begin{array}{c}10.46 \\
5\end{array}$ & 8.526 & 4.945 & 5.303 & 0.321 \\
\hline & Ho & 0.563 & 0.622 & 0.707 & 0.659 & 0.225 & 0.659 & 1.000 & 0.867 & 0.704 & 0.737 & 0.786 & 0.034 \\
\hline & $\mathrm{He}$ & 0.637 & 0.820 & 0.749 & 0.827 & 0.636 & 0.828 & 0.627 & 0.904 & 0.883 & 0.798 & 0.790 & 0.020 \\
\hline & HWE & $* * *$ & $* * *$ & $* * *$ & $* * *$ & $* * *$ & $* * *$ & $* * *$ & ns & $* * *$ & ns & & \\
\hline \multirow{6}{*}{ South Region } & $\mathrm{N}$ & 2 & 2 & 1 & 2 & 1 & 1 & 3 & 0 & 0 & 1 & 2.25 & 0.289 \\
\hline & $\mathrm{Na}$ & 2 & 2 & 2 & 3 & 1 & 2 & 2 & 0 & 0 & 2 & 2.6 & 0.358 \\
\hline & $\mathrm{Ne}$ & 2.000 & 1.600 & 2.000 & 2.667 & 1.000 & 2.000 & 2.000 & 0.000 & 0.000 & 2.000 & 2.272 & 0.298 \\
\hline & Ho & 1.000 & 0.500 & 1.000 & 1.000 & 0.000 & 1.000 & 1.000 & 0.000 & 0.000 & 1.000 & 0.708 & 0.091 \\
\hline & $\mathrm{He}$ & 0.500 & 0.375 & 0.500 & 0.625 & 0.000 & 0.500 & 0.500 & 0.000 & 0.000 & 0.500 & 0.490 & 0.053 \\
\hline & HWE & $\mathrm{ns}$ & ns & $\mathrm{ns}$ & $\mathrm{ns}$ & Mono & $\mathrm{ns}$ & ns & Mono & Mono & $\mathrm{ns}$ & & \\
\hline \multirow{6}{*}{ Chatham Rise } & $\mathrm{N}$ & 48 & 45 & 41 & 44 & 40 & 41 & 45 & 15 & 27 & 19 & 48.75 & 3.754 \\
\hline & $\mathrm{Na}$ & 8 & 19 & 15 & 10 & 9 & 12 & 6 & 15 & 14 & 12 & 11.5 & 0.838 \\
\hline & $\mathrm{Ne}$ & 2.753 & 5.556 & 3.979 & 5.779 & 2.747 & 5.827 & 2.682 & $\begin{array}{c}10.46 \\
5\end{array}$ & 8.526 & 4.945 & 5.074 & 0.486 \\
\hline & Ho & 0.563 & 0.622 & 0.707 & 0.659 & 0.225 & 0.659 & 1.000 & 0.867 & 0.704 & 0.737 & 0.698 & 0.037 \\
\hline & $\mathrm{He}$ & 0.637 & 0.820 & 0.749 & 0.827 & 0.636 & 0.828 & 0.627 & 0.904 & 0.883 & 0.798 & 0.768 & 0.022 \\
\hline & HWE & $* * *$ & $* * *$ & $* * *$ & $* * *$ & $* * *$ & $* * *$ & $* * *$ & ns & $* * *$ & ns & & \\
\hline \multirow{6}{*}{$\begin{array}{l}\text { Kermadec } \\
\text { Ridge }\end{array}$} & $\mathrm{N}$ & 10 & 9 & 10 & 11 & 9 & 12 & 3 & 3 & 5 & 7 & 9.35 & 0.658 \\
\hline & $\mathrm{Na}$ & 10 & 10 & 9 & 7 & 6 & 8 & 2 & 5 & 4 & 7 & 6.905 & 0.569 \\
\hline & $\mathrm{Ne}$ & 7.143 & 5.786 & 3.509 & 4.654 & 3.682 & 4.114 & 2.000 & 4.500 & 2.941 & 5.765 & 4.434 & 0.331 \\
\hline & Ho & 0.800 & 0.889 & 0.700 & 0.818 & 0.556 & 0.667 & 1.000 & 1.000 & 0.200 & 1.000 & 0.757 & 0.047 \\
\hline & $\mathrm{He}$ & 0.860 & 0.827 & 0.715 & 0.785 & 0.728 & 0.757 & 0.500 & 0.778 & 0.660 & 0.827 & 0.747 & 0.022 \\
\hline & HWE & ns & ns & $\mathrm{ns}$ & $\mathrm{ns}$ & ns & $\mathrm{ns}$ & ns & $\mathrm{ns}$ & $*$ & $\mathrm{~ns}$ & & \\
\hline \multirow{6}{*}{$\begin{array}{c}\text { Challenger } \\
\text { Plateau }\end{array}$} & $\mathrm{N}$ & 3 & 4 & 4 & 4 & 5 & 5 & 3 & 3 & 1 & 4 & 4.7 & 0.356 \\
\hline & $\mathrm{Na}$ & 4 & 5 & 6 & 6 & 7 & 4 & 3 & 4 & 1 & 5 & 5 & 0.348 \\
\hline & $\mathrm{Ne}$ & 3.600 & 4.000 & 4.571 & 4.571 & 6.250 & 3.846 & 2.571 & 3.600 & 1.000 & 3.200 & 4.026 & 0.303 \\
\hline & Ho & 1.000 & 0.750 & 1.000 & 1.000 & 0.800 & 0.600 & 1.000 & 1.000 & 0.000 & 1.000 & 0.797 & 0.062 \\
\hline & $\mathrm{He}$ & 0.722 & 0.750 & 0.781 & 0.781 & 0.840 & 0.740 & 0.611 & 0.722 & 0.000 & 0.688 & 0.705 & 0.041 \\
\hline & HWE & ns & ns & $\mathrm{ns}$ & $\mathrm{ns}$ & ns & $\mathrm{ns}$ & $\mathrm{ns}$ & $\mathrm{ns}$ & Mono & $\mathrm{ns}$ & & \\
\hline \multirow{6}{*}{$\begin{array}{c}\mathrm{NE} \\
\text { continental } \\
\text { slope }\end{array}$} & $\mathrm{N}$ & 3 & 4 & 3 & 3 & 1 & 3 & 3 & 0 & 4 & 3 & 3.15 & 0.233 \\
\hline & $\mathrm{Na}$ & 3 & 2 & 5 & 3 & 1 & 4 & 2 & 0 & 5 & 4 & 3.55 & 0.366 \\
\hline & $\mathrm{Ne}$ & 2.571 & 2.000 & 4.500 & 3.000 & 1.000 & 3.600 & 2.000 & 0.000 & 4.571 & 3.000 & 3.039 & 0.328 \\
\hline & Ho & 1.000 & 0.500 & 1.000 & 0.667 & 0.000 & 0.333 & 1.000 & 0.000 & 1.000 & 0.667 & 0.717 & 0.077 \\
\hline & $\mathrm{He}$ & 0.611 & 0.500 & 0.778 & 0.667 & 0.000 & 0.722 & 0.500 & 0.000 & 0.781 & 0.667 & 0.595 & 0.052 \\
\hline & HWE & $\mathrm{ns}$ & $\mathrm{ns}$ & $\mathrm{ns}$ & $\mathrm{ns}$ & Mono & $\mathrm{ns}$ & $\mathrm{ns}$ & Mono & ns & $\mathrm{ns}$ & & \\
\hline
\end{tabular}

$\mathrm{N}=$ number of individuals successfully amplified, $\mathrm{Na}=$ number of different alleles, $\mathrm{Ne}=$ number of effective alleles, Ho = observed heterozygosity, $\mathrm{He}=$ expected heterozygosity, HWE = test of deviation from HardyWeinberg equilibrium. Geomorphic feature groups were only included in this table when the number of individuals successfully amplified was larger than 4. Significant values of $p>0.05$ are marked as ns, $p<0.05$ are marked as $*, \mathrm{p}<0.01$ are marked as $* *$, and $\mathrm{p}<0.001$ are marked as $* * *$. Mono means the locus is monomorphic. 
Table 6.10 Basic population genetic information of microsatellite data at three spatial scales for

Madrepora oculata

\begin{tabular}{|c|c|c|c|c|c|c|c|c|c|c|c|c|c|c|}
\hline Population & Locus & c016 & g028 & c7 & g006 & g004 & g25 & c013 & g016 & g022 & a5 & g010 & Mean & SE \\
\hline \multirow{6}{*}{$\begin{array}{l}\text { Northern } \\
\text { province }\end{array}$} & $\mathrm{N}$ & 46 & 37 & 20 & 42 & 45 & 22 & 54 & 24 & 23 & 41 & 45 & $\begin{array}{c}36.27 \\
3\end{array}$ & 3.583 \\
\hline & $\mathrm{Na}$ & 14 & 18 & 13 & 11 & 16 & 5 & 11 & 14 & 15 & 18 & 13 & $\begin{array}{c}13.45 \\
5\end{array}$ & 1.107 \\
\hline & $\mathrm{Ne}$ & 9.51 & 8.322 & 5.442 & 5.219 & $\begin{array}{c}10.38 \\
5\end{array}$ & 4.19 & 2.602 & 5.62 & 5.75 & 4.344 & 6.853 & 0.814 & 0.024 \\
\hline & Но & 0.891 & 0.757 & 0.7 & 0.738 & 0.622 & 0.273 & 0.463 & 0.708 & 0.478 & 0.488 & 0.689 & 0.619 & 0.053 \\
\hline & $\mathrm{He}$ & 0.895 & 0.88 & 0.816 & 0.808 & 0.904 & 0.761 & 0.616 & 0.822 & 0.826 & 0.77 & 0.854 & 0.814 & 0.024 \\
\hline & HW & $* *$ & $* * *$ & $* * *$ & $* * *$ & $* * *$ & $* * *$ & $* * *$ & $* *$ & $* * *$ & $* * *$ & $* * *$ & & \\
\hline \multirow{6}{*}{$\begin{array}{l}\text { Southern } \\
\text { Province }\end{array}$} & $\mathrm{N}$ & 15 & 7 & 6 & 10 & 15 & 1 & 14 & 4 & 5 & 11 & 12 & 9.091 & 1.443 \\
\hline & $\mathrm{Na}$ & 11 & 6 & 5 & 4 & 13 & 1 & 4 & 4 & 5 & 9 & 9 & 6.455 & 1.082 \\
\hline & $\mathrm{Ne}$ & 6.818 & 4.261 & 3.789 & 3.39 & 8.036 & 1 & 2.78 & 2.909 & 3.125 & 5.902 & 4.364 & 0.683 & 0.072 \\
\hline & Но & 0.867 & 0.857 & 0.833 & 0.7 & 0.733 & 0 & 0.643 & 0.75 & 0.4 & 0.455 & 0.667 & 0.628 & 0.078 \\
\hline & $\mathrm{He}$ & 0.853 & 0.765 & 0.736 & 0.705 & 0.876 & 0 & 0.64 & 0.656 & 0.68 & 0.831 & 0.771 & 0.683 & 0.072 \\
\hline & HW & $*$ & $\mathrm{~ns}$ & $\mathrm{~ns}$ & ns & $*$ & Mono & $*$ & ns & ns & $* * *$ & $* *$ & & \\
\hline \multirow{6}{*}{$\begin{array}{l}\text { North } \\
\text { Region }\end{array}$} & $\mathrm{N}$ & 18 & 15 & 6 & 13 & 15 & 9 & 16 & 8 & 9 & 15 & 14 & $\begin{array}{c}12.54 \\
5\end{array}$ & 1.171 \\
\hline & $\mathrm{Na}$ & 14 & 14 & 8 & 8 & 11 & 5 & 7 & 8 & 6 & 13 & 11 & 9.545 & 0.966 \\
\hline & $\mathrm{Ne}$ & 9.127 & 9.184 & 6.545 & 5.2 & 7.5 & 4.378 & 2.197 & 4.923 & 3.375 & 6.522 & 6.323 & 5.934 & 0.664 \\
\hline & Ho & 0.944 & 0.8 & 0.667 & 0.615 & 0.733 & 0.111 & 0.438 & 0.625 & 0.444 & 0.533 & 0.786 & 0.609 & 0.068 \\
\hline & $\mathrm{He}$ & 0.89 & 0.891 & 0.847 & 0.808 & 0.867 & 0.772 & 0.545 & 0.797 & 0.704 & 0.847 & 0.842 & 0.801 & 0.03 \\
\hline & HW & $\mathrm{ns}$ & $\mathrm{ns}$ & $\mathrm{ns}$ & $* *$ & $\mathrm{~ns}$ & $* *$ & $\mathrm{~ns}$ & $\mathrm{~ns}$ & $*$ & $* * *$ & $\mathrm{~ns}$ & & \\
\hline \multirow{6}{*}{$\begin{array}{l}\text { Central } \\
\text { Region }\end{array}$} & $\mathrm{N}$ & 30 & 23 & 15 & 30 & 32 & 13 & 39 & 16 & 15 & 28 & 33 & $\begin{array}{c}24.90 \\
9\end{array}$ & 2.692 \\
\hline & $\mathrm{Na}$ & 12 & 14 & 9 & 8 & 16 & 5 & 10 & 12 & 11 & 14 & 11 & $\begin{array}{c}11.09 \\
1\end{array}$ & 0.929 \\
\hline & $\mathrm{Ne}$ & 8.145 & 6.491 & 4.018 & 4.945 & 9.752 & 3.25 & 2.873 & 5.447 & 6.338 & 3.401 & 6.6 & 5.569 & 0.652 \\
\hline & Ho & 0.867 & 0.739 & 0.733 & 0.8 & 0.563 & 0.385 & 0.462 & 0.75 & 0.467 & 0.464 & 0.636 & 0.624 & 0.049 \\
\hline & $\mathrm{He}$ & 0.877 & 0.846 & 0.751 & 0.798 & 0.897 & 0.692 & 0.652 & 0.816 & 0.842 & 0.706 & 0.848 & 0.793 & 0.025 \\
\hline & HW & $* *$ & $\mathrm{~ns}$ & $*$ & $* * *$ & $* * *$ & $* * *$ & $* * *$ & $\mathrm{~ns}$ & $* *$ & $* * *$ & $* * *$ & & \\
\hline \multirow{6}{*}{$\begin{array}{l}\text { South } \\
\text { Region }\end{array}$} & $\mathrm{N}$ & 13 & 6 & 5 & 9 & 13 & 1 & 13 & 4 & 4 & 9 & 10 & 7.909 & 1.261 \\
\hline & $\mathrm{Na}$ & 8 & 6 & 5 & 4 & 12 & 1 & 3 & 4 & 5 & 8 & 9 & 5.909 & 0.939 \\
\hline & $\mathrm{Ne}$ & 6.818 & 4.261 & 3.789 & 3.39 & 8.036 & 1 & 2.78 & 2.909 & 3.125 & 5.902 & 4.364 & 0.683 & 0.072 \\
\hline & Но & 0.846 & 0.833 & 0.8 & 0.667 & 0.769 & 0 & 0.692 & 0.75 & 0.5 & 0.444 & 0.7 & 0.637 & 0.074 \\
\hline & $\mathrm{He}$ & 0.825 & 0.778 & 0.74 & 0.698 & 0.876 & 0 & 0.589 & 0.656 & 0.75 & 0.84 & 0.815 & 0.688 & 0.073 \\
\hline & HW & $\mathrm{ns}$ & $\mathrm{ns}$ & ns & $\mathrm{ns}$ & $*$ & Mono & $\mathrm{ns}$ & $\mathrm{ns}$ & $\mathrm{ns}$ & $* * *$ & $*$ & & \\
\hline \multirow{6}{*}{$\begin{array}{l}\text { Chatham } \\
\text { Rise }\end{array}$} & $\mathrm{N}$ & 30 & 23 & 15 & 30 & 32 & 13 & 39 & 16 & 15 & 28 & 33 & 4.727 & 0.648 \\
\hline & $\mathrm{Na}$ & 12 & 14 & 9 & 8 & 16 & 5 & 10 & 12 & 11 & 14 & 11 & 5.364 & 0.812 \\
\hline & $\mathrm{Ne}$ & 8.145 & 6.491 & 4.018 & 4.945 & 9.752 & 3.25 & 2.873 & 5.447 & 6.338 & 3.401 & 6.6 & 5.569 & 0.652 \\
\hline & Ho & 0.867 & 0.739 & 0.733 & 0.8 & 0.563 & 0.385 & 0.462 & 0.75 & 0.467 & 0.464 & 0.636 & 0.623 & 0.106 \\
\hline & $\mathrm{He}$ & 0.877 & 0.846 & 0.751 & 0.798 & 0.897 & 0.692 & 0.652 & 0.816 & 0.842 & 0.706 & 0.848 & 0.689 & 0.053 \\
\hline & HW & $* *$ & $\mathrm{~ns}$ & $*$ & $* * *$ & $* * *$ & $* * *$ & $* * *$ & ns & $* *$ & $* * *$ & $* * *$ & & \\
\hline \multirow{6}{*}{$\begin{array}{c}\text { Campbell } \\
\text { Plateau }\end{array}$} & $\mathrm{N}$ & 14 & 7 & 6 & 9 & 15 & 1 & 14 & 4 & 4 & 10 & 11 & 8.636 & 1.403 \\
\hline & $\mathrm{Na}$ & 11 & 6 & 5 & 4 & 13 & 1 & 4 & 4 & 5 & 8 & 9 & 6.364 & 1.064 \\
\hline & $\mathrm{Ne}$ & 7.259 & 4.261 & 3.789 & 3.176 & 8.036 & 1.000 & 2.780 & 2.909 & 4.000 & 5.128 & 3.967 & 4.210 & 0.604 \\
\hline & Но & 0.857 & 0.857 & 0.833 & 0.667 & 0.733 & 0 & 0.643 & 0.750 & 0.500 & 0.500 & 0.636 & 0.634 & 0.074 \\
\hline & $\mathrm{He}$ & 0.862 & 0.765 & 0.736 & 0.685 & 0.876 & 0 & 0.640 & 0.656 & 0.750 & 0.805 & 0.748 & 0.684 & 0.072 \\
\hline & HW & $*$ & $\mathrm{~ns}$ & $\mathrm{~ns}$ & $\mathrm{~ns}$ & $*$ & Mono & $*$ & $\mathrm{~ns}$ & ns & $* *$ & $*$ & & \\
\hline \multirow{6}{*}{$\begin{array}{l}\text { Challenger } \\
\text { Plateau }\end{array}$} & $\mathrm{N}$ & 8 & 6 & 3 & 9 & 6 & 6 & 6 & 4 & 5 & 9 & 8 & 6.364 & 0.592 \\
\hline & $\mathrm{Na}$ & 9 & 7 & 6 & 6 & 6 & 3 & 6 & 4 & 2 & 11 & 8 & 6.182 & 0.784 \\
\hline & $\mathrm{Ne}$ & 5.333 & 6.000 & 6.000 & 4.765 & 4.500 & 2.571 & 2.667 & 2.909 & 1.471 & $\begin{array}{c}10.12 \\
5\end{array}$ & 4.129 & 4.588 & 0.712 \\
\hline & Но & 1.000 & 0.833 & 1.000 & 0.444 & 0.500 & 0 & 0.500 & 1.000 & 0.400 & 0.556 & 0.750 & 0.635 & 0.095 \\
\hline & $\mathrm{He}$ & 0.813 & 0.833 & 0.833 & 0.790 & 0.778 & 0.611 & 0.625 & 0.656 & 0.320 & 0.901 & 0.758 & 0.720 & 0.049 \\
\hline & HW & $\mathrm{ns}$ & $\mathrm{ns}$ & ns & $*$ & ns & $* *$ & ns & $\mathrm{ns}$ & ns & $*$ & ns & & \\
\hline \multirow{6}{*}{$\begin{array}{l}\text { Kermadec } \\
\text { Ridge }\end{array}$} & $\mathrm{N}$ & 8 & 7 & 2 & 3 & 6 & 3 & 8 & 4 & 3 & 4 & 4 & 4.727 & 0.648 \\
\hline & $\mathrm{Na}$ & 10 & 10 & 2 & 5 & 6 & 3 & 3 & 5 & 4 & 4 & 7 & 5.364 & 0.812 \\
\hline & $\mathrm{Ne}$ & 8.533 & 8.167 & 2.000 & 4.500 & 4.000 & 2.571 & 1.471 & 4.571 & 3.000 & 2.286 & 6.400 & 4.318 & 0.736 \\
\hline & Но & 0.875 & 0.857 & 0 & 1.000 & 1.000 & 0.333 & 0.375 & 0.250 & 0.667 & 0.500 & 1.000 & 0.623 & 0.106 \\
\hline & $\mathrm{He}$ & 0.883 & 0.878 & 0.500 & 0.778 & 0.750 & 0.611 & 0.320 & 0.781 & 0.667 & 0.563 & 0.844 & 0.689 & 0.053 \\
\hline & HW & ns & ns & ns & ns & ns & ns & ns & ns & ns & ns & ns & & \\
\hline
\end{tabular}

$\mathrm{N}=$ number of individuals successfully amplified, $\mathrm{Na}=$ number of different alleles, $\mathrm{Ne}=$ number of effective alleles, Ho = observed heterozygosity, $\mathrm{He}=$ expected heterozygosity, $\mathrm{HW}=$ test of deviation from HardyWeinberg equilibrium. Geomorphic feature groups were only included in this table when the number of individuals successfully amplified was larger than 4 . Significant values of $p>0.05$ are marked as ns, $p<0.05$ are marked as $*, \mathrm{p}<0.01$ are marked as $* *$, and $\mathrm{p}<0.001$ are marked as $* * *$. Mono means the locus is monomorphic. 


\subsubsection{Population genetic structure}

For all three coral species there was very strong concordance in AMOVA results for the reduced and for the all loci data sets, indicating that the presence or absence of a signal of genetic differentiation is conserved across all surveyed loci (Table 6.12). The AMOVA of genetic structure for G. dumosa detected large-scale structure amongst north, central, south regions and amongst the geomorphic features, but not between northern and southern provinces. The only AMOVA evidence of genetic structure in M. oculata was amongst the north, central and south regions (Table 6.13). The AMOVA for S. variabilis detected largescale structure between northern and southern provinces, amongst north, central, south regions and amongst the geomorphic features. Notably, a north-central-south regional differentiation was found in all three species for both data sets (Table 6.14).

For all three coral species, tests of differentiation ( $F_{\mathrm{ST}}$ values) amongst populations from the geomorphic features for both the reduced and all loci data sets showed similar patterns (Tables 6.14-6.16). In G. dumosa, the Kermadec Ridge population was significantly different from the Chatham Rise population. In $M$. oculata, no significant pairwise $F_{\text {ST }}$ values were observed for pairwise testing amongst all (Campbell Plateau, Challenger Plateau, Chatham Rise and Kermadec Ridge) populations (data not shown). For S. variabilis pairwise population $F_{\mathrm{ST}}$ values revealed no significant genetic differences between the Bounty Trough population and all other populations, and the Kermadec Ridge and Chatham Rise populations. 
Table 6.11 Basic population genetic information of microsatellite data at three spatial scales for

\section{Solenosmilia variabilis}

\begin{tabular}{|c|c|c|c|c|c|c|c|c|c|}
\hline Population & Locus & B106 & C107 & D5 & B1 & B116 & D103 & B123 & D213 \\
\hline \multirow{6}{*}{ Northern Province } & $\mathrm{N}$ & 163 & 159 & 160 & 162 & 148 & 135 & 161 & 160 \\
\hline & $\mathrm{Na}$ & 9 & 17 & 19 & 19 & 14 & 7 & 13 & 22 \\
\hline & $\mathrm{Ne}$ & 2.632 & 4.28 & 5.411 & 4.661 & 3.932 & 2.331 & 1.979 & 5.156 \\
\hline & Но & 0.957 & 0.736 & 0.788 & 0.901 & 0.682 & 0.548 & 0.304 & 0.988 \\
\hline & $\mathrm{He}$ & 0.62 & 0.766 & 0.815 & 0.785 & 0.746 & 0.571 & 0.495 & 0.806 \\
\hline & HWE & $* * *$ & $* * *$ & $* * *$ & $* * *$ & $* * *$ & $* * *$ & $* * *$ & $* * *$ \\
\hline \multirow{6}{*}{ Southern Province } & $\mathrm{N}$ & 38 & 37 & 38 & 39 & 35 & 32 & 38 & 39 \\
\hline & $\mathrm{Na}$ & 5 & 12 & 13 & 11 & 7 & 5 & 9 & 12 \\
\hline & $\mathrm{Ne}$ & 2.283 & 5.099 & 6.639 & 3.441 & 3.895 & 3.205 & 1.807 & 4.5 \\
\hline & Но & 0.895 & 0.946 & 0.737 & 0.744 & 0.514 & 0.625 & 0.289 & 0.949 \\
\hline & $\mathrm{He}$ & 0.562 & 0.804 & 0.849 & 0.709 & 0.743 & 0.688 & 0.447 & 0.778 \\
\hline & HWE & $* * *$ & $* * *$ & $*$ & $* * *$ & $* * *$ & $* *$ & $* * *$ & $* * *$ \\
\hline \multirow{6}{*}{ North Region } & $\mathrm{N}$ & 80 & 79 & 79 & 79 & 70 & 68 & 80 & 77 \\
\hline & $\mathrm{Na}$ & 8 & 13 & 18 & 17 & 13 & 7 & 12 & 21 \\
\hline & $\mathrm{Ne}$ & 3.197 & 4.411 & 5.33 & 7.048 & 4.048 & 2.169 & 2.162 & 6.566 \\
\hline & Ho & 0.925 & 0.709 & 0.785 & 0.962 & 0.729 & 0.5 & 0.313 & 0.987 \\
\hline & $\mathrm{He}$ & 0.687 & 0.773 & 0.812 & 0.858 & 0.753 & 0.539 & 0.538 & 0.848 \\
\hline & HWE & $* * *$ & $* * *$ & $* * *$ & $* * *$ & $* * *$ & $* * *$ & $* * *$ & $* * *$ \\
\hline \multirow{6}{*}{ Central Region } & $\overline{\mathrm{N}}$ & 83 & 80 & 81 & 83 & 78 & 67 & 81 & 83 \\
\hline & $\mathrm{Na}$ & 7 & 14 & 14 & 13 & 9 & 6 & 10 & 9 \\
\hline & $\mathrm{Ne}$ & 2.176 & 4.057 & 5.166 & 3.191 & 3.672 & 2.483 & 1.808 & 4.135 \\
\hline & Но & 0.988 & 0.763 & 0.79 & 0.843 & 0.641 & 0.597 & 0.296 & 0.988 \\
\hline & $\mathrm{He}$ & 0.54 & 0.754 & 0.806 & 0.687 & 0.728 & 0.597 & 0.447 & 0.758 \\
\hline & HWE & $* * *$ & $* * *$ & $* * *$ & $* * *$ & $* * *$ & $* * *$ & $* * *$ & $* * *$ \\
\hline \multirow{6}{*}{ South Region } & $\mathrm{N}$ & 38 & 37 & 38 & 39 & 35 & 32 & 38 & 39 \\
\hline & $\mathrm{Na}$ & 5 & 12 & 13 & 11 & 7 & 5 & 9 & 12 \\
\hline & $\mathrm{Ne}$ & 2.283 & 5.099 & 6.639 & 3.441 & 3.895 & 3.205 & 1.807 & 4.5 \\
\hline & Но & 0.895 & 0.946 & 0.737 & 0.744 & 0.514 & 0.625 & 0.289 & 0.949 \\
\hline & $\mathrm{He}$ & 0.562 & 0.804 & 0.849 & 0.709 & 0.743 & 0.688 & 0.447 & 0.778 \\
\hline & HWE & $* * *$ & $* * *$ & * & $* * *$ & $* * *$ & $* *$ & $* * *$ & $* * *$ \\
\hline & $\mathrm{N}$ & 83 & 80 & 81 & 83 & 78 & 67 & 81 & 83 \\
\hline & $\mathrm{Na}$ & 7 & 14 & 14 & 13 & 9 & 6 & 10 & 9 \\
\hline & $\mathrm{Ne}$ & 2.176 & 4.057 & 5.166 & 3.191 & 3.672 & 2.483 & 1.808 & 4.135 \\
\hline Chatham Rise & Но & 0.988 & 0.763 & 0.79 & 0.843 & 0.641 & 0.597 & 0.296 & 0.988 \\
\hline & $\mathrm{He}$ & 0.54 & 0.754 & 0.806 & 0.687 & 0.728 & 0.597 & 0.447 & 0.758 \\
\hline & HWE & $* * *$ & $* * *$ & $* * *$ & $* * *$ & $* * *$ & $* * *$ & $* * *$ & $* * *$ \\
\hline & $\mathrm{N}$ & 40 & 39 & 39 & 39 & 35 & 33 & 39 & 40 \\
\hline & $\mathrm{Na}$ & 7 & 9 & 15 & 15 & 8 & 7 & 10 & 17 \\
\hline & $\mathrm{Ne}$ & 2.576 & 4.702 & 4.061 & 7.141 & 2.984 & 2.679 & 2.227 & 4.769 \\
\hline Kermadec Ridge & Но & 0.925 & 0.795 & 0.744 & 0.923 & 0.629 & 0.515 & 0.282 & 0.975 \\
\hline & $\mathrm{He}$ & 0.612 & 0.787 & 0.754 & 0.86 & 0.665 & 0.627 & 0.551 & 0.79 \\
\hline & HWE & $*$ & $* * *$ & $* * *$ & $* * *$ & $* *$ & $* * *$ & $* * *$ & $* * *$ \\
\hline & $\mathrm{N}$ & 35 & 35 & 35 & 35 & 30 & 30 & 36 & 32 \\
\hline & $\mathrm{Na}$ & 6 & 9 & 13 & 12 & 10 & 5 & 8 & 15 \\
\hline Lowisville Seamount Chain & $\mathrm{Ne}$ & 4.077 & 3.137 & 5.889 & 5.53 & 4.455 & 1.654 & 1.799 & 9.143 \\
\hline Louisville Seamount Chain & Но & 0.943 & 0.571 & 0.8 & 1 & 0.833 & 0.433 & 0.278 & 1 \\
\hline & $\mathrm{He}$ & 0.755 & 0.681 & 0.83 & 0.819 & 0.776 & 0.396 & 0.444 & 0.891 \\
\hline & HWE & $* * *$ & $\mathrm{~ns}$ & $* * *$ & $* * *$ & $* * *$ & ns & $* * *$ & $* * *$ \\
\hline & $\mathrm{N}$ & 15 & 13 & 15 & 15 & 13 & 12 & 14 & 15 \\
\hline & $\mathrm{Na}$ & 2 & 8 & 10 & 6 & 5 & 5 & 5 & 7 \\
\hline Macauarie Ridge & $\mathrm{Ne}$ & 2 & 4.39 & 7.143 & 2.813 & 3.976 & 3.31 & 1.867 & 4.245 \\
\hline Macquarie Ridge & Но & 1 & 0.923 & 0.867 & 1 & 0.462 & 0.583 & 0.286 & 1 \\
\hline & $\mathrm{He}$ & 0.5 & 0.772 & 0.86 & 0.644 & 0.749 & 0.698 & 0.464 & 0.764 \\
\hline & HWE & $* * *$ & $*$ & ns & $* * *$ & $* *$ & $*$ & ns & $* * *$ \\
\hline & $\bar{N}$ & 5 & 5 & 5 & 5 & 5 & 5 & 5 & 5 \\
\hline & $\mathrm{Na}$ & 3 & 5 & 5 & 5 & 4 & 4 & 2 & 5 \\
\hline & $\mathrm{Ne}$ & 2.381 & 3.571 & 3.571 & 3.846 & 3.333 & 2.381 & 1.22 & 3.571 \\
\hline Bounty Trough & Ho & 1 & 1 & 0.6 & 0.6 & 0.6 & 0.4 & 0.2 & 1 \\
\hline & $\mathrm{He}$ & 0.58 & 0.72 & 0.72 & 0.74 & 0.7 & 0.58 & 0.18 & 0.72 \\
\hline & HWE & $\mathrm{ns}$ & $\mathrm{ns}$ & ns & ns & ns & ns & ns & ns \\
\hline & $\mathrm{N}$ & 4 & 4 & 4 & 4 & 4 & 4 & 4 & 4 \\
\hline & $\mathrm{Na}$ & 3 & 3 & 4 & 4 & 2 & 4 & 2 & 2 \\
\hline Bounty Plateau & $\mathrm{Ne}$ & 2.909 & 2.462 & 3.2 & 2.286 & 1.6 & 3.556 & 1.28 & 2 \\
\hline Bounty Plateau & Но & 0.75 & 1 & 0.5 & 0.5 & 0.5 & 0.75 & 0.25 & 1 \\
\hline & $\mathrm{He}$ & 0.656 & 0.594 & 0.688 & 0.563 & 0.375 & 0.719 & 0.219 & 0.5 \\
\hline & HWE & ns & ns & ns & ns & ns & ns & ns & ns \\
\hline & $\bar{N}$ & 4 & 4 & 4 & 4 & 4 & 4 & 4 & 4 \\
\hline & $\mathrm{Na}$ & 2 & 4 & 3 & 6 & 3 & 3 & 5 & 3 \\
\hline Hilosm & $\mathrm{Ne}$ & 2 & 3.2 & 2.462 & 4.571 & 2.909 & 2.133 & 4 & 2.462 \\
\hline Hikurangi Margin & Но & 1 & 1 & 1 & 1 & 0.75 & 0.75 & 0.75 & 1 \\
\hline & $\mathrm{He}$ & 0.5 & 0.688 & 0.594 & 0.781 & 0.656 & 0.531 & 0.75 & 0.594 \\
\hline & HWE & ns & ns & $\mathrm{ns}$ & $\mathrm{ns}$ & $\mathrm{ns}$ & $\mathrm{ns}$ & ns & $\mathrm{ns}$ \\
\hline & $\mathrm{N}$ & 8 & 8 & 8 & 8 & 7 & 7 & 8 & 8 \\
\hline & $\mathrm{Na}$ & 2 & 5 & 6 & 4 & 4 & 4 & 4 & 7 \\
\hline Camphell Plateay & $\mathrm{Ne}$ & 1.969 & 3.556 & 2.723 & 2.246 & 3.63 & 2.882 & 1.488 & 5.12 \\
\hline Campbell Plateau & Но & 0.875 & 0.875 & 0.75 & 0.375 & 0.857 & 0.857 & 0.25 & 0.875 \\
\hline & $\mathrm{He}$ & 0.492 & 0.719 & 0.633 & 0.555 & 0.724 & 0.653 & 0.328 & 0.805 \\
\hline & HWE & ns & ns & ns & ns & ns & $\mathrm{ns}$ & ns & ns \\
\hline
\end{tabular}


Continues

\begin{tabular}{|c|c|c|c|c|c|c|c|c|c|c|}
\hline Population & Locus & $\mathrm{C} 4$ & SVA1 & SVA3 & SVA7 & SVA2 & SVA14 & SVA15 & SVA4 & SVA12 \\
\hline \multirow{6}{*}{ Northern Province } & $\mathrm{N}$ & 159 & 132 & 132 & 123 & 133 & 88 & 159 & 117 & 70 \\
\hline & $\mathrm{Na}$ & 11 & 19 & 22 & 18 & 12 & 22 & 22 & 26 & 20 \\
\hline & $\mathrm{Ne}$ & 1.398 & 3.242 & 6.333 & 5.887 & 6.676 & 14.821 & 3.956 & 8.197 & 5.847 \\
\hline & Но & 0.245 & 0.598 & 0.659 & 0.943 & 0.677 & 0.727 & 0.887 & 0.846 & 0.471 \\
\hline & $\mathrm{He}$ & 0.285 & 0.692 & 0.842 & 0.83 & 0.85 & 0.933 & 0.747 & 0.878 & 0.829 \\
\hline & HWE & $* * *$ & $* * *$ & $* * *$ & $* * *$ & $* * *$ & $* * *$ & $* * *$ & $* * *$ & $* * *$ \\
\hline \multirow{6}{*}{ Southern Province } & $\mathrm{N}$ & 36 & 34 & 28 & 24 & 37 & 15 & 37 & 33 & 17 \\
\hline & $\mathrm{Na}$ & 6 & 12 & 11 & 10 & 9 & 13 & 13 & 18 & 12 \\
\hline & $\mathrm{Ne}$ & 1.624 & 2.953 & 6.817 & 7.432 & 4.265 & 9.375 & 5.024 & 10.573 & 7.049 \\
\hline & Но & 0.25 & 0.588 & 0.75 & 0.917 & 0.595 & 0.667 & 0.892 & 0.909 & 0.588 \\
\hline & $\mathrm{He}$ & 0.384 & 0.661 & 0.853 & 0.865 & 0.766 & 0.893 & 0.801 & 0.905 & 0.858 \\
\hline & HWE & $* * *$ & $* * *$ & ns & ns & ns & ns & $* * *$ & $* * *$ & $* *$ \\
\hline \multirow{6}{*}{ North Region } & $\mathrm{N}$ & 80 & 71 & 77 & 74 & 72 & 53 & 80 & 60 & 38 \\
\hline & $\mathrm{Na}$ & 8 & 18 & 16 & 16 & 10 & 21 & 14 & 16 & 14 \\
\hline & $\mathrm{Ne}$ & 1.298 & 4.211 & 5.01 & 5.77 & 7.175 & 13.872 & 3.514 & 7.385 & 7.166 \\
\hline & Ho & 0.213 & 0.648 & 0.623 & 0.959 & 0.708 & 0.868 & 0.9 & 0.783 & 0.553 \\
\hline & $\mathrm{He}$ & 0.23 & 0.763 & 0.8 & 0.827 & 0.861 & 0.928 & 0.715 & 0.865 & 0.86 \\
\hline & HWE & $* * *$ & $* * *$ & $* * *$ & $* * *$ & $* * *$ & $* * *$ & $* * *$ & $* * *$ & $* * *$ \\
\hline \multirow{6}{*}{ Central Region } & $\mathrm{N}$ & 79 & 61 & 55 & 49 & 61 & 35 & 79 & 57 & 32 \\
\hline & $\mathrm{Na}$ & 7 & 12 & 18 & 12 & 12 & 18 & 18 & 22 & 13 \\
\hline & $\mathrm{Ne}$ & 1.505 & 2.442 & 8.013 & 5.965 & 5.742 & 11.557 & 4.292 & 8.483 & 4.163 \\
\hline & Но & 0.278 & 0.541 & 0.709 & 0.918 & 0.639 & 0.514 & 0.873 & 0.912 & 0.375 \\
\hline & $\mathrm{He}$ & 0.335 & 0.59 & 0.875 & 0.832 & 0.826 & 0.913 & 0.767 & 0.882 & 0.76 \\
\hline & HWE & $* * *$ & $* *$ & $* * *$ & $* * *$ & $* * *$ & $* * *$ & $* * *$ & $* * *$ & $* * *$ \\
\hline & $\mathrm{N}$ & 36 & 34 & 28 & 24 & 37 & 15 & 37 & 33 & 17 \\
\hline & $\mathrm{Na}$ & 6 & 12 & 11 & 10 & 9 & 13 & 13 & 18 & 12 \\
\hline & $\mathrm{Ne}$ & 1.624 & 2.953 & 6.817 & 7.432 & 4.265 & 9.375 & 5.024 & 10.573 & 7.049 \\
\hline South Region & Но & 0.25 & 0.588 & 0.75 & 0.917 & 0.595 & 0.667 & 0.892 & 0.909 & 0.588 \\
\hline & $\mathrm{He}$ & 0.384 & 0.661 & 0.853 & 0.865 & 0.766 & 0.893 & 0.801 & 0.905 & 0.858 \\
\hline & HWE & $* * *$ & $* * *$ & ns & $\mathrm{ns}$ & $\mathrm{ns}$ & $\mathrm{ns}$ & $* * *$ & $* * *$ & $* *$ \\
\hline & $\mathrm{N}$ & 79 & 61 & 55 & 49 & 61 & 35 & 79 & 57 & 32 \\
\hline & $\mathrm{Na}$ & 7 & 12 & 18 & 12 & 12 & 18 & 18 & 22 & 13 \\
\hline Chatham Rise & $\mathrm{Ne}$ & 1.505 & 2.442 & 8.013 & 5.965 & 5.742 & 11.557 & 4.292 & 8.483 & 4.163 \\
\hline Chatham Rise & Но & 0.278 & 0.541 & 0.709 & 0.918 & 0.639 & 0.514 & 0.873 & 0.912 & 0.375 \\
\hline & $\mathrm{He}$ & 0.335 & 0.59 & 0.875 & 0.832 & 0.826 & 0.913 & 0.767 & 0.882 & 0.76 \\
\hline & HWE & $* * *$ & $* *$ & $* * *$ & $* * *$ & $* * *$ & $* * *$ & $* * *$ & $* * *$ & $* * *$ \\
\hline & $\mathrm{N}$ & 38 & 35 & 36 & 35 & 34 & 28 & 39 & 31 & 21 \\
\hline & $\mathrm{Na}$ & 7 & 10 & 12 & 12 & 9 & 18 & 12 & 14 & 7 \\
\hline & $\mathrm{Ne}$ & 1.282 & 1.989 & 4.662 & 6.447 & 6.165 & 13.635 & 3.723 & 7.364 & 4.594 \\
\hline Kermadec Ridge & Но & 0.211 & 0.486 & 0.639 & 1 & 0.735 & 0.857 & 0.949 & 0.839 & 0.476 \\
\hline & $\mathrm{He}$ & 0.22 & 0.497 & 0.785 & 0.845 & 0.838 & 0.927 & 0.731 & 0.864 & 0.782 \\
\hline & HWE & $* * *$ & $* * *$ & $* * *$ & $* * *$ & ns & ns & $* * *$ & $* *$ & $* * *$ \\
\hline & $\overline{\mathrm{N}}$ & 37 & 31 & 36 & 34 & 34 & 23 & 36 & 25 & 13 \\
\hline & $\mathrm{Na}$ & 4 & 16 & 13 & 11 & 10 & 15 & 11 & 9 & 10 \\
\hline Lowisville Seamount Chain & $\mathrm{Ne}$ & 1.284 & 9.856 & 3.216 & 4.817 & 5.766 & 9.281 & 3.256 & 6.25 & 5.729 \\
\hline Louisvinte seamount C nain & Ho & 0.189 & 0.871 & 0.583 & 0.912 & 0.735 & 0.87 & 0.833 & 0.72 & 0.692 \\
\hline & $\mathrm{He}$ & 0.221 & 0.899 & 0.689 & 0.792 & 0.827 & 0.892 & 0.693 & 0.84 & 0.825 \\
\hline & HWE & $* * *$ & $* * *$ & $* * *$ & $* * *$ & $* * *$ & $\mathrm{~ns}$ & $\mathrm{~ns}$ & $*$ & $* * *$ \\
\hline & $\mathrm{N}$ & 13 & 11 & 7 & 6 & 15 & 2 & 14 & 12 & 6 \\
\hline & $\mathrm{Na}$ & 5 & 6 & 7 & 6 & 6 & 3 & 9 & 10 & 8 \\
\hline Macourarie Ridor & $\mathrm{Ne}$ & 1.965 & 2.521 & 5.444 & 4.8 & 3.516 & 2.667 & 5.851 & 8.727 & 6.545 \\
\hline Macquarie Ridge & Но & 0.385 & 0.636 & 0.714 & 1 & 0.467 & 0.5 & 0.786 & 0.833 & 0.667 \\
\hline & $\mathrm{He}$ & 0.491 & 0.603 & 0.816 & 0.792 & 0.716 & 0.625 & 0.829 & 0.885 & 0.847 \\
\hline & HWE & $\mathrm{ns}$ & $\mathrm{ns}$ & ns & $\mathrm{ns}$ & $*$ & $\mathrm{~ns}$ & $* * *$ & $* *$ & ns \\
\hline & $\mathrm{N}$ & 5 & 5 & 5 & 4 & 5 & 4 & 5 & 5 & 3 \\
\hline & $\mathrm{Na}$ & 3 & 5 & 6 & 5 & 4 & 5 & 2 & 6 & 3 \\
\hline & $\mathrm{Ne}$ & 1.515 & 2.5 & 4.545 & 4 & 3.846 & 3.2 & 2 & 5.556 & 2.571 \\
\hline Bounty Trough & Но & 0.4 & 0.6 & 0.8 & 1 & 0.4 & 0.75 & 1 & 1 & 0.333 \\
\hline & $\mathrm{He}$ & 0.34 & 0.6 & 0.78 & 0.75 & 0.74 & 0.688 & 0.5 & 0.82 & 0.611 \\
\hline & HWE & $\mathrm{ns}$ & $\mathrm{ns}$ & $\mathrm{ns}$ & $\mathrm{ns}$ & $\mathrm{ns}$ & ns & $\mathrm{ns}$ & ns & ns \\
\hline & $\mathrm{N}$ & 4 & 4 & 4 & 4 & 4 & 3 & 4 & 4 & 3 \\
\hline & $\mathrm{Na}$ & 1 & 3 & 5 & 6 & 5 & 3 & 4 & 4 & 5 \\
\hline Bounty Plateau & $\mathrm{Ne}$ & 1 & 2.462 & 4.571 & 5.333 & 4 & 2.571 & 3.556 & 3.556 & 4.5 \\
\hline Bounty Plateau & Но & 0 & 0.5 & 0.75 & 1 & 0.75 & 0.333 & 1 & 1 & 1 \\
\hline & $\mathrm{He}$ & 0 & 0.594 & 0.781 & 0.813 & 0.75 & 0.611 & 0.719 & 0.719 & 0.778 \\
\hline & HWE & ns & $\mathrm{ns}$ & $\mathrm{ns}$ & $\mathrm{ns}$ & ns & ns & $\mathrm{ns}$ & $\mathrm{ns}$ & $\mathrm{ns}$ \\
\hline & $\bar{N}$ & 4 & 4 & 4 & 4 & 3 & 2 & 4 & 3 & 3 \\
\hline & $\mathrm{Na}$ & 2 & 2 & 6 & 4 & 3 & 4 & 3 & 3 & 3 \\
\hline Hilo so ti & $\mathrm{Ne}$ & 1.28 & 1.28 & 5.333 & 3.2 & 2.571 & 4 & 2.462 & 2.571 & 2 \\
\hline Hikurangi Margin & Но & 0.25 & 0.25 & 0.75 & 1 & 0.333 & 1 & 1 & 0.667 & 0.667 \\
\hline & $\mathrm{He}$ & 0.219 & 0.219 & 0.813 & 0.688 & 0.611 & 0.75 & 0.594 & 0.611 & 0.5 \\
\hline & HWE & $\mathrm{ns}$ & $\mathrm{ns}$ & ns & $\mathrm{ns}$ & $\mathrm{ns}$ & $\mathrm{ns}$ & $\mathrm{ns}$ & $\mathrm{ns}$ & $\mathrm{ns}$ \\
\hline & $\mathrm{N}$ & 8 & 8 & 8 & 8 & 8 & 4 & 8 & 7 & 4 \\
\hline & $\mathrm{Na}$ & 2 & 5 & 6 & 8 & 7 & 7 & 6 & 8 & 5 \\
\hline & $\mathrm{Ne}$ & 1.133 & 2 & 3.2 & 5.818 & 4.923 & 6.4 & 3.282 & 6.125 & 4 \\
\hline Campbell Plateau & Но & 0.125 & 0.375 & 0.75 & 0.75 & 0.875 & 0.75 & 1 & 1 & 0.5 \\
\hline & $\mathrm{He}$ & 0.117 & 0.5 & 0.688 & 0.828 & 0.797 & 0.844 & 0.695 & 0.837 & 0.75 \\
\hline & HWE & ns & $\mathrm{ns}$ & ns & $\mathrm{ns}$ & ns & ns & ns & ns & ns \\
\hline
\end{tabular}

Continues 


\begin{tabular}{|c|c|c|c|c|c|c|c|c|c|c|}
\hline Population & Locus & SVA16 & SVA13 & SVA11 & SVA10 & SVA5 & SVA18 & SVA8 & Mean & SE \\
\hline \multirow{6}{*}{ Northern Province } & $\bar{N}$ & 102 & 153 & 126 & 119 & 102 & 133 & 101 & 133.208 & 5.414 \\
\hline & $\mathrm{Na}$ & 13 & 15 & 22 & 23 & 12 & 31 & 26 & 18.083 & 1.21 \\
\hline & $\mathrm{Ne}$ & 2.459 & 2.689 & 3.967 & 9.824 & 7.368 & 7.125 & 8.064 & 5.343 & 0.612 \\
\hline & Но & 0.539 & 0.732 & 0.984 & 0.882 & 0.98 & 0.639 & 0.545 & 0.719 & 0.043 \\
\hline & $\mathrm{He}$ & 0.593 & 0.628 & 0.748 & 0.898 & 0.864 & 0.86 & 0.876 & 0.748 & 0.031 \\
\hline & HWE & $* * *$ & $* * *$ & $* * *$ & $* * *$ & $* * *$ & $* * *$ & $* * *$ & & \\
\hline \multirow{6}{*}{ Southern Province } & $\mathrm{N}$ & 24 & 36 & 30 & 28 & 23 & 28 & 23 & 31.208 & 1.455 \\
\hline & $\mathrm{Na}$ & 10 & 7 & 8 & 14 & 10 & 14 & 17 & 10.75 & 0.695 \\
\hline & $\mathrm{Ne}$ & 3.282 & 2.374 & 2.932 & 8.909 & 7.149 & 6.73 & 10.373 & 5.322 & 0.556 \\
\hline & Но & 0.583 & 0.778 & 1 & 0.857 & 1 & 0.643 & 0.696 & 0.725 & 0.042 \\
\hline & $\mathrm{He}$ & 0.695 & 0.579 & 0.659 & 0.888 & 0.86 & 0.851 & 0.904 & 0.75 & 0.029 \\
\hline & HWE & $* * *$ & $* * *$ & $* * *$ & * & $* * *$ & $* * *$ & ns & & \\
\hline \multirow{6}{*}{ North Region } & $\mathrm{N}$ & 58 & 76 & 64 & 69 & 56 & 72 & 53 & 69.375 & 2.282 \\
\hline & $\mathrm{Na}$ & 11 & 15 & 18 & 21 & 11 & 23 & 23 & 15.167 & 0.959 \\
\hline & $\mathrm{Ne}$ & 2.475 & 3.447 & 5.543 & 10.206 & 7.024 & 7.145 & 8.946 & 5.63 & 0.585 \\
\hline & Ho & 0.534 & 0.737 & 0.984 & 0.899 & 0.964 & 0.681 & 0.604 & 0.732 & 0.043 \\
\hline & $\mathrm{He}$ & 0.596 & 0.71 & 0.82 & 0.902 & 0.858 & 0.86 & 0.888 & 0.762 & 0.032 \\
\hline & HWE & $* * *$ & $* * *$ & $* * *$ & $* * *$ & $* * *$ & $* * *$ & $* * *$ & & \\
\hline \multirow{6}{*}{ Central Region } & $\mathrm{N}$ & 44 & 77 & 62 & 50 & 46 & 61 & 48 & 63.833 & 3.343 \\
\hline & $\mathrm{Na}$ & 11 & 8 & 13 & 17 & 11 & 23 & 21 & 13.25 & 0.984 \\
\hline & $\mathrm{Ne}$ & 2.344 & 2.103 & 2.815 & 8.803 & 7.425 & 6.615 & 6.847 & 4.825 & 0.543 \\
\hline & Но & 0.545 & 0.727 & 0.984 & 0.86 & 1 & 0.59 & 0.479 & 0.702 & 0.045 \\
\hline & $\mathrm{He}$ & 0.573 & 0.524 & 0.645 & 0.886 & 0.865 & 0.849 & 0.854 & 0.721 & 0.032 \\
\hline & HWE & $* * *$ & $* * *$ & $* * *$ & $* * *$ & $* * *$ & $* * *$ & $* * *$ & & \\
\hline & $\mathrm{N}$ & 24 & 36 & 30 & 28 & 23 & 28 & 23 & 31.208 & 1.455 \\
\hline & $\mathrm{Na}$ & 10 & 7 & 8 & 14 & 10 & 14 & 17 & 10.75 & 0.695 \\
\hline Soutb Region & $\mathrm{Ne}$ & 3.282 & 2.374 & 2.932 & 8.909 & 7.149 & 6.73 & 10.373 & 5.322 & 0.556 \\
\hline South Kegion & Но & 0.583 & 0.778 & 1 & 0.857 & 1 & 0.643 & 0.696 & 0.725 & 0.042 \\
\hline & $\mathrm{He}$ & 0.695 & 0.579 & 0.659 & 0.888 & 0.86 & 0.851 & 0.904 & 0.75 & 0.029 \\
\hline & HWE & $* * *$ & $* * *$ & $* * *$ & * & $* * *$ & $* * *$ & $\mathrm{~ns}$ & & \\
\hline & $\overline{\mathrm{N}}$ & 44 & 77 & 62 & 50 & 46 & 61 & 48 & 63.833 & 3.343 \\
\hline & $\mathrm{Na}$ & 11 & 8 & 13 & 17 & 11 & 23 & 21 & 13.25 & 0.984 \\
\hline Chatham Pice & $\mathrm{Ne}$ & 2.344 & 2.103 & 2.815 & 8.803 & 7.425 & 6.615 & 6.847 & 4.825 & 0.543 \\
\hline Chatnam Kise & Ho & 0.545 & 0.727 & 0.984 & 0.86 & 1 & 0.59 & 0.479 & 0.702 & 0.045 \\
\hline & $\mathrm{He}$ & 0.573 & 0.524 & 0.645 & 0.886 & 0.865 & 0.849 & 0.854 & 0.721 & 0.032 \\
\hline & HWE & $* * *$ & $* * *$ & $* * *$ & $* * *$ & $* * *$ & $* * *$ & $* * *$ & & \\
\hline & $\mathrm{N}$ & 31 & 37 & 28 & 33 & 30 & 36 & 28 & 34.333 & 0.992 \\
\hline & $\mathrm{Na}$ & 8 & 12 & 9 & 16 & 11 & 18 & 17 & 11.667 & 0.768 \\
\hline Kormodec Pidar & $\mathrm{Ne}$ & 2.379 & 3.271 & 2.872 & 8.25 & 7.004 & 6.529 & 8.385 & 4.987 & 0.571 \\
\hline Kermadec Kiage & Но & 0.548 & 0.811 & 1 & 1 & 0.933 & 0.667 & 0.571 & 0.73 & 0.047 \\
\hline & $\mathrm{He}$ & 0.58 & 0.694 & 0.652 & 0.879 & 0.857 & 0.847 & 0.881 & 0.73 & 0.033 \\
\hline & HWE & $* * *$ & $* * *$ & $* * *$ & $* * *$ & ns & $* * *$ & $* *$ & & \\
\hline & $\mathrm{N}$ & 23 & 34 & 32 & 32 & 23 & 31 & 22 & 30.583 & 1.231 \\
\hline & $\mathrm{Na}$ & 8 & 11 & 15 & 15 & 9 & 15 & 15 & 11.042 & 0.703 \\
\hline Louicuille Seamount $\mathrm{Ch}$ ain & $\mathrm{Ne}$ & 2.625 & 3.676 & 8.569 & 5.721 & 5.688 & 6.744 & 6.769 & 5.205 & 0.495 \\
\hline Louisville Seamount Chain & Но & 0.522 & 0.676 & 0.969 & 0.781 & 1 & 0.71 & 0.591 & 0.73 & 0.045 \\
\hline & $\mathrm{He}$ & 0.619 & 0.728 & 0.883 & 0.825 & 0.824 & 0.852 & 0.852 & 0.744 & 0.035 \\
\hline & HWE & $* * *$ & ns & $* *$ & $* * *$ & ns & ns & $* * *$ & & \\
\hline & $\mathrm{N}$ & 7 & 14 & 10 & 8 & 6 & 8 & 4 & 10.625 & 0.823 \\
\hline & $\mathrm{Na}$ & 6 & 3 & 3 & 7 & 8 & 7 & 3 & 6.042 & 0.452 \\
\hline Macaurie Pidce & $\mathrm{Ne}$ & 4.667 & 2.074 & 2.198 & 5.333 & 6.545 & 6.095 & 2.667 & 4.223 & 0.391 \\
\hline Macquarie Ridge & Но & 0.571 & 0.857 & 1 & 0.75 & 1 & 0.75 & 0 & 0.71 & 0.054 \\
\hline & $\mathrm{He}$ & 0.786 & 0.518 & 0.545 & 0.813 & 0.847 & 0.836 & 0.625 & 0.709 & 0.027 \\
\hline & HWE & $\mathrm{ns}$ & $*$ & * & $\mathrm{ns}$ & $\mathrm{ns}$ & $\mathrm{ns}$ & * & & \\
\hline & $\mathrm{N}$ & 5 & 5 & 4 & 4 & 5 & 5 & 4 & 4.708 & 0.112 \\
\hline & $\mathrm{Na}$ & 7 & 3 & 3 & 5 & 6 & 6 & 5 & 4.458 & 0.276 \\
\hline Bounty Trounh & $\mathrm{Ne}$ & 4.545 & 1.852 & 2.462 & 4 & 5 & 4.167 & 4.571 & 2.381 & 0.233 \\
\hline Bounty Irougn & Ho & 0.8 & 0.6 & 1 & 1 & 1 & 0.6 & 0.5 & 1 & 0.054 \\
\hline & $\mathrm{He}$ & 0.78 & 0.46 & 0.594 & 0.75 & 0.8 & 0.76 & 0.781 & 0.58 & 0.032 \\
\hline & HWE & ns & ns & ns & ns & ns & ns & ns & & \\
\hline & $\overline{\mathrm{N}}$ & 4 & 4 & 4 & 4 & 4 & 4 & 4 & 3.917 & 0.058 \\
\hline & $\mathrm{Na}$ & 2 & 2 & 5 & 7 & 6 & 5 & 5 & 3.833 & 0.317 \\
\hline Dert Dloge & $\mathrm{Ne}$ & 1.28 & 1.28 & 4.571 & 6.4 & 4.571 & 4 & 4.571 & 3.23 & 0.295 \\
\hline Bounty Plateau & Ho & 0.25 & 0.25 & 1 & 1 & 1 & 0.5 & 1 & 0.691 & 0.065 \\
\hline & $\mathrm{He}$ & 0.219 & 0.219 & 0.781 & 0.844 & 0.781 & 0.75 & 0.781 & 0.602 & 0.047 \\
\hline & HWE & ns & ns & ns & ns & ns & ns & ns & & \\
\hline & $\mathrm{N}$ & 3 & 4 & 3 & 3 & 3 & 4 & 2 & 3.542 & 0.134 \\
\hline & $\mathrm{Na}$ & 2 & 3 & 4 & 5 & 5 & 3 & 4 & 3.542 & 0.241 \\
\hline Hikurangi Maroin & $\mathrm{Ne}$ & 1.385 & 1.684 & 3.6 & 4.5 & 4.5 & 1.684 & 4 & 2.908 & 0.239 \\
\hline & Ho & 0.333 & 0.5 & 1 & 1 & 1 & 0.5 & 1 & 0.771 & 0.056 \\
\hline & $\mathrm{He}$ & 0.278 & 0.406 & 0.722 & 0.778 & 0.778 & 0.406 & 0.75 & 0.592 & 0.037 \\
\hline & HWE & ns & ns & ns & ns & $\mathrm{ns}$ & ns & ns & & \\
\hline & $\bar{N}$ & 7 & 8 & 8 & 8 & 7 & 8 & 8 & 7.458 & 0.233 \\
\hline & $\mathrm{Na}$ & 5 & 5 & 6 & 10 & 8 & 7 & 9 & 5.833 & 0.411 \\
\hline Camnhell Platean & $\mathrm{Ne}$ & 2.227 & 2.783 & 3.368 & 8 & 7 & 4.741 & 6.737 & 3.973 & 0.39 \\
\hline Campbell Plateau & Но & 0.571 & 0.875 & 1 & 0.875 & 1 & 0.625 & 0.875 & 0.736 & 0.051 \\
\hline & $\mathrm{He}$ & 0.551 & 0.641 & 0.703 & 0.875 & 0.857 & 0.789 & 0.852 & 0.676 & 0.037 \\
\hline & HWE & ns & ns & ns & ns & ns & ns & ns & & \\
\hline
\end{tabular}

$\mathrm{N}=$ number of individuals successfully amplified, $\mathrm{Na}=$ number of different alleles, $\mathrm{Ne}=$ number of effective alleles, $\mathrm{Ho}=$ observed heterozygosity, $\mathrm{He}=$ expected heterozygosity, $\mathrm{HWE}=$ test of deviation from Hardy-Weinberg equilibrium. Geomorphic feature groups were only included in this table when the number of individuals successfully amplified was larger than 4. Significant values of $\mathrm{p}>0.05$ are marked as ns, $\mathrm{p}<0.05$ are marked as $*, \mathrm{p}<0.01$ are marked as **, and $\mathrm{p}<$ 0.001 are marked as $* * *$. Mono means the locus is monomorphic. 
Table 6.12 Hierarchical AMOVA results for Goniocorella dumosa when tested for microsatellite genetic variation

\begin{tabular}{cccccccc}
\hline \multirow{2}{*}{ Source of variation } & \multicolumn{4}{c}{ All loci } & \multicolumn{3}{c}{ Neutral loci only } \\
\cline { 2 - 7 } & df & ss & Var. comp. & df & ss & Var. comp. \\
\hline Between Provinces & 1 & 9.373 & 0.000 & 1 & 3.629 & 0.000 \\
Amongst Individuals & 106 & 1058.678 & $2.390^{* *}$ & 106 & 422.144 & $0.769^{* *}$ \\
Within Individuals & 108 & 562.500 & $5.208^{* *}$ & 108 & 264.000 & $2.444^{* *}$ \\
Total & 215 & 1630.551 & 7.598 & 215 & 689.773 & 3.213 \\
\hline Amongst Regions & 2 & 27.947 & $0.088^{*}$ & 2 & 10.180 & $0.025^{*}$ \\
Amongst Individuals & 105 & 1040.104 & $2.349^{* *}$ & 105 & 415.593 & $0.757^{* *}$ \\
Within Individuals & 108 & 562.500 & $5.208^{* *}$ & 108 & 264.000 & $2.444^{* *}$ \\
Total & 215 & 1630.551 & 7.645 & 215 & 689.773 & 3.226 \\
\hline Amongst Geomorphic Features & 3 & 0.196 & $0.196^{* *}$ & 3 & 16.653 & $0.063^{* *}$ \\
Amongst Individuals & 96 & 2.318 & $2.318^{* *}$ & 96 & 377.757 & $0.740^{* *}$ \\
Within Individuals & 100 & 5.240 & $5.240^{* *}$ & 100 & 245.500 & $2.455^{* *}$ \\
Total & 199 & 7.754 & 7.754 & 199 & 639.910 & 3.258 \\
\hline
\end{tabular}

Significant values of $\mathrm{p}<0.05$ are marked as $*$, and $\mathrm{p}<0.01$ are marked as **.

Table 6.13 Hierarchical AMOVA results for Madrepora oculata when tested for microsatellite genetic variation

\begin{tabular}{ccccccc}
\hline \multirow{2}{*}{ Source of variation } & \multicolumn{4}{c}{ All loci } & \multicolumn{3}{c}{ Neutral loci only } \\
\cline { 2 - 7 } & df & ss & Var. comp. & df & ss & Var. comp. \\
\hline Between Provinces & 1 & 6.420 & 0.013 & 1 & 3.234 & 0.004 \\
Amongst Individuals & 91 & 507.634 & $1.926^{* *}$ & 91 & 269.846 & $0.934^{* *}$ \\
Within Individuals & 93 & 160.500 & $1.726^{* *}$ & 93 & 102 & $1.097^{* *}$ \\
Total & 185 & 674.554 & 3.665 & 185 & 375.081 & 2.035 \\
\hline Amongst Regions & 2 & 13.474 & $0.022^{*}$ & 2 & 7.581 & $0.016^{*}$ \\
Amongst Individuals & 90 & 500.580 & $1.918^{* *}$ & 90 & 265.5 & $0.927^{* *}$ \\
Within Individuals & 93 & 160.500 & $1.726^{* *}$ & 93 & 102 & $1.097^{* *}$ \\
Total & 185 & 674.554 & 3.666 & 185 & 375.081 & 2.039 \\
\hline Amongst Geomorphic Features & 3 & 18.763 & 0.017 & 3 & 11.377 & 0.029 \\
Amongst Individuals & 55 & 324.898 & $1.886^{* *}$ & 55 & 174.724 & $0.893^{* *}$ \\
Within Individuals & 59 & 126.000 & $2.136^{* *}$ & 59 & 82.000 & $1.390^{* *}$ \\
Total & 117 & 469.661 & 4.038 & 117 & 268.102 & 2.312 \\
\hline
\end{tabular}

Significant values of $\mathrm{p}<0.05$ are marked as $*$, and $\mathrm{p}<0.01$ are marked as $* *$. 
Table 6.14 Hierarchical AMOVA results for Solenosmilia variabilis when tested for microsatellite genetic variation

\begin{tabular}{ccccccc}
\hline \multirow{2}{*}{ Source of variation } & \multicolumn{3}{c}{ All } & \multicolumn{3}{c}{ Neutral only } \\
\cline { 2 - 7 } & df & ss & Var. comp. & df & ss & Var. comp. \\
\hline Between Provinces & 1 & 16.070 & $0.038^{*}$ & 1 & 11.039 & $0.044^{* *}$ \\
Amongst Individuals & 206 & 2322.236 & $2.207^{* *}$ & 206 & 1112.833 & $0.700^{* *}$ \\
Within Individuals & 208 & 1426.500 & $6.858^{* *}$ & 208 & 832.500 & $4.002^{* *}$ \\
Total & 415 & 3764.805 & 9.103 & 415 & 1956.373 & 4.747 \\
\hline Amongst Regions & 2 & 52.773 & $0.115^{* *}$ & 2 & 32.454 & $0.083^{* *}$ \\
Amongst Individuals & 205 & 2285.532 & $2.145^{* *}$ & 205 & 1091.419 & $0.661^{* *}$ \\
Within Individuals & 208 & 1426.500 & $6.858^{* *}$ & 208 & 832.500 & $4.002^{* *}$ \\
Total & 415 & 3764.805 & 9.119 & 415 & 1956.373 & 4.746 \\
\hline Amongst Geomorphic Features & 7 & 151.751 & $0.259^{* *}$ & 7 & 80.076 & $0.150^{* *}$ \\
Amongst Individuals & 192 & 2080.067 & $1.971^{* *}$ & 192 & 989.109 & $0.570^{* *}$ \\
Within Individuals & 200 & 1378.500 & $6.893^{* *}$ & 200 & 802.500 & $4.013^{* *}$ \\
Total & 399 & 3610.318 & 9.122 & 399 & 1871.685 & 4.732 \\
\hline
\end{tabular}

Significant values of $\mathrm{p}<0.05$ are marked as $*$, and $\mathrm{p}<0.01$ are marked as $* *$.

Table 6.15 Pairwise $F_{\text {ST }}$ values for all (below diagonal) and neutral (above diagonal) loci amongst Goniocorella dumosa populations

\begin{tabular}{ccccc}
\hline & NE Slope & Challenger Plateau & Chatham Rise & Kermadec Ridge \\
\hline NE Slope & & -0.018 & 0.033 & 0.009 \\
Challenger Plateau & -0.047 & & -0.006 & 0.015 \\
Chatham Rise & 0.001 & -0.014 & & $0.044^{*}$ \\
Kermadec Ridge & -0.012 & 0.003 & $0.024^{*}$ & \\
\hline
\end{tabular}

Table 6.16 Pairwise population genetic differentiation $\left(F_{S T}\right)$ of Madrepora oculata in microsatellites

\begin{tabular}{ccccc}
\hline & $\begin{array}{c}\text { Chatham } \\
\text { Rise }\end{array}$ & $\begin{array}{c}\text { Kermadec } \\
\text { Ridge }\end{array}$ & $\begin{array}{c}\text { Challenger } \\
\text { Plateau }\end{array}$ & $\begin{array}{c}\text { Campbell } \\
\text { Plateau }\end{array}$ \\
\hline Chatham Rise & 0.000 & -0.016 & -0.011 & 0.007 \\
Kermadec Ridge & & 0.000 & -0.025 & 0.021 \\
Challenger Plateau & & & 0.000 & -0.002 \\
Campbell Plateau & & & & 0.000 \\
\hline
\end{tabular}

Significant values of $\mathrm{p}<0.05$ are marked as $*$, and $\mathrm{p}<0.01$ are marked as $* *$. 
Table 6.17 Pairwise $F_{S T}$ values for all (below diagonal) and neutral (above diagonal) amongst Solenosmilia variabilis populations

\begin{tabular}{|c|c|c|c|c|c|c|c|c|c|c|}
\hline & $\begin{array}{c}\text { Bollons } \\
\text { Seamount }\end{array}$ & $\begin{array}{l}\text { Bounty } \\
\text { Plateau }\end{array}$ & $\begin{array}{l}\text { Bounty } \\
\text { Trough }\end{array}$ & $\begin{array}{c}\text { Campbell } \\
\text { Plateau }\end{array}$ & $\begin{array}{c}\text { Chatham } \\
\text { Rise }\end{array}$ & $\begin{array}{c}\text { Hikuran } \\
\text { gi } \\
\text { Margin }\end{array}$ & $\begin{array}{l}\text { Kermade } \\
\text { c Ridge }\end{array}$ & $\begin{array}{l}\text { Louisville } \\
\text { Seamount }\end{array}$ & $\begin{array}{l}\text { Macquari } \\
\text { e Ridge }\end{array}$ & $\begin{array}{c}\text { Tasman } \\
\text { Basin }\end{array}$ \\
\hline $\begin{array}{c}\text { Bollons } \\
\text { Seamount }\end{array}$ & 0 & -0.11 & 0.007 & -0.046 & -0.057 & 0.049 & -0.001 & 0.038 & -0.077 & -0.086 \\
\hline $\begin{array}{l}\text { Bounty } \\
\text { Plateau }\end{array}$ & -0.121 & 0 & -0.045 & -0.083 & -0.058 & -0.038 & -0.016 & 0.027 & -0.076 & -0.067 \\
\hline $\begin{array}{l}\text { Bounty } \\
\text { Trough }\end{array}$ & -0.023 & -0.070 & 0 & -0.024 & 0.015 & -0.055 & -0.026 & $0.046^{*}$ & $0.028 * *$ & 0.045 \\
\hline $\begin{array}{c}\text { Campbell } \\
\text { Plateau }\end{array}$ & -0.025 & -0.073 & -0.041 & 0 & -0.015 & -0.045 & 0.003 & $0.058 * *$ & -0.024 & -0.007 \\
\hline $\begin{array}{l}\text { Chatham } \\
\text { Rise }\end{array}$ & -0.038 & -0.053 & -0.006 & -0.013 & 0 & 0.034 & $0.024 * *$ & $0.070 * *$ & -0.013 & -0.005 \\
\hline $\begin{array}{c}\text { Hikurangi } \\
\text { Margin }\end{array}$ & 0.062 & -0.031 & -0.057 & -0.056 & $0.022^{*}$ & 0 & -0.011 & $0.068^{*}$ & $0.045^{*}$ & 0.070 \\
\hline $\begin{array}{l}\text { Kermadec } \\
\text { Ridge }\end{array}$ & -0.008 & -0.027 & -0.031 & -0.001 & $0.016 * *$ & -0.004 & 0 & $0.036^{* *}$ & $0.036^{* *}$ & 0.039 \\
\hline $\begin{array}{l}\text { Louisville } \\
\text { Seamount }\end{array}$ & 0.035 & 0.020 & 0.037 & $0.057 * *$ & $0.055^{* *}$ & $0.077^{*}$ & $0.029 * *$ & 0 & $0.076^{* *}$ & 0.042 \\
\hline $\begin{array}{l}\text { Macquarie } \\
\text { Ridge }\end{array}$ & -0.049 & -0.069 & -0.005 & -0.030 & -0.013 & $0.014 *$ & $0.020^{*}$ & $0.061 * *$ & 0 & -0.028 \\
\hline $\begin{array}{c}\text { Tasman } \\
\text { Basin }\end{array}$ & -0.091 & -0.092 & -0.010 & -0.037 & -0.019 & 0.022 & 0.005 & $0.041^{*}$ & -0.047 & 0 \\
\hline
\end{tabular}

Significant values of $\mathrm{p}<0.05$ are marked as $*$, and $\mathrm{p}<0.01$ are marked as **.

\subsubsection{Isolation by distance and isolation by depth}

Tests for isolation by distance and by depth identified only two significant results - in S. variabilis for distance and in G. dumosa for depth (Table 6.8).

\subsubsection{Post-hoc analyses}

\subsubsection{Geographic distribution}

Microsatellite variation revealed the presence of multiple genetic groups within each of the three corals. In G. dumosa, the DAPC scatter plot (Figure 6.4) and the Structure Harvester plot (Figure S6.3) provided evidence of more than one population, but the number of putative genetic clusters was inconsistent between Structure $(K=2)$ and DAPC $(K=3$, Northeast Slope, Kermadec Ridge and the rest of populations). In M. oculata, DAPC revealed that the Campbell Plateau and Chatham Rise populations were genetically similar to each other (Figure 6.5). The Structure Harvester plot indicated the presence of three groups (Figure S6.3). For S. variabilis, the DAPC differentiated S. variabilis into 5 genetic clusters, Campbell Plateau, Bounty Plateau, Bounty Trough, Louisville Seamount Chain, and all other populations (Figure 6.6). Depending on the Structure Harvester results used to determine the 
number of populations, $\mathrm{K}=3$ (based on $\Delta \mathrm{K}$ ) was the best grouping cluster number (Figure S6.3). All analyses provided support for the existence of a differentiated Louisville Seamount Chain population.

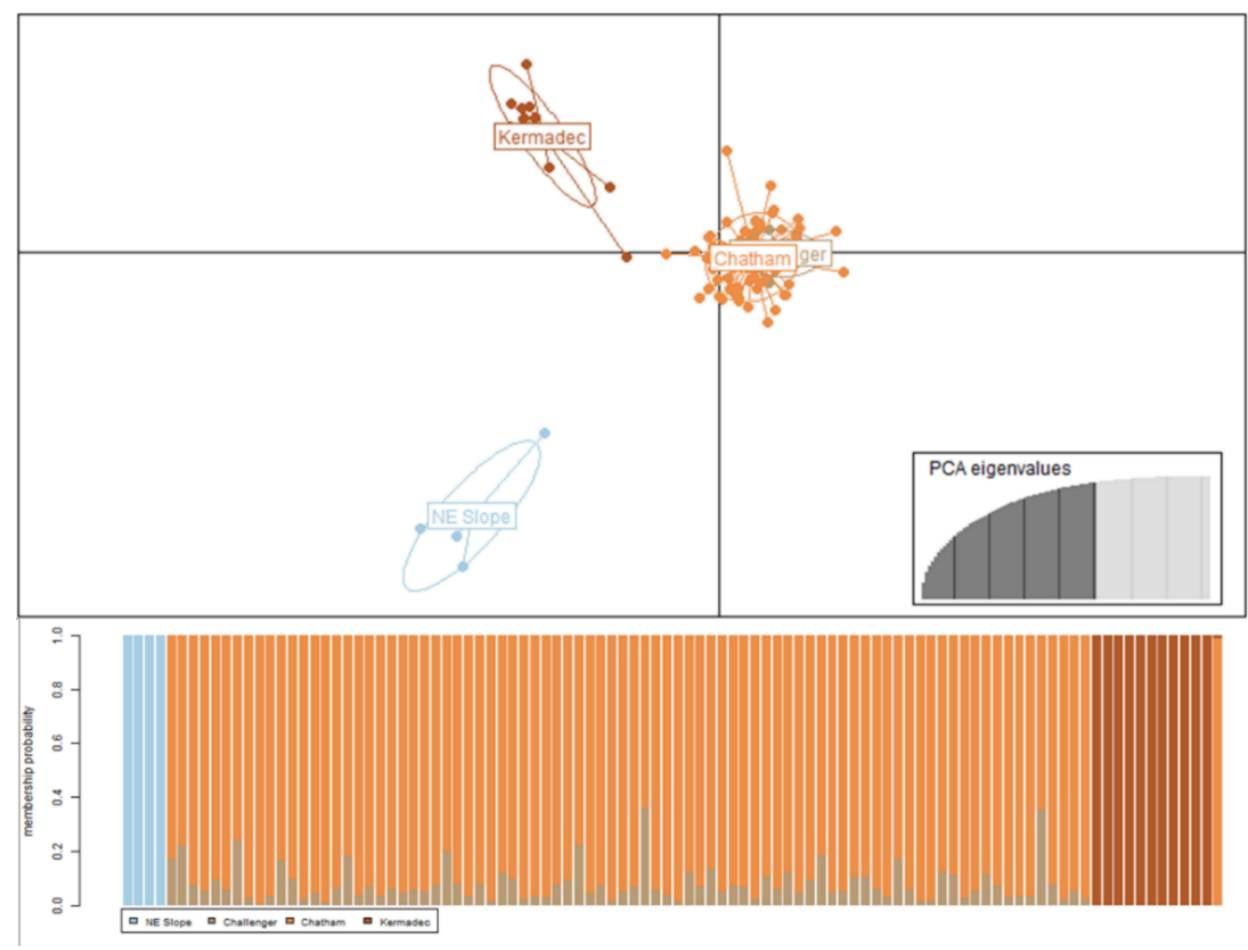

Figure 6.4 Scatterplot (above) and complot (below) of DAPC of Goniocorella dumosa based on variation at all microsatellite loci (60 PCs and 2 DAs)

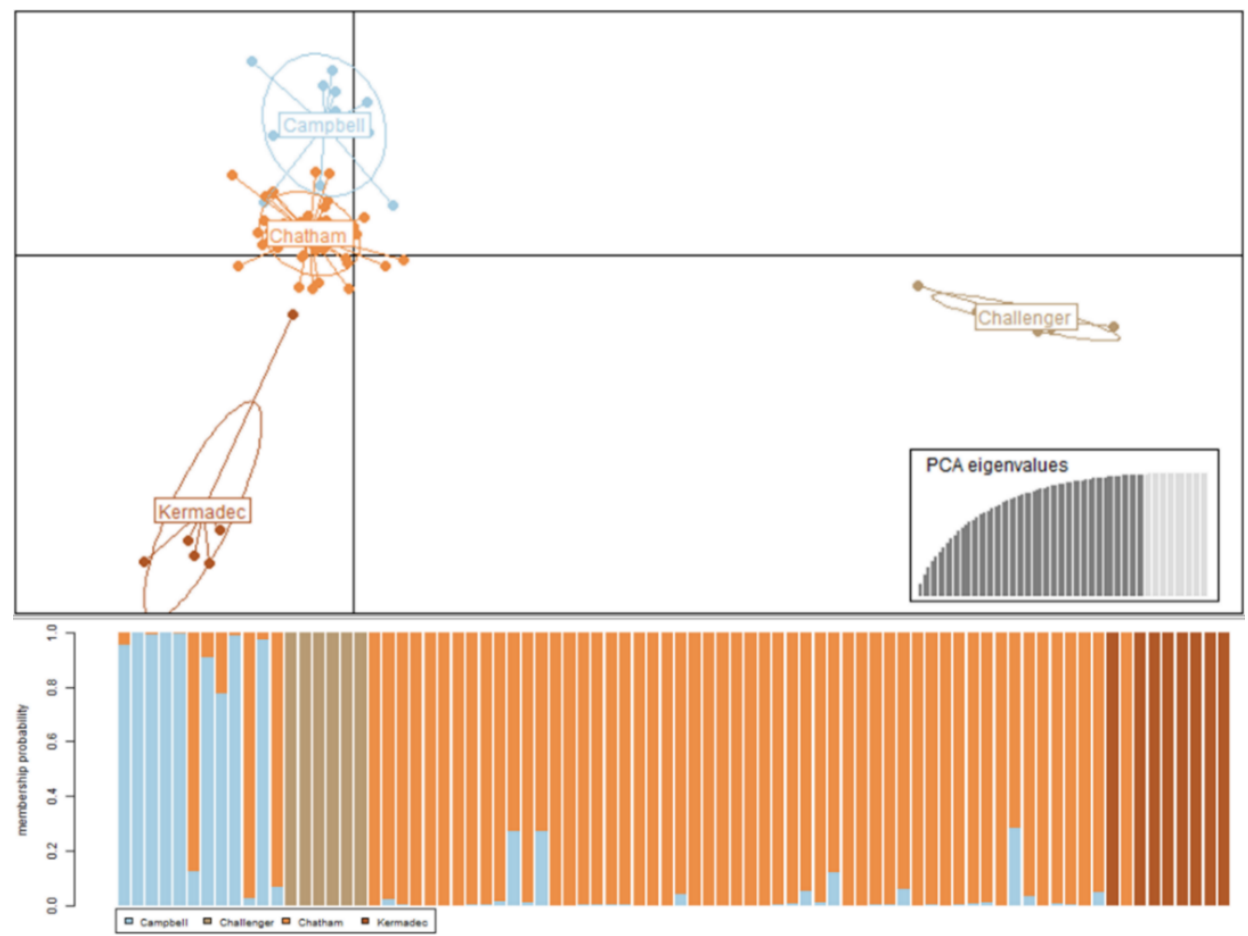

Figure 6.5 Scatterplot (above) and complot (below) of DAPC of Madrepora oculata based on variation at all microsatellite loci (60 PCs and 2 DAs) 


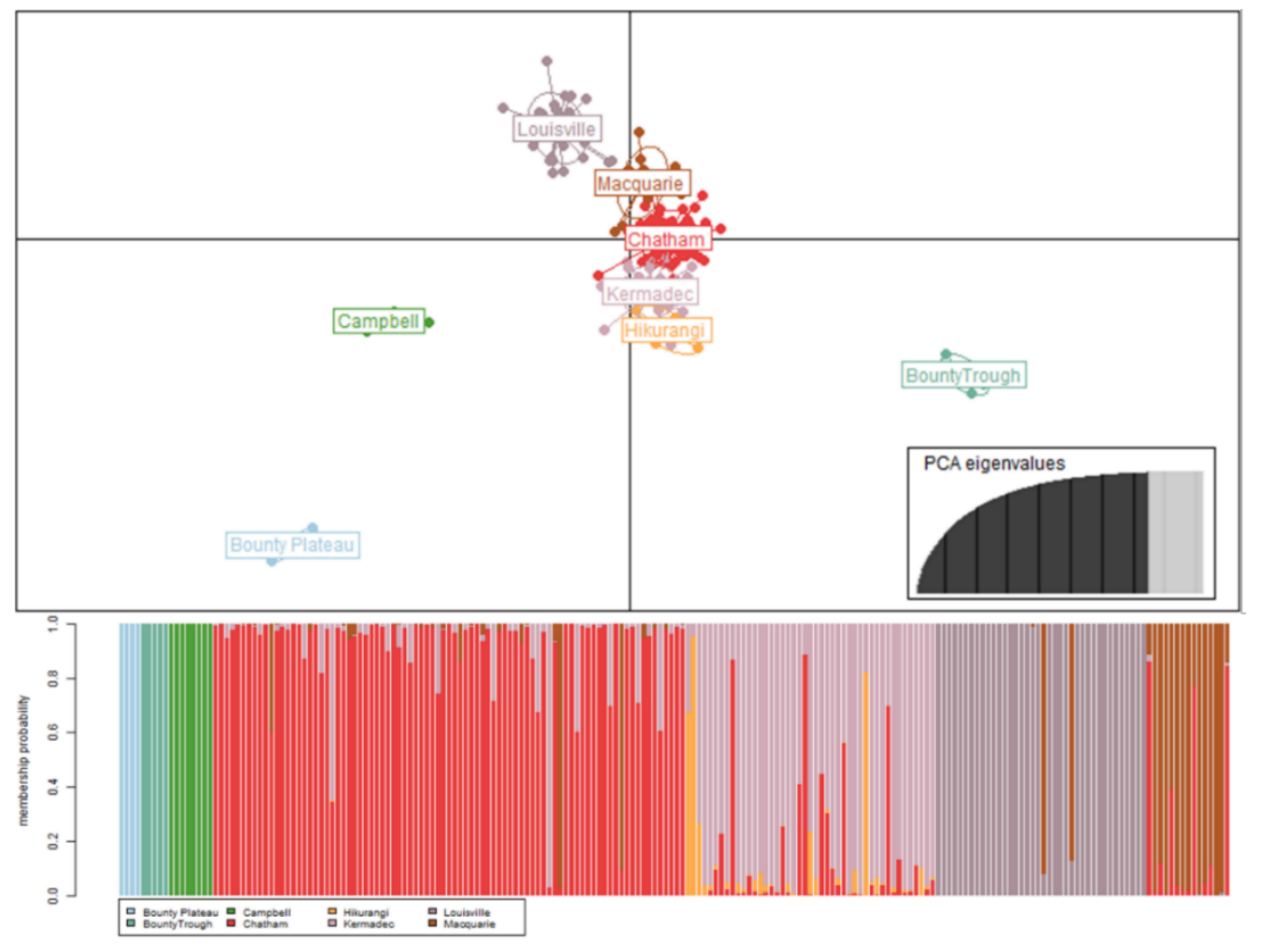

Figure 6.6 Scatterplot (above) and complot (below) of DAPC of Solenosmilia variabilis based on variation at all microsatellite loci (154 PCs and 2 DAs)

\subsubsection{Barriers to gene flow}

For G. dumosa, BARRIER software predicted that barriers to gene flow may exist for the Challenger Plateau and for the Chatham Rise populations (Figure 6.7A). In M. oculata, because there were no significant pairwise $F_{\mathrm{ST}}$ values calculated from the microsatellite data sets, the barriers to gene flow were predicted from the $\Phi_{\mathrm{ST}}$ values of the ITS sequence, which indicated that the Kermadec Ridge (to the north) and Chatham Rise populations are isolated (Figure 6.7B). There was generally very good agreement for the location of barriers to gene flow amongst populations of $S$. variabilis based on $\Phi_{\mathrm{ST}}$ values of the ITS sequence (Figure 6.7C) and $F_{\mathrm{ST}}$ values of the full microsatellite data set (Figure 6.7D). In both instances, four barriers to gene flow were predicted to occur, isolating the far north (Kermadec Ridge), the far east (Louisville Seamount Chain), the east (Chatham Rise), the south central (Campbell Plateau, Bounty Plateau, Bounty Trough), and the far south (Macquarie Ridge) populations. Comparisons of the locations of barriers to gene flow amongst species are somewhat limited by differences in sampling coverage for the three corals, but where spatial coverage overlaps, barriers to gene flow are common (Figure 6.7). In particular, regions such as the Kermadec Ridge and the Chatham Rise are identified as being bounded by barriers to gene flow, and 
other regions such as the south central (Campbell Plateau, Bounty Plateau, Bounty Trough) and also the far south (Macquarie Ridge) are also bounded by barriers. The status of populations on the Louisville Seamount Chain (to the east, outside the NZ EEZ) is hard to determine given that there only have samples of $S$. variabilis from this region, but there is strong evidence of its isolation based on two marker types (Figure 6.7).

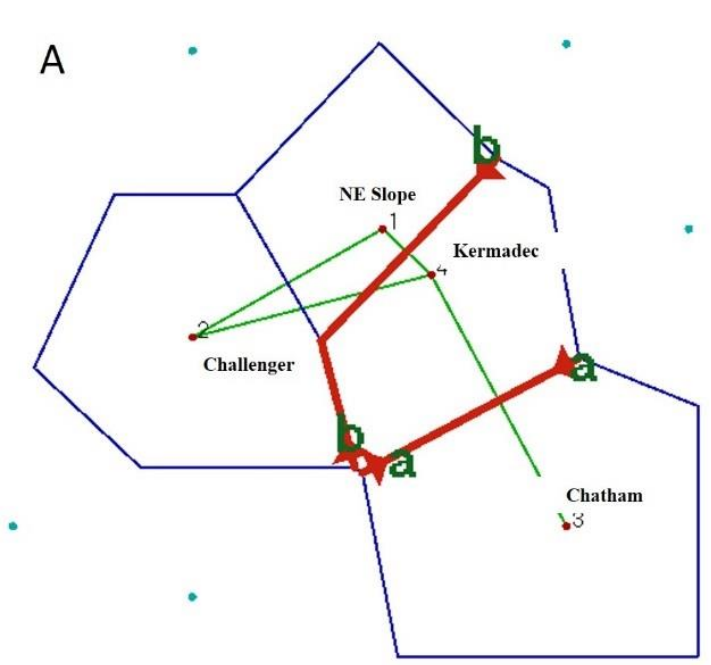

C

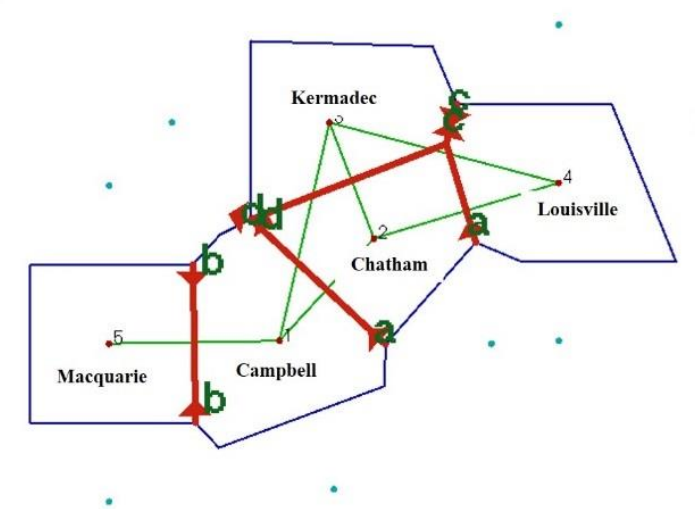

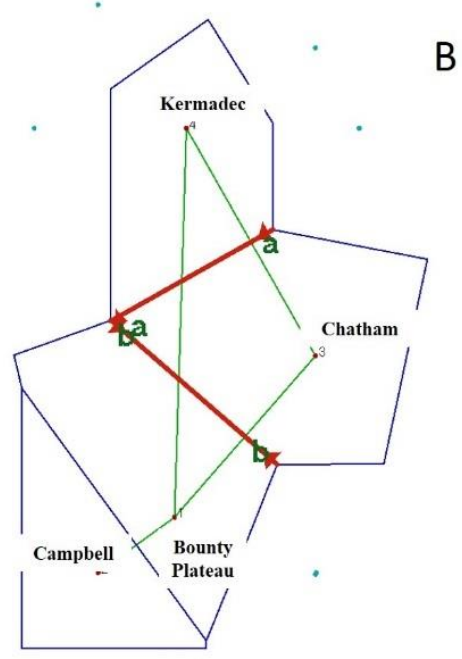

B

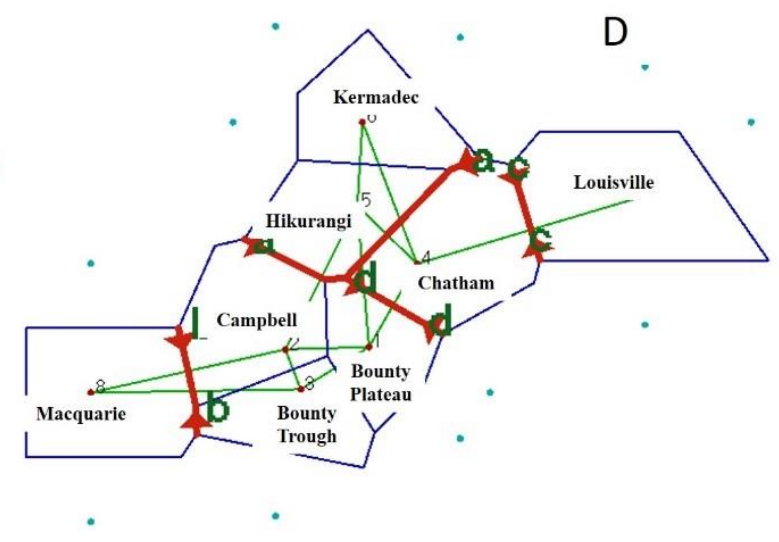

Figure 6.7 Barriers to gene flow indicated by software Barriers based on the full microsatellite data set for Goniocorella dumosa (A), ITS data for Madrepora oculata (B), ITS data for Solenosmilia variabilis (C) and the full microsatellite data set for Solenosmilia variabilis (D)

\subsubsection{Larval migration}

For G. dumosa, $\theta$ values estimated from MIGRATE ranged from 0.012 (Chatham Rise) to 0.962 (NE Slope) for all loci, and from 0.102 (NE Slope) to 0.931 (Challenger Plateau) for the neutral loci data set. All populations showed evidence of high levels of self- 
recruitment and there was evidence of only low levels of between-population gene flow in both microsatellite data sets. In both cases, populations on the Kermadec Ridge and the NE Slope were identified as source populations because they contributed but did not receive migrants. Additionally, both sets of analyses agreed that the populations on the Chatham Rise and the Challenger Plateau were not genetically connected with each other (Figure 6.8).
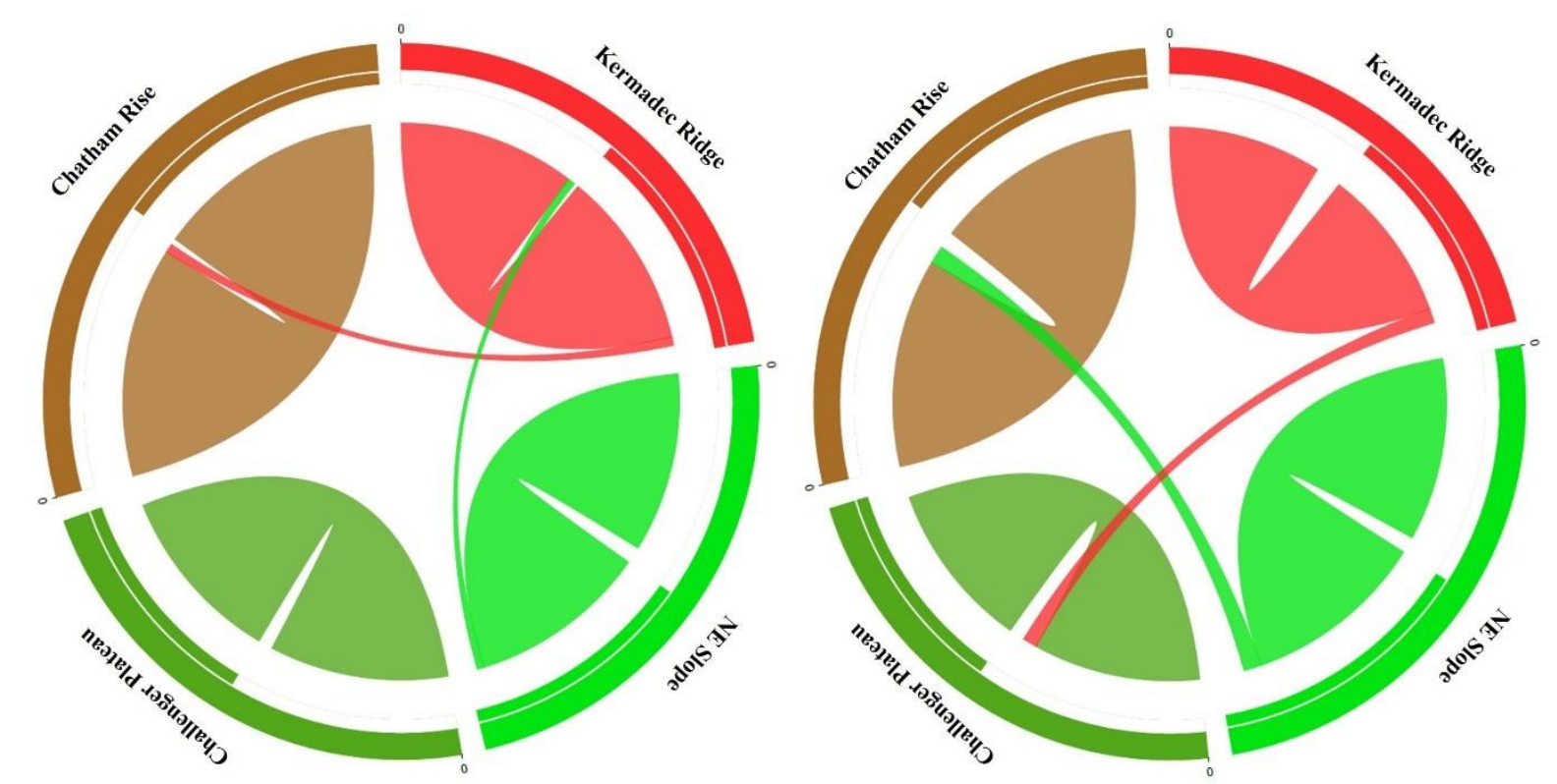

Figure 6.8 Circular plots of migration (gene flow) between and within populations of Goniocorella dumosa on geomorphic features based on all microsatellite loci (left) and neutral microsatellite loci (right)

For M. oculata, $\theta$ values estimated from the ITS data set ranged from 0.004 (Chatham Rise) to 0.265 (Bounty Plateau). Estimates of per generation migrants were variable amongst sample locations and suggested low to moderate gene flow. All populations showed evidence of some self-recruitment. The populations on the Kermadec Ridge and on the Chatham Rise did not exchange migrants with other populations. The Campbell Plateau and Bounty Plateau populations were well connected with each other (Figure 6.9). 


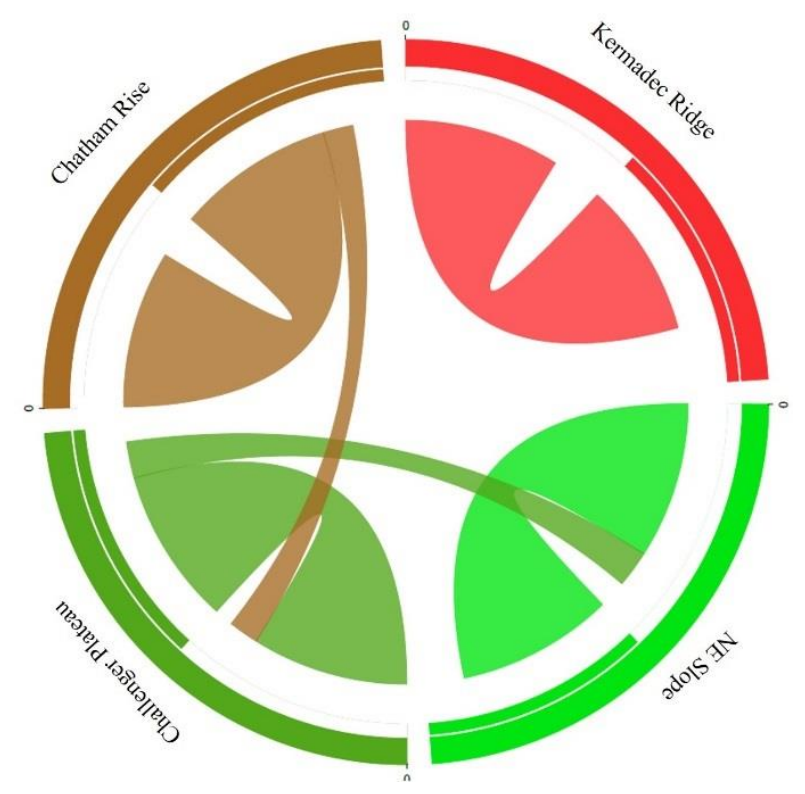

Figure 6.9 Circular plots of migration (gene flow) between and within populations of Madrepora oculata on geomorphic features based on ITS sequence data

For $S$. variabilis, $\theta$ values ranged from 0.076 (Bounty Trough) to 0.136 (Hikurangi Margin) in the full microsatellite dataset, and from 0.183 (Hikurangi Margin) to 1.880 (Macquarie Ridge) in the neutral microsatellite data set. Estimates of numbers of migrants per generation were variable amongst samples, and all populations showed evidence of medium to high levels of self-recruitment in both data sets (Figure 6.9). The Macquarie Ridge population made no contribution to the populations in the north (Kermadec Ridge, Louisville Seamount Chain and Hikurangi Margin), and the Louisville Seamount Chain population did not contribute migrants to the Macquarie Ridge population. Populations such as those on the Kermadec Ridge and the Bounty Trough were identified as being sources that contributed migrants to other populations but received limited or no migrants, whereas the Louisville Seamount Chain population was identified as a sink that received migrants from other populations but did not contribute to other populations. 

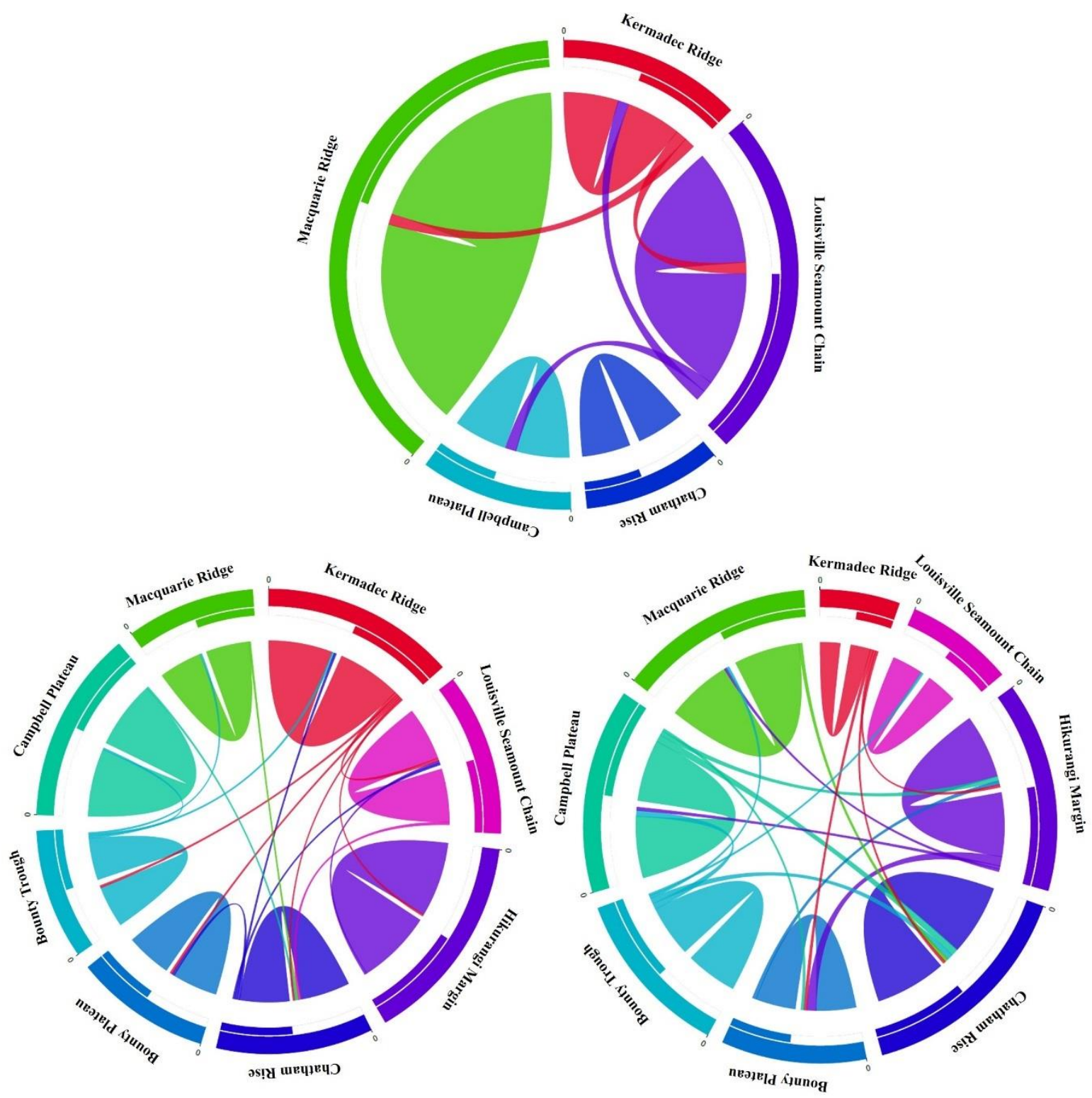

Figure 6.10 Circular plots of migration (gene flow) between and within populations of Solenosmilia variabilis on geomorphic features based on ITS sequence data (top), all microsatellite loci (bottom left) and neutral microsatellite loci (bottom right)

\subsection{Discussion}

Three habitat-forming deep-sea corals, G. dumosa, M. oculata and S. variabilis, were examined for genetic connectivity and gene flow amongst populations in the New Zealand region for the purpose of contributing to effective spatial management planning options to protect VMEs. An a priori hierarchical and spatially explicit approach was employed to hypothesis testing for population genetic structure, and identified barriers to gene flow as 
well as source and sink populations, all of which may contribute significantly to management decisions with conservation outcomes in the deep sea (e.g., Boschen et al., 2016). The testing framework is based on knowledge of environmental, and at times biological, differences between regions within and beyond the New Zealand EEZ, and also the possible influence of physical oceanographic currents that may influence genetic connectivity amongst geomorphic features such as ridges, plateaux and troughs. In addition, to maximise genetic information return, an approach that uses both a subset of putatively neutral microsatellite loci and also all loci was employed, regardless of possible causes of deviation from neutrality. This approach, whilst not common practice, has been shown to be of value (e.g., Wei et al., 2013; Gagnaire et al., 2015), in particular when loci that would ordinarily be discarded are informative, despite not meeting neutral expectations. The results for the full locus data set and for neutral loci subset are very much in agreement, and therefore provide some degree of independent confirmation of the findings.

\subsubsection{Influence of sample sizes and markers on structures detected}

As previously discussed for sponges in Chapter 5, small sample sizes for the three corals examined here are a challenge for population genetics studies. In practice, an understanding of the population genetics of many deep-sea taxa has to be pursued with less than perfect sample sets (resulting from logistic and cost difficulties of sample collection) because of the current management need to protect biodiversity, and particularly sensitive habitats such as VMEs. Although the limited sampling made it difficult to reveal small-scale population genetic structure, the approach employed here provides a broad spatial-scale view on the connectivity of these coral populations. Small samples sizes and an absence of fine spatial scale sampling are widely acknowledged as inevitable constraints in studies of deepsea organisms (Boschen et al., 2015, 2016). Although the sample sizes are acknowledged as a limitation that could fail to realise the full extent of genetic diversity within and between populations, they can still reveal information about patterns of genetic structure of sufficient magnitude to be useful to inform management decisions.

Due to low genetic diversity of nuclear (ITS) and mitochondrial regions (16s and Dloop), it found that they had limited application in S. variabilis and M. oculata population genetics study (Miller et al., 2010). In the present research, genetic diversities of ITS and Dloop regions were also low, but both of them provided evidence of population structure in all three examined species. While focusing on small-scale structures, sequencing markers were 
problematic to draw high resolution patterns. On the other hand, microsatellites, compared to sequences, provide better resolution of genetic structures, connectivity and isolation, but they require a larger sample size. Therefore, in the present study, to obtain a comprehensive understanding of the three examined corals both DNA sequence and microsatellite marker types were considered, and robust results were highlighted when both marker results were in agreement.

As the hierarchical spatial testing increased in definition, so the sample sizes decreased. Thus, the analyses have small sample sizes for most geomorphic features but larger sample sizes for the north-south biogeographic province level of testing. Estimates of, for example, migrants between populations on geomorphic features should be interpreted with care and are best viewed in combination with other analyses. However, larger-scale population groups (provinces and regions) and populations on some geomorphic features (such as the Chatham Rise) had large enough sample sizes to reveal patterns of genetic differentiation and connectivity. Overall, the analyses are probably best viewed as a conservative interpretation of patterns of population genetic differentiation.

Use of the software package STRUCTURE (Falush et al., 2007) to help identify the number of distinct genetic groups (i.e., identification of $\mathrm{K}$ ) is now widespread in population genetics analyses (e.g., Ruiz-Ramos et al., 2015). STRUCTURE is a parametric analysis, and is known to perform best when sample sizes are equal, when null alleles are absent and when populations are in HWE (Putman \& Carbone, 2014; Puechmaille, 2016). The analyses using STRUCTURE with no a priori groupings did not provide reliable results, most probably because of large differences in population/region sample sizes and the absence of HWE in several samples. Absence of HWE amongst deep-sea corals, in particular the presence of significant heterozygote deficiencies, is routinely reported (Le Goff \& Rogers, 2002; Baco et al., 2006; Lunden et al., 2014). One interpretation of multi-locus heterozygote deficiencies is that recruitment in many populations is locally derived with infrequent long-distance dispersal events. Such an interpretation is consistent with the results in this study for the three coral species were examined (see below). Because of the uncertainty associated with the STRUCTURE results, present study has focussed on other analyses for which there is greater robustness and more confirmation from other approaches.

Miller et al. (2010) noted that low genetic diversity of nuclear (ITS) and mitochondrial regions ( $16 \mathrm{~s}$ and D-loop) limits the utility of these markers in population genetics studies of $S$. variabilis and M. oculata. In the present study, genetic diversities of ITS and $D$-loop regions were low, and neither marker provided evidence of population structure 
in G. dumosa or S. variabilis, but ITS in M. oculata revealed a north-central-south regional pattern of differentiation. There were no unique haplotypes in any of the geomorphic feature populations and only four significant $\Phi_{S T}$ values were observed between pairs of populations on geomorphic features. These findings for the DNA sequences indicate that insufficient variation exists for these markers types to be informative at the scale of my work. In contrast, microsatellite variation provided much greater resolution of species-specific genetic structure, connectivity and isolation. Where significant results exist for both marker types for the same species they are in agreement (e.g., location of putative barriers to gene flow and estimation of exchange of migrants). In the present study, to obtain a comprehensive understanding of the population genetic structure of the three corals both DNA sequence and microsatellite marker types are considered, and robust results are highlighted when both marker results are in agreement.

\subsubsection{Patterns of genetic connectivity}

The DNA sequence markers provided only limited evidence of population genetic structure based on the hierarchical hypothesis testing framework, examination of microsatellite variation (full and reduced data sets) for all three species revealed structure at different spatial scales. Populations of M. oculata exhibited regional structure, populations of G. dumosa were differentiated at the regional and geomorphic features scale, and populations of S. variabilis exhibited structure between provinces, amongst regions and also amongst geomorphic features. In other comparable studies little or no evidence of province scale structure has been observed amongst populations of crustaceans and sponges in the New Zealand region (Bors et al., 2012; Chapter 5). Thus, it is concluded that there is little support for the hypothesis that water mass characteristics associated with biogeographic provinces within the New Zealand region result in genetic population structure of G. dumosa and $M$. oculata. However, a north-central-south regional pattern of structure was observed in another deep-sea Scleractinian, the cup coral, Desmophyllum dianthus, based on ITS variation (Miller et al., 2010) and also in the deep-sea sponge Poecillastra laminaris based COI, Cytb and microsatellite variation (Chapter 5). In combination, these findings provide evidence in support of the hypothesis that currents along the Chatham Rise act as a barrier to gene flow for several species across multiple phyla. Significant genetic differentiation has already been reported at the geomorphic feature population level in non-coral species between, for example, the Challenger Plateau and the Chatham Rise (Bors et al., 2012; Knox et al., 2012). 
These results, combined with those for the deep-sea corals, support the hypothesis that oceanic dynamics contribute to the formation of fine-scale population structure amongst populations on different geomorphic features within, and most likely also outside, the New Zealand EEZ.

\subsubsection{Oceanographic dynamics}

The patterns of multi-species genetic differentiation detected by the various markers may be attributable to a relationship between population distributions and oceanic current systems. Currents not only play a role in promoting long-distance transport of larvae, but may also act as barriers to gene flow (Rogers et al., 1998; Zane et al., 1998; Roques et al., 2002; Sutton \& Chereskin, 2002; Shaw et al., 2004; Plouviez et al., 2013). For example, currents acting as a 'soft' barrier to population connectivity have been indicated for octocorals in a recent study around New Zealand (Dueñas et al., 2016).

In this study, it was hypothesised that currents associated with the Subtropical Front would influence north-central-south regional scale population connectivity, which was indeed observed for all three corals. Surface and intermediate currents flow from northwest New Zealand in a southerly direction to the Chatham Rise following the eastern coastline of the North Island. Currents also flow from the southeast of New Zealand northward along the east coast of the South Island towards the Chatham Rise. The result is that currents from the warmer north and the cooler south meet and mix along the Chatham Rise, forming the Subtropical Front, before heading east into the Pacific Ocean (Chiswell et al., 2015). These two contrasting sets of currents, their associated eddies and the Subtropical Front may therefore impede larval migration between the south and north regions, and provide an explanation for the increased genetic diversity values observed amongst populations on the Chatham Rise (central region) for all three corals and also for some sponges (Chapter 5).

At the geomorphic features level, our analyses of the three corals provided specific evidence for a lack of genetic connectivity between populations on the Kermadec Ridge and the Chatham Rise (significant $F_{S T}$, clear split in DAPC plots and few if any migrants between these populations). No previous study has investigated connectivity between these two areas, but significant genetic subdivision between populations of $D$. dianthus was detected between the Auckland Island slope (far south) and seamounts on the northern Chatham Rise (Miller et al., 2010). This differentiation is consistent with patterns of flow at depth, with water from the far south flowing north until it reaches the southern edge of the Chatham Rise, before it is 
deflected eastward (Chiswell et al., 2015). In other words, migrants from the Auckland Island slope are not expected to reach the northern edge of the Chatham Rise, which will result in some degree of genetic differentiation, which is indeed what is observed. For S. variabilis, the most apparent difference between populations on geomorphic features was that between the Louisville Seamount Chain and all other populations. The general lack of current flow from the Louisville Seamount Chain towards most topographic features elsewhere in the New Zealand region (Chiswell \& Rickard, 2006) is likely to be the reason that populations of $S$. variabilis on this feature are differentiated genetically from those in the rest of the region.

\subsubsection{Depth and associated environmental gradients}

The influence of currents on population connectivity is expected to vary with depth, but generally we lack sufficient knowledge about the detail of depth-stratified flow to make robust predictions about how patterns of gene flow may vary with depth, in particular over large spatial scales. Nonetheless, depth is an important factor contributing to patterns of genetic connectivity for some benthic fauna, particularly for taxa that are distributed across a wide depth range (Chapter 2). In the present study, there was no evidence for isolation by depth (across the depth range 322-1805 m) for S. variabilis. Populations of S. variabilis from Australian waters were also not differentiated by depth, but populations were sampled within a more restricted depth range (1000-1400 m) (Miller \& Gunasekera, 2016). For populations of both G. dumosa (from 300-600 m) and M. oculata (from 700-1200 m) at least one marker revealed the existence of a pattern of isolation by depth. As neither G. dumosa nor M. oculata populations were genetically isolated by distance, this implies that depth and related environmental gradients may play an important role in the larval migration of these species. Within the New Zealand region Miller et al. (2011) reported that the stony cup coral $D$. dianthus from different depth strata $(<600 \mathrm{~m}, 1000-1500 \mathrm{~m},>1500 \mathrm{~m})$ are strongly differentiated based on DNA sequence variation. Unfortunately, the limited sample sizes of the present study precluded detailed analysis to determine at what depth(s) genetic differentiation for G. dumosa and M. oculata populations takes place (i.e., the response may just as easily be a gradient as an abrupt break). 


\subsubsection{Life history and dispersal strategies}

Species-specific differences in reproductive strategies may contribute to differences in patterns of gene flow and genetic structure observed across taxa in the deep sea (e.g., Bors et al., 2012; Samadi et al., 2006; Hilário et al., 2015). All three showed pronounced patterns of self-recruitment which doubtless have contributed to the patterns of genetic structure detected in all three species, as well as the limited connectivity that exist amongst regions. Interestingly, the migration estimates amongst populations on different geomorphic features for the three corals may be related to maximum oocyte size i.e., there is a positive relationship between increasing oocyte size and increasing connectivity (Table 6.18 summarises data for the three corals). M. oculata has the largest mean oocyte size (2 3 times larger than other two species) and was the only species for which significant differentiation amongst populations on geomorphic features was not observed, whereas $G$. dumosa and $S$. variabilis with a smaller mean oocyte diameter exhibited less connectivity (i.e., significant structure existed amongst populations on geomorphic features). Based on previous studies, $S$. variabilis and M. oculata are lecithotrophic, and they feed only after settlement and metamorphosis (Waller et al., 2005; Pires et al., 2014). Given its similarity to S. variabilis in terms of morphology and reproduction, the larvae of G. dumosa are likely to share the same characteristics. Based on this assumption, the larger oocyte size, providing more nutritional support, may promote a longer larval life and greater dispersal potential for M. oculata. Such a case would explain the MIGRATE result that $M$. oculata had a greater number of migrants amongst the geomorphic populations than either G. dumosa or S. variabilis, but will, of course, require testing. This result provides further support for the idea that life history characteristics may vary even between closely related deep-sea corals (Burgess \& Babcock, 2005 ) and therefore can influence patterns of population connectivity.

In the present study, none of the three coral species showed strong evidence of asexual reproduction when all specimens were assessed using multi-locus microsatellite genotypes. Whilst there was no direct evidence to indicate asexual reproduction in any of the three species, the smaller effective population size with heterozygote deficits at most loci of $S$. variabilis suggests that this species has a higher asexual or inbreeding rate in populations on geomorphic features than the other two species. Miller \& Gunasekera (2016) predicted that clonal reproduction may account for as much as $76 \%$ of recruitment in Australian populations of $S$. variabilis, and if applicable in the New Zealand context then this high asexual recruitment rate would explain the self-recruitment result in this study. Asexual reproduction 
has also been observed in G. dumosa, but the proportion of clonal reproduction in recruitment is unknown, whilst for M. oculata there is no evidence for asexual reproduction (Burgess \& Babcock, 2005). However, it has been reported that mixed clonal/sexual reproduction is nearly indistinguishable from strict sexual reproduction as long as the proportion of clonal reproduction is not large (Balloux et al., 2003).

Table 6.18 Information about reproductive life-history characteristics for Goniocorella dumosa,

Madrepora oculata and Solenosmilia variabilis

\begin{tabular}{|c|c|c|c|c|c|c|}
\hline Species & $\begin{array}{l}\text { Mode of Sexual } \\
\text { reproduction }\end{array}$ & $\begin{array}{c}\text { Max } \\
\text { Oocyte } \\
\text { diameter }\end{array}$ & $\begin{array}{l}\text { Fecundity } \\
\text { (oocytes per } \\
\text { polyp) }\end{array}$ & Methods & Production & Larvae \\
\hline $\begin{array}{c}\text { Goniocorella } \\
\text { dumosa }\end{array}$ & Gonochoric (1) & $135 \mu \mathrm{m}(1)$ & 480 (1) & Spawner (1) & Seasonal (1) & - \\
\hline $\begin{array}{c}\text { Madrepora } \\
\text { oculata }\end{array}$ & $\begin{array}{c}\text { Functional } \\
\text { Gonochoric } \\
(1,2,3)\end{array}$ & $\begin{array}{l}350 \mu \mathrm{m}(1) \\
650 \mu \mathrm{m}(2)\end{array}$ & $10(1)$ & $\begin{array}{c}\text { Broadcast } \\
\text { Spawner } \\
(1,2)\end{array}$ & $\begin{array}{c}\text { Periodic (1) } \\
\text { /Continuous (2) }\end{array}$ & $\begin{array}{c}\text { Lecithotrophic } \\
\qquad(1,2)\end{array}$ \\
\hline $\begin{array}{c}\text { Solenosmilia } \\
\text { variabilis }\end{array}$ & Gonochoric $(1,2)$ & $\begin{array}{l}165 \mu \mathrm{m}(1) \\
337 \mu \mathrm{m}(2)\end{array}$ & $290(1)$ & $\begin{array}{c}\text { Broadcast } \\
\text { Spawner } \\
(1,2)\end{array}$ & $\begin{array}{l}\text { Seasonal (1) / } \\
\text { Continuous (2, } \\
4)\end{array}$ & $\begin{array}{c}\text { Lecithotrophic } \\
(1,2)\end{array}$ \\
\hline
\end{tabular}

1. Waller (2005). 2. Pires et al. (2014). 3. Having a few hermaphroditic colonies. 4. A reproductive peak between April and September (Autumn-Spring).

\subsubsection{Conservation and management implications}

Seventeen seamount closure areas and 17 Benthic Protection Areas (BPAs) were established to protect benthic fauna, including VMEs such as coral reefs, from bottom trawl fisheries throughout the New Zealand EEZ in 2001 and 2007, respectively (Brodie \& Clark, 2002; Helson et al., 2010). These seamount closures and BPAs were not designed with population connectivity within and between these areas in mind, beyond the general principle that such protected areas should be large and be distributed amongst different environments throughout the EEZ. Understanding connectivity amongst areas is fundamental to designing an effective protected area network, or for modifying the existing distribution of protected areas to create a network (Palumbi, 2003). The maintenance of genetic diversity, and the protection of genetically distinct populations should also be considered as an integral component of protected area design (Bors et al., 2012).

The results of the present study for G. dumosa, M. oculata and S. variabilis provide new information useful for assessing the effectiveness of existing protection measures, and the design of future protected area networks in the EEZ as a proxy for VME indicator species with similar patterns of genetic population connectivity. The populations of the three coral species all showed evidence of pronounced self-recruitment within the scale of the 
geomorphic features examined. This finding suggests that additional protected areas will be required to maintain genetic diversity (through self-recruitment) of populations at features that do not already receive some protection. For S. variabilis, populations on all the geomorphic features examined were to some extent source populations for other populations, but those from the Kermadec Ridge and the Chatham Rise seem to be particularly important. Of particular significance as a migrant source for $G$. dumosa and $S$. variabilis populations was the Kermadec Ridge.

Currently, most geomorphic features examined contain one or more BPAs or seamount closures. The exceptions are the Hikurangi Margin, Bounty Trough and Macquarie Ridge, as well as the Lord Howe Rise and the Louisville Seamount Chain both of which are outside the New Zealand EEZ. The Hikurangi Margin is an area subject to bottom trawling (Clark \& O'Driscoll, 2003) and populations of VME indicator taxa, such as reef-forming corals, are therefore at risk from disturbance. The Bounty Trough is a geomorphic feature where deep-water drilling for hydrocarbons may occur in the future (Wood \& Davy, 2008), making populations of VME indicator taxa that exist there also at risk from anthropogenic activities. Although there are no BPAs on the Macquarie Ridge, two seamount closures to the west and east of the ridge, and a BPA to the south, may provide some protection for this southern source of genetic connectivity. However, whether these areas protect this genetic diversity will depend on whether they provide suitable habitat for the corals. Source populations of corals on the Kermadec Ridge are mainly protected from bottom trawling by the Kermadec and Tectonic Reach BPAs, and will likely receive additional protection should a proposed Kermadec Ocean Sanctuary be established in the near future (http://www.mfe.govt.nz/marine/kermadec-ocean-sanctuary). However, outside of the sanctuary area corals and other VME indicator taxa are still vulnerable to disturbance from future seabed mining of sulphide deposits in the region (Boschen et al., 2015). While there are two BPAs on the Chatham Rise, they do not cover the depth range of the two deeperdistributed species, in particular S. variabilis, nor do they provide much protection along the axis of the Rise, including some areas where G. dumosa forms its most dense thickets in an area of interest for phosphorite nodule mining. Considering the relative importance of the Chatham Rise as a sink population and as a hotspot area with the highest genetic diversity for the three coral species, additional protected areas should be considered here for the future management of human activities on this feature. This recommendation is the same as that made for deep-sea sponges in the New Zealand region (Chapter 5). 
The results of the present study, and those of previous genetic connectivity studies for various species around New Zealand (Smith et al., 2004b; Miller et al., 2010; Bors et al., 2012; Knox et al., 2012; Foltz et al., 2013; Boschen et al., 2015; Dueñas et al., 2016) provide genetic connectivity information at different scales. Not surprisingly, their connectivity patterns differ for the range of taxa with differing ecological characteristics. These variable patterns demonstrate the need for a flexible spatial management system that can be periodically adjusted to accommodate increased understanding about the connectivity of a range of benthic taxa at a variety of spatial scales. 


\section{Appendix}

Table S6.1 Success ratios of three corals for molecular experiments

\begin{tabular}{lccccc}
\hline & Total & Sampled & ITS & D-loop & SSR \\
\hline Goniocorella dumosa & 244 & $134(28.09 \%)$ & $133(27.85 \%)$ & $70(14.68 \%)$ & $108(22.64 \%)$ \\
Madrepora oculata & 164 & $96(20.13 \%)$ & $74(15.51 \%)$ & - & $93(19.50 \%)$ \\
Solenosmilia variabilis & 477 & $251(52.62 \%)$ & $186(38.99 \%)$ & $139(29.14 \%)$ & $208(43.61 \%)$ \\
\hline
\end{tabular}

Table S6.2 Locus by locus information for Goniocorella dumosa

\begin{tabular}{|c|c|c|c|c|c|}
\hline Locus & Success & $F_{I S}$ & $F_{S T}$ & Oosterhout & Selection \\
\hline B106 & $100.00 \%$ & -0.241 & 0.335 & -0.0278 & Neutral \\
\hline B1 & $100.00 \%$ & -0.794 & 0.031 & -0.4978 & Balancing \\
\hline $\mathrm{C} 107$ & $91.67 \%$ & 0.088 & 0.222 & 0.148 & Neutral \\
\hline D5 & $87.96 \%$ & -0.066 & 0.203 & 0.073 & Neutral \\
\hline B123 & $81.48 \%$ & -0.474 & 0.148 & 0.013 & Balancing \\
\hline D213 & $77.78 \%$ & -0.297 & 0.265 & -0.0046 & Neutral \\
\hline SVA13 & $70.37 \%$ & -0.419 & 0.331 & -0.0807 & Neutral \\
\hline $\mathrm{C} 4$ & $72.22 \%$ & -0.235 & 0.273 & 0.1009 & Positive \\
\hline SVA15 & $66.67 \%$ & -0.089 & 0.258 & 0.1327 & Neutral \\
\hline SVA10 & $60.19 \%$ & -0.172 & 0.482 & 0.0562 & Balancing \\
\hline SVA2 & $62.96 \%$ & -0.524 & 0.208 & 0.0314 & Balancing \\
\hline SVA18 & $60.19 \%$ & -0.276 & 0.434 & 0.0952 & Positive \\
\hline SVA8 & $56.48 \%$ & -0.417 & 0.364 & 0.0166 & Neutral \\
\hline D103 & $61.11 \%$ & -0.229 & 0.401 & 0.0737 & Neutral \\
\hline SVA1 & $52.78 \%$ & 0.046 & 0.629 & 0.2778 & Positive \\
\hline B116 & $59.26 \%$ & -0.156 & 0.352 & 0.0917 & Balancing \\
\hline SVA11 & $52.78 \%$ & -0.853 & 0.459 & -0.4397 & Balancing \\
\hline SVA4 & $43.52 \%$ & -0.43 & 0.553 & -0.0002 & Balancing \\
\hline SVA7 & $36.11 \%$ & 0.185 & 0.602 & 0.1387 & Positive \\
\hline SVA20 & $57.41 \%$ & -0.358 & 0.464 & 0.0137 & Neutral \\
\hline
\end{tabular}

Table S6.3 Locus by locus information for Madrepora oculata

\begin{tabular}{lccccc}
\hline \multicolumn{1}{c}{ Locus } & Success & $\boldsymbol{F}_{\boldsymbol{I S}}$ & $\boldsymbol{F}_{\boldsymbol{S} \boldsymbol{T}}$ & Oosterhout & Selection \\
\hline c016 & $65.59 \%$ & -0.445 & 0.247 & 0.0018 & Neutral \\
g028 & $47.31 \%$ & -0.392 & 0.460 & 0.0630 & Neutral \\
c7 & $27.96 \%$ & -0.539 & 0.604 & 0.0515 & Neutral \\
g006 & $55.91 \%$ & -0.423 & 0.417 & 0.0361 & Neutral \\
g004 & $64.52 \%$ & -0.182 & 0.358 & 0.1405 & Neutral \\
g25 & $24.73 \%$ & 0.572 & 0.850 & 0.3126 & Positive \\
c013 & $73.12 \%$ & -0.319 & 0.463 & 0.1130 & Neutral \\
g016 & $30.11 \%$ & -0.263 & 0.648 & 0.0718 & Neutral \\
g022 & $30.11 \%$ & -0.044 & 0.678 & 0.2185 & Neutral \\
a5 & $55.91 \%$ & 0.009 & 0.434 & 0.2003 & Neutral \\
g010 & $61.29 \%$ & -0.333 & 0.311 & 0.1030 & Neutral \\
\hline
\end{tabular}


Table S6.4 Locus by locus information for Solenosmilia variabilis

\begin{tabular}{lccccc}
\hline \multicolumn{1}{c}{ Locus } & Success & $\boldsymbol{F}_{\boldsymbol{I S}}$ & $\boldsymbol{F}_{\boldsymbol{S} \boldsymbol{T}}$ & Oosterhout & Selection \\
\hline B106 & $96.63 \%$ & -0.559 & 0.188 & -0.3679 & Neutral \\
C107 & $94.23 \%$ & -0.330 & 0.180 & -0.0057 & Neutral \\
D5 & $95.19 \%$ & -0.127 & 0.221 & 0.0257 & Neutral \\
B1 & $96.63 \%$ & -0.259 & 0.178 & -0.0799 & Neutral \\
B116 & $87.98 \%$ & -0.067 & 0.284 & 0.0523 & Positive \\
D103 & $80.29 \%$ & -0.138 & 0.285 & 0.0221 & Neutral \\
B123 & $95.67 \%$ & 0.062 & 0.234 & 0.1865 & Neutral \\
D213 & $95.67 \%$ & -0.420 & 0.162 & -0.1265 & Balance \\
C4 & $93.75 \%$ & -0.115 & 0.346 & 0.0819 & Positive \\
SVA1 & $79.81 \%$ & -0.176 & 0.181 & 0.0869 & Neutral \\
SVA3 & $76.92 \%$ & -0.090 & 0.217 & 0.1007 & Neutral \\
SVA7 & $70.67 \%$ & -0.350 & 0.249 & -0.0666 & Neutral \\
SVA2 & $81.73 \%$ & 0.057 & 0.215 & 0.1084 & Neutral \\
SVA14 & $49.52 \%$ & -0.045 & 0.363 & 0.1137 & Positive \\
SVA15 & $94.23 \%$ & -0.380 & 0.160 & -0.1153 & Neutral \\
SVA4 & $72.12 \%$ & -0.215 & 0.232 & 0.0131 & Neutral \\
SVA12 & $41.83 \%$ & 0.195 & 0.444 & 0.2038 & Neutral \\
SVA16 & $60.58 \%$ & -0.140 & 0.391 & 0.0598 & Balance \\
SVA13 & $90.87 \%$ & -0.444 & 0.221 & -0.1311 & Neutral \\
SVA11 & $75.00 \%$ & -0.532 & 0.248 & -0.2239 & Balance \\
SVA10 & $70.67 \%$ & -0.263 & 0.206 & 0.0137 & Balance \\
SVA5 & $60.10 \%$ & -0.256 & 0.359 & -0.0728 & Balance \\
SVA18 & $77.40 \%$ & -0.047 & 0.276 & 0.1269 & Neutral \\
SVA8 & $59.62 \%$ & -0.076 & 0.236 & 0.1823 & Neutral \\
\hline
\end{tabular}




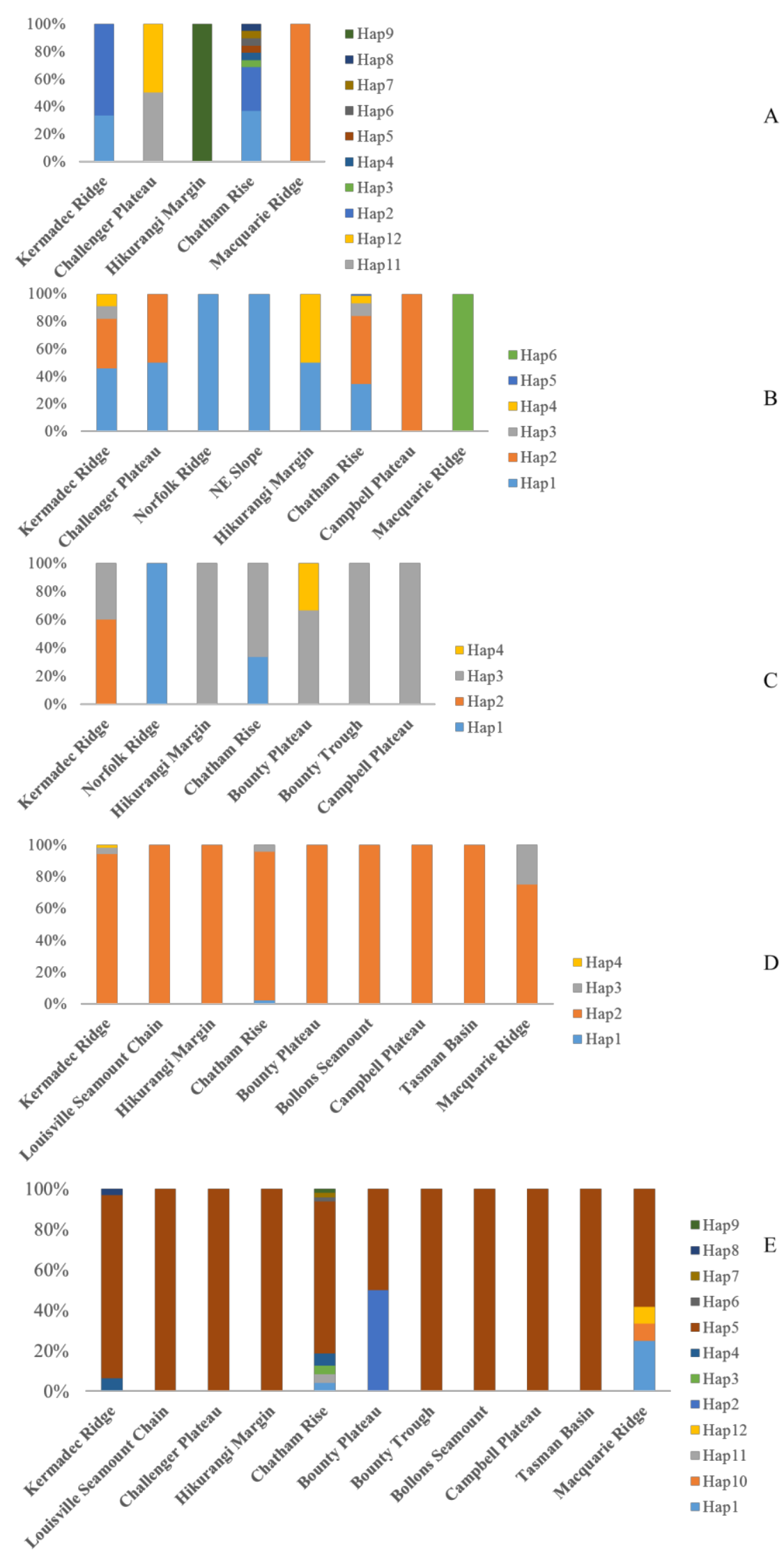

Figure S6.1 Haplotype distributions Goniocorella dumosa D-loop (A) ITS (B), Madrepora oculata ITS (C), Solenosmilia variabilis D-loop (D) and ITS (E) across populations on geomorphic features 


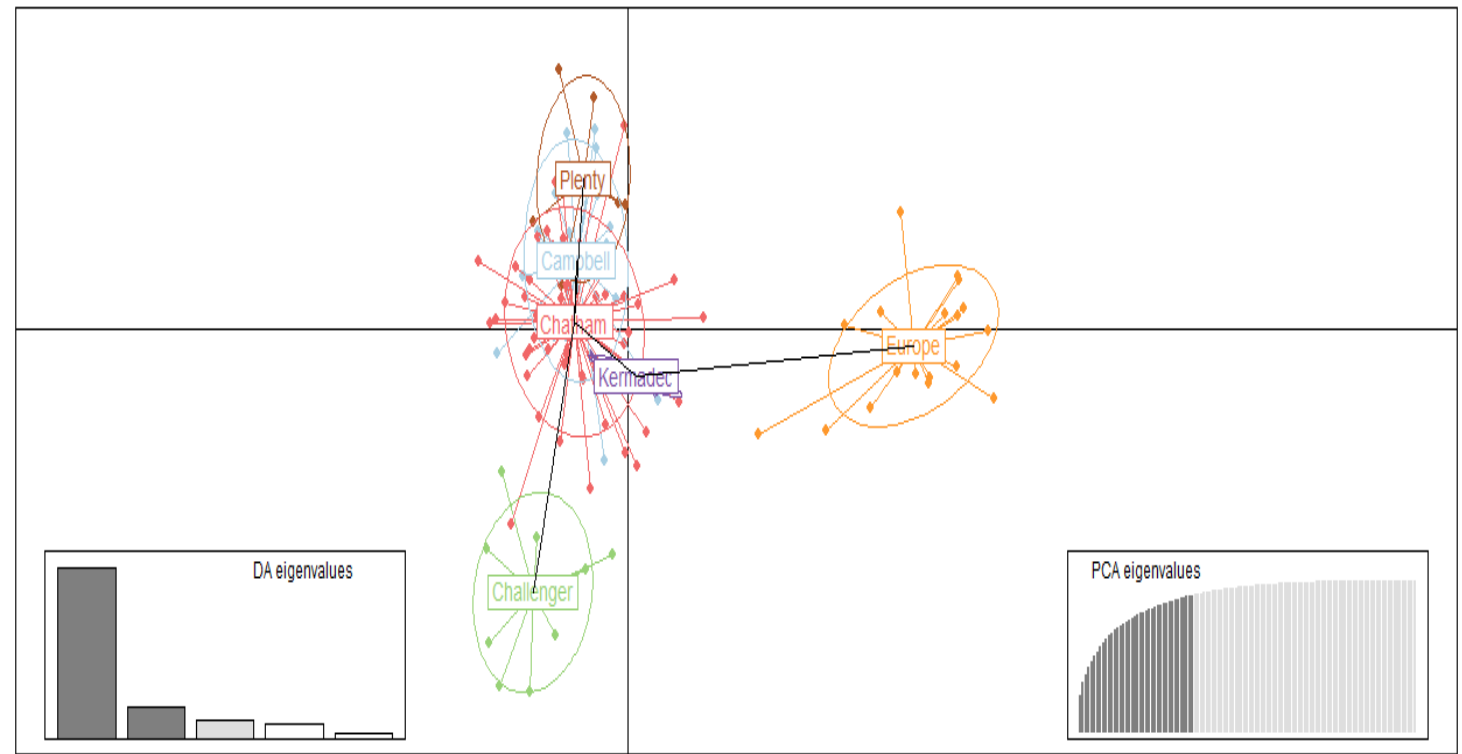

Figure S6.2 Scatterplot of DAPC of Madrepora oculata with New Zealand populations and the European population
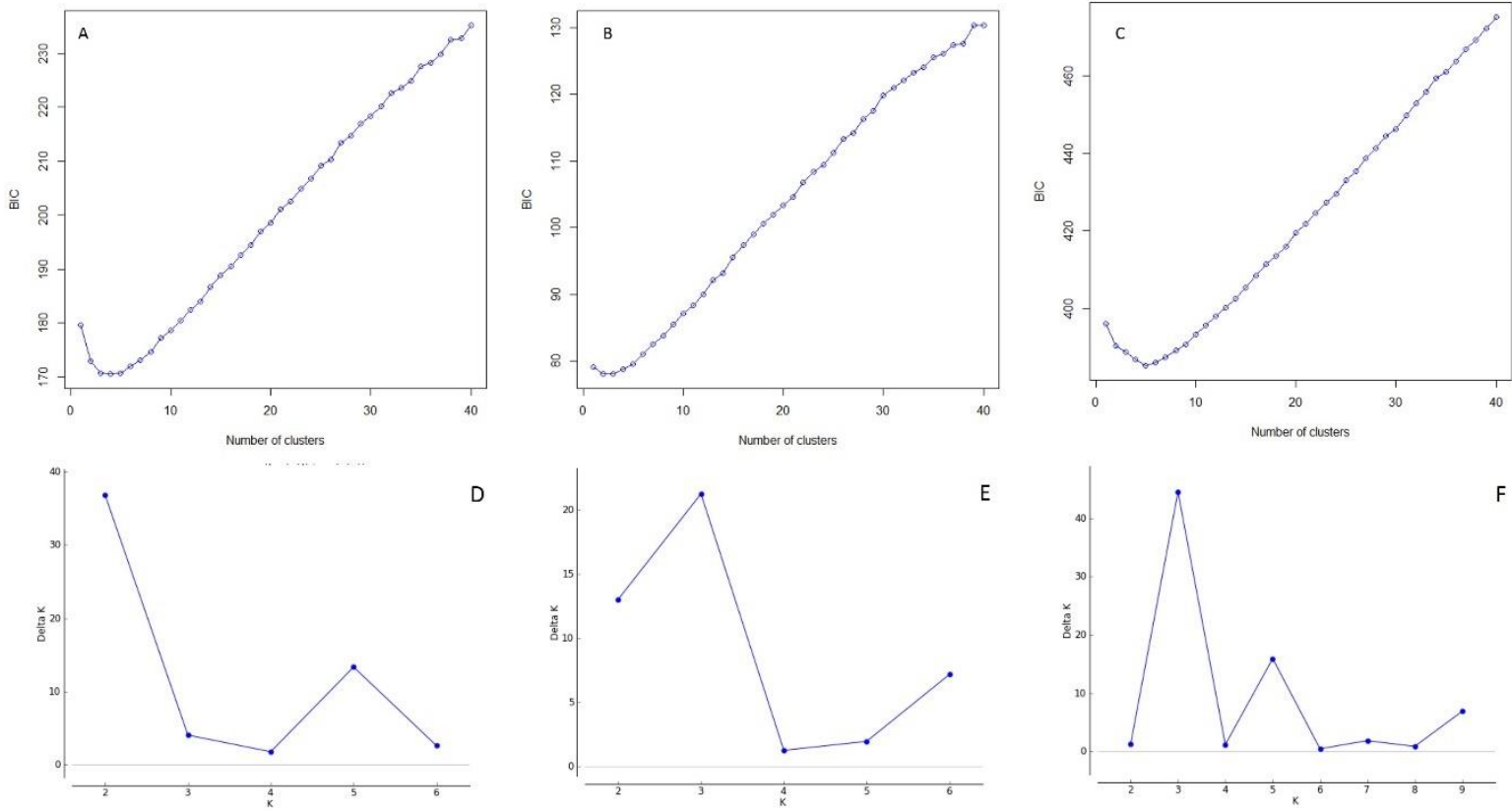

D
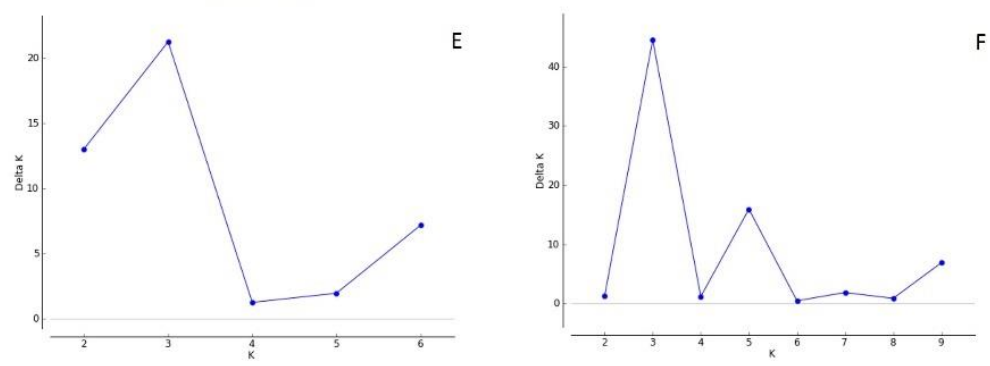

Fig S6.3 Optimal genetic clusters predicted by BIC for Goniocorella dumosa (A), Madrepora oculata (B) and Solenosmilia variabilis (C) and by Structure Harvester for Goniocorella dumosa (D), Madrepora oculata (E) and Solenosmilia variabilis (F) 

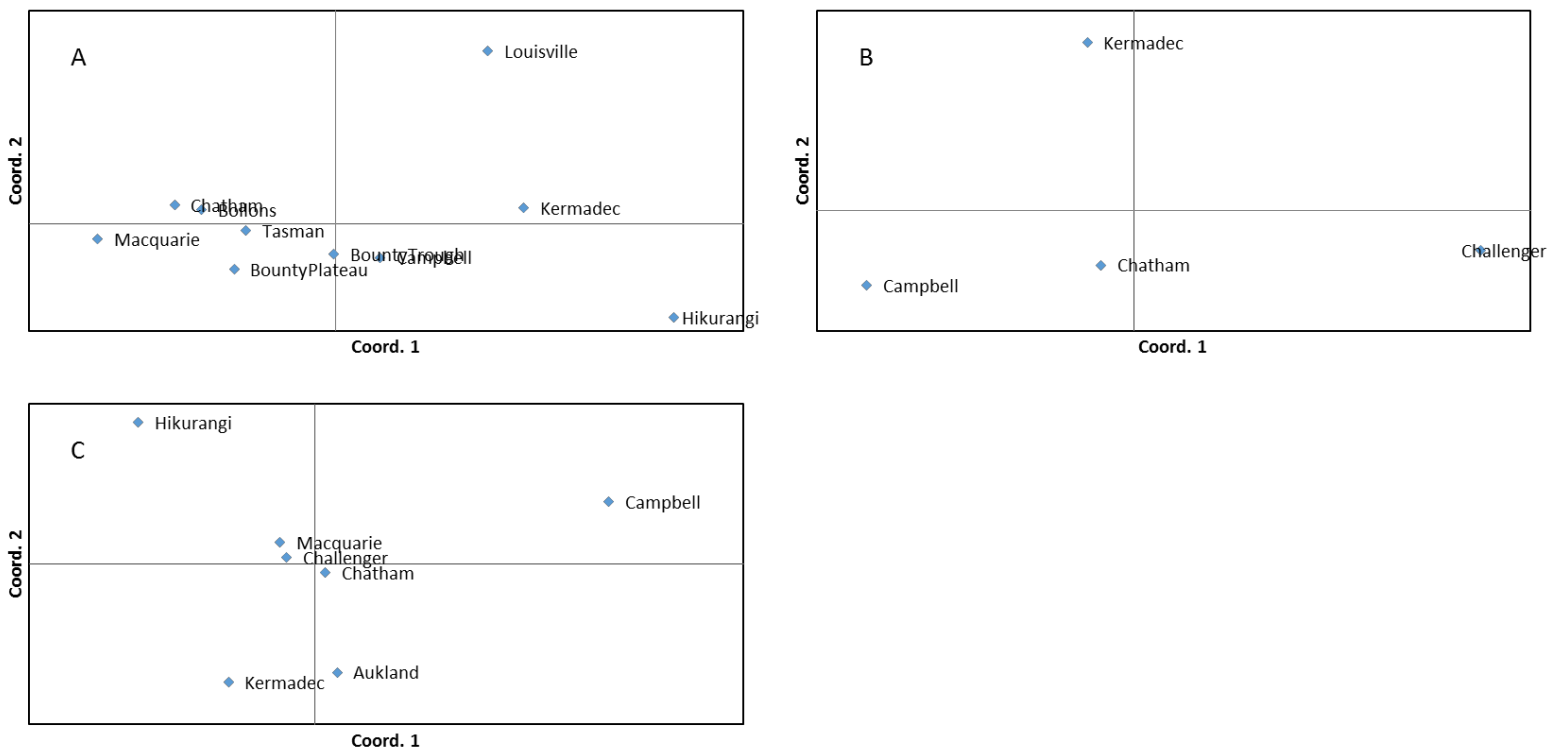

Figure S6.4 The $1^{\text {st }}$ and $2^{\text {nd }}$ coordinates of PCoA in Solenosmilia variabilis (A), Madrepora oculata (B) and Goniocorella dumosa (C)

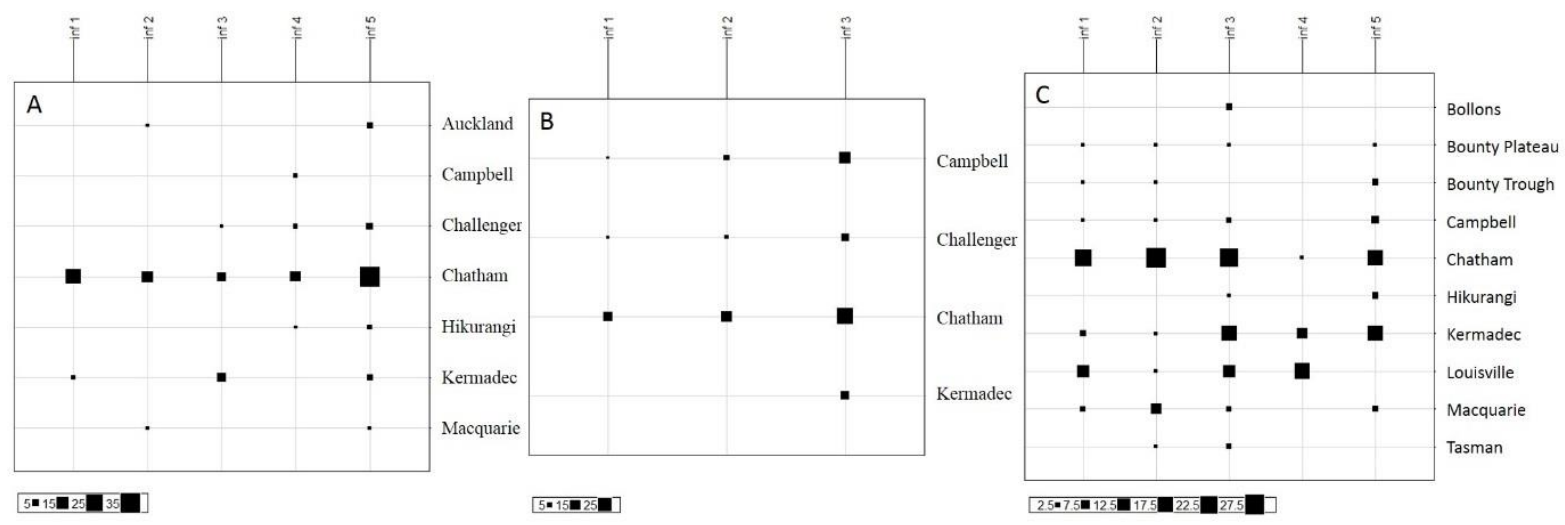

Fig S6.5 Re-assignments for Goniocorella dumosa (A), Madrepora oculata (B), Solenosmilia variabilis (C) based on genotyping results

Inf means the potential population based on $\mathrm{K}$ indicated from BIC in DAPC. 
Chapter 7. Identifying environmental factors associated with the genetic structure of Vulnerable Marine Ecosystem indicator taxa in the New Zealand region 


\subsection{Introduction}

Currently, deep sea biological and environmental processes are still poorly understood, especially in areas such as how spatially arrayed environmental and habitat features influence evolutionary processes and population genetic connectivity. The deep-sea environment experiences increasing pressure and acidicity, and generally decreasing temperature with increasing depth (Amils et al., 2007). Such gradients of physicochemical properties may strongly influence dispersal, settlement and recruitment patterns of deep-sea taxa. Additionally, oceanic currents (e.g. bottom currents (Chevaldonné et al., 1997) and surface currents (Arellano et al., 2014)) play an important role in shaping the patterns of genetic connectivity amongst populations in many deep-sea species (Miller et al., 2010; Miller, 2011; Baco \& Cairns, 2012), with the result that several different factors may act as barriers to gene flow in the deep sea (see Chapter 2).

Vulnerable marine ecosystems (VMEs) are those that are both easily disturbed by anthropologic activities and very slow to recover, or may never recover, and which are physically or functionally fragile (FAO, 2009). Coral reefs and sponge gardens are two recognised VMEs in the deep sea (FAO, 2009). Indicator taxa of these VMEs include scleractinian coral species that build reef frameworks (e.g. Lophelia pertusa Freiwald et al., 2004) and demosponges (e.g. Geodiidae NEAFC, 2014). Although there are no studies that have quantified the relationships between environmental factors and the maintenance of genetic structure in the deep sea, there are some studies from other research areas that might provide information about this interaction for VME indicator taxa of coral reefs and sponge gardens. Generally, depth, temperature, salinity, aragonite saturation state and particulate organic carbon (POC) have been recognised as influencing the formation of deep sea coral and sponge habitat (Adkins et al., 2003; Kiriakoulakis et al., 2005; Davies et al., 2008; Dullo et al., 2008; Dodds et al., 2009; Guinan et al., 2009; Roberts et al., 2009; Case et al., 2010). In addition, topography and sediment are also regarded as important factors influencing the distribution of coral and sponge habitat-forming deep-sea species (Dolan et al., 2008; Brooke et al., 2009). At the small scale, internal tidal mixing and $\mathrm{pH}$ variation may have significant effects on corals larvae and their ability to connect different populations (Frederiksen et al., 1992; McCulloch et al., 2012). However, the relative influence (weak, strong) and the mechanism of action by which these environmental variables moderate the interaction between habitat and population genetic structure and connectivity are still unknown. 
The discipline of seascape genetics is extended from landscape genetics, and attempts to explicitly integrate spatial ecological information with population genetic data to characterise marine spatial environmental factors that contribute to genetic connectivity and/or population genetic isolation (Manel et al., 2003). Selkoe et al. (2016) summarised seascape genetics studies over the last 10 years and found that temperature, oceanography and geography showed equal prevalence of influence on spatial genetic patterns of shallowsea species. However, no such studies have been conducted on deep-sea organisms. Within the New Zealand region, many species have distributions that encompass a range of environments, and are therefore amenable to quantification of the effect of environmental variation on genetic variation and population genetic structuring (e.g. Silva \& Gardner, 2016; Wei et al., 2013). Complex patterns of species-specific genetic variation in VME indicator taxa in New Zealand region have been described previously (Chapter 5 and Chapter 6). Such patterns may be caused by a number of different factors, including historical processes (phylogeography), connectivity (high versus low gene flow within and amongst populations), and environmental variation (contemporary selection pressure). Whilst there is no indication that this multi-taxon genetic structuring is attributable to local or regional adaptation, the recognition of its existence as a pre-existing condition is important. This recognition does not preclude the existence of other structuring, perhaps at a different spatial scale, which results from environmental variation across the New Zealand region.

Because of its highly variable seafloor topography and bathymetry and its complex patterns of oceanic dynamics, New Zealand presents a rare opportunity to test the possible effect of multi-factorial environmental influences on deep-sea genetic variation. In a global assessment of the biogeography of the deep sea based on depth, temperature, salinity, dissolved oxygen, particulate organic carbon (POC), hydrographic and bathymetric parameters (Watling et al., 2013), the New Zealand region was divided into two provinces, a northern (BY6) and a southern (BY10) province with a boundary at latitude $\sim 45^{\circ} \mathrm{S}$. The northern province includes plateaus around the North Island of New Zealand, and extends northward along the Kermadec and Colville Ridges almost to Tonga, as well as including the seamounts of the Louisville Seamount Chain (beyond New Zealand's Exclusive Economic Zone, the EEZ). The water mass is dominated by Antarctic Intermediate Water with temperatures averaging $2.8^{\circ} \mathrm{C}$ and with moderately high POC flux (4-6 $\left.\mathrm{g} \mathrm{m}^{-2} \mathrm{yr}^{-1}\right)$. The southern province includes the Campbell Plateau, Bounty Trough, and the Macquarie Ridge that extends to $\sim 60^{\circ} \mathrm{S}$. This province is characterised by Circumpolar Deep Water with a lower mean temperature $\left(1-2{ }^{\circ} \mathrm{C}\right)$ and lower POC flux $\left(1.5-3 \mathrm{~g} \mathrm{~m}^{-2} \mathrm{yr}^{-1}\right)$ than the northern 
province (Watling et al., 2013). The water mass characteristics of these two provinces is likely to influence the population distribution of deep-sea species across the New Zealand region, which in turn could be reflected in province-scale patterns of genetic structure. Three major currents and their associated fronts around New Zealand, the Tasman Front, the Subtropical Front and the Subantarctic Front, are expected to have the greatest influence on population connectivity of deep-sea populations in the region. These currents and frontal systems differentiate the water mass environments in the north and the south of the region, and also influence the direction of north and south currents across the Chatham Rise that may reduce or perhaps even block gene flow across this feature. For this reason, we identified a north, central and south region separated at $42^{\circ} \mathrm{S}$ and $45^{\circ} \mathrm{S}$ (above $42^{\circ} \mathrm{S}$ is north region, between $42-45^{\circ} \mathrm{S}$ is central region and below $45^{\circ} \mathrm{S}$ is south region) in this study. The seafloor of the New Zealand region is distinguished by different geomorphic features (such as seamounts, plateaux and ridges) which are characterised by different topographies and hydrodynamics that could influence genetic differentiation between populations on these geomorphic features (e.g. Bucklin et al., 1987; Yang et al., 2016). These three environmental-based classifications provide a background framework against which to test genetic differentiation amongst deep-sea populations of VME indicator taxa.

In the present study, data for $7 \mathrm{VME}$ indicator taxa, 4 sponges (Neoaulaxinia persicum, Penares sp., Pleroma menoui and Poecillastra laminaris) and 3 corals (Goniocorella dumosa, Madrepora oculata and Solenosmilia variabilis), collected around New Zealand across $\sim 34^{\circ}$ of latitude and $\sim 1750 \mathrm{~m}$ of depth, have been examined to achieve an understanding of the seascape genetics of these species. In total, 25 environmental variables and two different types of genetic data (sequencing and genotyping) were employed to quantify the relationships between environmental and genetic variation at different spatial scales. The results provide information about which environmental factors shape the genetic structure of the populations, and this information may directly contribute to understanding connectivity amongst VMEs and inform appropriate management measures.

\subsection{Materials and methods}

\subsubsection{Sample collection and molecular experiments}

All 4 sponges (Neoaulaxinia persicum, Penares sp., Pleroma menoui and Poecillastra laminaris) and 3 corals (Goniocorella dumosa, Madrepora oculata and Solenosmilia 
variabilis) were sampled from the NIWA invertebrate collection (NIC); details are presented in Chapter 5 and Chapter 6. Tissue samples from any one collection site were based on only one fragment or piece to avoid duplication of individuals because of colonial or asexual reproduction.

\subsubsection{Data collection and analyses}

\subsubsection{Data collection}

\subsection{DNA sequences}

A total of 480 samples ( $G$. dumosa $\mathrm{n}=108, M$. oculata $\mathrm{n}=93, N$. persicum $\mathrm{n}=30$, Penares sp. $\mathrm{n}=15$, P. menoui $\mathrm{n}=16$, Poecillastra laminaris $\mathrm{n}=63$ and $S$. variabilis $\mathrm{n}=208$ ) were sequenced for mitochondrial and/or nuclear DNA regions (Table 7.1). The protocols for and basic analyses of sequencing and genotyping of data are reported in the previous chapters. Individual pair-wise distances data from DNA sequences were generated from software MEGA (v 6).

Table 7.1 Numbers of specimens used for each genetic marker

\begin{tabular}{cccccccc}
\hline Species & I2S & COI & Cytb & D-loop & ITS & Microsatellites \\
\cline { 2 - 7 } & Goniocorella dumosa & - & - & - & 27 & 83 & 108 \\
Madrepora oculata & - & - & - & - & 40 & 93 \\
Solenosmilia variabilis & - & - & - & 141 & 121 & 208 \\
Neoaulaxinia persicum & 11 & 30 & - & - & - & - \\
Pleroma menoui & - & 16 & - & - & - & - \\
& Poecillastra laminaris & - & 53 & 51 & - & - & 63 \\
Penares sp. & 14 & 15 & - & - & - & - \\
\hline -, data not available & & & & & & &
\end{tabular}

\subsection{Microsatellite loci}

All loci used in this study are the same as described in Chapter 5 and Chapter 6, including all 10 loci and 4 neutral loci (ong8, ong26, ong27 and ong34) for Poecillastra laminaris; all 20 loci and 8 neutral loci (B106, B123, D5, D103, D213, SVA8, SVA13 and SVA20) for G. dumosa; all 11 loci and 6 neutral loci (c7, c016, g006, g010, g016 and g028) for M. oculata; all 24 loci and 12 neutral loci (B1, B106, C107, D5, D103, SVA1, SVA2, 
SVA3, SVA4, SVA7, SVA13 and SVA15) for S. variabilis. For each species, a pairwise individual-by-individual genotypic distance matrix was calculated from the microsatellite data using the Codom-Genotypic distance option within GenAlEx (Peakall \& Smouse 2012). Allelic frequencies were calculated using GenAlEx.

Table 7.2 Detailed information of environmental variables included in this study

\begin{tabular}{|c|c|c|c|c|c|}
\hline Type & Variable & $\begin{array}{c}\text { Abbrevia } \\
\text { tion }\end{array}$ & unit & Resolution & Source \\
\hline \multirow{5}{*}{$\begin{array}{l}\text { Topograhi } \\
\text { c variables }\end{array}$} & $\begin{array}{c}\text { Bathymetric Position Index - } \\
\text { Broad }\end{array}$ & bpi-broad & - & $25 \mathrm{~m}$ radius & Wright et al. (2005), 1+25 \\
\hline & Bathymetric Position Index - Fine & bpi-fine & - & $5 \mathrm{~m}$ radius & Wright et al. (2005), 1+5 \\
\hline & Seamount & smt & $\begin{array}{l}\text { Binomial } \\
(\text { yes/no) }\end{array}$ & $1 \mathrm{~km}$ & Rowden et al. (2008), \\
\hline & Slope in percent & $\begin{array}{l}\text { slope- } \\
\text { percent }\end{array}$ & $\%$ & 0.00001 & Jenness (2012) \\
\hline & Standard Deviation of Slope & $\begin{array}{l}\text { stdev- } \\
\text { slope }\end{array}$ & - & $3 \times 3$ window & Grohmann et al (2011), \\
\hline \multirow{17}{*}{$\begin{array}{l}\text { Physico- } \\
\text { chemical } \\
\text { variables }\end{array}$} & Sea surface temperature & sst & Degree & $1 \mathrm{~km}$ & NOAA satellite data \\
\hline & Sea surface temperature gradient & sstgrd & ${ }^{\circ} \mathrm{C} \mathrm{km-1}$ & $1 \mathrm{~km}$ & Uddstrom \& Oien (1999), \\
\hline & Bottom current speed & botspd & $\mathrm{m} \mathrm{s}-1$ & $1 \mathrm{~km}$ & Hadfield et al. (2002) \\
\hline & Bottom water temperature & tempbot & ${ }^{\circ} \mathrm{C}$ & $1 \mathrm{~km}$ & $\begin{array}{c}\text { CARS (2009) } \\
\text { (www.cmar.csiro.au/cars) }\end{array}$ \\
\hline & $\begin{array}{c}\text { Bottom water temperature } \\
\text { residuals }\end{array}$ & tempres & ${ }^{\circ} \mathrm{C} \mathrm{km-1}$ & $1 \mathrm{~km}$ & $\begin{array}{l}\text { CARS (2009), Leathwick } \\
\text { et al. }(2012)\end{array}$ \\
\hline & Temperature & $\begin{array}{l}\text { temperatu } \\
\text { re }\end{array}$ & ${ }^{\circ} \mathrm{C}$ & 0.001 & CARS (2009) \\
\hline & Tidal current speed & tidcurr & ms-1 & $1 \mathrm{~km}$ & $\begin{array}{l}\text { Walters et al. (2001), } \\
\text { Hadfield et al. (2002) }\end{array}$ \\
\hline & Density & $\begin{array}{l}\text { sigma- } \\
\text { theta }\end{array}$ & $\mathrm{kg} \mathrm{m}-3$ & 0.01 & NIWA \\
\hline & Dissolved organic matter & disorg & $\mathrm{m}-1$ & $1 \mathrm{~km}$ & NASA SeaDas \\
\hline & Dynamic topography & dynoc & $\mathrm{m}$ & $1 \mathrm{~km}$ & $\begin{array}{c}\text { AVISO } \\
\text { (http://www.aviso.oceanob } \\
\text { s.com) }\end{array}$ \\
\hline & Nitrate & woanitc & $\mu \mathrm{mol} 1-1$ & $1^{\circ}$ & Garcia et al. (2006) \\
\hline & Omega Aragonite & aragonite & $\Omega A R A G$ & - & Bostock et al. (2014) \\
\hline & Omega Calcite & calcite & $\Omega C A L C$ & - & Bostock et al. (2014) \\
\hline & Oxygen & oxygen & $\mathrm{ml} \mathrm{1-1}$ & - & CARS (2009) \\
\hline & Phosphate & woaphosc & $\mu \mathrm{mol} \mathrm{1-1}$ & $1^{\circ}$ & Garcia et al. (2006) \\
\hline & Salinity & salinity & psu & - & CARS (2009) \\
\hline & Silicate & silicate & $\mu \mathrm{mol} 1-1$ & $1^{\circ}$ & Garcia et al. (2006b) \\
\hline \multirow{3}{*}{$\begin{array}{l}\text { Biological } \\
\text { variables }\end{array}$} & $\begin{array}{c}\text { Chromophoric dissolved organic } \\
\text { matter }\end{array}$ & cdom & $\begin{array}{c}\text { aDOM } \\
(443) \mathrm{m}-1\end{array}$ & $1 \mathrm{~km}$ & Pinkerton et al. (2006) \\
\hline & Particulate organic carbon export & $\mathrm{POC}$ & $\begin{array}{l}\text { mg Corg.m- } \\
2 \mathrm{~d}-1\end{array}$ & - & Lutz et al. (2007) \\
\hline & Surface water primary productivity & vgpm & $\begin{array}{c}\mathrm{mg} \mathrm{C} \mathrm{m-2} \\
\mathrm{d}-1\end{array}$ & $1 \mathrm{~km}$ & $\begin{array}{l}\text { Behrenfield \& Falkowski } \\
\text { (1997) }\end{array}$ \\
\hline
\end{tabular}




\subsection{Environmental variables}

Data for 25 environmental variables - 5 topographic, 17 physicochemical and 3 biological variables - were obtained from various sources (Table 7.2). All the environmental variables have been shown from previous studies to potentially influence the distribution of coral and sponge species (see references in Introduction and Tracey et al., 2011). The variables calcite and aragonite were excluded from the analysis of sponge data, while silicate was excluded from the analysis of coral data.

\subsubsection{Statistical analysis}

Highly correlated environmental variables were identified and eliminated from subsequent analysis to reduce problems that arise for the interpretation of models that include a large number of collinear predictors (Graham, 2003). The Pearson correlation coefficients between all predictor variables were calculated for those data collected at the sponge and coral sample locations. Correlations were visualised, and where correlations were $>0.95$ one of the predicator variables was eliminated. Correlation analyses of environmental parameters in the entire dataset and their visualisation were conducted using the R project (v3.2.2).

Following the spatially hierarchical testing framework described in the previous chapters, the same approach was employed here. Specimens were grouped according to (1) the northern versus southern biogeographic provinces of Watling et al. (2013), (2) the northcentral-south regions, and (3) geomorphic features: NE continental slope, Bollons Seamount, Bounty Plateau, Bounty Trough, Campbell Plateau, Challenger Plateau, Chatham Rise, Hikurangi Margin, Kermadec Ridge, Louisville Seamount Chain, Macquarie Ridge and Tasman Basin. Not all species were collected from all geomorphic feature regions: spatial coverage was dictated by availability of archived material in the NIWA Inverterbrate Collection and newly collected material from recent research cruises.

A generalised linear model (GLM) was employed to test for the effect of environmental variables on genetic distance values calculated at the individual level on DNA sequence markers for all 7 species and on genotyping markers (microsatellites) for Poecillastra laminaris, G. dumosa, M. oculata and S. variabilis. A mean genetic distance value for each individual was calculated from all individual pairwise values, to be used as a dependent variable. To remove the effects of multi-collinearity amongst environmental variables, GLM analyses were performed using a stepwise model (both forwards and backwards), with variable inclusion/exclusion based on the Akaike Information Criterion 
(AIC) and Bayesian information criterion (BIC). The best fit model was chosen from the lowest AIC or BIC value model. All analyses were conducted using the package MASS in R (v3.2.2). Missing environmental values for the three species were replaced by means to reduce information loss. For all these analyses, the null hypothesis is that none of the environmental parameters (independent variables) explain significant variation in the genetic data set (dependent variable).

To test the null hypothesis from a population perspective, analyses were conducted using distance-based linear models (DistLM) using the statistical software PERMANOVA+ (Anderson et al., 2008). The DistLM routine is a semi-parametric, permutation-based method that does not rely on the assumption of normally distributed data (Anderson et al., 2008). The best-fitting relationship was chosen for the final regression models by comparing adjusted $r^{2}$ selection criterion. Relationships between environmental parameters (averaged for all sites within a population) were initially examined by analysing each predictor separately (marginal tests), and then sequentially using adjusted $r^{2}$ selection procedure. Similarity matrices in DistLM analyses were built using Bray-Curtis resemblance matrices of allele frequencies. The p-values for individual predictor variables were obtained using 9,999 permutations (Leduc et al., 2012). Distance-based redundancy analysis (dbRDA) plots were also generated using PERMANOVA+ to visualise the results once the best DistLM model of each species was obtained.

Based on microsatellite variation results from Chapter 5 and Chapter 6, significant genetic structure was detected at the northern-southern province level for $S$. variabilis, northcentral-south region level for all four species (G. dumosa, M. oculata, P. laminaris and $S$. variabilis), and at the level of geomorphic feature populations for G. dumosa, P. laminaris and $S$. variabilis. Only these structured populations were considered for the DistLM analysis. However, the province level was ultimately excluded from the analyses because the DistLM failed to build any models for $S$. variabilis for both the all loci and the neutral loci data sets.

Because the environmental variables data set is less complete beyond the New Zealand EEZ (no information for 10 variables, bottom speed, dissolved organic matter, sea surface temperature, sea surface temperature gradient, bottom water temperature, bottom water temperature residuals, tidal current speed and surface water primary productivity) for $S$. variabilis, averages of these 10 variables were used to replace the missing values in the Louiville Seamount Chain population (beyond the New Zealand EEZ) to complete the DistLM analyses at regional and geomorphic feature levels. 
The difference between the individual level (GLM) and the population level (DistLM) procedures, as employed in this study, is that the former is conducted on mean values of individual pairwise genetic distance and the latter on raw allele frequency data at the population level (all three analyses use the same environmental data). These analyses are different but complementary in their approach to determining if and in what way the observed genetic variation is explained by the environmental variables.

\subsection{Results}

Bivariate correlations showed that several of the topographic, physico-chemical and biological parameters were significantly correlated, especially the variables bpi-broad, depth, temperature, seamount, sea surface temperature and surface water primary productivity that were correlated with one or more of the other variables. Amongst the correlations, several of the physico-chemical variables were significantly correlated with each other (Figure 7.1). Notably, calcite and aragonite were highly correlated $\left(\mathrm{r}^{2}>0.99\right)$, as were temperature, aragonite, sigma-theta and calcite $\left(r^{2}>0.95\right)$; woanitc and woaphosc $\left(r^{2}>0.95\right)$; and tempbot and tempres $\left(r^{2}>0.95\right)$. Therefore, aragonite was selected to represent calcite, temperature and sigma-theta in the coral models, and sigma-theta for temperature in the sponge models. Tempbot was selected to represent tempres, and woanitc for woaphosc in models for both coral and sponge models. This selection procedure resulted in 20 variables (bpi-broad, bpifine, oxygen, POC, salinity, silicate, slope-percent, stdev-slope, sigma-theta, woanitc, botspd, cdom, disorg, dynoc, smt, sstgrd, sst, tempbot, tidcurr and vgpm) for the sponge data analysis, and 19 variables (bpi-broad, bpi-fine, aragonite, oxygen, POC, salinity, slope-percent, stdevslope, woanitc, botspd, cdom, disorg, dynoc, smt, sstgrd, sst, tempbot, tidcurr and vgpm) for the coral analysis.

\subsubsection{GLM analysis}

Amongst all populations, the GLM analysis established different models for different species and different genetic markers using 20 environmental variables for sponges and 19 variables for corals to explain the genetic variation at the individual level. BIC modelling was less successful at building models than AIC modelling, and AIC was therefore chosen to select environmental variables that explained the genetic variations. The AIC test removed the environmental variables that did not contribute to explaining genetic variation, such that 
the final model for each marker in each species represented the best fit subset of environmental variables that explained local genetic variation within populations.

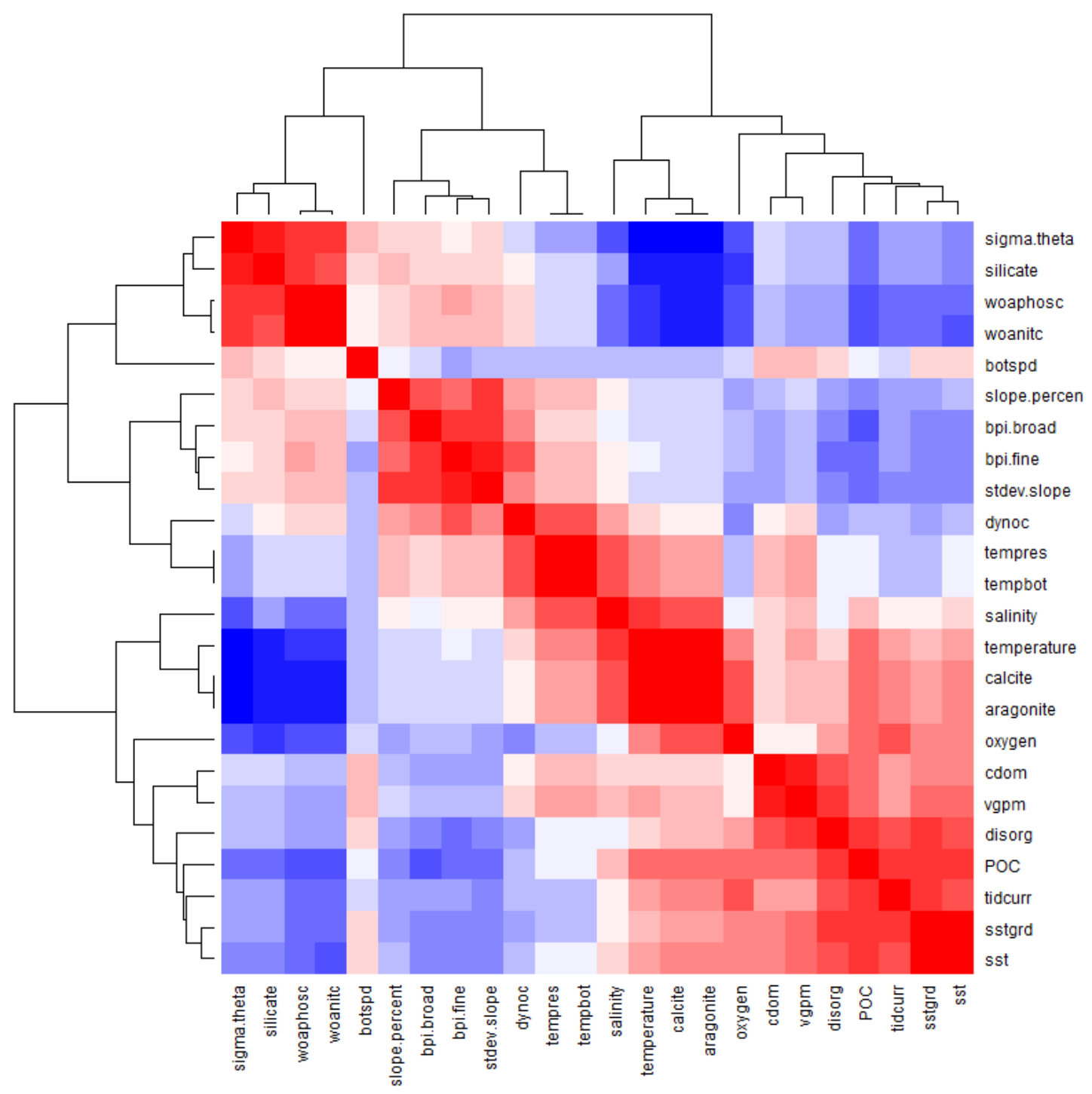

Figure 7.1 Heatmap of correlations between environmental variables

Colour represents the values of correlation coefficients: blue to red represents the value from -1 to 1 .

\subsubsection{GLM analyses for sponges}

Due to small sample sizes and/or low variation within the genetic markers, the GLM analysis of $C O I$ sequence variation in individuals of $N$. persicum and P. menoui failed to reveal a relationship between genetic and environmental variables (that is, model building failed). The model based on COI of Penares sp. was non-significant $(\mathrm{p}=0.124)$. Models for $N$. persicum, P. menoui and Penares sp. could not be built for the $12 S$ sequence datasets 
because of low DNA sequence variation. Only the models for Poecillastra laminaris could be built that were statistically significant $(\mathrm{p}<0.05)$ for both sequencing and genotyping datasets.

\subsection{Poecillastra laminaris}

The best fit model explaining variation in the COI sequence data contained 11 of the 20 variables, the best fit model for $C y t b$ variation contained 16 variables, whilst the optimal model for variation in the microsatellite data set contained 9 and 12 variables for all and neutral microsatellites, respectively. Three variables, sea surface temperature (sst), sea surface temperature gradient (sstgrd) and nitrate (woanitc), occurred in all three models (Table 7.3).

\subsection{Neoaulaxinia persicum, Pleroma menoui and Penares sp.}

Model fitting for these three sponge species was made difficult by small sample sizes and/or low levels of genetic variation. In the non-significant model for Penares sp., the environment variables POC and oxygen contributed to explaining variation in the $C O I$ sequence data set, whilst the model for $12 S$ sequence variation could not be fitted for Penares sp. or for N. persicum.

\subsubsection{2. $\quad$ GLM analyses for corals}

Eleven models were developed and tested for the three coral species for the DNA sequence and microsatellite (genotype) data. The multiple $\mathrm{R}^{2}$ values of the models were low $(<0.500)$ except for ITS of M. oculata (>0.900). However, all models, except D-loop for $G$. dumosa, neutral microsatellites for $M$. oculata and for $S$. variabilis, were statistically significant $(\mathrm{p}<0.05)$, indicating that a model with a low $r^{2}$ value can still help to predict how changes in the environmental variables may be associated with changes in genetic distance values amongst locations. Sea surface temperature gradient (sstgrd) was significant in explaining the genetic differentiation amongst individuals of all three corals for all models (except all microsatellite data for G. dumosa), even in non-significant models. This parameter may be considered as a common environmental variable that is related to genetic differentiation amongst individuals for the three stony coral species. The details of speciesspecific models are described below. 
Table 7.3 General linear model analysis results of genetic variation at the individual level for Poecillastra laminaris

\begin{tabular}{|c|c|c|c|c|}
\hline Variables & $\mathrm{COI}$ & Cytb & All SSR & Neutral SSR \\
\hline Multiple $\mathrm{R}^{2}$ & 0.544 & 0.803 & 0.44 & 0.441 \\
\hline Adjusted $\mathrm{R}^{2}$ & 0.371 & 0.66 & 0.311 & 0.872 \\
\hline p-value & 0.007 & $<0.001$ & 0.004 & 0.023 \\
\hline Intercept & $-1.544 \pm 0.456^{* *}$ & $-0.439 \pm 0.339 \mathrm{~ns}$ & $1761.000 \pm 662.700^{*}$ & $123.600 \pm 55.850^{*}$ \\
\hline botspd & $\mathrm{E}$ & $-0.068 \pm 0.029 *$ & $\mathrm{E}$ & $\mathrm{E}$ \\
\hline bpi broad & $0.000 \pm 0.000^{*}$ & $\mathrm{E}$ & $-0.007 \pm 0.004 \#$ & $-0.008 \pm 0.003 * *$ \\
\hline bpi fine & $\mathrm{E}$ & $0.000 \pm 0.000 \#$ & $\mathrm{E}$ & $\mathrm{E}$ \\
\hline cdom & $\mathrm{E}$ & $-0.022 \pm 0.008^{* *}$ & $\mathrm{E}$ & $5.185 \pm 1.813^{* *}$ \\
\hline disorg & $\mathrm{E}$ & $-0.660 \pm 0.181 * *$ & $\mathrm{E}$ & $111.400 \pm 51.740^{*}$ \\
\hline dynoc & $\mathrm{E}$ & $-0.053 \pm 0.012 * * *$ & $\mathrm{E}$ & $\mathrm{E}$ \\
\hline oxygen & $0.017 \pm 0.005 * *$ & $\mathrm{E}$ & $-15.120 \pm 3.870 * * *$ & $-2.120 \pm 1.025^{*}$ \\
\hline POC & $0.002 \pm 0.001 *$ & $0.000 \pm 0.000 \mathrm{~ns}$ & $-0.950 \pm 0.440^{*}$ & $-1.538 \pm 0.431 * *$ \\
\hline Salinity & $0.041 \pm 0.012 * *$ & $\mathrm{E}$ & $-28.510 \pm 11.910 *$ & $\mathrm{E}$ \\
\hline sigma theta & $\mathrm{E}$ & $0.015 \pm 0.011$ & $-20.480 \pm 7.410^{* *}$ & $-2.747 \pm 1.620 \#$ \\
\hline silicate & $0.001 \pm 0.000 * * *$ & $0.000 \pm 0.000^{*}$ & $\mathrm{E}$ & $\mathrm{E}$ \\
\hline slope percent & $0.000 \pm 0.000 \#$ & $0.000 \pm 0.000 \mathrm{~ns}$ & $\mathrm{E}$ & $-0.121 \pm 0.077 \mathrm{~ns}$ \\
\hline smt & $\mathrm{E}$ & $-0.003 \pm 0.002 \#$ & $\mathrm{E}$ & $\mathrm{E}$ \\
\hline sst & $1.980 \pm 0.853^{*}$ & $-2.616 \pm 0.551 * * *$ & $-15610.000 \pm 472.600 * *$ & $-794.200 \pm 245.700 * *$ \\
\hline sstgrd & $-5.432 \pm 2.254^{*}$ & $6.181 \pm 1.348 * * *$ & $2810.000 \pm 1152.000 *$ & $1337.000 \pm 551.000 *$ \\
\hline stdev slope & $\mathrm{E}$ & $-0.002 \pm 0.001 *$ & E & $0.587 \pm 0.286^{*}$ \\
\hline tempbot & $-0.002 \pm 0.001 \#$ & $-0.002 \pm 0.001 \#$ & $\mathrm{E}$ & $\mathrm{E}$ \\
\hline tidcurr & $-0.019 \pm 0.014 \mathrm{~ns}$ & $\mathrm{E}$ & $37.430 \pm 10.520 * * *$ & $\mathrm{E}$ \\
\hline vgpm & $\mathrm{E}$ & $0.000 \pm 0.000 * *$ & $\mathrm{E}$ & $29.800 \pm 7.295^{* * *}$ \\
\hline woanitc & $0.002 \pm 0.001 * *$ & $0.001 \pm 0.000 * *$ & $-1.090 \pm 0.306^{* * *}$ & $-0.666 \pm 0.168 * * *$ \\
\hline
\end{tabular}

Significance levels at $\mathrm{p}<0.001, \mathrm{p}<0.01, \mathrm{p}<0.05$ and $\mathrm{p}<0.1$ are labelled as ***,**,*,\#, and ns. Variable with "E" indicated that it was excluded from the modelling. Models for Neoaulaxinia persicum, Pleroma menoui and Penares sp. COI gene was failed to construct a model.

\subsection{Goniocorella dumosa}

The best fit model for the D-loop sequence data included 13 variables, but the whole model was not significant $(\mathrm{p}>0.05)$. For the ITS sequence data the best fit model contained only 3 variables, whilst for microsatellite variation the best fit model contained 3 variables for all loci and 5 variables for neutral loci. Amongst these three best fit models, dynamic topography (dynoc) was always included and contributed significantly, and sea surface temperature gradient (sstgrd) was also a significant contributor to the models except for the model based on all microsatellite data (Table 7.4). 
Table 7.4 General linear model analysis results of genetic variation for Goniocorella dumosa

\begin{tabular}{|c|c|c|c|c|}
\hline Variables & D-loop & ITS & All SSR & Neutral SSR \\
\hline Multiple R2 & 0.56 & 0.112 & 0.091 & 0.139 \\
\hline Adjusted R2 & 0.083 & 0.077 & 0.064 & 0.096 \\
\hline p-value & 0.394 & 0.028 & 0.021 & 0.009 \\
\hline Intercept & $-1.482 \pm 0.702 \#$ & $0.003 \pm 0.008 \mathrm{~ns}$ & $30.917 \pm 3.616^{* * *}$ & $-634.297 \pm 219.031 * *$ \\
\hline botspd & $-0.349 \pm 0.148^{*}$ & $\mathrm{E}$ & $\mathrm{E}$ & $\mathrm{E}$ \\
\hline bpi broad & $\mathrm{E}$ & E & $\mathrm{E}$ & $\mathrm{E}$ \\
\hline bpi fine & $0.000 \pm 0.000 \#$ & $\mathrm{E}$ & $\mathrm{E}$ & $\mathrm{E}$ \\
\hline cdom & $\mathrm{E}$ & E & $-1.249 \pm 0.749 \#$ & $\mathrm{E}$ \\
\hline disorg & $-1.276 \pm 0.403 * *$ & $\mathrm{E}$ & $46.423 \pm 20.917^{*}$ & $\mathrm{E}$ \\
\hline dynoc & $-0.484 \pm 0.169 *$ & $0.035 \pm 0.014 *$ & $18.578 \pm 6.005 * *$ & $11.412 \pm 3.987 * *$ \\
\hline oxygen & $0.012 \pm 0.009 \mathrm{~ns}$ & E & $\mathrm{E}$ & $5.885 \pm 1.931^{* *}$ \\
\hline POC & $\mathrm{E}$ & $\mathrm{E}$ & $\mathrm{E}$ & $\mathrm{E}$ \\
\hline salinity & $0.051 \pm 0.023 *$ & $\mathrm{E}$ & $\mathrm{E}$ & $18.411 \pm 6.249 * *$ \\
\hline aragonite & $\mathrm{E}$ & E & $\mathrm{E}$ & $-14.403 \pm 4.623 * *$ \\
\hline slope percent & $-0.002 \pm 0.001 \#$ & $\mathrm{E}$ & $\mathrm{E}$ & $\mathrm{E}$ \\
\hline smt & $-0.027 \pm 0.023 \mathrm{~ns}$ & E & $\mathrm{E}$ & $\mathrm{E}$ \\
\hline sst & $-1.378 \pm 0.565^{*}$ & $-0.428 \pm 0.199 *$ & $\mathrm{E}$ & $\mathrm{E}$ \\
\hline sstgrd & $6.689 \pm 2.098^{* *}$ & $1.029 \pm 0.382 * *$ & $\mathrm{E}$ & $73.295 \pm 31.874^{*}$ \\
\hline stdev slope & $-0.013 \pm 0.007 \#$ & $\mathrm{E}$ & $\mathrm{E}$ & $\mathrm{E}$ \\
\hline tempbot & $\mathrm{E}$ & E & $\mathrm{E}$ & $\mathrm{E}$ \\
\hline tidcurr & $-0.222 \pm 0.079 *$ & $\mathrm{E}$ & $\mathrm{E}$ & $\mathrm{E}$ \\
\hline vgpm & $0.000 \pm 0.000^{*}$ & E & $\mathrm{E}$ & $\mathrm{E}$ \\
\hline woanitc & $\mathrm{E}$ & E & $\mathrm{E}$ & E \\
\hline
\end{tabular}

Significance levels at $\mathrm{p}<0.001, \mathrm{p}<0.01, \mathrm{p}<0.05$ and $\mathrm{p}<0.1$ are labelled as $* * *, * *, *, \#$, and ns. Variable with "E" indicated that it was excluded from the modelling.

\subsection{Madrepora oculata}

For nuclear ITS DNA sequence data the best fit model contained 11 of the 19 environmental variables, whilst the best fit model for the microsatellite variation included 5 variables for all loci and 6 for the neutral loci. However, the model based on neutral microsatellites was not significant. For these nuclear DNA markers, 2 environmental variables (sea surface temperature gradient (sstgrd) and surface water primary productivity (vgpm)) were present in all models (Table 7.5).

\subsection{Solenosmilia variabilis}

For the D-loop analysis, the best fit model contained 14 variables, whilst a 7-variable model provided the best fit for variation for the ITS DNA sequence. The models for microsatellite variation included 5 variables for both the all loci and neutral loci datasets. 
These three models all shared an environmental variable - sea surface temperature gradient (sstgrd). The model based on the neutral loci was not statistically significant (Table 7.6).

Table 7.5 General linear model analysis results of genetic variation for Madrepora oculata

\begin{tabular}{|c|c|c|c|}
\hline Variables & ITS & All SSR & Neutral SSR \\
\hline Multiple $\mathrm{R}^{2}$ & 0.833 & 0.208 & 0.101 \\
\hline Adjusted $\mathrm{R}^{2}$ & 0.767 & 0.161 & 0.048 \\
\hline p-value & $<0.001$ & 0.001 & 0.104 \\
\hline Intercept & $0.765 \pm 0.209^{* *}$ & $22.070 \pm 1.572 * * *$ & $9.771 \pm 1.149 * * *$ \\
\hline botspd & $-0.648 \pm 0.240^{*}$ & $20.460 \pm 12.03 \#$ & $\mathrm{E}$ \\
\hline bpi broad & $0.000 \pm 0.000^{* * *}$ & E & E \\
\hline bpi fine & $\mathrm{E}$ & $\mathrm{E}$ & $\mathrm{E}$ \\
\hline cdom & $0.097 \pm 0.043^{*}$ & $-6.219 \pm 1.764 * * *$ & $\mathrm{E}$ \\
\hline disorg & $-9.132 \pm 2.022 * * *$ & E & $-83.280 \pm 36.540^{*}$ \\
\hline dynoc & $\mathrm{E}$ & $\mathrm{E}$ & E \\
\hline oxygen & $-0.100 \pm 0.039^{*}$ & $0.383 \pm 0.141 * *$ & $\mathrm{E}$ \\
\hline POC & $\mathrm{E}$ & $\mathrm{E}$ & $0.213 \pm 0.099 *$ \\
\hline salinity & $\mathrm{E}$ & E & E \\
\hline aragonite & $\mathrm{E}$ & $\mathrm{E}$ & $\mathrm{E}$ \\
\hline slope percent & E & E & E \\
\hline smt & $\mathrm{E}$ & $\mathrm{E}$ & $\mathrm{E}$ \\
\hline sst & $-6.616 \pm 5.270 \mathrm{~ns}$ & $\mathrm{E}$ & $\mathrm{E}$ \\
\hline sstgrd & $15.000 \pm 9.504 \mathrm{~ns}$ & $-504.200 \pm 131.800 * * *$ & $-162.500 \pm 72.320 *$ \\
\hline stdev slope & $\mathrm{E}$ & $\mathrm{E}$ & $\mathrm{E}$ \\
\hline tempbot & $-0.041 \pm 0.011^{* *}$ & $\mathrm{E}$ & $-0.694 \pm 0.341 *$ \\
\hline tidcurr & $-0.874 \pm 0.199 * * *$ & $\mathrm{E}$ & $\mathrm{E}$ \\
\hline vgpm & $0.001 \pm 0.000 * * *$ & $0.019 \pm 0.006^{* *}$ & $0.007 \pm 0.003 *$ \\
\hline woanitc & $-0.009 \pm 0.002 * * *$ & $\mathrm{E}$ & $\mathrm{E}$ \\
\hline
\end{tabular}

Significance levels at $\mathrm{p}<0.001, \mathrm{p}<0.01, \mathrm{p}<0.05$ and $\mathrm{p}<0.1$ are labelled as ${ }^{* * *},{ }^{* *},{ }^{*}, \#$, and ns. Variable with "E" indicated that it was excluded from the modelling.

\subsubsection{DistLM analyses}

\subsubsection{North-central-south region}

Marginal tests resulting from DISTLM analysis at the regional scale showed that the genetic structure of the four species was related to different environmental factors (Table 7.7). After removal of the variables that did not explain genetic variation, through sequential testing, only one variable was retained in each model. In G. dumosa, the biological factor surface water primary productivity (vgpm) explained genetic structure $\left(r^{2}>0.66\right)$ in both the all loci and the neutral loci data sets. Dynamic topography (dynoc) and seamount (smt) contributed to genetic differentiation in M. oculata in the all loci and the neutral loci data sets, 
respectively. The variable dynamic topography explained variation in the all loci and neutral loci data sets of S. variabilis. Physicochemical variables (silicate and bottom current speed) explained variation in the all loci and neutral loci data sets of Poecillastra laminaris, respectively.

Table 7.6 General linear model analysis results of genetic variation for Solenosmilia variabilis

\begin{tabular}{|c|c|c|c|c|}
\hline Variables & D-loop & ITS & All SSR & Neutral SSR \\
\hline Multiple R2 & 0.383 & 0.145 & 0.103 & 0.048 \\
\hline Adjusted R2 & 0.298 & 0.082 & 0.081 & 0.019 \\
\hline $\mathrm{p}$-value & $<0.001$ & 0.033 & $<0.001$ & 0.154 \\
\hline Intercept & $-1.002 \pm 0.320 * *$ & $-0.71 \pm 0.264 * *$ & $43.272 \pm 1.470 * * *$ & $19.577 \pm 1.093 * * *$ \\
\hline botspd & $\mathrm{E}$ & $\mathrm{E}$ & $-17.009 \pm 10.183 \#$ & $\mathrm{E}$ \\
\hline bpi broad & $0.000 \pm 0.000^{*}$ & $\mathrm{E}$ & $\mathrm{E}$ & E \\
\hline bpi fine & $0.000 \pm 0.000 \#$ & $0.000 \pm 0.000 \#$ & $\mathrm{E}$ & $\mathrm{E}$ \\
\hline cdom & $0.001 \pm 0.001 \mathrm{~ns}$ & $\mathrm{E}$ & $\mathrm{E}$ & $-2.200 \pm 0.961 *$ \\
\hline disorg & $0.062 \pm 0.027^{*}$ & $\mathrm{E}$ & E & $49.257 \pm 29.187 \#$ \\
\hline dynoc & $0.017 \pm 0.004 * * *$ & $\mathrm{E}$ & E & $6.451 \pm 2.328 * *$ \\
\hline oxygen & $0.023 \pm 0.005 * * *$ & $0.074 \pm 0.037 *$ & $\mathrm{E}$ & $\mathrm{E}$ \\
\hline $\mathrm{POC}$ & $\mathrm{E}$ & $\mathrm{E}$ & $-0.175 \pm 0.071^{*}$ & E \\
\hline salinity & $0.027 \pm 0.009^{* *}$ & $\mathrm{E}$ & $\mathrm{E}$ & E \\
\hline aragonite & $-0.046 \pm 0.010 * * *$ & $\mathrm{E}$ & $2.281 \pm 0.819^{* *}$ & $\mathrm{E}$ \\
\hline slope percent & $0.000 \pm 0.000^{*}$ & $\mathrm{E}$ & E & E \\
\hline smt & $-0.001 \pm 0.000 *$ & $0.055 \pm 0.026^{*}$ & $\mathrm{E}$ & $\mathrm{E}$ \\
\hline sst & $0.171 \pm 0.119 \mathrm{~ns}$ & $-13.960 \pm 7.574 \#$ & E & $-236.314 \pm 154.622 \mathrm{~ns}$ \\
\hline sstgrd & $-0.395 \pm 0.264 \mathrm{~ns}$ & $41.980 \pm 16.230^{*}$ & $353.840 \pm 156.672 *$ & $605.165 \pm 353.257 \#$ \\
\hline stdev slope & $\mathrm{E}$ & $0.015 \pm 0.007 *$ & $\mathrm{E}$ & E \\
\hline tempbot & $-0.001 \pm 0.000 *$ & $\mathrm{E}$ & $0.919 \pm 0.555 \#$ & E \\
\hline tidcurr & $0.007 \pm 0.005 \mathrm{~ns}$ & $\mathrm{E}$ & $\mathrm{E}$ & $\mathrm{E}$ \\
\hline vgpm & E & $\mathrm{E}$ & $\mathrm{E}$ & E \\
\hline woanitc & $\mathrm{E}$ & $0.010 \pm 0.004 *$ & E & E \\
\hline
\end{tabular}

Significance levels at $\mathrm{p}<0.001, \mathrm{p}<0.01, \mathrm{p}<0.05$ and $\mathrm{p}<0.1$ are labelled as $* * *, * *,{ }^{*}, \#$, and ns. Variable with "E" indicated that it was excluded from the modelling.

Table 7.7 Sequential test results of the DistLM analysis for microsatellite data amongst the NorthCentral-South regions

\begin{tabular}{ccccccc}
\hline Species & Loci & Variable & Adj R $^{2}$ & SS(trace) & p & Proportion \\
\hline \multirow{2}{*}{ Goniocorella dumosa } & All & + vgpm & 0.669 & 2429.8 & 0.169 & 0.834 \\
& Neutral & + vgpm & 0.739 & 2409.1 & 0.169 & 0.870 \\
\multirow{3}{*}{ Madrepora oculata } & All & + dynoc & 0.342 & 1406.2 & 0.159 & 0.671 \\
& Neutral & + smt & 0.324 & 1319.2 & 0.162 & 0.662 \\
\multirow{2}{*}{ Solenosmilia variabilis } & All & + dynoc & 0.284 & 353.02 & 0.167 & 0.642 \\
& Neutral & + dynoc & 0.394 & 360.18 & 0.167 & 0.697 \\
\multirow{2}{*}{ Poecillastra laminaris } & All & +silicate & 0.178 & 1870.8 & 0.161 & 0.589 \\
& Neutral & +botspd & 0.249 & 1515.2 & 0.162 & 0.624 \\
\hline
\end{tabular}




\subsubsection{Geomorphic feature populations}

Marginal tests resulting from DISTLM analysis showed that microsatellite variation of the sponge Poecillastra laminaris exhibited a significant relationship with nitrate, bottom water temperature, salinity and bottom current speed. When considered alone, nitrate explained $34.9 \%$ of the variability, bottom water temperature $29.3 \%$, salinity $23.0 \%$ in the all loci data set, and in the neutral loci data set bottom water temperature explained $34.9 \%$ of the variation, nitrate $35.8 \%$, and bottom current speed $18.8 \%$ (Table 7.8 and Figure 7.2 ). Despite the fact that each individual environmental factor was not significant, the selected best fit model from the sequential testing explained $87.2 \%$ and $89.5 \%$ of the variation observed in the full microsatellite and the neutral loci data sets, respectively (Table 7.7). Both models built for the all loci and neutral loci data sets agreed that nitrate was the variable that explained most of the genetic structure at the geomorphic feature spatial scale. The dbRDA results illustrated that the populations from the Campbell Plateau and the NE Slope were genetically different from all other populations, and that nitrate and bottom water temperature were the main contributors to this pattern in the two data sets (Figure 7.2).
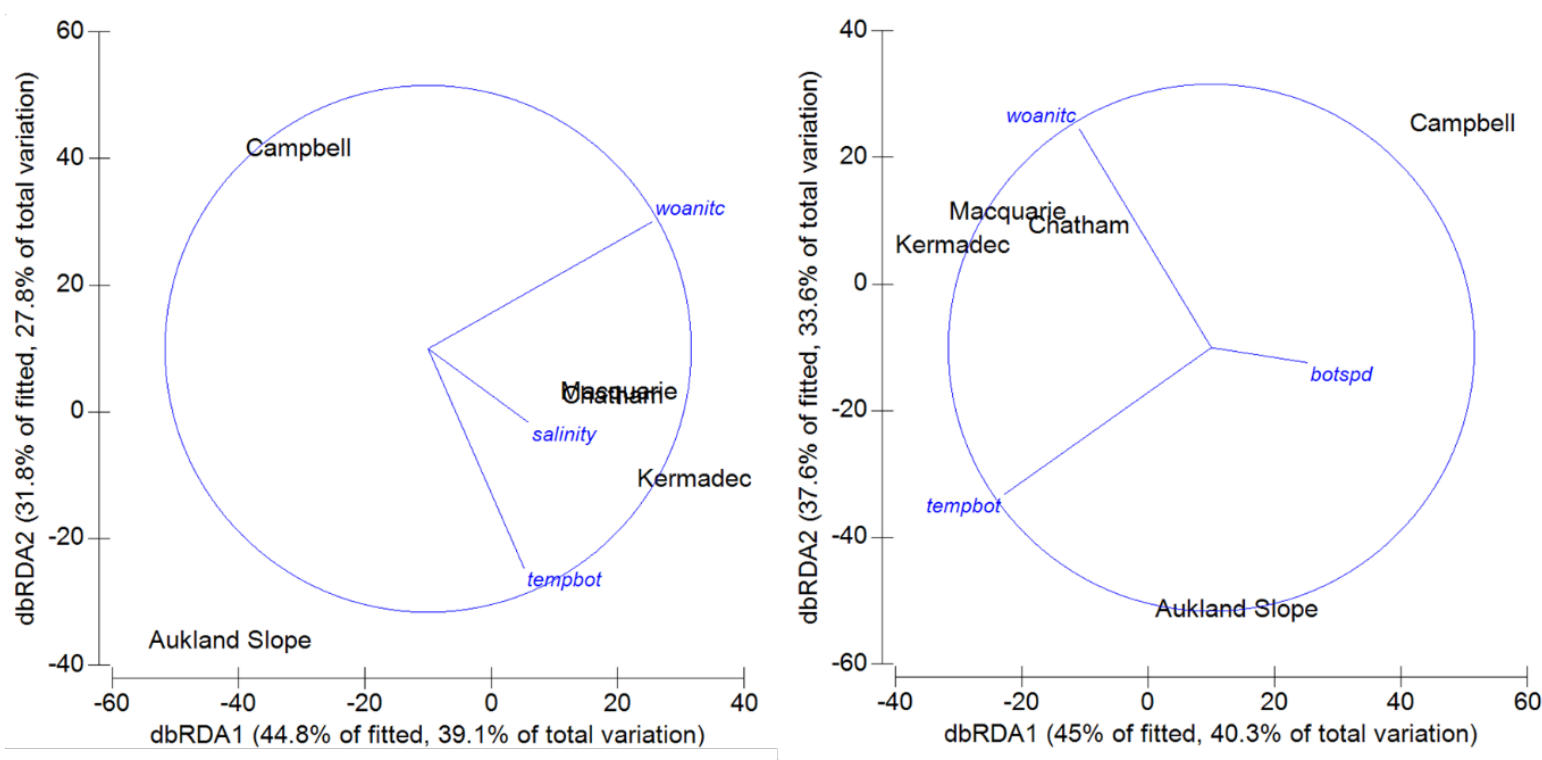

Figure 7.2 dbRDA analyses of all (left) and neutral (right) microsatellite variation for Poecillastra laminaris 
Table 7.8 Sequential test results of the DistLM analysis for microsatellite data amongst geomorphic features for all species

\begin{tabular}{|c|c|c|c|c|c|c|}
\hline Species & Loci & Variable & $\operatorname{Adj} R^{2}$ & SS(trace) & $\mathbf{p}$ & Proportion \\
\hline \multirow{4}{*}{ Goniocorella dumosa } & \multirow{2}{*}{ ALL } & +woanitc & 0.222 & 1783.0 & 0.090 & 0.482 \\
\hline & & +aragonite & 0.350 & 1117.5 & 0.393 & 0.302 \\
\hline & \multirow{2}{*}{ Neutral } & +woanitc & 0.233 & 1802.3 & 0.145 & 0.489 \\
\hline & & +aragonite & 0.477 & 1243.6 & 0.321 & 0.337 \\
\hline \multirow{10}{*}{ Solenosmilia variabilis } & \multirow{4}{*}{ ALL } & +stdevslope & 0.069 & 1242.1 & 0.103 & 0.202 \\
\hline & & + smt & 0.187 & 1334.8 & 0.026 & 0.217 \\
\hline & & +tempbot & 0.286 & 1065.5 & 0.080 & 0.173 \\
\hline & & +slopepercent & 0.363 & 829.2 & 0.211 & 0.135 \\
\hline & \multirow{6}{*}{ Neutral } & +stdevslope & 0.139 & 1345.4 & 0.028 & 0.262 \\
\hline & & + smt & 0.238 & 999.0 & 0.049 & 0.194 \\
\hline & & +tempbot & 0.304 & 751.2 & 0.149 & 0.146 \\
\hline & & +aragonite & 0.339 & 589.1 & 0.345 & 0.115 \\
\hline & & +tidcurr & 0.401 & 576.1 & 0.342 & 0.112 \\
\hline & & +salinity & 0.518 & 526.2 & 0.394 & 0.102 \\
\hline \multirow{6}{*}{ Poecillastra laminaris } & \multirow{3}{*}{ ALL } & +woanitc & 0.131 & 3963.5 & 0.037 & 0.349 \\
\hline & & +tempbot & 0.284 & 3336.3 & 0.287 & 0.293 \\
\hline & & +salinity & 0.487 & 2612.4 & 0.338 & 0.230 \\
\hline & \multirow{3}{*}{ Neutral } & +tempbot & 0.132 & 3675.6 & 0.073 & 0.349 \\
\hline & & +woanitc & 0.414 & 3768.4 & 0.083 & 0.358 \\
\hline & & + botspd & 0.578 & 1975.9 & 0.339 & 0.188 \\
\hline
\end{tabular}

For G. dumosa, the DistLM analysis from the all loci and neutral loci data sets showed that nitrate and aragonite were the main factors that explained microsatellite variation: these variables explained $78.34 \%$ of the all microsatellites genetic variation and $82.57 \%$ of the neutral loci genetic variation. The greatest single contributor was nitrate at $48.16 \%$ for all loci and $48.86 \%$ for neutral loci. The dbRDA plot showed that populations from Kermadec Ridge and Chatham Rise grouped together with similar levels of nitrate and aragonite. The Challenger Plateau and NE Continential Slope population were separated each other, as well as Chantham Rise and Kermadec Ridge populations, associated with a variation in nitrate (Figure 7.3). 

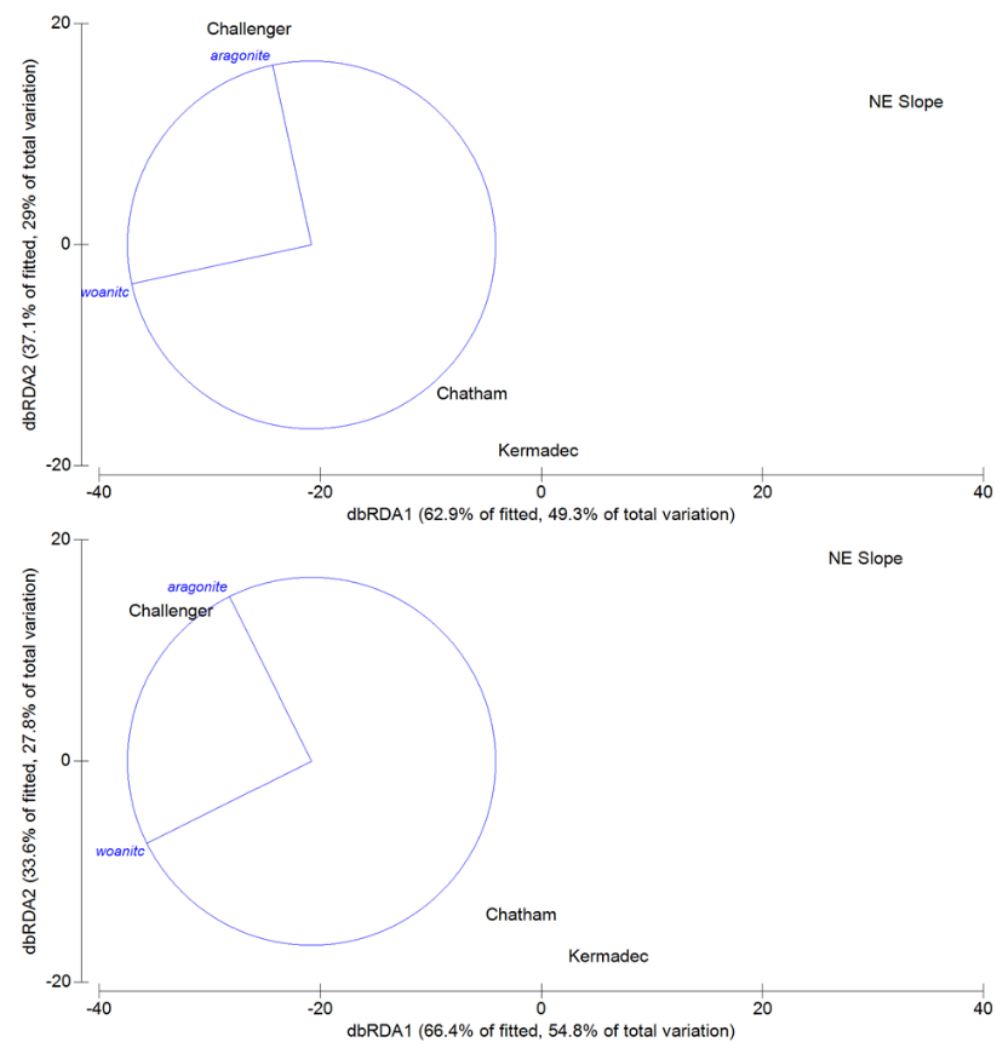

Figure 7.3 dbRDA analyses of all (above) and neutral (below) microsatellite variation for Goniocorella dumosa
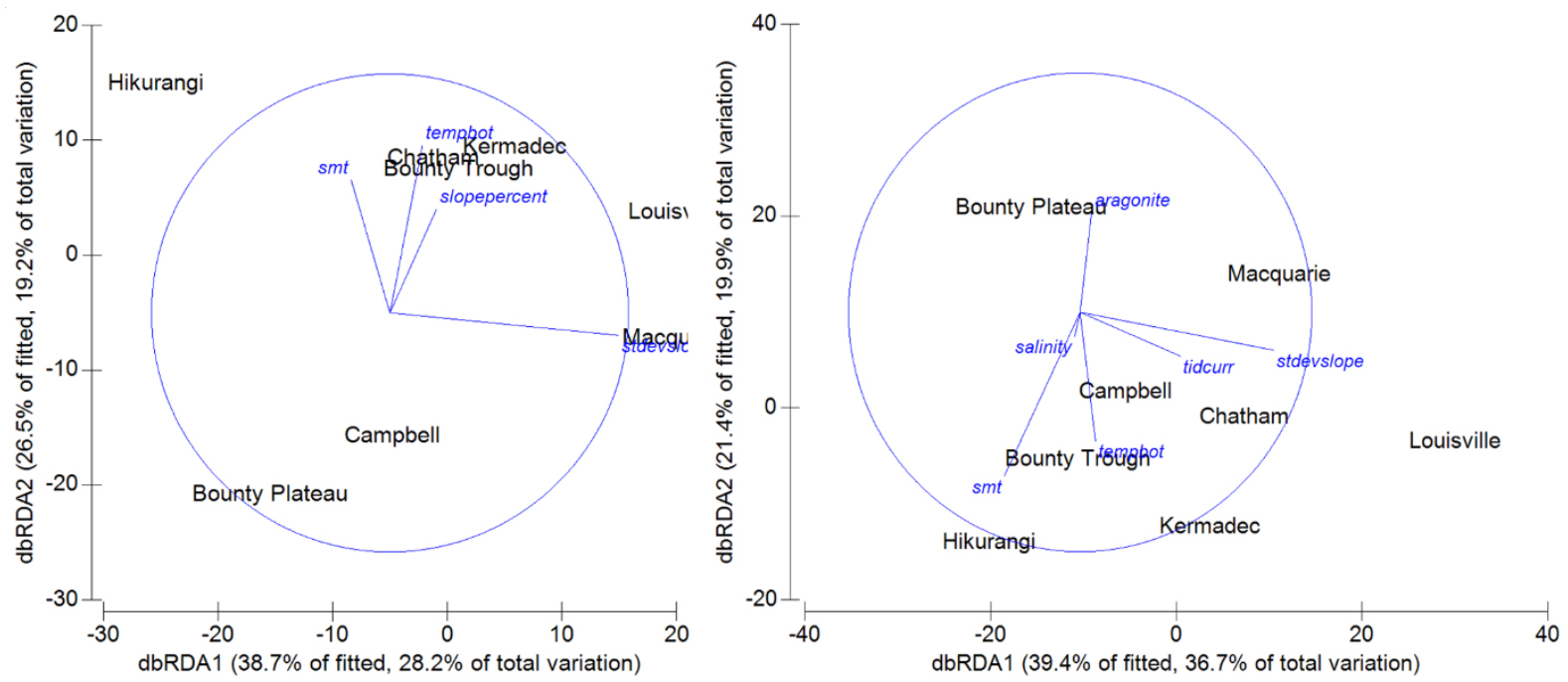

Figure 7.4 dbRDA analyses of all (left) and neutral (right) microsatellite variation for Solenosmilia variabilis

Microsatellite variation of all loci of $S$. variabilis was partially explained by standard deviation of slope, seamount percentage, bottom water temperature and slope percentage. 
These four variables explained $72.7 \%$ of the observed genetic variability, with the highest contribution being made by seamount percentage (Table 7.8). In the dbRDA plot, the Hikurangi Margin population was separated by standard deviation of slope from the other populations, and the other three environmental variables were separated populations into 6 groups. In the results of the neutral microsatellite loci analysis, standard deviation of slope, seamount percentage, bottom water temperature, aragonite, tidal current speed and salinity were the factors selected in the DistLM analysis, and these 6 variables accounted for $93.1 \%$ of the genetic variation. Amongst these variables, standard deviation of slope was the most significant variable in the DistLM analysis, and it explained $26.2 \%$ of the microsatellite genetic variation (Figure 7.4). The dbRDA plots revealed that the 8 geomorphic feature populations were genetically different from one another, and that this separation was influenced by the combination of these 6 environmental variables. Furthermore, the Bounty Plateau population was distinct from the other populations due to the influence of aragonite variation (Figure 7.4).

\subsection{Discussion}

Contrasting patterns of population genetic structure have been reported in previous chapters for the 4 sponge and 3 coral taxa throughout their distributional ranges in the New Zealand region. Here, environmental variability has been examined to help explain the taxonspecific genetic variation. Small sample size is however, a very big challenge that deep-sea researchers have to confront, and was a problem in the present study. Because sampling in the present study was based on availability of material collected by different research voyages over the years, rather than from a directed sampling programme, the distributions of the specimens analysed here had a spatial bias, with the highest density from the Chatham Rise (associated with opportunistic collecting during fisheries research surveys to the region). This bias may contribute to a failure to reveal seascape genetic patterns at a geomorphic feature population level. Moreover, because this is the first test of the role of environmental variability explaining genetic variability for deep-sea species, published information from previous studies for comparative purposes was entirely absent. Nonetheless, despite small population sample sizes in some cases (minimum $\mathrm{n}$ is 4 ) and a lack of comparative information about the relationships between environmental factors and genetic differentiation, the results generated here contribute to a more comprehensive understanding about environmental and genetic interactions at both small and large spatial scales. 


\subsubsection{Choices for markers and research scales}

The selected environmental variables varied amongst the different models for each species according to the different genetic markers and different spatial scales. For DNA sequence markers, mitochondrial markers had a better performance than nuclear markers, as judged by higher $r^{2}$ values in the one sponge and three corals models. There was no evidence to support the hypothesis that the environmental variables explained in absolute terms increased levels of genetic variation per se (e.g. the nucleotide diversity of the mitochondrial D-loop region was lower than that of the nuclear ITS region in S. variabilis, but the $\mathrm{R}^{2}$ value of the D-loop model was higher than that of the ITS model). One possible reason for this result is that the mitochondrial genome is more sensitive than the nuclear genome to environmental variability, perhaps because almost all of the mitogenome is coding whereas most of the nuclear genome is non-coding. For example, whole-organism metabolic rate has been shown to be affected by genetic polymorphism across mtDNA (Arnqvist et al., 2010). In the present study, oxygen and temperature variation significantly explained mtDNA variation in both corals and sponges (oxygen and temperature are directly related to metabolic rate (Gillooly et al., 2001; Seibel \& Drazen, 2007)), and this may be indirect evidence supporting why the model had a better explanation for the mtDNA markers. Microsatellites, which had a better performance in population genetic studies, were less sensitive to environmental factors, as might be expected for markers that are assumed to be selectively neutral. Whether there is a direct selection-based association between genetic and environmental variation, or whether the environmental variables simply act as barriers to gene flow (a more neutral rather than selection-based explanation) remains to be determined.

In the present study, analyses of data grouped at different spatial scales produced very different results. Outcomes varied as a result of different marker types. For example, at the local scale (individual level) most significant results involved dominant markers (DNA sequences), but at a broader scale (geomorphic feature population level) it was the codominant markers (microsatellites) that revealed significant relationships with environmental variables.

Of note is the fact that a single environmental variable may be included in all models for a single species, but for one marker the variable's contribution was positive and for another marker within the same species the variable's contribution was negative. Examples include aragonite and dissolved organic matter. Determining how a variable may have both a positive and negative contribution to genetic variation within the same species but for 
different markers will require further investigation. It may, however, be explained by direct selection pressure on one marker (e.g., the mitogenome) and an absence of selection pressure on another marker type (e.g., the nuclear genome). Given the linked nature of regions (genes) on the circular mitogenome, associations (either positive or negative) may be expected to be the same for two or more regions of mitochondrial DNA. The results here do not however, always support this expectation, indicating that different regions of the mitogenome may be responding differentially to the environmental variation, either as settled adults or as dispersive larvae.

\subsubsection{Environmental effects and genetic variations}

In general, topographic factors (such as bathymetric variation), physico-chemical factors (such as temperature, current speed, oxygen, aragonite saturation state, and nutrients), and biological factors (such as surface water Chl-a concentration) were examined directly in the present study. They have previously been identified as environmental drivers for controlling the large-scale distribution of deep-sea corals (Bryan \& Metaxas, 2006, 2007; Davies et al., 2008; Tittensor et al., 2009; Woodby et al., 2009; Fisher et al., 2014; Arellano et al., 2014).

At the north-central-south regional scale, the main variables driving the genetic structure in corals were dynamic topography, surface water primary productivity, seamount and bottom current speed. Dynamic topography, which is a measure of relative sea surface height, reflects the potential for large scale current movements (Tracey et al., 2011). Significant contributions of dynamic topography to explain genetic variation in M. oculata and S. variabilis supported the hypothesis in Chapter 6 that large-scale currents associated with the Tropical and Subtropical fronts shaped the regional genetic structure. For G. dumosa, the regional structure was influenced by surface water primary productivity, which is related to the amount of food available to suspension-feeding corals (Tracey et al., 2011). The importance of this variable for this species is also probably related to the influence of currents and fronts on genetic connectivity between the central region and the north and south region. That is, G. dumasa was more common in relatively shallower water (compared with $M$. oculata and S. variabilis) on the Chatham Rise of the central region, which is an area of particularly high sea surface primary productivity. In the sponge, Poecillastra laminaris, silicate was signification in explaining more half of the regional structure, but bottom current speed was the most important variable when tested against the neutral loci (it explained $>$ 
$60 \%$ ). This result might indicate that the genome of Poecillastra laminaris was under some sort of (unknown) selection pressure for silicate, and that the bottom current speed is acting as a genetic barrier by affecting larval settlement and/or the available food source. However, this hypothesis will require further evidence to test it. Overall, the current was an important factor that contributed to the formation of genetic structures for deep-sea corals and a sponge around New Zealand, and supports the primary explanation for the north-central-south regional genetic variation presented in Chapters 5 and 6 .

At the geomorphic feature level, the factors that affected genetic connectivity patterns varied across the different species. In G. dumosa, nitrate (co-correlate $=$ phosphate) and aragonite (co-correlates $=$ calcite, temperature, sigma-theta) were the factors that shaped the structure. These two variables have been shown to modify coral growth and calcification in other stony coral species (Marubini \& Atkinson, 1999; Leclercq et al., 2000; Turley et al., 2007; Silverman et al., 2007). In addition, temperature and water density (sigma-theta) have been documented as restricting the occurrence deep-sea stony corals (Freiwald et al., 2004; Roberts et al., 2006; Davies et al., 2008; Dullo et al., 2008). In S. variabilis, standard deviation of slope and seamount were the most important factors that influenced the genetic variation of populations at the geomorphic features scale, in both the all loci and neutral loci microsatellite data set. This indicates that the topography of different geomorphic features may affect the genetic structure of this species. This might provide an explanation for genetic isolations of populations from Kermadec Ridge, Louisville Seamount Chain and Macquarie Ridge. Previous studies of corals have detected similar patterns: genetic differentiation was significantly different between slope and ridges in Desmophyllum dianthus (Miller et al., 2010), and Narella (Baco \& Cairns, 2012). However, neither of these studies discussed the relationship between genetic differentiation and topography: further studies are required to verify this relationship. In Poecillastra laminaris, nitrate (co-correlate $=$ phosphate) and bottom water temperature (co-correlate $=$ bottom water temperature residuals) were the crucial explanators of population genetic variation at the geomorphic features scale, possibly reflecting that factors relating to energy were involved in the connectivity amongst populations (see next paragraph for discussion on food availability). Generally, one possible interpretation for all of the aforementioned variables acting as barriers to population connectivity relates to them reflecting the absence of a suitable environment for larval settlement and development amongst different geomorphic features. That is, the patterns of genetic structure could be driven by different conditions amongst geomorphic features that challenged the larvae from other populations to survive, colonise and/or reproduce once 
established as an adult, such that little or no genetic contribution amongst local gene pools induced genetic differentiation between local populations. However, there are no studies available for deep-sea species to provide evidence to support this suggestion.

At the individual level, the variables that elucidated the patterns of genetic variation differed not only amongst species but also by genetic marker. The important variables identified at this level were similar to those identified by habitat suitability or species distribution modelling, but the importance of each variable in the present study was different from those identified by these previous studies (Guinotte et al., 2006; Tittensor et al., 2009; Davies \& Guinotte, 2011; Tracey et al., 2011). In this study, sea surface temperature gradient was significant as an explanator of genetic variation in all three corals and the sponge Poecillastra laminaris for different markers, except the all loci data set of G. dumosa. In addition, a significant contribution of sea surface temperature was also detected as an explanator of genetic variation in P. laminaris and $S$. variabilis. Sea surface temperature and sea surface temperature gradient are both related to primary productivity, and the latter to the location of frontal systems (Hurst et al., 2012). Primary productivity and frontal systems are proxies for food availability (Abelson et al., 1993; Kaiser, 2011), and the genetic variation patterns of these two species might therefore be driven by food source availability. Food is closely related to an organism's growth, fecundity, larval dispersal and settlement (Thorson, 1950; Buss \& Jackson, 1981; Bertness \& Gaines, 1993; Qian, 1994; Pechenik et al., 1996; Fouzai et al., 2015), and thus food availability indirectly effects the genetic contribution made by one population to another population (by fecundity) and/or the genetic connectivity between populations (by larval dispersal and settlement). In Poecillastra laminaris, as well as sea surface temperature and sea surface temperature gradient, nitrate (co-correlate = phosphate) and particulate organic carbon export (POC) were included in all 4 individuallevel models. The significant relationship between POC and genetic structure were also detected in a shallow-sea reef sponge (Stylissa carteri) (Giles et al., 2015). A previous study has found that POC and nitrogen (PON) are very important food sources for sponges (Hadas et al., 2009), further suggesting that food source availability might be the reason for the genetic differentiation observed in Poecillastra laminaris. However, in modelling the distribution of the sponge Geodia, surface temperature did not contribute to the prediction (Knudby et al., 2013), indicating that this contradictory finding necessitates further investigation. Interestingly, dynamic topography was an important contribution to explaining the individual-level genetic variation of $G$. dumosa in all 4 models but not at large scales (regional and geomorphic feature level) for this species (it was important at these scales for 
the other two coral species). As noted above, dynamic topography is related to large-scale current dynamics, which have been shown previously to be related to the distribution of suspension-feeding organisms through infuencing food source availability and variability (Frederiksen et al., 1992; Tittensor et al., 2009), which provides more support for the explanation that food availability might explain the local genetic structure observed in $G$. dumosa. Generally, the results at the local scale from the present study suggest that food resource variability helps to explain small-scale genetic differentiation for both corals and sponges.

Interestingly, a previous study has found that deep-sea corals and sponges have similar environmental demands (Rooper et al., 2014), but despite this, the variables that influenced the patterns of genetic structure at different scales were species-specific. Nevertheless, how these different variables modified these species-specific patterns of genetic connectivity is still unclear. Additionally, high correlation coefficients leave as yet unresolved the question of whether species-specific genetic variation is correlated with a single environmental variable or multiple variables. Investigations of genotypic, phenotypic and environmental variability, as well as of gene-environment interactions are required to resolve this.

\subsubsection{Implications for management}

Marine environment managers are increasingly incorporating genetic information into management planning (Waples et al., 2008; McCook et al., 2009; Haig et al., 2016), and the results of seascape genetic modelling can help inform management options that need to respond to factors that influence genetic structure and connectivity. To maintain populationlevel genetic diversity and effective population size, it is essential to understand the patterns of genetic connectivity that exist amongst deep-sea populations. However, open spatial population structures, logistical sampling difficulties and unknown barriers to gene flow present challenges to connectivity research. To overcome these challenges, seascape genetic research may open another window to improve understanding of the linkages between genetics and the spatial ecology of populations (Selkoe et al., 2008).

The genetic separation of some deep-sea coral and sponge populations into regions or locations that are characterised by particular temperature or food availability regimes illustrates the importance of protecting these taxa via spatial management options that include locations (e.g. marine protected areas) that represent these environmental regimes. Additionally, the identification of variables that help to explain coral and sponge genetic 
variation indicates that networks of protected areas should include representatives of different geomorphic features, and conservation priorities could be allocated to sites that contain particularly complex topography. Related to the latter recommendation, the results from the present study indicate that the Kermadec region is important, because all examined species occurred there, and it has a complex bathymetry (slope, plateaux, seamounts, plains etc). Hence protecting this area serves to achieve a number of potential conservation objectives (representative populations and genetic diversity), something that currently being considered in the form of the Kermadec Ocean Sanctuary proposal (http://www.mfe.govt.nz/marine/ kermadec-ocean-sanctuary). 


\section{Appendix}

Table S7.1 Mean values of the environmental variables for the Poecillastra laminaris populations

\begin{tabular}{lcccccccc}
\hline \multicolumn{1}{c}{ Variable } & $\begin{array}{c}\text { Central } \\
\text { Region }\end{array}$ & $\begin{array}{c}\text { North } \\
\text { Region }\end{array}$ & $\begin{array}{c}\text { South } \\
\text { Region }\end{array}$ & $\begin{array}{c}\text { Campbell } \\
\text { Plateau }\end{array}$ & $\begin{array}{c}\text { Chatham } \\
\text { Rise }\end{array}$ & $\begin{array}{c}\text { Auckland } \\
\text { Slope }\end{array}$ & $\begin{array}{c}\text { Kermadec } \\
\text { Ridge }\end{array}$ & $\begin{array}{c}\text { Macquarie } \\
\text { Ridge }\end{array}$ \\
\hline bpi-broad & 120.526 & 618.438 & 996.882 & 8.750 & 120.526 & 440.750 & 927.200 & 1300.923 \\
bpi-fine & 59.789 & 246.625 & 164.588 & 14.500 & 59.789 & 225.250 & 422.800 & 210.769 \\
oxygen & 4.627 & 4.539 & 5.134 & 5.298 & 4.627 & 4.617 & 4.546 & 5.021 \\
POC & 14.475 & 9.237 & 5.326 & 11.125 & 14.475 & 12.721 & 5.410 & 3.817 \\
salinity & 34.440 & 34.770 & 34.380 & 34.385 & 34.440 & 34.882 & 34.852 & 34.383 \\
silicate & 28.732 & 13.620 & 35.789 & 31.394 & 28.732 & 8.899 & 6.724 & 38.939 \\
slope-percent & 2.312 & 7.060 & 10.518 & 1.158 & 2.312 & 8.833 & 8.585 & 13.398 \\
stdev-slope & 1.269 & 3.191 & 3.841 & 0.357 & 1.269 & 3.184 & 4.725 & 4.913 \\
sigma-theta & 31.684 & 31.299 & 31.778 & 31.731 & 31.684 & 31.196 & 31.141 & 31.793 \\
woanitc & 29.016 & 23.146 & 28.240 & 25.256 & 29.016 & 16.339 & 26.838 & 29.592 \\
botspd & 0.107 & 0.096 & 0.119 & 0.110 & 0.107 & 0.102 & 0.051 & 0.093 \\
cdom & 2.509 & 1.948 & 1.415 & 1.390 & 2.509 & 2.277 & 1.534 & 1.416 \\
disorg & 0.050 & 0.023 & 0.026 & 0.022 & 0.050 & 0.033 & 0.007 & 0.031 \\
dynoc & 0.524 & 0.693 & 0.361 & 0.329 & 0.524 & 0.597 & 0.813 & 0.438 \\
smt & 0.737 & 0.500 & 0.722 & 0.000 & 0.737 & 1.000 & 0.000 & 1.000 \\
sstgrd & 0.009 & 0.006 & 0.006 & 0.003 & 0.009 & 0.009 & 0.007 & 0.007 \\
sst & 0.017 & 0.014 & 0.010 & 0.006 & 0.017 & 0.021 & 0.013 & 0.013 \\
tempbot & -0.114 & 0.599 & -0.919 & -1.466 & -0.114 & 0.933 & -0.069 & -0.076 \\
tidcurr & 0.182 & 0.100 & 0.154 & 0.206 & 0.182 & 0.041 & 0.124 & 0.121 \\
vgpm & 640.344 & 538.315 & 317.396 & 284.656 & 640.344 & 672.843 & 351.955 & 355.189 \\
\hline
\end{tabular}

Table S7.2 Mean values of the environmental variables for the Goniocorella dumosa populations

\begin{tabular}{lccccccc}
\hline \multicolumn{1}{c}{ Variable } & $\begin{array}{c}\text { Central } \\
\text { Region }\end{array}$ & $\begin{array}{c}\text { North } \\
\text { Region }\end{array}$ & $\begin{array}{c}\text { South } \\
\text { Region }\end{array}$ & NE Slope & $\begin{array}{c}\text { Challenger } \\
\text { Plateau }\end{array}$ & $\begin{array}{c}\text { Chatham } \\
\text { Rise }\end{array}$ & $\begin{array}{c}\text { Kermadec } \\
\text { Ridge }\end{array}$ \\
bpi-broad & 45.435 & 267.375 & 274.600 & 124.500 & 79.800 & 45.435 & 399.083 \\
bpi-fine & 25.355 & 106.417 & 95.200 & 21.000 & 8.000 & 25.355 & 172.333 \\
aragonite & 1.627 & 1.739 & 1.468 & 1.954 & 1.939 & 1.627 & 1.582 \\
oxygen & 5.374 & 4.612 & 5.407 & 4.517 & 4.618 & 5.374 & 4.601 \\
POC & 25.135 & 15.711 & 10.278 & 15.824 & 16.279 & 25.135 & 14.003 \\
salinity & 34.518 & 34.817 & 34.443 & 34.994 & 34.978 & 34.518 & 34.703 \\
slope-percent & 1.665 & 8.757 & 5.343 & 2.266 & 3.250 & 1.665 & 12.132 \\
stdev-slope & 0.546 & 2.332 & 2.668 & 0.484 & 0.819 & 0.546 & 3.433 \\
woanitc & 19.275 & 18.164 & 20.895 & 13.868 & 20.375 & 19.275 & 18.777 \\
botspd & 0.073 & 0.085 & 0.045 & 0.085 & 0.052 & 0.073 & 0.098 \\
cdom & 2.388 & 2.317 & 1.430 & 1.930 & 2.388 & 2.388 & 2.288 \\
disorg & 0.063 & 0.039 & 0.026 & 0.039 & 0.041 & 0.063 & 0.033 \\
dynoc & 0.480 & 0.593 & 0.448 & 0.623 & 0.555 & 0.480 & 0.606 \\
smt & 0.145 & 0.333 & 0.200 & 0.000 & 0.000 & 0.145 & 0.583 \\
sstgrd & 0.013 & 0.008 & 0.006 & 0.009 & 0.006 & 0.013 & 0.008 \\
sst & 0.024 & 0.020 & 0.013 & 0.021 & 0.017 & 0.024 & 0.021 \\
tempbot & -0.371 & 1.349 & -0.489 & 1.580 & 2.395 & -0.371 & 0.950 \\
tidcurr & 0.382 & 0.094 & 0.266 & 0.147 & 0.205 & 0.382 & 0.048 \\
vgpm & 653.412 & 705.329 & 326.955 & 684.132 & 636.341 & 653.412 & 681.477 \\
\hline
\end{tabular}


Table S7.3 Mean values of the environmental variables for the Madrepora oculata populations

\begin{tabular}{lccc}
\hline \multicolumn{1}{c}{ Variable } & Central Region & North Region & South Region \\
\hline bpi-broad & 61.048 & 373.533 & -6.929 \\
bpi-fine & 8.476 & 123.067 & 0.571 \\
aragonite & 1.092 & 1.518 & 1.061 \\
oxygen & 4.375 & 4.424 & 4.773 \\
POC & 13.869 & 11.733 & 7.831 \\
salinity & 34.425 & 34.733 & 34.358 \\
slope-percent & 2.858 & 4.357 & 1.214 \\
stdev-slope & 0.765 & 2.010 & 0.106 \\
woanitc & 27.707 & 24.164 & 30.024 \\
botspd & 0.113 & 0.085 & 0.119 \\
cdom & 2.392 & 2.118 & 1.365 \\
disorg & 0.050 & 0.032 & 0.022 \\
dynoc & 0.514 & 0.610 & 0.284 \\
smt & 0.238 & 0.400 & 0.000 \\
sstgrd & 0.009 & 0.005 & 0.007 \\
sst & 0.016 & 0.013 & 0.013 \\
tempbot & -0.244 & 1.014 & -1.658 \\
tidcurr & 0.173 & 0.123 & 0.120 \\
vgpm & 624.800 & 611.207 & 292.567 \\
\hline
\end{tabular}

Table S7.4 Mean values of the environmental variables for the Solenosmilia variabilis populations at regional scale

\begin{tabular}{lccc}
\hline \multicolumn{1}{c}{ Variable } & $\begin{array}{c}\text { Central } \\
\text { Region }\end{array}$ & $\begin{array}{c}\text { North } \\
\text { Region }\end{array}$ & $\begin{array}{c}\text { South } \\
\text { Region }\end{array}$ \\
\hline bpibroad & 92.912 & 1131.808 & 251.088 \\
bpifine & 9.147 & 315.673 & 58.853 \\
aragonite & 1.207 & 1.327 & 1.189 \\
oxygen & 4.319 & 4.117 & 4.580 \\
POC & 13.967 & 5.043 & 5.638 \\
salinity & 34.456 & 34.469 & 34.402 \\
slopepercent & 3.131 & 13.529 & 4.263 \\
stdevslope & 0.820 & 5.077 & 1.785 \\
woanitc & 28.835 & 32.498 & 31.602 \\
botspd & 0.120 & 0.080 & 0.093 \\
cdom & 2.504 & 1.902 & 1.382 \\
disorg & 0.052 & 0.024 & 0.024 \\
dynoc & 0.524 & 0.693 & 0.366 \\
smt & 0.265 & 0.868 & 0.176 \\
sstgrd & 0.008 & 0.003 & 0.006 \\
sst & 0.016 & 0.007 & 0.011 \\
tempbot & -0.074 & 0.282 & -0.874 \\
tidcurr & 0.162 & 0.092 & 0.120 \\
vgpm & 649.818 & 540.369 & 312.129 \\
\hline & & &
\end{tabular}


Table S7.4 Mean values of the environmental variables for the Solenosmilia variabilis geomorphic feature populations

\begin{tabular}{lcccccccc}
\hline \multicolumn{1}{c}{ Variable } & $\begin{array}{c}\text { Bounty } \\
\text { Plateau }\end{array}$ & $\begin{array}{c}\text { Bounty } \\
\text { Trough }\end{array}$ & $\begin{array}{c}\text { Campbell } \\
\text { Plateau }\end{array}$ & $\begin{array}{c}\text { Chatham } \\
\text { Rise }\end{array}$ & $\begin{array}{c}\text { Hikurangi } \\
\text { Margin }\end{array}$ & $\begin{array}{c}\text { Kermade } \\
\text { c Ridge }\end{array}$ & $\begin{array}{c}\text { Louisville } \\
\text { Seamoun } \\
\text { t Chain }\end{array}$ & $\begin{array}{c}\text { Macquarie } \\
\text { Ridge }\end{array}$ \\
\hline bpibroad & 27.000 & 74.800 & 6.750 & 92.912 & 603.500 & 909.429 & 2016.917 & 538.733 \\
bpifine & -0.500 & 38.600 & -2.625 & 9.147 & 53.250 & 344.171 & 330.833 & 122.067 \\
aragonite & 1.467 & 1.078 & 1.142 & 1.207 & 1.256 & 1.238 & 1.595 & 1.213 \\
oxygen & 4.851 & 4.355 & 4.698 & 4.319 & 4.604 & 4.004 & 4.323 & 4.484 \\
POC & 9.822 & 5.712 & 6.976 & 13.967 & 9.559 & 5.108 & 2.621 & 4.406 \\
salinity & 34.324 & 34.450 & 34.313 & 34.456 & 34.463 & 34.489 & 34.402 & 34.391 \\
slopepercent & 0.958 & 2.026 & 1.405 & 3.131 & 8.304 & 13.047 & 16.740 & 7.979 \\
stdevslope & 0.179 & 0.769 & 0.490 & 0.820 & 1.727 & 4.797 & 7.071 & 3.478 \\
woanitc & 30.027 & 32.940 & 31.687 & 28.835 & 25.291 & 33.364 & 32.060 & 31.263 \\
botspd & 0.091 & 0.097 & 0.132 & 0.120 & 0.191 & 0.069 & 0.105 & 0.072 \\
cdom & 1.356 & 1.283 & 1.402 & 2.504 & 2.365 & 1.819 & 1.799 & 1.411 \\
disorg & 0.029 & 0.023 & 0.019 & 0.052 & 0.039 & 0.019 & 0.031 & 0.026 \\
dynoc & 0.223 & 0.221 & 0.308 & 0.524 & 0.589 & 0.713 & 0.466 & 0.484 \\
smt & 0.000 & 0.200 & 0.000 & 0.265 & 0.750 & 0.857 & 1.000 & 0.333 \\
sstgrd & 0.007 & 0.007 & 0.005 & 0.008 & 0.005 & 0.003 & 0.006 & 0.005 \\
sst & 0.015 & 0.012 & 0.009 & 0.016 & 0.011 & 0.006 & 0.011 & 0.010 \\
tempbot & -2.315 & -0.779 & -1.559 & -0.074 & 0.341 & 0.282 & -0.478 & -0.157 \\
tidcurr & 0.142 & 0.107 & 0.111 & 0.162 & 0.109 & 0.090 & 0.123 & 0.122 \\
vgpm & 343.394 & 318.312 & 252.408 & 649.818 & 730.177 & 503.669 & 462.871 & 333.582 \\
\hline & & & & & & & & \\
\end{tabular}


Chapter 8. General discussion 
New Zealand's exclusive economic zone (EEZ) covers approximately fifteen times the land area of the country, with most areas being greater than $200 \mathrm{~m}$ in depth, and is comprised of various topographical habitats and complex hydrodynamic systems. However, deep-sea organisms around the world are poorly researched and the New Zealand region is no exception. Meanwhile, the increasing exploitation of marine resources and human-induced environmental changes brings a threat to this relatively unknown system. As such, there are growing concerns about protection in the deep sea and the impacts of anthropological activities, mainly fisheries (Ramirez-Llodra et al., 2011). The vulnerable marine ecosystem (VME) concept originated from discussions at the United Nations General Assembly (UNGA), as established in UNGA Resolution 61/105. The FAO International Guidelines for the Management of Deep-sea Fisheries in the High Seas expanded on the VME concept for fisheries management (FAO, 2009). VME indicator taxa are defined as those that are unique or rare, functionally significant, fragile, structurally complex, slow to recover or may never recover, exhibit slow growth, late age of maturity, low or unpredictable recruitment, and are long lived (e.g. Parker et al., 2009). In New Zealand, it has been reported that VME indicator taxa such as deep-sea corals are impacted by commercial trawling, both within and outside New Zealand's EEZ (Anderson \& Clark, 2003; Clark \& Rowden, 2009). However, limited information about the genetic structure and population dynamics of deep-sea species, especially VME indicator taxa, constrains effective management and conservation of marine resources. Population genetics integrated with ecological data is considered to be a powerful approach to address questions about population structure, and gain an understanding of connectivity patterns amongst populations to better inform managers who make recommendations about spatial planning actions that may include marine protected areas (Palumbi, 2003).

The general aims of this thesis were to investigate the genetic structure of VME indicator taxa, specifically 4 sponges (Neoaulaxinia persicum, Penares sp., Pleroma menoui and Poecillastra laminaris) and 3 corals (Goniocorella dumosa, Madrepora oculata and Solenosmilia variabilis), and their relationships with environmental variation, and provide information for deep-sea conservation around New Zealand. For this first population genetics study of deep-sea sponges, and one of only a few studies of deep-sea stony corals, a multidisciplinary approach was used with different genetic markers (mainly developed in this study) and environmental data. This study provides important information for management of these VME indicator taxa, especially the poorly-understood sponges, it contributes to a 
broader understanding of the patterns of gene flow around New Zealand and it identifies potential factors that shape the patterns of population genetic structure and connectivity.

In this general discussion, the major findings of this study are reviewed and summarised, and considered with respect to their contribution to the field of deep-sea population genetics. The management implications of the research are also highlighted, and finally future research needs are identified.

\subsection{Major findings}

\subsubsection{Marker performance}

In the present study, three different types of genetic markers were involved, sequence (COI, Cytb, 12S, D-loop, ITS), genotype (microsatellites) and single nucleotide polymorphism (SNPs). Sequence markers, both mitochondrial and nuclear, were developed for Poecillastra laminaris (0) and Solenosmilia variabilis (Chapter 4) in this study. For microsatellites, 10 polymorphic microsatellite markers were developed for Poecillastra laminaris, and 18 polymorphic microsatellites were successfully generated from 454 next generation sequencing data for S. variabilis. Cross-amplification of these microsatellites and another 9 microsatellites from previous studies were tested in three stony corals (Desmophyllum dianthus, Goniocorella dumosa, Madrepora oculata). A significant number of SNPs $(102,678)$ and indels $(846)$ were identified based on specific filter criteria for $S$. variablis, and the variations amongst individuals were tested.

In the sponges, although COI was successfully amplified in four species, only for Poecillastra laminaris was genetic structure detected. Surprisingly, the nucleotide diversities of $C O I$ were higher than have been reported for shallow water sponges (Duran et al., 2004; Lavrov et al., 2005; Wörheide, 2006). However, these higher genetic diversities did not contribute to structure discovery, because genetic structure has been detected in shallow water sponges with much lower nucleotide diversity levels (Duran et al., 2003; Wörheide 2006; DeBiasse et al., 2009; López-Legentil and Pawlik 2009). This result also supported the hypothesis in Chapter 2 that genetic variation is not related to the ability to detect barriers. In the corals, the sequence data did not reveal evidence of genetic structure in G. dumosa or $S$. variabilis for either ITS or D-loop. Variations of these two non-coding genes still could not provide enough resolution to show evidence of genetic structure, consistent with findings in a previous study (Miller et al., 2011). In general, COI and Cytb in P. laminaris and ITS in M. 
oculata provided enough resolution to reveal population structure, and they could be used for future studies. Additionally, ITS markers for sponges and COI markers for corals were tested at the beginning of this project, but multicopy PCR products and poor quality of sequencing meant that these markers were excluded from subsequent research.

For microsatellites, major issues for both sponges and corals were missing values in genotypes and small sample sizes. These issues resulted in inconsistent results between different analytical approaches, despite lots of effort for data quality control and post-hoc testing. Small sample size is a common challenge in deep-sea research (see Chapter 2), and the balance between accuracy and information loss from valued samples is now widely appreciated. Depending on the availability of samples from the archive for the present study, minimum sample size was set at 4 individuals. Meanwhile, many loci exhibited null alleles in all three corals, caused by missed alleles in the genotyping. Considering the DNA quality available from archived samples in a study such as this one, this phenomenon might be resolved by extensive (time consuming and expensive) testing of new PCR protocols. Although there were some disadvantages to the use of microsatellite data sets, different spatial structures were still revealed and their presence supported by different analytical approaches.

SNPs are a product of next generation sequencing, but so far have not been widely used in deep-sea population genetics studies. In the present study, the developed SNPs failed to reveal a clear split between different populations (Austrialia and the Louisville Seamount Chain), and this indicated a further filter of these SNPs is required. When designing the primers, many SNPs exhibited polyadenylations of upstream and/or downstream sequences: these could be a technical challenge to the use of these SNPs. Further experiments are required to characterise these candidate SNPs.

\subsubsection{Common patterns of genetic structure in the New Zealand deep sea}

As reviewed for coastal species, there is a north-south genetic differentiation in New Zealand, and a putative barrier to gene flow at about $42^{\circ} \mathrm{S}$ (Gardner et al., 2010). However, no reports from deep-sea organisms have discussed this pattern of differentiation. Based on the Watling et al. (2013) definition of deep-sea biogeographic provinces, there was potentially a north-south differentiation around $45^{\circ} \mathrm{S}$, where the province boundary exists. In the present study, the patterns of genetic variation observed for collected species around New Zealand reflected different hierarchical degrees of differentiation from north to south and 
different geomorphic feature populations (Table 8.1). The results showed that the patterns varied amongst different species, as well as with different genetic markers.

Table 8.1 Genetic differentiation patterns amongst different species in the present study

\begin{tabular}{|c|c|c|c|c|c|c|}
\hline Species & Marker & $\begin{array}{c}\text { Between } \\
\text { Provinces }\end{array}$ & $\begin{array}{l}\text { Amongst } \\
\text { Regions }\end{array}$ & $\begin{array}{c}\text { Amongst Geomorphic } \\
\text { Feature Populations }\end{array}$ & $\begin{array}{c}\text { Isolation by } \\
\text { Distance }\end{array}$ & $\begin{array}{c}\text { Isolation by } \\
\text { Depth }\end{array}$ \\
\hline \multirow{4}{*}{ Poecillastra laminaris } & COI & $\mathrm{Y}$ & $\mathrm{Y}$ & $\mathrm{Y}$ & $\mathrm{Y}$ & $\mathrm{N}$ \\
\hline & $C y t b$ & $\mathrm{Y}$ & $\mathrm{Y}$ & $\mathrm{Y}$ & $\mathrm{N}$ & $\mathrm{N}$ \\
\hline & All SSR & $\mathrm{N}$ & $\mathrm{N}$ & $\mathrm{Y}$ & $\mathrm{N}$ & $\mathrm{N}$ \\
\hline & Neutral SSR & $\mathrm{N}$ & $\mathrm{Y}$ & $\mathrm{N}$ & $\mathrm{N}$ & $\mathrm{N}$ \\
\hline \multirow{2}{*}{ Penares sp. } & COI & $\mathrm{N}$ & $\mathrm{N}$ & $\mathrm{N}$ & $\mathrm{N}$ & $\mathrm{N}$ \\
\hline & $12 S$ & $\mathrm{~N}$ & $\mathrm{~N}$ & $\mathrm{~N}$ & $\mathrm{~N}$ & $\mathrm{~N}$ \\
\hline \multirow{2}{*}{ Neoaulaxinia persicum } & COI & $\mathrm{N}$ & $\mathrm{N}$ & $\mathrm{N}$ & $\mathrm{N}$ & $\mathrm{N}$ \\
\hline & $12 S$ & $\mathrm{~N}$ & $\mathrm{~N}$ & $\mathrm{~N}$ & $\mathrm{~N}$ & $\mathrm{~N}$ \\
\hline \multirow[t]{2}{*}{ Pleroma menoui } & COI & $\mathrm{N}$ & $\mathrm{N}$ & $\mathrm{N}$ & $\mathrm{N}$ & $\mathrm{N}$ \\
\hline & ITS & $\mathrm{N}$ & $\mathrm{N}$ & $\mathrm{N}$ & $\mathrm{N}$ & $\mathrm{Y}$ \\
\hline \multirow{3}{*}{ Goniocorella dumosa } & D-loop & $\mathrm{N}$ & $\mathrm{N}$ & $\mathrm{N}$ & $\mathrm{N}$ & $\mathrm{N}$ \\
\hline & All SSR & $\mathrm{N}$ & $\mathrm{Y}$ & $\mathrm{Y}$ & $\mathrm{N}$ & $\mathrm{Y}$ \\
\hline & Neutral SSR & $\mathrm{N}$ & $\mathrm{Y}$ & Y & $\mathrm{N}$ & $\mathrm{N}$ \\
\hline \multirow{3}{*}{ Madrepora oculata } & ITS & $\mathrm{N}$ & $\mathrm{Y}$ & $\mathrm{N}$ & $\mathrm{N}$ & $\mathrm{Y}$ \\
\hline & All SSR & $\mathrm{N}$ & $\mathrm{Y}$ & $\mathrm{N}$ & $\mathrm{N}$ & $\mathrm{N}$ \\
\hline & Neutral SSR & $\mathrm{N}$ & $\mathrm{Y}$ & $\mathrm{N}$ & $\mathrm{N}$ & $\mathrm{N}$ \\
\hline \multirow{4}{*}{ Solenosmilia variabilis } & ITS & $\mathrm{N}$ & $\mathrm{N}$ & $\mathrm{N}$ & $\mathrm{N}$ & $\mathrm{N}$ \\
\hline & D-loop & $\mathrm{N}$ & $\mathrm{N}$ & $\mathrm{N}$ & $\mathrm{N}$ & $\mathrm{N}$ \\
\hline & All SSR & Y & Y & Y & Y & $\mathrm{N}$ \\
\hline & Neutral SSR & $\mathrm{Y}$ & $\mathrm{Y}$ & $\mathrm{Y}$ & $\mathrm{N}$ & $\mathrm{N}$ \\
\hline
\end{tabular}

$\mathrm{Y}$ means significant relationship at $\mathrm{p}<0.05$, and $\mathrm{N}$ means $\mathrm{p}>0.05$.

As hypothesised in Chapter 1, some common patterns were expected to be observed amongst the examined species. Due to issues with small sample sizes and uninformative markers, no spatial structure was observed in Penares sp., Neoaulaxinium persicum or Pleroma menoui, and these species are not involved in the following discussion. Indeed, variation in the mitochondrial DNA markers $C O I$ and $C y t b$ for P. laminaris, nuclear DNA marker ITS for M. oculata, microsatellites for Poecillastra laminaris, G. dumosa, M. oculata and S. variabilis have all revelaed the existence of pronounced north-central-south regional structure in deep-sea organisms (Table 8.1). However, despite this common pattern, there were more species-specific patterns discovered. Mitochondrial genes of $P$. laminaris and microsatellites of $S$. variabilis revealed a northern-southern provinces structure, and only $M$. oculata exhibited no evidence of genetic structure at the geomorphic feature level. Isolation by distance was observed in P. laminaris and S. variabilis, and isolation by depth was found in G. dumosa and M. oculata (Table 8.1). These shared and different patterns could be contributed to interaction between the species themselves and their environments, as discussed in the subsequent sections. 


\subsubsection{Connectivity amongst geomorphic feature populations}

Overall, less population genetic structure was detected among geomorphic feature populations within regions (north region: Kermadec Ridge, Challenger Plateau and Hikurangi Margin and south region: Bounty Trough, Campbell Plateau, Macquarie Ridge) than amongst regions for both the sponge and corals (Table 8.2). This result indicates that more connection exists within regions and less or no connections exist amongst regions; this is also in agreement with the north-central-south region structure. Amongst the geomorphic feature populations, populations from the Kermadec and Macquarie ridges were genetically isolated (Table 8.2). Furthermore, populations of $S$. variabilis from the Louisville Seamount Chain were also genetically distinct from other populations. The Kermadec and Macquarie ridge populations are located at the northern-most and southern-most extremes of the New Zealand EEZ, and the Louisville Seamount Chain occurs to the northeast of New Zealand, beyond the EEZ.

Table 8.2 Summary of significant genetic differentiation amongst different geomorphic feature populations of sponges (above diagonal) and corals (below diagonal)

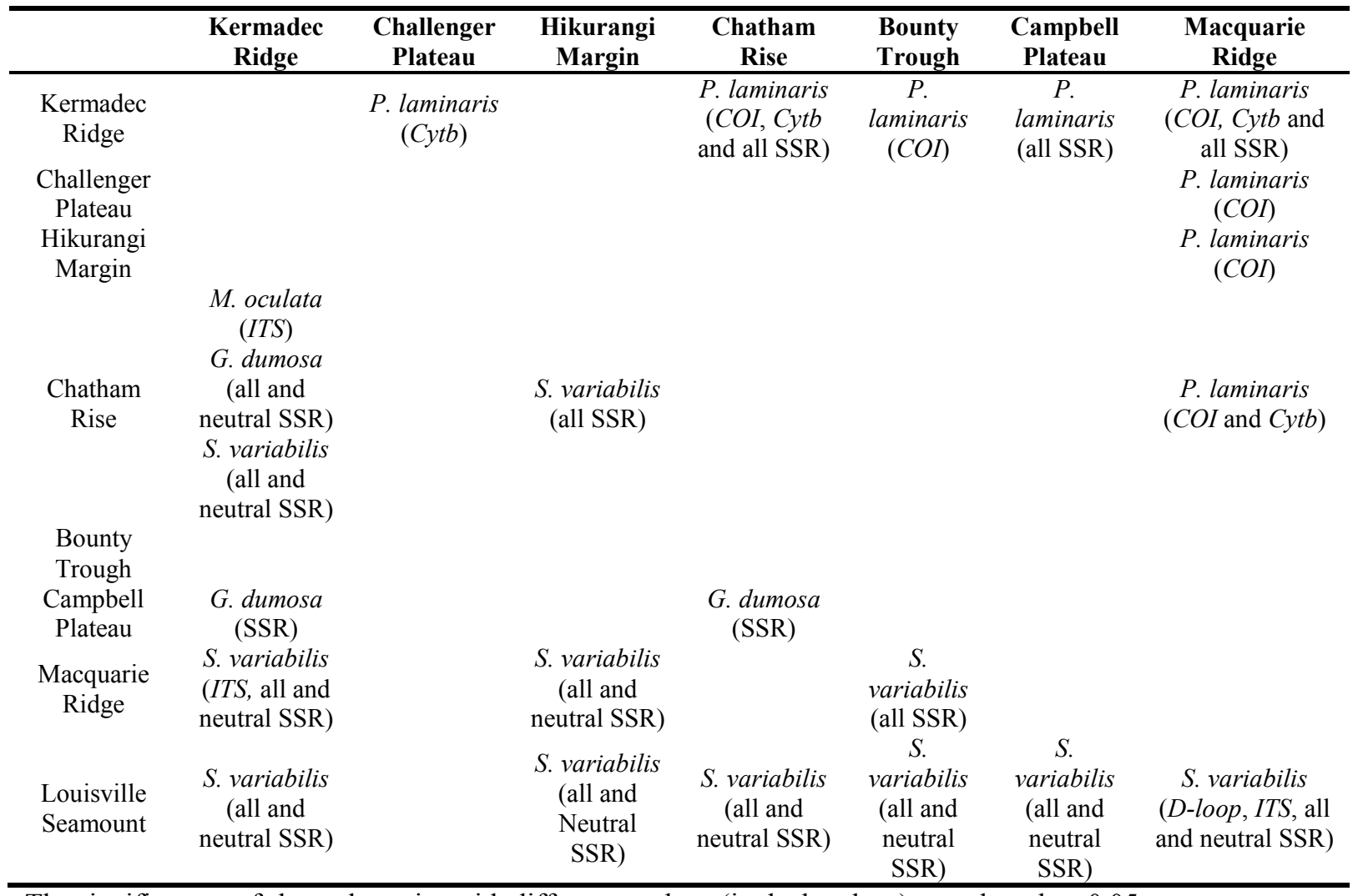

The significances of showed species with different markers (in the brackets) were less than 0.05 . 
Migration analysis of four species ( 3 corals, 1 sponge) showed that connectivity amongst geomorphic feature populations is very complicated. Chiefly, all populations had strong self-recruitment, and limited connections between populations (Chapter 5 and Chapter 6). On a broad scale, migration between the north and south regions was less than that within regions in all four species. The population from the Kermedec Ridge is acting as a source population (providing migrants to other populations but receiving few if any migrants from other populations), whilst the population from the Chatham Rise was both a source and a sink population. Populations on geomorphic features in the south region (such as Bounty Plateau, Campbell Plateau, Macquarie Ridge) were relatively well connected with each other.

\subsubsection{Factors contributing to genetic differentiation}

Understanding the deep-sea environmental and biological processes that shape spatial genetic patterns of species is critical for predicting evolutionary dynamics and defining significant evolutionary and/or management units. In this study, the potential role of environmental and biological factors in modifying the genetic structure of deep-sea habitatforming species in the New Zealand region was first evaluated based on the findings of Chapters 5 and 6 , and then the role of environmental factors was investigated using a seascape genetics approach in Chapter 7 .

\subsubsection{Depth and Distance}

Depth was reported as the most important factor as a genetic barrier for deep-sea organisms in the meta-analysis (Chapter 2), but most of the species in this study did not exhibit a pattern of genetic isolation by depth. In the 7 examined species across different markers, only microsatellites and ITS of G. dumosa and ITS of M. oculata revealed a weak pattern of isolation by depth. The distribution of collected specimens showed that G. dumosa had a relatively narrow and comparatively shallow distribution and $M$. oculata had a moderate distribution (Figure 8.1): the depth distribution did not support that genetic isolation preferentially occurred in wide depth ranges. This result could indicate that the influence of depth on genetic connectivity is different amongst species, even taxonomically close species. Furthermore, variation in depth was significantly associated with nuclear marker type (ITS and microsatellites) but not mitochondrial marker (D-loop), which indicated that different marker types exhibit different results. 


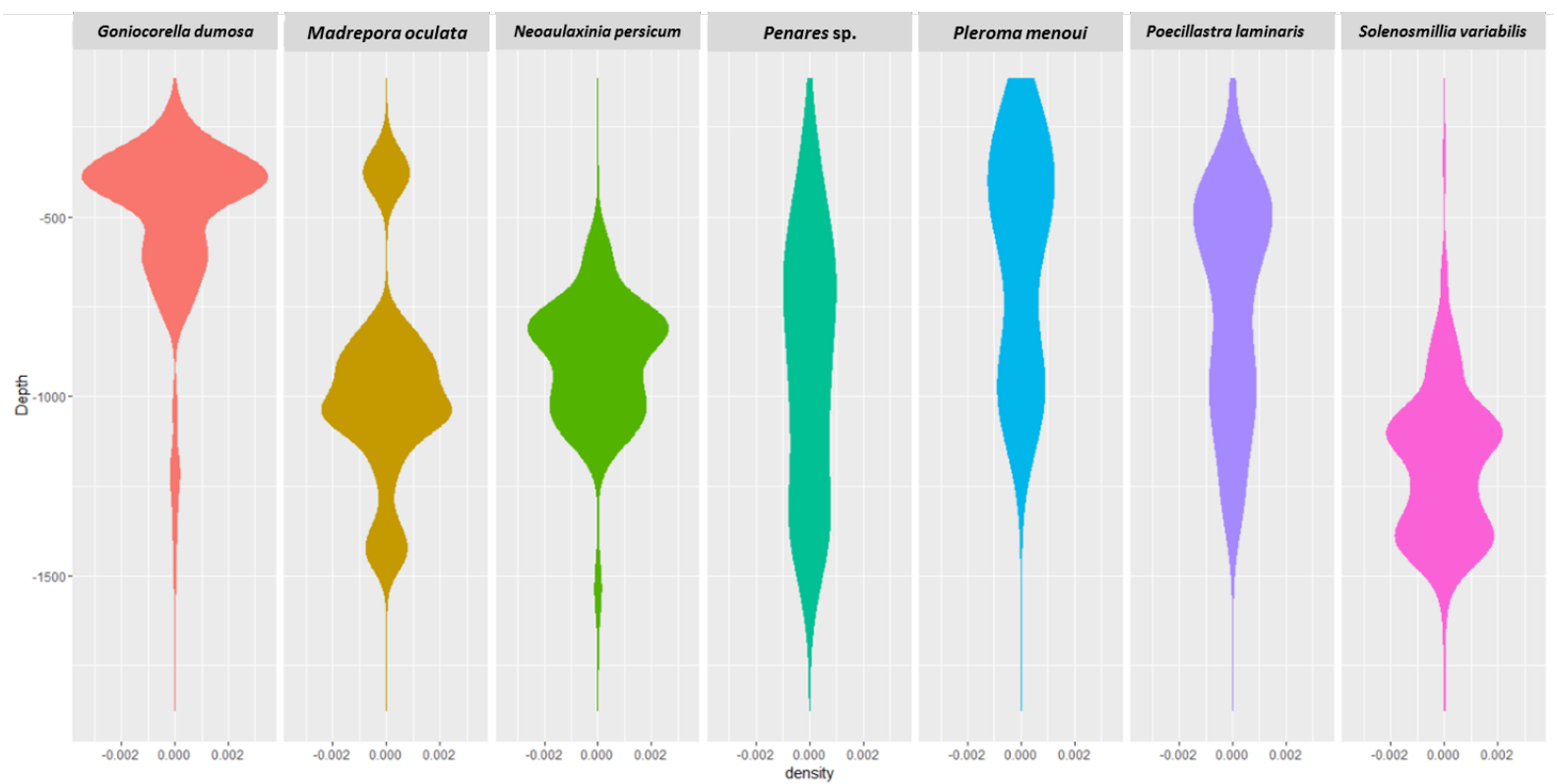

Figure 8.1 Sample collection depth distributions of all examined species

Distance is a barrier to gene flow in some deep-sea organisms (Chapter 2), however, isolation by distance (IBD) only occurred in Poecillastra laminaris (COI) and S. variabilis (microsatellites), and non-significant values were observed for the other marker types of the other species (Table 8.1). Previous study has showed that deep-sea organisms have a long planktonic larval duration (Baco et al., 2016; Hilário et al., 2015), and this might assist most deep-sea species to achieve large-scale genetic connectivity. Lack of distance as a barrier to gene flow for these species indicated that the studied species achieved colonisation and genetic connectivity in patchy habitats. However, whilst there was a possibility that the distances between two sampling sites might not be great enough to reveal the genetic isolation caused by distance, this explanation seems to be unlikely given the spatial scales involved and the fact that patterns of IBD were observed for Poecillastra laminaris and $S$. variabilis.

\subsubsection{Currents and Topograghy}

In the meta-analysis (Chapter 2) currents and topography were identified as significant barriers to genetic connectivity. In the present study, dynamic topography (a proxy for large-scale current flow) and standard deviation of slope were the explanators in the seascapes models for genetic variation in three corals at different spatial levels, providing direct evidence that currents and topography affect genetic connectivity. 
The hydrodynamic system may not only act as a 'soft' barrier for genetic connectivity, but it also can promote larval dispersal and achieving long-distance genetic connection (Rogers et al., 1998; Zane et al., 1998; Roques et al., 2002; Sutton \& Chereskin, 2002; Shaw et al., 2004; Plouviez et al., 2013). Three major currents and their associated fronts around New Zealand, the Tasman Front, the Subtropical Front and the Subantarctic Front, are expected to have the greatest potential influence on population connectivity of deep-sea populations in the region. These currents and frontal systems differentiate the water mass environments in the north and the south of the region, and also influence the direction of north and south currents across the Chatham Rise that may reduce or perhaps even block gene flow across this feature. Populations of three coral species and the sponge, Poecillastra laminaris, from the Kermadec Ridge were shown to be genetically differentiated from other populations in the New Zealand region, and the East Auckland Current and its associated eddies may be responsible for this relative isolation (Chiswell \& Roemmich, 1998). Significant genetic differentiations were detected between the populations from Louisville Seamount Chain and other populations within New Zealand EEZ (Chapter 6), and the general lack of current flow from the Louisville Seamount Chain towards New Zealand (Chiswell \& Rickard, 2006) may act to separate populations on this feature from those in the rest of the region. The D'Urville Current (Chiswell, 2000) might assist M. oculata larvae to connect between geomorphic features to the west, and explain the lack of genetic differentiation between Challenger Plateau and Chatham Rise observed for G. dumosa and M. oculata. Furthermore, genetic differentiation between biogeographic provinces was found for $S$. variabilis, and this was possibly caused by the different currents and frontal systems in the north (Tropical Front) and south (Subtropical Front and Subantarctic Front) of the region (Chiswell et al., 2015), which may impede larval dispersal and reduce or perhaps even block gene flow across the Chatham Rise.

On the role of currents in promoting gene flow, the haplotypic distribution of all species and panmixia patterns at different scales in different species could be affected by ocean current dynamics, and the results of this study indicate that two main population connection routes may exist. First, a northern route from the northwest of the North Island with the southerly flow of the East Auckland Current down the east coast of North Island with the East Cape Current, and then eastwards immediately north of the Chatham Rise and the Subtropical Front. Second, a southern route from south of the South Island northwards with the Southland Current, then eastwards immediately south of the Chatham Rise and the 
Subtropical Front. However, hydrodynamic modelling that includes simulated larval dispersal is required to further substantiate this hypothesis.

The observed isolation of most populations on the Kermadec Ridge, Macquarie Ridge and Louisville Seamount Chain could possibly be explained by their relative spatial isolation. However, only the genetic structure of two study species (P. laminaris and S. variabilis) showed evidence for isolation by distance. Another explanation for the isolation of populations on the Louisville Seamount Chain, and Kermadec and Macquarie ridges relates to the populations being sampled from seamounts on these features. Although there was no direct evidence to show that there is a relationship between seamount and the presence of genetic barriers, topographic controlled current flows and other hydrodynamic features associated with seamounts are thought likely to influence the genetic isolation of populations (Shank, 2010). However, evidence for such patterns is equivocal (Chapter 2), and the influence of the interaction of topography and currents at the seamount-scale on genetic structure requires further investigation.

\subsubsection{3. $\quad$ Life features}

Reproduction and larval dispersal strategies are considered important factors in shaping genetic connectivity patterns in many deep-sea invertebrates (for examples see Hilário et al. (2015)), and species-specific differences in reproductive strategies may contribute to differences in patterns of gene flow and genetic structure observed across taxa (e.g. Bors et al., 2012; Samadi et al., 2006). Deep-sea demosponges and corals have a widerange of reproductive strategies (Witte, 1996; Waller 2005; Lemoine et al., 2007; Pires et al., 2014), but very few studies have determined empirically the reproductive strategies or mode of larval dispersal for the four species examined in the present study. Studies of these species or closely related species have reported different reproduction and dispersal strategies, to the extent that these markedly different strategies corresponded directly with the different genetic connectivity patterns amongst different species (Waller, 2005; Lemoine et al., 2007; Miller, 2011; Pires et al., 2014).

Detailed information on the reproductive biology of the four species would be highly beneficial for assessing the importance of reproductive and dispersal strategies on population structure and genetic connectivity. Unfortunately, there is no reproduction information available for sponges in the present study. The three corals are thought to exhibit a similar 
reproductive strategy - continuous reproduction, lecithotrophic larvae and feeding after settlement and metamorphosis (Waller, 2005; Pires et al., 2014).

One of the unexpected results of the seascape analysis was the finding that food availability proxies (nitrate and surface water primary productivity) were related to genetic structure at regional and individual level. Food availability may influence population size through colonisation success, which in turn may influence the dispersal potential of a population. However, this study was the first to examine the relationships between genetic variation and environmental variation in the deep sea, and so there is limited context in which to place the finding.

\subsection{Management implications and future directions}

Findings of this study contribute to VME management, with significant new knowledge about deep-sea connectivity in New Zealand and important findings regarding the processes that can shape the population genetic structure of VME indicator taxa. New genetic markers were developed for sponges and corals, and these powerful tools were used to address ecological questions in the deep sea. The multidisciplinary approach was used to investigate the effects of environmental factors on population genetic connectivity. In general, these findings can be applied to the management of VMEs around New Zealand, and provide a background to other benthic species with similar life history characteristics and inhabiting similar habitats.

Examination of genetic population structure for sponge and coral species illustrates that the connectivity within regions is species-specific. Although connectivity patterns vary, this research provides strong evidence across multiple species that the Kermadec Ridge and Macquarie Ridge regions are genetically differentiated from other parts of the New Zealand EEZ. Moreover, the genetic differentiation between the Louisville Seamount Chain and other populations within the EEZ indicates that genetic disconnections exist between inside and outside of the New Zealand EEZ. These results indicate that areas on the Kermadec and Macquarie ridges and the Louisville Seamount Chain need to be represented in a New Zealand region-scale network of marine protected areas. The current Kermadec Benthic Protection Area (BPA) and the proposed establishment of the Kermadec Ocean Sanctuary (http://www.mfe.govt.nz/marine/kermadec-ocean-sanctuary) will contribute towards ensuring the protection of VME indicator taxa in that region. However, there are presently no large protection areas on the Macquarie Ridge or the Louisville Seamount Chain, which could 
bring a risk that the populations from these two features might not recovery after major anthropogenic disturbance events. While bottom trawling on seamount features on the Macquarie Ridge is relatively limited, such disturbance is more evident on some seamounts on the Louisville Seamount Chain where only limited protection measures are in place (Penney \& Guinotte 2013).

The Chatham Rise (central region) was identified as containing sink populations for two VME indicator taxa (P. laminaris and $S$. variabilis) and an area of high genetic diversity for 5 taxa (P. laminaris, N. persicum, G. dumosa, M. oculata and S. variabilis). Currently, there are two BPAs on the Chatham Rise, and they cover very limited areas along the Rise. Because of the relative importance of the Chatham Rise for genetic variation in the region, further protected areas, or an enlarging of the current BPAs (e.g. to extend across the rise), should be considered to afford greater protection to VMEs.

Amongst the examined species, the connectivity patterns, as well as the factors that influenced the patterns, varied from species and between spatial scales. Previous studies in the New Zealand region have also revealed different connectivity patterns in other species (Smith et al., 2004b; Miller et al., 2010; Bors et al., 2012; Knox et al., 2012; Foltz et al., 2013; Boschen et al., 2015; Dueñas et al., 2016). Not only is more knowledge about genetic connectivity patterns required for the management of VME indicator taxa but the current findings and previous findings argue that a management system will need to be flexible to change with the advent of new information.

\subsection{Future research directions}

In the present study, a hierarchical province, region and geomorphic feature framework was employed to test spatial genetic variation of VME indicator taxa in the New Zealand region. For future research, it will be useful to use additional frameworks. For example, the Marine Environment Classification (MEC) of the New Zealand region (Snelder et al., 2007). This classification could provide a more comprehensive spatial framework for understanding spatial genetic variation because it synthesises a greater deal of environemtal variation than the framework employed in this study, and the hierarchical level that can be applied is flexible (from 1- 290 classes). Unfortunately, samples included in this study were sufficiently unbalanced to allow for a suitable coverage of classes, and thus the MEC framework was not adopted. In the future, directed sampling might allow for the use of the 
MEC framework to examine genetic spatial structure across the New Zealand in a way that is more directly applicable to management.

As a new genetic marker type, SNPs have been developed for S. variabilis, although this is an unfinished part of this project. Characterisation of these markers is essential before they are usable. Primary characterisation found that polyadenylation was linked to many of the SNPs and this caused primer design issues. In Chapter 4, however, no clear genetic splits were observed between populations of $S$. variabilis if all SNPs were included in the analysis. Unfortunately, it is not financially feasible to genotype all SNPs across all individuals, and large SNP data sets bring other issues, such as computationally intensive analyses (for details see Helyar et al., 2011). Despite obstacles to the use of this marker type, the low-scoring error rates, relative ease of calibration amongst laboratories compared to length-based markers and the associated ability to assemble combined temporal and spatial data sets from multiple laboratories make SNPs very attractive in population genetic studies (Brumfield et al., 2003; Morin et al., 2004). This is especially the case in terms of the advantages of using SNPs for poor quality DNA samples, which is common for deep-sea samples (Morin \& McCarthy, 2007; Smith et al., 2011). Thus, future genetic research in the deep sea around New Zealand should include this marker type and further development work is planned for the SNPs developed here.

Increasing attempts are made to understand the factors responsible for genetic connectivity in deep-sea populations (Hilário et al., 2015; Baco et al., 2016). In the present study, the use of seascape genetics aims to determine a spatially explicit correlation between genetic population parameters and environmental factors, and it provides a glimpse of the relations between genetic variation and environmental variation. This study identified that broad-scale current patterns were an important factor that contributes to genetic connectivity in the region. Integration of genetic information with hydrodynamic dispersal modelling (e.g. Viard et al., 2006; Berry et al., 2012) will yield a more comprehensive understanding of which currents, and how currents, influence genetic connectivity around New Zealand. Such a study should be considered as the next-step in the investigation of the genetic patterns determined by this study.

Building a genetic connectivity model to predict genetic variation in unsampled areas is advantageous from a management aspect (Epps et al., 2007). However, genetic connectivity is a descriptive variable, and how to best introduce genetic parameters into predictive models that represent genetic connectivity has not been widely addressed. Dolgener et al. (2014) employed an environmental niche factor analysis (ENFA) to quantify 
genetic diversity and its relationship with environmental factors, and found that the ENFAbased landscape genetics approach allows researchers to put various aspects of long-versus short-term effective population size and genetic connectivity into an ecological and spatially explicit context, enabling forecast assessments to be made under future environmental scenarios. However, they did not use this approach to make predictions into environmental space. Models that couple genetic and environmental information, and predict into environmental space, may not only guide the design of contemporary spatial protection measures, but may also predict long term changes, which is very informative for deep-sea management and conversation. 


\section{References}

Abbiati M, Santangelo G, Novelli S (1993) Genetic variation within and between two Tyrrhenian populations of the Mediterranean alcyonarian Corallium rubrum. Marine Ecology Progress Series, 95, 245-250.

Abelson A, Miloh T, Loya Y (1993) Flow patterns induced by substrata and body morphologies of benthic organisms, and their roles in determining availability of food particles. Limnology and Oceanography, 38, 1116-1124.

Addamo AM, García-Jiménez R, Taviani M et al. (2015) Development of microsatellite markers in the deep-sea cup coral Desmophyllum dianthus by 454 sequencing and crossspecies amplifications in scleractinia order. Journal of Heredity, 106, 322-330.

Addamo AM, Reimer JD, Taviani M et al. (2012) Desmophyllum dianthus (Esper, 1794) in the Scleractinian phylogeny and its intraspecific diversity. PLOS ONE, 7, e50215.

Adkins JF, Boyle EA, Curry WB et al. (2003) Stable isotopes in deep-sea corals and a new mechanism for "vital effects". Geochimica et Cosmochimica Acta, 67, 1129-1143.

Agardy T (2000) Information needs for marine protected areas: Scientific and societal. Bulletin of Marine Science, 66, 875-888.

Almany GR, Connolly SR, Heath DD et al. (2009) Connectivity, biodiversity conservation and the design of marine reserve networks for coral reefs. Coral Reefs, 28, 339-351.

Althaus F, Williams A, Schlacher TA et al. (2009) Impacts of bottom trawling on deep-coral ecosystems of seamounts are long-lasting. Marine Ecology Progress Series, 397, 279294.

Anadón JD, del Mar Mancha-Cisneros M, Best BD et al. (2013) Habitat-specific larval dispersal and marine connectivity: implications for spatial conservation planning. Ecosphere, 4, 1-15.

Anderson OF, Clark MR (2003) Analysis of bycatch in the fishery for orange roughy, Hoplostethus atlanticus, on the South Tasman Rise. Marine and Freshwater Research, 54, 643-652.

Anderson CD, Epperson BK, Fortin MJ et al. (2010) Considering spatial and temporal scale in landscape-genetic studies of gene flow. Molecular Ecology, 19, 3565-3575.

Anderson MJ, Gorley RN, Clarke KR (2008) PERMANOVA+ for PRIMER: Guide to software and statistical methods. 2008, 214.

Antao T, Lopes A, Lopes RJ et al.(2008) LOSITAN: A workbench to detect molecular 
adaptation based on a $F_{S T}$-outlier method. BMC Bioinformatics, 9, 1-5.

Arango CP, Soler-Membrives A, Miller KJ (2011) Genetic differentiation in the circumAntarctic sea spider Nymphon australe (Pycnogonida; Nymphonidae). Deep-Sea Research Part II: Topical Studies in Oceanography, 58, 212-219.

Ardron J, Druel E, Gjerde K et al. (2013) Advancing governance of the high seas. IIDRI Policy Brief, 6, 1-8.

Arellano SM, van Gaest AL, Johnson SB et al.(2014) Larvae from deep-sea methane seeps disperse in surface waters. Proceedings of The Royal Society B, 281, 20133276.

Arnqvist G, Dowling DK, Eady P et al. (2010) Genetic architecture of metabolic rate: Environment specific epistasis between mitochondrial and nuclear genes in an insect. Evolution, 64, 3354-3363.

Auster PJ, Gjerde K, Heupel E et al. (2011) Definition and detection of vulnerable marine ecosystems on the high seas: Problems with the "move-on" rule. ICES Journal of Marine Science, 68, 254-264.

Ayala FJ, Valentine JW (1974) Genetic variability in the cosmopolitan deep-water ophiuran Ophiomusium lymani. Marine Biology, 27, 51-57.

Ayala FJ, Valentine JW, Hedgecock D et al. (1975) Deep-sea asteroids: high genetic variability in a Stable Environment. Evolution, 29, 203-212.

Baco AR, Cairns SD (2012) Comparing molecular variation to morphological species designations in the deep-sea coral Narella reveals new insights into seamount coral ranges. PLoS ONE, 7, e45555.

Baco AR, Etter RJ, Ribeiro PA et al. (2016) A synthesis of genetic connectivity in deep-sea fauna and implications for marine reserve design. Molecular Ecology, 25, 3276-3298.

Baird HP, Miller KJ, Stark JS (2011) Evidence of hidden biodiversity, ongoing speciation and diverse patterns of genetic structure in giant Antarctic amphipods. Molecular Ecology, 20, 3439-3454.

Ball AO, Sedberry GR, Zatcoff MS et al.(2000) Population structure of the wreckfish Polyprion americanus determined with microsatellite genetic markers. Marine Biology, 137, 1077-1090.

Banks SC, Piggott MP, Williamson JE et al. (2007) Oceanic variability and coastal topography shape genetic structure in a long-dispersing sea urchin. Ecology, 88, 30553064.

Becerro MA, Uriz MJ, Maldonado M et al.(2012) Advances in sponge science: phylogeny, systematics, ecology. Preface. Advances in marine biology, 61, ix-x. 
Beedessee G, Watanabe H, Ogura T et al. (2013) High connectivity of animal populations in deep-sea hydrothermal vent fields in the central indian ridge relevant to its geological setting. PLoS ONE, 8, e81570.

Beerli P (2006) Comparison of Bayesian and maximum-likelihood inference of population genetic parameters. Bioinformatics, 22, 341-345.

Beerli P, Felsenstein J (1999) Maximum-likelihood estimation of migration rates and effective population numbers in two populations using a coalescent approach. Genetics, 152, 763-773.

Bell JJ, Smith D, Hannan D et al. (2014) Resilience to disturbance despite limited dispersal and self-recruitment in tropical barrel sponges: implications for conservation and management. PLoS ONE, 9, e91635.

Bentlage B, Wörheide G (2007) Low genetic structuring among Pericharax heteroraphis (Porifera: Calcarea) populations from the Great Barrier Reef (Australia), revealed by analysis of nrDNA and nuclear intron sequences. Coral Reefs, 26, 807-816.

Benzie JAH, Sandusky C, Wilkinson CR (1994) Genetic structure of dictyoceratid sponge populations on the western Coral Sea reefs. Marine Biology, 119, 335-345.

Berry O, England P, Marriott RJ, et al. (2012) Understanding age-specific dispersal in fishes through hydrodynamic modelling, genetic simulations and microsatellite DNA analysis. Molecular Ecology, 21, 2145-2159.

Bertness MD, Gaines SD (1993) Larval dispersal and local adaptation in acorn barnacles. Evolution, 47, 316-320.

Bisol P, Costa R, Sibuet M (1984) Ecological and genetical survey on two deep-sea holothurians: Benthogone rosea and Benthodytes typica. Marine Ecology Progress Series, 15, 275-281.

Black MB, Lutz RA, Vrijenhoek RC (1994) Gene flow among vestimentiferan tube worm (Riftia pachyptila) populations from hydrothermal vents of the eastern Pacific. Marine Biology, 120, 33-39.

Black MB, Trivedi A, Maas PAY et al. (1998) Population genetics and biogeography of vestimentiferan tube worms. Deep-Sea Research Part II: Topical Studies in Oceanography, 45, 365-382.

Blanquer A, Uriz MJ, Caujapé-Castells J (2009) Small-scale spatial genetic structure in Scopalina lophyropoda, an encrusting sponge with philopatric larval dispersal and frequent fission and fusion events. Marine Ecology Progress Series, 380, 95-102.

Blanquer A Uriz MJ (2010) Population genetics at three spatial scales of a rare sponge living 
in fragmented habitats. BMC Evolutionary Biology, 10, 1.

Bolch CJS, Elliott NG, Ward RD (1993) Enzyme variation in south-eastern Australian samples of the blue-eye or deepsea trevalla, Hyperoglyphe antarctica Carmichael 1818 (Teleostei: Stromateoidei). Australian Journal of Marine and Feshwater Research, 44, 687-697.

Bongiorni L, Mea M, Gambi C et al. (2010) Deep-water scleractinian corals promote higher biodiversity in deep-sea meiofaunal assemblages along continental margins. Biological Conservation, 143, 1687-1700.

Boom R, Sol CJA, Salimans MMM et al. (1990) Rapid and simple method for purification of nucleic acids. Journal of Clinical Microbiology, 28, 495-503.

Bors EK, Rowden AA, Maas EW et al. (2012) Patterns of deep-sea genetic connectivity in the new zealand region: implications for management of benthic ecosystems. PLoS ONE, 7, e49474.

Boschen RE, Collins PC, Tunnicliffe V et al. (2016) A primer for use of genetic tools in selecting and testing the suitability of set-aside sites protected from deep-sea seafloor massive sulfide mining activities. Ocean and Coastal Management, 122, 37-48.

Boschen RE, Rowden AA, Clark MR et al. (2013) Mining of deep-sea seafloor massive sulfides: A review of the deposits, their benthic communities, impacts from mining, regulatory frameworks and management strategies. Ocean and Coastal Management, 84, 54-67.

Boschen RE, Rowden AA, Clark MR et al. (2015) Limitations in the use of archived vent mussel samples to assess genetic connectivity among seafloor massive sulfide deposits: A case study with implications for environmental management. Frontiers in Marine Science, 2, 1-14.

Brandão SN, Sauer J, Schön I (2010) Circumantarctic distribution in Southern Ocean benthos? A genetic test using the genus Macroscapha (Crustacea, Ostracoda) as a model. Molecular Phylogenetics And Evolution, 55, 1055-1069.

Brandt A, Gooday AJ, Brandão SN et al. (2007) First insights into the biodiversity and biogeography of the Southern Ocean deep sea. Nature, 447, 307-311.

Briggs JC, Bowen BW (2012) A realignment of marine biogeographic provinces with particular reference to fish distributions. Journal of Biogeography, 39, 12-30.

Brodie S, Clark M (2002) The New Zealand Seamount Management Strategy - steps towards conserving offshore marine habitat. In: Beumer, J.P.; Grant, A.; Smith, D.C. (Eds). Aquatic Protected Areas: what works best and how do we know? Proceedings of the 
World Congress on Aquatic Protected Areas, 664-673.

Brooke SD, Holmes MW, Young CM (2009) Sediment tolerance of two different morphotypes of the deep-sea coral Lophelia pertusa from the Gulf of Mexico. Marine Ecology Progress Series, 390, 137-144.

Brooke S, Ross SW, Bane JM et al. (2013) Temperature tolerance of the deep-sea coral Lophelia pertusa from the southeastern United States. Deep-Sea Research Part II: Topical Studies in Oceanography, 92, 240-248.

Brown SDJ, Collins RA, Boyer S et al. (2012) Spider: An R package for the analysis of species identity and evolution, with particular reference to DNA barcoding. Molecular Ecology Resources, 12, 562-565.

Brumfield RT, Beerli P, Nickerson DA et al. (2003) Single nucleo-tide polymorphisms (SNPs) as markers in phylogeography. Trends in Ecology and Evolution, 18, 249-256.

Lo Brutto S, Maggio T, Arculeo M (2013) Isolation by distance (IBD) signals in the deepwater rose shrimp Parapenaeus longirostris (Lucas, 1846) (Decapoda, Panaeidae) in the Mediterranean Sea. Marine Environmental Research, 90, 1-8.

Bryan TL, Metaxas A (2006) Distribution of deep-water corals along the North American continental margins: Relationships with environmental factors. Deep-Sea Research Part I: Oceanographic Research Papers, 53, 1865-1879.

Bryan TL, Metaxas A (2007) Predicting suitable habitat for deep-water gorgonian corals on the Atlantic and Pacific Continental Margins of North America. Marine Ecology Progress Series, 330, 113-126.

Bucklin A, Wilson RR, Smith KL (1987) Genetic differentiation of seamount and basin populations of the deep-sea amphipod Eurythenes gryllus. Deep Sea Research Part A. Oceanographic Research Papers, 34, 1795-1810.

Bucklin A (1988) Allozymic variability of Riftia pachyptila populations from the Galapogos Rift and 21 N hydrothermal vents. Deep-Sea Research, 35, 1759-1768.

Burgess S, Babcock R (2005) Reproductive ecology of three reef-forming, deep-sea corals in the New Zealand region. Cold-Water Corals and Ecosystems, 701-713.

Buss LW, Jackson JB (1981) Planktonic food availability and suspension-feeder abundance: evidence of in situ depletion. Journal of Experimental Marine Biology and Ecology, 49, 151-161.

Buston PM, D'Aloia CC (2013) Marine ecology: reaping the benefits of local dispersal. Current Biology, 23, 351-353.

Calderón I, Ortega N, Duran S, et al. (2007) Finding the relevant scale: clonality and genetic 
structure in a marine invertebrate (Crambe crambe, Porifera). Molecular ecology, 16, 1799-1810.

Casado-Amezúa P, García-Jiménez R, Kersting DK et al. (2011). Development of microsatellite markers as a molecular tool for conservation studies of the Mediterranean reef builder coral Cladocora caespitosa (Anthozoa, Scleractinia). Journal of Heredity, 102, 622-626.

Casado-Amezú P, Goffredo S, Templado J et al. (2012) Genetic assessment of population structure and connectivity in the threatened Mediterranean coral Astroides calycularis (Scleractinia, Dendrophylliidae) at different spatial scales. Molecular Ecology, 21, 36713685 .

Case DH, Robinson LF, Auro ME et al. (2010) Environmental and biological controls on Mg and Li in deep-sea scleractinian corals. Earth and Planetary Science Letters, 300, 215225.

Catarino D, Knutsen H, Veríssimo A et al. (2015) The Pillars of Hercules as a bathymetric barrier to gene flow promoting isolation in a global deep-sea shark (Centroscymmus coelolepis). Molecular Ecology, 24, 6061-6079.

Chakraborty R, De Andrade M, Daiger SP et al. (1992) Apparent heterozygote deficiencies observed in DNA typing data and their implications in forensic applications. Annals of Human Genetics, 56, 45-57.

Chape S, Blyth S, Fish L et al. (2003) 2003 United Nations list of protected areas. UNEPWCMC: Cambridge, UK, 44.

Chase MR, Etter RJ, Rex MA et al. (1998) Bathymetric patterns of genetic variation in a deep-sea protobranch bivalve, Deminucula atacellana. Marine Biology, 131, 301-308.

Chen CA, Yu JK (2000) Universal primers for amplification of mitochondrial small subunit ribosomal RNA-encoding gene in scleractinian corals. Marine Biotechnology, 2, 146153.

Chevaldonné P, Joilivet D, Vangriesheim A et al. (1997) Hydrothermal-vent alvinellid polychaete dispersal in the eastern Pacific. 1. Influence of vent site distribution, bottom currents, and biological patterns. Limnology and Oceanography, 42, 67-80.

Chiappone M, Dienes H, Swanson DW et al. (2005) Impacts of lost fishing gear on coral reef sessile invertebrates in the Florida Keys National Marine Sanctuary. Biological Conservation, 121, 221-230.

Chiswell SM (2000) The Wairarapa coastal current. New Zealand Journal of Marine and Freshwater Research, 34, 303-315. 
Chiswell SM, Bostock HC, Sutton PJ et al. (2015) Physical oceanography of the deep seas around New Zealand: a review. New Zealand Journal of Marine and Freshwater Research, 49, 1-32.

Chiswell SM, Rickard GJ (2006) Comparison of model and observational ocean circulation climatologies for the New Zealand region. Journal of Geophysical Research: Oceans, 111, C10.

Chiswell SM, Roemmich D (1998) The East Cape Current and two eddies: A mechanism for larval retention? New Zealand Journal of Marine and Freshwater Research, 32, 385-397.

Cho W, Shank TM (2010) Incongruent patterns of genetic connectivity among four ophiuroid species with differing coral host specificity on North Atlantic seamounts. Marine Ecology, 31, 121-143.

Clark M, Anderson O, Bowden D et al. (2014) Voyage report of a survey of deep-sea habitats of the Louisville Seamount Chain (TAN1402). NIWA Preliminary Project report VMES133

Clark MR, Dunn MR (2012) Spatial management of deep-sea seamount fisheries: balancing sustainable exploitation and habitat conservation. Environmental Conservation, 39, 204214.

Clark M, O’Driscoll R (2003) Deepwater fisheries and aspects of their impact on seamount habitat in New Zealand. Journal of Northwest Atlantic Fishery Science, 31, 441-458.

Clark MR, Rowden AA (2009) Effect of deepwater trawling on the macro-invertebrate assemblages of seamounts on the Chatham Rise, New Zealand. Deep-Sea Research Part I: Oceanographic Research Papers, 56, 1540-1554.

Clark MR, Rowden AA, Schlacher T et al. (2010) The ecology of seamounts: structure, function, and human impacts. Annual Review of Marine Science, 2, 253-278.

Clark MR, Tittensor DP (2010) An index to assess the risk to stony corals from bottom trawling on seamounts. Marine Ecology, 31, 200-211.

Clarke KR, Gorley RN (2006) PRIMER v6: User manual/tutorial. PRIMER-E, Plymouth.

CCAMLR (2009) CCAMLR VME Taxa Classification Guide. Hobart, Australia.

Costantini F, Fauvelot C, Abbiati M (2007) Fine-scale genetic structuring in Corallium rubrum: Evidence of inbreeding and limited effective larval dispersal. Marine Ecology Progress Series, 340, 109-119.

Costantini F, Taviani M, Remia A et al. (2010) Deep-water Corallium rubrum (L., 1758) from the Mediterranean Sea: Preliminary genetic characterisation. Marine Ecology, 31, 261-269. 
Cowart DA, Huang C, Arnaud-Haond S et al. (2013) Restriction to large-scale gene flow vs. regional panmixia among cold seep Escarpia spp. (Polychaeta, Siboglinidae). Molecular Ecology, 22, 4147-4162.

Cowen RK, Gawarkiewicz G, Pineda J et al. (2002) Population connectivity in marine systems. Report of a workshop to develop science recommendations for the National Science Foundation.

Cowen RK, Sponaugle S (2009) Larval dispersal and marine population connectivity. Marine Science, 1, 443-466.

Coykendall DK, Johnson SB, Karl S et al. (2011) Genetic diversity and demographic instability in Riftia pachyptila tubeworms from eastern Pacific hydrothermal vents. BMC Evolutionary Biology, 11, 96.

Craddock C, Hoeh WR, Lutz RA et al. (1995) Extensive gene flow among mytilid (Bathymodiolus thermophilus) populations from hydrothermal vents of the eastern Pacific. Marine Biology, 124, 137-146.

Craddock C, Lutz RA, Vrijenhoek RC (1997) Patterns of dispersal and larval development of archaeogastropod limpets at hydrothermal vents in the eastern Pacific. Journal of Experimental Marine Biology and Ecology, 210, 37-51.

Creasey S, Rogers D, Tyler P (1996) Genetic comparison of two populations of the deep-sea vent shrimp Rimicaris exoculata (Decapoda: Bresiliidae) from the Mid-Atlantic Ridge. Marine Biology, 125, 473-482.

Cullen DJ (1970) A tectonic analysis of the south-west Pacific. New Zealand Journal of Geology and Geophysics, 13, 7-20.

Dailianis T, Tsigenopoulos CS, Dounas C et al. (2011) Genetic diversity of the imperilled bath sponge Spongia officinalis Linnaeus, 1759 across the Mediterranean Sea: patterns of population differentiation and implications for taxonomy and conservation. Molecular Ecology, 20, 3757-3772.

Davies AJ, Guinotte JM (2011) Global habitat suitability for framework-forming cold-water corals. PLOS ONE, 6, e18483.

Davies AJ, Roberts JM, Hall-Spencer J (2007) Preserving deep-sea natural heritage: Emerging issues in offshore conservation and management. Biological Conservation, 138, 299-312.

Davies AJ, Wisshak M, Orr JC et al. (2008) Predicting suitable habitat for the cold-water coral Lophelia pertusa (Scleractinia). Deep-Sea Research Part I: Oceanographic Research Papers, 55, 1048-1062. 
Davies SW, Rahman M, Meyer E et al. (2013) Novel polymorphic microsatellite markers for population genetics of the endangered Caribbean star coral, Montastraea faveolata. Marine Biodiversity, 43, 167-172.

Dawson E (1984) The benthic fauna of the Chatham Rise: An assessment relative to possible effects of phosphorite mining. Geologisches Jahrbuch, D65, 209-231.

DeBiasse MB, Richards VP, Shivji MS (2010) Genetic assessment of connectivity in the common reef sponge, Callyspongia vaginalis (Demospongiae: Haplosclerida) reveals high population structure along the Florida reef tract. Coral Reefs, 29, 47-55.

Dellicour S, Mardulyn P (2014) spads 1.0: A toolbox to perform spatial analyses on DNA sequence data sets. Molecular Ecology Resources, 14, 647-651.

Dey KL, Weatherhead MA (2004) User Manual for the New Zealand Marine Environment Classification. Unpublished report to the Ministry for the Environment by the National Institute of Water \& Atmospheric Research Ltd.

Dodds LA, Black KD, Orr H et al. (2009) Lipid biomarkers reveal geographical differences in food supply to the cold-water coral Lophelia pertusa (Scleractinia). Marine Ecology Progress Series, 397, 113-124.

Dolan MFJ, Grehan AJ, Guinan JC et al. (2008) Modelling the local distribution of coldwater corals in relation to bathymetric variables: Adding spatial context to deep-sea video data. Deep-Sea Research Part I: Oceanographic Research Papers, 55, 1564-1579.

Dolgener N, Freudenberger L, Schluck M et al. (2014). Environmental niche factor analysis (ENFA) relates environmental parameters to abundance and genetic diversity in an endangered amphibian, the fire-bellied toad (Bombina bombina). Conservation Genetics, 15, 11-21.

van Dover CL (2014) Impacts of anthropogenic disturbances at deep-sea hydrothermal vent ecosystems: A review. Marine Environmental Research. 102, 59-72.

van Dover CL, German CR, Speer KG et al. (2002) Evolution and biogeography of deep-sea vent and seep invertebrates. Science, 295, 1253-1257.

Drummond AJ, Ashton B, Buxton S et al. (2013) Geneious v7.0.5. Available at: http://www.geneious.com/.

Dueñas LF, Sánchez JA (2009) Character lability in deep-sea bamboo corals (Octocorallia, Isididae, Keratoisidinae). Marine Ecology Progress Series, 397, 11-23.

Dueñas LF, Tracey DM, Crawford AJ et al. (2016) The Antarctic Circumpolar Current as a diversification trigger for deep-sea octocorals. BMC Evolutionary Biology, 16, 2.

Dullo WC, Flögel S, Rüggeberg A (2008) Cold-water coral growth in relation to the 
hydrography of the Celtic and Nordic European continental margin. Marine Ecology Progress Series, 371, 165-176.

Duran S, Giribet G, Turon X (2004a) Phylogeographical history of the sponge Crambe crambe (Porifera, Poecilosclerida): range expansion and recent invasion of the Macaronesian islands from the Mediterranean Sea. Molecular Ecology, 13, 109-122.

Duran S, Pascual M, Turon X (2004b) Low levels of genetic variation in mtDNA sequences over the western Mediterranean and Atlantic range of the sponge Crambe crambe (Poecilosclerida). Marine Biology, 144, 31-35.

Duran S, PascualM, Estoup A et al. (2004c) Strong population structure in the marine sponge Crambe crambe (Poecilosclerida) as revealed by microsatellite markers. Molecular Ecology, 13, 511-522.

Earl DA, vonHoldt BM (2012) STRUCTURE HARVESTER: A website and program for visualizing STRUCTURE output and implementing the Evanno method. Conservation Genetics Resources, 4, 359-361.

Elbrecht V, Poettker L, John U et al. (2013) The complete mitochondrial genome of the stonefly Dinocras cephalotes. Mitochondrial DNA, 1736, 1-2.

Emerson KJ, Merz CR, Catchen JM et al. (2010) Resolving postglacial phylogeography using high-throughput sequencing. Proceedings of the National Academy of Sciences of the United States of America, 107, 16196-16200.

Epps CW, Wehausen JD, Bleich VC, et al. (2007) Optimizing dispersal and corridor models using landscape genetics. Journal of applied ecology, 44, 714-724.

Excoffier L, Lischer HEL (2010) Arlequin suite ver 3.5: A new series of programs to perform population genetics analyses under Linux and Windows. Molecular Ecology Resources, 10, 564-567.

Fallon SJ, Thresher RE, Adkins J (2014) Age and growth of the cold-water scleractinian Solenosmilia variabilis and its reef on SW Pacific seamounts. Coral Reefs, 33, 31-38.

Falush D, Stephens M, Pritchard JK (2007) Inference of population structure using multilocus genotype data: Dominant markers and null alleles. Molecular Ecology Notes, 7, 574-578.

FAO (2009) International guidelines for the management of deep-sea fisheries in the high seas. Rome, Italy.

Faure B, Jollivet D, Tanguy A et al. (2009) Speciation in the deep sea: Multi-locus analysis of divergence and gene flow between two hybridizing species of hydrothermal vent mussels. PLoS ONE, 4, e6485.

Fevolden SE, Schneppenheim R (1989) Genetic homogeneity of Krill (Euphausia superba 
Dana) in the Southern-Ocean. Polar Biology, 9, 533-539.

Fisher CR, Hsing PY, Kaiser CL et al. (2014) Footprint of Deepwater Horizon blowout impact to deep-water coral communities. Proceedings of the National Academy of Sciences of the United States of America, 111, 11744-9.

Flot JF, Dahl M, André C (2013) Lophelia pertusa corals from the Ionian and Barents seas share identical nuclear ITS2 and near-identical mitochondrial genome sequences. BMC Research Notes, 6, 144.

Foltz DW, Fatland SD, Eléaume M et al. (2013) Global population divergence of the sea star Hippasteria phrygiana corresponds to the onset of the last glacial period of the Pleistocene. Marine Biology, 160, 1285-1296.

de Forges BR, Koslow J, Poore GC (2000) Diversity and endemism of the benthic seamount fauna in the southwest Pacific. Nature, 405, 944-947.

Fouzai N, Opdal AF, Jørgensen C et al. (2015) Effects of temperature and food availability on larval cod survival: a model for behaviour in vertical gradients. Marine Ecology Progress Series, 529, 199-212.

France SC, Hessler RR, Vrijenhoek RC (1992) Genetic differentiation between spatiallydisjunct populations of the deep-sea, hydrothermal vent-endemic amphipod Ventiella sulfuris. Marine Biology, 114, 551-559.

France SC, Hoover LL (2002) DNA sequences of the mitochondrial COI gene have low levels of divergence among deep-sea octocorals (Cnidaria: Anthozoa). Hidrobiologia, 471, 149-155.

France SC, Kocher TD (1996) Geographic and bathymetric patterns of mitochondrial $16 S$ rRNA sequence divergence among deep-sea amphipods, Eurythenes gryllus. Marine Biology, 126, 633-643.

Frederiksen R, Jensen A, Westerberg H (1992) The distribution of the scleractinian coral Lophelia pertusa around the Faroe Islands and the relation to internal tidal mixing. Sarsia, 77, 157-171.

Freiwald A, Fossa JH, Grehan A et al. (2004) Cold-water coral reefs. UNEP-WCMC, Cambridge, UK

Friess C, Sedberry GR (2011) Genetic evidence for a single stock of the deep-sea teleost Beryx decadactylus in the North Atlantic Ocean as inferred from mtDNA control region analysis. Journal of Fish Biology, 78, 466-478.

Gage JD (1996) Why are there so many species in deep-sea sediments? Journal of Experimental Marine Biology and Ecology, 200, 257-286. 
Gagneux P, Boesch C, Woodruff DS (1997) Microsatellite scoring errors associated with noninvasive genotyping based on nuclear DNA amplified from shed hair. Molecular Ecology, 6, 861-868.

Gardner JPA, Bell JJ, Constable HB, Hannan DA, Ritchie PA, Zuccarello GC. 2010. Multispecies coastal marine connectivity: a literature review with recommendations for further research. New Zealand Aquatic Environment \& Biodiversity Report, 58, 1-47. ISSN 1176-9440.

Gass SE, Roberts JM (2006) The occurrence of the cold-water coral Lophelia pertusa (Scleractinia) on oil and gas platforms in the North Sea: Colony growth, recruitment and environmental controls on distribution. Marine Pollution Bulletin, 52, 549-559.

Giles EC, Saenz-Agudelo P, Hussey NE, et al. (2015) Exploring seascape genetics and kinship in the reef sponge Stylissa carteri in the Red Sea. Ecology and evolution, 5, 2487-2502.

Gillooly JF, Brown JH, West GB (2001) Effects of size and temperature on metabolic rate. Science, 293, 2248-2252.

Glover AG, Smith CR (2003) The deep-sea floor ecosystem: current status and prospects of anthropogenic change by the year 2025. Environmental Conservation, 30, 219-241.

Le Goff MC, Rogers AD (2002) Characterization of 10 microsatellite loci for the deep-sea coral Lophelia pertusa (Linnaeus 1758). Molecular Ecology Notes, 2, 164-166.

Le Goff-Vitry MC, Rogers AD, Baglow D (2004) A deep-sea slant on the molecular phylogeny of the Scleractinia. Molecular Phylogenetics and Evolution, 30, 167-177.

González-Romero S, Reyes-Bonilla H, Cairns SD (2009) Range extensions of three species of the Family Caryophylliidae (Scleractinia) in the eastern Pacific Ocean. Biodiversity Records, 2, e108.

Gordeeva NV (2014) Phylogeography, genetic isolation, and migration of deep-sea fishes in the South Atlantic. Journal of Ichthyology, 54, 642-659.

Gotelli NJ, Colwell RK (2001) Quantifying biodiversity: Procedures and pitfalls in the measurement and comparison of species richness. Ecology Letters, 4, 379-391.

Graham M (2003) Confronting multicollinearity in ecological multiple regression. Ecology, 8, 2809-2815.

Gu Z, Gu L, Eils R et al. (2014) Circlize implements and enhances circular visualization in R. Bioinformatics, 30, 2811-2812.

Guardiola M, Frotscher J, Uriz MJ (2012) Genetic structure and differentiation at a short-time scale of the introduced calcarean sponge Paraleucilla magna to the western 
Mediterranean. Hydrobiologia, 687, 71-84.

Guinan J, Grehan AJ, Dolan MFJ et al. (2009) Quantifying relationships between video observations of cold-water coral cover and seafloor features in Rockall Trough, west of Ireland. Marine Ecology Progress Series, 375, 125-138.

Guinotte JM, Orr J, Cairns S et al. (2006) Will human-induced changes in seawater chemistry alter the distribution of deep-sea scleractinian corals? Frontiers in Ecology and the Environment, 4, 141-146.

Haig SM, Miller M, Bellinger R, et al. (2016). The conservation genetics juggling act: integrating genetics and ecology, science and policy. Evolutionary Applications, 9, 181195.

Hain S, Corcoran E (2004) The status of the cold-water coral reefs of the world. In: Wilkinson, C (Ed.) Status of Coral Reefs of the World: 2004, 1, 115-136.

Hadas E, Shpigel M, Ilan M (2009) Particulate organic matter as a food source for a coral reef sponge. Journal of Experimental Biology, 212, 3643-3650.

Hurst RJ, Renwick JA, Sutton PJH, et al. (2012) Climate and ocean trends of potential relevance to fisheries in the New Zealand region. New Zealand Aquatic Environment and Biodiversity Report No. 90.202p.

Havermans C, Sonet G, d'Udekem d'Acoz C et al. (2013) Genetic and morphological divergences in the cosmopolitan deep-sea amphipod Eurythenes gryllus reveal a diverse abyss and a bipolar species. PLOS ONE, 8, e74218.

Hedgecock D, Barbe PH, Edmands S (2007) Genetic approaches to measuring connectivity. Oceanography, 20, 70-79.

Hellberg ME, Burton RS, Neigel JE et al. (2002) Genetic assessment of connectivity among marine populations. Bulletin of marine science, 70, 273-290.

Helson J, Leslie S, Clement G et al. (2010) Private rights, public benefits: Industry-driven seabed protection. Marine Policy, 34, 557-566.

Henry LA, Roberts JM (2007) Biodiversity and ecological composition of macrobenthos on cold-water coral mounds and adjacent off-mound habitat in the bathyal Porcupine Seabight, NE Atlantic. Deep-Sea Research Part I: Oceanographic Research Papers, 54, 654-672.

Hensley RT, Beardmore J, Tyler P (1995) Genetic variance in Ophiomusium lymani (Ophiuroidea, Echinodermata) from lower bathyal depths in the Rockall Trough (Northeast Atlantic). Marine Biology, 121, 469-475.

Herrera S, Shank TM, Sánchez JA (2012) Spatial and temporal patterns of genetic variation 
in the widespread antitropical deep-sea coral Paragorgia arborea. Molecular Ecology, 21, 6053-6067.

Herrera S, Watanabe H, Shank TM (2015) Evolutionary and biogeographical patterns of barnacles from deep-sea hydrothermal vents. Molecular Ecology, 24, 673-689.

Helyar SJ, Hemmer-Hansen J, Bekkevold D et al. (2011) Application of SNPs for population genetics of nonmodel organisms: new opportunities and challenges. Molecular Ecology Resources, 11, 123-136.

Hilário A, Metaxas A, Gaudron SM et al. (2015) Estimating dispersal distance in the deep sea: challenges and applications to marine reserves. Frontiers in Marine Science, 2, 1-14.

Hoarau G, Borsa P (2000) Extensive gene flow within sibling species in the deep-sea fish Beryx splendens. Comptes Rendus de l'Academie des Sciences - Serie III, 323, 315-325.

Hohenlohe PA, Bassham S, Etter PD et al. (2010) Population genomics of parallel adaptation in threesppine stickleback using sequenced RAD tags. PLoS Genetics, 6, e1000862.

Holleley CE, Geerts PG (2009) Multiplex Manager 1.0: A cross-platform computer program that plans and optimizes multiplex PCR. BioTechniques, 46, 511-517.

Hourigan TF, Lumsden SE, Dorr G et al. (2007) State of deep coral ecosystems of the United States: Introduction and national overview. In: The State of Deep Coral Ecosystems of the United States. NOAA Technical Memorandum CRCP-3. Silver Spring MD, 1-64.

Howell KL, Rogers AD, Tyler PA et al. (2004) Reproductive isolation among morphotypes of the Atlantic seastar species Zoroaster fulgens (Asteroidea: Echinodermata). Marine Biology, 144, 977-984.

Hurtado LA, Lutz RA, Vrijenhoek RC (2004) Distinct patterns of genetic differentiation among annelids of eastern Pacific hydrothermal vents. Molecular Ecology, 13, 26032615.

Quesada H, Beynon CM, Skibinski DO (1995) A mitochondrial DNA discontinuity in the mussel Mytilus galloprovincialis Lmk: Pleistocene vicariance biogeography and secondary intergradation. Molecular Biology and Evolution, 12, 521-524.

Ishida Y (2009) Sewall Wright and Gustave Malécot on Isolation by Distance. Philosophy of Science, 76, 784-796.

Jackson AM, Munguía-Vega A, Beldade R et al. (2015) Incorporating historical and ecological genetic data for leopard grouper (Mycteroperca rosacea) into marine reserve design in the Gulf of California. Conservation Genetics, 16, 811-822.

Jensen JL, Bohonak AJ, Kelley ST (2005) Isolation by Distance, web service. BMC genetics, 6, 13. 
Jollivet D, Desbruyeres D, Ladrat C et al. (1995) Evidence for differences in the allozyme thermostability of deep-sea hydrothermal vent polychaetes (Alvinellidae): A possible selection by habitat. Marine Ecology Progress Series, 123, 125-136.

Jombart T (2008) Adegenet: A R package for the multivariate analysis of genetic markers. Bioinformatics, 24, 1403-1405.

Jombart T, Devillard S, Balloux F (2010) Discriminant analysis of principal components: a new method for the analysis of genetically structured populations. BMC Genetics, 11, 94.

Jorde PE, Søvik G, Westgaard JI et al. (2015) Genetically distinct populations of northern shrimp, Pandalus borealis, in the North Atlantic: Adaptation to different temperatures as an isolation factor. Molecular Ecology, 24, 1742-1757.

Jorquera-Paegelow E (2014) Genetic analyses on the deep-sea shrimp Acanthephyra pelagica in the North-West Atlantic. Dalhousie University.

Kaiser MJ (2011) Marine ecology: processes, systems, and impacts. Oxford University Press.

Kelly M, Buckeridge JS (2005) An early Paleogene sponge fauna, Chatham Island, New Zealand. New Zealand Journal of Marine and Freshwater Research, 39, 899-914.

Kelly M, Lee D, Kelly S et al.(2003) A recent sponge, Pleroma aotea Kelly (Order Lithistida: Family Pleromidae), in the late Eocene Ototara Limestone of Otago, New Zealand. New Zealand Journal of Marine and Freshwater Research, 37, 129-138.

Kelly M (2007) The marine fauna of New Zealand: Porifera: lithistid Demospongiae (Rock Sponges). NIWA Biodiversity Memoir, 121, 1-100.

Kiriakoulakis K, Fisher EL, Wolff GA et al. (2005) Lipids and nitrogen isotopes of two deepwater corals from the North-East Atlantic: initial results and implications for their nutrition. In: Cold-water Corals and Ecosystems, 715-729.

Knox MA, Hogg ID, Pilditch CA et al. (2012) Mitochondrial DNA (COI) analyses reveal that amphipod diversity is associated with environmental heterogeneity in deep-sea habitats. Molecular Ecology, 21, 4885-4897.

Knudby A, Kenchington E, Murillo FJ (2013) Modeling the distribution of Geodia sponges and sponge grounds in the Northwest Atlantic. PLoS ONE, 8, e82306.

Kojima S, Ohta S, Yamamoto T et al. (2003) Molecular taxonomy of vestimentiferans of the western Pacific, and their phylogenetic relationship to species of the eastern Pacific III. Alaysia-like vestimentiferans and relationships among families. Marine Biology, 142, 625-635.

Kojima S, Watanabe H, Tsuchida S et al. (2006) Phylogenetic relationships of a tube worm (Lamellibrachia juni) from three hydrothermal vent fields in the South Pacific. Journal 
of the Marine Biological Association of the UK, 86, 1357.

Kudrass HR, Rad U Von (1984) Underwater television and photography observations, sidescan sonar and acoustic reflectivity measurements of phosphorite-rich areas on the Chatham Rise (New Zealand). Geology Journal, 65, 69-89.

Kumar S, Banks TW, Cloutier S (2012) SNP discovery through next-generation sequencing and its applications. International Journal of Plant Genomics, 2012, 1-15.

Kwok S, Kellogg DE, McKinney N et al. (1990) Effects of primer-template mismatches on the polymerase chain reaction: human immunodeficiency virus type 1 model studies. Nucleic acids research, 18, 999-1005.

Lavrov DV, Forget L, Kelly M et al. (2005) Mitochondrial genomes of two demosponges provide insights into an early stage of animal evolution. Molecular Biology and Evolution, 22, 1231-1239.

Leclercq N, Gattuso JP, Jaubert J (2000) CO2 partial pressure controls the calcification rate of a coral community. Global Change Biology, 6, 329-334.

Lecroq B, Gooday AJ, Pawlowski J (2009) Global genetic homogeneity in the deep-sea foraminiferan Epistominella exigua (Rotaliida: Pseudoparrellidae). Zootaxa, 32, 23-32.

Leduc D, Rowden AA, Bowden DA et al. (2012) Unimodal relationship between biomass and species richness of deep-sea nematodes: Implications for the link between productivity and diversity. Marine Ecology Progress Series, 454, 53-64.

Lemoine N, Buell N, Hill A et al. (2007) Porifera research: biodiversity, innovation and sustainability. Museu Nacional.

Levin LA, Baco AR, Bowden DA et al. (2016) Hydrothermal vents and methane seeps: Rethinking the sphere of influence. Frontiers in Marine Science, 3, 72.

Lévy-Hartmann L, Roussel V, Letourneur Y et al. (2011) Global and New Caledonian patterns of population genetic variation in the deep-sea splendid alfonsino, Beryx splendens, inferred from mtDNA. Genetica, 139, 1349-1365.

Li H, Durbin R (2009) Fast and accurate short read alignment with Burrows-Wheeler transform. Bioinformatics, 25, 1754-1760.

Li H, Handsaker B, Wysoker A et al. (2009) The sequence alignment/map format and SAMtools. Bioinformatics, 25, 2078-2079.

Lin MF, Kitahara MV, Tachikawa $\mathrm{H}$ et al. (2012) Novel organization of the mitochondrial genome in the deep-sea coral, Madrepora oculata (Hexacorallia, Scleractinia, Oculinidae) and its taxonomic implications. Molecular phylogenetics and evolution, $\mathbf{6 5}$, 323-328. 
Liu L, Li Y, Li S et al. (2012) Comparison of next-generation sequencing systems. Journal of Biomedicine and Biotechnology, 2012, 251311-251364.

Longmore C, Trueman CN, Neat F et al. (2014) Ocean-scale connectivity and life cycle reconstruction in a deep-sea fish. Canadian Journal of Fisheries and Aquaculture Science, 12, 1-12.

López-Legentil S, Pawlik JR (2009) Genetic structure of the Caribbean giant barrel sponge Xestospongia muta using the I3-M11 partition of COI. Coral Reefs, 28, 157-165.

Lotterhos KE, Dick SJ, Haggarty DR (2014) Evaluation of rockfish conservation area networks in the United States and Canada relative to the dispersal distance for black rockfish (Sebastes melanops). Evolutionary Applications, 7, 238-259.

Lowe WH, Allendorf FW (2010) What can genetics tell us about population connectivity? Molecular Ecology, 19, 3038-3051.

Lunden JJ, McNicholl CG, Sears CR et al. (2014) Acute survivorship of the deep-sea coral Lophelia pertusa from the Gulf of Mexico under acidification, warming, and deoxygenation. Frontiers in Marine Science, 1, 78.

Mackay KA, Mitchell JS, Neil HL et al. (2015) New Zealand's marine realm. NIWA chart, Miscellaneous Series, No.94.

Maggio T, Lo Brutto S, Cannas R et al. (2009) Environmental features of deep-sea habitats linked to the genetic population structure of a crustacean species in the Mediterranean Sea. Marine Ecology, 30, 354-365.

Maldonado M, Bergquist PL (2002) Phylum Porifera. In: Young CM (Ed.), Atlas of marine invertebrate larvae. Academic Press, San Diego, 21-50.

Mantel N (1967) The detection of disease clustering and a generalized regression approach. Cancer Research, 27, 209-220.

Manel S, Schwartz MK, Luikart G et al. (2003) Landscape genetics: Combining landscape ecology and population genetics. Trends in Ecology and Evolution, 18, 189-197.

Manni F, Guerard E, Heyer E (2004) Geographic patterns of (genetic, morphologic, linguistic) variation: How barriers can be detected by using Monmonier's algorithm. Human Biology, 76, 173-190.

Marshall TC, Slate J, Kruuk LEB et al. (1998) Statistical confidence for likelihood-based paternity inference in natural populations. Molecular Ecology, 7, 639-655.

Mariani S, Uriz MJ, Turon X et al. (2006) Dispersal strategies in sponge larvae: integrating the life history of larvae and the hydrologic component. Oecologia, 149, 174-184.

Marubini F, Atkinson MJ (1999) Effects of lowered pH and elevated nitrate on coral 
calcification. Marine Ecology Progress Series, 188, 117-121.

Matabos M, Thiébaut E, Le Guen D et al. (2008) Geographic clines and stepping-stone patterns detected along the East Pacific Rise in the vetigastropod Lepetodrilus elevatus reflect species crypticism. Marine Biology, 153, 545-563.

McClain CR, Hardy SM (2010) The dynamics of biogeographic ranges in the deep sea. Proceedings of the Royal Society B: Biological Sciences, 277, 3533-3546.

McCook LJ, Almany GR, Berumen ML, et al. (2009). Management under uncertainty: guidelines for incorporating connectivity into the protection of coral reefs. Coral Reefs, $\mathbf{2 8}$, 353-366.

McCulloch M, Trotter J, Montagna P et al. (2012) Resilience of cold-water scleractinian corals to ocean acidification: Boron isotopic systematics of $\mathrm{pH}$ and saturation state upregulation. Geochimica et Cosmochimica Acta, 87, 21-34.

McMullin ER, Hourdez S, Schaeffer SW et al. (2003) Phylogeny and biogeography of deep sea vestimentiferan tubworms and their bacterial symbionts. Symbiosis, 34, 1-41.

Meer MH, Berumen ML, Hobbs JPA et al. (2014) Population connectivity and the effectiveness of marine protected areas to protect vulnerable, exploited and endemic coral reef fishes at an endemic hotspot. Coral Reefs, 34, 393-402.

Miller KJ, Rowden AA, Williams A et al. (2011) Out of their depth? Isolated deep populations of the cosmopolitan coral Desmophyllum dianthus may be highly vulnerable to environmental change. PLOS ONE, 6, e19004.

Miller KJ, Williams A, Rowden AA et al. (2010) Conflicting estimates of connectivity among deep-sea coral populations. Marine Ecology, 31, 144-157.

Montoya-Maya PH, Macdonald AHH, Schleyer MH (2014) Cross-amplification and characterization of microsatellite loci in Acropora austera from the south-western Indian Ocean. Genetics and Molecular Research, 13, 1244-1250.

Morin PA, McCarthy M (2007) Highly accurate SNP genotyping fromhistorical and lowquality samples. Molecular Ecology Notes, 7, 937-946.

Morin PA, Luikart G, Wayne RK et al. (2004) SNPs inecology, evolution and conservation. Trends in Ecology and Evolution, 19, 208-216.

Morrison CL, Eackles MS, Johnson RL et al. (2008) Characterization of 13 microsatellite loci for the deep-sea coral, Lophelia pertusa (Linnaeus 1758), from the western North Atlantic Ocean and Gulf of Mexico. Molecular Ecology Resources, 8, 1037-1039.

Murillo FJ, Muñoz PD, Cristobo J et al. (2012) Deep-sea sponge grounds of the Flemish Cap, Flemish Pass and the Grand Banks of Newfoundland (Northwest Atlantic Ocean): 
Distribution and species composition. Marine Biology Research, 8, 842-854.

Nakajima Y, Nishikawa A, Isomura N et al. (2009). Genetic connectivity in the broadcastspawning coral Acropora digitifera analyzed by microsatellite markers on the Sekisei Reef, Southwestern Japan. Zoological science, 26, 209-215.

Neal L, Wiklund H, Muir AI et al. (2014) The identity of juvenile Polynoidae (Annelida) in the Southern Ocean revealed by DNA taxonomy, with notes on the status of Herdmanella gracilis Ehlers sensu Augener. Memoirs of Museum Victoria, 71, 203-216.

NEAFC (2014) Recommendation on the protection of vulnerable marine ecosystems in the NEAFC Regulatory Area. Recommendation 2014/19.

O'Hara TD, England PR, Gunasekera RM et al. (2014) Limited phylogeographic structure for five bathyal ophiuroids at continental scales. Deep-Sea Research Part I: Oceanographic Research Papers, 84, 18-28.

O'Hara TD, Rowden AA, Williams A (2008) Cold-water coral habitats on seamounts: Do they have a specialist fauna? Diversity and Distributions, 14, 925-934.

Oldham P, Hall S, Barnes C et al. (2014) Valuing the Deep : Marine Genetic Resources in Areas Beyond National Jurisdiction. Defra Contract MB0128 Final Report.

O'Leary BC, Brown RL, Johnson DE, et al. (2012) The first network of marine protected areas (MPAs) in the high seas: the process, the challenges and where next. Marine Policy, 36, 598-605.

Oliver TA, Garfield DA, Manier MK et al. (2010) Whole-genome positive selection and habitat-driven evolution in a shallow and a deep-sea urchin. Genome Biology and Evolution, 2, 800-814.

Pritchard JK, Stephens M, Donnelly P (2000) Inference of population structure using multilocus genotype data. Genetics, 155, 945-59.

van Oosterhout C, Hutchinson WF, Wills DPM et al. (2004) MICRO-CHECKER: Software for identifying and correcting genotyping errors in microsatellite data. Molecular Ecology Notes, 4, 535-538.

van Oppen MJH, Underwood JN, Muirhead AN et al.(2007) Ten microsatellite loci for the reef-building coral Acropora millepora (Cnidaria, Scleractinia) from the Great Barrier Reef, Australia: Primer note. Molecular Ecology Notes, 7, 436-438.

Palumbi SR (2003) Population genetics, demographic connectivity, and the design of marine reserves. Ecological Applications, 13, 146-158.

Paradis E (2010) Pegas: An R package for population genetics with an integrated-modular approach. Bioinformatics, 26, 419-420. 
Parker SJ, Bowden DA (2010) Identifying taxonomic groups vulnerable to bottom longline fishing gear in the Ross Sea region. CCAMLR Science, 17, 105-127.

Parker SJ, Penney AJ, Clark MR (2009) Detection criteria for managing trawl impacts on vulnerable marine ecosystems in high seas fisheries of the South Pacific Ocean. Marine Ecology Progress Series, 397, 309-317.

Pawlowski J, Bowser SS, Gooday AJ (2007a) A note on the genetic similarity between shallow- and deep-water Epistominella vitrea (Foraminifera) in the Antarctic. Deep-Sea Research Part II: Topical Studies in Oceanography, 54, 1720-1726.

Pawlowski J, Fahrni J, Lecroq B et al. (2007b) Bipolar gene flow in deep-sea benthic foraminifera. Molecular Ecology, 16, 4089-4096.

Pawson D, Alvariño A, Hosmer SC et al. (1983) Biology of the Antarctic Seas XI (Kornicker LS, Ed.). American Geophysical Union, Washington DC.

Peakall R, Smouse PE (2012) GenALEx 6.5: Genetic analysis in Excel. Population genetic software for teaching and research-an update. Bioinformatics, 28, 2537-2539.

Pechenik JA, Estrella MS, Hammer K (1996) Food limitation stimulates metamorphosis of competent larvae and alters postmetamorphic growth rate in the marine prosobranch gastropod Crepidula fornicata. Marine Biology, 127, 267-275.

Penney AJ (2014) Review of the biodiversity component of the New Zealand Vulnerable Marine Ecosystem Evidence Process. New Zealand Aquatic Environment and Biodiversity Report, 135.

Penney AJ, Guinotte JM (2013) Evaluation of New Zealand's high-seas bottom trawl closures using predictive habitat models and quantitative risk assessment. PLoS ONE, $\mathbf{8}$, e82273.

Petersen JM, Ramette A, Lott C et al. (2010) Dual symbiosis of the vent shrimp Rimicaris exoculata with filamentous gamma- and epsilonproteobacteria at four Mid-Atlantic Ridge hydrothermal vent fields. Environmental Microbiology, 12, 2204-2218.

Pibernat RA, Ellis-Evans C, Hinghofer-Szalkay HG (Eds.) (2007) Life in extreme environments. Springer Science and Business Media.

Pires DO, Silva JC, Bastos ND (2014) Reproduction of deep-sea reef-building corals from the southwestern Atlantic. Deep-Sea Research Part II: Topical Studies in Oceanography, 99, 51-63.

Plouviez S, Faure B, Le Guen D et al. (2013) A new barrier to dispersal trapped old genetic clines that escaped the Easter Microplate tension zone of the Pacific vent mussels. PLoS ONE, 8, e81555. 
Plouviez S, Le Guen D, Lecompte O et al. (2010) Determining gene flow and the influence of selection across the equatorial barrier of the East Pacific Rise in the tube-dwelling polychaete Alvinella pompejana. BMC Evolutionary Biology, 10, 220.

Plouviez S, Shank TM, Faure B et al. (2009) Comparative phylogeography among hydrothermal vent species along the East Pacific Rise reveals vicariant processes and population expansion in the South. Molecular Ecology, 18, 3903-3917.

Portnoy DS, Hollenbeck CM, Belcher CN et al. (2014) Contemporary population structure and post-glacial genetic demography in a migratory marine species, the blacknose shark, Carcharhinus acronotus. Molecular ecology, 23, 5480-95.

Probert PK, McKnight DG, Grove SL (1997) Benthic invertebrate bycatch from a deep-water trawl fishery, Chatham Rise, New Zealand. Aquatic Conservation: Marine and Freshwater Ecosystems, 7, 27-40.

Puechmaille SJ (2016) The program structure does not reliably recover the correct population structure when sampling is uneven: subsampling and new estimators alleviate the problem. Molecular Ecology Resources, 16, 608-27.

Putman AI, Carbone I (2014) Challenges in analysis and interpretation of microsatellite data for population genetic studies. Ecology and Evolution, 4, 4399-4428.

Qian PY (1994) Effect of food quantity on growth and reproductive characteristics of Capitella sp.(Annelids: Polychaeta). Invertebrate Reproduction and Development, 26, 175-185.

Quattrini AM (2014) Genetic connectivity of octocorallia across abiotic gradients in the deep Gulf of Mexico. Temple University.

Quattro J, Chase M, Rex M et al. (2001) Extreme mitochondrial DNA divergence within populations of the deep-sea gastropod Frigidoalvania brychia. Marine Biology, 139, 1107-1113.

Ramirez-Llodra E, Brandt A, Danovaro R et al. (2010) Deep, diverse and definitely different: Unique attributes of the world's largest ecosystem. Biogeosciences, 7, 2851-2899.

Ramirez-Llodra E, Tyler PA, Baker MC et al. (2011) Man and the last great wilderness: Human impact on the deep sea. PLoS ONE, 6, e22588.

Raupach MJ, Thatje S, Dambach J et al. (2010) Genetic homogeneity and circum-Antarctic distribution of two benthic shrimp species of the Southern Ocean, Chorismus antarcticus and Nematocarcinus lanceopes. Marine Biology, 157, 1783-1797.

Reisser CMO, Bell JJ, Gardner JPA (2014) Correlation between pelagic larval duration and realised dispersal: Long-distance genetic connectivity between northern New Zealand 
and the Kermadec Islands archipelago. Marine Biology, 161, 297-312.

Riehl T, Kaiser S (2012) Conquered from the Deep Sea? A new deep-sea isopod species from the Antarctic shelf shows pattern of recent colonization. PLoS ONE, 7, 36-38.

Roberts JM, Davies AJ, Henry LA et al. (2009) Mingulay reef complex: An interdisciplinary study of cold-water coral habitat, hydrography and biodiversity. Marine Ecology Progress Series, 397, 139-151.

Roberts JM, Wheeler AJ, Freiwald A (2006) Reefs of the deep: The biology and geology of cold-water coral ecosystems. Science, 312, 543-547.

Rogers AD (1999) The biology of Lophelia pertusa (Linnaeus 1758) and other deep-water reef-forming corals and impacts from human activities. International Review of Hydrobiology, 84, 315-406.

Rogers A, Blanchard JL, Mumby PJ (2014) Vulnerability of coral reef fisheries to a loss of structural complexity. Current Biology, 24, 1000-1005.

Rogers AD, Clarke A, Peck LS (1998) Population genetics of the Antarctic hetronemertean Parbolasia corrugatus from the South Orkney Islands. Marine Biology, 131, 1-13.

Rooper CN, Zimmermann M, Prescott MM et al. (2014) Predictive models of coral and sponge distribution, abundance and diversity in bottom trawl surveys of the Aleutian Islands, Alaska. Marine Ecology Progress Series, 503, 157-176.

Roques S, Sévigny JM, Bernatchez L (2002) Genetic structure of deep-water redfish, Sebastes mentella, populations across the North Atlantic. Marine Biology, 140, 297-307.

Roterman CN, Copley JT, Linse KT et al. (2016) Connectivity in the cold: The comparative population genetics of vent-endemic fauna in the Scotia Sea, Southern Ocean. Molecular Ecology, 25, 1073-1088.

Ruiz-Ramos DV., Saunders M, Fisher CR et al. (2015) Home bodies and wanderers: Sympatric lineages of the deep-sea black coral Leiopathes glaberrima. PLoS ONE, 10.

Sá-Pinto A, Branco MS, Alexandrino PB et al. (2012) Barriers to gene flow in the marine environment: insights from two common intertidal limpet species of the Atlantic and Mediterranean. PLoS ONE, 7, e50330.

Sacco F, Marcias S, Deiana AM et al. (2011) Population genetics of the red and blue shrimp Aristeus antennatus in the western Mediterranean Sea. Biologia Marina Mediterranea, 18, 364-365.

Samadi S, Bottan L, Macpherson E et al. (2006) Seamount endemism questioned by the geographic distribution and population genetic structure of marine invertebrates. Marine Biology, 149, 1463-1475. 
Schuelke M (2000) An economic method for the fluorescent labeling of PCR fragments. Nature Biotechnology, 18, 233-234.

Schüller M (2011) Evidence for a role of bathymetry and emergence in speciation in the genus Glycera (Glyceridae, Polychaeta) from the deep Eastern Weddell Sea. Polar Biology, 34, 549-564.

Sedberry GR, Carlin J (1996) Population structure in the panoceanic wreckfish, Polyprion americanus (Teleostei: Polyprionidae), as indicated by mtDNA variation. Journal of Fish Biology, 49, 318-329.

Seibel BA, Drazen JC (2007) The rate of metabolism in marine animals: environmental constraints, ecological demands and energetic opportunities. Philosophical transactions of the Royal Society of London. Series B, Biological Sciences, 362, 2061-78.

Selkoe KA, Aloia CC, Crandall ED, et al. (2016) A decade of seascape genetics: Contributions to basic and applied marine connectivity. Marine Ecology Progress Series, 554, 1-19.

Selkoe KA, Gaggiotti OE, Bowen BW et al. (2014) Emergent patterns of population genetic structure for a coral reef community. Molecular Ecology, 23, 3064-3079.

Selkoe KA, Henzler CM, Gaines SD (2008) Seascape genetics and the spatial ecology of marine populations. Fish and Fisheries, 9, 363-377.

Shank T (2010) Seamounts: Deep-ocean laboratories of faunal connectivity, evolution, and endemism. Oceanography, 23, 108-122.

Shank TM, Halanych KM (2007) Toward a mechanistic understanding of larval dispersal: Insights from genomic fingerprinting of the deep-sea hydrothermal vent tubeworm Riftia pachyptila. Marine Ecology, 28, 25-35.

Sharma R, Goossens B, Kun-Rodrigues C et al. (2012) Two different high throughput sequencing approaches identify thousands of de novo genomic markers for the genetically depleted bornean elephant. PLoS ONE, 7, e49533.

Shaw PW, Arkhipkin AI, Al-Khairulla H (2004) Genetic structuring of Patagonian toothfish populations in the Southwest Atlantic Ocean: The effect of the Antarctic Polar Front and deep-water troughs as barriers to genetic exchange. Molecular Ecology, 13, 3293-3303.

Shearer TL, Oppen MJH Van, Romano SL et al. (2002) Slow mitochondria DNA sequence evolution in the Anthozoa. Molecular ecology, 11, 2475-2487.

Shinzato C, Shoguchi E, Kawashima T et al. (2011) Using the Acropora digitifera genome to understand coral responses to environmental change. Nature, 476, 320-323.

Shinzato C, Yasuoka Y, Mungpakdee S et al. (2014) Development of novel, cross-species 
microsatellite markers for Acropora corals using next-generation sequencing technology. Frontiers in Marine Science, 1, 11.

Shmida A, Wilson M V. (1985) Biological determinants of species diversity. Journal of Biogeography, 12, 1-20.

Sibuet M, Olu K (1998) Biogeography, biodiversity and fluid dependence of deep-sea coldseep communities at active and passive margins. Deep-Sea Research Part II: Topical Studies in Oceanography, 45, 517-567.

Siebenaller JF (1978) Genetic variation in deep-sea invertebrate populations: The bathyal gastropod Bathybembix bairdii. Marine Biology, 47, 265-275.

Silva CNS, Gardner JPA (201) Identifying environmental factors associated with the genetic structure of the New Zealand scallop: linking seascape genetics and ecophysiological tolerance. ICES Journal of Marine Science. 73, 1924-1934.

Silverman J, Lazar B, Erez J (2007) Effect of aragonite saturation, temperature, and nutrients on the community calcification rate of a coral reef. Journal of Geophysical Research: Oceans, 112, C5.

Slatkin M, Hudson RR (1991) Pairwise comparison of mitochondrial DNA sequences in stable and exponetially growing populations. Genetics, 129, 555-562.

Smith M, Pascal C, Grauvogel Z et al. (2011) Multiplex preamplification PCR and microsatellite validation allows accurate single nucleotide polymorphism (SNP) genotyping of historical fish scales. Molecular Ecology Resources, 11, 257-266.

Smith PJ, McVeagh SM, Ede A (1996) Genetically isolated stocks of orange-roughy (Hoplostethus atlanticus), but not of Hoki (Macruronus novaezelandiae), in the Tasman Sea and Southwest Pacific Ocean around New Zealand. Marine Biology, 125, 783-793.

Smith PJ, McVeagh SM, Mingoia JT et al. (2004a) Mitochondrial DNA sequence variation in deep-sea bamboo coral (Keratoisidinae) species in the southwest and northwest Pacific Ocean. Marine Biology, 144, 253-261.

Smith PJ, McVeagh SM, Won Y et al. (2004b) Genetic heterogeneity among New Zealand species of hydrothermal vent mussels (Mylilidae: Bathymodiolus). Marine Biology, 144, 537-545.

Snelder TH, Leathwick JR, Dey KL et al. (2007) Development of an ecological marine classification in the New Zealand region. Environmental Management, 39, 12-29.

van Soest R, Boury-Esnault N, Janussen D et al. (2005) World Porifera database. Available at: http://www.vliz.be/vmdcdata/porifera.

Sutton PJH, Chereskin TK (2002) Absolute geostrophic currents in the East Auckland 
Current region. New Zealand Journal of Marine and Freshwater Research, 36, 751-762.

Tang PC, Wei NV, Chen CW et al. (2010). Comparative study of genetic variability of AAT and CT/GT microsatellites in staghorn coral, Acropora (Scleractinia: Acroporidae). Zoological Studies, 49, 657-668.

Tóth G, Gáspári Z, Jurka J (2000) Microsatellites in different eukaryotic genomes: Surveys and analysis. Genome Research, 10, 967-981.

Teixeira S, Serrão EA, Arnaud-Haond S (2012) Panmixia in a fragmented and unstable environment: The hydrothermal shrimp Rimicaris exoculata disperses extensively along the Mid-Atlantic ridge. PLoS ONE, 7, e38521.

Thiagarajan N, Gerlach D, Roberts ML et al. (2013) Movement of deep-sea coral populations on climatic timescales. Paleoceanography, 28, 227-236.

Thorson G (1950) Reproductive and larval ecology of marine bottom invertebrates. Biological Reviews, 25, 1-45.

Tittensor DP, Baco AR, Brewin PE et al. (2009) Predicting global habitat suitability for stony corals on seamounts. Journal of Biogeography, 36, 1111-1128.

Tracey DM, Anderson OF, Oliver MD (2007) A guide to common deepsea invertebrates in New Zealand waters. New Zealand Aquatic Environment and Biodiversity Report, 1.

Tracey DM, Rowden AA, Mackay KA et al. (2011) Habitat-forming cold-water corals show affinity for seamounts in the New Zealand region. Marine Ecology Progress Series, 430, $1-22$.

Truelove NK, Griffiths S, Ley-Cooper K et al. (2015) Genetic evidence from the spiny lobster fishery supports international cooperation among Central American marine protected areas. Conservation Genetics, 16, 347-358.

Turley CM, Roberts JM, Guinotte JM (2007) Corals in deep-water: Will the unseen hand of ocean acidification destroy cold-water ecosystems? Coral Reefs, 26, 445-448.

Underwood JN, Souter PB, Ballment ER et al. (2006) Development of 10 polymorphic microsatellite markers from herbicide-bleached tissues of the brooding pocilloporid coral Seriatopora hystrix. Molecular Ecology Notes, 6, 176-178.

Varela AI, Ritchie PA, Smith PJ (2012) Low levels of global genetic differentiation and population expansion in the deep-sea teleost Hoplostethus atlanticus revealed by mitochondrial DNA sequences. Marine Biology, 159, 1049-1060.

Varela AI, Ritchie PA, Smith PJ (2013) Global genetic population structure in the commercially exploited deep-sea teleost orange roughy (Hoplostethus atlanticus) based on microsatellite DNA analyses. Fisheries Research, 140, 83-90. 
Viard F, Ellien C, Dupont L (2006) Dispersal ability and invasion success of Crepidula fornicata in a single gulf: insights from genetic markers and larval-dispersal model. Helgoland Marine Research, 60, 144.

Vinogradova NG (1997) Zoogeography of the abyssal and hadal zones. Advances in Marine Biology, 32, 325-387.

Vrijenhoek RC (2010) Genetic diversity and connectivity of deep-sea hydrothermal vent metapopulations. Molecular Ecology, 19, 4391-4411.

Waller RG (2005) Deep-water Scleractinia (Cnidaria: Anthozoa): current knowledge of reproductive processes. Cold-water Corals and Ecosystems, 691-700.

Waller RG, Tyler PA (2005) The reproductive biology of two deep-water, reef-building scleractinians from the NE Atlantic Ocean. Coral Reefs, 24, 514-522.

Waller RG, Tyler P, Gage JD (2002) Reproductive ecology of the deep-sea scleractinian coral Fungiacyathus marenzelleri (Vaughan, 1906) in the northeast Atlantic Ocean. Coral Reefs, 21, 325-331.

Waller RG, Tyler PA, Gage JD (2005) Sexual reproduction in three hermaphroditic deep-sea Caryophyllia species (Anthozoa: Scleractinia) from the NE Atlantic Ocean. Coral Reefs, 24, 594-602.

Wandeler P, Hoeck PE, Keller LF (2007) Back to the future: museum specimens in population genetics. Trends in Ecology and Evolution, 22, 634-642.

Wang S, Zhang L, Matz M (2009). Microsatellite characterization and marker development from public EST and WGS databases in the reef-building coral Acropora millepora (Cnidaria, Anthozoa, Scleractinia). Journal of Heredity,100, 329-337.

Waples RS, Punt AE, Cope JM (2008) Integrating genetic data into management of marine resources: how can we do it better? Fish and Fisheries, 9, 423-449.

Watling L, Guinotte J, Clark MR et al. (2013) A proposed biogeography of the deep ocean floor. Progress in Oceanography, 111, 91-112.

Wattier R, Engel CR, Saumitou-Laprade P et al. (1998) Short allele dominance as a source of heterozygote deficiency at microsatellite loci: Experimental evidence at the dinucleotide locus Gv1CT in Gracilaria gracilis (Rhodophyta). Molecular Ecology, 7, 1569-1573.

Wei K, Wood AR, Gardner JPA (2013) Seascape genetics of the New Zealand greenshell mussel: Sea surface temperature explains macrogeographic scale genetic variation. Marine Ecology Progress Series, 477, 107-121.

Whalan S, de Nys R, Smith-Keune C et al. (2008a) Low genetic variability within and among populations of the brooding sponge Rhopaloeides odorabile on the central Great Barrier. 
Reef Aquatic Biology, 3, 111-119.

Whalan S, Ettinger-Epstein P, de Nys R (2008b) The effect of temperature on larval presettlement duration and metamorphosis for the sponge, Rhopaloeides odorabile. Coral Reefs, 27, 783-786.

Whalan S, Johnson MS, Harvey E et al.(2005) Mode of reproduction, recruitment, and genetic subdivision in the brooding sponge Haliclona sp.. Marine Biology, 146, 425-433.

White M, Mohn C (2004) Seamounts: a review of physical processes and their influence on the seamount ecosystem. OASIS Report 37.

Wilson JR, Harrison PL (1998) Settlement-competency periods of larvae of three species of scleractinian corals. Marine Biology, 131, 339-345.

Wilson NG, Hunter RL, Lockhart SJ et al. (2007) Multiple lineages and absence of panmixia in the "circumpolar" crinoid Promachocrinus kerguelensis from the Atlantic sector of Antarctica. Marine Biology, 152, 895-904.

Winkelmann I, Campos PF, Strugnell J et al. (2013) Mitochondrial genome diversity and population structure of the giant squid Architeuthis: genetics sheds new light on one of the most enigmatic marine species. Proceedings of the Royal Society of London B: Biological Sciences, 280, 20130273.

Witte U (1996) Seasonal reproduction in deep-sea sponges-triggered by vertical particle flux? Marine Biology, 124, 571-581.

Won Y, Young CR, Lutz RA et al. (2003) Dispersal barriers and isolation among deep-sea mussel populations (Mytilidae: Bathymodiolus) from eastern Pacific hydrothermal vents. Molecular Ecology, 12, 169-184.

Woodby D, Carlile D, Hulbert L (2009) Predictive modeling of coral distribution in the central Aleutian Islands, USA. Marine Ecology Progress Series, 397, 227-240.

Woolley SNC, Tittensor DP, Dunstan PK et al. (2016) Deep-sea diversity patterns are shaped by energy availability. Nature, 533, 1-17.

Wörheide G (2006) Low variation in partial cytochrome oxidase subunit I (COI) mitochondrial sequences in the coralline demosponge Astrosclera willeyana across the Indo-Pacific. Marine Biology, 148, 907-912.

Xavier JR, Soest RWM, Breeuwer JA et al. (2010) Phylogeography, genetic diversity and structure of the poecilosclerid sponge Phorbas fictitius at oceanic islands. Contributions to Zoology, 79, 119-129.

Yabe H, Eguchi M (1936) Deep-water corals from off Owasi, Mie Prefecture. Proceedings of the Imperial Academy, 12, 167-168. 
Yang CH, Tsuchida S, Fujikura K et al. (2016) Connectivity of the squat lobsters Shinkaia crosnieri (Crustacea: Decapoda: Galatheidae) between cold seep and hydrothermal vent habitats. Bulletin of Marine Science, 92, 17-31.

Young AG (2000) Genetics, demography and viability of fragmented populations. Cambridge University Press.

Young CM (2003) Reproduction, development and life-history traits. Ecosystems of the the Deep Oceans, 381-426.

Zane L, Ostellari L, Maccatrozzo L et al. (1998) Molecular evidence for genetic subdivision of Antarctic krill (Euphausia superba Dana) populations. Proceedings of the Royal Society of London B. Biological Sciences, 265, 2387-2391.

Zawada DG, Piniak GA, Hearn JC (2010) Topographic complexity and roughness of a tropical benthic seascape. Geophysical Research Letters, 37, L14604.

Zeng C, Kelly M, Gardner JPA (2016) Development and characterization of ten highly polymorphic microsatellite markers for the demosponge Poecillastra laminaris (Sollas). Marine Biodiversity, 1-3.

Zeng C, Thomas LJ, Kelly M et al. (2014a) The complete mitochondrial genome of the deepsea sponge Poecillastra laminaris (Astrophorida, Vulcanellidae). Mitochondrial DNA, 27, 1658-1659.

Zeng C, Tracey DM, Clark MR et al. (2014b) The complete mitochondrial genome of the deep-sea stony coral Solenosmilia variabilis (Scleractinia, Caryophylliidae) and its interindividual variation. Mitochondrial DNA, 27, 1959-1960.

Zenk W, Siedler G, Ishida A et al. (2005) Pathways and variability of the Antarctic Intermediate Water in the western equatorial Pacific Ocean. Progress in Oceanography, 67, 245-281.

Zezina ON (1997) Biogeography of the bathyal zone. Advances in Marine Biology, 32, 389426.

Zilberberg C, Peluso L, Marques JA et al. (2014) Polymorphic microsatellite loci for endemic Mussismilia corals (Anthozoa: Scleractinia) of the southwest Atlantic Ocean. Journal of Heredity, esu023. 